\title{
CLIMATOLOGIC, SOIL-WATER, GROUND-WATER, GEOLOGIC, SURFACE-WATER, AND WATER-QUALITY DATA FOR A SURFACE COAL MINE IN NORTHWESTERN COLORADO
}

by Robert S. Williams, Jr., Gregory M. Clark, and Norman E. Spahr

U.S. GEOLOGICAL SURVEY

Open-File Report 92-122

Prepared in cooperation with the

U.S. BUREAU OF LAND MANAGEMENT and the

COLORADO DEPARTMENT OF NATURAL RESOURCES,

MINED LAND RECLAMATION DIVISION

Denver, Colorado

1993 


\section{U.S. DEPARTMENT OF THE INTERIOR BRUCE BABBITT, Secretary \\ U.S. GEOLOGICAL SURVEY \\ Dallas L. Peck, Director}

For additional information write to:

District Chief U.S. Geological Survey Box 25046, Mail Stop 415 Federal Center Denver, CO 80225-0046
Copies of this report can be purchased from:

U.S. Geological Survey Books and Open-File Reports Section Federal Center

Box 25425

Denver, CO 80225 


\section{FIGURES}

Figure 1. Map showing location of the study area-1

2. Map showing study area and data-collection sites-_...... 4

3. Diagram showing general lithologic log for a typical well in the study area-... 6

4. Schematic diagram showing hydrogeology of reclaimed spoil and bedrock at the study area-1 7

5. Map showing data-collection points and instrumentation at the Spring Creek and Zuli sites-a 8

6. Map showing data-collection points and instrumentation at the Cow Camp Creek site-1 9

7. Map showing data-collection points and instrumentation at the Bond Creek site-10 10

8. Schematic diagram of a lysimeter and a soil-water access tube in the reclaimed spoil 11

9. Diagram showing typical bedrock well completion-_... 12

10-13. Diagrams showing geophysical and lithologic logs for:

10. Well SSD487 at the Spring Creek site-_. 13

11. Well SCU287 at the Cow Camp Creek site-.......... 14

12. Well SZU287 at the Zuli site-_. 15

13. Well SBU287 at the Bond Creek site-_. 16

14-17. Hydrographs showing hydraulic head for bedrock wells at the:

14. Spring Creek site-17

15. Cow Camp Creek site-18

16. Zuli site-19

17. Bond Creek site-10 20

18-21. Hydrographs showing:

18. Water levels for reclaimed-spoil wells at the Spring Creek site--

19. Water levels for reclaimed-spoil wells at the Cow Camp Creek site-..... 22

20. Mean daily water levels for reclaimed-spoil well SSS487-60 at the Spring Creek site-23

21. Mean daily water levels for reclaimed-spoil well SCS487-63 at the Cow Camp Creek site-...... 24 
Figures 22-25. Diagrams showing core-sample identification, depth, and

lithology for the:

22. Spring Creek core hole-25

23. Cow Camp Creek core hole-16 26

24. Zuli core hole-1 27

25. Bond Creek core hole-... 28

26. Hydrograph showing mean daily discharge for the spring

at the Spring Creek site, water years 1988 and 1989-..-

27. Hydrograph showing mean daily discharge for the spring

at the Cow Camp Creek site, water years 1988 and 1989--

28. Graph showing identification of depth and lithology of

Cow Camp Creek core samples used for batch-mixing

experiments--..-

TABLES

Table 1. Air-temperature, relative-humidity, solar-radiation, and

precipitation data, October 1987-December 1989-
2. Windspeed and wind-vector data, October 1987-December 1989--

3 . Wind-run and wind-duration histogram data, October 1987-

December 1989--

4. Snow-course information for 1988 and 1989 at the Spring Creek, Cow Camp Creek, and soil-water control sites-------.--

5. Soil-water content at the Spring Creek site during 1988 and $1989-116$

6. Soil-water content at the Cow Camp Creek site during 1988 and 1989

7. Soil-water content at the soil-water control site during 1988 and 1989-1...

8. Soil dry density at the Spring Creek, Cow Camp Creek, and soil-water control sites, May 11, 1989-_ 130

9. Well information--. 131

10. Summary of geophysical and lithologic logs-_. 132

11. Hydraulic-head data for bedrock wells-

12. Water-level data for reclaimed spoil wells-_a 139

13. Mean daily water levels for reclaimed spoil well SSS487-60 at the Spring Creek site-14

14. Mean daily water levels for reclaimed spoil well SCS487-63 at the Cow Camp Creek site-142

15-18. Porosity, grain density, and permeability of selected core samples from the

15. Spring Creek site core hole-co

16. Cow Camp Creek site core hole-1

17. Zuli site core hole-(

18. Bond Creek site core hole-1ing

19. Mineral composition of core samples from selected lithologic units at the Cow Camp Creek site-147

20. Spring and lysimeter information- 148 
Tables 21-24. Mean daily discharge from the spring at the:

21. Spring Creek site during water year 1988_...... 149

22. Spring Creek site during water year 1989-_... 150

23. Cow Camp Creek site during water year 1988-...... 151

24. Cow Camp Creek site during water year 1989_..... 152

25. Water-quality analyses for wells-_ 153

26. Water-quality analyses for springs and lysimeters---.-- 209

27. Water-quality information from Cow Camp Creek batch-mixing samples collected after 90 days of water-rock contact--- 217

\section{CONVERSION FACTORS AND RELATED INFORMATION}

\section{Multiply}

acre-foot (acre-ft)

cubic foot per second

foot ( $f t$ )

gram per cubic centimeter $\left(\mathrm{g} / \mathrm{cm}^{3}\right)$

inch (in.)

inch per year (in./yr)

micrometer $(\mu \mathrm{m})$

mile (mi)

mile per hour

millidarcy $(\mathrm{mD})$
By

0.001233
0.02832
0.3048
62.4
2.54
2.54
$39.37 \times 10^{-6}$
1.609
1.609
$3.17 \times 10^{-8}$

To obtain

cubic hectometer cubic meter per second meter

pound per cubic foot centimeter centimeter per year inch kilometer kilometer per hour foot per second

Degree Celsius $\left({ }^{\circ} \mathrm{C}\right)$ can be converted to degree Fahrenheit $\left({ }^{\circ} \mathrm{F}\right)$ by using the following equation:

$$
{ }^{\circ} \mathrm{C}=5 / 9\left({ }^{\circ} \mathrm{F}-32\right) \text {. }
$$

The following terms and abbreviations also are used in this report:

micrograms per liter $(\mu \mathrm{g} / \mathrm{L})$

microsiemens per centimeter at 25 degrees Celsius $(\mu \mathrm{S} / \mathrm{cm})$

milligrams per liter $(\mathrm{mg} / \mathrm{L})$

National Geodetic Vertical Datum of 1929 (NGVD of 1929): A geodetic datum derived from a general adjustment of the first-order level nets of both the United States and Canada, formerly called Sea Level Datum of 1929. 
CLIMATOLOGIC, SOIL-WATER, GROUND-WATER, GEOLOGIC, SURFACE-WATER, AND WATER-QUALITY DATA FOR A SURFACE COAL MINE

IN NORTHWESTERN COLORADO

By Robert S. Williams, Jr., Gregory M. Clark, and Norman E. Spahr

\section{ABSTRACT}

Climatologic, soil-water, ground-water, geologic, surface-water, and water-quality data were collected at five sites at a surface coal mine in northwestern Colorado. The data were collected to help describe the hydrogeology and geochemistry of an area that is being surface mined for coal.

The study area was at the Seneca II Coal Mine. The Lennox coal and the Wadge coal are the major seams being mined. Confined aquifers in bedrock and unconfined aquifers in reclaimed spoil are present in the area. Springs are present at the toe of the reclaimed spoil slope.

Climatologic data were collected at two sites. Air temperature, relative humidity, solar radiation, windspeed, and wind duration were collected at one site. Precipitation was measured at three rain gages. Snow-course data were collected at three sites. Soil-water content and soil dry density were measured.

Eighteen wells in the confined aquifer and seven in the unconfined aquifer were installed at the study area. Geophysical and geologic logs were recorded for selected cored boreholes. Water levels were measured at the wells. Porosity, grain density, and permeability of selected core were estimated. Mineral composition also was determined.

Ground-water samples were collected for water-quality analysis. Spring discharge and water quality were measured for two springs. Water quantity and quality were measured for two lysimeters.

\section{INTRODUCTION}

During surface mining of coal, the vegetation and overburden are removed, the coal is mined, the overburden is replaced, and the surface is reclaimed. After mining, the reclaimed spoil undergoes weathering, settling, and compacting for an unknown period of time before reaching equilibrium. Full evaluation of this disturbed and changing system and understanding of reclaimed-spoil hydrogeologic processes is essential to regulatory agencies for support of leasing, permitting, and reclamation related to coal mining on Federal lands. 
In 1986, the U.S. Geological Survey in cooperation with the U.S. Bureau of Land Management and the Colorado Department of Natural Resources, Mined Land Reclamation Division, began a study to determine the processes controlling the hydrology of reclaimed coal spoils at a surface coal mine in northwestern Colorado. However, in order to evaluate this disturbed and changing system, climatologic, soil-water, ground-water, geologic, surface-water, and water-quality data needed to be collected and compiled to aid researchers, regulators, and land managers in conceptualizing the hydrology of potential coal-spoil sites.

\section{Purpose and Scope}

This report lists climatologic, soil-water, ground-water, geologic, surface-water, and water-quality data collected at five sites as part of a study to evaluate the hydrology and geochemistry of a surface coal mine in northwestern Colorado. Monitoring equipment and instrumentation were installed in the summer of 1987. Data were collected during 1988 and 1989. The monitoring equipment and instrumentation were placed on undisturbed areas and reclaimed spoil areas. Reclaimed spoil, as described in this report, is material that has been excavated from a mine pit and then has been replaced, regraded, topsoiled, and revegetated.

The study area was at the Seneca II Coal Mine. The mine is divided approximately in half by an anticline. The Lennox coal and the Wadge coal are the major seams being mined. Confined aquifers in bedrock and unconfined aquifers in reclaimed spoil are present in the area. Springs are present at the toe of the reclaimed spoil slope.

Air temperature, relative humidity, solar radiation, windspeed, and wind duration were collected at one site. Precipitation was measured at three rain gages. Snow-course data were collected at three sites.

Lysimeters were installed at two sites, and water quantity and quality were measured. Soil-water content was measured using soil-water access tubes and a neutron probe. Soil dry density was measured once with a density probe.

Eighteen wells in the confined aquifer and seven wells in the unconfined aquifer were installed. Geophysical and geologic logs were recorded for selected cored boreholes. Water levels were measured at the wells. Porosity, grain density, and permeability of selected core were estimated. Mineral composition also was determined. Ground-water samples were collected for water-quality analysis. Two springs that flowed from the reclaimed spoil were monitored, and discharge and water quality were measured.

\section{Description of Study Area}

The study area is located at the Seneca II Coal Mine in northwestern Colorado (fig. 1). The site is about $20 \mathrm{mi}$ west and $5 \mathrm{mi}$ south of Steamboat Springs, Colorado. The area is shown on the U.S. Geological Survey topographic maps of the Milner and Mount Harris quadrangles (fig. 2). The area ranges in altitude from about 6,800 to $8,000 \mathrm{ft}$. The vegetation primarily is sagebrush and grasses with scattered stands of scrub oak, serviceberry, and 


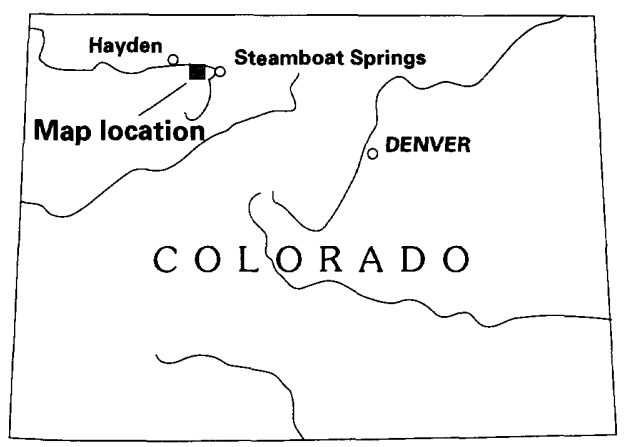

\section{EXPLANATION}

AXIS OF ANTICLINE--Showing direction of plunge (from Tweto, 1976)

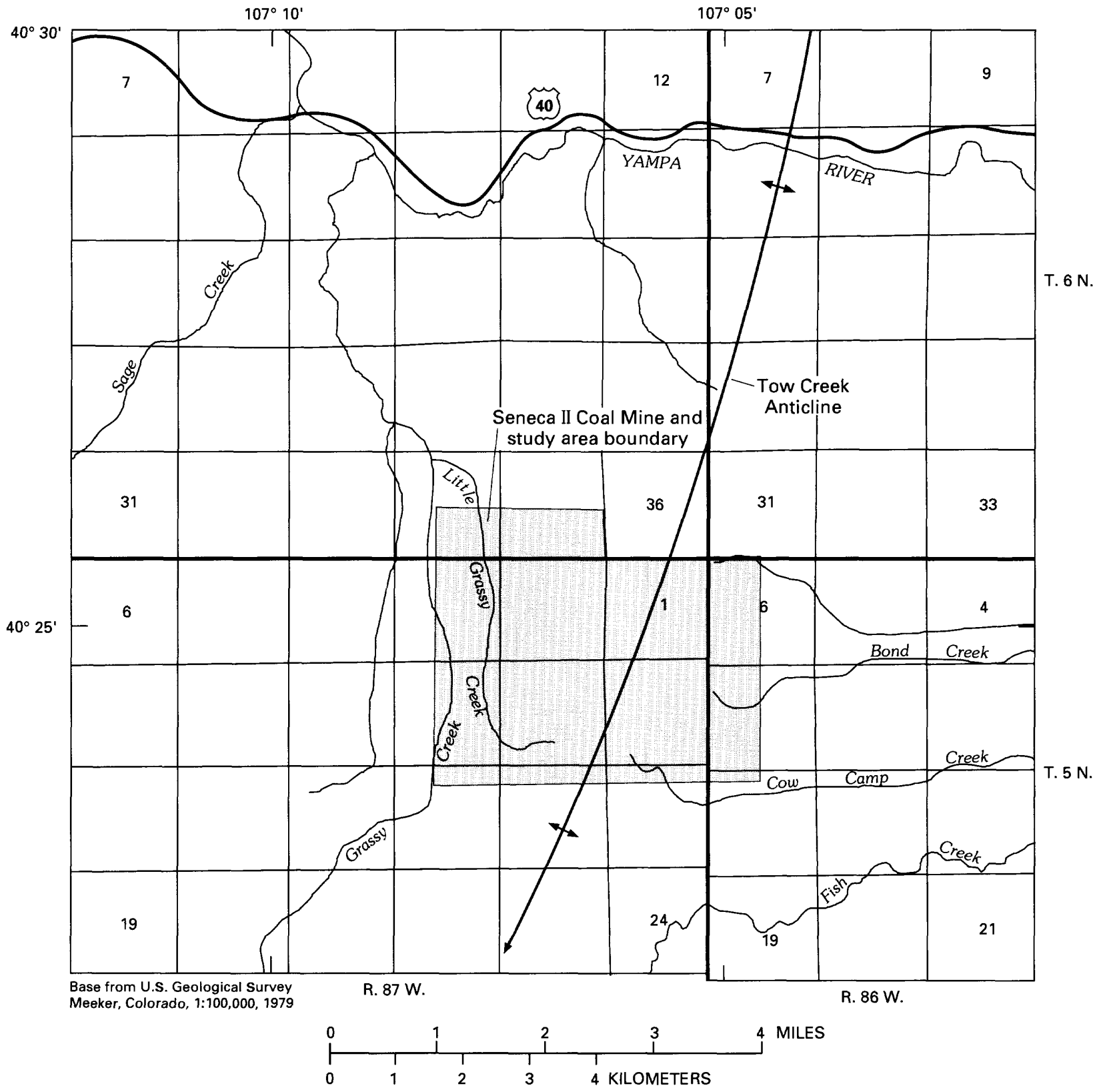

Figure 1.--Location of the study area (modified from Clark and Williams, 1990). 


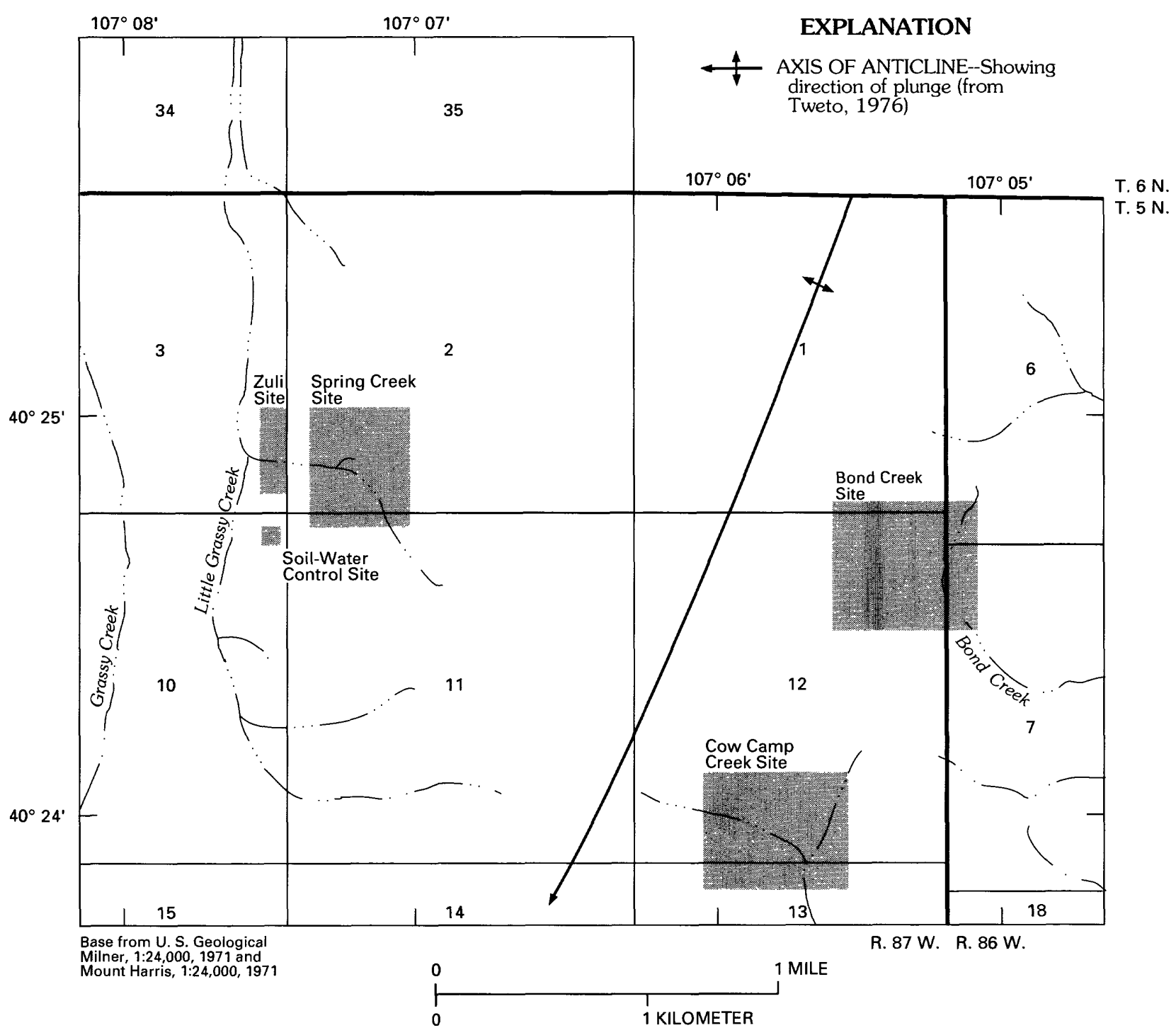

Figure 2.--Study area and data-collection sites. 
aspen. Average yearly rainfall for the area is 16 in. (ENMAP Corporation, 1981). Warm days and cool nights characterize the summer; winter is relatively cold. The average date of the first killing frost is September 14, and the average date of the last killing frost is June 10 .

A prominent feature in the study area is the Tow Creek anticline, a northeastward-trending asymmetrical fold (Bass and others, 1955) (fig. 1). The axis of the anticline extends through the central part of the mine. Rocks east of the axis of the anticline dip to the east, and rocks west of the axis dip to the west (Bass and others, 1955) (fig. 1). The eastern part of the area is drained by Bond Creek, Cow Camp Creek, and other unnamed streams that are tributary to Fish Creek. The western part of the area is drained by Little Grassy Creek, which is tributary to Grassy Creek. In the southern part of the study area at the plunging, southern tip of the anticline, rocks dip to the south (Bass and others, 1955) (fig. 1). The coal seams of interest in this study are the Lennox and the Wadge (fig. 3) of the Williams Fork Formation of Cretaceous age.

Unconfined aquifers develop in the reclaimed spoil during and following mining (fig. 4). The aquifers generally are at the toe of the slope at the location of the mine pit low wall. A spring(s) will form when the water level rises above the reclaimed spoil surface.

\section{Acknowledgments}

Personnel from the Peabody Coal Company at the Flagstaff, Arizona, headquarters office and at the Seneca II Coal Mine supplied technical information and machinery throughout the study. Bulldozers and backhoes were provided during well construction, roads were snowplowed and graded as needed, and wells were surveyed and mapped at all sites. This work and logistical assistance was a significant contribution to the completion of the investigation and is appreciated.

\section{SAMPLING-SITE LOCATIONS AND DATA-COLLECTION INFORMATION}

Data were collected at five sites in the study area. The sites are Spring Creek (figs. 2 and 5), Cow Camp Creek (figs. 2 and 6), Zuli (figs. 2 and 5), Bond Creek (figs. 2 and 7), and soil-water control site (fig. 2).

Climatologic information was monitored at one climate station (fig. 5) and three weighing-bucket precipitation gages (figs. 5 and 6). Air temperature and relative humidity were measured using a ${ }^{1}$ Campbell Scientific 201 sensor (table 1; tables 1-27 are in the "Data" section at the back of the report). Incoming short wave solar radiation was measured with an Eppley PSP (precision spectral pyranometer) radiometer (table 1). Windspeed, wind vector, wind run, and wind duration were measured using a MET ONE anemometer and a MET ONE wind vane (tables 2 and 3). All sensors were measured every 10 seconds and were averaged daily by a Campbell Scientific data logger.

${ }^{1}$ The use of trade or product names in this report is for identification purposes only, and does not constitute endorsement by the U.S. Geological Survey. 


\section{LITHOLOGY}

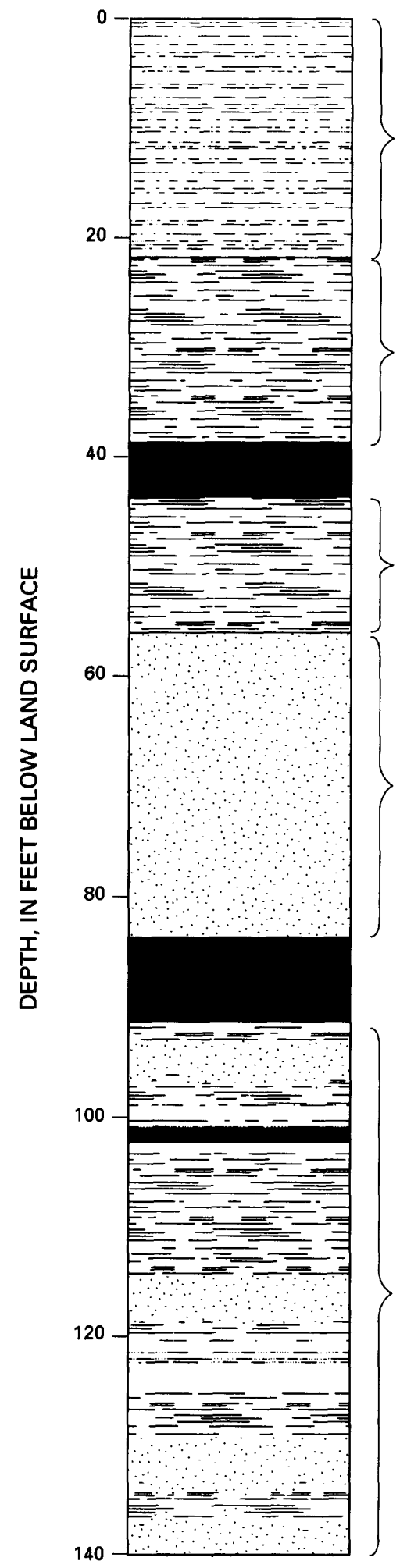

\section{DESCRIPTION} Silt and clay: Light to dark brown, moist
soil and root zone

Shale: Dark grey, well indurated, very fine grained, very well sorted, interbedded sandstone lenses

Lennox coal: Black, hard, fractured

Shale: Dark grey, well indurated, very fine grained, well sorted, small fractures

Sandstone: Grey to light grey, very fine grained, well sorted, calcareous, shale and siltstone lenses present

Wadge coal: Black, hard, fractured

Shale, sandstone, siltstone: Interbedded, light to dark grey, well indurated, very fine grained, small, black, coal seam present

\section{EXPLANATION}

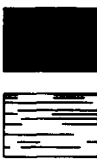

COAL

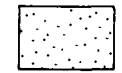

SANDSTONE

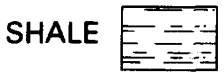

SILTSTONE

AND CLAY

Figure 3.--General lithologic log for a typical well in the study area. 


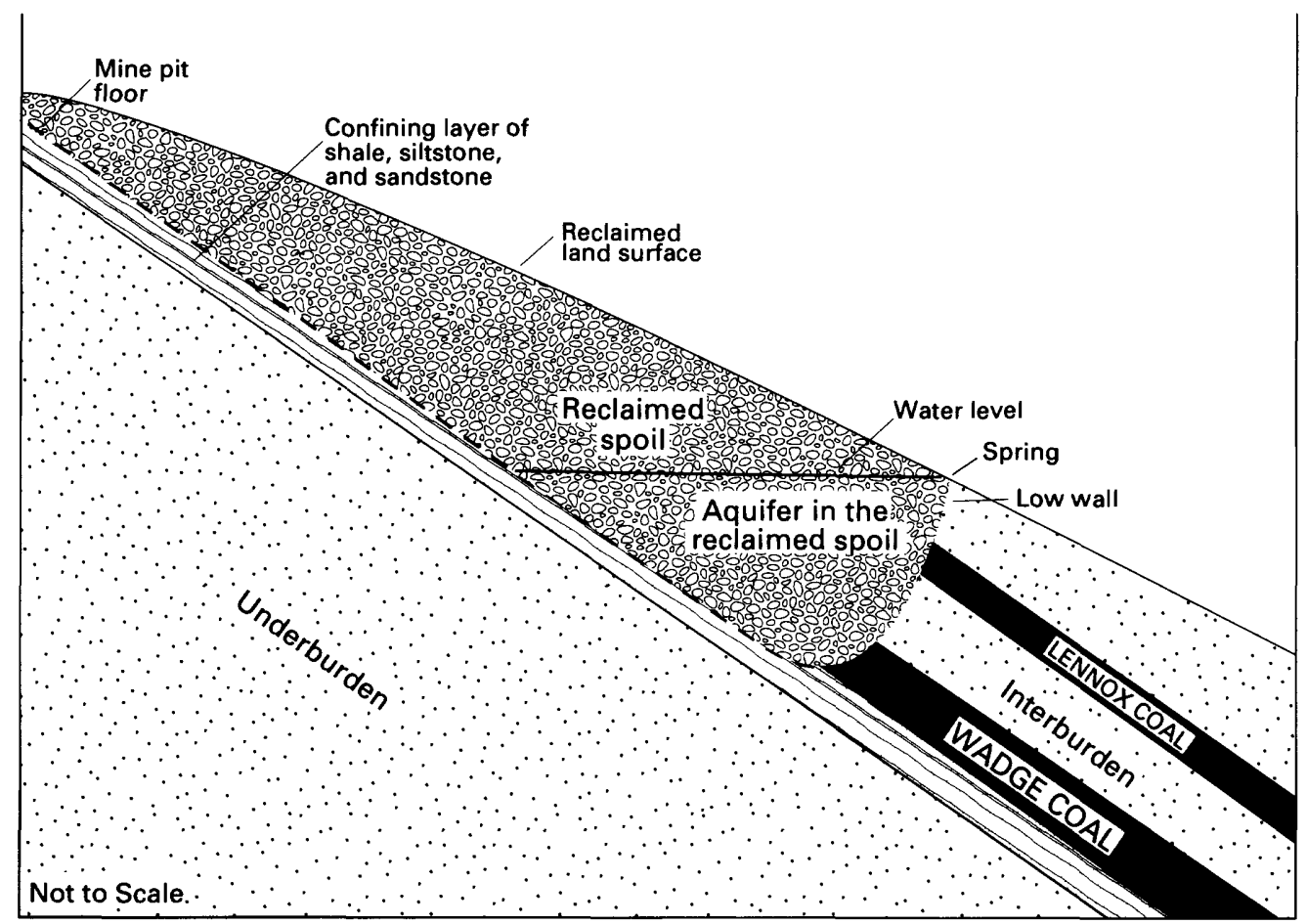

Figure 4.--Schematic diagram showing hydrogeology of reclaimed spoil and bedrock at the study area (modified from Clark and Williams, 1990).

Precipitation was recorded using three weighing type precipitation gages (table 1). Snow courses were run monthly at three sites when snowpack was present (table 4). Snow tubes were used to measure snow depth and snow water content.

Water was collected from lysimeters at the Spring Creek site (figs. 2 and 5) and the Cow Camp Creek site (figs. 2 and 6). A schematic diagram of a lysimeter and soil-water access tube is shown in figure 8.

Soil-water access tubes were installed at the Spring Creek (figs. 2 and 5), Cow Camp Creek (figs. 2 and 6), and soil-water control sites (fig. 2). Soil-water content was measured using a neutron probe (tables 5 to 7). Soil dry density was measured once on May 11, 1989, using a density probe (table 8).

Wells are located at four sites at the study area. Two sites, Spring Creek (figs. 2 and 5) and Cow Camp Creek (figs. 2 and 6), have some wells completed in bedrock and some in reclaimed spoil. Two other sites, Zuli (figs. 2 and 5) and Bond Creek (figs. 2 and 7), have wells completed only in the bedrock. A typical bedrock well completion is shown in figure 9. The Zuli site is downdip from the Spring Creek site. The Bond Creek site was being mined during the period of data collection. The mine pit is the area of active mining, and the spoil ridge is the unreclaimed spoil area (fig. 7). 


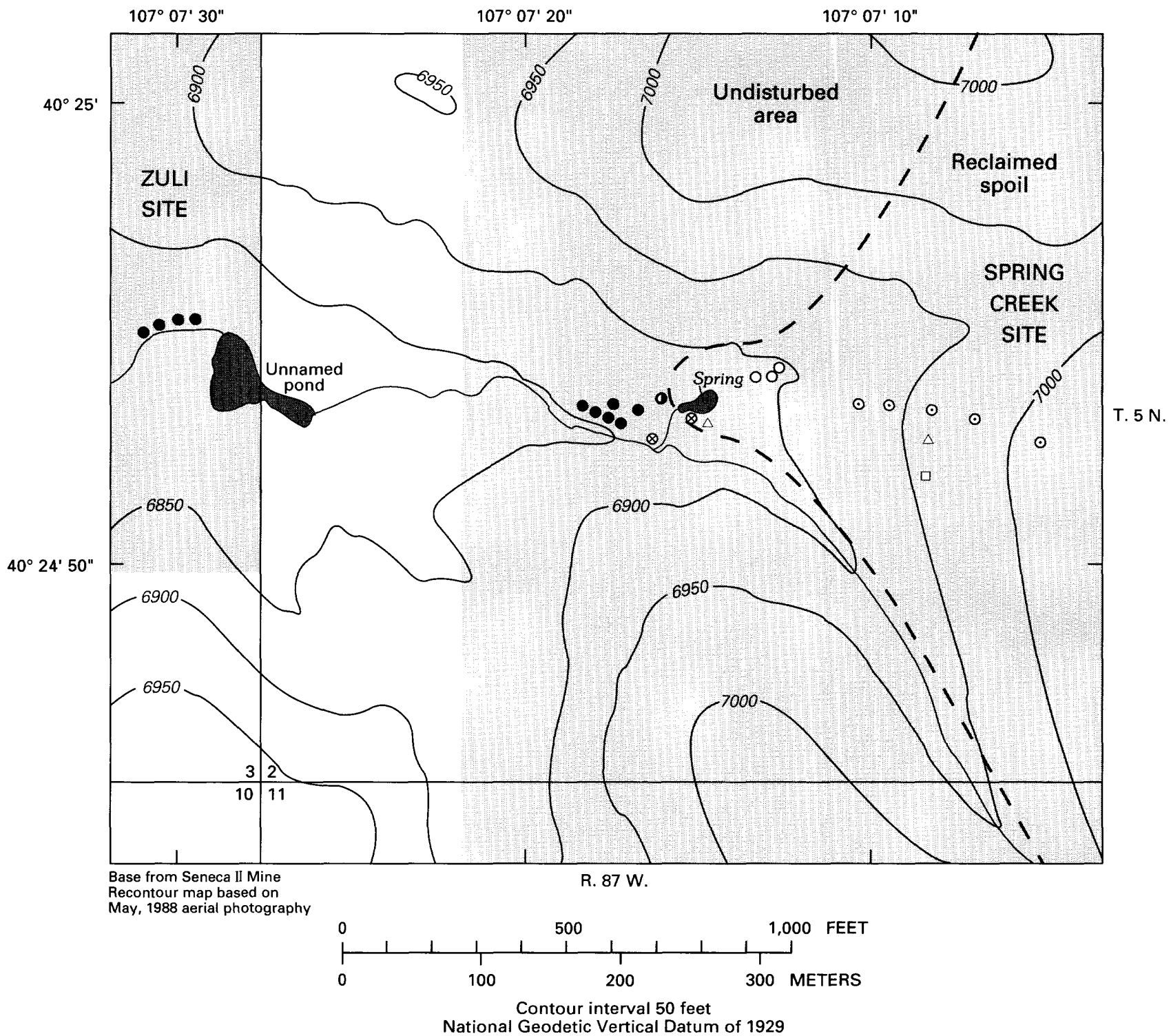

\section{EXPLANATION}

- WELL COMPLETED IN BEDROCK

- WELL COMPLETED IN RECLAIMED SPOIL

- - - APPROXIMATE BOUNDARY OF RECLAIMED SPOIL STUDY SITES

- LYSIMETER AND SOIL-WATER ACCESS TUBE WEIGHING-BUCKET

PRECIPITATION GAGE

$\odot \quad$ SOIL-WATER ACCESS TUBE

ه SURFACE-WATER GAGE

- ClimATE STATION

Figure 5.--Data-collection points and instrumentation at the Spring Creek and $\mathrm{Zuli}$ sites. 

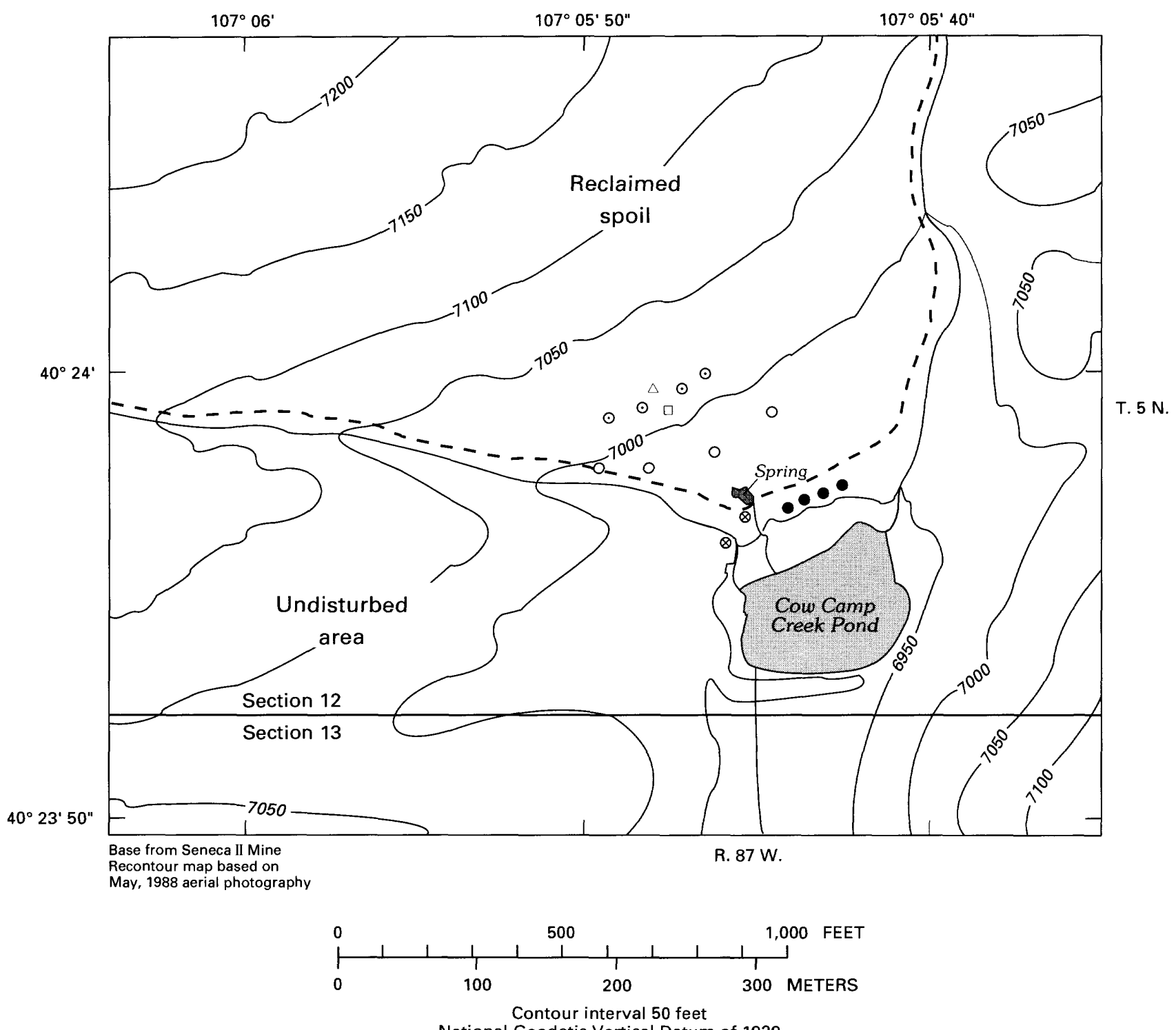

National Geodetic Vertical Datum of 1929

\section{EXPLANATION}

- WELL COMPLETED IN BEDROCK

O WELL COMPLETED IN RECLAIMED SPOIL

- - - APPROXIMATE BOUNDARY

OF RECLAIMED SPOIL

$\square \quad$ LYSIMETER AND SOIL-WATER

ACCESS TUBE

$\triangle \quad$ WEIGHING-BUCKET

PRECIPITATION GAGE

$\odot \quad$ SOIL-WATER ACCESS TUBE

$\otimes \quad$ SURFACE-WATER GAGE

Figure 6.--Data-collection points and instrumentation at the Cow Camp Creek site. 


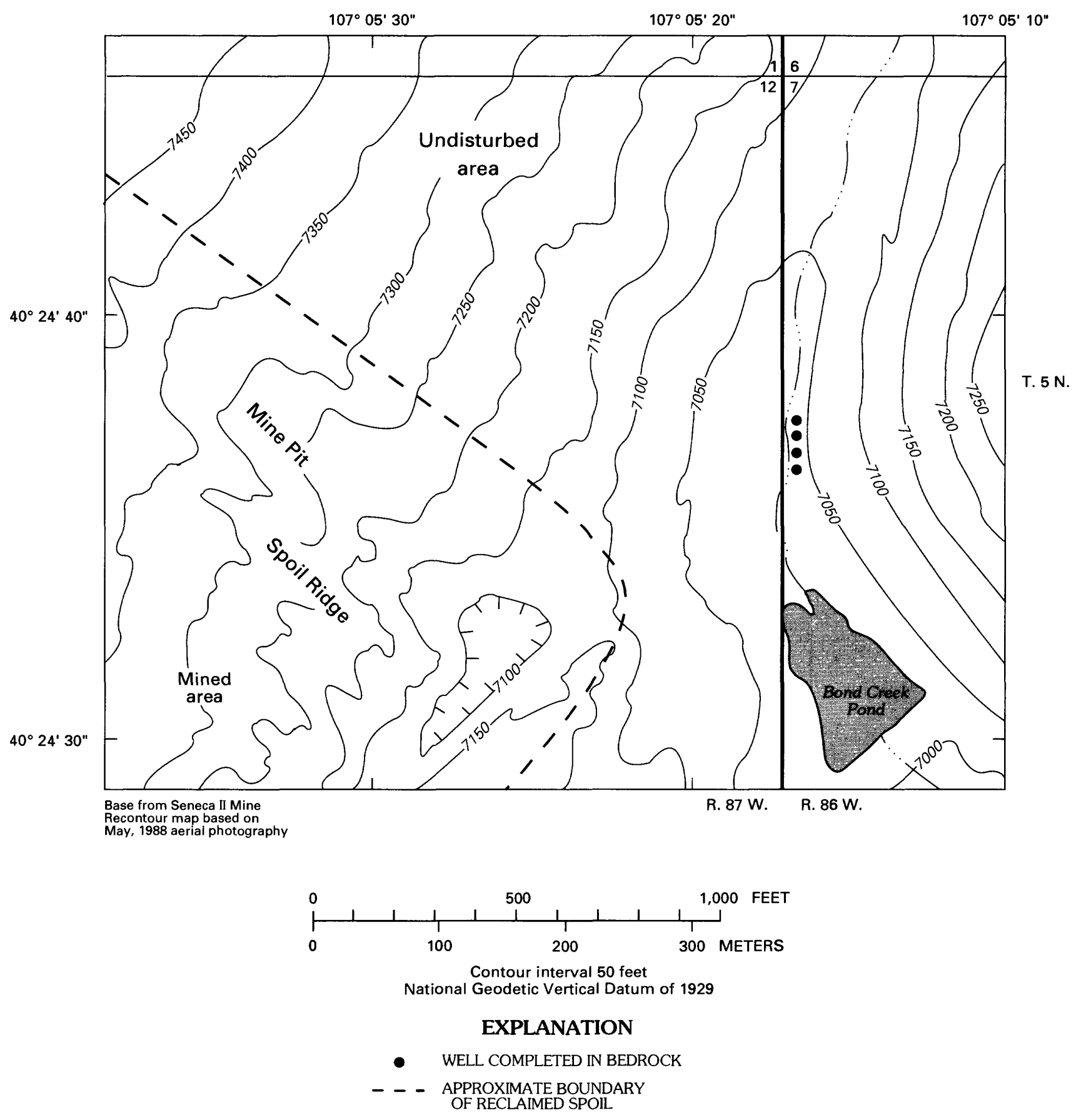

Figure 7.--Data-collection points and instrumentation at the Bond Creek site. 


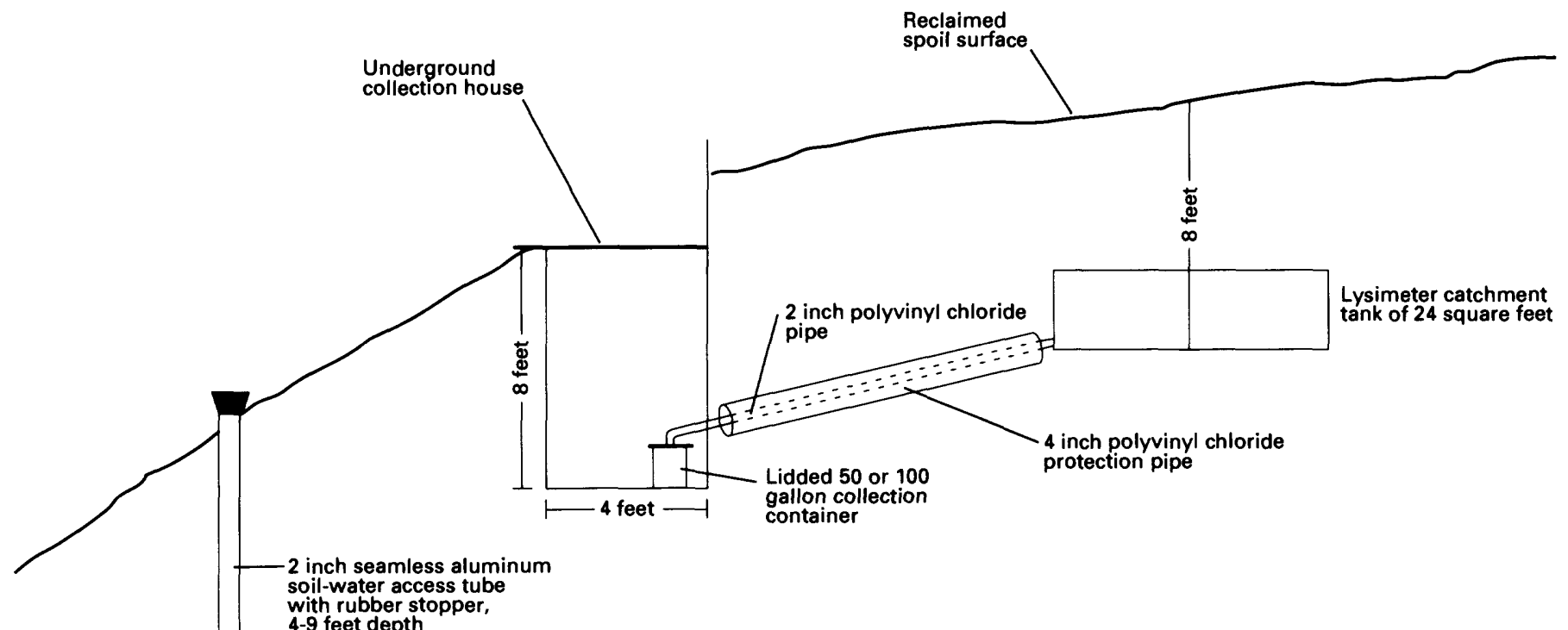

with rubber stopper,
4-9 feet depth

Figure 8.--Schematic diagram of a lysimeter and a soil-water access tube in the reclaimed spoil.

Eighteen bedrock wells are installed in the study area. At four sites within the study area, a bedrock well is completed in the Lennox coal, the interburden (bedrock zone between the Lennox coal and the Wadge coal), the Wadge coal, and the underburden (bedrock zone below the Wadge coal). In addition, two bedrock wells (SSU287 and SSD487, table 9) are completed deeper in the underburden at the Spring Creek site, each in separate aquifers.

Seven wells were installed in reclaimed spoil in the study area. Three reclaimed spoil wells are at the Spring Creek site (fig. 5) and four reclaimed spoil wells are at the Cow Camp Creek site (fig. 6).

Locations and names of wells (bedrock and reclaimed spoil wells) are listed in table 9. Well depths and intervals and zones of completion also are listed in table 9. Information about geophysical and lithologic logs is listed in table 10 and shown in figures 10 through 13 . Water levels at the bedrock wells were measured with a pressure gage if the hydraulic head was above the top of the well casing and with a steel tape if the water level was below the top of the well casing during site visits (table 11). Hydrographs showing hydraulic head for the bedrock wells are shown in figures 14 to 17 . All of the bedrock aquifers in which wells were completed during this study were under confined conditions. Hydraulic head in all of these bedrock wells was above land surface. Water levels in the reclaimed spoil wells were measured with a steel tape (table 12). Hydrographs for the reclaimed spoil wells are shown in figures 18 and 19. Hydrographs for the reclaimed spoil wells equipped with continuous recorders are shown in figures 20 and 21 . Mean daily water levels for the reclaimed spoil wells equipped with continuous recorders are listed in tables 13 and 14 . 


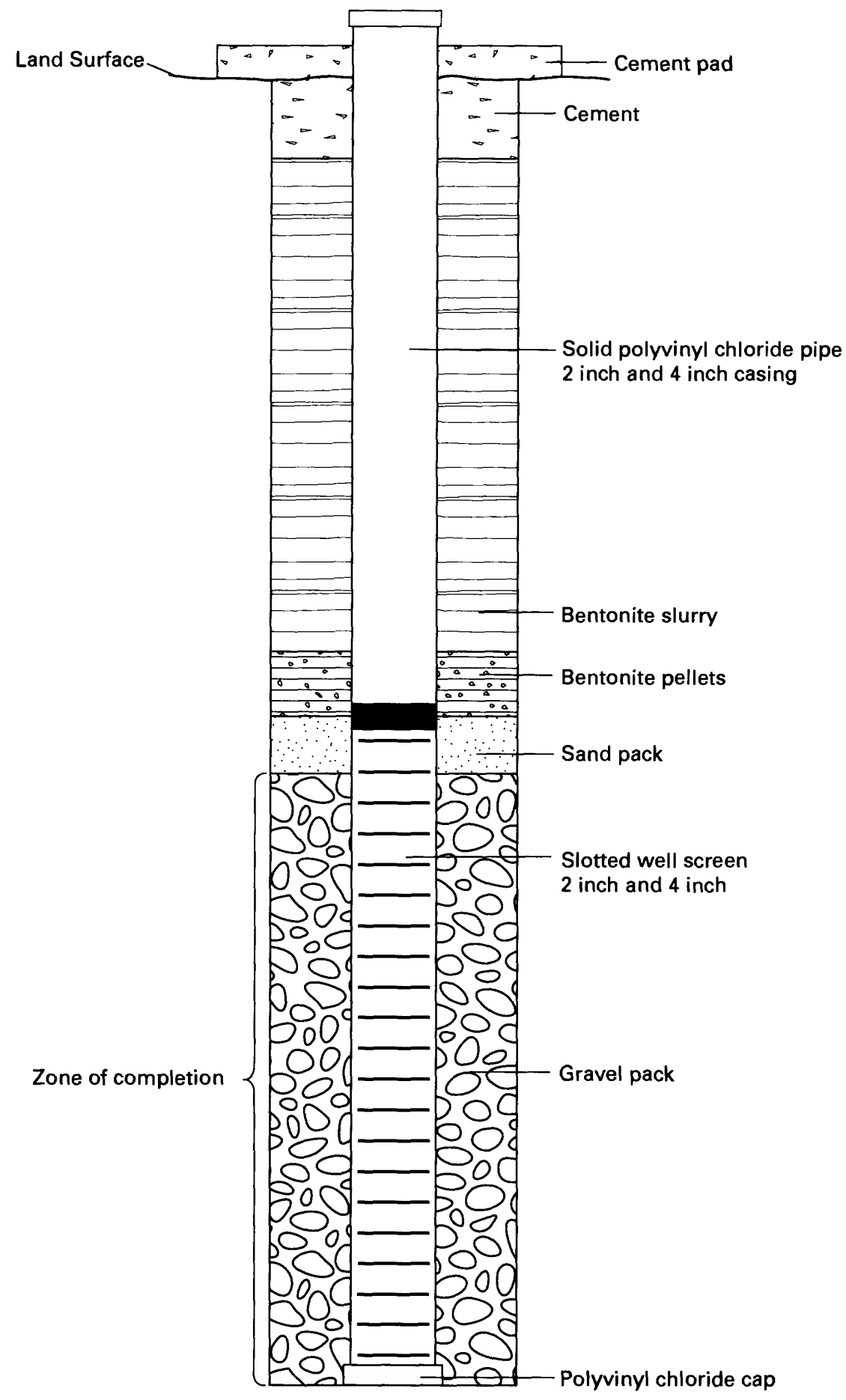

Figure 9.--Typical bedrock well completion. 


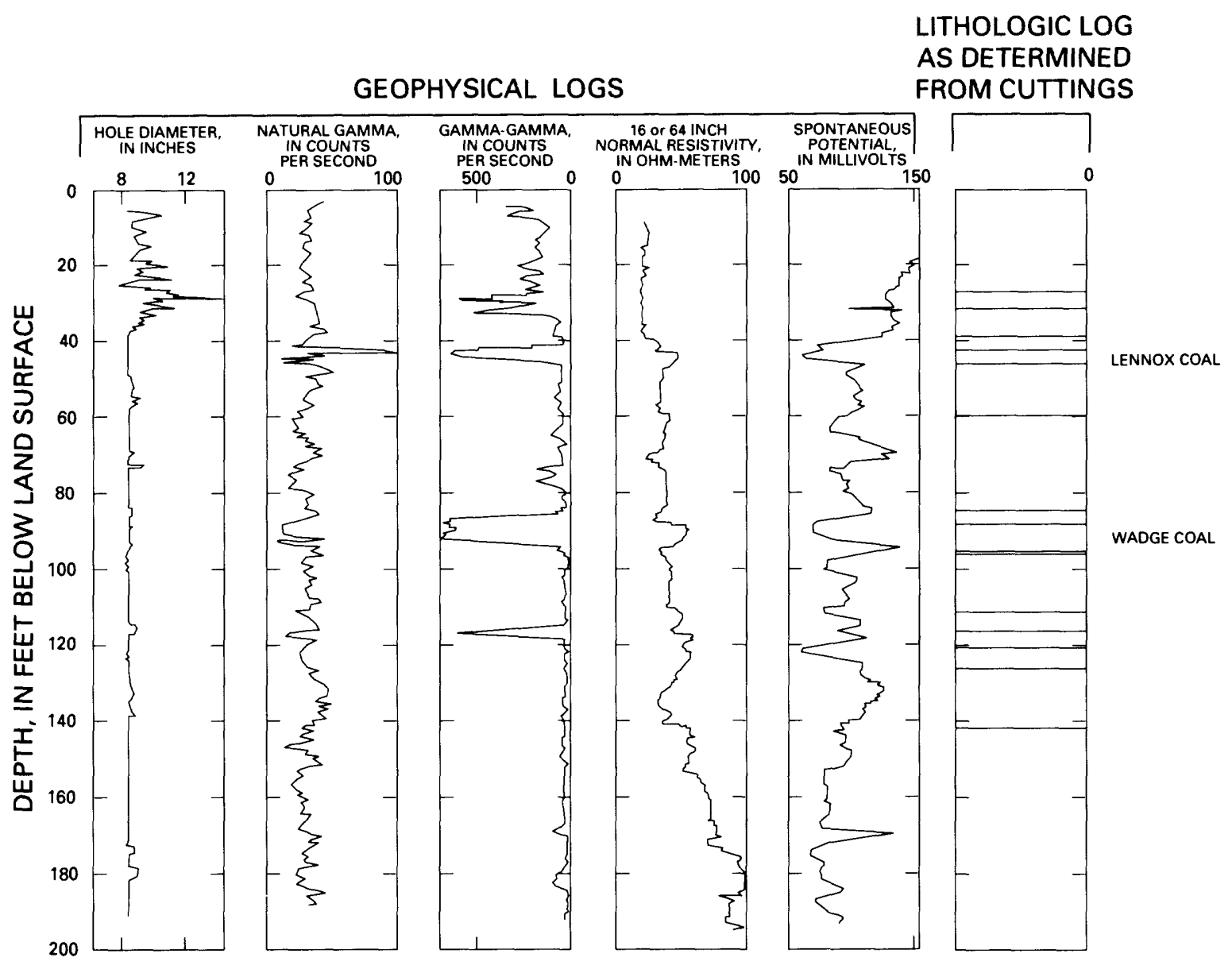

Figure 10.--Geophysical and lithologic logs for well SSD487

at the Spring Creek site. 


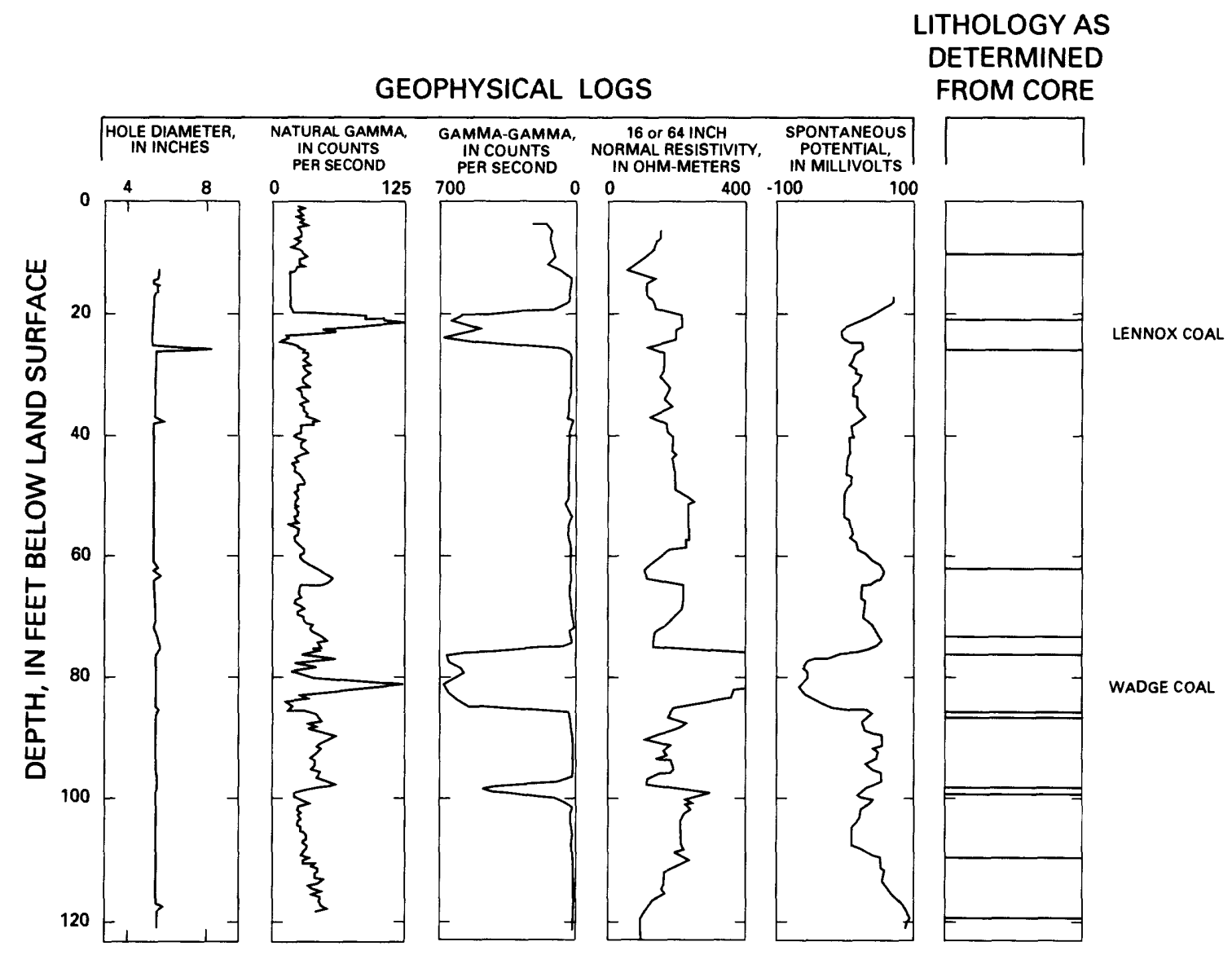

Figure 11.--Geophysical and lithologic logs for well SCU287 at the Cow Camp Creek site. 


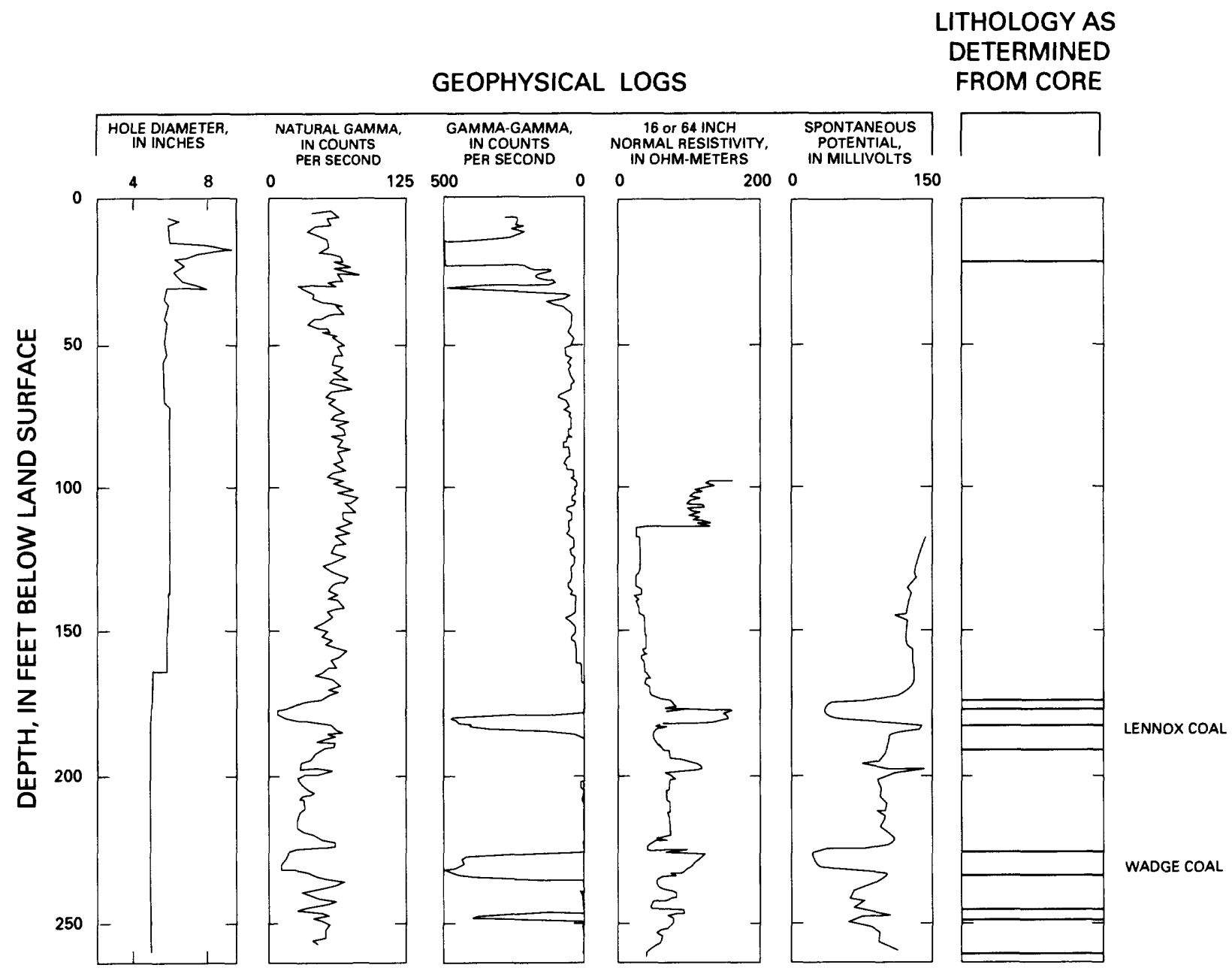

Figure 12.--Geophysical and lithologic logs for well SZU287 at the Zuli site. 


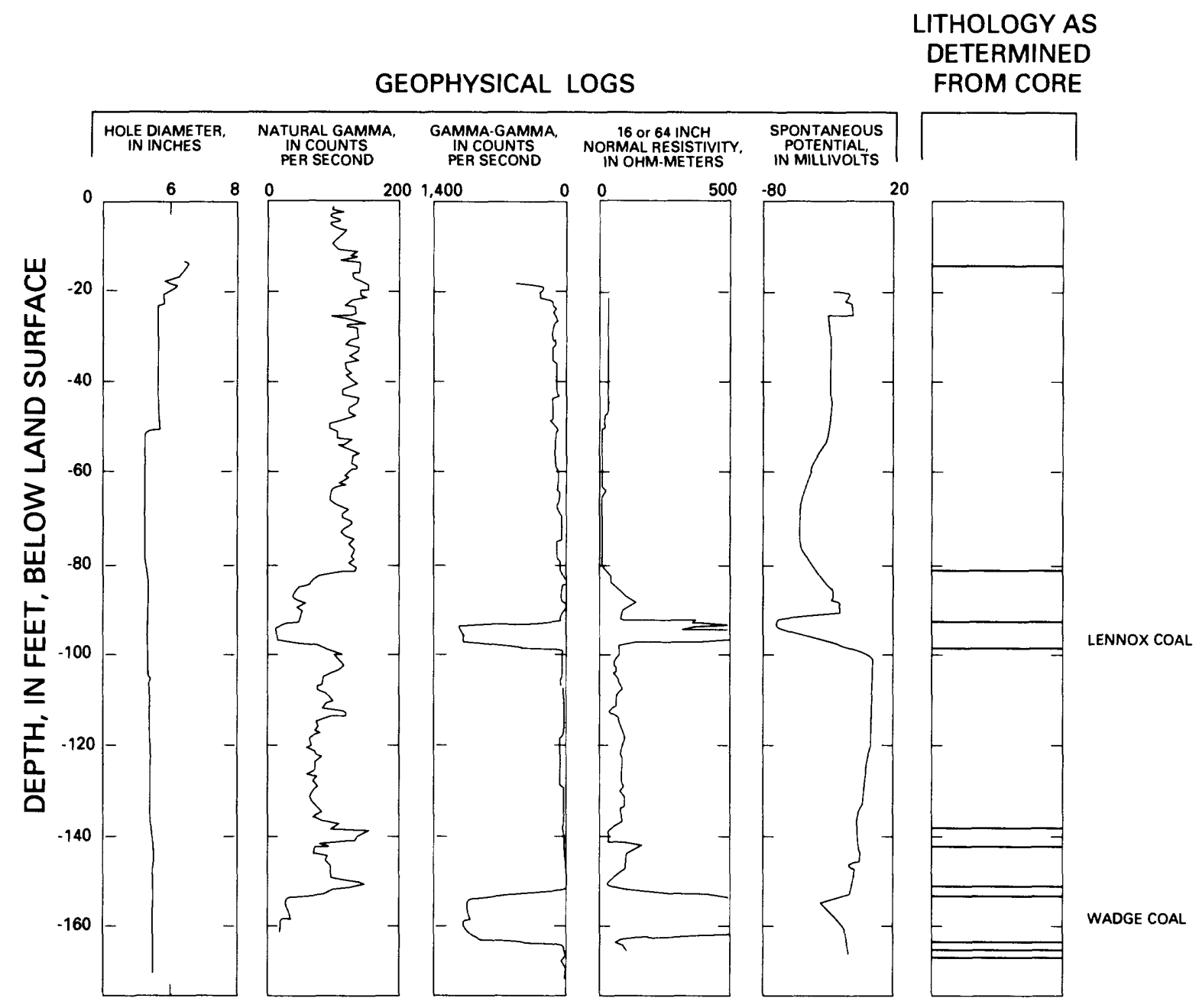

Figure 13.--Geophysical and lithologic logs for well SBU287 at the Bond Creek site. 


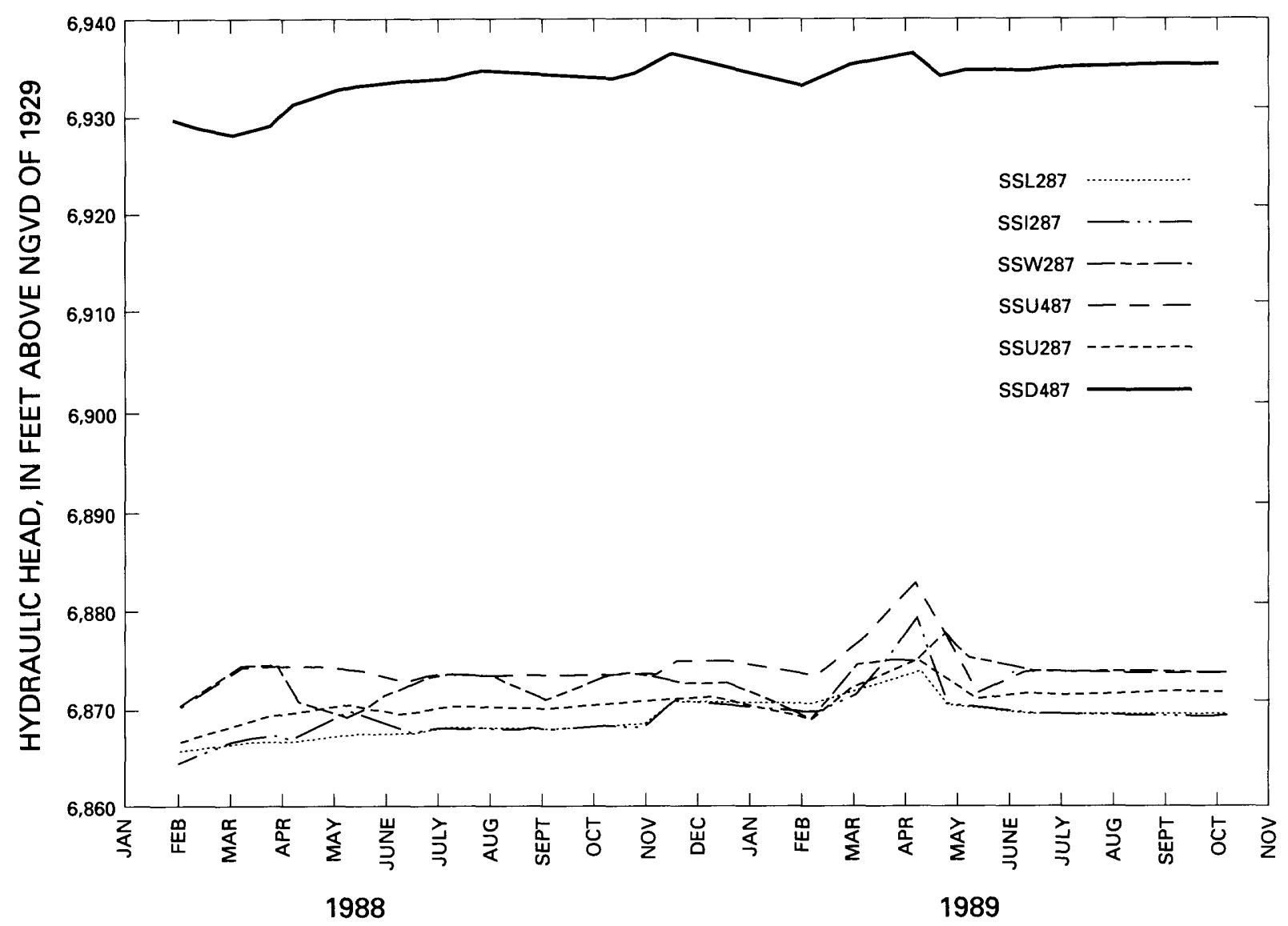

Figure 14.--Hydraulic head for bedrock wells at the Spring Creek site. 


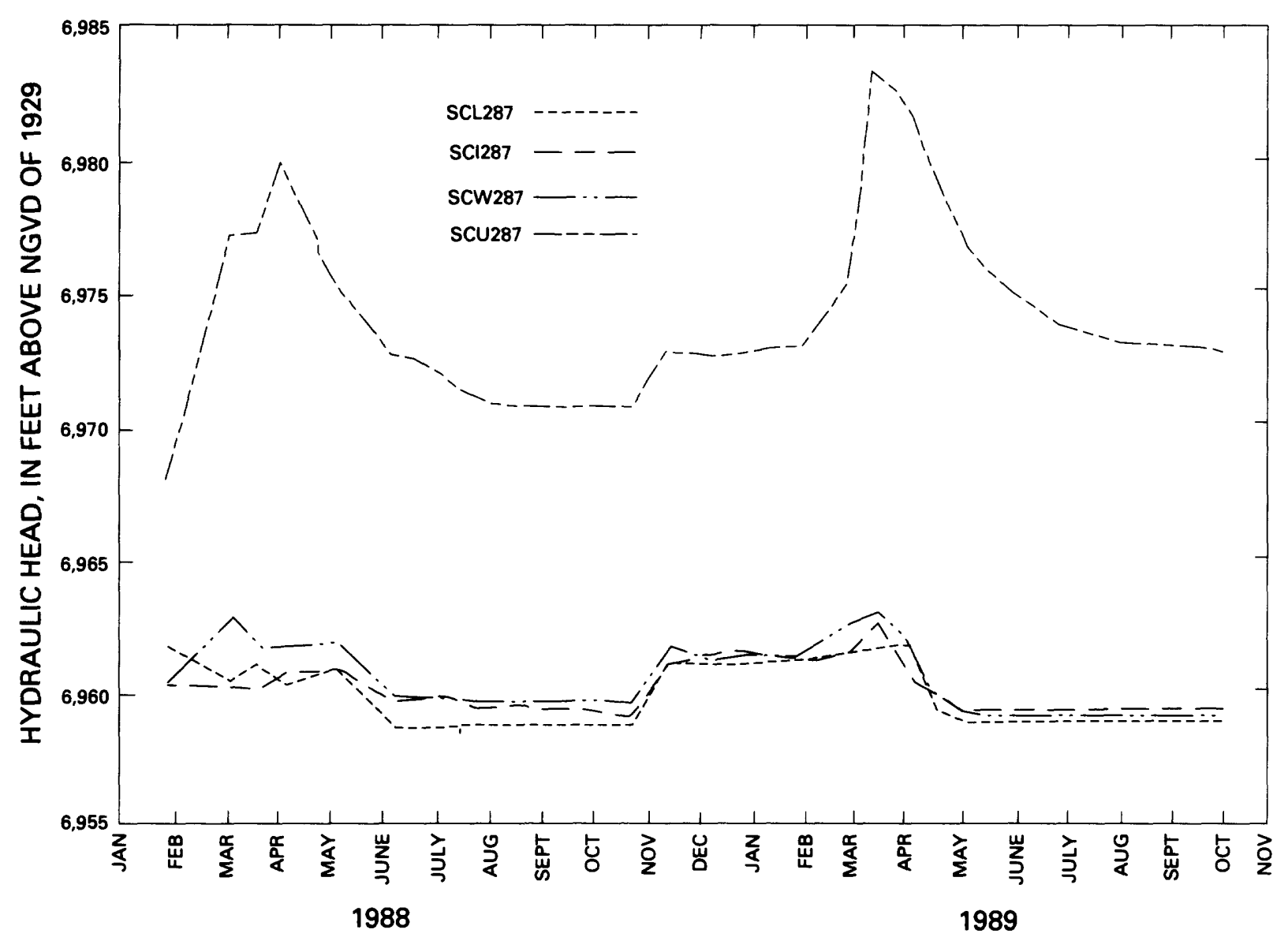

Figure 15.--Hydraulic head for bedrock wells at the Cow Camp Creek site. 


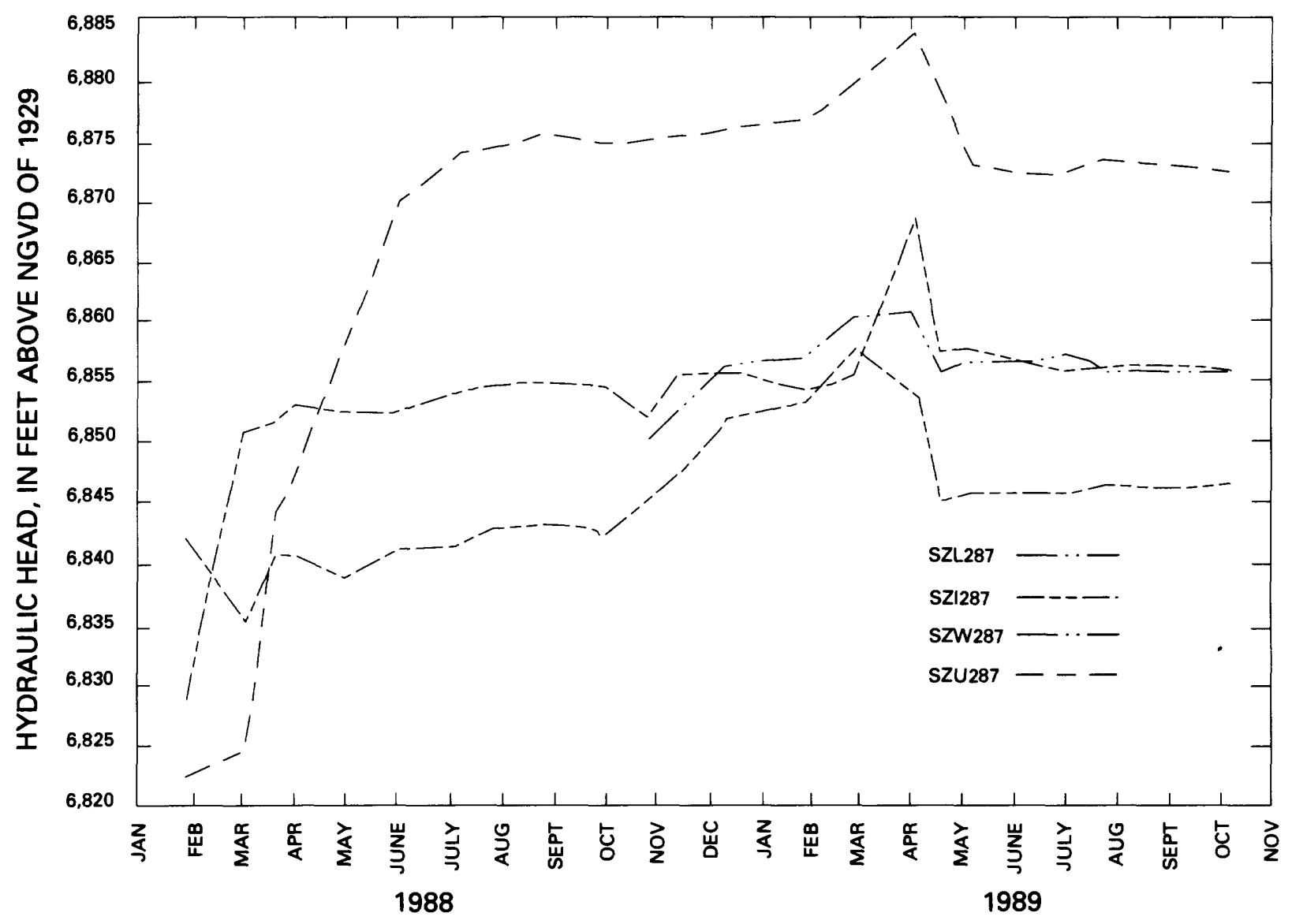

Figure 16.--Hydraulic head for bedrock wells at the Zuli site. 


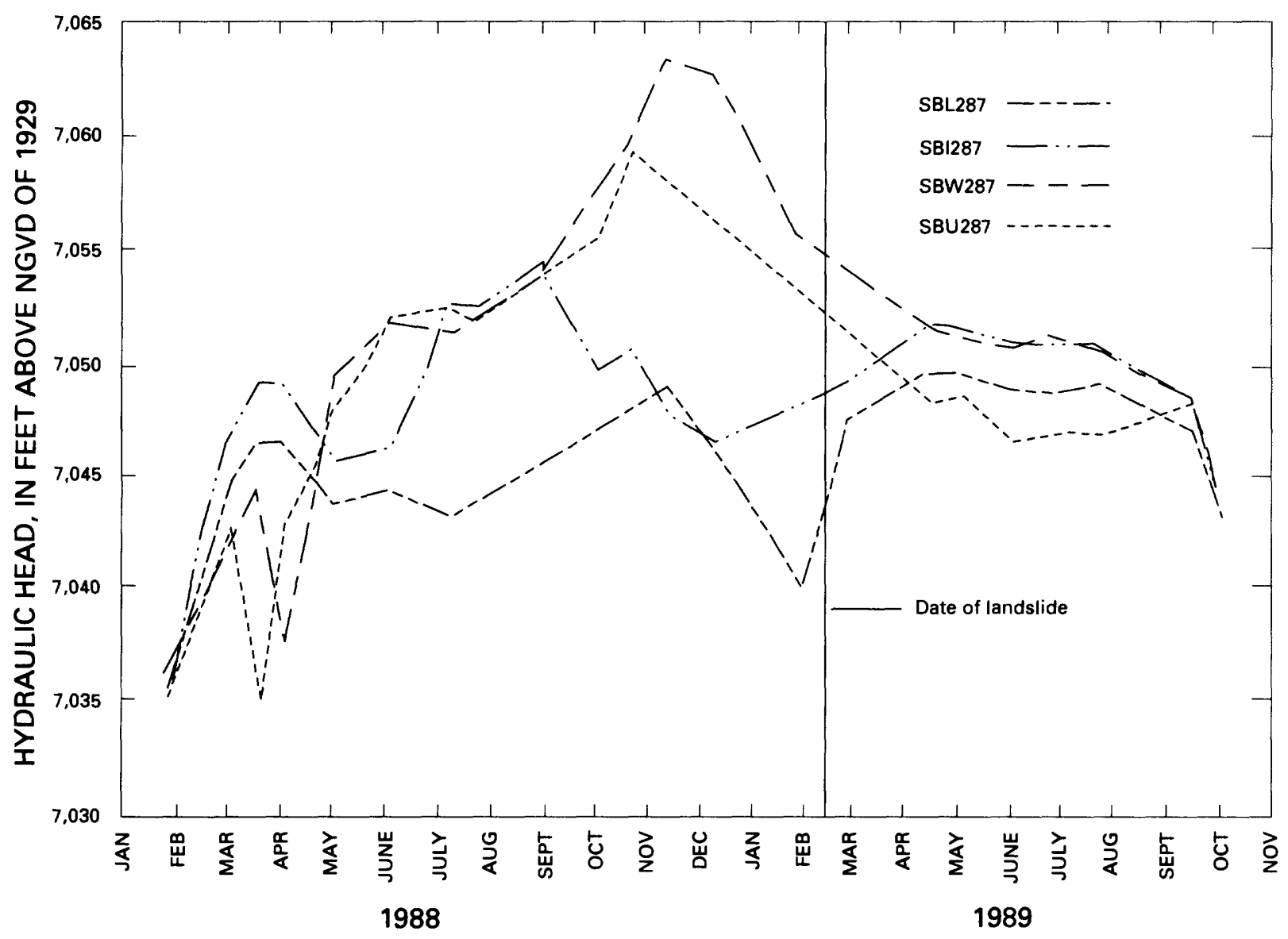

Figure 17.--Hydraulic head for bedrock wells at the Bond Creek site. 


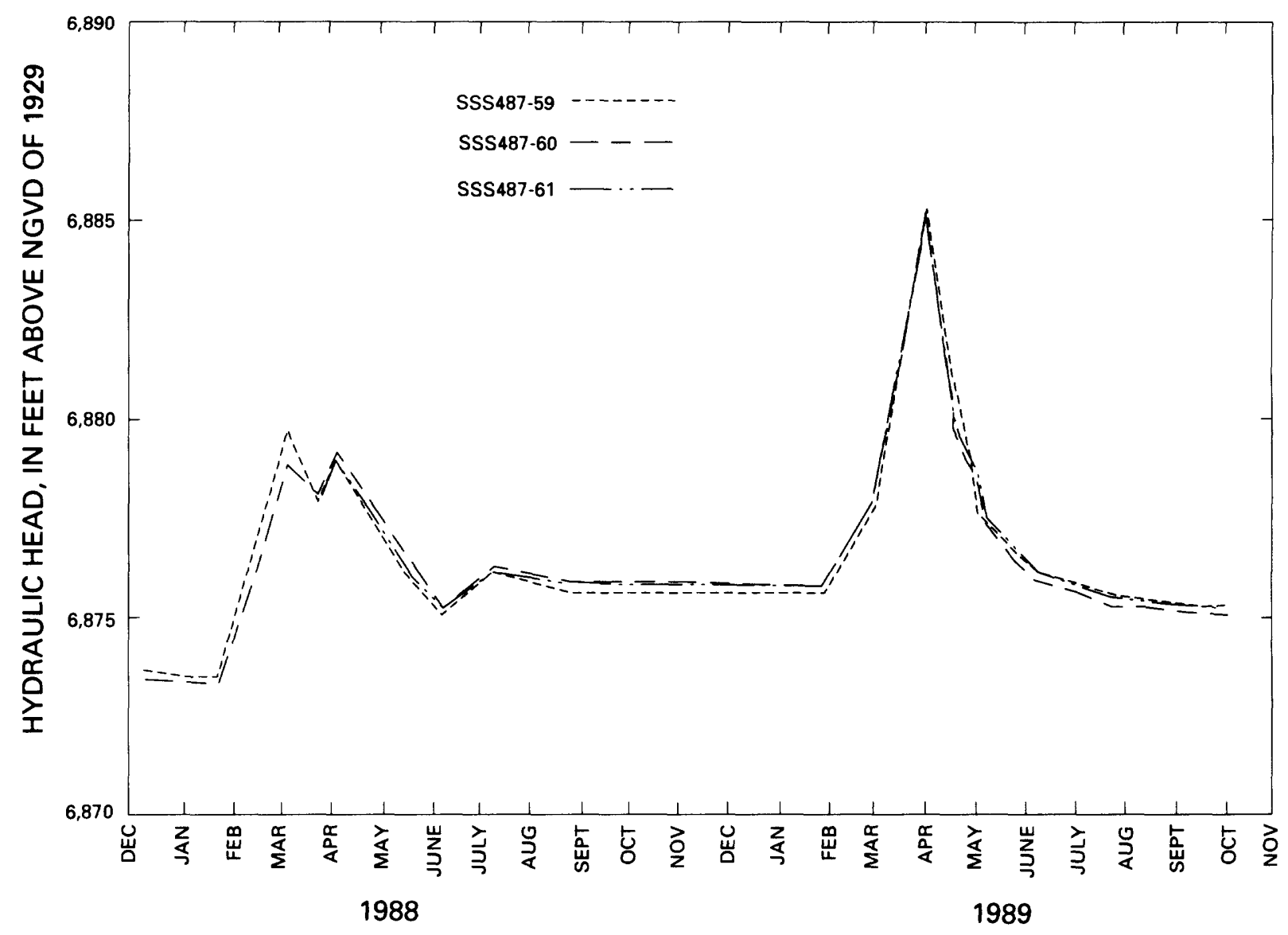

Figure 18.--Water levels for reclaimed-spoil wells at the Spring Creek site. 


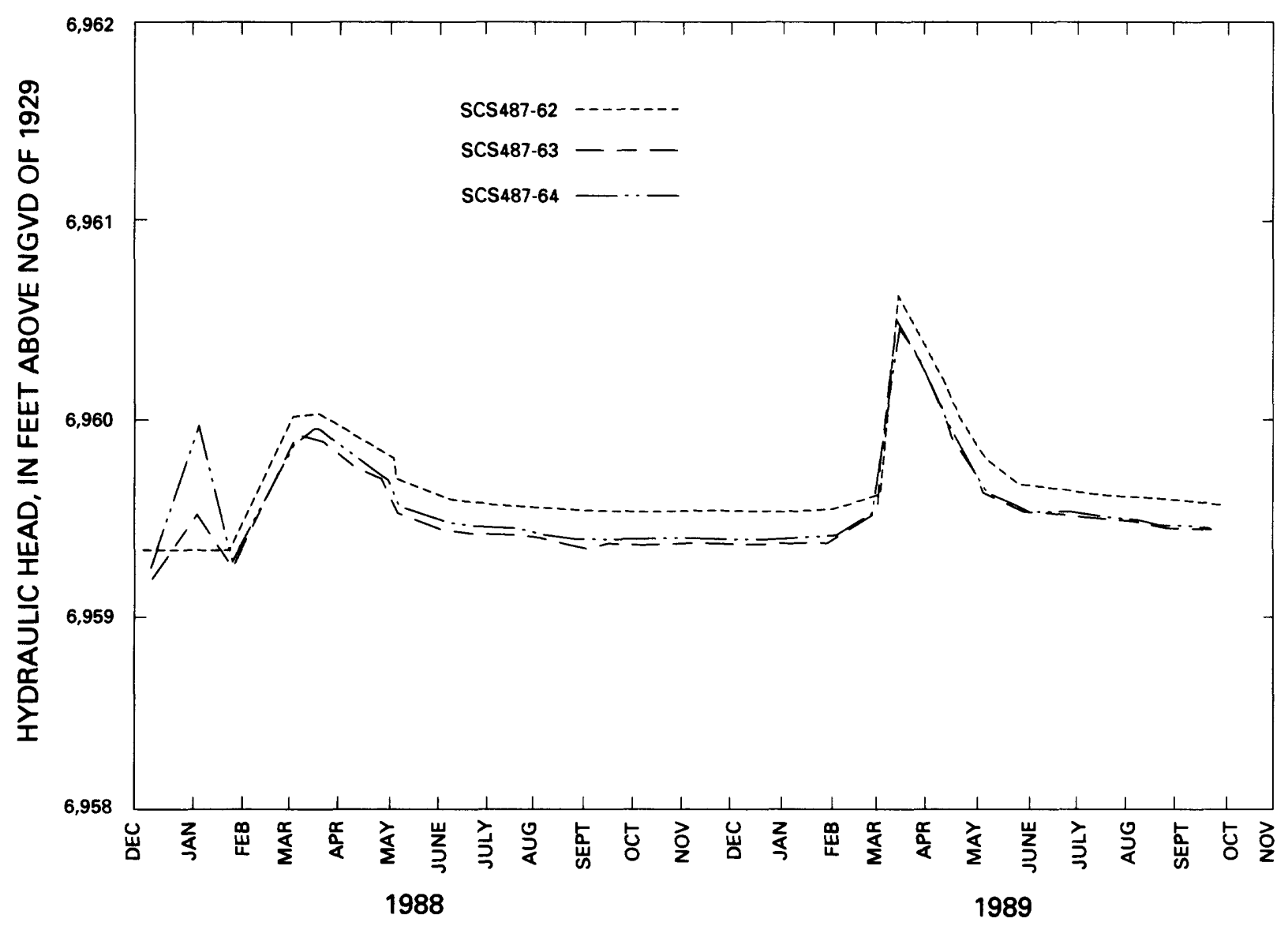

Figure 19.--Water levels for reclaimed-spoil wells at the Cow Camp Creek site. 


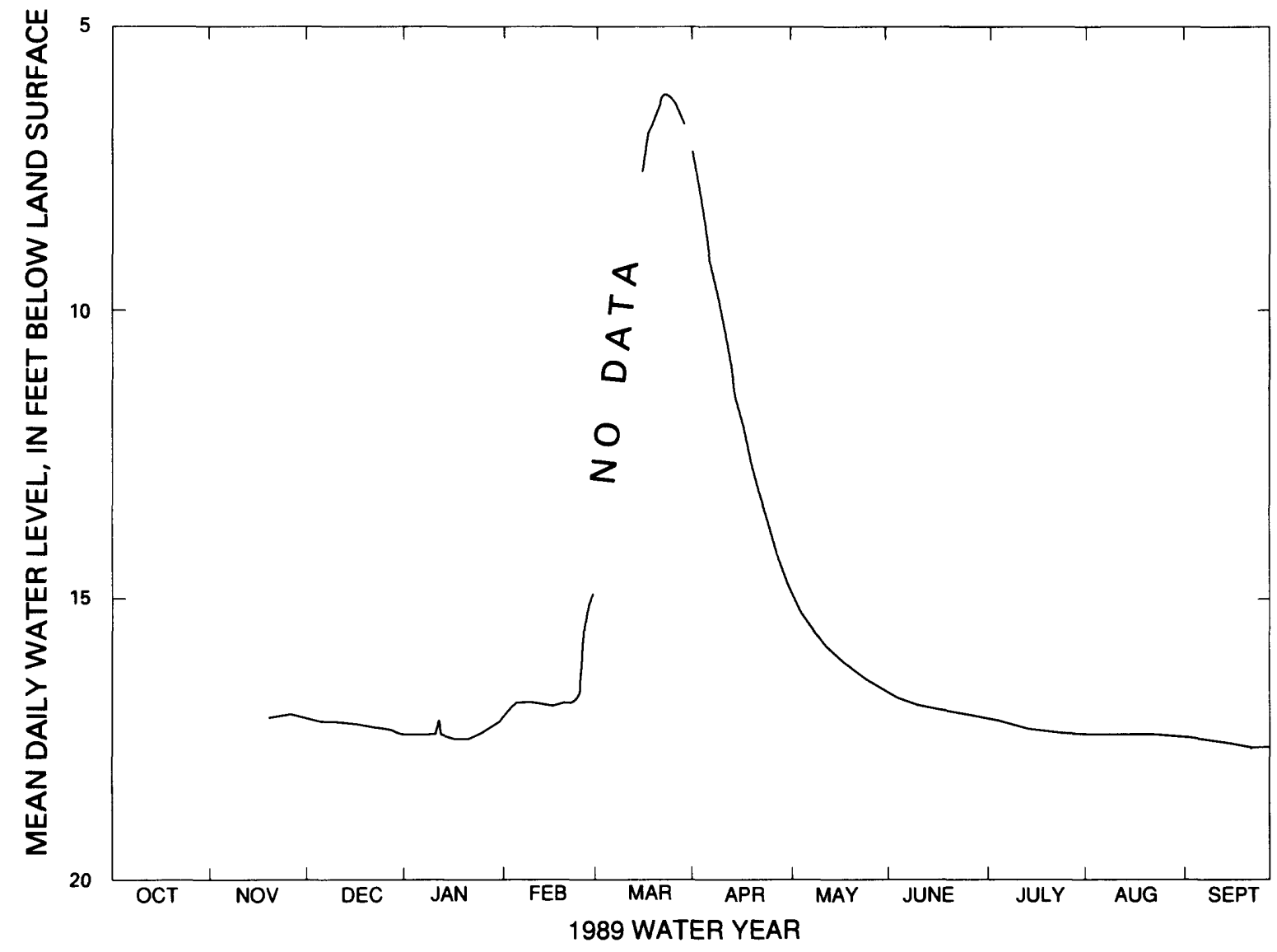

Figure 20.--Mean daily water levels for reclaimed-spoil well SSS487-60 at the Spring Creek site. 


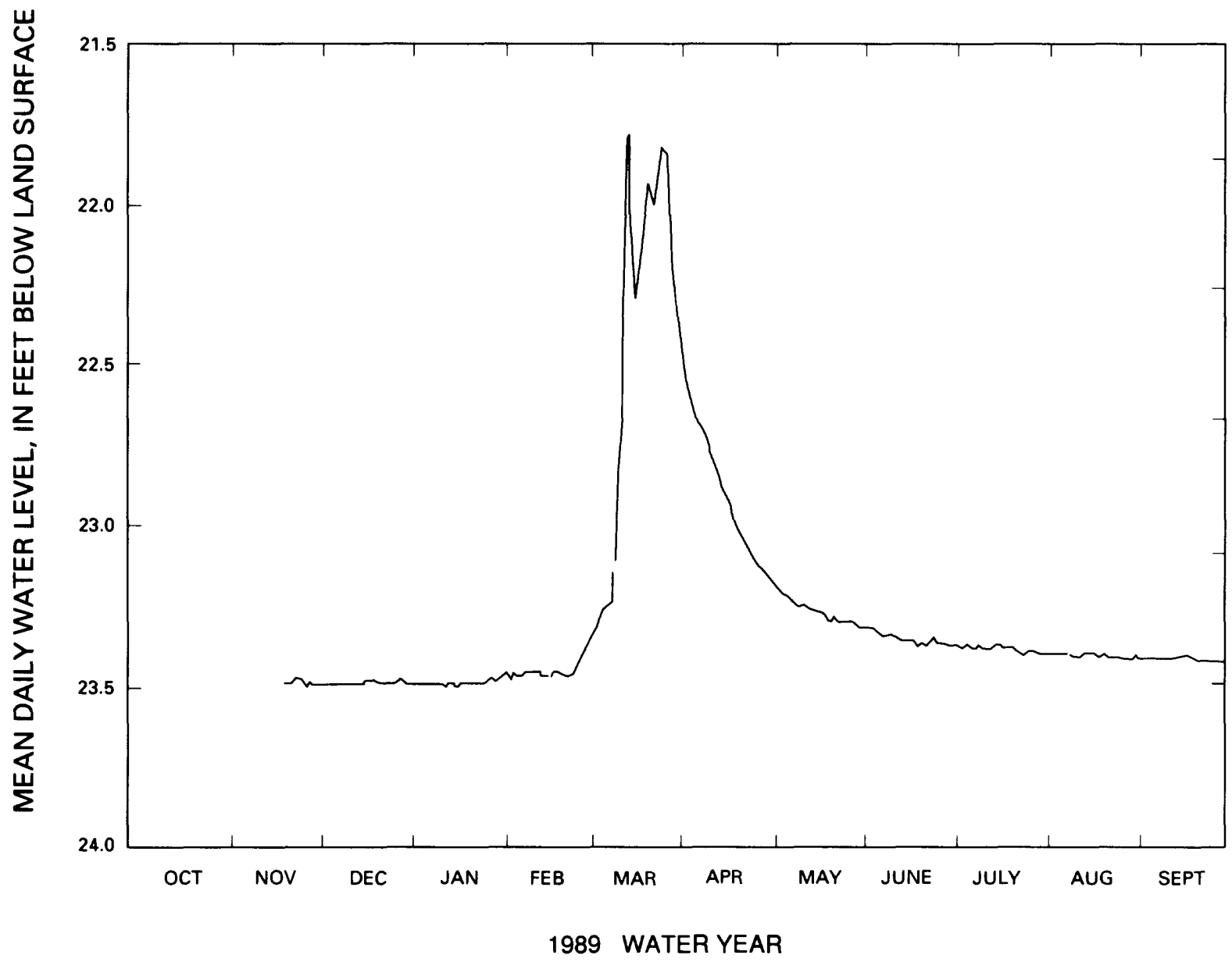

Figure 21.--Mean daily water levels for reclaimed-spoil well SCS487-63 at the Cow Camp Creek site.

For selected core samples (figs. 22 to 25), porosity, grain density, and permeability were analyzed by Core Laboratories, Aurora, Colorado (tables 15 to 18). Mineralogic composition of core samples from selected lithologic units at the Cow Camp Creek site was analyzed by the Office of Energy and Marine Geology, Branch of Petroleum Geology, U.S. Department of the Interior (table 19). 


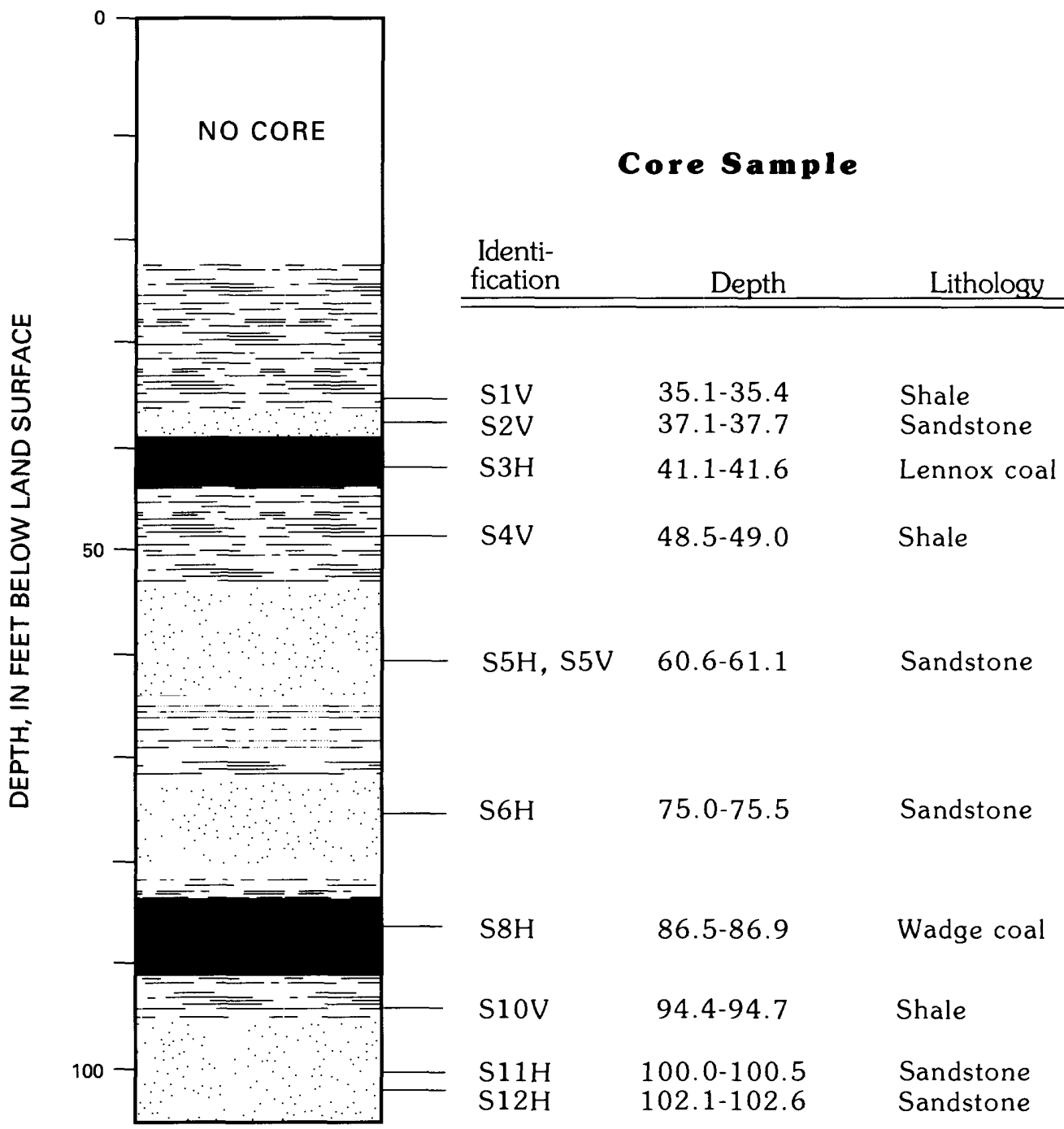

EXPLANATION

$\begin{array}{ll}\because \because \ddots 6 \text { SANDSTONE } & \\ \text { COAL } & \end{array}$

Figure 22.--Core-sample identification, depth, and lithology for the Spring Creek core hole. 


\section{Core Sample}

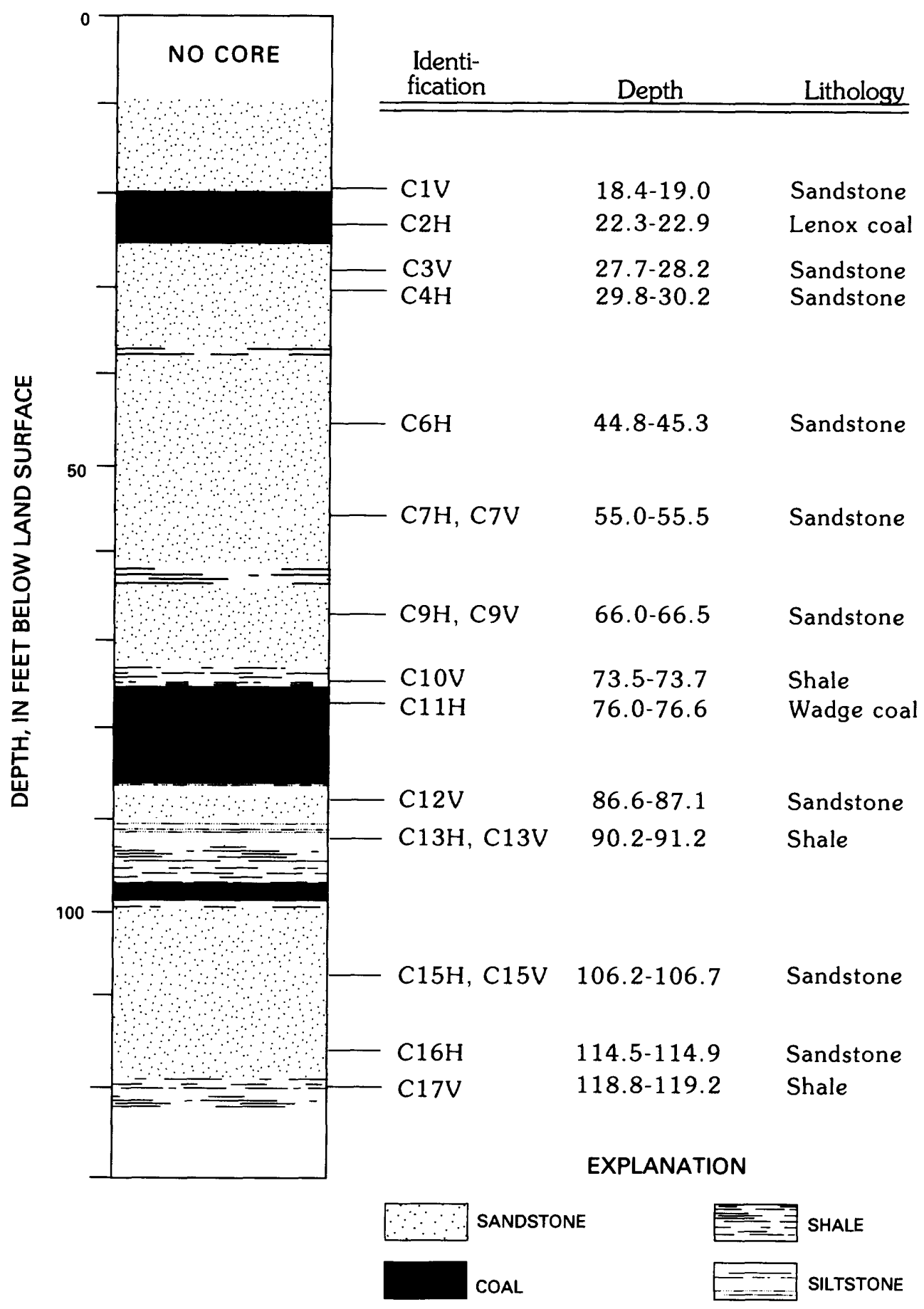

Figure 23.--Core-sample identification, depth, and lithology for the Cow Camp Creek core hole. 


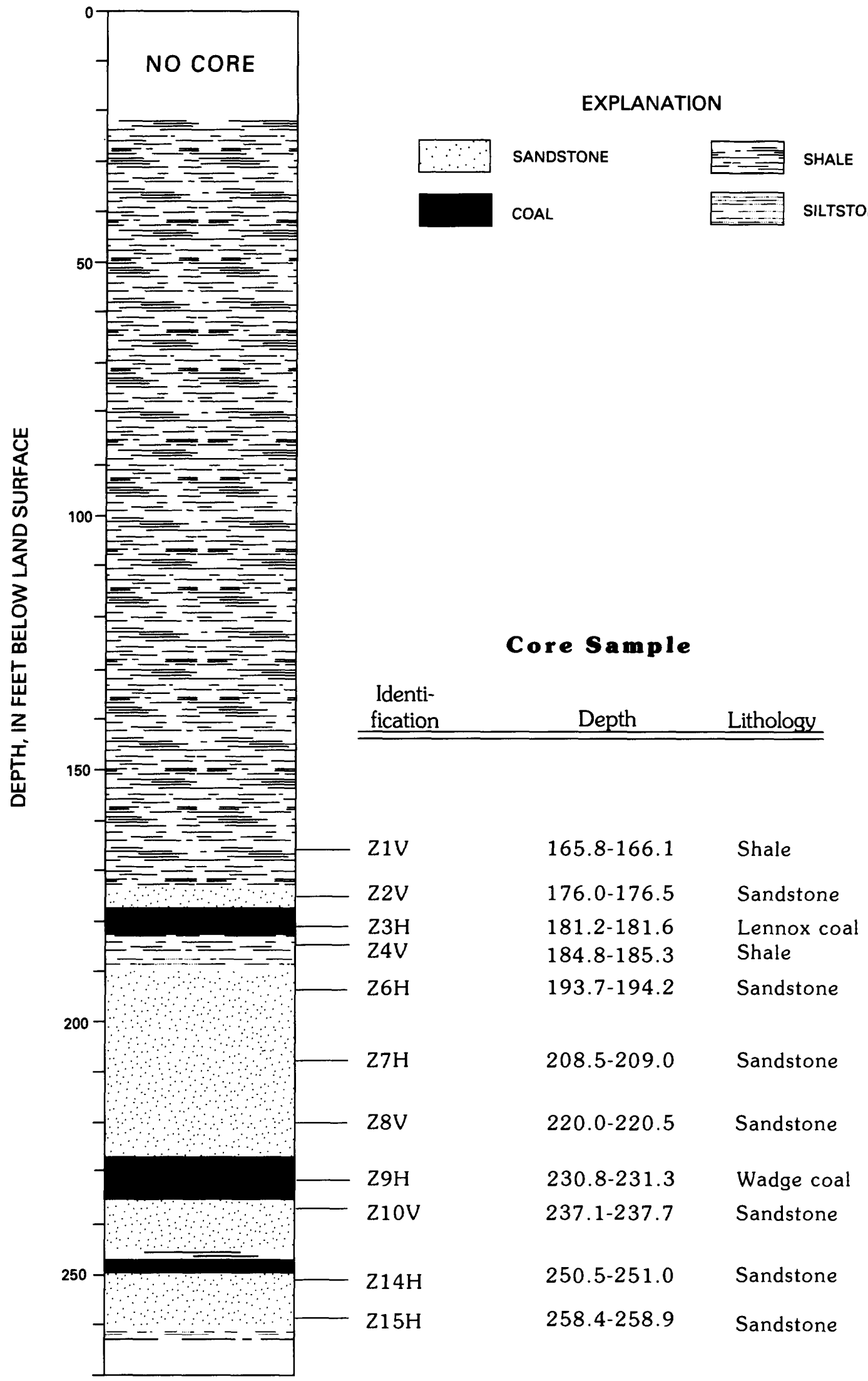

Figure 24.--Core-sample identification, depth, and lithology for the Zuli core hole. 


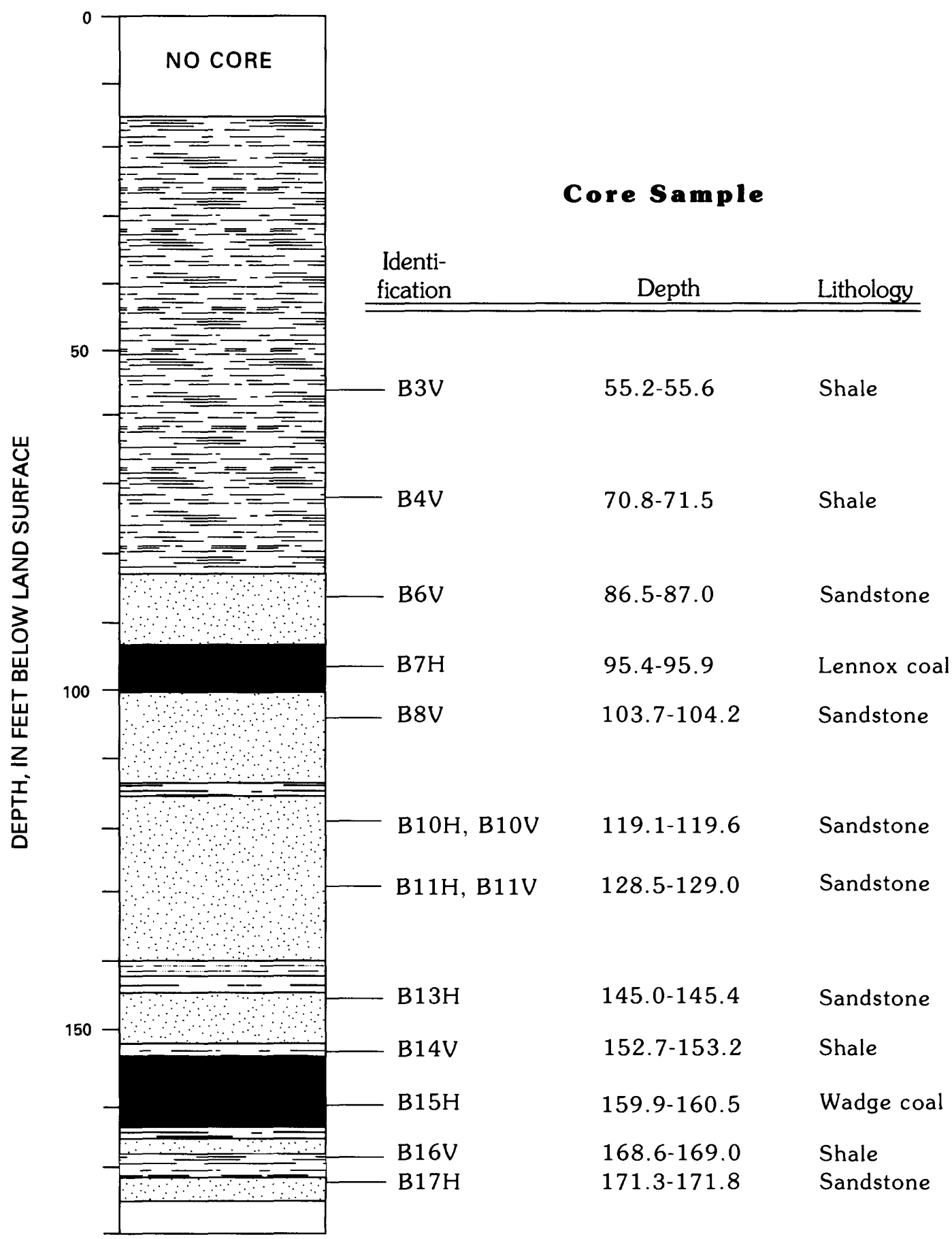

EXPLANATION

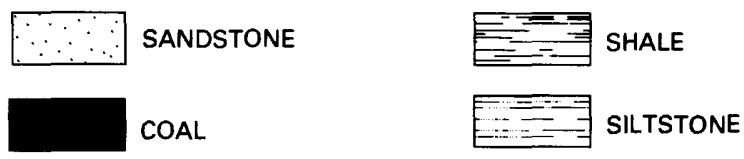

Figure 25.--Core-sample identification, depth, and lithology for the Bond Creek core hole. 
Springs were monitored at the Spring Creek site (figs. 2 and 5; table 20) and at the Cow Camp Creek site (figs. 2 and 6; table 20). Spring stage was monitored with a staff gage and recorded every one-half hour on a punch tape with a digital recorder. Discharge measurements were made by collecting the discharge water in calibrated buckets. Hydrographs for the springs are plotted (figs. 26 and 27), and mean daily discharge information is listed (tables 21 to 24 ).

Water samples were collected for water-quality analysis from the wells except for well SCS487-65, which was dry during the period of sample collection (table 25). Water samples were collected for water-quality analysis from the springs and the lysimeters (table 26). The core samples also were combined with water and used for batch-mixing experiments (fig. 28; table 27). The batch-mixing experiments are discussed in Clark and Williams (1991).

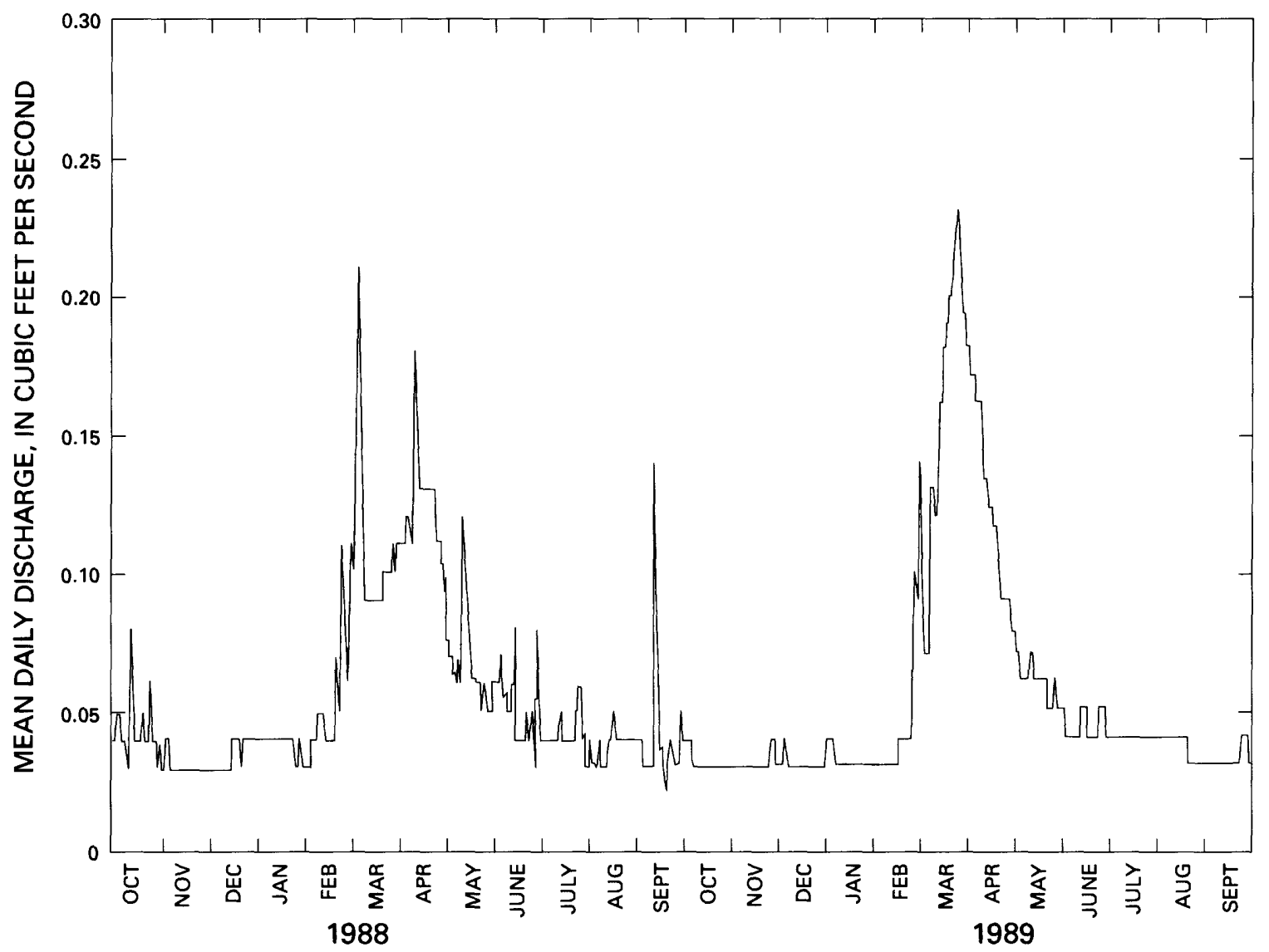

Figure 26.--Hydrograph showing mean daily discharge for the spring at the Spring Creek site, water years 1988 and 1989. 


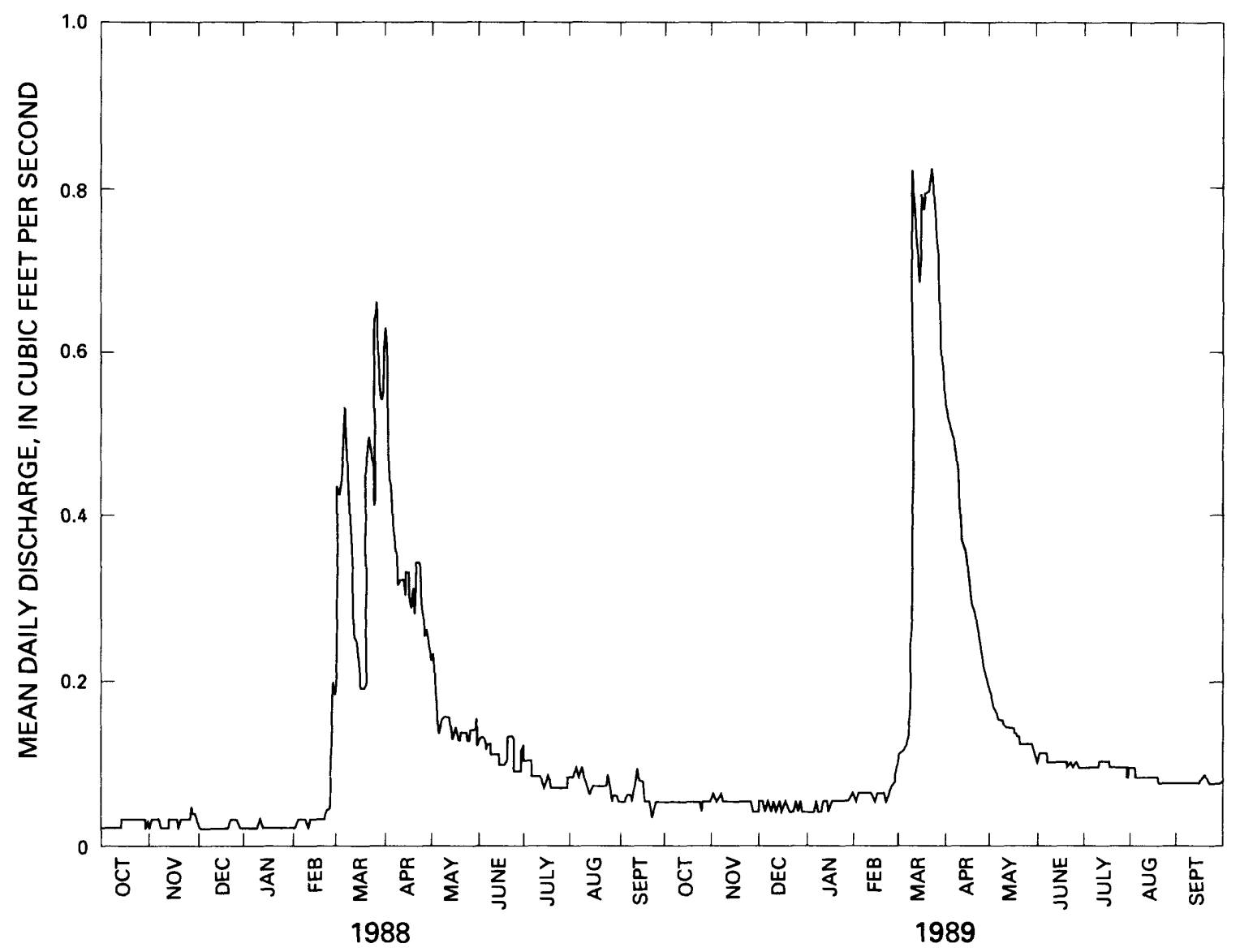

Figure 27.--Hydrograph showing mean daily discharge for the spring at the Cow Camp Creek site, water years 1988 and 1989.

\section{REFERENCES CITED}

Bass, N.W., Eby, J.B., and Campbell, M.R., 1955 [1956], Geology and mineral fuels of parts of Routt and Moffat Counties, Colorado: U.S. Geological Survey Bulletin 1027-D, p. 143-250.

Clark, G.M., and Williams, R.S., Jr., 1990, Hydrologic and geochemical characterization of recharge and groundwater flow in a reclaimedcoal-mined land, northwestern Colorado, in Fifth Billings Symposium on Disturbed Land Rehabilitation--volume II, Hazardous waste management; wildlife; hydrology; drainages, erosion and wetlands; soils, minesoils and overburden; linear disturbances; oil and gas [1990 Proceedings]: Bozeman, Montana State University, Reclamation Research Unit Publication 9003 , p. 173-185.

1991, Identification of dissolved-constituent sources in mine-site ground water using batch mixing: Water Resources Bulletin, v. 27, no. 1, p. 93-100.

ENMAP Corporation, 1981, Guide to Colorado's weather and climate: Boulder, Colo., map pamphlet.

Tweto, Ogden, 1976, Geologic map of the Craig $1^{\circ} \times 2^{\circ}$ quadrangle, northwestern Colorado: U.S. Geological Survey Miscellaneous Investigations Series Map I-972, scale $1: 250,000$. 


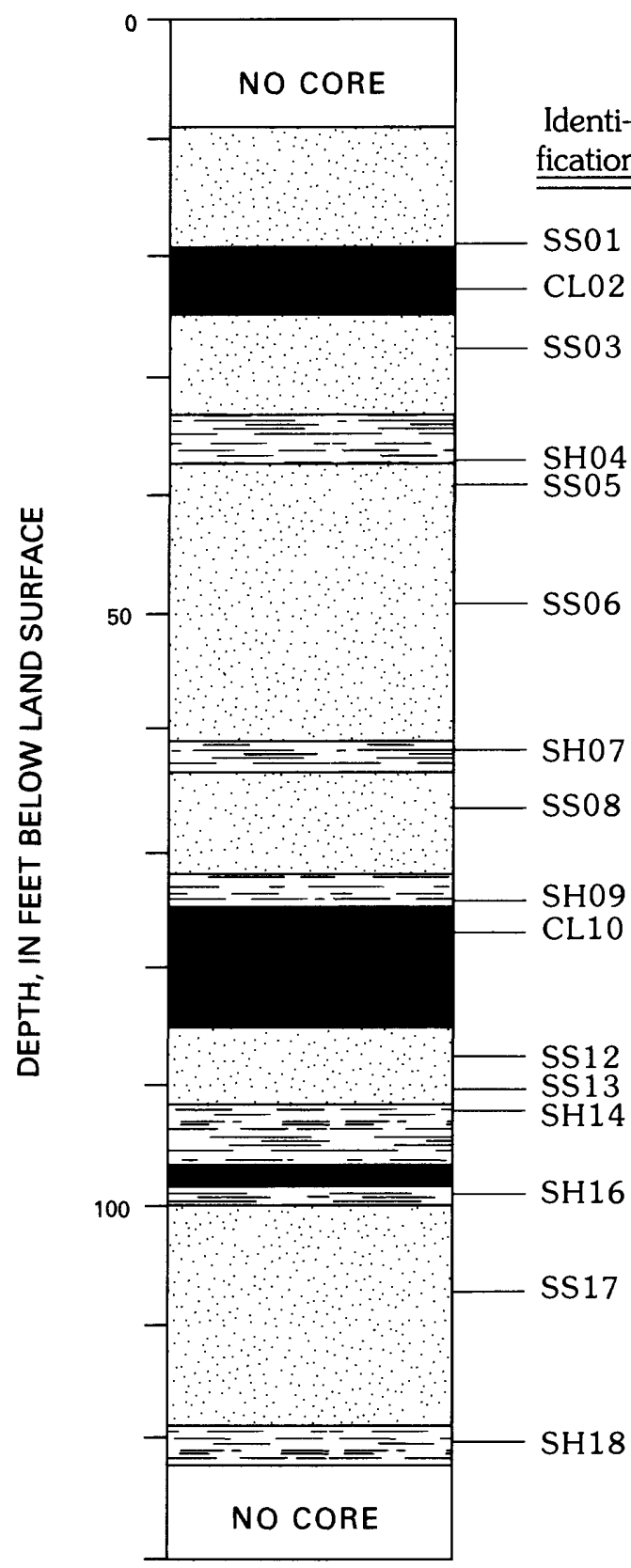

\section{Core Sample}

Identi-

\begin{tabular}{lll} 
fication & Depth & Lithology \\
\hline SS01 & $18.4-18.7$ & Sandstone \\
CL02 & $22.6-22.9$ & Lennox coal \\
SS03 & $27.9-28.2$ & Sandstone
\end{tabular}

27.9-28.2 Sandstone

36.7-37.0 Shale

39.2-39.5 Sandstone

49.0-49.3 Sandstone

61.0-61.3 Shale

66.1-66.5 Sandstone

73.5-73.7 Shale

76.0-76.4 Wadge coal

87.1-87.4 Sandstone

89.7-90.0 Sandstone

90.9-91.2 Shale

98.3-98.6 Shale

106.2-106.5 Sandstone

118.0-119.2 Shale

\section{EXPLANATION}

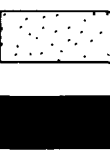

SANDSTONE

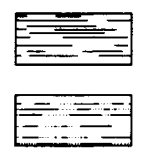

SHALE

COAL

SILTSTONE

Figure 28.--Identification of depth and lithology of Cow Camp Creek core samples used for batch-mixing experiments. 
DATA 
Table 1.--Air-temperature, relative-humidity, solar-radiation, and precipitation data, October 1987-December 1989

$[--$, no data $]$

\begin{tabular}{|c|c|c|c|c|c|c|}
\hline \multirow{2}{*}{$\begin{array}{l}\text { Day } \\
\text { of } \\
\text { month }\end{array}$} & Air temperature & \multirow{2}{*}{$\begin{array}{l}\text { Average } \\
\text { relative } \\
\text { humidity } \\
\text { (percent) }\end{array}$} & \multirow{2}{*}{$\begin{array}{c}\text { Solar } \\
\text { radiation } \\
\text { (langleys) }\end{array}$} & \multicolumn{3}{|c|}{$\begin{array}{c}\text { Precipitation } \\
\text { (inches) }\end{array}$} \\
\hline & $\begin{array}{c}\text { Minimum Average Maximum } \\
\text { (degrees Celsius) }\end{array}$ & & & $\begin{array}{l}\text { Spring } \\
\text { Creek } 1\end{array}$ & $\begin{array}{l}\text { Spring } \\
\text { Creek } 2\end{array}$ & $\begin{array}{c}\text { Cow } \\
\text { Camp } \\
\text { Creek }\end{array}$ \\
\hline
\end{tabular}

OCTOBER 1987

\begin{tabular}{|c|c|c|c|c|c|c|c|c|}
\hline 1 & -- & -- & -- & -- & -- & -- & -- & -- \\
\hline 2 & -- & -- & -- & -- & -- & -- & -- & -- \\
\hline 3 & -- & -- & -- & -- & -- & -- & -- & -- \\
\hline 4 & -- & -- & -- & -- & -- & -- & -- & - \\
\hline 5 & -- & -- & -- & -- & -- & -- & -- & -- \\
\hline 6 & - & -- & - & -- & -- & -- & -- & -- \\
\hline 7 & - & -- & -- & -- & -- & -- & -- & -- \\
\hline 8 & -- & -- & -- & -- & -- & -- & -- & -- \\
\hline 9 & -- & -- & -- & -- & -- & -- & -- & - \\
\hline 10 & -- & -- & -- & -- & -- & -- & -- & - \\
\hline 11 & -- & -- & -- & -- & -- & -- & -- & -- \\
\hline 12 & -- & -- & -- & -- & -- & -- & -- & -- \\
\hline 13 & -- & -- & -- & -- & -- & -- & -- & -- \\
\hline 14 & -- & -- & -- & -- & -- & -- & -- & - \\
\hline 15 & -- & -- & -- & -- & -- & -- & -- & -- \\
\hline 16 & -- & -- & -- & -- & -- & -- & -- & -- \\
\hline 17 & -- & -- & -- & -- & -- & -- & -- & -- \\
\hline 18 & -- & -- & -- & -- & -- & -- & -- & -- \\
\hline 19 & -- & -- & -- & -- & -- & -- & -- & -- \\
\hline 20 & -- & -- & -- & -- & -- & -- & -- & -- \\
\hline 21 & -- & -- & -- & -- & -- & -- & -- & -- \\
\hline 22 & -- & -- & -- & -- & -- & -- & -- & -- \\
\hline 23 & -- & -- & -- & -- & -- & -- & -- & -- \\
\hline 24 & -- & -- & -- & -- & -- & -- & -- & -- \\
\hline 25 & -- & -- & -- & -- & -- & -- & -- & -- \\
\hline 26 & -- & -- & -- & -- & -- & -- & -- & -- \\
\hline 27 & -- & -- & -- & -- & -- & -- & -- & -- \\
\hline 28 & -- & -- & -- & -- & -- & -- & -- & -- \\
\hline 29 & 1 & 9 & 19 & 54 & 272 & 0.00 & -- & -- \\
\hline 30 & 4 & 6 & 8 & 89 & 59 & .30 & - & - \\
\hline 31 & 1 & 7 & 14 & 79 & 284 & .00 & -- & -- \\
\hline
\end{tabular}


Table 1.--Air-temperature, relative-humidity, solar-radiation, and precipitation data, October 1987-December 1989--Continued

\begin{tabular}{|c|c|c|c|c|c|c|}
\hline \multirow{2}{*}{$\begin{array}{l}\text { Day } \\
\text { of } \\
\text { month }\end{array}$} & Air temperature & \multirow{2}{*}{$\begin{array}{l}\text { Average } \\
\text { relative } \\
\text { humidity } \\
\text { (percent) }\end{array}$} & \multirow{2}{*}{$\begin{array}{c}\text { Solar } \\
\text { radiation } \\
\text { (langleys) }\end{array}$} & \multicolumn{3}{|c|}{$\begin{array}{c}\text { Precipitation } \\
\text { (inches) }\end{array}$} \\
\hline & $\begin{array}{c}\text { Minimum Average Maximum } \\
\text { (degrees Celsius) }\end{array}$ & & & $\begin{array}{l}\text { Spring } \\
\text { Creek } 1\end{array}$ & $\begin{array}{l}\text { Spring } \\
\text { Creek } 2\end{array}$ & $\begin{array}{c}\text { Cow } \\
\text { Camp } \\
\text { Creek }\end{array}$ \\
\hline
\end{tabular}

NOVEMBER 1987

\begin{tabular}{|c|c|c|c|c|c|c|c|c|}
\hline 1 & 3 & 8 & 16 & 79 & 185 & 0.12 & -- & -- \\
\hline 2 & 3 & 6 & 12 & 84 & 155 & .14 & -- & -- \\
\hline 3 & 2 & 6 & 11 & 81 & 160 & .00 & -- & -- \\
\hline 4 & 0 & 6 & 14 & 74 & 308 & .00 & - & -- \\
\hline 5 & -1 & 8 & 18 & 58 & 298 & .00 & -- & -- \\
\hline 6 & -1 & 6 & 12 & 73 & 161 & .03 & -- & -- \\
\hline 7 & -1 & 1 & 5 & 92 & 83 & .08 & - & - \\
\hline 8 & -2 & 2 & 10 & 82 & 276 & .00 & -- & -- \\
\hline 9 & -4 & 3 & 12 & 62 & 293 & .00 & -- & -- \\
\hline 10 & -5 & 0 & 10 & 75 & 137 & .05 & -- & -- \\
\hline 11 & -7 & -1 & 6 & 88 & 351 & .06 & -- & -- \\
\hline 12 & -6 & 1 & 8 & 74 & 208 & .00 & -- & -- \\
\hline 13 & -1 & 4 & 11 & 63 & 187 & .00 & - & -- \\
\hline 14 & -4 & 0 & 3 & 97 & 56 & .27 & -- & -- \\
\hline 15 & -12 & -5 & -3 & 92 & 81 & .13 & -- & -- \\
\hline 16 & -14 & -8 & -3 & 82 & 154 & .00 & -- & -- \\
\hline 17 & -14 & -7 & -3 & 88 & 125 & .10 & - & -- \\
\hline 18 & -12 & -8 & -1 & 71 & 283 & .00 & -- & -- \\
\hline 19 & -10 & -3 & 5 & 61 & 264 & .00 & -- & -- \\
\hline 20 & -5 & 2 & 9 & 54 & 257 & .00 & 0.00 & 0.00 \\
\hline 21 & -7 & 0 & 7 & 60 & 165 & .00 & .00 & .00 \\
\hline 22 & -7 & -2 & 2 & 83 & 124 & .00 & .00 & .03 \\
\hline 23 & -5 & -2 & 5 & 86 & 192 & .15 & .15 & .25 \\
\hline 24 & -12 & -6 & 1 & 76 & 161 & .00 & .00 & .00 \\
\hline 25 & -10 & -4 & 4 & 64 & 209 & .00 & .00 & .00 \\
\hline 26 & -5 & -3 & 1 & 73 & 143 & .00 & .00 & .00 \\
\hline 27 & -10 & -6 & -1 & 84 & 158 & .00 & .01 & .00 \\
\hline 28 & -11 & -5 & 4 & 67 & 239 & .00 & .00 & .00 \\
\hline 29 & -9 & -5 & 3 & 67 & 207 & .00 & .00 & .00 \\
\hline 30 & -11 & -5 & 2 & 71 & 214 & .00 & .00 & .00 \\
\hline
\end{tabular}


Table 1.--Air-temperature, relative-humidity, solar-radiation, and precipitation data, October 1987-December 1989--Continued

\begin{tabular}{|c|c|c|c|c|c|c|c|c|}
\hline \multirow{2}{*}{$\begin{array}{l}\text { Day } \\
\text { of } \\
\text { month }\end{array}$} & \multicolumn{3}{|c|}{ Air temperature } & \multirow{2}{*}{$\begin{array}{l}\text { Average } \\
\text { relative } \\
\text { humidity } \\
\text { (percent) }\end{array}$} & \multirow{2}{*}{$\begin{array}{c}\text { Solar } \\
\text { radiation } \\
\text { (langleys) }\end{array}$} & \multicolumn{3}{|c|}{$\begin{array}{c}\text { Precipitation } \\
\text { (inches) }\end{array}$} \\
\hline & $\begin{array}{r}\text { Minimum } \\
\text { (deg }\end{array}$ & $\begin{array}{l}\text { Averag } \\
\text { rees Ce }\end{array}$ & $\begin{array}{l}\text { Maximum } \\
\text { ius ) }\end{array}$ & & & $\begin{array}{l}\text { Spring } \\
\text { Creek } 1\end{array}$ & $\begin{array}{l}\text { Spring } \\
\text { Creek } 2\end{array}$ & $\begin{array}{l}\text { Cow } \\
\text { Camp } \\
\text { Creek }\end{array}$ \\
\hline \multicolumn{9}{|c|}{ DECEMBER 1987} \\
\hline 1 & -12 & -4 & 1 & 68 & 179 & 0.02 & 0.03 & 0.00 \\
\hline 2 & -4 & 0 & 4 & 76 & 119 & .00 & .00 & .01 \\
\hline 3 & -2 & 3 & 11 & 66 & 230 & .00 & .00 & .00 \\
\hline 4 & -3 & 4 & 14 & 61 & 206 & .00 & .00 & .00 \\
\hline 5 & -2 & 1 & 6 & 91 & 37 & .25 & .27 & .35 \\
\hline 6 & -3 & 1 & 9 & 88 & 230 & .00 & .00 & .00 \\
\hline 7 & -7 & -1 & 8 & 89 & 143 & .21 & .11 & .37 \\
\hline 8 & -11 & -6 & -1 & 80 & 233 & .00 & .00 & .00 \\
\hline 9 & -9 & -4 & -1 & 82 & 101 & .03 & .03 & .03 \\
\hline 10 & -3 & 2 & 9 & 79 & 111 & .05 & .02 & .11 \\
\hline 11 & -8 & -4 & 1 & 59 & 201 & .00 & .00 & .00 \\
\hline 12 & -14 & -10 & -6 & 67 & 192 & .00 & .00 & .00 \\
\hline 13 & -13 & -10 & -6 & 56 & 175 & .00 & .00 & .00 \\
\hline 14 & -17 & -13 & -9 & 69 & 207 & .00 & .00 & .00 \\
\hline 15 & -20 & -13 & -6 & 63 & 228 & .00 & .00 & .00 \\
\hline 16 & -14 & -7 & -3 & 51 & 128 & .00 & .00 & .00 \\
\hline 17 & -7 & -3 & 2 & 88 & 105 & .00 & .00 & .00 \\
\hline 18 & -8 & -4 & -2 & 97 & 70 & .07 & .06 & .10 \\
\hline 19 & -13 & -6 & -3 & 95 & 42 & .08 & .09 & .13 \\
\hline 20 & -17 & -11 & -3 & 74 & 137 & .00 & .00 & .00 \\
\hline 21 & -11 & -7 & 0 & 76 & 64 & .05 & .07 & .07 \\
\hline 22 & -11 & -6 & -3 & 90 & 45 & .80 & .75 & .45 \\
\hline 23 & -11 & -8 & -4 & 98 & 19 & .44 & .43 & .89 \\
\hline 24 & -- & -- & -- & -- & -- & .01 & .02 & .00 \\
\hline 25 & -- & -- & -- & -. & $\ldots$ & -- & .00 & .00 \\
\hline 26 & -28 & -20 & -14 & 81 & 82 & -- & .03 & .03 \\
\hline 27 & -17 & -10 & -6 & 88 & 34 & -- & .07 & .08 \\
\hline 28 & -12 & -7 & 1 & 83 & 114 & -- & .00 & .00 \\
\hline 29 & -12 & -5 & 4 & 71 & 221 & .00 & .00 & .00 \\
\hline 30 & -12 & -8 & 2 & 87 & 152 & .15 & .14 & .25 \\
\hline 31 & -14 & -10 & -6 & 82 & 56 & .04 & .03 & .02 \\
\hline
\end{tabular}


Table 1.--Air-temperature, relative-humidity, solar-radiation, and precipitation data, October 1987-December 1989--Continued

\begin{tabular}{|c|c|c|c|c|c|c|c|c|}
\hline \multirow{2}{*}{$\begin{array}{l}\text { Day } \\
\text { of } \\
\text { month }\end{array}$} & \multicolumn{3}{|c|}{ Air temperature } & \multirow{2}{*}{$\begin{array}{l}\text { Average } \\
\text { relative } \\
\text { humidity } \\
\text { (percent) }\end{array}$} & \multirow{2}{*}{$\begin{array}{c}\text { Solar } \\
\text { radiation } \\
\text { (langleys) }\end{array}$} & \multicolumn{3}{|c|}{$\begin{array}{c}\text { Precipitation } \\
\text { (inches) }\end{array}$} \\
\hline & $\begin{array}{r}\overline{\text { Minimum }} \\
\text { (deg }\end{array}$ & $\begin{array}{l}\text { Average } \\
\text { rees Cels }\end{array}$ & $\begin{array}{l}\text { Maximum } \\
\text { ius) }\end{array}$ & & & $\begin{array}{l}\text { Spring } \\
\text { Creek } 1\end{array}$ & $\begin{array}{l}\text { Spring } \\
\text { Creek } 2\end{array}$ & $\begin{array}{c}\text { Cow } \\
\text { Camp } \\
\text { Creek }\end{array}$ \\
\hline & & & & JANUARY 1 & 988 & & & \\
\hline 1 & -- & -- & -- & -- & -- & 0.02 & 0.02 & 0.02 \\
\hline 2 & -- & -- & -- & -- & -- & .00 & .00 & .00 \\
\hline 3 & -23 & -15 & -9 & 62 & 200 & .00 & .00 & .00 \\
\hline 4 & -17 & -10 & -6 & 72 & 152 & .27 & .32 & .32 \\
\hline 5 & -9 & -6 & -4 & 97 & 59 & .16 & .25 & .20 \\
\hline 6 & -10 & -7 & -4 & 98 & 35 & .22 & .14 & .27 \\
\hline 7 & -10 & -9 & -5 & 94 & 59 & .03 & .05 & .06 \\
\hline 8 & -10 & -8 & -4 & 96 & 27 & .25 & .24 & .23 \\
\hline 9 & -11 & -8 & -5 & 96 & 21 & .45 & .40 & .50 \\
\hline 10 & -7 & -3 & 2 & 89 & 30 & .06 & .05 & .07 \\
\hline 11 & -9 & -3 & 6 & 82 & 158 & .19 & .11 & .39 \\
\hline 12 & -16 & -10 & -7 & 69 & 190 & .00 & .00 & .00 \\
\hline 13 & -18 & -11 & -6 & 73 & 178 & .00 & .00 & .05 \\
\hline 14 & -13 & -7 & -2 & 76 & 180 & .00 & .00 & .00 \\
\hline 15 & -10 & -3 & 5 & 74 & 177 & .05 & .05 & .11 \\
\hline 16 & -13 & -9 & -3 & 75 & 233 & .00 & .00 & .00 \\
\hline 17 & -12 & -7 & -2 & 85 & 104 & .10 & .10 & .13 \\
\hline 18 & -11 & -7 & -3 & 90 & 125 & .29 & .25 & .22 \\
\hline 19 & -22 & -12 & -6 & 68 & 143 & .00 & .00 & .00 \\
\hline 20 & -25 & -16 & -10 & 73 & 220 & .01 & .02 & .02 \\
\hline 21 & -18 & -12 & -7 & 84 & 106 & .04 & .06 & .06 \\
\hline 22 & -19 & -12 & -6 & 77 & 205 & .00 & .00 & .00 \\
\hline 23 & -12 & -8 & -4 & 80 & 167 & .00 & .00 & .05 \\
\hline 24 & -17 & -12 & -8 & 80 & 283 & .00 & .00 & .00 \\
\hline 25 & -19 & -11 & -5 & 69 & 200 & .00 & .00 & .00 \\
\hline 26 & -12 & -7 & -1 & 67 & 287 & .00 & .00 & .00 \\
\hline 27 & -10 & -3 & 5 & 66 & 292 & .00 & .00 & .00 \\
\hline 28 & -9 & -1 & 4 & 60 & 210 & .00 & .00 & .00 \\
\hline 29 & -7 & 0 & 6 & 64 & 248 & .00 & .00 & .08 \\
\hline 30 & -6 & -2 & 2 & 95 & 186 & .24 & .23 & .20 \\
\hline 31 & -7 & -5 & -2 & 99 & 66 & .58 & .55 & .66 \\
\hline
\end{tabular}


Table 1.--Air-temperature, relative-humidity, solar-radiation, and precipitation data, October 1987-December 1989--Continued

\begin{tabular}{|c|c|c|c|c|c|c|c|c|}
\hline \multirow{2}{*}{$\begin{array}{l}\text { Day } \\
\text { of } \\
\text { month }\end{array}$} & \multicolumn{3}{|c|}{ Air temperature } & \multirow{2}{*}{$\begin{array}{l}\text { Average } \\
\text { relative } \\
\text { humidity } \\
\text { (percent) }\end{array}$} & \multirow{2}{*}{$\begin{array}{c}\text { Solar } \\
\text { radiation } \\
\text { (langleys) }\end{array}$} & \multicolumn{3}{|c|}{$\begin{array}{c}\text { Precipitation } \\
\text { (inches) }\end{array}$} \\
\hline & $\begin{array}{r}\text { Minimum } \\
\text { (deg }\end{array}$ & $\begin{array}{l}\text { Averag } \\
\text { rees Ce }\end{array}$ & $\begin{array}{l}\text { Maximum } \\
\text { ius ) }\end{array}$ & & & $\begin{array}{l}\text { Spring } \\
\text { Creek } 1\end{array}$ & $\begin{array}{l}\text { Spring } \\
\text { Creek } 2\end{array}$ & $\begin{array}{c}\text { Cow } \\
\text { Camp } \\
\text { Creek }\end{array}$ \\
\hline \multicolumn{9}{|c|}{ FEBRUARY 1988} \\
\hline 1 & -12 & -7 & -3 & 95 & 124 & 0.27 & 0.26 & 0.30 \\
\hline 2 & -8 & -6 & -3 & 96 & 46 & .15 & .14 & .13 \\
\hline 3 & -17 & -10 & -4 & 75 & 69 & .00 & .00 & .01 \\
\hline 4 & -19 & -12 & -1 & 68 & 117 & .00 & .00 & .02 \\
\hline 5 & -17 & -10 & -1 & 67 & 348 & .00 & .00 & .00 \\
\hline 6 & -16 & -8 & 0 & 66 & 330 & .00 & .00 & .00 \\
\hline 7 & -12 & -6 & 2 & 69 & 289 & .00 & .00 & .01 \\
\hline 8 & -6 & -3 & -1 & 89 & 78 & .08 & .09 & .09 \\
\hline 9 & -7 & -2 & 4 & 82 & 213 & .07 & .04 & .15 \\
\hline 10 & -6 & -3 & 1 & 83 & 290 & .00 & .00 & .00 \\
\hline 11 & -7 & -4 & 0 & 85 & 162 & .00 & .00 & .00 \\
\hline 12 & -10 & -4 & 5 & 73 & 358 & .00 & .00 & .00 \\
\hline 13 & -10 & -4 & 4 & 73 & 258 & .05 & .05 & .17 \\
\hline 14 & -9 & -6 & -3 & 79 & 305 & .00 & .00 & .00 \\
\hline 15 & -8 & -3 & 3 & 71 & 331 & .00 & .00 & .09 \\
\hline 16 & -11 & -7 & -2 & 86 & 275 & .04 & .03 & .00 \\
\hline 17 & -13 & -9 & -2 & 79 & 390 & .00 & .00 & .00 \\
\hline 18 & -14 & -10 & -5 & 73 & 260 & .00 & .00 & .00 \\
\hline 19 & -14 & -9 & -3 & 82 & 398 & .00 & .00 & .00 \\
\hline 20 & -8 & -4 & 0 & 81 & 213 & .00 & .00 & .00 \\
\hline 21 & -9 & -3 & 5 & 77 & 387 & .00 & .00 & .00 \\
\hline 22 & -9 & -5 & 2 & 71 & 237 & .00 & .00 & .00 \\
\hline 23 & -10 & -5 & 5 & 67 & 410 & .00 & .00 & .00 \\
\hline 24 & -10 & -3 & 6 & 59 & 411 & .00 & .00 & .00 \\
\hline 25 & -8 & -1 & 8 & 57 & 420 & .00 & .00 & .00 \\
\hline 26 & -8 & -1 & 7 & 58 & 423 & .00 & .00 & .00 \\
\hline 27 & -8 & 0 & 10 & 58 & 424 & .00 & .00 & .00 \\
\hline 28 & -3 & 0 & 3 & 90 & 174 & .10 & .12 & .12 \\
\hline 29 & 0 & 4 & 9 & 68 & 307 & .00 & .00 & .00 \\
\hline
\end{tabular}


Table 1.--Air-temperature, relative-humidity, solar-radiation, and precipitation data, October 1987-December 1989--Continued

\begin{tabular}{|c|c|c|c|c|c|c|c|c|}
\hline \multirow{2}{*}{$\begin{array}{l}\text { Day } \\
\text { of } \\
\text { month }\end{array}$} & \multicolumn{3}{|c|}{ Air temperature } & \multirow{2}{*}{$\begin{array}{l}\text { Average } \\
\text { relative } \\
\text { humidity } \\
\text { (percent) }\end{array}$} & \multirow{2}{*}{$\begin{array}{c}\text { Solar } \\
\text { radiation } \\
\text { (langleys) }\end{array}$} & \multicolumn{3}{|c|}{$\begin{array}{c}\text { Precipitation } \\
\text { (inches) }\end{array}$} \\
\hline & $\begin{array}{r}\text { Minimum } \\
\text { (deg) }\end{array}$ & $\begin{array}{l}\text { Average } \\
\text { rees Cels }\end{array}$ & $\begin{array}{l}\text { Maximum } \\
\text { ius ) }\end{array}$ & & & $\begin{array}{l}\text { Spring } \\
\text { Creek } 1\end{array}$ & $\begin{array}{l}\text { Spring } \\
\text { Creek } 2\end{array}$ & $\begin{array}{c}\text { Cow } \\
\text { Camp } \\
\text { Creek }\end{array}$ \\
\hline \multicolumn{9}{|c|}{ MARCH 1988} \\
\hline 1 & -4 & 1 & 7 & 87 & 338 & 0.20 & 0.19 & 0.10 \\
\hline 2 & -5 & -2 & 3 & 98 & 122 & .02 & .05 & .05 \\
\hline 3 & -4 & -1 & 3 & 86 & 413 & .00 & .00 & .01 \\
\hline 4 & -7 & -2 & 2 & 76 & 388 & .07 & .05 & .13 \\
\hline 5 & -9 & -2 & 4 & 60 & 454 & .00 & .00 & .00 \\
\hline 6 & -6 & 1 & 8 & 63 & 383 & .21 & .08 & .54 \\
\hline 7 & -11 & -7 & -4 & 92 & 387 & .06 & .10 & .03 \\
\hline 8 & -12 & -6 & 0 & 74 & 494 & .00 & .00 & .00 \\
\hline 9 & -8 & 0 & 8 & 65 & 495 & .00 & .00 & .00 \\
\hline 10 & -9 & -5 & 1 & 94 & 163 & .17 & .18 & .25 \\
\hline 11 & -12 & -8 & -5 & 85 & 384 & .00 & .00 & .00 \\
\hline 12 & -15 & -10 & -6 & 87 & 373 & .02 & .02 & .04 \\
\hline 13 & -15 & -11 & -7 & 78 & 467 & .03 & .02 & .01 \\
\hline 14 & -15 & -7 & -1 & 67 & 503 & .00 & .00 & .00 \\
\hline 15 & -10 & -2 & 4 & 71 & 401 & .02 & .01 & .01 \\
\hline 16 & -11 & -6 & -1 & 86 & 277 & .17 & .10 & .26 \\
\hline 17 & -16 & -10 & -4 & 85 & 497 & .02 & .02 & .00 \\
\hline 18 & -14 & -7 & 1 & 67 & 538 & .00 & .00 & .00 \\
\hline 19 & -10 & -2 & 7 & 60 & 551 & .00 & .00 & .01 \\
\hline 20 & -7 & 2 & 10 & 55 & 543 & .00 & .00 & .00 \\
\hline 21 & -3 & 5 & 15 & 46 & 559 & .00 & .00 & .00 \\
\hline 22 & -2 & 3 & 9 & 61 & 394 & .00 & .00 & .00 \\
\hline 23 & -5 & 2 & 11 & 67 & 344 & .15 & .10 & .35 \\
\hline 24 & -6 & -3 & 1 & 78 & 429 & .01 & .00 & .03 \\
\hline 25 & -3 & 3 & 7 & 54 & 188 & .00 & .00 & .00 \\
\hline 26 & -2 & 5 & 11 & 61 & 577 & .00 & .00 & .00 \\
\hline 27 & -7 & 5 & 16 & 52 & 487 & .10 & .09 & .14 \\
\hline 28 & -12 & -7 & -2 & 57 & 488 & .00 & .00 & .00 \\
\hline 29 & -11 & -4 & 2 & 53 & 445 & .00 & .00 & .01 \\
\hline 30 & -5 & -3 & -1 & 86 & 188 & .11 & .16 & .10 \\
\hline 31 & -5 & -1 & 5 & 57 & 575 & .00 & .00 & .00 \\
\hline
\end{tabular}


Table 1.--Air-temperature, relative-humidity, solar-radiation, and precipitation data, October 1987-December 1989--Continued

\begin{tabular}{|c|c|c|c|c|c|c|c|}
\hline \multirow{2}{*}{$\begin{array}{l}\text { Day } \\
\text { of } \\
\text { month }\end{array}$} & \multicolumn{2}{|c|}{ Air temperature } & \multirow{2}{*}{$\begin{array}{l}\text { Average } \\
\text { relative } \\
\text { humidity } \\
\text { (percent) }\end{array}$} & \multirow{2}{*}{$\begin{array}{c}\text { Solar } \\
\text { radiation } \\
\text { (langleys) }\end{array}$} & \multicolumn{3}{|c|}{$\begin{array}{c}\text { Precipitation } \\
\text { (inches) }\end{array}$} \\
\hline & $\begin{array}{r}\overline{\text { Minimum }} \\
(\text { deg }\end{array}$ & $\begin{array}{l}\text { Average Maximum } \\
\text { rees Celsius) }\end{array}$ & & & $\begin{array}{l}\text { Spring } \\
\text { Creek } 1\end{array}$ & $\begin{array}{l}\text { Spring } \\
\text { Creek } 2\end{array}$ & $\begin{array}{c}\text { Cow } \\
\text { Camp } \\
\text { Creek }\end{array}$ \\
\hline
\end{tabular}

\begin{tabular}{|c|c|c|c|c|c|c|c|c|}
\hline \multicolumn{9}{|c|}{ APRIL 1988} \\
\hline 1 & -10 & -2 & 7 & 69 & 631 & 0.00 & 0.00 & 0.00 \\
\hline 2 & -6 & 2 & 12 & 57 & 606 & .00 & .00 & .00 \\
\hline 3 & -3 & 7 & 15 & 40 & 523 & .00 & .00 & .00 \\
\hline 4 & -4 & 5 & 12 & 57 & 287 & .05 & .04 & .07 \\
\hline 5 & -8 & 1 & 10 & 57 & 687 & .08 & .06 & .13 \\
\hline 6 & -2 & 7 & 15 & 40 & 615 & .00 & .00 & .00 \\
\hline 7 & -1 & 11 & 20 & 33 & 619 & .00 & .00 & .00 \\
\hline 8 & -5 & 4 & 15 & 33 & 465 & .00 & .00 & .02 \\
\hline 9 & -7 & -3 & 4 & 66 & 461 & .00 & .00 & .02 \\
\hline 10 & -9 & 0 & 10 & 43 & 649 & .00 & .00 & .00 \\
\hline 11 & -3 & 7 & 16 & 29 & 636 & .00 & .00 & .00 \\
\hline 12 & -1 & 10 & 19 & 28 & 630 & .00 & .00 & .00 \\
\hline 13 & 3 & 12 & 21 & 25 & 573 & .00 & .00 & .00 \\
\hline 14 & 5 & 12 & 20 & 29 & 520 & .00 & .00 & .00 \\
\hline 15 & 2 & 8 & 16 & 68 & 363 & .00 & .03 & .02 \\
\hline 16 & 5 & 11 & 21 & 47 & 618 & .00 & .00 & .00 \\
\hline 17 & 3 & 8 & 13 & 57 & 298 & .00 & .00 & .00 \\
\hline 18 & -2 & 7 & 16 & 61 & 477 & .02 & .03 & .05 \\
\hline 19 & 2 & 7 & 14 & 74 & 330 & .15 & .14 & .15 \\
\hline 20 & 3 & 10 & 18 & 47 & 567 & .00 & .00 & .00 \\
\hline 21 & 1 & 6 & 12 & 66 & 224 & .02 & .02 & .23 \\
\hline 22 & -1 & 2 & 9 & 76 & 269 & .00 & .00 & .00 \\
\hline 23 & -3 & 2 & 9 & 75 & 410 & .00 & .00 & .02 \\
\hline 24 & -3 & 3 & 9 & 79 & 452 & .01 & .00 & .00 \\
\hline 25 & -5 & 0 & 8 & 94 & 186 & .26 & .22 & .38 \\
\hline 26 & -9 & 0 & 9 & 60 & 709 & .00 & .00 & .08 \\
\hline 27 & 0 & 7 & 16 & 43 & 435 & .00 & .00 & .00 \\
\hline 28 & 3 & 11 & 18 & 35 & 417 & .00 & .00 & .00 \\
\hline 29 & 2 & 11 & 19 & 44 & 648 & .00 & .00 & .00 \\
\hline 30 & 2 & 14 & 23 & 28 & 658 & .00 & .00 & .00 \\
\hline
\end{tabular}


Table 1.--Air-temperature, relative-humidity, solar-radiation, and precipitation data, October 1987-December 1989--Continued

\begin{tabular}{|c|c|c|c|c|c|c|c|c|}
\hline \multirow{2}{*}{$\begin{array}{l}\text { Day } \\
\text { of } \\
\text { month }\end{array}$} & \multicolumn{3}{|c|}{ Air temperature } & \multirow{2}{*}{$\begin{array}{l}\text { Average } \\
\text { relative } \\
\text { humidity } \\
\text { (percent) }\end{array}$} & \multirow{2}{*}{$\begin{array}{c}\text { Solar } \\
\text { radiation } \\
\text { (langleys) }\end{array}$} & \multicolumn{3}{|c|}{$\begin{array}{c}\text { Precipitation } \\
\text { (inches) }\end{array}$} \\
\hline & $\begin{array}{l}\text { Minimum } \\
\text { (degr }\end{array}$ & $\begin{array}{l}\text { Average } \\
\text { rees Cels }\end{array}$ & $\begin{array}{l}\text { Maximum } \\
\text { ius ) }\end{array}$ & & & $\begin{array}{l}\text { Spring } \\
\text { Creek } 1\end{array}$ & $\begin{array}{l}\text { Spring } \\
\text { Creek } 2\end{array}$ & $\begin{array}{r}\text { Cow } \\
\text { Camp } \\
\text { Creek }\end{array}$ \\
\hline \multicolumn{9}{|c|}{ MAY 1988} \\
\hline 1 & -5 & 7 & 16 & 48 & 265 & 0.17 & 0.17 & 0.23 \\
\hline 2 & -5 & -2 & 4 & 85 & 301 & .06 & .01 & .22 \\
\hline 3 & -4 & 3 & 12 & 56 & 696 & .00 & .00 & .00 \\
\hline 4 & 2 & 10 & 20 & 32 & 553 & .00 & .00 & .00 \\
\hline 5 & 7 & 15 & 23 & 19 & 587 & .00 & .00 & .00 \\
\hline 6 & 0 & 6 & 14 & 37 & 415 & .00 & .00 & .00 \\
\hline 7 & -5 & 4 & 12 & 51 & 508 & .14 & .12 & .17 \\
\hline 8 & -7 & -1 & 8 & 94 & 325 & .17 & .11 & .27 \\
\hline 9 & 0 & 7 & 16 & 63 & 572 & .00 & .00 & .00 \\
\hline 10 & 5 & 8 & 15 & 53 & 536 & .00 & .00 & .00 \\
\hline 11 & 3 & 11 & 21 & 37 & 747 & .00 & .00 & .00 \\
\hline 12 & 4 & 15 & 24 & 29 & 733 & .00 & .00 & .00 \\
\hline 13 & 5 & 17 & 26 & 24 & 644 & .00 & .00 & .00 \\
\hline 14 & 1 & 14 & 22 & 28 & 738 & .00 & .00 & .00 \\
\hline 15 & 3 & 14 & 23 & 29 & 745 & .00 & .00 & .00 \\
\hline 16 & 8 & 18 & 27 & 22 & 641 & .00 & .00 & .00 \\
\hline 17 & 5 & 14 & 23 & 50 & 426 & .29 & .30 & .26 \\
\hline 18 & 4 & 8 & 15 & 90 & 375 & .32 & .36 & .40 \\
\hline 19 & 3 & 5 & 7 & 94 & 144 & .24 & .24 & .34 \\
\hline 20 & 0 & 6 & 13 & 68 & 552 & .00 & .00 & .00 \\
\hline 21 & -1 & 7 & 14 & 55 & 581 & .00 & .00 & .00 \\
\hline 22 & -1 & 9 & 17 & 47 & 609 & .00 & .00 & .00 \\
\hline 23 & 1 & 12 & 21 & 41 & 754 & .00 & .00 & .00 \\
\hline 24 & 5 & 15 & 25 & 36 & 749 & .00 & .00 & .00 \\
\hline 25 & 5 & 13 & 22 & 55 & 433 & .02 & .02 & .05 \\
\hline 26 & 4 & 15 & 22 & 50 & 570 & .00 & .00 & .00 \\
\hline 27 & 9 & 17 & 25 & 32 & 610 & .00 & .00 & .00 \\
\hline 28 & 6 & 17 & 25 & 29 & 654 & .00 & .00 & .00 \\
\hline 29 & 11 & 17 & 25 & 23 & $56 i$ & .00 & .00 & .00 \\
\hline 30 & 0 & 5 & 11 & 73 & 271 & .10 & .10 & .10 \\
\hline 31 & -2 & 7 & 14 & 59 & 584 & .00 & .00 & .00 \\
\hline
\end{tabular}


Table 1.--Air-temperature, relative-humidity, solar-radiation, and precipitation data, October 1987-December 1989--Continued

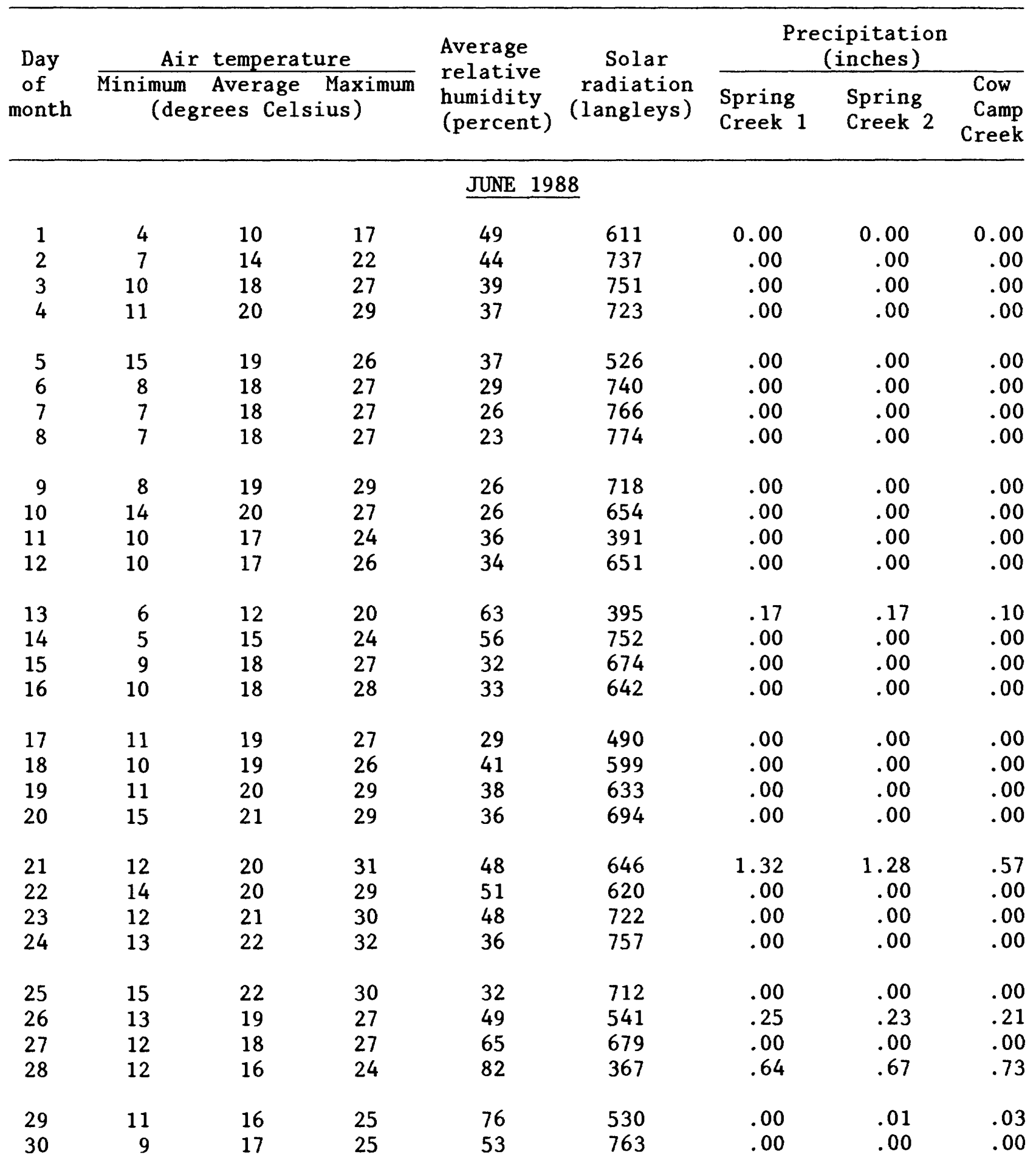


Table 1.--Air-temperature, relative-humidity, solar-radiation, and precipitation data, October 1987-December 1989--Continued

\begin{tabular}{|c|c|c|c|c|c|c|c|c|}
\hline \multirow{2}{*}{$\begin{array}{l}\text { Day } \\
\text { of } \\
\text { month }\end{array}$} & \multicolumn{3}{|c|}{ Air temperature } & \multirow{2}{*}{$\begin{array}{l}\text { Average } \\
\text { relative } \\
\text { humidity } \\
\text { (percent) }\end{array}$} & \multirow{2}{*}{$\begin{array}{c}\text { Solar } \\
\text { radiation } \\
\text { (langleys) }\end{array}$} & \multicolumn{3}{|c|}{$\begin{array}{c}\text { Precipitation } \\
\text { (inches) }\end{array}$} \\
\hline & $\begin{array}{l}\text { Minimum } \\
(\text { degr }\end{array}$ & $\begin{array}{l}\text { Average } \\
\text { rees Cels }\end{array}$ & $\begin{array}{l}\text { Maximum } \\
\text { ius ) }\end{array}$ & & & $\begin{array}{l}\text { Spring } \\
\text { Creek } 1\end{array}$ & $\begin{array}{l}\text { Spring } \\
\text { Creek } 2\end{array}$ & $\begin{array}{c}\text { Cow } \\
\text { Camp } \\
\text { Creek }\end{array}$ \\
\hline \multicolumn{9}{|c|}{ JULY 1988} \\
\hline 1 & 8 & 19 & 29 & 37 & 682 & 0.00 & 0.00 & 0.00 \\
\hline 2 & 11 & 19 & 28 & 42 & 561 & .00 & .00 & .00 \\
\hline 3 & 10 & 20 & 29 & 40 & 584 & .00 & .00 & .00 \\
\hline 4 & 10 & 16 & 23 & 75 & 404 & .11 & .12 & .26 \\
\hline 5 & 11 & 19 & 28 & 50 & 600 & .00 & .00 & .00 \\
\hline 6 & 13 & 20 & 27 & 48 & 555 & .00 & .00 & .00 \\
\hline 7 & 11 & 20 & 29 & 45 & 637 & .00 & .00 & .00 \\
\hline 8 & 11 & 20 & 28 & 32 & 694 & .00 & .00 & .00 \\
\hline 9 & 10 & 19 & 27 & 31 & 626 & .00 & .00 & .00 \\
\hline 10 & 13 & 19 & 26 & 33 & 599 & .00 & .00 & .00 \\
\hline 11 & 9 & 19 & 27 & 35 & 595 & .00 & .00 & .00 \\
\hline 12 & 10 & 20 & 28 & 32 & 549 & .00 & .00 & .00 \\
\hline 13 & 11 & 21 & 31 & 26 & 745 & .00 & .00 & .00 \\
\hline 14 & 16 & 23 & 31 & 23 & 691 & .00 & .00 & .00 \\
\hline 15 & 11 & 21 & 30 & 21 & 598 & .00 & .00 & .00 \\
\hline 16 & 15 & 21 & 32 & 27 & 584 & .00 & .00 & .00 \\
\hline 17 & 11 & 20 & 30 & 29 & 610 & .00 & .00 & .00 \\
\hline 18 & 9 & 19 & 29 & 21 & 740 & .00 & .00 & .00 \\
\hline 19 & 7 & 17 & 27 & 31 & 709 & .00 & .00 & .00 \\
\hline 20 & 8 & 18 & 27 & 29 & 724 & .00 & .00 & .00 \\
\hline 21 & 6 & 20 & 31 & 20 & 739 & .00 & .00 & .00 \\
\hline 22 & 11 & 21 & 32 & 16 & 668 & .00 & .00 & .00 \\
\hline 23 & 13 & 21 & 29 & 16 & 547 & .00 & .00 & .00 \\
\hline 24 & 10 & 21 & 31 & 21 & 667 & .00 & .00 & .00 \\
\hline 25 & 13 & 22 & 32 & 18 & 657 & .00 & .00 & .00 \\
\hline 26 & 15 & 21 & 29 & 22 & 447 & .00 & .00 & .00 \\
\hline 27 & 12 & 19 & 28 & 40 & 514 & .00 & .00 & .00 \\
\hline 28 & 11 & 18 & 28 & 52 & 515 & .00 & .00 & .02 \\
\hline 29 & 10 & 18 & 29 & 58 & 578 & .00 & .00 & .00 \\
\hline 30 & 11 & 20 & 30 & 44 & 617 & .00 & .00 & .03 \\
\hline 31 & 12 & 20 & 31 & 43 & 549 & .00 & .00 & .00 \\
\hline
\end{tabular}


Table 1.--Air-temperature, relative-humidity, solar-radiation, and precipitation data, October 1987-December 1989--Continued

\begin{tabular}{|c|c|c|c|c|c|c|c|c|}
\hline \multirow{2}{*}{$\begin{array}{l}\text { Day } \\
\text { of } \\
\text { month }\end{array}$} & \multicolumn{3}{|c|}{ Air temperature } & \multirow{2}{*}{$\begin{array}{l}\text { Average } \\
\text { relative } \\
\text { humidity } \\
\text { (percent) }\end{array}$} & \multirow{2}{*}{$\begin{array}{c}\text { Solar } \\
\text { radiation } \\
\text { (langleys) }\end{array}$} & \multicolumn{3}{|c|}{$\begin{array}{c}\text { Precipitation } \\
\text { (inches) }\end{array}$} \\
\hline & $\begin{array}{l}\text { Minimum } \\
\text { (deg }\end{array}$ & $\begin{array}{l}\text { Average } \\
\text { rees Cels }\end{array}$ & $\begin{array}{l}\text { Maximum } \\
\text { ius ) }\end{array}$ & & & $\begin{array}{l}\text { Spring } \\
\text { Creek } 1\end{array}$ & $\begin{array}{l}\text { Spring } \\
\text { Creek } 2\end{array}$ & $\begin{array}{c}\text { Cow } \\
\text { Camp } \\
\text { Creek }\end{array}$ \\
\hline \multicolumn{9}{|c|}{ AUGUST 1988} \\
\hline 1 & 9 & 17 & 28 & 53 & 507 & 0.05 & 0.04 & 0.00 \\
\hline 2 & 7 & 20 & 31 & 44 & 614 & .00 & .05 & .03 \\
\hline 3 & 11 & 17 & 27 & 66 & 463 & .33 & .32 & .23 \\
\hline 4 & 10 & 16 & 24 & 61 & 710 & .00 & .00 & .00 \\
\hline 5 & 6 & 18 & 28 & 37 & 676 & .00 & .00 & .00 \\
\hline 6 & 12 & 17 & 25 & 50 & 314 & .00 & .00 & .00 \\
\hline 7 & 8 & 15 & 23 & 72 & 484 & .21 & .20 & .20 \\
\hline 8 & 8 & 16 & 25 & 49 & 588 & .00 & .00 & .00 \\
\hline 9 & 7 & 17 & 27 & 31 & 675 & .00 & .00 & .00 \\
\hline 10 & 6 & 19 & 29 & 23 & 633 & .00 & .00 & .00 \\
\hline 11 & 8 & 20 & 29 & 21 & 570 & .00 & .00 & .00 \\
\hline 12 & 11 & 17 & 23 & 45 & 409 & .02 & .02 & .19 \\
\hline 13 & 7 & 18 & 28 & 38 & 588 & .00 & .00 & .00 \\
\hline 14 & 10 & 22 & 32 & 18 & 683 & .00 & .00 & .00 \\
\hline 15 & 12 & 20 & 30 & 32 & 406 & .28 & .30 & .45 \\
\hline 16 & 12 & 18 & 27 & 60 & 555 & .00 & .00 & .00 \\
\hline 17 & 12 & 19 & 26 & 45 & 414 & .00 & .00 & .00 \\
\hline 18 & 11 & 20 & 30 & 34 & 592 & .00 & .00 & .00 \\
\hline 19 & 10 & 20 & 30 & 25 & 630 & .00 & .00 & .00 \\
\hline 20 & 10 & 18 & 28 & 36 & 386 & .03 & .03 & .05 \\
\hline 21 & 11 & 17 & 25 & 67 & 351 & .00 & .00 & .00 \\
\hline 22 & 11 & 19 & 27 & 42 & 623 & .00 & .00 & .00 \\
\hline 23 & 7 & 19 & 30 & 23 & 647 & .00 & .00 & .00 \\
\hline 24 & 13 & 22 & 31 & 18 & 625 & .00 & .00 & .00 \\
\hline 25 & 10 & 21 & 31 & 16 & 590 & .00 & .00 & .00 \\
\hline 26 & 9 & 19 & 30 & 26 & 417 & .02 & .03 & .00 \\
\hline 27 & 12 & 18 & 25 & 37 & 596 & .00 & .00 & .00 \\
\hline 28 & 11 & 18 & 27 & 26 & 591 & .00 & .00 & .00 \\
\hline 29 & 10 & 19 & 28 & 21 & 535 & .00 & .00 & .00 \\
\hline 30 & 10 & 19 & 29 & 26 & 561 & .00 & .00 & .00 \\
\hline 31 & 10 & 18 & 29 & 26 & 482 & .00 & .00 & .00 \\
\hline
\end{tabular}


Table 1.--Air-temperature, relative-humidity, solar-radiation, and precipitation data, October 1987-December 1989--Continued

\begin{tabular}{|c|c|c|c|c|c|c|c|c|}
\hline \multirow{2}{*}{$\begin{array}{l}\text { Day } \\
\text { of } \\
\text { month }\end{array}$} & \multicolumn{3}{|c|}{ Air temperature } & \multirow{2}{*}{$\begin{array}{l}\text { Average } \\
\text { relative } \\
\text { humidity } \\
\text { (percent) }\end{array}$} & \multirow{2}{*}{$\begin{array}{c}\text { Solar } \\
\text { radiation } \\
\text { (langleys) }\end{array}$} & \multicolumn{3}{|c|}{$\begin{array}{c}\text { Precipitation } \\
\text { (inches) }\end{array}$} \\
\hline & $\begin{array}{r}\text { Minimum } \\
\text { (deg }\end{array}$ & $\begin{array}{l}\text { Average } \\
\text { rees Cels }\end{array}$ & $\begin{array}{l}\text { Maximum } \\
\text { ius ) }\end{array}$ & & & $\begin{array}{l}\text { Spring } \\
\text { Creek } 1\end{array}$ & $\begin{array}{l}\text { Spring } \\
\text { Creek } 2\end{array}$ & $\begin{array}{c}\text { Cow } \\
\text { Camp } \\
\text { Creek }\end{array}$ \\
\hline \multicolumn{9}{|c|}{ SEPTEMBER 1988} \\
\hline 1 & 10 & 18 & 29 & 24 & 587 & 0.00 & 0.00 & 0.00 \\
\hline 2 & 11 & 19 & 28 & 25 & 524 & .00 & .00 & .00 \\
\hline 3 & 9 & 18 & 27 & 17 & 593 & .00 & .00 & .00 \\
\hline 4 & 7 & 17 & 27 & 18 & 605 & .00 & .00 & .00 \\
\hline 5 & 5 & 17 & 26 & 17 & 593 & .00 & .00 & .00 \\
\hline 6 & 6 & 17 & 28 & 16 & 523 & .00 & .00 & .00 \\
\hline 7 & 10 & 19 & 29 & 16 & 540 & .00 & .00 & .00 \\
\hline 8 & 8 & 17 & 28 & 16 & 574 & .00 & .00 & .00 \\
\hline 9 & 5 & 18 & 29 & 15 & 551 & .00 & .00 & .00 \\
\hline 10 & 12 & 17 & 22 & 30 & 285 & .10 & .07 & .04 \\
\hline 11 & -1 & 6 & 14 & 86 & 95 & 1.13 & 1.14 & 1.23 \\
\hline 12 & 2 & 5 & 8 & 93 & 131 & .75 & .86 & .55 \\
\hline 13 & 4 & 8 & 14 & 78 & 272 & .00 & .00 & .02 \\
\hline 14 & 1 & 6 & 11 & 85 & 233 & .07 & .10 & .06 \\
\hline 15 & 1 & 8 & 16 & 67 & 396 & .00 & .00 & .00 \\
\hline 16 & 2 & 12 & 23 & 47 & 537 & .00 & .00 & .00 \\
\hline 17 & 5 & 15 & 25 & 29 & 542 & .00 & .00 & .00 \\
\hline 18 & -2 & 9 & 16 & 24 & 412 & .00 & .00 & .00 \\
\hline 19 & -4 & 7 & 20 & 31 & 538 & .00 & .00 & .00 \\
\hline 20 & 4 & 16 & 24 & 17 & 528 & .00 & .00 & .00 \\
\hline 21 & 8 & 13 & 21 & 56 & 339 & .02 & .02 & .07 \\
\hline 22 & 3 & 9 & 16 & 69 & 293 & .00 & .00 & .06 \\
\hline 23 & 1 & 10 & 19 & 56 & 505 & .00 & .00 & .00 \\
\hline 24 & 2 & 12 & 22 & 40 & 443 & .00 & .00 & .00 \\
\hline 25 & 4 & 14 & 22 & 28 & 492 & .00 & .00 & .00 \\
\hline 26 & 3 & 13 & 22 & 23 & 360 & .00 & .00 & .00 \\
\hline 27 & 5 & 12 & 21 & 40 & 314 & .00 & .00 & .00 \\
\hline 28 & -1 & 4 & 10 & 48 & 288 & .00 & .00 & .00 \\
\hline 29 & -5 & 5 & 15 & 42 & 485 & .00 & .00 & .00 \\
\hline 30 & -1 & 9 & 20 & 37 & 456 & .00 & .00 & .00 \\
\hline
\end{tabular}


Table 1.--Air-temperature, relative-humidity, solar-radiation, and precipitation data, October 1987-December 1989--Continued

\begin{tabular}{|c|c|c|c|c|c|c|c|c|}
\hline \multirow{2}{*}{$\begin{array}{l}\text { Day } \\
\text { of } \\
\text { month }\end{array}$} & \multicolumn{3}{|c|}{ Air temperature } & \multirow{2}{*}{$\begin{array}{l}\text { Average } \\
\text { relative } \\
\text { humidity } \\
\text { (percent) }\end{array}$} & \multirow{2}{*}{$\begin{array}{c}\text { Solar } \\
\text { radiation } \\
\text { (langleys) }\end{array}$} & \multicolumn{3}{|c|}{$\begin{array}{c}\text { Precipitation } \\
\text { (inches) }\end{array}$} \\
\hline & $\begin{array}{c}\text { Minimum } \\
(\text { degr }\end{array}$ & $\begin{array}{l}\text { Average } \\
\text { cees Cels }\end{array}$ & $\begin{array}{l}\text { Maximum } \\
\text { ius ) }\end{array}$ & & & $\begin{array}{l}\text { Spring } \\
\text { Creek } 1\end{array}$ & $\begin{array}{l}\text { Spring } \\
\text { Creek } 2\end{array}$ & $\begin{array}{c}\text { Cow } \\
\text { Camp } \\
\text { Creek }\end{array}$ \\
\hline \multicolumn{9}{|c|}{ OCTOBER 1988} \\
\hline 1 & 3 & 12 & 21 & 35 & 470 & 0.00 & 0.00 & 0.00 \\
\hline 2 & 4 & 13 & 23 & 29 & 464 & .00 & .00 & .00 \\
\hline 3 & 3 & 13 & 23 & 25 & 408 & .00 & .00 & .00 \\
\hline 4 & 5 & 12 & 22 & 36 & 241 & .00 & .00 & .00 \\
\hline 5 & 5 & 11 & 20 & 50 & 354 & .00 & .00 & .00 \\
\hline 6 & 4 & 11 & 19 & 47 & 435 & .00 & .00 & .00 \\
\hline 7 & 1 & 9 & 17 & 52 & 333 & .00 & .00 & .00 \\
\hline 8 & 1 & 9 & 19 & 52 & 390 & .00 & .00 & .00 \\
\hline 9 & 2 & 7 & 13 & 51 & 255 & .02 & .01 & .00 \\
\hline 10 & 0 & 8 & 18 & 39 & 428 & .00 & .00 & .00 \\
\hline 11 & -1 & 9 & 20 & 30 & 421 & .00 & .00 & .00 \\
\hline 12 & 0 & 11 & 20 & 25 & 311 & .00 & .00 & .00 \\
\hline 13 & 2 & 9 & 18 & 49 & 308 & .00 & .00 & .00 \\
\hline 14 & 4 & 10 & 19 & 45 & 250 & .00 & .00 & .01 \\
\hline 15 & 2 & 9 & 18 & 50 & 398 & .00 & .00 & .00 \\
\hline 16 & 2 & 9 & 19 & 40 & 369 & .00 & .00 & .00 \\
\hline 17 & 2 & 11 & 21 & 36 & 351 & .00 & .00 & .00 \\
\hline 18 & 6 & 12 & 19 & 35 & 315 & .00 & .00 & .00 \\
\hline 19 & 2 & 7 & 13 & 58 & 155 & .06 & .06 & .07 \\
\hline 20 & -1 & 7 & 16 & 59 & 374 & .00 & .00 & .00 \\
\hline 21 & 0 & 9 & 19 & 34 & 379 & .00 & .00 & .00 \\
\hline 22 & 0 & 9 & 18 & 27 & 293 & .00 & .00 & .00 \\
\hline 23 & -1 & 8 & 18 & 28 & 367 & .00 & .00 & .00 \\
\hline 24 & 1 & 8 & 18 & 28 & 351 & .00 & .00 & .00 \\
\hline 25 & -1 & 8 & 17 & 29 & 343 & .00 & .00 & .00 \\
\hline 26 & 1 & 10 & 20 & 28 & 360 & .00 & .00 & .00 \\
\hline 27 & 0 & 8 & 16 & 24 & 338 & .00 & .00 & .00 \\
\hline 28 & 1 & 9 & 20 & 19 & 244 & .00 & .00 & .00 \\
\hline 29 & 4 & 9 & 17 & 31 & 214 & .00 & .00 & .00 \\
\hline 30 & 1 & 8 & 17 & 47 & 316 & .00 & .00 & .00 \\
\hline 31 & -1 & 6 & 17 & 30 & 339 & .00 & .00 & .00 \\
\hline
\end{tabular}


Table 1.--Air-temperature, relative-humidity, solar-radiation, and precipitation data, October 1987-December 1989--Continued

\begin{tabular}{|c|c|c|c|c|c|c|c|c|}
\hline \multirow{2}{*}{$\begin{array}{l}\text { Day } \\
\text { of } \\
\text { month }\end{array}$} & \multicolumn{3}{|c|}{ Air temperature } & \multirow{2}{*}{$\begin{array}{l}\text { Average } \\
\text { relative } \\
\text { humidity } \\
\text { (percent) }\end{array}$} & \multirow{2}{*}{$\begin{array}{c}\text { Solar } \\
\text { radiation } \\
\text { (langleys) }\end{array}$} & \multicolumn{3}{|c|}{$\begin{array}{c}\text { Precipitation } \\
\text { (inches) }\end{array}$} \\
\hline & $\begin{array}{r}\text { Minimum } \\
\text { (degr }\end{array}$ & $\begin{array}{l}\text { Average } \\
\text { rees Cel }\end{array}$ & $\begin{array}{l}\text { Maximum } \\
\text { ius ) }\end{array}$ & & & $\begin{array}{l}\text { Spring } \\
\text { Creek } 1\end{array}$ & $\begin{array}{l}\text { Spring } \\
\text { Creek } 2\end{array}$ & $\begin{array}{c}\text { Cow } \\
\text { Camp } \\
\text { Creek }\end{array}$ \\
\hline \multicolumn{9}{|c|}{ NOVEMBER 1988} \\
\hline 1 & -3 & 7 & 17 & 29 & 258 & 0.00 & 0.00 & 0.00 \\
\hline 2 & 3 & 8 & 15 & 41 & 211 & .00 & .00 & .01 \\
\hline 3 & 2 & 6 & 11 & 69 & 35 & .33 & .35 & .37 \\
\hline 4 & 1 & 3 & 7 & 56 & 150 & .00 & .00 & .00 \\
\hline 5 & -2 & 2 & 8 & 48 & 306 & .00 & .00 & .00 \\
\hline 6 & -4 & 6 & 15 & 41 & 242 & .00 & .00 & .00 \\
\hline 7 & -2 & 3 & 10 & 36 & 305 & .00 & .00 & .00 \\
\hline 8 & -7 & 0 & 6 & 69 & 34 & .17 & .10 & -- \\
\hline 9 & -16 & -5 & -1 & 93 & 50 & - & .50 & -- \\
\hline 10 & -6 & -1 & 4 & 85 & 120 & .00 & .00 & 1.25 \\
\hline 11 & -5 & 0 & 5 & 91 & 87 & .24 & .20 & -- \\
\hline 12 & -7 & -2 & 2 & 74 & 236 & .00 & .00 & -- \\
\hline 13 & -1 & 2 & 6 & 81 & 138 & .00 & .00 & -- \\
\hline 14 & -4 & 4 & 11 & 71 & 256 & -- & .22 & .00 \\
\hline 15 & -11 & -6 & -3 & 91 & 209 & -- & .22 & .00 \\
\hline 16 & -12 & -6 & 1 & 83 & 139 & .60 & .02 & .00 \\
\hline 17 & -9 & -4 & -1 & 85 & 128 & -- & .09 & .05 \\
\hline 18 & -12 & -6 & -1 & 91 & 83 & .00 & .00 & .02 \\
\hline 19 & -13 & -8 & -2 & 85 & 122 & .00 & .00 & .00 \\
\hline 20 & -10 & -6 & 0 & 76 & 289 & .00 & .00 & .10 \\
\hline 21 & -10 & -5 & 1 & 76 & 179 & .00 & .00 & .42 \\
\hline 22 & -9 & -3 & 3 & 72 & 216 & .00 & .00 & .00 \\
\hline 23 & -3 & 5 & 12 & 63 & 255 & .00 & .00 & .00 \\
\hline 24 & -9 & -2 & 10 & 74 & 216 & -- & .03 & .05 \\
\hline 25 & -9 & -6 & -1 & 84 & 212 & -- & .02 & .00 \\
\hline 26 & -9 & -7 & -5 & 89 & 192 & -- & .09 & .00 \\
\hline 27 & -11 & -9 & -5 & 74 & 209 & .45 & .06 & .00 \\
\hline 28 & -10 & -6 & -2 & 74 & 95 & -- & .05 & .00 \\
\hline 29 & -9 & -6 & -4 & 81 & 139 & -- & .02 & .00 \\
\hline 30 & -9 & -5 & 0 & 85 & 161 & -- & .04 & .03 \\
\hline
\end{tabular}


Table 1.--Air-temperature, relative-humidity, solar-radiation, and precipitation data, October 1987-December 1989--Continued

\begin{tabular}{|c|c|c|c|c|c|c|c|c|}
\hline \multirow{2}{*}{$\begin{array}{l}\text { Day } \\
\text { of } \\
\text { month }\end{array}$} & \multicolumn{3}{|c|}{ Air temperature } & \multirow{2}{*}{$\begin{array}{l}\text { Average } \\
\text { relative } \\
\text { humidity } \\
\text { (percent) }\end{array}$} & \multirow{2}{*}{$\begin{array}{c}\text { Solar } \\
\text { radiation } \\
\text { (langleys) }\end{array}$} & \multicolumn{3}{|c|}{$\begin{array}{c}\text { Precipitation } \\
\text { (inches) }\end{array}$} \\
\hline & $\begin{array}{r}\text { Minimum } \\
\text { (deg }\end{array}$ & $\begin{array}{l}\text { Average } \\
\text { rees Cels }\end{array}$ & $\begin{array}{l}\text { Maximum } \\
\text { ius ) }\end{array}$ & & & $\begin{array}{l}\text { Spring } \\
\text { Creek } 1\end{array}$ & $\begin{array}{l}\text { Spring } \\
\text { Creek } 2\end{array}$ & $\begin{array}{c}\text { Cow } \\
\text { Camp } \\
\text { Creek }\end{array}$ \\
\hline \multicolumn{9}{|c|}{ DECEMBER 1988} \\
\hline 1 & -11 & -4 & 0 & 75 & 261 & 0.00 & 0.00 & 0.00 \\
\hline 2 & -4 & -1 & 3 & 66 & 259 & .00 & .00 & .02 \\
\hline 3 & -8 & -2 & 5 & 66 & 257 & .00 & .00 & .00 \\
\hline 4 & -9 & -3 & 3 & 66 & 253 & .00 & .00 & .19 \\
\hline 5 & -9 & -2 & 5 & 62 & 252 & .00 & .00 & .13 \\
\hline 6 & -10 & -5 & 0 & 77 & 241 & .00 & .00 & .08 \\
\hline 7 & -8 & -4 & 1 & 82 & 46 & .13 & .15 & .02 \\
\hline 8 & -16 & -11 & -5 & 85 & 233 & .00 & .00 & .00 \\
\hline 9 & -15 & -7 & -2 & 74 & 125 & .00 & .00 & .00 \\
\hline 10 & -8 & -3 & 1 & 78 & 233 & .00 & .00 & .16 \\
\hline 11 & -10 & -5 & -1 & 93 & 67 & .04 & .04 & .00 \\
\hline 12 & -10 & -3 & 3 & 78 & 191 & .00 & .00 & .06 \\
\hline 13 & -7 & -2 & 4 & 82 & 139 & .00 & .00 & .00 \\
\hline 14 & -7 & -4 & 0 & 80 & 92 & .00 & .00 & .12 \\
\hline 15 & -14 & -8 & -5 & 82 & 168 & -- & .23 & .06 \\
\hline 16 & -15 & -10 & -3 & 86 & 244 & -- & .03 & .00 \\
\hline 17 & -14 & -10 & -3 & 85 & 263 & -- & .00 & .00 \\
\hline 18 & -13 & -5 & 3 & 73 & 259 & -- & .00 & .00 \\
\hline 19 & -7 & -4 & -1 & 93 & 45 & -- & .09 & .20 \\
\hline 20 & -10 & -5 & 1 & 86 & 123 & -- & .16 & .24 \\
\hline 21 & -10 & -4 & 1 & 79 & 127 & - & .00 & .00 \\
\hline 22 & -8 & -6 & -3 & 68 & 236 & -- & .08 & .19 \\
\hline 23 & -13 & -7 & -4 & 88 & 117 & 1.00 & .09 & .00 \\
\hline 24 & -15 & -10 & -5 & 75 & 152 & -- & .08 & .18 \\
\hline 25 & -8 & -2 & 4 & 82 & 133 & -- & .10 & .02 \\
\hline 26 & -16 & -10 & -6 & 84 & 191 & -- & .07 & .05 \\
\hline 27 & -22 & -16 & -11 & 73 & 215 & -- & .04 & .18 \\
\hline 28 & -20 & -15 & -8 & 64 & 175 & -- & .00 & .08 \\
\hline 29 & -19 & -13 & -7 & 74 & 238 & -- & .00 & .00 \\
\hline 30 & -12 & -8 & -3 & 82 & 134 & -- & .02 & .00 \\
\hline 31 & -13 & -8 & 0 & 77 & 242 & -- & .00 & .00 \\
\hline
\end{tabular}


Table 1.--Air-temperature, relative-humidity, solar-radiation, and precipitation data, October 1987-December 1989--Continued

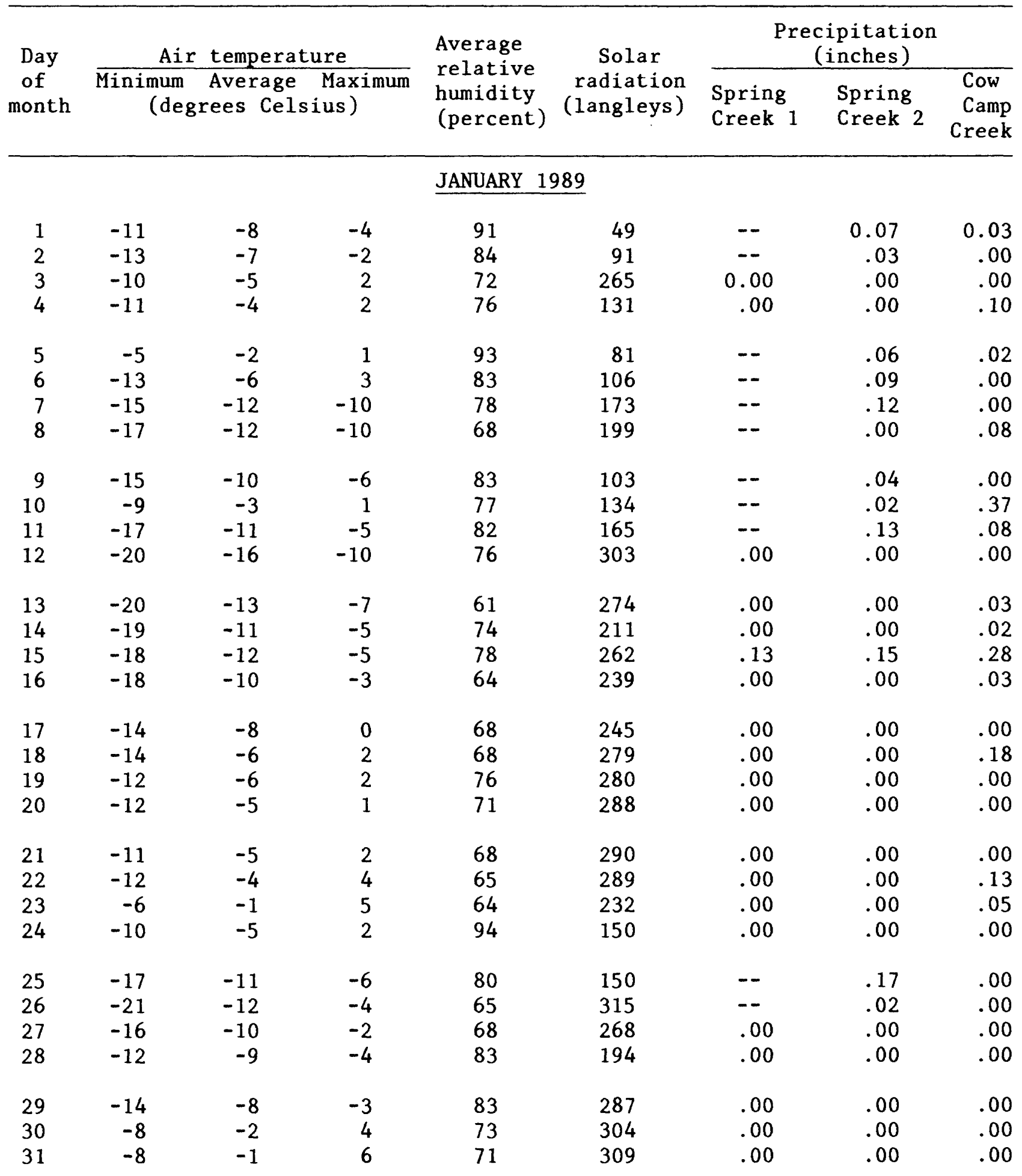


Table 1.--Air-temperature, relative-humidity, solar-radiation, and precipitation data, October 1987-December 1989--Continued

\begin{tabular}{|c|c|c|c|c|c|c|c|c|}
\hline \multirow{2}{*}{$\begin{array}{l}\text { Day } \\
\text { of } \\
\text { month }\end{array}$} & \multicolumn{3}{|c|}{ Air temperature } & \multirow{2}{*}{$\begin{array}{l}\text { Average } \\
\text { relative } \\
\text { humidity } \\
\text { (percent) }\end{array}$} & \multirow{2}{*}{$\begin{array}{c}\text { Solar } \\
\text { radiation } \\
\text { (langleys) }\end{array}$} & \multicolumn{3}{|c|}{$\begin{array}{c}\text { Precipitation } \\
\text { (inches) }\end{array}$} \\
\hline & $\begin{array}{l}\text { Minimum } \\
\text { (deg) }\end{array}$ & $\begin{array}{l}\text { Average } \\
\text { rees Cels }\end{array}$ & $\begin{array}{l}\text { Maximum } \\
\text { ius ) }\end{array}$ & & & $\begin{array}{l}\text { Spring } \\
\text { Creek } 1\end{array}$ & $\begin{array}{l}\text { Spring } \\
\text { Creek } 2\end{array}$ & $\begin{array}{c}\text { Cow } \\
\text { Camp } \\
\text { Creek }\end{array}$ \\
\hline \multicolumn{9}{|c|}{ FEBRUARY 1989} \\
\hline 1 & -1 & 5 & 8 & 48 & 288 & 0.00 & 0.00 & 0.00 \\
\hline 2 & -17 & -8 & 5 & 83 & 180 & -- & .07 & .11 \\
\hline 3 & -20 & -18 & -16 & 85 & 35 & -- & .58 & .57 \\
\hline 4 & -28 & -24 & -19 & 80 & 39 & -- & .35 & .50 \\
\hline 5 & -34 & -28 & -17 & 76 & 186 & -- & .00 & .00 \\
\hline 6 & -36 & -29 & -18 & 75 & 219 & -- & .00 & .00 \\
\hline 7 & -31 & -24 & -14 & 79 & 262 & -- & .00 & .00 \\
\hline 8 & -26 & -20 & -10 & 80 & 299 & -- & .00 & .00 \\
\hline 9 & -19 & -8 & -2 & 83 & 199 & -- & .00 & .00 \\
\hline 10 & -5 & -2 & 3 & 94 & 165 & -- & .12 & .09 \\
\hline 11 & -9 & -4 & 0 & 97 & 169 & -- & .28 & .27 \\
\hline 12 & -12 & -7 & -4 & 96 & 221 & -- & .11 & .18 \\
\hline 13 & -16 & -10 & -4 & 93 & 280 & -- & .03 & .04 \\
\hline 14 & -11 & -10 & -7 & 93 & 85 & -- & .22 & .23 \\
\hline 15 & -15 & -10 & -4 & 82 & 138 & -- & .02 & .04 \\
\hline 16 & -17 & -7 & 1 & 71 & 448 & -- & .00 & .00 \\
\hline 17 & -12 & -4 & 5 & 73 & 366 & -- & .00 & .00 \\
\hline 18 & -7 & -2 & 2 & 87 & 227 & -- & .08 & -- \\
\hline 19 & -10 & -4 & 1 & 97 & 273 & -- & .27 & -- \\
\hline 20 & -6 & -5 & -3 & 96 & 240 & -- & .12 & -- \\
\hline 21 & -10 & -6 & 1 & 86 & 176 & -- & .05 & -- \\
\hline 22 & -11 & -5 & 3 & 73 & 376 & -- & .00 & .00 \\
\hline 23 & -6 & -1 & 8 & 71 & 407 & -- & .00 & .00 \\
\hline 24 & -8 & 1 & 7 & 73 & 420 & -- & .00 & .00 \\
\hline 25 & 2 & 4 & 11 & 70 & 369 & -- & .00 & .00 \\
\hline 26 & -3 & 1 & 4 & 84 & 90 & -- & .10 & .17 \\
\hline 27 & -10 & -5 & -2 & 70 & 312 & -- & .00 & .00 \\
\hline 28 & -6 & -3 & 3 & 62 & 446 & -- & .00 & .00 \\
\hline
\end{tabular}


Table 1.--Air-temperature, relative-humidity, solar-radiation, and precipitation data, October 1987-December 1989--Continued

\begin{tabular}{|c|c|c|c|c|c|c|c|c|}
\hline \multirow{2}{*}{$\begin{array}{l}\text { Day } \\
\text { of } \\
\text { month }\end{array}$} & \multicolumn{3}{|c|}{ Air temperature } & \multirow{2}{*}{$\begin{array}{l}\text { Average } \\
\text { relative } \\
\text { humidity } \\
\text { (percent) }\end{array}$} & \multirow{2}{*}{$\begin{array}{c}\text { Solar } \\
\text { radiation } \\
\text { (langleys) }\end{array}$} & \multicolumn{3}{|c|}{$\begin{array}{c}\text { Precipitation } \\
\text { (inches) }\end{array}$} \\
\hline & $\begin{array}{r}\text { Minimum } \\
\text { (deg }\end{array}$ & $\begin{array}{l}\text { Average } \\
\text { rees Cel }\end{array}$ & $\begin{array}{l}\text { Maximum } \\
\text { ius) }\end{array}$ & & & $\begin{array}{l}\text { Spring } \\
\text { Creek } 1\end{array}$ & $\begin{array}{l}\text { Spring } \\
\text { Creek } 2\end{array}$ & $\begin{array}{c}\text { Cow } \\
\text { Camp } \\
\text { Creek }\end{array}$ \\
\hline \multicolumn{9}{|c|}{ MARCH 1989} \\
\hline 1 & -- & -- & -- & -- & - & 0.00 & 0.00 & 0.00 \\
\hline 2 & -- & -- & -- & -- & -- & .16 & .18 & .16 \\
\hline 3 & -16 & -8 & 0 & 93 & 213 & .36 & .25 & .49 \\
\hline 4 & -22 & -17 & -11 & 79 & 545 & .01 & .00 & .01 \\
\hline 5 & -20 & -10 & -2 & 63 & 482 & .00 & .00 & .00 \\
\hline 6 & -8 & -2 & 4 & 70 & 303 & .00 & .00 & .00 \\
\hline 7 & -3 & 2 & 6 & 79 & 190 & .00 & .00 & .00 \\
\hline 8 & 2 & 6 & 11 & 79 & 384 & .00 & .00 & .00 \\
\hline 9 & 3 & 8 & 16 & 64 & 471 & .00 & .00 & .00 \\
\hline 10 & 4 & 9 & 17 & 50 & 459 & .00 & .00 & .00 \\
\hline 11 & 1 & 7 & 17 & 57 & 383 & .00 & .00 & .00 \\
\hline 12 & 2 & 7 & 15 & 58 & 386 & .00 & .00 & .00 \\
\hline 13 & -4 & 5 & 14 & 61 & 394 & .00 & .02 & .02 \\
\hline 14 & -8 & -4 & 1 & 65 & 378 & .00 & .00 & .00 \\
\hline 15 & -10 & -2 & 5 & 59 & 364 & .00 & .00 & .00 \\
\hline 16 & -3 & 6 & 15 & 44 & 418 & .00 & .00 & .00 \\
\hline 17 & -5 & 0 & 11 & 51 & 558 & .03 & .03 & .09 \\
\hline 18 & -6 & 3 & 11 & 47 & 434 & .00 & .00 & .00 \\
\hline 19 & -6 & 1 & 12 & 94 & 179 & .15 & .15 & .23 \\
\hline 20 & -7 & -3 & 2 & 84 & 572 & .07 & .03 & .14 \\
\hline 21 & -7 & -1 & 7 & 65 & 528 & .00 & .00 & .00 \\
\hline 22 & -5 & 4 & 11 & 70 & 387 & .02 & .01 & .01 \\
\hline 23 & -2 & 5 & 9 & 71 & 309 & .00 & .00 & .00 \\
\hline 24 & 1 & 7 & 14 & 54 & 528 & .00 & .00 & .00 \\
\hline 25 & 1 & 9 & 16 & 36 & 551 & .00 & .00 & .00 \\
\hline 26 & 3 & 8 & 14 & 52 & 359 & .00 & .00 & .00 \\
\hline 27 & 0 & 4 & 8 & 73 & 277 & .00 & .00 & .00 \\
\hline 28 & -4 & 6 & 15 & 58 & 507 & .03 & .03 & .02 \\
\hline 29 & -6 & -1 & 4 & 93 & 213 & .18 & .12 & .35 \\
\hline 30 & -6 & -2 & 3 & 77 & 500 & .05 & .02 & .13 \\
\hline 31 & -7 & 2 & 12 & 64 & 353 & .09 & .09 & .14 \\
\hline
\end{tabular}


Table 1.--Air-temperature, relative-humidity, solar-radiation, and precipitation data, October 1987-December 1989--Continued

\begin{tabular}{|c|c|c|c|c|c|c|c|c|}
\hline \multirow{2}{*}{$\begin{array}{l}\text { Day } \\
\text { of } \\
\text { month }\end{array}$} & \multicolumn{3}{|c|}{ Air temperature } & \multirow{2}{*}{$\begin{array}{l}\text { Average } \\
\text { relative } \\
\text { humidity } \\
\text { (percent) }\end{array}$} & \multirow{2}{*}{$\begin{array}{c}\text { Solar } \\
\text { radiation } \\
\text { (langleys) }\end{array}$} & \multicolumn{3}{|c|}{$\begin{array}{c}\text { Precipitation } \\
\text { (inches) }\end{array}$} \\
\hline & $\begin{array}{r}\text { Minimum } \\
\text { (deg) }\end{array}$ & $\begin{array}{l}\text { Average } \\
\text { rees Cels }\end{array}$ & $\begin{array}{l}\text { Maximum } \\
\text { ius) }\end{array}$ & & & $\begin{array}{l}\text { Spring } \\
\text { Creek } 1\end{array}$ & $\begin{array}{l}\text { Spring } \\
\text { Creek } 2\end{array}$ & $\begin{array}{c}\text { Cow } \\
\text { Camp } \\
\text { Creek }\end{array}$ \\
\hline \multicolumn{9}{|c|}{ APRIL 1989} \\
\hline 1 & -5 & 0 & 6 & 88 & 292 & 0.10 & 0.06 & 0.11 \\
\hline 2 & -5 & 3 & 11 & 75 & 227 & .11 & .08 & .02 \\
\hline 3 & -5 & -2 & 4 & 84 & 300 & .04 & .02 & .16 \\
\hline 4 & -4 & 0 & 5 & 59 & 453 & .00 & .00 & .00 \\
\hline 5 & -1 & 6 & 14 & 55 & 280 & -- & .00 & .00 \\
\hline 6 & 1 & 9 & 18 & 42 & 574 & -- & .00 & .00 \\
\hline 7 & 2 & 11 & 20 & 38 & 395 & -- & .00 & .00 \\
\hline 8 & 3 & 10 & 16 & 37 & 351 & -- & .00 & .00 \\
\hline 9 & -7 & 0 & 6 & 51 & 653 & -- & .00 & .00 \\
\hline 10 & -11 & 0 & 9 & 34 & 626 & -- & .00 & .00 \\
\hline 11 & -1 & 4 & 12 & 53 & 427 & -- & .02 & .00 \\
\hline 12 & -3 & 5 & 14 & 31 & 657 & -- & .00 & .00 \\
\hline 13 & -4 & 6 & 16 & 32 & 642 & -- & .00 & .00 \\
\hline 14 & 1 & 9 & 19 & 31 & 620 & -- & .00 & .00 \\
\hline 15 & 2 & 11 & 20 & 38 & 406 & -- & .02 & .06 \\
\hline 16 & 4 & 10 & 17 & 64 & 375 & -- & .07 & .00 \\
\hline 17 & 5 & 13 & 21 & 44 & 456 & -- & .00 & .00 \\
\hline 18 & 3 & 11 & 21 & 40 & 631 & -- & .00 & .00 \\
\hline 19 & 3 & 14 & 23 & 33 & 468 & -- & .00 & .00 \\
\hline 20 & 8 & 16 & 24 & 27 & 449 & -- & .00 & .00 \\
\hline 21 & 6 & 16 & 23 & 21 & 337 & -- & .00 & .00 \\
\hline 22 & 8 & 17 & 23 & 18 & 557 & -- & .00 & .00 \\
\hline 23 & 7 & 16 & 23 & 17 & 670 & -- & .00 & .00 \\
\hline 24 & 7 & 14 & 21 & 17 & 624 & -- & .00 & .00 \\
\hline 25 & 4 & 13 & 21 & 21 & 643 & -- & .00 & .00 \\
\hline 26 & 0 & 8 & 14 & 32 & 476 & -- & .00 & .00 \\
\hline 27 & -3 & 2 & 9 & 56 & 353 & -- & .00 & .00 \\
\hline 28 & -3 & 2 & 9 & 70 & 402 & -- & .00 & .00 \\
\hline 29 & -5 & 1 & 7 & 64 & 397 & -- & .00 & .00 \\
\hline 30 & -4 & 2 & 10 & 58 & 526 & -- & .00 & .00 \\
\hline
\end{tabular}


Table 1.--Air-temperature, relative-humidity, solar-radiation, and precipitation data, October 1987-December 1989--Continued

\begin{tabular}{|c|c|c|c|c|c|c|c|c|}
\hline \multirow{2}{*}{$\begin{array}{l}\text { Day } \\
\text { of } \\
\text { month }\end{array}$} & \multicolumn{3}{|c|}{ Air temperature } & \multirow{2}{*}{$\begin{array}{l}\text { Average } \\
\text { relative } \\
\text { humidity } \\
\text { (percent) }\end{array}$} & \multirow{2}{*}{$\begin{array}{c}\text { Solar } \\
\text { radiation } \\
\text { (langleys) }\end{array}$} & \multicolumn{3}{|c|}{$\begin{array}{c}\text { Precipitation } \\
\text { (inches) }\end{array}$} \\
\hline & $\begin{array}{r}\text { Minimum } \\
(\text { degr }\end{array}$ & $\begin{array}{l}\text { Average } \\
\text { rees Cel. }\end{array}$ & $\begin{array}{l}\text { Maximum } \\
\text { ius ) }\end{array}$ & & & $\begin{array}{l}\text { Spring } \\
\text { Creek } 1\end{array}$ & $\begin{array}{l}\text { Spring } \\
\text { Creek } 2\end{array}$ & $\begin{array}{c}\text { Cow } \\
\text { Camp } \\
\text { Creek }\end{array}$ \\
\hline \multicolumn{9}{|c|}{ MAY 1989} \\
\hline 1 & -3 & 7 & 16 & 46 & 577 & -- & 0.04 & 0.04 \\
\hline 2 & 3 & 8 & 15 & 74 & 417 & -- & .23 & .27 \\
\hline 3 & 2 & 7 & 14 & 63 & 386 & -- & .23 & .20 \\
\hline 4 & 0 & 8 & 15 & 52 & 443 & -- & .00 & .00 \\
\hline 5 & 0 & 10 & 20 & 44 & 717 & -- & .00 & .00 \\
\hline 6 & 4 & 13 & 23 & 31 & 710 & -- & .00 & .00 \\
\hline 7 & 6 & 16 & 24 & 30 & 511 & -- & .00 & .00 \\
\hline 8 & 6 & 14 & 23 & 54 & 528 & -- & .00 & .00 \\
\hline 9 & 4 & 15 & 25 & 51 & 544 & -- & .00 & .00 \\
\hline 10 & 9 & 14 & 22 & 51 & 443 & -- & .03 & .02 \\
\hline 11 & 5 & 13 & 22 & 43 & 545 & -- & .00 & .00 \\
\hline 12 & -1 & 3 & 9 & 88 & 320 & -- & .11 & .12 \\
\hline 13 & -1 & 7 & 14 & 64 & 567 & -- & .00 & .00 \\
\hline 14 & 1 & 4 & 11 & 85 & 217 & 0.49 & .47 & .59 \\
\hline 15 & 2 & 6 & 13 & 82 & 394 & .05 & .02 & .03 \\
\hline 16 & 1 & 6 & 12 & 85 & 396 & .01 & .01 & .00 \\
\hline 17 & 2 & 9 & 17 & 69 & 679 & .00 & .00 & .00 \\
\hline 18 & 2 & 14 & 23 & 43 & 741 & .00 & .00 & .00 \\
\hline 19 & 1 & 8 & 16 & 36 & 735 & .00 & .00 & .00 \\
\hline 20 & -1 & 11 & 22 & 32 & 753 & .00 & .00 & .00 \\
\hline 21 & 6 & 14 & 21 & 31 & 491 & .00 & .00 & .00 \\
\hline 22 & 4 & 16 & 26 & 29 & 735 & .00 & .00 & .00 \\
\hline 23 & 9 & 18 & 25 & 18 & 579 & .00 & .00 & .00 \\
\hline 24 & 3 & 9 & 17 & 32 & 692 & .00 & .00 & .00 \\
\hline 25 & 1 & 7 & 14 & 40 & 700 & .00 & .00 & .00 \\
\hline 26 & -2 & 8 & 17 & 35 & 770 & .00 & .00 & .00 \\
\hline 27 & 0 & 14 & 25 & 26 & 743 & .00 & .00 & .00 \\
\hline 28 & 9 & 19 & 26 & 16 & 770 & .00 & .00 & .00 \\
\hline 29 & 11 & 19 & 25 & 17 & 758 & .00 & .00 & .00 \\
\hline 30 & 3 & 13 & 21 & 36 & 614 & .00 & .00 & .00 \\
\hline 31 & 1 & 9 & 16 & 60 & 725 & .00 & .00 & .00 \\
\hline
\end{tabular}


Table 1.--Air-temperature, relative-humidity, solar-radiation, and precipitation data, October 1987-December 1989--Continued

\begin{tabular}{|c|c|c|c|c|c|c|c|c|}
\hline \multirow{2}{*}{$\begin{array}{l}\text { Day } \\
\text { of } \\
\text { month }\end{array}$} & \multicolumn{3}{|c|}{ Air temperature } & \multirow{2}{*}{$\begin{array}{l}\text { Average } \\
\text { relative } \\
\text { humidity } \\
\text { (percent) }\end{array}$} & \multirow{2}{*}{$\begin{array}{c}\text { Solar } \\
\text { radiation } \\
\text { (langleys) }\end{array}$} & \multicolumn{3}{|c|}{$\begin{array}{c}\text { Precipitation } \\
\text { (inches) }\end{array}$} \\
\hline & $\begin{array}{l}\text { Minimum } \\
\text { (degr }\end{array}$ & $\begin{array}{l}\text { Average } \\
\text { rees Cel. }\end{array}$ & $\begin{array}{l}\text { Maximum } \\
\text { ius) }\end{array}$ & & & $\begin{array}{l}\text { Spring } \\
\text { Creek } 1\end{array}$ & $\begin{array}{l}\text { Spring } \\
\text { Creek } 2\end{array}$ & $\begin{array}{l}\text { Cow } \\
\text { Camp } \\
\text { Creek }\end{array}$ \\
\hline \multicolumn{9}{|c|}{ JUNE 1989} \\
\hline 1 & 2 & 11 & 21 & 40 & 741 & 0.00 & 0.00 & 0.00 \\
\hline 2 & 2 & 13 & 23 & 30 & 748 & .00 & .00 & .00 \\
\hline 3 & 4 & 12 & 19 & 45 & 350 & .00 & .00 & .00 \\
\hline 4 & 2 & 10 & 21 & 69 & 501 & .15 & .14 & .09 \\
\hline 5 & 3 & 13 & 22 & 60 & 704 & .00 & .00 & .00 \\
\hline 6 & 6 & 11 & 18 & 60 & 358 & .00 & .00 & .00 \\
\hline 7 & 3 & 14 & 23 & 54 & 756 & .00 & .00 & .00 \\
\hline 8 & 7 & 13 & 21 & 62 & 348 & .09 & .15 & .38 \\
\hline 9 & 5 & 13 & 21 & 65 & 535 & .00 & .00 & .00 \\
\hline 10 & 6 & 13 & 20 & 67 & 363 & .10 & .10 & .14 \\
\hline 11 & 7 & 15 & 23 & 61 & 682 & .00 & .00 & .00 \\
\hline 12 & 6 & 12 & 19 & 64 & 504 & .00 & .00 & .00 \\
\hline 13 & 6 & 13 & 21 & 58 & 630 & .00 & .00 & .00 \\
\hline 14 & 6 & 15 & 23 & 39 & 758 & .00 & .00 & .00 \\
\hline 15 & 6 & 18 & 29 & 34 & 754 & .00 & .00 & .00 \\
\hline 16 & 9 & 20 & 29 & 35 & 726 & .10 & .10 & .10 \\
\hline 17 & 3 & 14 & 23 & 57 & 785 & .00 & .00 & .00 \\
\hline 18 & 6 & 19 & 30 & 38 & 758 & .00 & .00 & .00 \\
\hline 19 & 12 & 23 & 33 & 25 & 711 & .00 & .00 & .00 \\
\hline 20 & 10 & 22 & 28 & 19 & 625 & .00 & .00 & .00 \\
\hline 21 & 1 & 8 & 15 & 33 & 561 & .00 & .00 & .00 \\
\hline 22 & -1 & 10 & 19 & 40 & 703 & .00 & .00 & .00 \\
\hline 23 & 6 & 12 & 17 & 45 & 421 & .00 & .00 & .00 \\
\hline 24 & 4 & 13 & 21 & 57 & 749 & .00 & .00 & .00 \\
\hline 25 & 3 & 14 & 25 & 57 & 629 & .00 & .00 & .00 \\
\hline 26 & 5 & 15 & 24 & 53 & 762 & .00 & .00 & .00 \\
\hline 27 & 5 & 18 & 28 & 34 & 729 & .00 & .00 & .00 \\
\hline 28 & 11 & 19 & 27 & 33 & 625 & .00 & .00 & .00 \\
\hline 29 & 10 & 19 & 27 & 38 & 613 & .01 & .01 & .00 \\
\hline 30 & 9 & 21 & 30 & 27 & 758 & .00 & .00 & .00 \\
\hline
\end{tabular}


Table 1.--Air-temperature, relative-humidity, solar-radiation, and precipitation data, October 1987-December 1989--Continued

\begin{tabular}{|c|c|c|c|c|c|c|c|c|}
\hline \multirow{2}{*}{$\begin{array}{l}\text { Day } \\
\text { of } \\
\text { month }\end{array}$} & \multicolumn{3}{|c|}{ Air temperature } & \multirow{2}{*}{$\begin{array}{l}\text { Average } \\
\text { relative } \\
\text { humidity } \\
\text { (percent) }\end{array}$} & \multirow{2}{*}{$\begin{array}{c}\text { Solar } \\
\text { radiation } \\
\text { (langleys) }\end{array}$} & \multicolumn{3}{|c|}{$\begin{array}{c}\text { Precipitation } \\
\text { (inches) }\end{array}$} \\
\hline & $\begin{array}{r}\text { Minimum } \\
\text { (deg }\end{array}$ & $\begin{array}{l}\text { Average } \\
\text { rees Cel }\end{array}$ & $\begin{array}{l}\text { Maximum } \\
\text { ius) }\end{array}$ & & & $\begin{array}{l}\text { Spring } \\
\text { Creek } 1\end{array}$ & $\begin{array}{l}\text { Spring } \\
\text { Creek } 2\end{array}$ & $\begin{array}{c}\text { Cow } \\
\text { Camp } \\
\text { Creek }\end{array}$ \\
\hline \multicolumn{9}{|c|}{ JULY 1989} \\
\hline 1 & 9 & 20 & 29 & 21 & 781 & 0.00 & 0.00 & 0.00 \\
\hline 2 & 8 & 20 & 31 & 19 & 775 & .00 & .00 & .00 \\
\hline 3 & 7 & 21 & 32 & 21 & 771 & .00 & .00 & -- \\
\hline 4 & 12 & 24 & 34 & 16 & 766 & .00 & .00 & -- \\
\hline 5 & 11 & 23 & 34 & 16 & 755 & .00 & .00 & -- \\
\hline 6 & 10 & 23 & 35 & 20 & 749 & .00 & .00 & -- \\
\hline 7 & 13 & 25 & 35 & 20 & 646 & .00 & .00 & -- \\
\hline 8 & 12 & 24 & 33 & 21 & 536 & .00 & .00 & -- \\
\hline 9 & 17 & 25 & 35 & 19 & 692 & .00 & .00 & -- \\
\hline 10 & 11 & 20 & 30 & 49 & 561 & .09 & .10 & -- \\
\hline 11 & 11 & 18 & 28 & 66 & 417 & .04 & .05 & -- \\
\hline 12 & 11 & 16 & 21 & 82 & 200 & .57 & .58 & -- \\
\hline 13 & 10 & 18 & 26 & 68 & 639 & .03 & .03 & -- \\
\hline 14 & 9 & 18 & 27 & 52 & 699 & .00 & .00 & -- \\
\hline 15 & 11 & 21 & 29 & 34 & 667 & .00 & .00 & -- \\
\hline 16 & 12 & 21 & 30 & 27 & 674 & .00 & .00 & -- \\
\hline 17 & 10 & 19 & 29 & 28 & 744 & .00 & .00 & .00 \\
\hline 18 & 8 & 18 & 28 & 36 & 728 & .00 & .00 & .00 \\
\hline 19 & 12 & 22 & 31 & 29 & 718 & .00 & .00 & .00 \\
\hline 20 & 14 & 23 & 32 & 29 & 693 & .00 & .00 & .00 \\
\hline 21 & 15 & 24 & 33 & 28 & 679 & .00 & .00 & .00 \\
\hline 22 & 14 & 20 & 29 & 52 & 469 & .29 & .28 & .25 \\
\hline 23 & 13 & 17 & 25 & 80 & 453 & .40 & .42 & .42 \\
\hline 24 & 9 & 16 & 25 & 76 & 557 & .00 & .00 & .00 \\
\hline 25 & 12 & 18 & 27 & 60 & 566 & .00 & .00 & .00 \\
\hline 26 & 12 & 19 & 28 & 52 & 635 & .00 & .00 & .00 \\
\hline 27 & 14 & 19 & 27 & 58 & 523 & .00 & .00 & .00 \\
\hline 28 & 11 & 19 & 26 & 68 & 386 & .20 & .10 & .19 \\
\hline 29 & 11 & 16 & 24 & 79 & 503 & .00 & .00 & .00 \\
\hline 30 & 10 & 18 & 27 & 65 & 603 & .00 & .00 & .00 \\
\hline 31 & 11 & 21 & 30 & 51 & 704 & .00 & .00 & .00 \\
\hline
\end{tabular}


Table 1.--Air-temperature, relative-humidity, solar-radiation, and precipitation data, October 1987-December 1989--Continued

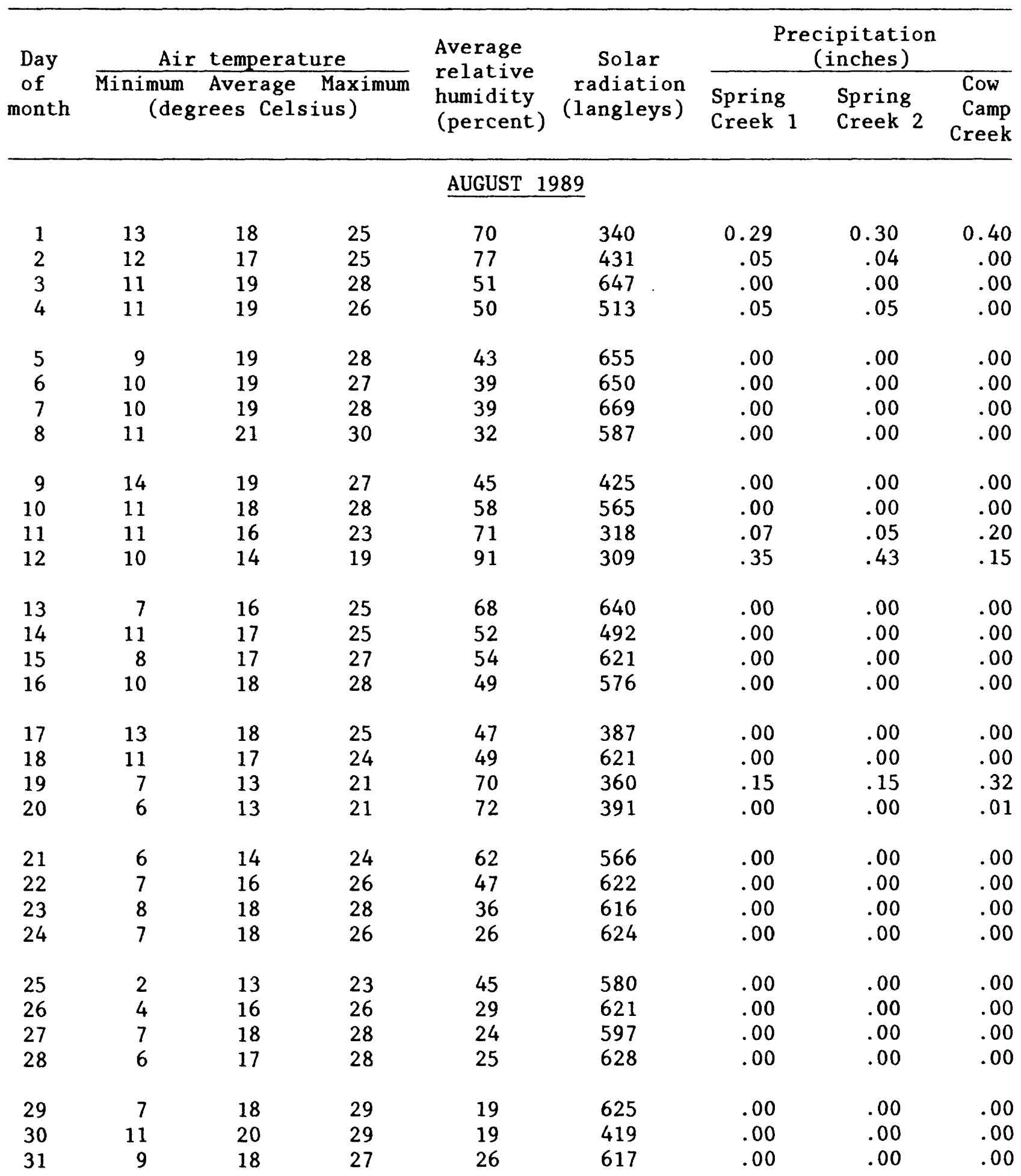


Table 1.--Air-temperature, relative-humidity, solar-radiation, and precipitation data, October 1987-December 1989--Continued

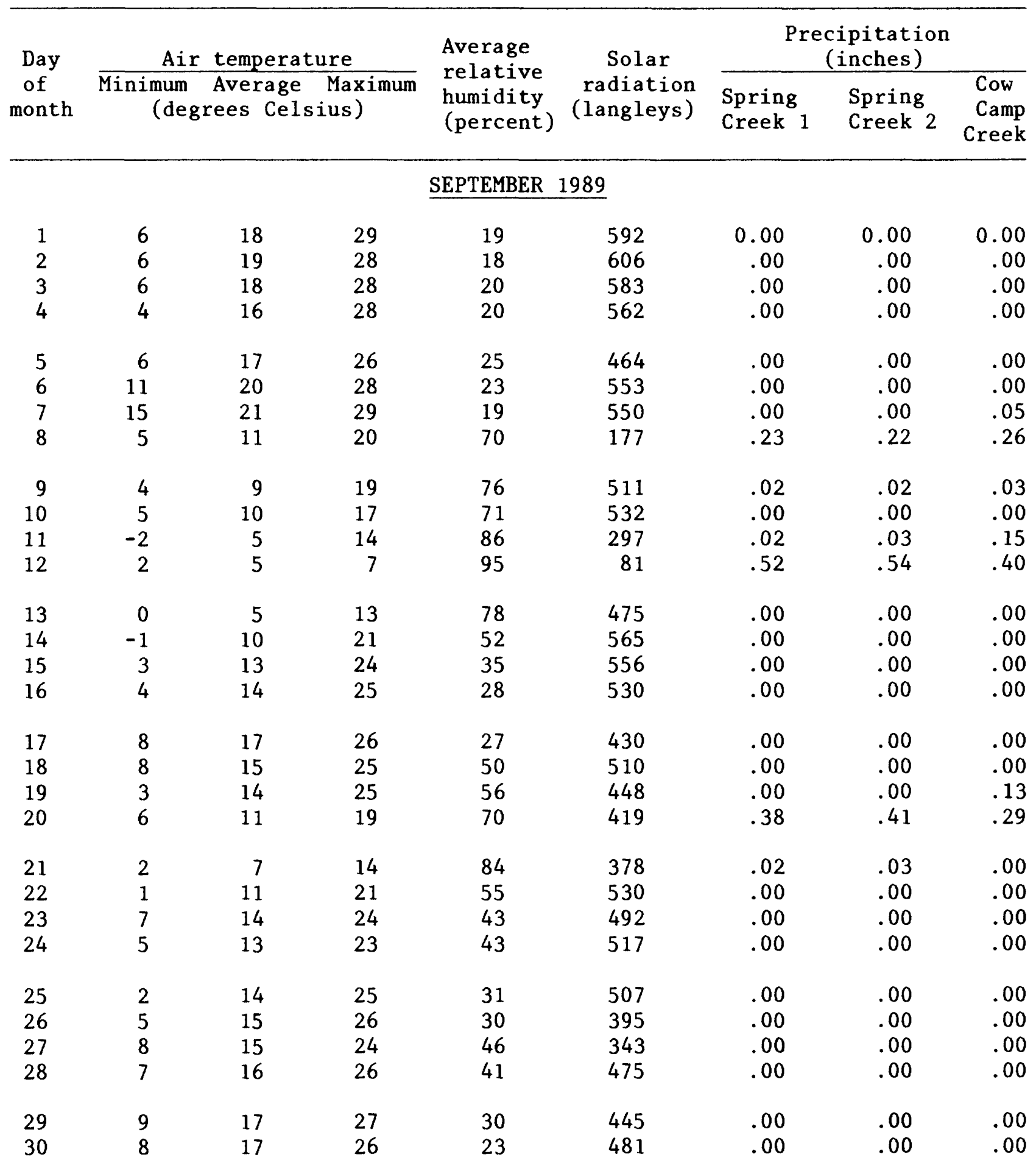


Table 1.--Air-temperature, relative-humidity, solar-radiation, and precipitation data, October 1987-December 1989--Continued

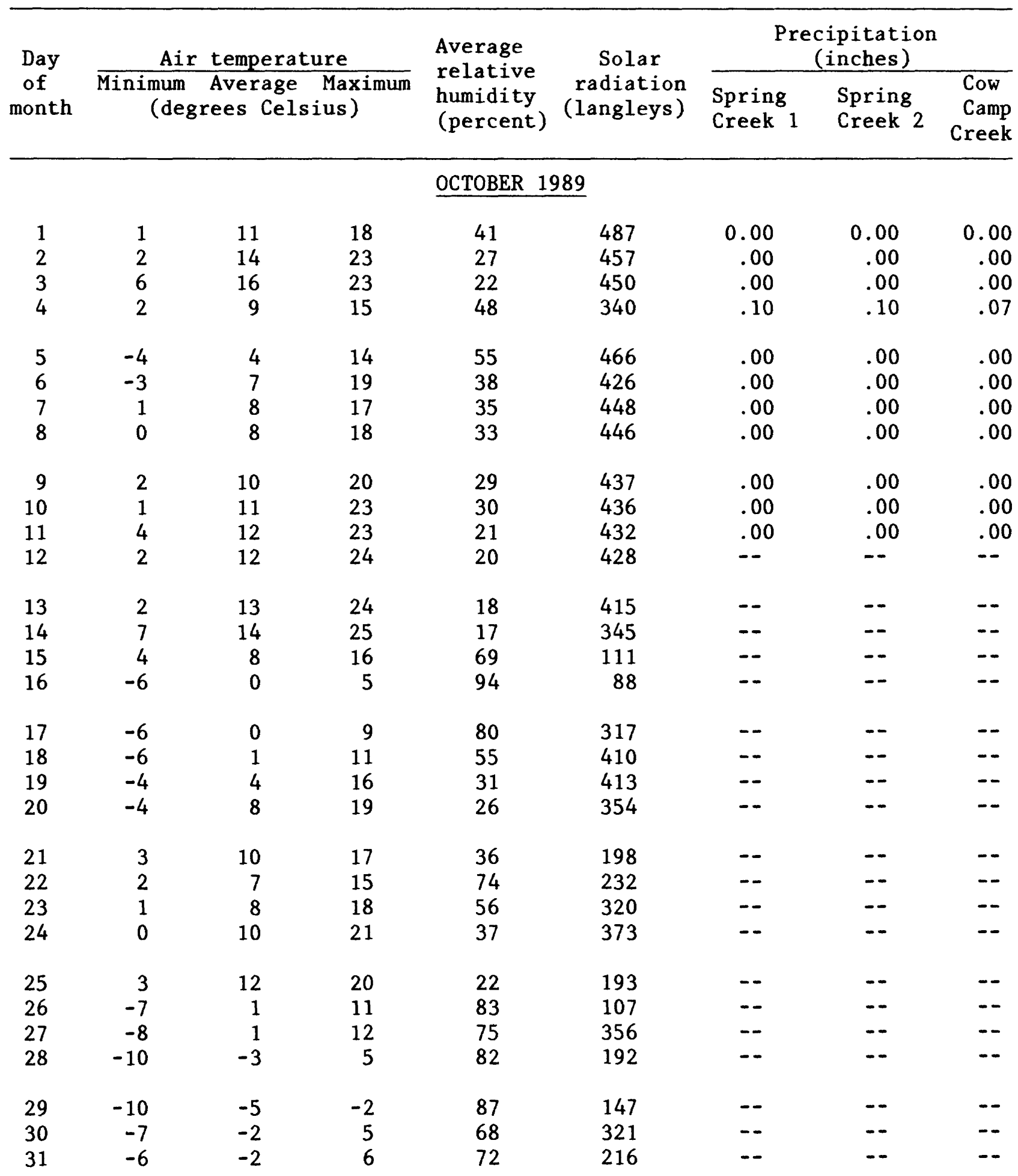


Table 1.--Air-temperature, relative-humidity, solar-radiation, and precipitation data, October 1987-December 1989--Continued

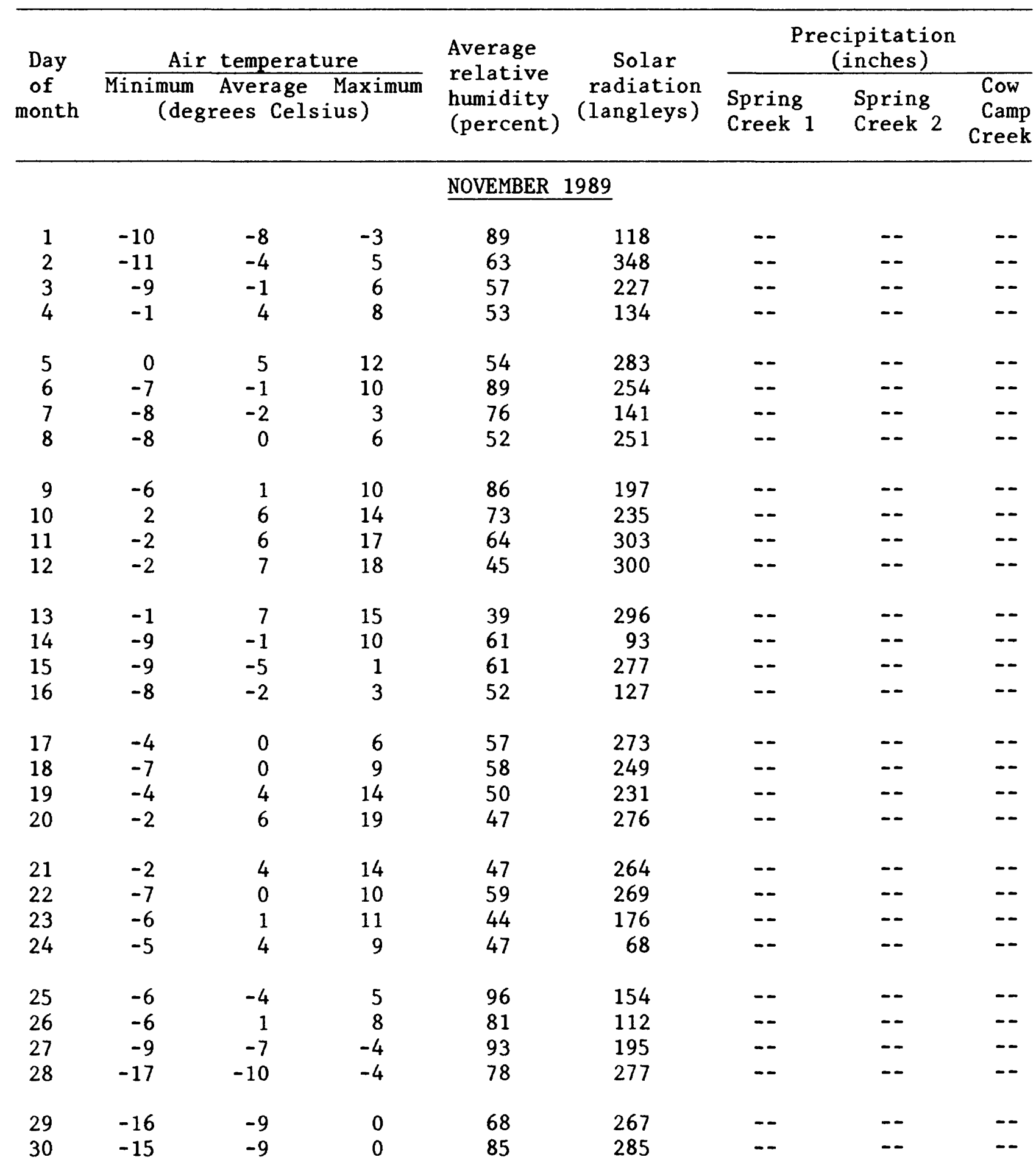


Table 1.--Air-temperature, relative-humidity, solar-radiation, and precipitation data, October 1987-December 1989--Continued

\begin{tabular}{|c|c|c|c|c|c|c|c|c|}
\hline \multirow{2}{*}{$\begin{array}{l}\text { Day } \\
\text { of } \\
\text { month }\end{array}$} & \multicolumn{3}{|c|}{ Air temperature } & \multirow{2}{*}{$\begin{array}{l}\text { Average } \\
\text { relative } \\
\text { humidity } \\
\text { (percent) }\end{array}$} & \multirow{2}{*}{$\begin{array}{c}\text { Solar } \\
\text { radiation } \\
\text { (langleys) }\end{array}$} & \multicolumn{3}{|c|}{$\begin{array}{c}\text { Precipitation } \\
\text { (inches) }\end{array}$} \\
\hline & $\begin{array}{l}\text { Minimum } \\
(\text { degi }\end{array}$ & $\begin{array}{l}\text { Average } \\
\text { rees Cel }\end{array}$ & $\begin{array}{l}\text { Maximum } \\
\text { ius ) }\end{array}$ & & & $\begin{array}{l}\text { Spring } \\
\text { Creek } 1\end{array}$ & $\begin{array}{l}\text { Spring } \\
\text { Creek } 2\end{array}$ & $\begin{array}{c}\text { Cow } \\
\text { Camp } \\
\text { Creek }\end{array}$ \\
\hline \multicolumn{9}{|c|}{ DECEMBER 1989} \\
\hline 1 & -13 & -8 & 0 & 84 & 311 & -- & -- & -- \\
\hline 2 & -14 & -7 & 1 & 72 & 270 & -- & -- & -- \\
\hline 3 & -10 & -3 & 5 & 59 & 255 & -- & -- & -- \\
\hline 4 & -10 & -2 & 5 & 60 & 190 & -- & -- & -- \\
\hline 5 & -4 & 1 & 6 & 75 & 112 & -- & -- & -- \\
\hline 6 & -5 & -2 & 2 & 91 & 159 & -- & -- & -- \\
\hline 7 & -10 & -5 & -1 & 87 & 76 & -- & -- & -- \\
\hline 8 & -12 & -5 & 3 & 68 & 226 & -- & -- & -- \\
\hline 9 & -5 & -1 & 5 & 70 & 127 & -- & -- & -- \\
\hline 10 & -15 & -8 & -5 & 94 & 203 & -- & -- & -- \\
\hline 11 & -18 & -14 & -9 & 87 & 86 & -- & -- & -- \\
\hline 12 & -16 & -10 & -5 & 72 & 136 & -- & -- & -- \\
\hline 13 & -15 & -9 & -4 & 92 & 66 & -- & -- & -- \\
\hline 14 & -7 & -4 & 0 & 85 & 45 & -- & -- & -- \\
\hline 15 & -8 & -5 & -3 & 98 & 88 & -- & -- & -- \\
\hline 16 & -12 & -9 & -6 & 96 & 47 & -- & -- & -- \\
\hline 17 & -14 & -10 & -7 & 95 & 23 & -- & -- & -- \\
\hline 18 & -18 & -13 & -8 & 92 & 38 & -- & -- & -- \\
\hline 19 & -18 & -11 & -6 & 87 & 40 & -- & -- & -- \\
\hline 20 & -11 & -7 & 1 & 74 & 54 & -- & -- & -- \\
\hline 21 & -12 & -6 & 1 & 78 & 128 & -- & -- & -- \\
\hline 22 & -20 & -11 & -5 & 91 & 64 & - & -- & -- \\
\hline 23 & -11 & -5 & 1 & 80 & 241 & -- & -- & -- \\
\hline 24 & -10 & -6 & 1 & 84 & 241 & -- & -- & -- \\
\hline 25 & -13 & -7 & 1 & 75 & 249 & -- & -- & -- \\
\hline 26 & -13 & -6 & 1 & 63 & 243 & -- & -- & -- \\
\hline 27 & -10 & -4 & 3 & 61 & 214 & -- & -- & -- \\
\hline 28 & -11 & -5 & 0 & 67 & 175 & -- & -- & -- \\
\hline 29 & -8 & -5 & -2 & 93 & 141 & -- & -- & -- \\
\hline 30 & -14 & -8 & -5 & 94 & 72 & -- & -- & -- \\
\hline 31 & -12 & -8 & -4 & 87 & 100 & -- & -- & -- \\
\hline
\end{tabular}


Table 2.--Windspeed and wind-vector data,

october 1987-December 1989

$[--$, no data $]$

\begin{tabular}{ccccc}
$\begin{array}{c}\text { Day of } \\
\text { month }\end{array}$ & $\begin{array}{c}\text { Average } \\
\text { windspeed } \\
\text { (miles per } \\
\text { hour) }\end{array}$ & $\begin{array}{c}\text { Wind-vector } \\
\text { magnitude } \\
\text { (miles per } \\
\text { hour) }\end{array}$ & $\begin{array}{c}\text { Wind-vector } \\
\text { direction } \\
\text { (degrees from } \\
\text { north) }\end{array}$ & $\begin{array}{c}\text { Standard deviation } \\
\text { of wind-vector } \\
\text { direction } \\
\text { (degrees) }\end{array}$ \\
\hline
\end{tabular}

OCTOBER 1987

\begin{tabular}{|c|c|c|c|c|}
\hline 1 & - & - & -- & -- \\
\hline 2 & -- & -- & -- & -- \\
\hline 3 & -- & -- & -- & -- \\
\hline 4 & -- & -- & -- & - \\
\hline 5 & -- & -- & -- & -- \\
\hline 6 & -- & -- & -- & -- \\
\hline 7 & -- & -- & -- & - \\
\hline 8 & -- & -- & -- & -- \\
\hline 9 & -- & -- & - & - \\
\hline 10 & -- & - & -- & -- \\
\hline 11 & -- & -- & -- & -- \\
\hline 12 & - & - & -- & -- \\
\hline 13 & -- & -- & - & - \\
\hline 14 & -- & -- & -- & -- \\
\hline 15 & -- & -- & -- & - \\
\hline 16 & -- & -- & -- & -- \\
\hline 17 & -- & -- & -- & - \\
\hline 18 & -- & -- & -- & - \\
\hline 19 & -- & -- & -- & - \\
\hline 20 & -- & -- & -- & -- \\
\hline 21 & -- & -- & -- & - \\
\hline 22 & -- & -- & -- & -- \\
\hline 23 & -- & -- & -- & -- \\
\hline 24 & -- & -- & -- & -- \\
\hline 25 & -- & -- & -- & -- \\
\hline 26 & -- & -- & - & - \\
\hline 27 & -- & -- & -- & -- \\
\hline 28 & -- & -- & -- & -- \\
\hline 29 & 4.25 & 2.51 & 87 & 52 \\
\hline 30 & 3.28 & 1.91 & 95 & 52 \\
\hline 31 & 4.74 & 2.84 & 88 & 51 \\
\hline
\end{tabular}


Table 2.--Windspeed and wind-vector data, october 1987-December 1989--Continued

\begin{tabular}{|c|c|c|c|c|}
\hline $\begin{array}{r}\text { Day of } \\
\text { month }\end{array}$ & $\begin{array}{c}\text { Average } \\
\text { windspeed } \\
\text { (miles per } \\
\text { hour) }\end{array}$ & $\begin{array}{l}\text { Wind-vector } \\
\text { magnitude } \\
\text { (miles per } \\
\text { hour) }\end{array}$ & $\begin{array}{c}\text { Wind-vector } \\
\text { direction } \\
\text { (degrees from } \\
\text { north) }\end{array}$ & $\begin{array}{l}\text { Standard deviation } \\
\text { of wind-vector } \\
\text { direction } \\
\text { (degrees) }\end{array}$ \\
\hline \multicolumn{5}{|c|}{ NOVEMBER 1987} \\
\hline $\begin{array}{l}1 \\
2 \\
3 \\
4\end{array}$ & $\begin{array}{l}5.46 \\
3.58 \\
3.28 \\
4.36\end{array}$ & $\begin{array}{r}3.19 \\
1.33 \\
.43 \\
2.30\end{array}$ & $\begin{array}{r}98 \\
264 \\
52 \\
97\end{array}$ & $\begin{array}{l}52 \\
64 \\
76 \\
56\end{array}$ \\
\hline $\begin{array}{l}5 \\
6 \\
7 \\
8\end{array}$ & $\begin{array}{l}5.90 \\
5.03 \\
4.03 \\
4.14\end{array}$ & $\begin{array}{r}3.43 \\
.36 \\
3.25 \\
2.81\end{array}$ & $\begin{array}{r}85 \\
296 \\
273 \\
88\end{array}$ & $\begin{array}{l}52 \\
78 \\
35 \\
46\end{array}$ \\
\hline $\begin{array}{r}9 \\
10 \\
11 \\
12\end{array}$ & $\begin{array}{l}5.17 \\
5.76 \\
4.91 \\
4.31\end{array}$ & $\begin{array}{l}2.68 \\
1.58 \\
2.10 \\
2.10\end{array}$ & $\begin{array}{r}89 \\
78 \\
109 \\
79\end{array}$ & $\begin{array}{l}56 \\
69 \\
61 \\
58\end{array}$ \\
\hline $\begin{array}{l}13 \\
14 \\
15 \\
16\end{array}$ & $\begin{array}{l}5.09 \\
3.88 \\
5.83 \\
4.72\end{array}$ & $\begin{array}{l}1.65 \\
1.98 \\
3.92 \\
3.61\end{array}$ & $\begin{array}{r}122 \\
268 \\
274 \\
97\end{array}$ & $\begin{array}{l}67 \\
57 \\
46 \\
39\end{array}$ \\
\hline $\begin{array}{l}17 \\
18 \\
19 \\
20\end{array}$ & $\begin{array}{l}6.35 \\
8.36 \\
7.07 \\
8.71\end{array}$ & $\begin{array}{l}4.14 \\
7.48 \\
5.36 \\
6.98\end{array}$ & $\begin{array}{r}277 \\
86 \\
101 \\
87\end{array}$ & $\begin{array}{l}48 \\
26 \\
40 \\
36\end{array}$ \\
\hline $\begin{array}{l}21 \\
22 \\
23 \\
24\end{array}$ & $\begin{array}{l}5.64 \\
4.48 \\
4.33 \\
4.38\end{array}$ & $\begin{array}{r}2.18 \\
2.67 \\
.62 \\
1.65\end{array}$ & $\begin{array}{r}97 \\
104 \\
140 \\
107\end{array}$ & $\begin{array}{l}63 \\
51 \\
75 \\
64\end{array}$ \\
\hline $\begin{array}{l}25 \\
26 \\
27 \\
28\end{array}$ & $\begin{array}{l}6.44 \\
4.92 \\
3.92 \\
7.35\end{array}$ & $\begin{array}{r}4.38 \\
2.91 \\
.61 \\
6.18\end{array}$ & $\begin{array}{r}92 \\
89 \\
120 \\
95\end{array}$ & $\begin{array}{l}46 \\
52 \\
74 \\
32\end{array}$ \\
\hline $\begin{array}{l}29 \\
30\end{array}$ & $\begin{array}{l}4.81 \\
4.71\end{array}$ & $\begin{array}{l}2.62 \\
2.57\end{array}$ & $\begin{array}{r}105 \\
99\end{array}$ & $\begin{array}{l}55 \\
55\end{array}$ \\
\hline
\end{tabular}


Table 2.--Windspeed and wind-vector data, October 1987-December 1989--Continued

\begin{tabular}{ccccc}
$\begin{array}{c}\text { Day of } \\
\text { month }\end{array}$ & $\begin{array}{c}\text { Average } \\
\text { windspeed } \\
\text { (miles per } \\
\text { hour) }\end{array}$ & $\begin{array}{c}\text { Wind-vector } \\
\text { magnitude } \\
\text { (miles per } \\
\text { hour) }\end{array}$ & $\begin{array}{c}\text { Wind-vector } \\
\text { direction } \\
\text { (degrees from } \\
\text { north) }\end{array}$ & $\begin{array}{c}\text { Standard deviation } \\
\text { of wind-vector } \\
\text { direction } \\
\text { (degrees) }\end{array}$ \\
\hline
\end{tabular}

\section{DECEMBER 1987}

\begin{tabular}{|c|c|c|c|c|}
\hline 1 & 4.98 & 3.28 & 105 & 47 \\
\hline 2 & 4.78 & 3.72 & 99 & 38 \\
\hline 3 & 4.77 & 3.97 & 102 & 33 \\
\hline 4 & 5.12 & 3.00 & 110 & 52 \\
\hline 5 & 3.17 & 1.84 & 101 & 52 \\
\hline 6 & 4.66 & 3.37 & 97 & 43 \\
\hline 7 & 7.57 & 2.40 & 240 & 67 \\
\hline 8 & 5.39 & .47 & 57 & 77 \\
\hline 9 & 6.07 & 3.70 & 86 & 51 \\
\hline 10 & 8.98 & 4.09 & 254 & 60 \\
\hline 11 & 12.22 & 11.54 & 272 & 19 \\
\hline 12 & 5.48 & 1.51 & 259 & 69 \\
\hline 13 & 10.82 & 10.54 & 80 & 13 \\
\hline 14 & 5.05 & 1.25 & 267 & 70 \\
\hline 15 & 5.85 & 4.78 & 103 & 35 \\
\hline 16 & 6.60 & 4.61 & 92 & 44 \\
\hline 17 & 3.56 & 1.46 & 98 & 62 \\
\hline 18 & 3.24 & .16 & 93 & 79 \\
\hline 19 & 4.47 & 3.27 & 276 & 42 \\
\hline 20 & 5.60 & 4.56 & 103 & 35 \\
\hline 21 & 6.51 & .46 & 217 & 78 \\
\hline 22 & 5.05 & 3.52 & 91 & 45 \\
\hline 23 & 5.91 & 1.25 & 65 & 72 \\
\hline 24 & -- & -- & -- & -- \\
\hline 25 & - & -- & - &.- \\
\hline 26 & 3.62 & 2.37 & 98 & 48 \\
\hline 27 & 4.40 & .88 & 294 & 72 \\
\hline 28 & 6.58 & 5.31 & 78 & 36 \\
\hline 29 & 8.83 & 6.72 & 80 & 40 \\
\hline 30 & 7.08 & 4.11 & 263 & 52 \\
\hline 31 & 4.97 & 3.47 & 271 & \\
\hline
\end{tabular}


Table 2.--Windspeed and wind-vector data, october 1987-December 1989--Continued

\begin{tabular}{|c|c|c|c|c|}
\hline $\begin{array}{l}\text { Day of } \\
\text { month }\end{array}$ & $\begin{array}{c}\text { Average } \\
\text { windspeed } \\
\text { (miles per } \\
\text { hour) }\end{array}$ & $\begin{array}{l}\text { Wind-vector } \\
\text { magnitude } \\
\text { (miles per } \\
\text { hour) }\end{array}$ & $\begin{array}{l}\text { Wind-vector } \\
\text { direction } \\
\text { (degrees from } \\
\text { north) }\end{array}$ & $\begin{array}{l}\text { Standard deviation } \\
\text { of wind-vector } \\
\text { direction } \\
\text { (degrees) }\end{array}$ \\
\hline \multicolumn{5}{|c|}{ JANUARY 1988} \\
\hline 1 & -- & -- & -- & -- \\
\hline 2 & -- & -- & -- & -- \\
\hline 3 & 5.30 & 3.86 & 102 & 42 \\
\hline 4 & 6.41 & 4.06 & 96 & 49 \\
\hline 5 & 6.00 & 4.83 & 113 & 36 \\
\hline 6 & 4.22 & 2.00 & 285 & 59 \\
\hline 7 & 4.19 & 3.22 & 79 & 39 \\
\hline 8 & 3.09 & 1.39 & 306 & 60 \\
\hline 9 & 3.20 & .54 & 320 & 74 \\
\hline 10 & 4.96 & 2.95 & 81 & 51 \\
\hline 11 & 9.78 & 3.99 & 254 & 62 \\
\hline 12 & 8.29 & 4.85 & 280 & 52 \\
\hline 13 & 8.53 & 6.24 & 81 & 42 \\
\hline 14 & 6.53 & 4.79 & 104 & 42 \\
\hline 15 & 8.97 & 1.60 & 228 & 73 \\
\hline 16 & 8.42 & 2.07 & 80 & 70 \\
\hline 17 & 7.54 & 5.17 & 90 & 45 \\
\hline 18 & 9.11 & 6.74 & 83 & 41 \\
\hline 19 & 5.48 & 3.57 & 79 & 48 \\
\hline 20 & 8.32 & 6.43 & 105 & 39 \\
\hline 21 & 3.87 & 1.40 & 63 & 65 \\
\hline 22 & 9.15 & 7.53 & 106 & 34 \\
\hline 23 & 11.00 & 8.03 & 291 & 42 \\
\hline 24 & 4.81 & 1.79 & 329 & 64 \\
\hline 25 & 6.59 & 4.47 & 115 & 46 \\
\hline 26 & 8.08 & 6.78 & 126 & 32 \\
\hline 27 & 6.91 & 4.62 & 108 & 47 \\
\hline 28 & 7.90 & 5.98 & 91 & 40 \\
\hline 29 & 5.31 & 1.67 & 121 & 67 \\
\hline 30 & 5.89 & 4.81 & 296 & 35 \\
\hline 31 & 3.57 & .80 & 336 & 71 \\
\hline
\end{tabular}


Table 2.--Windspeed and wind-vector data, October 1987-December 1989--Continued

\begin{tabular}{ccccc}
$\begin{array}{c}\text { Day of } \\
\text { month }\end{array}$ & $\begin{array}{c}\text { Average } \\
\text { windspeed } \\
\text { (miles per } \\
\text { hour) }\end{array}$ & $\begin{array}{c}\text { Wind-vector } \\
\text { magnitude } \\
\text { (miles per } \\
\text { hour) }\end{array}$ & $\begin{array}{c}\text { Wind-vector } \\
\text { direction } \\
\text { (degrees from } \\
\text { north) }\end{array}$ & $\begin{array}{c}\text { Standard deviation } \\
\text { of wind-vector } \\
\text { direction } \\
\text { (degrees) }\end{array}$ \\
\hline
\end{tabular}

\section{FEBRUARY 1988}

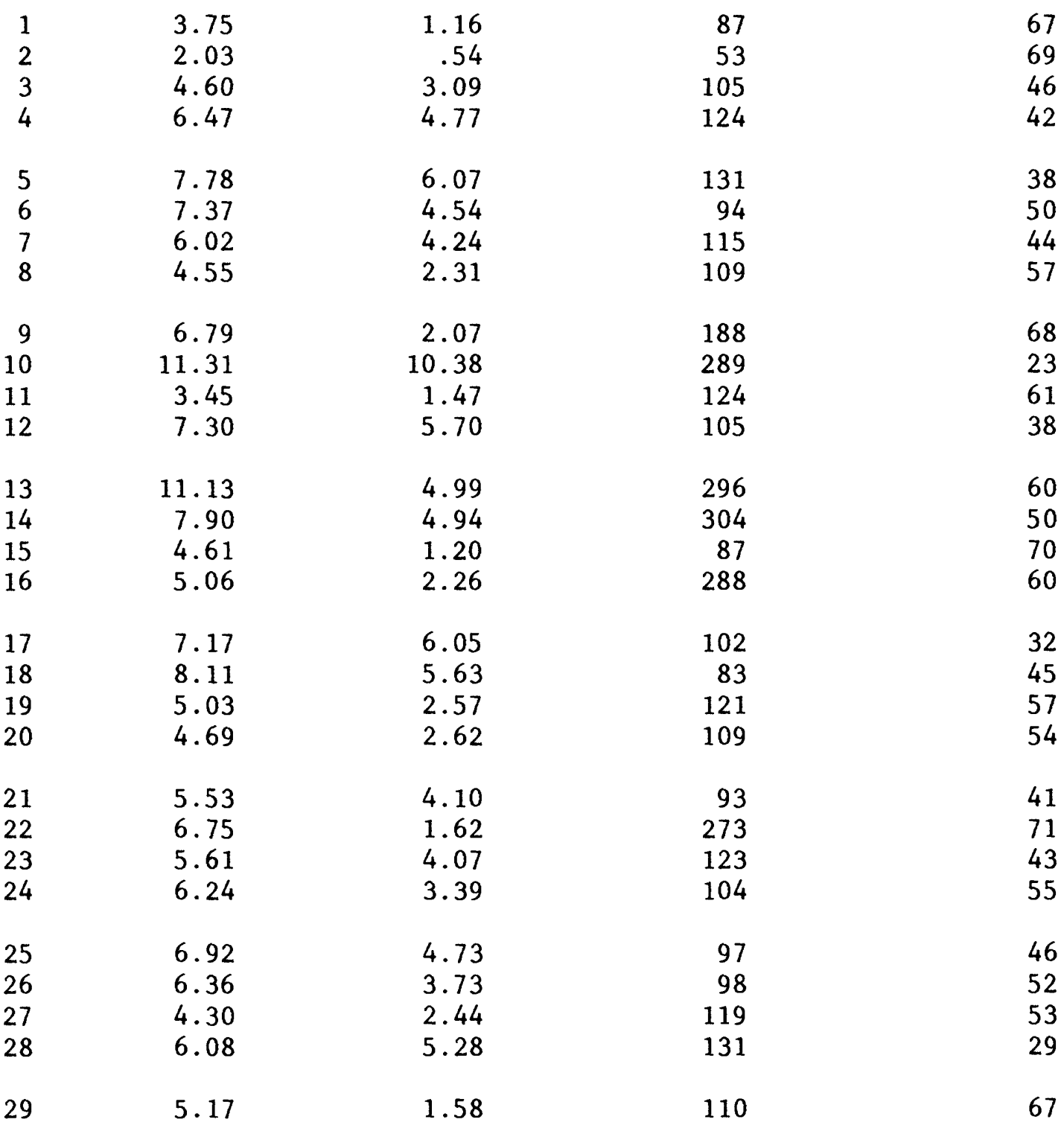


Table 2.--Windspeed and wind-vector data, October 1987-December 1989--Continued

\begin{tabular}{|c|c|c|c|c|}
\hline $\begin{array}{l}\text { Day of } \\
\text { month }\end{array}$ & $\begin{array}{c}\text { Average } \\
\text { windspeed } \\
\text { (miles per } \\
\text { hour) }\end{array}$ & $\begin{array}{l}\text { Wind-vector } \\
\text { magnitude } \\
\text { (miles per } \\
\text { hour) }\end{array}$ & $\begin{array}{c}\text { Wind-vector } \\
\text { direction } \\
\text { (degrees from } \\
\text { north) }\end{array}$ & $\begin{array}{l}\text { Standard deviation } \\
\text { of wind-vector } \\
\text { direction } \\
\text { (degrees) }\end{array}$ \\
\hline \multicolumn{5}{|c|}{ MARCH 1988} \\
\hline $\begin{array}{l}1 \\
2 \\
3 \\
4\end{array}$ & $\begin{array}{l}7.04 \\
3.38 \\
4.34 \\
7.95\end{array}$ & $\begin{array}{r}5.06 \\
.82 \\
1.79 \\
5.21\end{array}$ & $\begin{array}{l}100 \\
274 \\
270 \\
282\end{array}$ & $\begin{array}{l}43 \\
71 \\
62 \\
48\end{array}$ \\
\hline $\begin{array}{l}5 \\
6 \\
7 \\
8\end{array}$ & $\begin{array}{r}6.48 \\
6.94 \\
10.07 \\
6.33\end{array}$ & $\begin{array}{l}4.49 \\
1.54 \\
8.80 \\
4.10\end{array}$ & $\begin{array}{l}113 \\
165 \\
285 \\
108\end{array}$ & $\begin{array}{l}45 \\
71 \\
29 \\
48\end{array}$ \\
\hline $\begin{array}{r}9 \\
10 \\
11 \\
12\end{array}$ & $\begin{array}{l}9.36 \\
5.30 \\
7.45 \\
4.36\end{array}$ & $\begin{array}{l}7.36 \\
1.86 \\
6.43 \\
1.12\end{array}$ & $\begin{array}{r}91 \\
310 \\
284 \\
275\end{array}$ & $\begin{array}{l}37 \\
65 \\
30 \\
70\end{array}$ \\
\hline $\begin{array}{l}13 \\
14 \\
15 \\
16\end{array}$ & $\begin{array}{r}6.59 \\
7.26 \\
11.47 \\
8.44\end{array}$ & $\begin{array}{r}.48 \\
5.52 \\
10.31 \\
1.68\end{array}$ & $\begin{array}{r}235 \\
116 \\
94 \\
40\end{array}$ & $\begin{array}{l}78 \\
40 \\
26 \\
73\end{array}$ \\
\hline $\begin{array}{l}17 \\
18 \\
19 \\
20\end{array}$ & $\begin{array}{l}4.27 \\
6.62 \\
6.38 \\
6.68\end{array}$ & $\begin{array}{l}1.17 \\
3.46 \\
4.20 \\
4.81\end{array}$ & $\begin{array}{l}289 \\
115 \\
102 \\
123\end{array}$ & $\begin{array}{l}69 \\
56 \\
47 \\
43\end{array}$ \\
\hline $\begin{array}{l}21 \\
22 \\
23 \\
24\end{array}$ & $\begin{array}{r}5.56 \\
4.94 \\
7.30 \\
10.03\end{array}$ & $\begin{array}{l}4.15 \\
2.18 \\
1.40 \\
9.20\end{array}$ & $\begin{array}{l}129 \\
275 \\
264 \\
286\end{array}$ & $\begin{array}{l}41 \\
61 \\
73 \\
23\end{array}$ \\
\hline $\begin{array}{l}25 \\
26 \\
27 \\
28\end{array}$ & $\begin{array}{r}9.42 \\
6.63 \\
10.65 \\
5.38\end{array}$ & $\begin{array}{l}7.74 \\
5.35 \\
5.54 \\
3.57\end{array}$ & $\begin{array}{l}280 \\
121 \\
249 \\
280\end{array}$ & $\begin{array}{l}34 \\
36 \\
56 \\
47\end{array}$ \\
\hline $\begin{array}{l}29 \\
30 \\
31\end{array}$ & $\begin{array}{r}5.39 \\
8.35 \\
12.49\end{array}$ & $\begin{array}{r}.64 \\
7.91 \\
12.00\end{array}$ & $\begin{array}{r}177 \\
95 \\
95\end{array}$ & $\begin{array}{l}76 \\
19 \\
16\end{array}$ \\
\hline
\end{tabular}


Table 2.--Windspeed and wind-vector data, october 1987-December 1989--Continued

\begin{tabular}{|c|c|c|c|c|}
\hline $\begin{array}{r}\text { Day of } \\
\text { month }\end{array}$ & $\begin{array}{c}\text { Average } \\
\text { windspeed } \\
\text { (miles per } \\
\text { hour) }\end{array}$ & $\begin{array}{l}\text { Wind-vector } \\
\text { magnitude } \\
\text { (miles per } \\
\text { hour) }\end{array}$ & $\begin{array}{l}\text { Wind-vector } \\
\text { direction } \\
\text { (degrees from } \\
\text { north) }\end{array}$ & $\begin{array}{l}\text { Standard deviation } \\
\text { of wind-vector } \\
\text { direction } \\
\text { (degrees) }\end{array}$ \\
\hline \multicolumn{5}{|c|}{ APRIL 1988} \\
\hline $\begin{array}{l}1 \\
2 \\
3 \\
4\end{array}$ & $\begin{array}{l}4.69 \\
6.35 \\
7.24 \\
6.46\end{array}$ & $\begin{array}{l}1.31 \\
2.79 \\
3.39 \\
2.81\end{array}$ & $\begin{array}{l}250 \\
139 \\
199 \\
252\end{array}$ & $\begin{array}{l}69 \\
61 \\
59 \\
61\end{array}$ \\
\hline $\begin{array}{l}5 \\
6 \\
7 \\
8\end{array}$ & $\begin{array}{l}6.02 \\
8.55 \\
6.88 \\
8.69\end{array}$ & $\begin{array}{l}3.18 \\
7.32 \\
4.09 \\
6.16\end{array}$ & $\begin{array}{l}277 \\
107 \\
132 \\
279\end{array}$ & $\begin{array}{l}56 \\
31 \\
52 \\
44\end{array}$ \\
\hline $\begin{array}{r}9 \\
10 \\
11 \\
12\end{array}$ & $\begin{array}{l}6.06 \\
8.04 \\
8.86 \\
6.79\end{array}$ & $\begin{array}{l}2.53 \\
4.31 \\
7.43 \\
5.36\end{array}$ & $\begin{array}{l}282 \\
103 \\
103 \\
108\end{array}$ & $\begin{array}{l}62 \\
55 \\
33 \\
37\end{array}$ \\
\hline $\begin{array}{l}13 \\
14 \\
15 \\
16\end{array}$ & $\begin{array}{r}8.87 \\
11.01 \\
5.58 \\
12.80\end{array}$ & $\begin{array}{r}7.33 \\
10.32 \\
2.88 \\
12.39\end{array}$ & $\begin{array}{r}99 \\
98 \\
108 \\
93\end{array}$ & $\begin{array}{l}34 \\
20 \\
56 \\
14\end{array}$ \\
\hline $\begin{array}{l}17 \\
18 \\
19 \\
20\end{array}$ & $\begin{array}{l}7.32 \\
5.60 \\
5.31 \\
6.93\end{array}$ & $\begin{array}{r}4.94 \\
.97 \\
3.26 \\
2.32\end{array}$ & $\begin{array}{r}92 \\
110 \\
274 \\
176\end{array}$ & $\begin{array}{l}46 \\
74 \\
50 \\
66\end{array}$ \\
\hline $\begin{array}{l}21 \\
22 \\
23 \\
24\end{array}$ & $\begin{array}{l}5.53 \\
6.80 \\
5.68 \\
4.94\end{array}$ & $\begin{array}{l}1.52 \\
5.33 \\
3.33 \\
2.18\end{array}$ & $\begin{array}{l}114 \\
274 \\
266 \\
270\end{array}$ & $\begin{array}{l}69 \\
38 \\
52 \\
60\end{array}$ \\
\hline $\begin{array}{l}25 \\
26 \\
27 \\
28\end{array}$ & $\begin{array}{l}7.73 \\
5.00 \\
6.18 \\
7.31\end{array}$ & $\begin{array}{r}4.13 \\
.36 \\
1.10 \\
4.48\end{array}$ & $\begin{array}{r}285 \\
300 \\
292 \\
89\end{array}$ & $\begin{array}{l}55 \\
78 \\
73 \\
50\end{array}$ \\
\hline $\begin{array}{l}29 \\
30\end{array}$ & $\begin{array}{l}6.98 \\
7.85\end{array}$ & $\begin{array}{r}.24 \\
4.10\end{array}$ & $\begin{array}{l}298 \\
131\end{array}$ & $\begin{array}{l}80 \\
56\end{array}$ \\
\hline
\end{tabular}


Table 2.--Windspeed and wind-vector data,

october 1987-December 1989--Continued

\begin{tabular}{|c|c|c|c|c|}
\hline $\begin{array}{l}\text { Day of } \\
\text { month }\end{array}$ & $\begin{array}{c}\text { Average } \\
\text { windspeed } \\
\text { (miles per } \\
\text { hour) }\end{array}$ & $\begin{array}{l}\text { Wind-vector } \\
\text { magnitude } \\
\text { (miles per } \\
\text { hour) }\end{array}$ & $\begin{array}{l}\text { Wind-vector } \\
\text { direction } \\
\text { (degrees from } \\
\text { north) }\end{array}$ & $\begin{array}{l}\text { Standard deviation } \\
\text { of wind-vector } \\
\text { direction } \\
\text { (degrees) }\end{array}$ \\
\hline \multicolumn{5}{|c|}{ MAY 1988} \\
\hline 1 & 8.52 & 4.76 & 272 & 54 \\
\hline 2 & 9.02 & 7.75 & 282 & 30 \\
\hline 3 & 5.29 & 2.10 & 92 & 63 \\
\hline 4 & 10.12 & 6.77 & 103 & 47 \\
\hline 5 & 10.26 & 6.21 & 115 & 51 \\
\hline 6 & 10.71 & 6.49 & 243 & 51 \\
\hline 7 & 13.36 & 11.87 & 272 & 27 \\
\hline 8 & 6.17 & 4.70 & 284 & 40 \\
\hline 9 & 6.59 & 1.97 & 276 & 68 \\
\hline 10 & 6.06 & .47 & 275 & 78 \\
\hline 11 & 8.49 & 4.42 & 96 & 56 \\
\hline 12 & 5.31 & .36 & 7 & 78 \\
\hline 13 & 8.28 & .88 & 188 & 77 \\
\hline 14 & 8.24 & 5.46 & 286 & 47 \\
\hline 15 & 6.23 & .30 & 150 & 79 \\
\hline 16 & 8.21 & 2.80 & 101 & 66 \\
\hline 17 & 6.49 & 1.30 & 152 & 72 \\
\hline 18 & 6.38 & .50 & 314 & 78 \\
\hline 19 & 5.62 & 4.61 & 282 & 34 \\
\hline 20 & 4.55 & .50 & 7 & 76 \\
\hline 21 & 6.19 & 2.39 & 86 & 63 \\
\hline 22 & 5.87 & 1.18 & 107 & 72 \\
\hline 23 & 5.95 & 1.13 & 104 & 73 \\
\hline 24 & 6.10 & .45 & 169 & 78 \\
\hline 25 & 7.01 & 3.65 & 100 & 56 \\
\hline 26 & 7.38 & 4.19 & 104 & 53 \\
\hline 27 & 9.48 & 4.44 & 99 & 59 \\
\hline 28 & 5.68 & 2.50 & 99 & 61 \\
\hline 29 & 8.65 & 5.19 & 104 & 51 \\
\hline 30 & 7.04 & 2.10 & 281 & 68 \\
\hline 31 & 6.36 & 2.64 & 287 & 62 \\
\hline
\end{tabular}


Table 2.--Windspeed and wind-vector data, October 1987-December 1989--Continued

\begin{tabular}{|c|c|c|c|c|}
\hline $\begin{array}{l}\text { Day of } \\
\text { month }\end{array}$ & $\begin{array}{c}\text { Average } \\
\text { windspeed } \\
\text { (miles per } \\
\text { hour) }\end{array}$ & $\begin{array}{l}\text { Wind-vector } \\
\text { magnitude } \\
\text { (miles per } \\
\text { hour) }\end{array}$ & $\begin{array}{c}\text { Wind-vector } \\
\text { direction } \\
\text { (degrees from } \\
\text { north) }\end{array}$ & $\begin{array}{l}\text { Standard deviation } \\
\text { of wind-vector } \\
\text { direction } \\
\text { (degrees) }\end{array}$ \\
\hline
\end{tabular}

JUNE 1988

\begin{tabular}{|c|c|c|c|}
\hline 4.97 & 3.35 & 284 & 46 \\
\hline 4.40 & .78 & 287 & 73 \\
\hline 8.42 & 5.30 & 93 & 49 \\
\hline 6.10 & 1.95 & 89 & 67 \\
\hline 9.80 & 7.81 & 100 & 37 \\
\hline 7.24 & 3.05 & 104 & 62 \\
\hline 5.22 & 1.69 & 138 & 67 \\
\hline 5.69 & .68 & 238 & 76 \\
\hline 9.02 & 3.46 & 100 & 64 \\
\hline 11.56 & 4.52 & 118 & 63 \\
\hline 7.90 & 6.22 & 100 & 37 \\
\hline 8.98 & 7.03 & 98 & 38 \\
\hline 6.42 & 3.58 & 93 & 54 \\
\hline 4.41 & 1.17 & 68 & 69 \\
\hline 7.56 & 3.26 & 76 & 61 \\
\hline 6.13 & 1.53 & 91 & 70 \\
\hline 8.53 & 5.71 & 101 & 47 \\
\hline 4.48 & 1.68 & 139 & 64 \\
\hline 6.30 & 4.11 & 103 & 48 \\
\hline 6.83 & 4.11 & 105 & 51 \\
\hline 7.36 & 4.49 & 94 & 51 \\
\hline 8.71 & 7.05 & 94 & 35 \\
\hline 5.26 & 2.73 & 92 & 56 \\
\hline 6.71 & 4.06 & 91 & 51 \\
\hline 10.96 & 10.43 & 94 & 18 \\
\hline 7.29 & 5.58 & 100 & 39 \\
\hline 6.65 & 3.96 & 106 & 51 \\
\hline 4.27 & 1.23 & 162 & 68 \\
\hline 4.73 & 1.22 & 101 & 70 \\
\hline 4.82 & 1.64 & 125 & 66 \\
\hline
\end{tabular}


Table 2.--Windspeed and wind-vector data, October 1987-December 1989--Continued

\begin{tabular}{|c|c|c|c|c|}
\hline $\begin{array}{l}\text { Day of } \\
\text { month }\end{array}$ & $\begin{array}{c}\text { Average } \\
\text { windspeed } \\
\text { (miles per } \\
\text { hour) }\end{array}$ & $\begin{array}{l}\text { Wind-vector } \\
\text { magnitude } \\
\text { (miles per } \\
\text { hour) }\end{array}$ & $\begin{array}{l}\text { Wind-vector } \\
\text { direction } \\
\text { (degrees from } \\
\text { north) }\end{array}$ & $\begin{array}{l}\text { Standard deviation } \\
\text { of wind-vector } \\
\text { direction } \\
\text { (degrees) }\end{array}$ \\
\hline \multicolumn{5}{|c|}{ JULY 1988} \\
\hline 1 & 4.46 & 2.73 & 85 & 50 \\
\hline 2 & 6.73 & 4.65 & 108 & 45 \\
\hline 3 & 7.07 & 4.46 & 93 & 49 \\
\hline 4 & 4.75 & 1.96 & 99 & 62 \\
\hline 5 & 9.04 & 7.35 & 99 & 35 \\
\hline 6 & 5.80 & 3.40 & 110 & 52 \\
\hline 7 & 5.50 & 1.45 & 155 & 70 \\
\hline 8 & 6.01 & .33 & 241 & 79 \\
\hline 9 & 6.16 & 2.32 & 89 & 64 \\
\hline 10 & 10.37 & 8.94 & 96 & 30 \\
\hline 11 & 7.25 & 2.82 & 117 & 63 \\
\hline 12 & 6.14 & 2.25 & 210 & 64 \\
\hline 13 & 5.88 & 3.45 & 107 & 52 \\
\hline 14 & 8.21 & 3.11 & 131 & 64 \\
\hline 15 & 7.23 & 4.94 & 107 & 46 \\
\hline 16 & 7.65 & 5.66 & 102 & 41 \\
\hline 17 & 6.30 & 1.28 & 213 & 72 \\
\hline 18 & 5.79 & .38 & 109 & 78 \\
\hline 19 & 5.59 & .49 & 275 & 77 \\
\hline 20 & 5.76 & 2.10 & 102 & 65 \\
\hline 21 & 5.42 & 1.13 & 216 & 72 \\
\hline 22 & 5.87 & 1.89 & 133 & 67 \\
\hline 23 & 5.50 & 1.80 & 129 & 66 \\
\hline 24 & 4.93 & 2.39 & 122 & 58 \\
\hline 25 & 5.69 & 2.10 & 113 & 64 \\
\hline 26 & 6.96 & 3.95 & 106 & 53 \\
\hline 27 & 5.57 & 3.14 & 93 & 54 \\
\hline 28 & 5.72 & 3.75 & 72 & 47 \\
\hline 29 & 6.61 & 4.78 & 28 & 43 \\
\hline 30 & 6.18 & 4.50 & 25 & 42 \\
\hline 31 & 6.10 & 2.76 & 97 & 60 \\
\hline
\end{tabular}


Table 2.--Windspeed and wind-vector data,

october 1987-December 1989--Continued

\begin{tabular}{|c|c|c|c|c|}
\hline $\begin{array}{l}\text { Day of } \\
\text { month }\end{array}$ & $\begin{array}{c}\text { Average } \\
\text { windspeed } \\
\text { (miles per } \\
\text { hour) }\end{array}$ & $\begin{array}{l}\text { Wind-vector } \\
\text { magnitude } \\
\text { (miles per } \\
\text { hour) }\end{array}$ & $\begin{array}{l}\text { Wind-vector } \\
\text { direction } \\
\text { (degrees from } \\
\text { north) }\end{array}$ & $\begin{array}{l}\text { Standard deviation } \\
\text { of wind-vector } \\
\text { direction } \\
\text { (degrees) }\end{array}$ \\
\hline \multicolumn{5}{|c|}{ AUGUST 1988} \\
\hline $\begin{array}{l}1 \\
2 \\
3 \\
4\end{array}$ & $\begin{array}{l}5.84 \\
5.48 \\
4.40 \\
4.29\end{array}$ & $\begin{array}{r}1.01 \\
1.78 \\
1.99 \\
.17\end{array}$ & $\begin{array}{r}153 \\
78 \\
305 \\
136\end{array}$ & $\begin{array}{l}74 \\
67 \\
60 \\
79\end{array}$ \\
\hline $\begin{array}{l}5 \\
6 \\
7 \\
8\end{array}$ & $\begin{array}{l}4.61 \\
5.42 \\
5.33 \\
6.57\end{array}$ & $\begin{array}{r}.62 \\
.79 \\
3.79 \\
3.43\end{array}$ & $\begin{array}{r}150 \\
183 \\
89 \\
46\end{array}$ & $\begin{array}{l}75 \\
75 \\
44 \\
56\end{array}$ \\
\hline $\begin{array}{r}9 \\
10 \\
11 \\
12\end{array}$ & $\begin{array}{l}5.73 \\
5.49 \\
4.96 \\
6.05\end{array}$ & $\begin{array}{r}.86 \\
.81 \\
1.21 \\
2.85\end{array}$ & $\begin{array}{r}59 \\
224 \\
101 \\
103\end{array}$ & $\begin{array}{l}75 \\
75 \\
70 \\
59\end{array}$ \\
\hline $\begin{array}{l}13 \\
14 \\
15 \\
16\end{array}$ & $\begin{array}{l}5.67 \\
6.81 \\
5.80 \\
6.18\end{array}$ & $\begin{array}{l}2.65 \\
1.93 \\
1.04 \\
4.55\end{array}$ & $\begin{array}{r}154 \\
206 \\
90 \\
101\end{array}$ & $\begin{array}{l}59 \\
69 \\
73 \\
42\end{array}$ \\
\hline $\begin{array}{l}17 \\
18 \\
19 \\
20\end{array}$ & $\begin{array}{l}7.09 \\
6.16 \\
5.45 \\
5.30\end{array}$ & $\begin{array}{r}5.25 \\
4.72 \\
1.81 \\
.41\end{array}$ & $\begin{array}{r}54 \\
38 \\
93 \\
184\end{array}$ & $\begin{array}{l}41 \\
39 \\
66 \\
78\end{array}$ \\
\hline $\begin{array}{l}21 \\
22 \\
23 \\
24\end{array}$ & $\begin{array}{l}5.39 \\
5.52 \\
5.27 \\
7.97\end{array}$ & $\begin{array}{r}2.94 \\
.45 \\
1.93 \\
2.91\end{array}$ & $\begin{array}{r}89 \\
271 \\
133 \\
95\end{array}$ & $\begin{array}{l}55 \\
78 \\
64 \\
65\end{array}$ \\
\hline $\begin{array}{l}25 \\
26 \\
27 \\
28\end{array}$ & $\begin{array}{l}5.95 \\
5.53 \\
6.14 \\
6.91\end{array}$ & $\begin{array}{r}1.75 \\
1.27 \\
.64 \\
1.80\end{array}$ & $\begin{array}{r}128 \\
255 \\
302 \\
73\end{array}$ & $\begin{array}{l}68 \\
71 \\
77 \\
70\end{array}$ \\
\hline $\begin{array}{l}29 \\
30 \\
31\end{array}$ & $\begin{array}{l}6.85 \\
5.66 \\
4.85\end{array}$ & $\begin{array}{r}3.28 \\
.51 \\
1.00\end{array}$ & $\begin{array}{r}96 \\
226 \\
108\end{array}$ & $\begin{array}{l}58 \\
77 \\
72\end{array}$ \\
\hline
\end{tabular}


Table 2.--Windspeed and wind-vector data, october 1987-December 1989--Continued

\begin{tabular}{ccccc}
$\begin{array}{c}\text { Day of } \\
\text { month }\end{array}$ & $\begin{array}{c}\text { Average } \\
\text { windspeed } \\
\text { (miles per } \\
\text { hour) }\end{array}$ & $\begin{array}{c}\text { Wind-vector } \\
\text { magnitude } \\
\text { (miles per } \\
\text { hour) }\end{array}$ & $\begin{array}{c}\text { Wind-vector } \\
\text { direction } \\
\text { (degrees from } \\
\text { north) }\end{array}$ & $\begin{array}{c}\text { Standard deviation } \\
\text { of wind-vector } \\
\text { direction } \\
\text { (degrees) }\end{array}$ \\
\hline
\end{tabular}

SEPTEMBER 1988

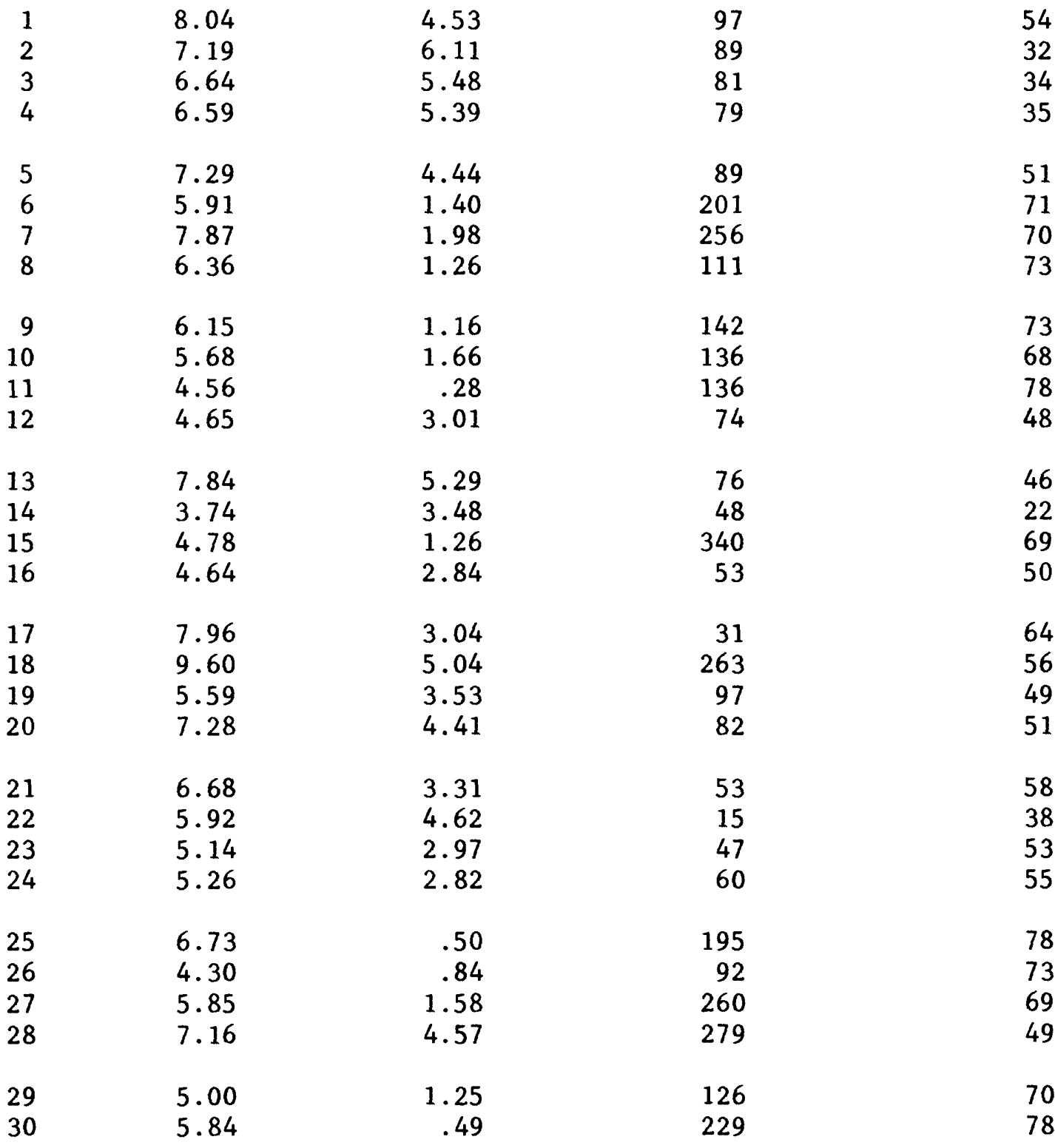


Table 2.--Windspeed and wind-vector data, October 1987-December 1989--Continued

\begin{tabular}{ccccc}
$\begin{array}{c}\text { Day of } \\
\text { month }\end{array}$ & $\begin{array}{c}\text { Average } \\
\text { windspeed } \\
\text { (miles per } \\
\text { hour) }\end{array}$ & $\begin{array}{c}\text { Wind-vector } \\
\text { magnitude } \\
\text { (miles per } \\
\text { hour) }\end{array}$ & $\begin{array}{c}\text { Wind-vector } \\
\text { direction } \\
\text { (degrees from } \\
\text { north) }\end{array}$ & $\begin{array}{c}\text { Standard deviation } \\
\text { of wind-vector } \\
\text { direction } \\
\text { (degrees) }\end{array}$ \\
\hline
\end{tabular}

\section{OCTOBER 1988}

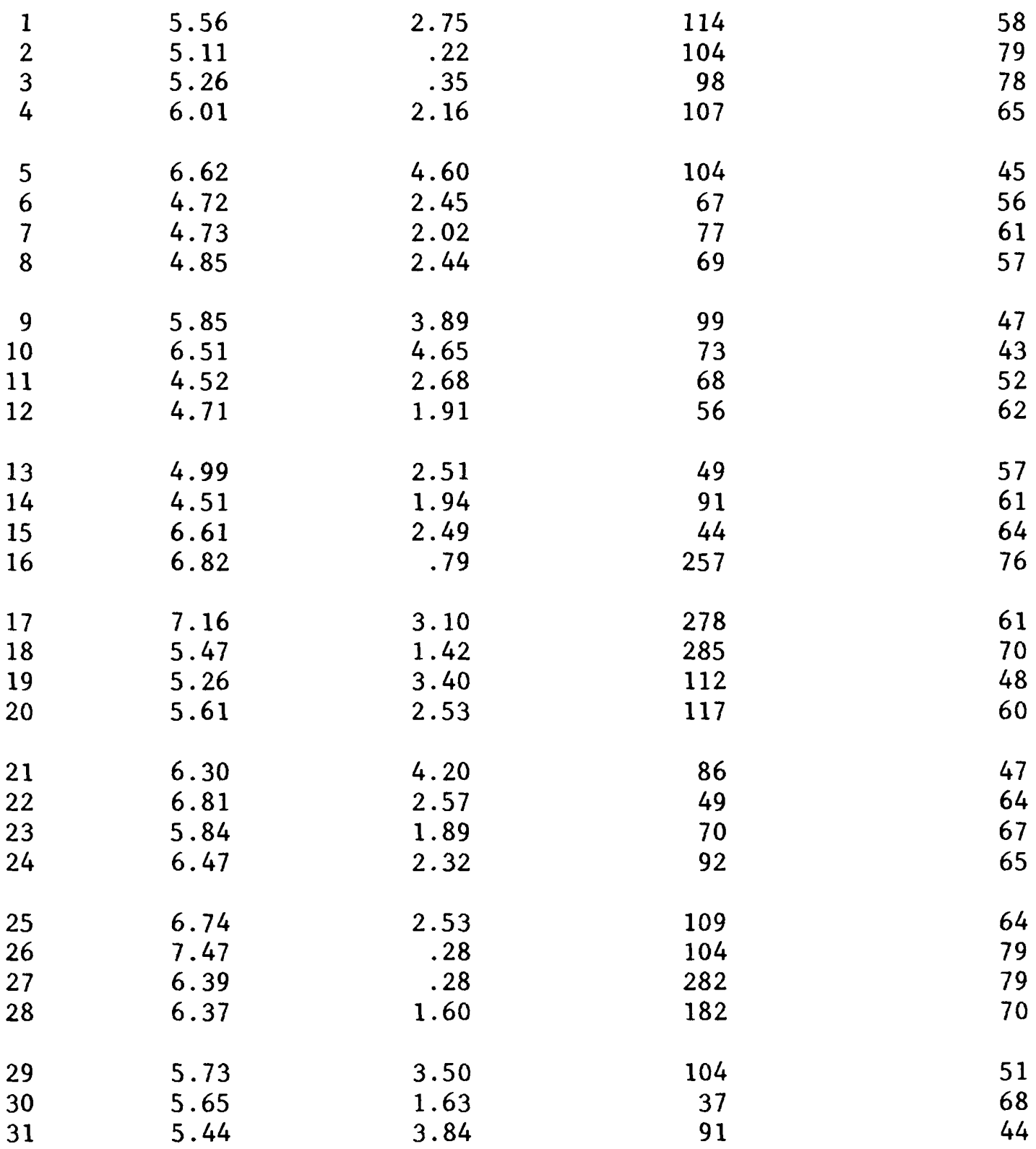


Table 2.--Windspeed and wind-vector data, October 1987-December 1989--Continued

\begin{tabular}{|c|c|c|c|c|}
\hline $\begin{array}{l}\text { Day of } \\
\text { month }\end{array}$ & $\begin{array}{c}\text { Average } \\
\text { windspeed } \\
\text { (miles per } \\
\text { hour) }\end{array}$ & $\begin{array}{l}\text { Wind-vector } \\
\text { magnitude } \\
\text { (miles per } \\
\text { hour) }\end{array}$ & $\begin{array}{l}\text { Wind-vector } \\
\text { direction } \\
\text { (degrees from } \\
\text { north) }\end{array}$ & $\begin{array}{l}\text { Standard deviation } \\
\text { of wind-vector } \\
\text { direction } \\
\text { (degrees) }\end{array}$ \\
\hline \multicolumn{5}{|c|}{ NOVEMBER 1988} \\
\hline $\begin{array}{l}1 \\
2 \\
3 \\
4\end{array}$ & $\begin{array}{r}5.11 \\
5.26 \\
7.70 \\
12.05\end{array}$ & $\begin{array}{r}1.50 \\
.19 \\
5.47 \\
11.04\end{array}$ & $\begin{array}{r}48 \\
153 \\
288 \\
287\end{array}$ & $\begin{array}{l}68 \\
80 \\
44 \\
23\end{array}$ \\
\hline $\begin{array}{l}5 \\
6 \\
7 \\
8\end{array}$ & $\begin{array}{l}6.21 \\
6.46 \\
5.50 \\
4.76\end{array}$ & $\begin{array}{l}2.71 \\
2.83 \\
1.02 \\
1.17\end{array}$ & $\begin{array}{l}290 \\
335 \\
292 \\
123\end{array}$ & $\begin{array}{l}61 \\
61 \\
73 \\
70\end{array}$ \\
\hline $\begin{array}{r}9 \\
10 \\
11 \\
12\end{array}$ & $\begin{array}{l}5.84 \\
6.35 \\
9.79 \\
5.14\end{array}$ & $\begin{array}{c}1.90 \\
3.99 \\
-- \\
--\end{array}$ & $\begin{array}{r}285 \\
121 \\
-- \\
--\end{array}$ & $\begin{array}{l}67 \\
49 \\
-- \\
--\end{array}$ \\
\hline $\begin{array}{l}13 \\
14 \\
15 \\
16\end{array}$ & $\begin{array}{l}5.34 \\
6.89 \\
6.40 \\
5.06\end{array}$ & $\begin{array}{l}-- \\
-- \\
-- \\
--\end{array}$ & $\begin{array}{l}-- \\
-- \\
--\end{array}$ & $\begin{array}{l}-- \\
-- \\
--\end{array}$ \\
\hline $\begin{array}{l}17 \\
18 \\
19 \\
20\end{array}$ & $\begin{array}{l}5.42 \\
3.97 \\
3.62 \\
5.19\end{array}$ & $\begin{array}{l}-- \\
-- \\
--\end{array}$ & $\begin{array}{l}-- \\
-- \\
-- \\
--\end{array}$ & $\begin{array}{l}-- \\
-- \\
-- \\
--\end{array}$ \\
\hline $\begin{array}{l}21 \\
22 \\
23 \\
24\end{array}$ & $\begin{array}{l}4.92 \\
5.73 \\
5.75 \\
9.13\end{array}$ & $\begin{array}{l}-- \\
-- \\
-- \\
--\end{array}$ & $\begin{array}{l}-- \\
-- \\
-- \\
--\end{array}$ & $\begin{array}{l}-- \\
-- \\
-- \\
--\end{array}$ \\
\hline $\begin{array}{l}25 \\
26 \\
27 \\
28\end{array}$ & $\begin{array}{l}3.45 \\
7.49 \\
9.20 \\
8.23\end{array}$ & $\begin{array}{l}-- \\
-- \\
-- \\
--\end{array}$ & $\begin{array}{l}-- \\
-- \\
--\end{array}$ & $\begin{array}{l}-- \\
-- \\
-- \\
--\end{array}$ \\
\hline $\begin{array}{l}29 \\
30\end{array}$ & $\begin{array}{l}8.08 \\
5.01\end{array}$ & -- & -- & -- \\
\hline
\end{tabular}


Table 2.--Windspeed and wind-vector data,

october 1987-December 1989--Continued

\begin{tabular}{ccccc}
\hline $\begin{array}{c}\text { Day of } \\
\text { month }\end{array}$ & $\begin{array}{c}\text { Average } \\
\text { windspeed } \\
\text { (miles per } \\
\text { hour) }\end{array}$ & $\begin{array}{c}\text { Wind-vector } \\
\text { magnitude } \\
\text { (miles per } \\
\text { hour) }\end{array}$ & $\begin{array}{c}\text { Wind-vector } \\
\text { direction } \\
\text { (degrees from } \\
\text { north) }\end{array}$ & $\begin{array}{c}\text { Standard deviation } \\
\text { of wind-vector } \\
\text { direction } \\
\text { (degrees) }\end{array}$ \\
\hline
\end{tabular}

\section{DECEMBER 1988}

1

3

4

5

6

7

8

9

10

11

12

13

14

15

16

17

18

19

20

21

22

23

24

25

26

27

28

29

30

31
8.88

11.44

5.77

6.07

7.04

6.08

6.80

3.94

7.17

3.63

4.14

5.87

5.39

5.57

9.45

4.20

3.07

4.26

4.37

5.49

9.42

8.53

7.63

8.57

10.07

8.49

6.79

7.47

4.85

6.31

6.13

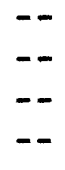

--

$--$

$-$

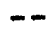

-

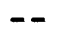

$-$

$\because$

$-$

$-$

$-$

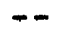

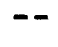

-

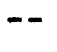

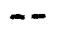

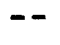

$-$

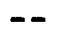

$-$

$-$

$-$

$\because$
$-$

$-$

$--$

$-$

$-$

$-$

$--$

$-$

$-$

$-$

$--$

-

$--$

$-$

$--$

$--$

$-$

$-$

$-$

$-$

$-$

$-$

--

$-$

$-$

$-$

--

-

$\because$

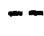

-

-

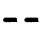

$-$

$-$

$-$

$-$

-

$-$

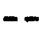

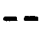

$-$

-

-

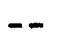

$-$

$-$

-.

--

$-$

-.

$-$

--

$-$

$-$

-.

$-$

$-$

$-$

$--$ 
Table 2.--Windspeed and wind-vector data, October 1987-December 1989--Continued

\begin{tabular}{|c|c|c|c|c|}
\hline $\begin{array}{c}\text { Day of } \\
\text { month }\end{array}$ & $\begin{array}{c}\text { Average } \\
\text { windspeed } \\
\text { (miles per } \\
\text { hour) }\end{array}$ & $\begin{array}{l}\text { Wind-vector } \\
\text { magnitude } \\
\text { (miles per } \\
\text { hour) }\end{array}$ & $\begin{array}{c}\text { Wind-vector } \\
\text { direction } \\
\text { (degrees from } \\
\text { north) }\end{array}$ & $\begin{array}{l}\text { Standard deviation } \\
\text { of wind-vector } \\
\text { direction } \\
\text { (degrees) }\end{array}$ \\
\hline \multicolumn{5}{|c|}{ JANUARY 1989} \\
\hline 1 & 2.81 & - & -- & -- \\
\hline 2 & 3.63 & -- & -- & -- \\
\hline 3 & 5.95 & -- & -- & -- \\
\hline 4 & 7.49 & -- & -- & -- \\
\hline 5 & 4.31 & -- & - & -- \\
\hline 6 & 8.04 & -- & -- & -- \\
\hline 7 & 6.26 & -- & -- & -- \\
\hline 8 & 7.80 & -- & -- & -- \\
\hline 9 & 5.50 & -- & $\cdots$ & -- \\
\hline 10 & 6.36 & -- & -- & -- \\
\hline 11 & 7.70 & -- & -- & -- \\
\hline 12 & 8.87 & -- & -- & -- \\
\hline 13 & 8.31 & -- & -- & -- \\
\hline 14 & 4.91 & -- & -- & -- \\
\hline 15 & 5.82 & -- & -- & -- \\
\hline 16 & 6.03 & -- & -- & -- \\
\hline 17 & 5.91 & -- & -- & -- \\
\hline 18 & 7.30 & -- & -- & -- \\
\hline 19 & 6.22 & -- & -- & -- \\
\hline 20 & 6.51 & -- & -- & -- \\
\hline 21 & 6.90 & -- & -- & -- \\
\hline 22 & 5.48 & -- & -- & - \\
\hline 23 & 4.76 & -- & -- & -- \\
\hline 24 & 4.36 & -- & -- & -- \\
\hline 25 & 3.98 & -- & -- & -- \\
\hline 26 & 8.53 & -- & -- & -- \\
\hline 27 & 4.34 & -- & -- & -- \\
\hline 28 & 3.36 & -- & -- & -- \\
\hline 29 & 7.06 & -- & -- & -- \\
\hline 30 & 5.98 & -- & -- & -- \\
\hline 31 & 5.20 & -- & -- & -- \\
\hline
\end{tabular}


Table 2.--Windspeed and wind-vector data,

october 1987-December 1989--Continued

\begin{tabular}{ccccc}
\hline $\begin{array}{c}\text { Day of } \\
\text { month }\end{array}$ & $\begin{array}{c}\text { Average } \\
\text { windspeed } \\
\text { (miles per } \\
\text { hour) }\end{array}$ & $\begin{array}{c}\text { Wind-vector } \\
\text { magnitude } \\
\text { (miles per } \\
\text { hour) }\end{array}$ & $\begin{array}{c}\text { Wind-vector } \\
\text { direction } \\
\text { (degrees from } \\
\text { north) }\end{array}$ & $\begin{array}{c}\text { Standard deviation } \\
\text { of wind-vector } \\
\text { direction } \\
\text { (degrees) }\end{array}$ \\
\hline
\end{tabular}

FEBRUARY 1989

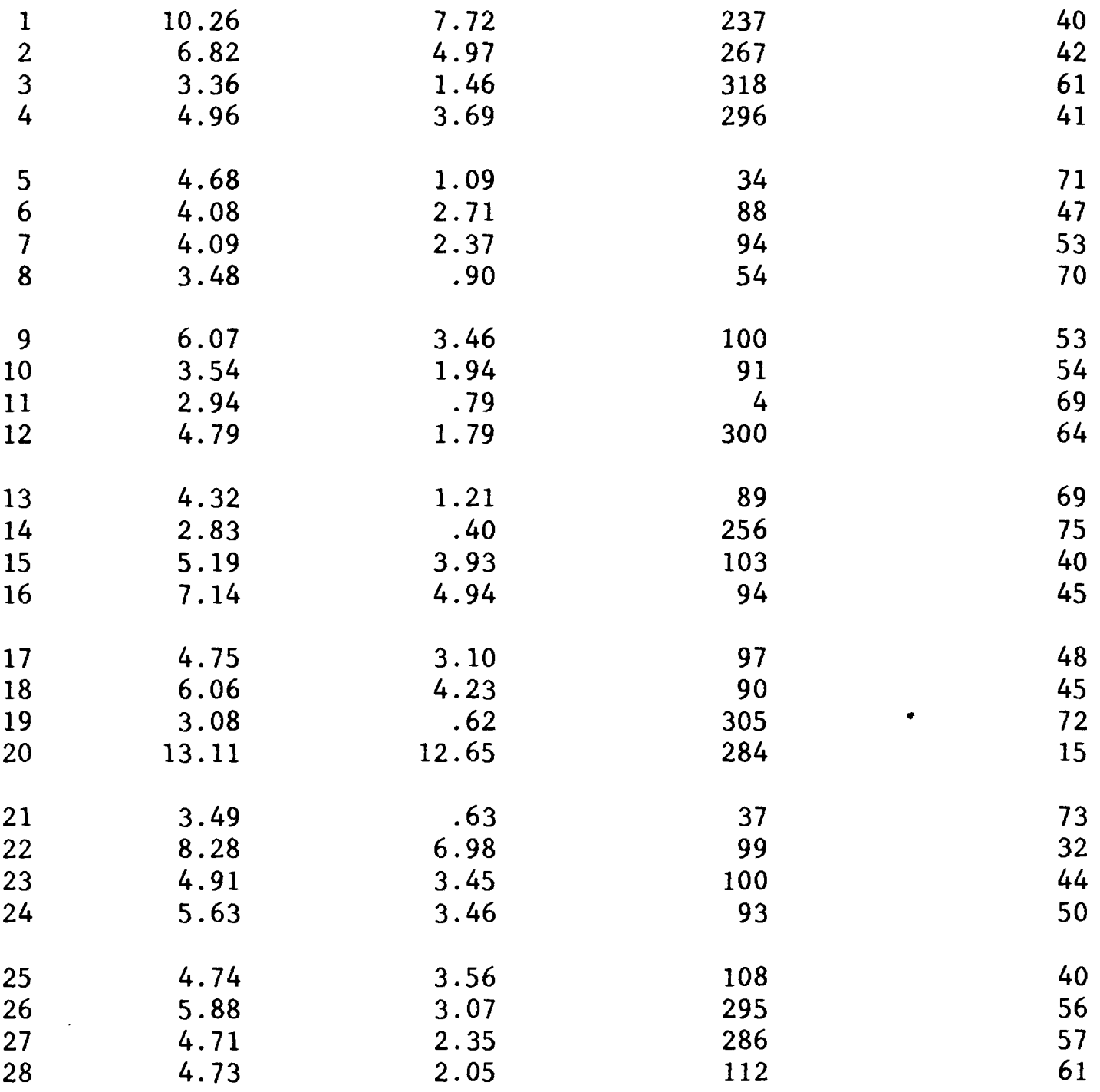


Table 2.--Windspeed and wind-vector data, october 1987-December 1989--Continued

\begin{tabular}{|c|c|c|c|c|}
\hline $\begin{array}{r}\text { Day of } \\
\text { month }\end{array}$ & $\begin{array}{c}\text { Average } \\
\text { windspeed } \\
\text { (miles per } \\
\text { hour) }\end{array}$ & $\begin{array}{l}\text { Wind-vector } \\
\text { magnitude } \\
\text { (miles per } \\
\text { hour) }\end{array}$ & $\begin{array}{l}\text { Wind-vector } \\
\text { direction } \\
\text { (degrees from } \\
\text { north) }\end{array}$ & $\begin{array}{l}\text { Standard deviation } \\
\text { of wind-vector } \\
\text { direction } \\
\text { (degrees) }\end{array}$ \\
\hline \multicolumn{5}{|c|}{ MARCH 1989} \\
\hline 1 & - & -- & -- & - \\
\hline 2 & -- & -- & -- & -- \\
\hline 3 & 6.15 & 3.86 & 292 & 49 \\
\hline 4 & 4.64 & 2.34 & 59 & 57 \\
\hline 5 & 6.98 & 4.71 & 88 & 46 \\
\hline 6 & 8.10 & 6.74 & 93 & 33 \\
\hline 7 & 8.86 & 7.71 & 87 & 29 \\
\hline 8 & 6.14 & 4.45 & 98 & 43 \\
\hline 9 & 5.92 & 4.24 & 124 & 43 \\
\hline 10 & 7.34 & 5.34 & 131 & 42 \\
\hline 11 & 4.73 & 2.68 & 107 & 53 \\
\hline 12 & 5.77 & 3.85 & 115 & 47 \\
\hline 13 & 8.81 & 3.96 & 255 & 60 \\
\hline 14 & 11.40 & 10.54 & 281 & 22 \\
\hline 15 & 3.96 & 1.44 & 275 & 65 \\
\hline 16 & 5.65 & 1.82 & 171 & 67 \\
\hline 17 & 11.09 & 8.50 & 274 & 39 \\
\hline 18 & 6.31 & 2.51 & 121 & 63 \\
\hline 19 & 4.57 & .16 & 205 & 80 \\
\hline 20 & 5.53 & 1.62 & 280 & 68 \\
\hline 21 & 6.23 & 4.55 & 103 & 42 \\
\hline 22 & 6.48 & 3.15 & 102 & 58 \\
\hline 23 & 7.90 & 3.80 & 85 & 58 \\
\hline 24 & 5.38 & 3.01 & 113 & 54 \\
\hline 25 & 6.80 & 3.11 & 128 & 60 \\
\hline 26 & 7.08 & 2.05 & 95 & 68 \\
\hline 27 & 5.14 & 2.04 & 261 & 63 \\
\hline 28 & 5.98 & .58 & 162 & 77 \\
\hline 29 & 5.84 & 4.35 & 270 & 41 \\
\hline 30 & 7.47 & 4.22 & 278 & 53 \\
\hline 31 & 7.04 & .72 & 164 & 77 \\
\hline
\end{tabular}


Table 2.--Windspeed and wind-vector data, October 1987-December 1989--Continued

\begin{tabular}{|c|c|c|c|c|}
\hline $\begin{array}{l}\text { Day of } \\
\text { month }\end{array}$ & $\begin{array}{c}\text { Average } \\
\text { windspeed } \\
\text { (miles per } \\
\text { hour) }\end{array}$ & $\begin{array}{l}\text { Wind-vector } \\
\text { magnitude } \\
\text { (miles per } \\
\text { hour) }\end{array}$ & $\begin{array}{c}\text { Wind-vector } \\
\text { direction } \\
\text { (degrees from } \\
\text { north) }\end{array}$ & $\begin{array}{l}\text { Standard deviation } \\
\text { of wind-vector } \\
\text { direction } \\
\text { (degrees) }\end{array}$ \\
\hline \multicolumn{5}{|c|}{ APRIL 1989} \\
\hline $\begin{array}{l}1 \\
2 \\
3 \\
4\end{array}$ & $\begin{array}{r}3.83 \\
8.79 \\
9.32 \\
11.85\end{array}$ & $\begin{array}{r}1.95 \\
7.44 \\
8.55 \\
10.67\end{array}$ & $\begin{array}{r}98 \\
267 \\
277 \\
281\end{array}$ & $\begin{array}{l}57 \\
32 \\
23 \\
26\end{array}$ \\
\hline $\begin{array}{l}5 \\
6 \\
7 \\
8\end{array}$ & $\begin{array}{r}5.64 \\
9.61 \\
8.90 \\
10.79\end{array}$ & $\begin{array}{l}3.70 \\
6.23 \\
6.00 \\
9.83\end{array}$ & $\begin{array}{l}282 \\
280 \\
281 \\
281\end{array}$ & $\begin{array}{l}48 \\
48 \\
46 \\
24\end{array}$ \\
\hline $\begin{array}{r}9 \\
10 \\
11 \\
12\end{array}$ & $\begin{array}{l}9.18 \\
6.28 \\
6.38 \\
5.81\end{array}$ & $\begin{array}{l}7.04 \\
3.45 \\
1.34 \\
4.02\end{array}$ & $\begin{array}{r}280 \\
87 \\
101 \\
93\end{array}$ & $\begin{array}{l}39 \\
54 \\
72 \\
45\end{array}$ \\
\hline $\begin{array}{l}13 \\
14 \\
15 \\
16\end{array}$ & $\begin{array}{l}5.71 \\
7.33 \\
7.56 \\
7.06\end{array}$ & $\begin{array}{l}.07 \\
1.52 \\
2.13 \\
3.18\end{array}$ & $\begin{array}{l}113 \\
149 \\
234 \\
281\end{array}$ & $\begin{array}{l}81 \\
72 \\
69 \\
60\end{array}$ \\
\hline $\begin{array}{l}17 \\
18 \\
19 \\
20\end{array}$ & $\begin{array}{l}8.73 \\
7.86 \\
6.42 \\
6.48\end{array}$ & $\begin{array}{l}4.77 \\
2.62 \\
2.13 \\
3.79\end{array}$ & $\begin{array}{r}273 \\
281 \\
122 \\
96\end{array}$ & $\begin{array}{l}55 \\
66 \\
66 \\
52\end{array}$ \\
\hline $\begin{array}{l}21 \\
22 \\
23 \\
24\end{array}$ & $\begin{array}{l}7.33 \\
8.85 \\
8.21 \\
7.49\end{array}$ & $\begin{array}{l}2.62 \\
3.82 \\
5.16 \\
4.93\end{array}$ & $\begin{array}{l}159 \\
159 \\
121 \\
121\end{array}$ & $\begin{array}{l}65 \\
61 \\
49 \\
47\end{array}$ \\
\hline $\begin{array}{l}25 \\
26 \\
27 \\
28\end{array}$ & $\begin{array}{l}6.94 \\
8.06 \\
5.87 \\
5.84\end{array}$ & $\begin{array}{r}2.02 \\
1.73 \\
3.78 \\
.68\end{array}$ & $\begin{array}{l}139 \\
235 \\
271 \\
224\end{array}$ & $\begin{array}{l}68 \\
72 \\
48 \\
76\end{array}$ \\
\hline $\begin{array}{l}29 \\
30\end{array}$ & $\begin{array}{l}5.73 \\
6.43\end{array}$ & $\begin{array}{l}3.04 \\
3.07\end{array}$ & $\begin{array}{l}280 \\
271\end{array}$ & $\begin{array}{l}56 \\
59\end{array}$ \\
\hline
\end{tabular}


Table 2.--Windspeed and wind-vector data, October 1987-December 1989--Continued

\begin{tabular}{|c|c|c|c|c|}
\hline $\begin{array}{r}\text { Day of } \\
\text { month }\end{array}$ & $\begin{array}{c}\text { Average } \\
\text { windspeed } \\
\text { (miles per } \\
\text { hour) }\end{array}$ & $\begin{array}{l}\text { Wind-vector } \\
\text { magnitude } \\
\text { (miles per } \\
\text { hour) }\end{array}$ & $\begin{array}{c}\text { Wind-vector } \\
\text { direction } \\
\text { (degrees from } \\
\text { north) }\end{array}$ & $\begin{array}{l}\text { Standard deviation } \\
\text { of wind-vector } \\
\text { direction } \\
\text { (degrees) }\end{array}$ \\
\hline \multicolumn{5}{|c|}{ MAY 1989} \\
\hline $\begin{array}{l}1 \\
2 \\
3 \\
4\end{array}$ & $\begin{array}{l}7.65 \\
7.19 \\
7.84 \\
8.85\end{array}$ & $\begin{array}{l}3.63 \\
1.65 \\
4.48 \\
7.20\end{array}$ & $\begin{array}{r}275 \\
96 \\
272 \\
282\end{array}$ & $\begin{array}{l}59 \\
71 \\
53 \\
35\end{array}$ \\
\hline $\begin{array}{l}5 \\
6 \\
7 \\
8\end{array}$ & $\begin{array}{l}6.69 \\
6.38 \\
7.27 \\
8.14\end{array}$ & $\begin{array}{r}1.70 \\
.27 \\
.51 \\
1.37\end{array}$ & $\begin{array}{r}289 \\
277 \\
165 \\
78\end{array}$ & $\begin{array}{l}70 \\
79 \\
78 \\
74\end{array}$ \\
\hline $\begin{array}{r}9 \\
10 \\
11 \\
12\end{array}$ & $\begin{array}{r}8.70 \\
11.39 \\
8.84 \\
4.94\end{array}$ & $\begin{array}{l}5.34 \\
9.67 \\
5.60 \\
2.23\end{array}$ & $\begin{array}{r}82 \\
92 \\
89 \\
269\end{array}$ & $\begin{array}{l}50 \\
31 \\
49 \\
60\end{array}$ \\
\hline $\begin{array}{l}13 \\
14 \\
15 \\
16\end{array}$ & $\begin{array}{l}7.31 \\
9.93 \\
7.89 \\
5.08\end{array}$ & $\begin{array}{r}2.88 \\
7.90 \\
4.92 \\
.94\end{array}$ & $\begin{array}{r}89 \\
86 \\
84 \\
103\end{array}$ & $\begin{array}{l}63 \\
37 \\
50 \\
73\end{array}$ \\
\hline $\begin{array}{l}17 \\
18 \\
19 \\
20\end{array}$ & $\begin{array}{l}4.83 \\
6.23 \\
8.12 \\
5.45\end{array}$ & $\begin{array}{r}1.44 \\
2.14 \\
5.60 \\
.06\end{array}$ & $\begin{array}{l}277 \\
252 \\
275 \\
309\end{array}$ & $\begin{array}{l}68 \\
66 \\
45 \\
81\end{array}$ \\
\hline $\begin{array}{l}21 \\
22 \\
23 \\
24\end{array}$ & $\begin{array}{l}6.49 \\
6.37 \\
8.18 \\
8.75\end{array}$ & $\begin{array}{r}1.02 \\
.84 \\
3.81 \\
7.71\end{array}$ & $\begin{array}{l}183 \\
168 \\
231 \\
279\end{array}$ & $\begin{array}{l}74 \\
75 \\
59 \\
28\end{array}$ \\
\hline $\begin{array}{l}25 \\
26 \\
27 \\
28\end{array}$ & $\begin{array}{l}6.93 \\
5.48 \\
7.10 \\
8.40\end{array}$ & $\begin{array}{r}3.20 \\
.80 \\
1.45 \\
3.98\end{array}$ & $\begin{array}{r}279 \\
70 \\
199 \\
170\end{array}$ & $\begin{array}{l}59 \\
75 \\
72 \\
59\end{array}$ \\
\hline $\begin{array}{l}29 \\
30 \\
31\end{array}$ & $\begin{array}{l}7.44 \\
7.24 \\
7.01\end{array}$ & $\begin{array}{l}2.69 \\
3.36 \\
3.15\end{array}$ & $\begin{array}{l}186 \\
243 \\
274\end{array}$ & $\begin{array}{l}65 \\
59 \\
60\end{array}$ \\
\hline
\end{tabular}


Table 2.--Windspeed and wind-vector data, October 1987-December 1989--Continued

\begin{tabular}{|c|c|c|c|c|}
\hline $\begin{array}{c}\text { Day of } \\
\text { month }\end{array}$ & $\begin{array}{c}\text { Average } \\
\text { windspeed } \\
\text { (miles per } \\
\text { hour) }\end{array}$ & $\begin{array}{l}\text { Wind-vector } \\
\text { magnitude } \\
\text { (miles per } \\
\text { hour) }\end{array}$ & $\begin{array}{l}\text { Wind-vector } \\
\text { direction } \\
\text { (degrees from } \\
\text { north) }\end{array}$ & $\begin{array}{l}\text { Standard deviation } \\
\text { of wind-vector } \\
\text { direction } \\
\text { (degrees) }\end{array}$ \\
\hline \multicolumn{5}{|c|}{ JUNE 1989} \\
\hline $\begin{array}{l}1 \\
2 \\
3 \\
4\end{array}$ & $\begin{array}{l}5.69 \\
6.64 \\
6.79 \\
6.46\end{array}$ & $\begin{array}{l}0.76 \\
1.56 \\
2.35 \\
2.47\end{array}$ & $\begin{array}{r}308 \\
119 \\
83 \\
89\end{array}$ & $\begin{array}{l}75 \\
71 \\
65 \\
64\end{array}$ \\
\hline $\begin{array}{l}5 \\
6 \\
7 \\
8\end{array}$ & $\begin{array}{l}6.33 \\
6.66 \\
5.73 \\
9.07\end{array}$ & $\begin{array}{l}1.82 \\
1.25 \\
2.39 \\
7.01\end{array}$ & $\begin{array}{l}98 \\
81 \\
90 \\
88\end{array}$ & $\begin{array}{l}68 \\
73 \\
62 \\
39\end{array}$ \\
\hline $\begin{array}{r}9 \\
10 \\
11 \\
12\end{array}$ & $\begin{array}{l}8.06 \\
6.87 \\
6.14 \\
5.56\end{array}$ & $\begin{array}{r}5.09 \\
4.00 \\
.29 \\
2.64\end{array}$ & $\begin{array}{r}86 \\
82 \\
3 \\
83\end{array}$ & $\begin{array}{l}49 \\
52 \\
79 \\
59\end{array}$ \\
\hline $\begin{array}{l}13 \\
14 \\
15 \\
16\end{array}$ & $\begin{array}{l}4.58 \\
6.37 \\
5.77 \\
8.55\end{array}$ & $\begin{array}{r}1.46 \\
.92 \\
1.24 \\
4.41\end{array}$ & $\begin{array}{l}275 \\
247 \\
111 \\
248\end{array}$ & $\begin{array}{l}67 \\
75 \\
72 \\
56\end{array}$ \\
\hline $\begin{array}{l}17 \\
18 \\
19 \\
20\end{array}$ & $\begin{array}{l}6.25 \\
6.28 \\
7.40 \\
9.46\end{array}$ & $\begin{array}{l}.58 \\
1.48 \\
1.22 \\
6.26\end{array}$ & $\begin{array}{l}347 \\
109 \\
167 \\
239\end{array}$ & $\begin{array}{l}77 \\
71 \\
74 \\
47\end{array}$ \\
\hline $\begin{array}{l}21 \\
22 \\
23 \\
24\end{array}$ & $\begin{array}{l}7.45 \\
5.56 \\
6.62 \\
6.61\end{array}$ & $\begin{array}{l}3.79 \\
1.68 \\
1.82 \\
1.74\end{array}$ & $\begin{array}{r}281 \\
75 \\
73 \\
283\end{array}$ & $\begin{array}{l}57 \\
68 \\
69 \\
70\end{array}$ \\
\hline $\begin{array}{l}25 \\
26 \\
27 \\
28\end{array}$ & $\begin{array}{l}6.44 \\
6.04 \\
6.21 \\
7.13\end{array}$ & $\begin{array}{r}1.91 \\
1.20 \\
.51 \\
1.34\end{array}$ & $\begin{array}{l}262 \\
286 \\
192 \\
212\end{array}$ & $\begin{array}{l}68 \\
72 \\
78 \\
73\end{array}$ \\
\hline $\begin{array}{l}29 \\
30\end{array}$ & $\begin{array}{l}7.14 \\
8.06\end{array}$ & $\begin{array}{l}5.60 \\
2.08\end{array}$ & $\begin{array}{r}89 \\
108\end{array}$ & $\begin{array}{l}38 \\
70\end{array}$ \\
\hline
\end{tabular}


Table 2.--Windspeed and wind-vector data, october 1987-December 1989--Continued

\begin{tabular}{|c|c|c|c|c|}
\hline $\begin{array}{r}\text { Day of } \\
\text { month }\end{array}$ & $\begin{array}{c}\text { Average } \\
\text { windspeed } \\
\text { (miles per } \\
\text { hour) }\end{array}$ & $\begin{array}{l}\text { Wind-vector } \\
\text { magnitude } \\
\text { (miles per } \\
\text { hour) }\end{array}$ & $\begin{array}{c}\text { Wind-vector } \\
\text { direction } \\
\text { (degrees from } \\
\text { north) }\end{array}$ & $\begin{array}{l}\text { Standard deviation } \\
\text { of wind-vector } \\
\text { direction } \\
\text { (degrees) }\end{array}$ \\
\hline \multicolumn{5}{|c|}{ JULY 1989} \\
\hline $\begin{array}{l}1 \\
2 \\
3 \\
4\end{array}$ & $\begin{array}{l}7.24 \\
5.71 \\
5.78 \\
6.69\end{array}$ & $\begin{array}{l}1.86 \\
3.45 \\
1.81 \\
1.50\end{array}$ & $\begin{array}{r}191 \\
89 \\
99 \\
90\end{array}$ & $\begin{array}{l}70 \\
51 \\
67 \\
71\end{array}$ \\
\hline $\begin{array}{l}5 \\
6 \\
7 \\
8\end{array}$ & $\begin{array}{l}5.09 \\
6.04 \\
6.99 \\
7.02\end{array}$ & $\begin{array}{l}.91 \\
1.68 \\
3.59 \\
4.64\end{array}$ & $\begin{array}{r}101 \\
82 \\
96 \\
88\end{array}$ & $\begin{array}{l}73 \\
69 \\
56 \\
47\end{array}$ \\
\hline $\begin{array}{r}9 \\
10 \\
11 \\
12\end{array}$ & $\begin{array}{l}8.98 \\
6.49 \\
7.11 \\
7.69\end{array}$ & $\begin{array}{l}6.83 \\
2.69 \\
6.11 \\
6.17\end{array}$ & $\begin{array}{l}86 \\
77 \\
84 \\
88\end{array}$ & $\begin{array}{l}40 \\
62 \\
30 \\
36\end{array}$ \\
\hline $\begin{array}{l}13 \\
14 \\
15 \\
16\end{array}$ & $\begin{array}{l}6.65 \\
5.04 \\
7.56 \\
6.91\end{array}$ & $\begin{array}{r}2.66 \\
.84 \\
3.49 \\
2.41\end{array}$ & $\begin{array}{r}96 \\
91 \\
92 \\
193\end{array}$ & $\begin{array}{l}63 \\
74 \\
59 \\
65\end{array}$ \\
\hline $\begin{array}{l}17 \\
18 \\
19 \\
20\end{array}$ & $\begin{array}{l}7.98 \\
5.08 \\
6.42 \\
7.55\end{array}$ & $\begin{array}{l}2.09 \\
1.55 \\
2.35 \\
5.28\end{array}$ & $\begin{array}{r}270 \\
91 \\
94 \\
83\end{array}$ & $\begin{array}{l}70 \\
68 \\
64 \\
44\end{array}$ \\
\hline $\begin{array}{l}21 \\
22 \\
23 \\
24\end{array}$ & $\begin{array}{l}8.46 \\
6.40 \\
6.31 \\
5.00\end{array}$ & $\begin{array}{l}6.45 \\
2.35 \\
2.53 \\
2.70\end{array}$ & $\begin{array}{l}83 \\
79 \\
94 \\
89\end{array}$ & $\begin{array}{l}39 \\
64 \\
63 \\
55\end{array}$ \\
\hline $\begin{array}{l}25 \\
26 \\
27 \\
28\end{array}$ & $\begin{array}{l}8.52 \\
9.19 \\
5.35 \\
5.97\end{array}$ & $\begin{array}{l}6.75 \\
8.60 \\
2.49 \\
4.33\end{array}$ & $\begin{array}{l}85 \\
82 \\
99 \\
92\end{array}$ & $\begin{array}{l}37 \\
21 \\
59 \\
42\end{array}$ \\
\hline $\begin{array}{l}29 \\
30 \\
31\end{array}$ & $\begin{array}{l}5.64 \\
6.05 \\
6.79\end{array}$ & $\begin{array}{l}2.37 \\
4.61 \\
5.35\end{array}$ & $\begin{array}{l}92 \\
84 \\
80\end{array}$ & $\begin{array}{l}62 \\
40 \\
37\end{array}$ \\
\hline
\end{tabular}


Table 2.--Windspeed and wind-vector data, october 1987-December 1989--Continued

\begin{tabular}{|c|c|c|c|c|}
\hline $\begin{array}{l}\text { Day of } \\
\text { month }\end{array}$ & $\begin{array}{c}\text { Average } \\
\text { windspeed } \\
\text { (miles per } \\
\text { hour) }\end{array}$ & $\begin{array}{l}\text { Wind-vector } \\
\text { magnitude } \\
\text { (miles per } \\
\text { hour) }\end{array}$ & $\begin{array}{c}\text { Wind-vector } \\
\text { direction } \\
\text { (degrees from } \\
\text { north) }\end{array}$ & $\begin{array}{l}\text { Standard deviation } \\
\text { of wind-vector } \\
\text { direction } \\
\text { (degrees) }\end{array}$ \\
\hline \multicolumn{5}{|c|}{ AUGUST 1989} \\
\hline $\begin{array}{l}1 \\
2 \\
3 \\
4\end{array}$ & $\begin{array}{l}5.86 \\
5.43 \\
7.23 \\
6.84\end{array}$ & $\begin{array}{r}3.37 \\
2.08 \\
.85 \\
2.17\end{array}$ & $\begin{array}{r}89 \\
89 \\
171 \\
109\end{array}$ & $\begin{array}{l}53 \\
64 \\
76 \\
67\end{array}$ \\
\hline $\begin{array}{l}5 \\
6 \\
7 \\
8\end{array}$ & $\begin{array}{l}6.09 \\
6.67 \\
7.33 \\
7.43\end{array}$ & $\begin{array}{l}3.08 \\
3.56 \\
2.89 \\
4.99\end{array}$ & $\begin{array}{l}90 \\
85 \\
70 \\
84\end{array}$ & $\begin{array}{l}57 \\
55 \\
63 \\
46\end{array}$ \\
\hline $\begin{array}{r}9 \\
10 \\
11 \\
12\end{array}$ & $\begin{array}{l}5.63 \\
4.99 \\
4.22 \\
4.09\end{array}$ & $\begin{array}{r}.19 \\
.66 \\
1.28 \\
2.17\end{array}$ & $\begin{array}{r}240 \\
97 \\
128 \\
101\end{array}$ & $\begin{array}{l}80 \\
75 \\
68 \\
55\end{array}$ \\
\hline $\begin{array}{l}13 \\
14 \\
15 \\
16\end{array}$ & $\begin{array}{l}4.92 \\
6.98 \\
5.15 \\
6.90\end{array}$ & $\begin{array}{r}2.05 \\
.84 \\
.78 \\
3.41\end{array}$ & $\begin{array}{r}77 \\
51 \\
280 \\
84\end{array}$ & $\begin{array}{l}62 \\
76 \\
75 \\
58\end{array}$ \\
\hline $\begin{array}{l}17 \\
18 \\
19 \\
20\end{array}$ & $\begin{array}{r}10.45 \\
7.43 \\
5.85 \\
5.20\end{array}$ & $\begin{array}{l}9.91 \\
3.70 \\
1.75 \\
1.09\end{array}$ & $\begin{array}{r}86 \\
249 \\
73 \\
77\end{array}$ & $\begin{array}{l}18 \\
57 \\
68 \\
72\end{array}$ \\
\hline $\begin{array}{l}21 \\
22 \\
23 \\
24\end{array}$ & $\begin{array}{l}5.81 \\
4.78 \\
5.58 \\
7.94\end{array}$ & $\begin{array}{r}.79 \\
.21 \\
.98 \\
2.00\end{array}$ & $\begin{array}{r}300 \\
23 \\
186 \\
193\end{array}$ & $\begin{array}{l}75 \\
79 \\
74 \\
70\end{array}$ \\
\hline $\begin{array}{l}25 \\
26 \\
27 \\
28\end{array}$ & $\begin{array}{l}5.06 \\
5.63 \\
6.53 \\
5.69\end{array}$ & $\begin{array}{r}1.13 \\
.52 \\
1.50 \\
.82\end{array}$ & $\begin{array}{l}289 \\
288 \\
230 \\
326\end{array}$ & $\begin{array}{l}71 \\
77 \\
71 \\
75\end{array}$ \\
\hline $\begin{array}{l}29 \\
30 \\
31\end{array}$ & $\begin{array}{l}6.02 \\
7.17 \\
6.90\end{array}$ & $\begin{array}{l}1.14 \\
1.53 \\
1.54\end{array}$ & $\begin{array}{r}96 \\
173 \\
235\end{array}$ & $\begin{array}{l}73 \\
72 \\
71\end{array}$ \\
\hline
\end{tabular}


Table 2.--Windspeed and wind-vector data, October 1987-December 1989--Continued

\begin{tabular}{|c|c|c|c|c|}
\hline $\begin{array}{l}\text { Day of } \\
\text { month }\end{array}$ & $\begin{array}{c}\text { Average } \\
\text { windspeed } \\
\text { (miles per } \\
\text { hour) }\end{array}$ & $\begin{array}{l}\text { Wind-vector } \\
\text { magnitude } \\
\text { (miles per } \\
\text { hour) }\end{array}$ & $\begin{array}{l}\text { Wind-vector } \\
\text { direction } \\
\text { (degrees from } \\
\text { north) }\end{array}$ & $\begin{array}{l}\text { Standard deviation } \\
\text { of wind-vector } \\
\text { direction } \\
\text { (degrees) }\end{array}$ \\
\hline \multicolumn{5}{|c|}{ SEPTEMBER 1989} \\
\hline $\begin{array}{l}1 \\
2 \\
3 \\
4\end{array}$ & $\begin{array}{l}6.29 \\
7.42 \\
7.62 \\
6.07\end{array}$ & $\begin{array}{r}1.20 \\
2.41 \\
2.27 \\
.96\end{array}$ & $\begin{array}{r}99 \\
235 \\
271 \\
92\end{array}$ & $\begin{array}{l}73 \\
67 \\
68 \\
74\end{array}$ \\
\hline $\begin{array}{l}5 \\
6 \\
7 \\
8\end{array}$ & $\begin{array}{l}6.02 \\
8.16 \\
7.65 \\
5.13\end{array}$ & $\begin{array}{r}1.63 \\
2.93 \\
3.14 \\
.87\end{array}$ & $\begin{array}{l}147 \\
136 \\
163 \\
227\end{array}$ & $\begin{array}{l}69 \\
65 \\
62 \\
74\end{array}$ \\
\hline $\begin{array}{r}9 \\
10 \\
11 \\
12\end{array}$ & $\begin{array}{l}4.43 \\
5.79 \\
5.95 \\
5.13\end{array}$ & $\begin{array}{l}1.90 \\
1.95 \\
1.14 \\
3.25\end{array}$ & $\begin{array}{r}281 \\
285 \\
75 \\
83\end{array}$ & $\begin{array}{l}61 \\
66 \\
73 \\
49\end{array}$ \\
\hline $\begin{array}{l}13 \\
14 \\
15 \\
16\end{array}$ & $\begin{array}{l}3.78 \\
5.01 \\
5.66 \\
5.31\end{array}$ & $\begin{array}{r}1.17 \\
1.29 \\
.38 \\
2.60\end{array}$ & $\begin{array}{r}281 \\
105 \\
356 \\
87\end{array}$ & $\begin{array}{l}67 \\
70 \\
78 \\
58\end{array}$ \\
\hline $\begin{array}{l}17 \\
18 \\
19 \\
20\end{array}$ & $\begin{array}{l}6.68 \\
6.54 \\
4.36 \\
7.03\end{array}$ & $\begin{array}{r}2.16 \\
.54 \\
.56 \\
.97\end{array}$ & $\begin{array}{r}150 \\
256 \\
37 \\
332\end{array}$ & $\begin{array}{l}67 \\
78 \\
76 \\
75\end{array}$ \\
\hline $\begin{array}{l}21 \\
22 \\
23 \\
24\end{array}$ & $\begin{array}{l}4.74 \\
8.12 \\
7.12 \\
6.26\end{array}$ & $\begin{array}{l}2.76 \\
7.23 \\
1.71 \\
1.30\end{array}$ & $\begin{array}{r}282 \\
87 \\
60 \\
129\end{array}$ & $\begin{array}{l}52 \\
27 \\
71 \\
72\end{array}$ \\
\hline $\begin{array}{l}25 \\
26 \\
27 \\
28\end{array}$ & $\begin{array}{l}6.50 \\
6.71 \\
5.70 \\
5.44\end{array}$ & $\begin{array}{r}2.78 \\
.89 \\
3.10 \\
.96\end{array}$ & $\begin{array}{r}81 \\
353 \\
93 \\
71\end{array}$ & $\begin{array}{l}61 \\
75 \\
55 \\
74\end{array}$ \\
\hline $\begin{array}{l}29 \\
30\end{array}$ & $\begin{array}{l}7.98 \\
7.31\end{array}$ & $\begin{array}{l}4.15 \\
3.09\end{array}$ & $\begin{array}{r}82 \\
194\end{array}$ & $\begin{array}{l}56 \\
62\end{array}$ \\
\hline
\end{tabular}


Table 2.--Windspeed and wind-vector data, october 1987-December 1989--Continued

\begin{tabular}{|c|c|c|c|c|}
\hline $\begin{array}{r}\text { Day of } \\
\text { month }\end{array}$ & $\begin{array}{c}\text { Average } \\
\text { windspeed } \\
\text { (miles per } \\
\text { hour) }\end{array}$ & $\begin{array}{l}\text { Wind-vector } \\
\text { magnitude } \\
\text { (miles per } \\
\text { hour) }\end{array}$ & $\begin{array}{c}\text { Wind-vector } \\
\text { direction } \\
\text { (degrees from } \\
\text { north) }\end{array}$ & $\begin{array}{l}\text { Standard deviation } \\
\text { of wind-vector } \\
\text { direction } \\
\text { (degrees) }\end{array}$ \\
\hline \multicolumn{5}{|c|}{ OCTOBER 1989} \\
\hline 1 & 6.80 & 3.05 & 276 & 60 \\
\hline 2 & 7.15 & 1.90 & 168 & 69 \\
\hline 3 & 7.19 & 3.51 & 158 & 58 \\
\hline 4 & 6.87 & 3.18 & 250 & 59 \\
\hline 5 & 5.31 & 1.44 & 85 & 69 \\
\hline 6 & 5.85 & .34 & 12 & 79 \\
\hline 7 & 6.03 & .37 & 88 & 78 \\
\hline 8 & 5.63 & .92 & 89 & 74 \\
\hline 9 & 6.18 & 1.44 & 101 & 71 \\
\hline 10 & 6.40 & 1.22 & 106 & 73 \\
\hline 11 & 7.71 & 1.67 & 285 & 72 \\
\hline 12 & 5.48 & .71 & 24 & 76 \\
\hline 13 & 5.58 & .48 & 61 & 77 \\
\hline 14 & 5.66 & 1.35 & 116 & 71 \\
\hline 15 & 3.70 & .25 & 292 & 78 \\
\hline 16 & 3.78 & .77 & 304 & 72 \\
\hline 17 & 4.60 & 1.00 & 97 & 72 \\
\hline 18 & 4.43 & 1.82 & 94 & 62 \\
\hline 19 & 7.40 & 5.45 & 86 & 42 \\
\hline 20 & 4.47 & 1.33 & 71 & 68 \\
\hline 21 & 4.54 & .88 & 146 & 73 \\
\hline 22 & 3.99 & .52 & 344 & 76 \\
\hline 23 & 5.32 & 1.44 & 111 & 69 \\
\hline 24 & 5.68 & 1.70 & 105 & 68 \\
\hline 25 & 8.49 & 5.93 & 103 & 45 \\
\hline 26 & 5.87 & 3.39 & 264 & 53 \\
\hline 27 & 5.83 & 4.68 & 102 & 36 \\
\hline 28 & 6.88 & 2.69 & 268 & 63 \\
\hline 29 & 4.10 & 2.06 & 273 & 57 \\
\hline 30 & 4.30 & .67 & 94 & 74 \\
\hline 31 & 4.20 & .42 & 219 & 77 \\
\hline
\end{tabular}


Table 2.--Windspeed and wind-vector data, october 1987-December 1989--Continued

\begin{tabular}{|c|c|c|c|c|}
\hline $\begin{array}{l}\text { Day of } \\
\text { month }\end{array}$ & $\begin{array}{c}\text { Average } \\
\text { windspeed } \\
\text { (miles per } \\
\text { hour) }\end{array}$ & $\begin{array}{l}\text { Wind-vector } \\
\text { magnitude } \\
\text { (miles per } \\
\text { hour) }\end{array}$ & $\begin{array}{l}\text { Wind-vector } \\
\text { direction } \\
\text { (degrees from } \\
\text { north) }\end{array}$ & $\begin{array}{l}\text { Standard deviation } \\
\text { of wind-vector } \\
\text { direction } \\
\text { (degrees) }\end{array}$ \\
\hline \multicolumn{5}{|c|}{ NOVEMBER 1989} \\
\hline $\begin{array}{l}1 \\
2 \\
3 \\
4\end{array}$ & $\begin{array}{l}4.04 \\
5.07 \\
4.65 \\
6.11\end{array}$ & $\begin{array}{l}0.67 \\
2.92 \\
1.34 \\
3.82\end{array}$ & $\begin{array}{l}277 \\
103 \\
118 \\
269\end{array}$ & $\begin{array}{l}74 \\
53 \\
68 \\
50\end{array}$ \\
\hline $\begin{array}{l}5 \\
6 \\
7 \\
8\end{array}$ & $\begin{array}{l}5.92 \\
4.97 \\
5.04 \\
6.04\end{array}$ & $\begin{array}{l}3.98 \\
1.55 \\
2.82 \\
3.08\end{array}$ & $\begin{array}{l}279 \\
273 \\
290 \\
295\end{array}$ & $\begin{array}{l}46 \\
67 \\
54 \\
57\end{array}$ \\
\hline $\begin{array}{r}9 \\
10 \\
11 \\
12\end{array}$ & $\begin{array}{l}2.97 \\
3.31 \\
4.38 \\
6.37\end{array}$ & $\begin{array}{l}1.03 \\
1.09 \\
2.83 \\
1.36\end{array}$ & $\begin{array}{r}100 \\
87 \\
99 \\
149\end{array}$ & $\begin{array}{l}65 \\
66 \\
48 \\
72\end{array}$ \\
\hline $\begin{array}{l}13 \\
14 \\
15 \\
16\end{array}$ & $\begin{array}{l}6.63 \\
8.55 \\
6.49 \\
4.86\end{array}$ & $\begin{array}{l}.96 \\
7.03 \\
3.38 \\
3.49\end{array}$ & $\begin{array}{l}219 \\
279 \\
289 \\
116\end{array}$ & $\begin{array}{l}75 \\
34 \\
56 \\
43\end{array}$ \\
\hline $\begin{array}{l}17 \\
18 \\
19 \\
20\end{array}$ & $\begin{array}{l}4.85 \\
5.05 \\
5.06 \\
5.81\end{array}$ & $\begin{array}{l}1.02 \\
2.44 \\
3.51 \\
4.18\end{array}$ & $\begin{array}{r}85 \\
100 \\
83 \\
96\end{array}$ & $\begin{array}{l}72 \\
58 \\
45 \\
43\end{array}$ \\
\hline $\begin{array}{l}21 \\
22 \\
23 \\
24\end{array}$ & $\begin{array}{l}5.61 \\
5.38 \\
4.59 \\
6.60\end{array}$ & $\begin{array}{l}1.63 \\
2.15 \\
2.71 \\
3.06\end{array}$ & $\begin{array}{r}91 \\
86 \\
96 \\
244\end{array}$ & $\begin{array}{l}68 \\
63 \\
52 \\
59\end{array}$ \\
\hline $\begin{array}{l}25 \\
26 \\
27 \\
28\end{array}$ & $\begin{array}{l}5.19 \\
8.22 \\
8.90 \\
5.53\end{array}$ & $\begin{array}{r}.73 \\
2.51 \\
8.35 \\
2.69\end{array}$ & $\begin{array}{r}301 \\
270 \\
284 \\
87\end{array}$ & $\begin{array}{l}75 \\
68 \\
20 \\
58\end{array}$ \\
\hline $\begin{array}{l}29 \\
30\end{array}$ & $\begin{array}{l}5.68 \\
4.49\end{array}$ & $\begin{array}{l}3.40 \\
2.90\end{array}$ & $\begin{array}{l}96 \\
92\end{array}$ & $\begin{array}{l}51 \\
48\end{array}$ \\
\hline
\end{tabular}


Table 2.--Windspeed and wind-vector data, October 1987-December 1989--Continued

\begin{tabular}{|c|c|c|c|c|}
\hline $\begin{array}{r}\text { Day of } \\
\text { month }\end{array}$ & $\begin{array}{c}\text { Average } \\
\text { windspeed } \\
\text { (miles per } \\
\text { hour) }\end{array}$ & $\begin{array}{l}\text { Wind-vector } \\
\text { magnitude } \\
\text { (miles per } \\
\text { hour) }\end{array}$ & $\begin{array}{c}\text { Wind-vector } \\
\text { direction } \\
\text { (degrees from } \\
\text { north) }\end{array}$ & $\begin{array}{l}\text { Standard deviation } \\
\text { of wind-vector } \\
\text { direction } \\
\text { (degrees) }\end{array}$ \\
\hline \multicolumn{5}{|c|}{ DECEMBER 1989} \\
\hline 1 & 4.02 & 2.14 & 108 & 55 \\
\hline 2 & 4.61 & 2.92 & 114 & 49 \\
\hline 3 & 6.05 & 4.02 & 104 & 47 \\
\hline 4 & 4.55 & 2.61 & 110 & 53 \\
\hline 5 & 3.89 & 2.43 & 94 & 50 \\
\hline 6 & 5.31 & 2.26 & 285 & 61 \\
\hline 7 & 4.67 & 2.00 & 304 & 61 \\
\hline 8 & 6.30 & 4.44 & 94 & 44 \\
\hline 9 & 5.40 & .87 & 205 & 74 \\
\hline 10 & 5.18 & 2.89 & 296 & 54 \\
\hline 11 & 4.20 & 1.01 & 320 & 71 \\
\hline 12 & 4.99 & 3.37 & 120 & 46 \\
\hline 13 & 3.14 & .70 & 328 & 71 \\
\hline 14 & 7.39 & 6.45 & 286 & 29 \\
\hline 15 & 5.38 & 4.18 & 295 & 38 \\
\hline 16 & 2.67 & .95 & 326 & 65 \\
\hline 17 & 1.94 & .64 & 57 & 66 \\
\hline 18 & 4.12 & .66 & 329 & 74 \\
\hline 19 & 4.85 & 3.27 & 113 & 46 \\
\hline 20 & 5.52 & 4.28 & 108 & 38 \\
\hline 21 & 6.10 & 1.02 & 230 & 74 \\
\hline 22 & 5.76 & 4.74 & 105 & 34 \\
\hline 23 & 5.46 & 3.03 & 102 & 54 \\
\hline 24 & 5.98 & 4.89 & 103 & 35 \\
\hline 25 & 6.54 & 4.12 & 80 & 49 \\
\hline 26 & 6.85 & 4.65 & 91 & 46 \\
\hline 27 & 8.80 & 6.91 & 80 & 38 \\
\hline 28 & 5.13 & 3.41 & 101 & 47 \\
\hline 29 & 4.44 & 1.84 & 83 & 62 \\
\hline 30 & 4.28 & 2.49 & 306 & 52 \\
\hline 31 & 6.72 & 6.00 & 79 & 26 \\
\hline
\end{tabular}


Table 3.--Wind-rm and wind-duration histogran data, Octobor 1987-Decenber 1989

$$
[--, \text { no data }]
$$

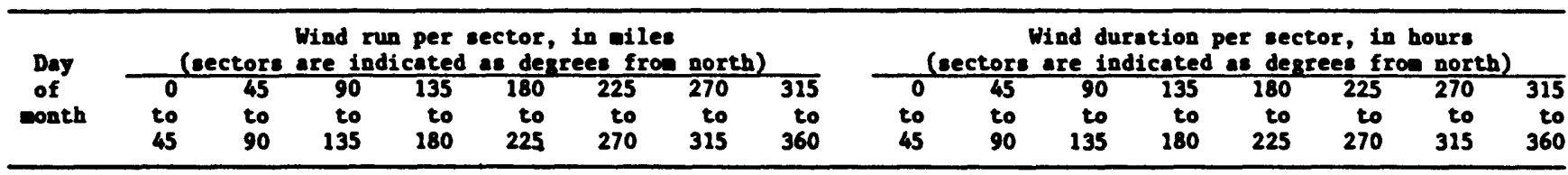

\section{OCTOBER 2987}

\begin{tabular}{|c|c|c|c|c|c|c|c|c|c|c|c|c|c|c|c|c|}
\hline 1 & -- & -- & -- & -- & -- & -- & -- & -- & -- & -- & -- & -- & -- & -- & -- & -- \\
\hline 2 & $=$ & 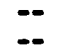 & $=-$ & $=$ & $\begin{array}{l}-- \\
--\end{array}$ & $\begin{array}{l}-- \\
--\end{array}$ & $\begin{array}{l}-- \\
--\end{array}$ & $\begin{array}{l}-- \\
--\end{array}$ & $\begin{array}{l}-- \\
--\end{array}$ & $=$ & 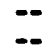 & $=-$ & $=$ & $=$ & $=$ & $=$ \\
\hline 4 & -- & -- & -- & -- & -- & -- & -- & -- & -. & - & - & - & -- & $\ldots$ & -. & - \\
\hline 5 & -- & -- & -- & -- & -- & -- & -- & -- & -- & -- & -- & -- & -- & - & -- & -- \\
\hline 6 & -- & -- & -- & -- & -- & -- & $\cdots$ & -- & -- & -- & -- & -- & -- & -- & -- & -- \\
\hline 8 & -- & -- & -- & -- & -- & -- & -- & -- & -- & -- & -- & -- & -- & -- & -- & -. \\
\hline 9 & -- & -- & -- & -- & -- & -- & -- & -- & -- & -- & -- & -- & - & - & -- & $\because$ \\
\hline 10 & -- & -- & -- & -- & -- & -- & -- & -- & -- & -- & -- & $\cdots$ & -- & -- & -- & -- \\
\hline 11 & -- & -- & -- & -- & -- & -- & -- & -- & - & -- & - & $\cdots$ & - & - & $\cdots$ & 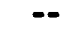 \\
\hline 12 & -- & -- & -- & -- & -- & -- & $\cdots$ & -- & -- & -- & -- & -- & -- & -- & -- & - \\
\hline 13 & -- & -- & -- & -- & -- & -- & -- & -- & - & - & -- & -- & - & -. & .. & -- \\
\hline 14 & $\cdots$ & $\cdots$ & -- & -- & -- & -- & -- & -- & -- & -- & -- & -- & - & - & -- & -- \\
\hline 15 & -- & -- & -- & -- & -- & -- & -- & -- & -- & -- & $\cdots$ & -- & -- & -- & -- & - \\
\hline 16 & -- & -- & -- & -- & - & -- & - & - & -- & - & $\cdots$ & $\cdots$ & $\cdots$ & $\cdots$ & $\cdots$ & -- \\
\hline 17 & - & - & -- & -- & -- & -. & - & -- & -- & -- & -- & -- & -- & - & -- & - \\
\hline 18 & -- & -- & -- & -- & -- & -- & -- & -- & -- & -- & -- & -- & -- & -- & -- & $\cdots$ \\
\hline $\begin{array}{l}19 \\
20\end{array}$ & $=$ & $\overline{--}$ & $\ddot{-}$ & $\overline{-}$ & $\ddot{-}$ & 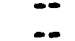 & $\ddot{-}$ & 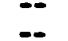 & $=-$ & $\overline{--}$ & $=$ & $=$ & $=$ & $=$ & $=$ & $\therefore$ \\
\hline & & & & & & & & & & & & & & & & \\
\hline 21 & -- & -- & -- & -- & -- & $\cdots$ & -- & -- & -- & - & -- & -- & -- & -- & -- & - \\
\hline 22 & $\because$ & $\overline{-}$ & 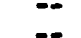 & $\because$ & $\ddot{-}$ & $\because$ & $\overline{-}$ & $\overline{-}$ & 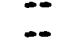 & $\overline{-}$ & 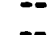 & 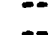 & 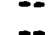 & $\overline{-}$ & $\because$ & \\
\hline 24 & $=$ & -- & $=$ & $=$ & $=$ & $=$ & $=$ & $=-$ & $=$ & $=$ & $=$ & $=$ & $=$ & $=-$ & $\therefore$ & 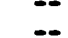 \\
\hline & & & & & & & & & & & & & & & & \\
\hline 25 & -- & -- & $\cdots$ & -- & -- & $\cdots$ & -- & -- & -- & -- & -- & -- & - & -- & -- & -- \\
\hline $\begin{array}{l}26 \\
27\end{array}$ & $=$ & -- & $=$ & $=$ & $=$ & $\because$ & 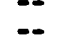 & $\cdots$ & -- & $\cdots$ & -- & - & $\cdots$ & $\therefore$ & $\therefore$ & \\
\hline 28 & -. & -- & $\ldots$ & -- & -- & -- &.- & -- & .. & 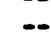 & $=$ & .. & 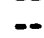 & .. & - & $\ldots$ \\
\hline 29 & 4.99 & 11.69 & 32.38 & 4.34 & & 7.51 & 4.78 & 3.65 & 1.9 & 7.8 & 7.0 & 1.2 & 0.9 & 2.2 & 1.5 & 1.4 \\
\hline 30 & 1.20 & 7.82 & $\begin{array}{r}31.80 \\
0\end{array}$ & 1.42 & $\begin{array}{l}2.64 \\
2.40\end{array}$ & $\begin{array}{r}7.10 \\
12.89\end{array}$ & 5.16 & $\begin{array}{l}1.42 \\
1.80\end{array}$ & .9 & 6.0 & 7.5 & .7 & 1.3 & 3.5 & 3.0 & 1.0 \\
\hline & & & & & & & & & & & & & & & & \\
\hline
\end{tabular}


Table 3.--Wind-ren and wind-duration histogra data, October 1987-Docuber 1989--Continued

\begin{tabular}{|c|c|c|c|c|c|c|c|c|c|c|c|c|c|c|c|c|}
\hline $\begin{array}{l}\text { Dey } \\
\text { of } \\
\text { onth }\end{array}$ & $\begin{array}{l}0 \\
0 \\
20 \\
45\end{array}$ & $\frac{\text { sectors }}{45}$ & $\begin{array}{l}\text { Wind r } \\
\text { are ind } \\
90 \\
\text { to } \\
135\end{array}$ & $\begin{array}{c}\text { per } \\
\text { cated } \\
135 \\
\text { to } \\
180\end{array}$ & $\begin{array}{c}\text { ector, } \\
\text { ders } \\
180 \\
\text { to } \\
225\end{array}$ & $\begin{array}{l}\text { In aile } \\
\text { ees fro } \\
225 \\
\text { to } \\
270\end{array}$ & $\begin{array}{l}\text { north } \\
270 \\
\text { to } \\
315\end{array}$ & $\begin{array}{r}315 \\
20 \\
360\end{array}$ & $\begin{array}{r}0 \\
t 0 \\
45\end{array}$ & $\begin{array}{c}\text { ctors } \\
45 \\
\text { to } \\
90\end{array}$ & $\begin{array}{r}\text { ind du } \\
\text { are in } \\
\begin{array}{r}90 \\
\text { to } \\
135\end{array}\end{array}$ & $\begin{array}{l}\text { tion } \\
\text { sated } \\
135 \\
\text { to } \\
180\end{array}$ & 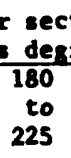 & $\begin{array}{r}\text { in } \\
225 \\
\text { to } \\
270\end{array}$ & $\begin{array}{c}\text { ours } \\
\text { nort } \\
270 \\
\text { to } \\
315\end{array}$ & $\begin{array}{r}315 \\
t 0 \\
360\end{array}$ \\
\hline \multicolumn{17}{|c|}{ MOVEMBER 1987} \\
\hline $\begin{array}{l}1 \\
2 \\
3 \\
4\end{array}$ & $\begin{array}{l}6.38 \\
3.26 \\
4.25 \\
3.48\end{array}$ & $\begin{array}{r}41.42 \\
7.94 \\
21.50 \\
25.13\end{array}$ & $\begin{array}{l}49.70 \\
11.26 \\
16.51 \\
47.11\end{array}$ & $\begin{array}{r}11.09 \\
2.09 \\
.79 \\
5.98\end{array}$ & $\begin{array}{l}3.62 \\
5.78 \\
2.11 \\
1.75\end{array}$ & $\begin{array}{r}10.94 \\
29.33 \\
16.61 \\
9.22\end{array}$ & $\begin{array}{r}5.18 \\
21.60 \\
12.77 \\
7.27\end{array}$ & $\begin{array}{l}2.45 \\
4.51 \\
3.86 \\
4.42\end{array}$ & $\begin{array}{l}1.5 \\
1.7 \\
1.8 \\
1.0\end{array}$ & $\begin{array}{l}6.5 \\
3.8 \\
7.1 \\
5.6\end{array}$ & $\begin{array}{l}8.0 \\
4.7 \\
4.8 \\
9.1\end{array}$ & $\begin{array}{r}2.3 \\
1.1 \\
.5 \\
1.5\end{array}$ & $\begin{array}{r}1.0 \\
1.8 \\
.9 \\
.7\end{array}$ & $\begin{array}{l}2.4 \\
5.6 \\
4.2 \\
2.4\end{array}$ & $\begin{array}{l}1.5 \\
3.8 \\
3.4 \\
1.9\end{array}$ & $\begin{array}{l}0.7 \\
1.4 \\
1.2 \\
1.5\end{array}$ \\
\hline $\begin{array}{l}5 \\
6 \\
7 \\
8\end{array}$ & $\begin{array}{l}4.25 \\
5.14 \\
1.06 \\
4.56\end{array}$ & $\begin{array}{r}56.66 \\
25.66 \\
1.82 \\
42.24\end{array}$ & $\begin{array}{r}47.54 \\
20.98 \\
2.21 \\
35.23\end{array}$ & $\begin{array}{r}4.06 \\
2.42 \\
.29 \\
4.70\end{array}$ & $\begin{array}{l}2.78 \\
5.57 \\
2.57 \\
1.20\end{array}$ & $\begin{array}{r}8.52 \\
29.90 \\
41.50 \\
6.26\end{array}$ & $\begin{array}{r}9.55 \\
23.11 \\
41.21 \\
3.31\end{array}$ & $\begin{array}{l}8.04 \\
7.73 \\
5.86 \\
1.73\end{array}$ & $\begin{array}{r}1.1 \\
1.8 \\
.8 \\
1.6\end{array}$ & $\begin{array}{l}6.8 \\
4.3 \\
1.3 \\
8.2\end{array}$ & $\begin{array}{l}7.1 \\
3.9 \\
1.4 \\
7.2\end{array}$ & $\begin{array}{r}1.1 \\
1.0 \\
.2 \\
1.4\end{array}$ & $\begin{array}{r}1.0 \\
1.6 \\
1.4 \\
.6\end{array}$ & $\begin{array}{l}2.3 \\
5.1 \\
7.9 \\
2.5\end{array}$ & $\begin{array}{l}2.5 \\
4.0 \\
8.5 \\
1.4\end{array}$ & $\begin{array}{l}2.1 \\
2.3 \\
2.3 \\
1.0\end{array}$ \\
\hline $\begin{array}{r}9 \\
10 \\
11 \\
12\end{array}$ & $\begin{array}{l}4.03 \\
3.89 \\
2.64 \\
5.18\end{array}$ & $\begin{array}{l}45.84 \\
49.85 \\
25.51 \\
39.00\end{array}$ & $\begin{array}{l}41.09 \\
30.70 \\
47.64 \\
28.82\end{array}$ & $\begin{array}{r}4.22 \\
3.62 \\
10.03 \\
4.20\end{array}$ & $\begin{array}{l}3.38 \\
2.38 \\
2.47 \\
2.81\end{array}$ & $\begin{array}{r}11.74 \\
22.08 \\
15.58 \\
6.74\end{array}$ & $\begin{array}{r}9.10 \\
20.14 \\
10.56 \\
9.29\end{array}$ & $\begin{array}{l}4.49 \\
5.38 \\
3.29 \\
7.01\end{array}$ & $\begin{array}{l}1.1 \\
1.0 \\
1.0 \\
1.4\end{array}$ & $\begin{array}{l}7.0 \\
7.5 \\
5.3 \\
6.6\end{array}$ & $\begin{array}{l}7.1 \\
5.3 \\
8.3 \\
6.0\end{array}$ & $\begin{array}{l}1.2 \\
1.0 \\
2.0 \\
1.5\end{array}$ & $\begin{array}{r}1.1 \\
.8 \\
.8 \\
1.1\end{array}$ & $\begin{array}{l}2.7 \\
3.4 \\
3.0 \\
2.4\end{array}$ & $\begin{array}{l}2.2 \\
3.5 \\
2.4 \\
2.9\end{array}$ & $\begin{array}{l}1.4 \\
1.3 \\
1.1 \\
2.0\end{array}$ \\
\hline $\begin{array}{l}13 \\
14 \\
15 \\
16\end{array}$ & $\begin{array}{l}2.16 \\
2.57 \\
2.66 \\
2.35\end{array}$ & $\begin{array}{r}21.58 \\
7.56 \\
7.54 \\
38.52\end{array}$ & $\begin{array}{r}47.54 \\
7.63 \\
7.25 \\
54.19\end{array}$ & $\begin{array}{r}8.21 \\
3.17 \\
.38 \\
8.30\end{array}$ & $\begin{array}{l}4.82 \\
3.77 \\
3.55 \\
1.32\end{array}$ & $\begin{array}{r}21.62 \\
33.77 \\
55.80 \\
3.10\end{array}$ & $\begin{array}{r}12.89 \\
30.58 \\
54.34 \\
3.07\end{array}$ & $\begin{array}{l}3.24 \\
3.96 \\
8.02 \\
2.30\end{array}$ & $\begin{array}{r}.8 \\
1.5 \\
.9 \\
.9\end{array}$ & $\begin{array}{l}3.9 \\
2.7 \\
1.8 \\
7.2\end{array}$ & $\begin{array}{r}7.8 \\
2.4 \\
1.6 \\
10.0\end{array}$ & $\begin{array}{r}1.7 \\
1.7 \\
.1 \\
2.0\end{array}$ & $\begin{array}{r}1.3 \\
1.7 \\
.6 \\
.7\end{array}$ & $\begin{array}{l}4.2 \\
7.1 \\
8.2 \\
1.1\end{array}$ & $\begin{array}{l}3.1 \\
5.3 \\
8.6 \\
1.1\end{array}$ & $\begin{array}{r}1.1 \\
1.4 \\
2.0 \\
.9\end{array}$ \\
\hline $\begin{array}{l}17 \\
18 \\
19 \\
20\end{array}$ & $\begin{array}{l}3.38 \\
2.33 \\
5.26 \\
8.11\end{array}$ & $\begin{array}{r}7.97 \\
127.85 \\
46.10 \\
104.45\end{array}$ & $\begin{array}{r}8.86 \\
59.42 \\
87.53 \\
74.86\end{array}$ & $\begin{array}{r}.86 \\
3.31 \\
15.62 \\
5.90\end{array}$ & $\begin{array}{l}4.61 \\
1.13 \\
4.08 \\
4.13\end{array}$ & $\begin{array}{r}55.66 \\
1.87 \\
3.05 \\
3.19\end{array}$ & $\begin{array}{r}60.17 \\
1.87 \\
3.36 \\
3.67\end{array}$ & $\begin{array}{r}10.42 \\
2.74 \\
4.44 \\
4.56\end{array}$ & $\begin{array}{r}1.1 \\
.6 \\
1.1 \\
1.2\end{array}$ & $\begin{array}{r}2.1 \\
12.0 \\
5.6 \\
9.9\end{array}$ & $\begin{array}{r}2.2 \\
7.9 \\
10.4 \\
8.4\end{array}$ & $\begin{array}{r}.5 \\
.8 \\
2.7 \\
1.0\end{array}$ & $\begin{array}{r}1.4 \\
.4 \\
1.1 \\
.8\end{array}$ & $\begin{array}{r}6.8 \\
.7 \\
.9 \\
.8\end{array}$ & $\begin{array}{r}7.1 \\
.6 \\
1.0 \\
.9\end{array}$ & $\begin{array}{r}2.6 \\
.8 \\
1.1 \\
1.0\end{array}$ \\
\hline $\begin{array}{l}21 \\
22 \\
23 \\
24\end{array}$ & $\begin{array}{r}4.58 \\
.82 \\
1.92 \\
1.78\end{array}$ & $\begin{array}{l}20.90 \\
29.09 \\
19.30 \\
20.64\end{array}$ & $\begin{array}{l}61.39 \\
48.50 \\
30.19 \\
42.36\end{array}$ & $\begin{array}{l}7.90 \\
9.70 \\
6.98 \\
9.17\end{array}$ & $\begin{array}{l}1.99 \\
1.56 \\
2.33 \\
2.71\end{array}$ & $\begin{array}{r}10.97 \\
6.91 \\
23.54 \\
9.26 \\
.\end{array}$ & $\begin{array}{r}17.16 \\
8.18 \\
17.62 \\
16.46\end{array}$ & $\begin{array}{r}10.08 \\
2.54 \\
1.94 \\
2.42\end{array}$ & $\begin{array}{r}1.2 \\
.4 \\
1.0 \\
.8\end{array}$ & $\begin{array}{l}4.4 \\
6.1 \\
4.5 \\
5.7\end{array}$ & $\begin{array}{l}9.0 \\
9.6 \\
6.9 \\
8.0\end{array}$ & $\begin{array}{l}1.4 \\
2.1 \\
1.8 \\
2.0\end{array}$ & $\begin{array}{l}.7 \\
.6 \\
.7 \\
.8\end{array}$ & $\begin{array}{l}2.0 \\
1.8 \\
4.7 \\
2.1\end{array}$ & $\begin{array}{l}2.9 \\
2.3 \\
3.5 \\
3.6\end{array}$ & 2.2 \\
\hline $\begin{array}{l}25 \\
26 \\
27 \\
28\end{array}$ & $\begin{array}{r}3.84 \\
.89 \\
1.97 \\
2.64\end{array}$ & $\begin{array}{l}62.95 \\
58.01 \\
29.71 \\
84.58\end{array}$ & $\begin{array}{l}55.75 \\
33.58 \\
13.27 \\
68.16\end{array}$ & $\begin{array}{r}12.12 \\
2.30 \\
8.38 \\
12.86\end{array}$ & $\begin{array}{l}1.10 \\
1.87 \\
6.96 \\
2.30\end{array}$ & $\begin{array}{r}5.47 \\
13.78 \\
18.22 \\
3.12\end{array}$ & $\begin{array}{r}7.20 \\
6.34 \\
11.50 \\
.86\end{array}$ & $\begin{array}{l}5.93 \\
1.10 \\
3.98 \\
1.70\end{array}$ & $\begin{array}{r}1.0 \\
.3 \\
1.0 \\
.7\end{array}$ & $\begin{array}{l}6.9 \\
8.8 \\
4.9 \\
8.2\end{array}$ & $\begin{array}{r}8.2 \\
6.5 \\
3.4 \\
10.1\end{array}$ & $\begin{array}{l}1.9 \\
1.1 \\
1.9 \\
2.4\end{array}$ & $\begin{array}{r}.4 \\
.9 \\
2.0 \\
.7\end{array}$ & $\begin{array}{r}1.7 \\
4.0 \\
5.5 \\
.8\end{array}$ & $\begin{array}{r}2.1 \\
1.8 \\
3.5 \\
.4\end{array}$ & $\begin{array}{r}1.7 \\
.5 \\
1.5 \\
.5\end{array}$ \\
\hline $\begin{array}{l}29 \\
30\end{array}$ & $\begin{array}{l}1.85 \\
2.90\end{array}$ & $\begin{array}{l}25.63 \\
32.18\end{array}$ & $\begin{array}{l}56.57 \\
48.38\end{array}$ & $\begin{array}{l}6.48 \\
6.46\end{array}$ & $\begin{array}{l}1.70 \\
1.73\end{array}$ & $\begin{array}{l}13.94 \\
11.16\end{array}$ & $\begin{array}{l}6.86 \\
7.58\end{array}$ & $\begin{array}{l}2.11 \\
2.42\end{array}$ & $\begin{array}{r}.6 \\
1.0\end{array}$ & $\begin{array}{l}6.4 \\
6.6\end{array}$ & $\begin{array}{r}10.4 \\
8.6\end{array}$ & $\begin{array}{l}1.2 \\
1.4\end{array}$ & $\begin{array}{l}.5 \\
.8\end{array}$ & $\begin{array}{l}2.5 \\
2.8\end{array}$ & $\begin{array}{l}1.4 \\
1.9\end{array}$ & .7 \\
\hline
\end{tabular}


Table 3.--Nind-rm and wind-duration histogren data, October 1987-Decerber 1989--Continued

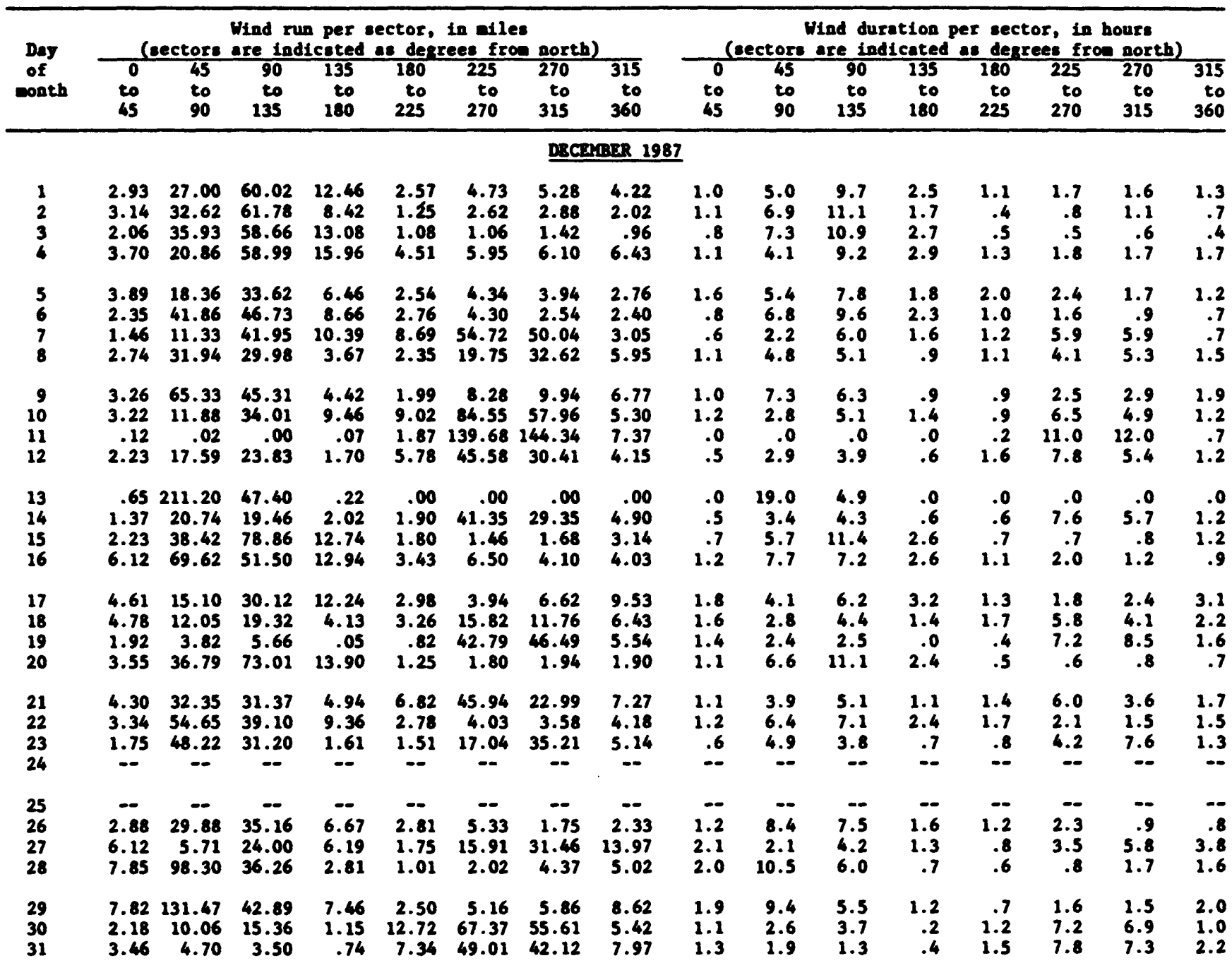


Table 3.--Wind-rum and wind-duration histogren deta, Octobor 1987-Decuber 1989--Cont inued

\begin{tabular}{|c|c|c|c|c|c|c|c|c|c|c|c|c|c|c|c|c|}
\hline \multirow{2}{*}{$\begin{array}{c}\text { Dey } \\
\text { of } \\
\text { eonth }\end{array}$} & \multicolumn{8}{|c|}{$\begin{array}{l}\text { Wind run per sector, in ailes } \\
\text { sectors are indicated as derrees from north) }\end{array}$} & \multicolumn{8}{|c|}{$\begin{array}{l}\text { Wind duration per sector, in hours } \\
\text { tors are indicated as degrees from north) }\end{array}$} \\
\hline & $\begin{array}{l}0 \\
\text { to } \\
45\end{array}$ & $\begin{array}{l}45 \\
t 0 \\
90\end{array}$ & $\begin{array}{r}90 \\
t 0 \\
135\end{array}$ & $\begin{array}{r}135 \\
60 \\
180\end{array}$ & $\begin{array}{r}180 \\
t 0 \\
225\end{array}$ & $\begin{array}{r}225 \\
20 \\
270\end{array}$ & $\begin{array}{r}270 \\
\text { to } \\
315\end{array}$ & $\begin{array}{r}315 \\
t 0 \\
360\end{array}$ & $\begin{array}{l}0 \\
\text { to } \\
45\end{array}$ & $\begin{array}{l}45 \\
t 0 \\
90\end{array}$ & $\begin{array}{r}90 \\
t 0 \\
135\end{array}$ & $\begin{array}{r}135 \\
t 0 \\
180\end{array}$ & $\begin{array}{r}180 \\
t 0 \\
225\end{array}$ & $\begin{array}{r}225 \\
t 0 \\
270\end{array}$ & $\begin{array}{r}270 \\
\text { to } \\
315\end{array}$ & $\begin{array}{r}315 \\
t 0 \\
360\end{array}$ \\
\hline \multicolumn{17}{|c|}{ JANUARY 1988} \\
\hline $\begin{array}{l}1 \\
2 \\
3 \\
4\end{array}$ & $\begin{array}{c}-- \\
-0 \\
4.37 \\
5.18\end{array}$ & $\begin{array}{c}\ldots \\
\ldots- \\
31.63 \\
51.84\end{array}$ & $\begin{array}{c}\ldots \\
66.19 \\
58.80\end{array}$ & $\begin{array}{c}-0 \\
12.89 \\
14.30\end{array}$ & $\begin{array}{l}\ldots \\
\ldots- \\
1.66 \\
4.51\end{array}$ & $\begin{array}{c}\ldots \\
\ldots- \\
1.92 \\
5.23\end{array}$ & $\begin{array}{l}-0 \\
-- \\
3.55 \\
5.86\end{array}$ & $\begin{array}{l}\ldots \\
\ldots . \\
4.63 \\
7.75\end{array}$ & $\begin{array}{l}\ldots \\
-. \\
1.3 \\
1.3\end{array}$ & $\begin{array}{l}\ldots \\
-. \\
6.1 \\
5.5\end{array}$ & $\begin{array}{r}-- \\
10.1 \\
7.8\end{array}$ & $\begin{array}{l}-. \\
-. \\
2.4 \\
2.7\end{array}$ & $\begin{array}{l}\ldots- \\
0.6 \\
1.3\end{array}$ & $\begin{array}{l}-- \\
-- \\
0.8 \\
1.5\end{array}$ & $\begin{array}{l}\ldots \\
\ldots \\
1.2 \\
1.7\end{array}$ & $\begin{array}{l}\ldots- \\
1.3 \\
2.0\end{array}$ \\
\hline $\begin{array}{l}5 \\
6 \\
7 \\
8\end{array}$ & $\begin{array}{l}2.26 \\
1.99 \\
5.64 \\
3.65\end{array}$ & $\begin{array}{r}19.63 \\
6.22 \\
60.50 \\
9.14\end{array}$ & $\begin{array}{r}90.05 \\
13.92 \\
22.10 \\
6.84\end{array}$ & $\begin{array}{r}22.32 \\
3.89 \\
3.02 \\
1.42\end{array}$ & $\begin{array}{r}1.70 \\
.91 \\
.89 \\
.86\end{array}$ & $\begin{array}{r}1.66 \\
17.95 \\
2.21 \\
10.46\end{array}$ & $\begin{array}{r}1.82 \\
49.90 \\
2.90 \\
31.03\end{array}$ & $\begin{array}{r}4.30 \\
6.38 \\
2.95 \\
10.58\end{array}$ & $\begin{array}{l}.8 \\
1.4 \\
2.4 \\
2.3\end{array}$ & $\begin{array}{l}3.5 \\
3.0 \\
9.7 \\
4.4\end{array}$ & $\begin{array}{r}12.6 \\
4.3 \\
5.9 \\
2.6\end{array}$ & $\begin{array}{r}3.5 \\
1.0 \\
1.2 \\
.7\end{array}$ & $\begin{array}{l}.6 \\
.8 \\
.4 \\
.6\end{array}$ & $\begin{array}{l}.8 \\
3.6 \\
1.3 \\
3.2\end{array}$ & $\begin{array}{l}.8 \\
8.1 \\
1.7 \\
7.1\end{array}$ & $\begin{array}{l}1.3 \\
1.7 \\
1.6 \\
2.9\end{array}$ \\
\hline $\begin{array}{r}9 \\
10 \\
11 \\
12\end{array}$ & $\begin{array}{l}5.45 \\
9.26 \\
5.66 \\
2.83\end{array}$ & $\begin{array}{l}13.73 \\
46.94 \\
34.61 \\
18.43\end{array}$ & $\begin{array}{l}13.08 \\
34.94 \\
17.14 \\
14.02\end{array}$ & $\begin{array}{r}1.15 \\
6.67 \\
5.60 \\
.98\end{array}$ & $\begin{array}{r}1.78 \\
2.64 \\
23.02 \\
4.27\end{array}$ & $\begin{array}{r}16.08 \\
4.85 \\
89.16 \\
54.74\end{array}$ & $\begin{array}{r}18.86 \\
5.26 \\
50.76 \\
93.65\end{array}$ & $\begin{array}{r}6.19 \\
7.61 \\
8.76 \\
10.03\end{array}$ & $\begin{array}{l}2.1 \\
2.8 \\
1.2 \\
1.0\end{array}$ & $\begin{array}{l}4.0 \\
6.7 \\
3.8 \\
4.0\end{array}$ & $\begin{array}{l}3.7 \\
4.8 \\
2.6 \\
2.7\end{array}$ & $\begin{array}{r}.5 \\
1.3 \\
.9 \\
.2\end{array}$ & $\begin{array}{r}1.0 \\
1.2 \\
1.9 \\
.5\end{array}$ & $\begin{array}{l}5.3 \\
2.0 \\
7.1 \\
4.8\end{array}$ & $\begin{array}{l}5.2 \\
2.2 \\
4.8 \\
9.0\end{array}$ & $\begin{array}{l}2.0 \\
2.7 \\
1.7 \\
1.8\end{array}$ \\
\hline $\begin{array}{l}13 \\
14 \\
15 \\
16\end{array}$ & $\begin{array}{l}9.38 \\
6.72 \\
5.42 \\
4.80\end{array}$ & $\begin{array}{r}120.67 \\
33.55 \\
26.21 \\
40.20\end{array}$ & $\begin{array}{l}36.36 \\
78.43 \\
40.68 \\
74.95\end{array}$ & $\begin{array}{r}18.50 \\
24.26 \\
18.10 \\
6.48\end{array}$ & $\begin{array}{r}1.94 \\
.86 \\
11.93 \\
.48\end{array}$ & $\begin{array}{r}1.97 \\
2.09 \\
59.16 \\
9.50\end{array}$ & $\begin{array}{r}8.98 \\
3.91 \\
39.98 \\
58.25\end{array}$ & $\begin{array}{r}6.60 \\
6.36 \\
13.13 \\
7.08\end{array}$ & $\begin{array}{l}1.9 \\
1.9 \\
1.2 \\
1.3\end{array}$ & $\begin{array}{l}8.6 \\
4.5 \\
2.9 \\
3.4\end{array}$ & $\begin{array}{l}4.9 \\
9.5 \\
4.1 \\
9.8\end{array}$ & $\begin{array}{l}2.7 \\
3.4 \\
2.1 \\
1.2\end{array}$ & $\begin{array}{r}.6 \\
.4 \\
1.5 \\
.2\end{array}$ & $\begin{array}{r}.7 \\
.7 \\
5.2 \\
1.2\end{array}$ & $\begin{array}{l}2.6 \\
1.4 \\
6.3 \\
5.2\end{array}$ & $\begin{array}{l}1.7 \\
1.8 \\
2.5 \\
1.6\end{array}$ \\
\hline $\begin{array}{l}17 \\
18 \\
19 \\
20\end{array}$ & $\begin{array}{r}10.34 \\
3.96 \\
21.34 \\
3.86\end{array}$ & $\begin{array}{r}67.51 \\
103.92 \\
38.18 \\
32.06\end{array}$ & $\begin{array}{r}56.42 \\
78.86 \\
47.52 \\
120.26\end{array}$ & $\begin{array}{r}25.70 \\
1.32 \\
10.15 \\
26.14\end{array}$ & $\begin{array}{r}1.54 \\
.43 \\
.65 \\
1.90\end{array}$ & $\begin{array}{l}2.90 \\
2.33 \\
1.73 \\
2.64\end{array}$ & $\begin{array}{r}4.37 \\
16.20 \\
6.86 \\
4.20\end{array}$ & $\begin{array}{r}11.64 \\
11.35 \\
4.78 \\
8.18\end{array}$ & $\begin{array}{l}2.1 \\
1.1 \\
3.8 \\
1.0\end{array}$ & $\begin{array}{l}6.2 \\
7.7 \\
5.8 \\
3.7\end{array}$ & $\begin{array}{r}6.7 \\
7.5 \\
8.7 \\
11.4\end{array}$ & $\begin{array}{r}3.5 \\
.5 \\
2.2 \\
3.1\end{array}$ & $\begin{array}{l}.5 \\
.3 \\
.2 \\
.5\end{array}$ & $\begin{array}{l}.9 \\
.9 \\
.5 \\
.8\end{array}$ & \begin{tabular}{l|}
1.3 \\
3.3 \\
1.2 \\
1.3
\end{tabular} & $\begin{array}{l}2.6 \\
2.5 \\
1.3 \\
2.0\end{array}$ \\
\hline $\begin{array}{l}21 \\
22 \\
23 \\
24\end{array}$ & $\begin{array}{l}8.88 \\
6.26 \\
7.97 \\
8.59\end{array}$ & $\begin{array}{r}17.47 \\
34.56 \\
10.32 \\
9.50\end{array}$ & $\begin{array}{r}30.65 \\
134.83 \\
10.70 \\
20.18\end{array}$ & $\begin{array}{r}4.58 \\
33.07 \\
4.06 \\
3.82\end{array}$ & $\begin{array}{r}1.01 \\
.60 \\
2.26 \\
1.08\end{array}$ & $\begin{array}{r}3.38 \\
1.37 \\
35.90 \\
5.40\end{array}$ & $\begin{array}{r}14.54 \\
2.35 \\
169.44 \\
42.26\end{array}$ & $\begin{array}{r}11.90 \\
5.93 \\
22.73 \\
24.07\end{array}$ & $\begin{array}{l}3.0 \\
1.3 \\
1.7 \\
2.3\end{array}$ & $\begin{array}{l}3.8 \\
4.1 \\
1.6 \\
2.4\end{array}$ & $\begin{array}{r}5.2 \\
12.2 \\
1.2 \\
4.5\end{array}$ & $\begin{array}{r}1.1 \\
3.3 \\
.7 \\
.7\end{array}$ & $\begin{array}{l}.7 \\
.3 \\
.7 \\
.5\end{array}$ & $\begin{array}{r}1.5 \\
.5 \\
3.1 \\
1.3\end{array}$ & $\begin{array}{r}4.4 \\
.8 \\
12.0 \\
7.1\end{array}$ & $\begin{array}{l}3.8 \\
1.4 \\
2.8 \\
5.0\end{array}$ \\
\hline $\begin{array}{l}25 \\
26 \\
27 \\
28\end{array}$ & $\begin{array}{l}5.98 \\
2.66 \\
6.26 \\
5.35\end{array}$ & $\begin{array}{l}26.64 \\
21.22 \\
40.18 \\
68.18\end{array}$ & $\begin{array}{l}60.31 \\
71.38 \\
61.37 \\
84.60\end{array}$ & $\begin{array}{l}48.31 \\
92.16 \\
37.58 \\
12.55\end{array}$ & $\begin{array}{l}1.56 \\
1.27 \\
3.72 \\
2.06\end{array}$ & $\begin{array}{l}2.64 \\
1.13 \\
2.88 \\
3.50\end{array}$ & $\begin{array}{l}4.82 \\
1.82 \\
4.46 \\
5.88\end{array}$ & $\begin{array}{l}7.37 \\
2.14 \\
8.95 \\
7.15\end{array}$ & $\begin{array}{r}1.6 \\
.7 \\
1.8 \\
1.5\end{array}$ & $\begin{array}{l}3.6 \\
2.8 \\
3.8 \\
6.6\end{array}$ & $\begin{array}{l}7.7 \\
8.6 \\
7.1 \\
8.2\end{array}$ & $\begin{array}{l}5.8 \\
9.6 \\
5.1 \\
2.1\end{array}$ & $\begin{array}{r}.6 \\
.4 \\
1.0 \\
.8\end{array}$ & $\begin{array}{r}1.0 \\
.5 \\
1.0 \\
1.2\end{array}$ & $\begin{array}{r}1.5 \\
.6 \\
1.6 \\
1.8\end{array}$ & $\begin{array}{r}2.0 \\
.7 \\
2.6 \\
1.7\end{array}$ \\
\hline $\begin{array}{l}29 \\
30 \\
31\end{array}$ & $\begin{array}{l}6.38 \\
3.34 \\
6.96\end{array}$ & $\begin{array}{r}20.11 \\
2.88 \\
7.22\end{array}$ & $\begin{array}{r}33.43 \\
.86 \\
14.90\end{array}$ & $\begin{array}{r}26.71 \\
.36 \\
8.35\end{array}$ & $\begin{array}{l}4.03 \\
2.66 \\
1.80\end{array}$ & $\begin{array}{r}14.81 \\
21.19 \\
4.97\end{array}$ & $\begin{array}{l}14.06 \\
80.40 \\
24.84\end{array}$ & $\begin{array}{r}7.49 \\
29.42 \\
16.22\end{array}$ & $\begin{array}{l}1.8 \\
1.0 \\
2.7\end{array}$ & $\begin{array}{l}4.4 \\
1.1 \\
1.9\end{array}$ & $\begin{array}{r}6.1 \\
.5 \\
3.4\end{array}$ & $\begin{array}{r}3.1 \\
.1 \\
2.2\end{array}$ & $\begin{array}{l}.9 \\
.4 \\
.8\end{array}$ & $\begin{array}{l}2.7 \\
2.8 \\
1.8\end{array}$ & $\begin{array}{r}2.9 \\
12.5 \\
6.4\end{array}$ & $\begin{array}{l}1.9 \\
5.5 \\
4.7\end{array}$ \\
\hline
\end{tabular}


Table 3.--Wind-run and wind-duration histogran data, octobor 1987-Decenber 1989--Continued

\begin{tabular}{|c|c|c|c|c|c|c|c|c|c|c|c|c|c|c|c|c|}
\hline $\begin{array}{l}\text { Day } \\
\text { of } \\
\text { nonth }\end{array}$ & \multicolumn{2}{|c|}{ (eectors } & \multicolumn{6}{|c|}{$\begin{array}{l}\text { Wind run per sector, in ailes } \\
\text { are indicated as desrees froe a }\end{array}$} & \multicolumn{8}{|c|}{$\begin{array}{l}\text { Wind duration per sector, in bours } \\
\text { (sectors are indicated as degrees from north) }\end{array}$} \\
\hline \multicolumn{17}{|c|}{ FEBRUARY 1988} \\
\hline $\begin{array}{l}1 \\
2 \\
3 \\
4\end{array}$ & $\begin{array}{l}5.11 \\
7.85 \\
6.02 \\
3.86\end{array}$ & $\begin{array}{r}15.24 \\
9.24 \\
15.10 \\
18.38\end{array}$ & $\begin{array}{r}31.51 \\
9.77 \\
61.68 \\
61.80\end{array}$ & $\begin{array}{r}8.42 \\
4.13 \\
13.73 \\
57.50\end{array}$ & $\begin{array}{l}1.61 \\
1.54 \\
1.18 \\
2.86\end{array}$ & $\begin{array}{l}4.20 \\
4.78 \\
2.66 \\
2.93\end{array}$ & $\begin{array}{r}15.98 \\
4.94 \\
4.54 \\
3.62\end{array}$ & $\begin{array}{l}7.58 \\
5.78 \\
5.21 \\
4.06\end{array}$ & $\begin{array}{l}1.9 \\
3.6 \\
2.0 \\
1.1\end{array}$ & $\begin{array}{l}3.4 \\
4.8 \\
3.0 \\
3.4\end{array}$ & $\begin{array}{r}6.6 \\
4.2 \\
11.1 \\
8.4\end{array}$ & $\begin{array}{l}2.9 \\
1.5 \\
2.3 \\
6.6\end{array}$ & $\begin{array}{r}0.9 \\
1.1 \\
.5 \\
1.0\end{array}$ & $\begin{array}{l}1.5 \\
2.9 \\
1.2 \\
1.1\end{array}$ & $\begin{array}{l}3.7 \\
2.9 \\
2.0 \\
1.2\end{array}$ & $\begin{array}{l}2.8 \\
2.8 \\
1.9 \\
1.1\end{array}$ \\
\hline $\begin{array}{l}5 \\
6 \\
7 \\
8\end{array}$ & $\begin{array}{l}3.65 \\
8.76 \\
4.27 \\
3.43\end{array}$ & $\begin{array}{l}18.38 \\
64.08 \\
18.07 \\
20.09\end{array}$ & $\begin{array}{l}55.92 \\
50.54 \\
66.65 \\
43.15\end{array}$ & $\begin{array}{l}94.66 \\
25.85 \\
39.89 \\
18.86\end{array}$ & $\begin{array}{l}2.45 \\
2.76 \\
1.82 \\
1.73\end{array}$ & $\begin{array}{l}4.44 \\
8.02 \\
1.94 \\
6.38\end{array}$ & $\begin{array}{l}2.69 \\
7.37 \\
4.82 \\
9.77\end{array}$ & $\begin{array}{l}4.37 \\
9.10 \\
6.96 \\
5.59\end{array}$ & $\begin{array}{l}.8 \\
1.7 \\
1.3 \\
1.4\end{array}$ & $\begin{array}{l}2.4 \\
4.9 \\
3.5 \\
3.5\end{array}$ & $\begin{array}{l}6.8 \\
6.0 \\
9.5 \\
7.3\end{array}$ & $\begin{array}{l}9.0 \\
3.4 \\
4.8 \\
3.2\end{array}$ & $\begin{array}{r}.8 \\
1.0 \\
.6 \\
.8\end{array}$ & $\begin{array}{l}1.8 \\
2.5 \\
.7 \\
2.4\end{array}$ & $\begin{array}{l}1.0 \\
2.2 \\
1.6 \\
3.2\end{array}$ & $\begin{array}{l}1.2 \\
2.2 \\
2.0 \\
2.2\end{array}$ \\
\hline $\begin{array}{r}9 \\
10 \\
11 \\
12\end{array}$ & $\begin{array}{l}4.10 \\
2.54 \\
1.85 \\
4.32\end{array}$ & $\begin{array}{r}12.58 \\
.79 \\
3.31 \\
54.31\end{array}$ & $\begin{array}{r}34.87 \\
.10 \\
40.70 \\
66.43\end{array}$ & $\begin{array}{r}29.71 \\
.07 \\
12.98 \\
40.37\end{array}$ & $\begin{array}{l}9.43 \\
1.22 \\
2.30 \\
1.01\end{array}$ & $\begin{array}{r}50.93 \\
40.39 \\
6.60 \\
1.58\end{array}$ & $\begin{array}{r}17.78 \\
198.96 \\
10.70 \\
2.95\end{array}$ & $\begin{array}{r}3.43 \\
27.05 \\
4.18 \\
4.15\end{array}$ & $\begin{array}{r}1.4 \\
.6 \\
1.1 \\
1.2\end{array}$ & $\begin{array}{r}2.8 \\
.2 \\
1.3 \\
5.9\end{array}$ & $\begin{array}{r}5.5 \\
.0 \\
7.6 \\
8.8\end{array}$ & $\begin{array}{r}4.8 \\
.0 \\
2.9 \\
4.8\end{array}$ & $\begin{array}{r}1.1 \\
.2 \\
1.5 \\
.3\end{array}$ & $\begin{array}{r}4.7 \\
3.6 \\
3.2 \\
.6\end{array}$ & $\begin{array}{r}2.5 \\
15.3 \\
4.4 \\
1.0\end{array}$ & $\begin{array}{l}1.0 \\
3.9 \\
1.8 \\
1.2\end{array}$ \\
\hline $\begin{array}{l}17 \\
18 \\
19 \\
20\end{array}$ & $\begin{array}{l}.82 \\
6.22 \\
2.47 \\
2.42\end{array}$ & $\begin{array}{l}50.54 \\
95.69 \\
16.27 \\
23.38\end{array}$ & $\begin{array}{l}93.91 \\
57.94 \\
44.21 \\
46.66\end{array}$ & $\begin{array}{r}17.90 \\
5.54 \\
29.88 \\
17.40\end{array}$ & $\begin{array}{r}1.20 \\
.48 \\
2.88 \\
2.11\end{array}$ & $\begin{array}{l}2.40 \\
3.70 \\
7.68 \\
9.43\end{array}$ & $\begin{array}{r}3.46 \\
11.90 \\
10.44 \\
6.14\end{array}$ & $\begin{array}{r}1.80 \\
12.67 \\
6.43 \\
4.61\end{array}$ & $\begin{array}{r}.4 \\
1.2 \\
.9 \\
1.1\end{array}$ & $\begin{array}{l}4.9 \\
6.3 \\
2.5 \\
3.1\end{array}$ & $\begin{array}{r}11.5 \\
6.9 \\
7.5 \\
7.0\end{array}$ & $\begin{array}{l}3.3 \\
1.1 \\
4.6 \\
3.5\end{array}$ & $\begin{array}{r}.5 \\
.2 \\
1.2 \\
1.0\end{array}$ & $\begin{array}{l}1.2 \\
1.3 \\
2.4 \\
3.8\end{array}$ & $\begin{array}{l}1.3 \\
3.4 \\
2.8 \\
2.4\end{array}$ & $\begin{array}{l}.8 \\
3.2 \\
2.0 \\
1.8\end{array}$ \\
\hline $\begin{array}{l}21 \\
22 \\
23 \\
24\end{array}$ & $\begin{array}{l}4.99 \\
1.75 \\
1.56 \\
5.83\end{array}$ & $\begin{array}{r}47.42 \\
6.02 \\
10.75 \\
36.22\end{array}$ & $\begin{array}{l}59.06 \\
49.08 \\
69.65 \\
48.74\end{array}$ & $\begin{array}{r}7.18 \\
2.50 \\
37.49 \\
29.81\end{array}$ & $\begin{array}{l}2.64 \\
1.49 \\
1.32 \\
3.12\end{array}$ & $\begin{array}{r}3.02 \\
30.00 \\
5.04 \\
6.77\end{array}$ & $\begin{array}{r}3.38 \\
63.36 \\
5.54 \\
8.35\end{array}$ & $\begin{array}{r}4.80 \\
7.51 \\
3.26 \\
10.34\end{array}$ & $\begin{array}{r}1.3 \\
.6 \\
.5 \\
1.2\end{array}$ & $\begin{array}{l}6.4 \\
1.8 \\
1.8 \\
4.1\end{array}$ & $\begin{array}{r}9.7 \\
10.0 \\
11.4 \\
6.6\end{array}$ & $\begin{array}{r}1.7 \\
.8 \\
5.4 \\
4.0\end{array}$ & $\begin{array}{r}1.1 \\
.4 \\
.5 \\
1.0\end{array}$ & $\begin{array}{l}1.1 \\
3.2 \\
1.7 \\
2.2\end{array}$ & $\begin{array}{l}1.1 \\
6.0 \\
1.6 \\
2.4\end{array}$ & $\begin{array}{l}1.5 \\
1.1 \\
1.0 \\
2.5\end{array}$ \\
\hline
\end{tabular}


Table 3.--Wind-rum and wind-chration histogran data, octobor 1987-Decenber 1989--Continued

\begin{tabular}{|c|c|c|c|c|c|c|c|c|c|c|c|c|c|c|c|c|}
\hline \multirow{2}{*}{$\begin{array}{l}\text { Dey } \\
\text { of } \\
\text { conth }\end{array}$} & \multicolumn{8}{|c|}{$\begin{array}{l}\text { Wind run per sector, in ailes } \\
\text { sectors sre indicated as desrees froe north) }\end{array}$} & \multicolumn{8}{|c|}{$\begin{array}{l}\text { Wind duration per sector, in hours } \\
\text { tors are indicated as degrees froe north) }\end{array}$} \\
\hline & $\begin{array}{r}0 \\
t 0 \\
45\end{array}$ & $\begin{array}{l}45 \\
t 0 \\
90\end{array}$ & $\begin{array}{r}90 \\
t 0 \\
135\end{array}$ & $\begin{array}{r}135 \\
\text { to } \\
180\end{array}$ & $\begin{array}{r}180 \\
t 0 \\
225\end{array}$ & $\begin{array}{r}225 \\
\text { to } \\
270\end{array}$ & $\begin{array}{r}270 \\
t 0 \\
315\end{array}$ & $\begin{array}{r}315 \\
t 0 \\
360\end{array}$ & $\begin{array}{r}0 \\
t 0 \\
45\end{array}$ & $\begin{array}{l}45 \\
t 0 \\
90\end{array}$ & $\begin{array}{r}90 \\
t 0 \\
135\end{array}$ & $\begin{array}{r}135 \\
t 0 \\
180\end{array}$ & $\begin{array}{r}180 \\
t 0 \\
225\end{array}$ & $\begin{array}{r}225 \\
t 0 \\
270\end{array}$ & $\begin{array}{r}270 \\
t 0 \\
315\end{array}$ & $\begin{array}{r}315 \\
t 0 \\
360\end{array}$ \\
\hline \multicolumn{17}{|c|}{ MARCA 1988} \\
\hline $\begin{array}{l}1 \\
2 \\
3 \\
4\end{array}$ & $\begin{array}{l}2.86 \\
2.21 \\
2.45 \\
1.46\end{array}$ & $\begin{array}{r}57.50 \\
4.87 \\
5.14 \\
2.35\end{array}$ & $\begin{array}{l}69.72 \\
13.15 \\
10.61 \\
18.77\end{array}$ & $\begin{array}{r}20.11 \\
9.72 \\
10.56 \\
4.73\end{array}$ & $\begin{array}{l}2.66 \\
2.47 \\
3.26 \\
2.81\end{array}$ & $\begin{array}{r}6.17 \\
13.51 \\
28.51 \\
40.80\end{array}$ & $\begin{array}{r}4.63 \\
24.89 \\
33.05 \\
102.41\end{array}$ & $\begin{array}{r}5.16 \\
10.20 \\
10.34 \\
17.28\end{array}$ & $\begin{array}{r}0.7 \\
.9 \\
.8 \\
.5\end{array}$ & $\begin{array}{r}5.4 \\
1.5 \\
1.2 \\
.9\end{array}$ & $\begin{array}{l}8.6 \\
3.8 \\
2.5 \\
4.2\end{array}$ & $\begin{array}{l}3.4 \\
2.7 \\
2.2 \\
1.1\end{array}$ & $\begin{array}{r}0.9 \\
1.1 \\
1.1 \\
.6\end{array}$ & $\begin{array}{l}1.8 \\
4.4 \\
6.1 \\
4.4\end{array}$ & $\begin{array}{r}1.5 \\
6.6 \\
7.4 \\
10.0\end{array}$ & $\begin{array}{l}1.5 \\
3.0 \\
2.6 \\
2.2\end{array}$ \\
\hline $\begin{array}{l}5 \\
6 \\
7 \\
8\end{array}$ & $\begin{array}{l}3.70 \\
1.97 \\
1.63 \\
2.26\end{array}$ & $\begin{array}{r}27.86 \\
15.55 \\
1.49 \\
21.74\end{array}$ & $\begin{array}{r}68.59 \\
36.65 \\
5.52 \\
79.46\end{array}$ & $\begin{array}{r}36.86 \\
37.73 \\
1.01 \\
25.20\end{array}$ & $\begin{array}{l}3.58 \\
9.43 \\
1.56 \\
1.61\end{array}$ & $\begin{array}{r}4.10 \\
21.48 \\
43.01 \\
5.26\end{array}$ & $\begin{array}{r}4.49 \\
33.62 \\
172.08 \\
9.00\end{array}$ & $\begin{array}{r}6.17 \\
9.89 \\
15.12 \\
7.27\end{array}$ & $\begin{array}{r}1.0 \\
.6 \\
.5 \\
.8\end{array}$ & $\begin{array}{r}3.3 \\
1.7 \\
.7 \\
2.5\end{array}$ & $\begin{array}{l}8.8 \\
4.7 \\
1.5 \\
9.7\end{array}$ & $\begin{array}{r}5.3 \\
4.9 \\
.3 \\
4.1\end{array}$ & $\begin{array}{r}1.1 \\
2.2 \\
.4 \\
.7\end{array}$ & $\begin{array}{l}1.3 \\
3.8 \\
4.3 \\
1.8\end{array}$ & $\begin{array}{r}1.3 \\
4.2 \\
14.1 \\
2.2\end{array}$ & $\begin{array}{l}1.7 \\
1.7 \\
2.0 \\
2.0\end{array}$ \\
\hline $\begin{array}{r}9 \\
10 \\
11 \\
12\end{array}$ & $\begin{array}{l}4.10 \\
9.82 \\
5.71 \\
2.16\end{array}$ & $\begin{array}{r}90.48 \\
18.65 \\
1.20 \\
4.06\end{array}$ & $\begin{array}{r}102.50 \\
10.85 \\
.46 \\
23.81\end{array}$ & $\begin{array}{r}6.65 \\
5.71 \\
.10 \\
8.16\end{array}$ & $\begin{array}{l}1.10 \\
3.19 \\
2.06 \\
1.13\end{array}$ & $\begin{array}{r}7.20 \\
16.85 \\
46.66 \\
18.14\end{array}$ & $\begin{array}{r}7.63 \\
48.41 \\
107.74 \\
36.91\end{array}$ & $\begin{array}{r}4.85 \\
13.42 \\
14.50 \\
9.98\end{array}$ & $\begin{array}{r}.9 \\
2.1 \\
1.8 \\
.7\end{array}$ & $\begin{array}{r}7.8 \\
3.0 \\
.5 \\
1.6\end{array}$ & $\begin{array}{r}9.3 \\
2.0 \\
.2 \\
7.2\end{array}$ & $\begin{array}{r}1.2 \\
1.3 \\
.1 \\
1.8\end{array}$ & $\begin{array}{r}.4 \\
1.1 \\
.4 \\
.6\end{array}$ & $\begin{array}{l}1.5 \\
3.8 \\
5.4 \\
3.7\end{array}$ & $\begin{array}{r}1.6 \\
7.7 \\
12.3 \\
6.2\end{array}$ & $\begin{array}{l}1.2 \\
2.8 \\
3.3 \\
2.1\end{array}$ \\
\hline $\begin{array}{l}13 \\
14 \\
15 \\
16\end{array}$ & $\begin{array}{r}1.46 \\
1.87 \\
.65 \\
2.50\end{array}$ & $\begin{array}{r}10.63 \\
29.28 \\
107.66 \\
21.60\end{array}$ & $\begin{array}{r}31.46 \\
86.14 \\
150.22 \\
82.70\end{array}$ & $\begin{array}{r}32.95 \\
40.63 \\
6.96 \\
.05\end{array}$ & $\begin{array}{r}1.49 \\
5.33 \\
1.15 \\
.31\end{array}$ & $\begin{array}{r}11.11 \\
2.40 \\
2.62 \\
12.00\end{array}$ & $\begin{array}{r}50.06 \\
3.65 \\
4.06 \\
58.75\end{array}$ & $\begin{array}{r}18.67 \\
4.70 \\
1.92 \\
24.65\end{array}$ & $\begin{array}{l}.5 \\
.6 \\
.1 \\
.8\end{array}$ & $\begin{array}{l}1.4 \\
3.0 \\
8.4 \\
2.1\end{array}$ & $\begin{array}{r}5.2 \\
9.8 \\
12.6 \\
7.2\end{array}$ & $\begin{array}{r}4.9 \\
6.0 \\
.9 \\
.0\end{array}$ & $\begin{array}{r}.6 \\
1.2 \\
.3 \\
.1\end{array}$ & $\begin{array}{r}1.9 \\
.8 \\
.4 \\
1.9\end{array}$ & $\begin{array}{r}6.3 \\
1.2 \\
.8 \\
7.8\end{array}$ & $\begin{array}{r}3.0 \\
1.3 \\
.4 \\
3.9\end{array}$ \\
\hline $\begin{array}{l}17 \\
18 \\
19 \\
20\end{array}$ & $\begin{array}{r}3.12 \\
2.66 \\
10.54 \\
2.06\end{array}$ & $\begin{array}{l}10.13 \\
29.86 \\
35.54 \\
31.18\end{array}$ & $\begin{array}{l}19.49 \\
58.56 \\
68.06 \\
52.30\end{array}$ & $\begin{array}{r}3.10 \\
32.64 \\
20.28 \\
57.84\end{array}$ & $\begin{array}{l}1.61 \\
4.37 \\
4.32 \\
2.71\end{array}$ & $\begin{array}{r}18.22 \\
11.52 \\
4.61 \\
7.73\end{array}$ & $\begin{array}{r}36.96 \\
10.39 \\
4.01 \\
3.00\end{array}$ & $\begin{array}{l}9.70 \\
8.57 \\
5.35 \\
3.31\end{array}$ & $\begin{array}{r}1.0 \\
.6 \\
1.9 \\
.6\end{array}$ & $\begin{array}{l}2.2 \\
3.2 \\
5.0 \\
4.2\end{array}$ & $\begin{array}{l}5.5 \\
6.7 \\
8.5 \\
7.3\end{array}$ & $\begin{array}{r}.9 \\
4.2 \\
3.0 \\
7.3\end{array}$ & $\begin{array}{r}.5 \\
1.2 \\
1.2 \\
.9\end{array}$ & $\begin{array}{l}3.9 \\
3.0 \\
1.4 \\
1.7\end{array}$ & $\begin{array}{l}7.2 \\
2.7 \\
1.3 \\
1.0\end{array}$ & $\begin{array}{l}2.7 \\
2.2 \\
1.5 \\
1.0\end{array}$ \\
\hline $\begin{array}{l}21 \\
22 \\
23 \\
24\end{array}$ & $\begin{array}{r}2.23 \\
2.33 \\
1.68 \\
.65\end{array}$ & $\begin{array}{r}12.89 \\
5.93 \\
17.47 \\
.14\end{array}$ & $\begin{array}{r}53.06 \\
18.14 \\
38.93 \\
.05\end{array}$ & $\begin{array}{r}51.62 \\
3.07 \\
11.95 \\
.10\end{array}$ & $\begin{array}{l}3.79 \\
3.10 \\
3.86 \\
2.11\end{array}$ & $\begin{array}{r}4.44 \\
33.91 \\
33.14 \\
48.82\end{array}$ & $\begin{array}{r}2.86 \\
40.56 \\
59.86 \\
164.40\end{array}$ & $\begin{array}{r}2.45 \\
11.40 \\
8.18 \\
24.36\end{array}$ & $\begin{array}{l}.7 \\
.8 \\
.5 \\
.1\end{array}$ & $\begin{array}{r}2.8 \\
1.8 \\
2.8 \\
.0\end{array}$ & $\begin{array}{r}9.0 \\
4.1 \\
6.2 \\
.0\end{array}$ & $\begin{array}{r}7.3 \\
1.0 \\
2.1 \\
.0\end{array}$ & $\begin{array}{r}1.2 \\
.9 \\
.8 \\
.2\end{array}$ & $\begin{array}{l}1.4 \\
5.7 \\
4.2 \\
5.2\end{array}$ & $\begin{array}{r}.9 \\
6.9 \\
5.8 \\
15.6\end{array}$ & $\begin{array}{l}.7 \\
2.5 \\
1.4 \\
2.7\end{array}$ \\
\hline $\begin{array}{l}25 \\
26 \\
27 \\
28\end{array}$ & $\begin{array}{r}2.66 \\
1.01 \\
.22 \\
.94\end{array}$ & $\begin{array}{r}1.87 \\
20.98 \\
1.68 \\
2.14\end{array}$ & $\begin{array}{r}5.88 \\
79.18 \\
28.80 \\
12.72\end{array}$ & $\begin{array}{r}.96 \\
46.97 \\
35.78 \\
2.35\end{array}$ & $\begin{array}{r}3.74 \\
3.22 \\
11.57 \\
1.68\end{array}$ & $\begin{array}{r}67.15 \\
3.96 \\
80.38 \\
30.22\end{array}$ & $\begin{array}{r}125.50 \\
1.51 \\
91.56 \\
69.86\end{array}$ & $\begin{array}{r}18.07 \\
2.21 \\
5.59 \\
8.95\end{array}$ & $\begin{array}{l}.6 \\
.4 \\
.0 \\
.5\end{array}$ & $\begin{array}{r}.5 \\
3.4 \\
.4 \\
1.1\end{array}$ & $\begin{array}{r}1.5 \\
11.3 \\
3.7 \\
4.3\end{array}$ & $\begin{array}{r}.3 \\
6.1 \\
3.8 \\
.9\end{array}$ & $\begin{array}{r}.6 \\
.7 \\
1.3 \\
.6\end{array}$ & $\begin{array}{l}6.5 \\
1.0 \\
6.7 \\
4.7\end{array}$ & $\begin{array}{r}11.5 \\
.4 \\
7.4 \\
9.6\end{array}$ & $\begin{array}{r}2.3 \\
.6 \\
.6 \\
2.0\end{array}$ \\
\hline $\begin{array}{l}29 \\
30 \\
31\end{array}$ & $\begin{array}{r}2.06 \\
.91 \\
.19\end{array}$ & $\begin{array}{l}13.97 \\
65.81 \\
99.94\end{array}$ & $\begin{array}{r}35.50 \\
129.89 \\
194.64\end{array}$ & $\begin{array}{r}13.85 \\
2.33 \\
3.05\end{array}$ & $\begin{array}{r}4.58 \\
.07 \\
.34\end{array}$ & $\begin{array}{r}22.94 \\
.43 \\
.14\end{array}$ & $\begin{array}{r}26.62 \\
.74 \\
.98\end{array}$ & $\begin{array}{r}9.53 \\
.31 \\
.22\end{array}$ & $\begin{array}{l}.7 \\
.2 \\
.0\end{array}$ & $\begin{array}{l}2.2 \\
8.3 \\
7.5\end{array}$ & $\begin{array}{r}5.9 \\
14.1 \\
15.4\end{array}$ & $\begin{array}{r}3.0 \\
.5 \\
.6\end{array}$ & $\begin{array}{r}1.2 \\
.0 \\
.1\end{array}$ & $\begin{array}{r}4.0 \\
.2 \\
.0\end{array}$ & $\begin{array}{r}4.5 \\
.3 \\
.2\end{array}$ & $\begin{array}{r}2.4 \\
.2 \\
.0\end{array}$ \\
\hline
\end{tabular}


Table 3.--Wind-run and wind-curation histogran data, October 1987-Decanber 1989--Continued

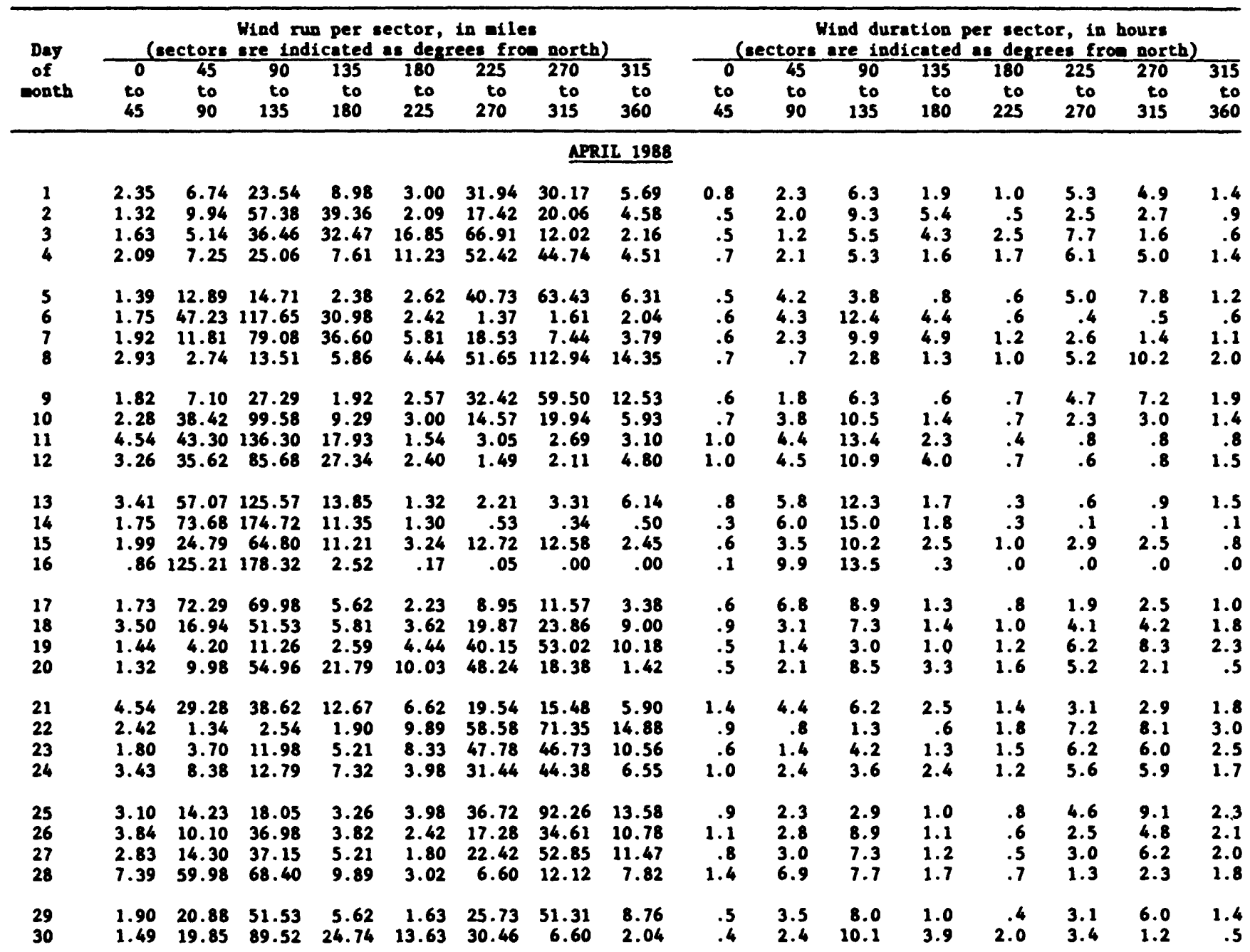


Table 3.--Wind-rum and wind-curation histogra data, october 1987-Decu-ber 1989--Continued

\begin{tabular}{|c|c|c|c|c|c|c|c|c|c|c|c|c|c|c|c|c|}
\hline \multirow{2}{*}{$\begin{array}{c}\text { Day } \\
\text { of } \\
\text { nonth }\end{array}$} & \multicolumn{4}{|c|}{$\begin{array}{l}\text { Wind run per } \\
\text { eectore ere indicated }\end{array}$} & \multicolumn{4}{|c|}{$\begin{array}{l}\text { sector, in ailes } \\
\text { a desrees fron north) }\end{array}$} & \multicolumn{8}{|c|}{$\begin{array}{l}\text { Wind duration per sector, in hours } \\
\text { (sectors are indicated a degrees fron north) }\end{array}$} \\
\hline & $\begin{array}{l}0 \\
20 \\
45\end{array}$ & $\begin{array}{l}45 \\
\text { to } \\
90\end{array}$ & $\begin{array}{r}90 \\
\text { to } \\
135\end{array}$ & $\begin{array}{r}135 \\
10 \\
180\end{array}$ & $\begin{array}{r}180 \\
\text { to } \\
225\end{array}$ & $\begin{array}{r}225 \\
\text { to } \\
270\end{array}$ & $\begin{array}{r}270 \\
\text { to } \\
315\end{array}$ & $\begin{array}{r}315 \\
t 0 \\
360\end{array}$ & $\begin{array}{l}0 \\
t 0 \\
45\end{array}$ & $\begin{array}{l}45 \\
\text { to } \\
90\end{array}$ & $\begin{array}{r}90 \\
\text { to } \\
135\end{array}$ & $\begin{array}{r}135 \\
t 0 \\
180\end{array}$ & $\begin{array}{r}180 \\
t 0 \\
225\end{array}$ & $\begin{array}{r}225 \\
\text { to } \\
270\end{array}$ & $\begin{array}{r}270 \\
t 0 \\
315\end{array}$ & $\begin{array}{r}315 \\
\text { to } \\
360\end{array}$ \\
\hline \multicolumn{17}{|c|}{ MAI 1988} \\
\hline $\begin{array}{l}1 \\
2 \\
3 \\
4\end{array}$ & $\begin{array}{r}.53 \\
.98 \\
5.54 \\
1.92\end{array}$ & $\begin{array}{r}5.57 \\
1.51 \\
20.59 \\
65.52\end{array}$ & $\begin{array}{r}17.21 \\
6.38 \\
55.46 \\
124.42\end{array}$ & $\begin{array}{r}15.58 \\
1.68 \\
8.06 \\
11.71\end{array}$ & $\begin{array}{r}10.97 \\
1.03 \\
2.16 \\
6.72\end{array}$ & $\begin{array}{r}41.98 \\
45.91 \\
9.62 \\
23.40\end{array}$ & $\begin{array}{r}96.77 \\
148.25 \\
15.53 \\
8.35\end{array}$ & $\begin{array}{r}15.96 \\
10.82 \\
9.58 \\
.77\end{array}$ & $\begin{array}{r}0.1 \\
.4 \\
1.6 \\
.3\end{array}$ & $\begin{array}{r}0.8 \\
.6 \\
3.8 \\
5.2\end{array}$ & $\begin{array}{r}2.7 \\
1.6 \\
7.9 \\
11.0\end{array}$ & $\begin{array}{r}2.6 \\
.5 \\
1.8 \\
2.0\end{array}$ & $\begin{array}{r}1.8 \\
.3 \\
.7 \\
1.1\end{array}$ & $\begin{array}{l}4.6 \\
4.6 \\
2.3 \\
3.0\end{array}$ & $\begin{array}{r}9.6 \\
13.8 \\
3.2 \\
1.1\end{array}$ & $\begin{array}{r}1.8 \\
2.0 \\
2.6 \\
.1\end{array}$ \\
\hline $\begin{array}{l}5 \\
6 \\
7 \\
8\end{array}$ & $\begin{array}{l}.46 \\
.74 \\
.22 \\
.89\end{array}$ & $\begin{array}{r}52.61 \\
5.76 \\
.12 \\
2.18\end{array}$ & $\begin{array}{r}117.79 \\
28.18 \\
.19 \\
5.86\end{array}$ & $\begin{array}{r}22.75 \\
17.64 \\
.41 \\
1.94\end{array}$ & $\begin{array}{r}16.20 \\
24.82 \\
13.44 \\
3.07\end{array}$ & $\begin{array}{r}29.90 \\
123.60 \\
131.06 \\
36.50\end{array}$ & $\begin{array}{r}5.98 \\
52.75 \\
157.68 \\
78.31\end{array}$ & $\begin{array}{r}.53 \\
3.65 \\
17.38 \\
19.08\end{array}$ & $\begin{array}{l}.1 \\
.2 \\
.0 \\
.3\end{array}$ & $\begin{array}{r}3.8 \\
.7 \\
.0 \\
.7\end{array}$ & $\begin{array}{r}10.4 \\
3.9 \\
.0 \\
1.4\end{array}$ & $\begin{array}{r}3.1 \\
2.3 \\
.0 \\
.6\end{array}$ & $\begin{array}{r}2.2 \\
2.4 \\
1.1 \\
.6\end{array}$ & $\begin{array}{l}3.4 \\
9.7 \\
9.4 \\
5.2\end{array}$ & $\begin{array}{r}.7 \\
4.3 \\
11.6 \\
11.3\end{array}$ & $\begin{array}{r}.1 \\
.5 \\
1.8 \\
3.7\end{array}$ \\
\hline $\begin{array}{r}9 \\
10 \\
11 \\
12\end{array}$ & $\begin{array}{l}2.54 \\
2.52 \\
2.21 \\
6.14\end{array}$ & $\begin{array}{r}8.33 \\
18.82 \\
49.30 \\
19.66\end{array}$ & $\begin{array}{l}34.56 \\
39.38 \\
99.43 \\
29.02\end{array}$ & $\begin{array}{l}9.19 \\
6.19 \\
4.66 \\
7.94\end{array}$ & $\begin{array}{l}1.34 \\
1.82 \\
2.30 \\
4.34\end{array}$ & $\begin{array}{l}26.69 \\
21.48 \\
17.54 \\
17.74\end{array}$ & $\begin{array}{l}64.54 \\
47.66 \\
23.16 \\
28.13\end{array}$ & $\begin{array}{r}10.80 \\
7.30 \\
5.06 \\
14.02\end{array}$ & $\begin{array}{r}.8 \\
.8 \\
.5 \\
1.6\end{array}$ & $\begin{array}{l}2.3 \\
3.5 \\
4.4 \\
3.3\end{array}$ & $\begin{array}{r}6.6 \\
7.8 \\
10.0 \\
4.9\end{array}$ & $\begin{array}{l}1.8 \\
1.6 \\
1.0 \\
1.7\end{array}$ & $\begin{array}{r}.3 \\
.6 \\
.6 \\
1.1\end{array}$ & $\begin{array}{l}3.0 \\
3.0 \\
2.8 \\
3.1\end{array}$ & $\begin{array}{l}7.2 \\
5.4 \\
3.6 \\
4.8\end{array}$ & $\begin{array}{l}2.0 \\
1.2 \\
1.1 \\
3.3\end{array}$ \\
\hline $\begin{array}{l}13 \\
14 \\
15 \\
16\end{array}$ & $\begin{array}{l}3.26 \\
3.19 \\
1.94 \\
4.70\end{array}$ & $\begin{array}{r}43.92 \\
6.38 \\
12.82 \\
53.83\end{array}$ & $\begin{array}{l}39.31 \\
18.19 \\
53.16 \\
65.33\end{array}$ & $\begin{array}{l}8.40 \\
1.06 \\
8.57 \\
7.82\end{array}$ & $\begin{array}{r}12.29 \\
1.68 \\
1.51 \\
7.10\end{array}$ & $\begin{array}{l}54.50 \\
40.58 \\
21.07 \\
30.86\end{array}$ & $\begin{array}{r}31.63 \\
111.17 \\
39.48 \\
20.88\end{array}$ & $\begin{array}{r}5.38 \\
15.36 \\
10.85 \\
6.31\end{array}$ & $\begin{array}{l}.8 \\
.9 \\
.6 \\
.9\end{array}$ & $\begin{array}{l}3.9 \\
1.5 \\
2.6 \\
4.5\end{array}$ & $\begin{array}{l}4.5 \\
3.5 \\
9.0 \\
6.9\end{array}$ & $\begin{array}{r}1.5 \\
.4 \\
1.4 \\
1.5\end{array}$ & $\begin{array}{r}2.0 \\
.4 \\
.4 \\
1.2\end{array}$ & $\begin{array}{l}6.3 \\
4.3 \\
2.9 \\
4.2\end{array}$ & $\begin{array}{r}3.7 \\
10.3 \\
5.1 \\
3.1\end{array}$ & $\begin{array}{l}1.2 \\
2.6 \\
1.9 \\
1.4\end{array}$ \\
\hline $\begin{array}{l}17 \\
18 \\
19 \\
20\end{array}$ & $\begin{array}{l}3.19 \\
2.71 \\
1.03 \\
6.84\end{array}$ & $\begin{array}{r}17.71 \\
17.23 \\
4.94 \\
19.70\end{array}$ & $\begin{array}{r}48.41 \\
45.38 \\
3.31 \\
24.77\end{array}$ & $\begin{array}{r}16.99 \\
4.39 \\
.41 \\
3.79\end{array}$ & $\begin{array}{r}8.62 \\
2.62 \\
.41 \\
2.71\end{array}$ & $\begin{array}{l}30.77 \\
20.16 \\
32.30 \\
17.66\end{array}$ & $\begin{array}{l}21.19 \\
49.39 \\
86.86 \\
24.74\end{array}$ & $\begin{array}{r}8.74 \\
10.97 \\
5.54 \\
8.59\end{array}$ & $\begin{array}{r}.9 \\
.8 \\
.4 \\
1.5\end{array}$ & $\begin{array}{l}2.7 \\
2.2 \\
1.8 \\
4.4\end{array}$ & $\begin{array}{l}6.8 \\
5.0 \\
1.7 \\
5.1\end{array}$ & $\begin{array}{r}2.8 \\
1.2 \\
.3 \\
1.3\end{array}$ & $\begin{array}{r}1.5 \\
.7 \\
.3 \\
1.2\end{array}$ & $\begin{array}{l}4.3 \\
3.6 \\
6.0 \\
4.1\end{array}$ & $\begin{array}{r}3.3 \\
7.7 \\
11.3 \\
4.2\end{array}$ & $\begin{array}{l}1.5 \\
2.6 \\
2.1 \\
2.1\end{array}$ \\
\hline $\begin{array}{l}21 \\
22 \\
23 \\
24\end{array}$ & $\begin{array}{l}6.94 \\
3.07 \\
2.30 \\
3.43\end{array}$ & $\begin{array}{l}43.15 \\
29.16 \\
21.72 \\
16.73\end{array}$ & $\begin{array}{l}49.54 \\
43.54 \\
55.70 \\
46.13\end{array}$ & $\begin{array}{l}6.24 \\
9.62 \\
3.96 \\
8.78\end{array}$ & $\begin{array}{l}2.28 \\
3.34 \\
2.33 \\
4.27\end{array}$ & $\begin{array}{l}16.66 \\
21.84 \\
22.82 \\
24.74\end{array}$ & $\begin{array}{l}19.22 \\
24.29 \\
26.81 \\
34.34\end{array}$ & $\begin{array}{l}4.39 \\
5.95 \\
6.84 \\
7.94\end{array}$ & $\begin{array}{r}1.4 \\
.8 \\
.6 \\
.8\end{array}$ & $\begin{array}{l}6.5 \\
4.5 \\
2.9 \\
3.0\end{array}$ & $\begin{array}{l}7.8 \\
7.4 \\
8.4 \\
7.2\end{array}$ & $\begin{array}{l}1.3 \\
2.3 \\
1.3 \\
1.7\end{array}$ & $\begin{array}{r}.6 \\
.8 \\
.7 \\
1.1\end{array}$ & $\begin{array}{l}2.6 \\
3.1 \\
3.9 \\
3.7\end{array}$ & $\begin{array}{l}2.9 \\
3.6 \\
4.4 \\
4.7\end{array}$ & $\begin{array}{l}.9 \\
1.4 \\
1.6 \\
1.7\end{array}$ \\
\hline $\begin{array}{l}25 \\
26 \\
27 \\
28\end{array}$ & $\begin{array}{l}3.74 \\
3.53 \\
1.30 \\
4.20\end{array}$ & $\begin{array}{l}35.52 \\
48.00 \\
55.06 \\
30.60\end{array}$ & $\begin{array}{r}79.75 \\
74.42 \\
105.17 \\
55.15\end{array}$ & $\begin{array}{r}11.47 \\
14.14 \\
5.93 \\
9.58\end{array}$ & $\begin{array}{l}3.12 \\
7.85 \\
3.17 \\
3.89\end{array}$ & $\begin{array}{l}11.64 \\
15.02 \\
23.52 \\
11.06\end{array}$ & $\begin{array}{r}16.10 \\
8.74 \\
29.21 \\
14.52\end{array}$ & $\begin{array}{l}6.86 \\
5.30 \\
4.27 \\
7.15\end{array}$ & $\begin{array}{r}.9 \\
.8 \\
.3 \\
1.0\end{array}$ & $\begin{array}{l}4.1 \\
5.2 \\
4.7 \\
3.8\end{array}$ & $\begin{array}{l}9.5 \\
8.4 \\
9.9 \\
8.2\end{array}$ & $\begin{array}{l}2.0 \\
2.4 \\
1.4 \\
2.5\end{array}$ & $\begin{array}{r}.8 \\
1.6 \\
.7 \\
1.2\end{array}$ & $\begin{array}{l}2.1 \\
2.5 \\
2.8 \\
2.4\end{array}$ & $\begin{array}{l}2.8 \\
1.8 \\
3.3 \\
3.0\end{array}$ & $\begin{array}{r}1.5 \\
1.3 \\
.8 \\
1.7\end{array}$ \\
\hline $\begin{array}{l}29 \\
30 \\
31\end{array}$ & $\begin{array}{r}.72 \\
2.16 \\
3.31\end{array}$ & $\begin{array}{l}39.94 \\
20.83 \\
10.66\end{array}$ & $\begin{array}{r}111.53 \\
29.54 \\
23.69\end{array}$ & $\begin{array}{r}15.89 \\
3.65 \\
4.75\end{array}$ & $\begin{array}{l}3.89 \\
3.41 \\
1.99\end{array}$ & $\begin{array}{l}10.49 \\
36.58 \\
26.71\end{array}$ & $\begin{array}{l}19.99 \\
62.71 \\
67.90\end{array}$ & $\begin{array}{r}5.26 \\
10.01 \\
13.30\end{array}$ & $\begin{array}{l}.2 \\
.5 \\
.9\end{array}$ & $\begin{array}{l}3.6 \\
2.3 \\
2.3\end{array}$ & $\begin{array}{r}11.3 \\
3.9 \\
5.2\end{array}$ & $\begin{array}{r}2.8 \\
.9 \\
1.3\end{array}$ & $\begin{array}{l}.8 \\
.9 \\
.7\end{array}$ & $\begin{array}{l}1.6 \\
5.6 \\
3.4\end{array}$ & $\begin{array}{l}2.7 \\
8.2 \\
7.7\end{array}$ & $\begin{array}{l}.9 \\
1.6 \\
2.4\end{array}$ \\
\hline
\end{tabular}


Table 3.--Wind-run and wind-curation histogran data, octobor 1987-Decambor 1989--Continued

\begin{tabular}{|c|c|c|c|c|c|c|c|c|c|c|c|c|c|c|c|c|}
\hline $\begin{array}{l}\text { Day } \\
\text { of } \\
\text { eonth }\end{array}$ & $\begin{array}{l}0 \\
t 0 \\
45\end{array}$ & $\begin{array}{c}\text { sectors } \\
45 \\
\text { to } \\
90\end{array}$ & $\begin{array}{r}\text { Wind } \\
\text { are in } \\
90 \\
\text { to } \\
135\end{array}$ & $\begin{array}{c}\begin{array}{c}\text { per } \\
\text { cated }\end{array} \\
135 \\
\text { to } \\
180\end{array}$ & $\begin{array}{c}\text { sector, } \\
\text { as degr } \\
180 \\
\text { to } \\
225\end{array}$ & $\begin{aligned} \text { in ail } \\
\frac{\text { ees } \mathrm{fx}}{225} \\
\text { to } \\
270\end{aligned}$ & $\begin{array}{l}\text { north } \\
270 \\
\text { to } \\
315\end{array}$ & $\begin{array}{r}315 \\
\text { to } \\
360\end{array}$ & $\begin{array}{l}0 \\
t 0 \\
45\end{array}$ & $\begin{array}{l}\text { ctore } \\
45 \\
\text { to } \\
90\end{array}$ & $\begin{array}{l}\text { ad du } \\
\text { re in } \\
90 \\
\text { to } \\
135\end{array}$ & $\begin{array}{l}\text { tion } \\
\text { cated } \\
135 \\
\text { to } \\
180\end{array}$ & $\begin{array}{c}18 \text { degt } \\
180 \\
\text { to } \\
225\end{array}$ & $\begin{array}{l}x \text {, in } \\
\text { es fre } \\
225 \\
\text { to } \\
270\end{array}$ & $\begin{array}{l}\text { Dours } \\
\text { nortb } \\
270 \\
\text { to } \\
315\end{array}$ & $\begin{array}{r}315 \\
\text { to } \\
360\end{array}$ \\
\hline $\begin{array}{l}1 \\
2 \\
3 \\
4\end{array}$ & $\begin{array}{l}5.45 \\
5.23 \\
2.47 \\
4.97\end{array}$ & $\begin{array}{r}2.30 \\
9.26 \\
67.03 \\
38.95\end{array}$ & $\begin{array}{r}6.07 \\
24.07 \\
89.95 \\
45.48\end{array}$ & $\begin{array}{l}1.56 \\
4.10 \\
6.60 \\
6.94\end{array}$ & $\begin{array}{l}3.36 \\
2.64 \\
2.69 \\
4.85\end{array}$ & $\begin{array}{r}32.74 \\
21.17 \\
9.89 \\
15.46\end{array}$ & $\begin{array}{l}53.1 \frac{\pi}{6} \\
29.04 \\
17.33 \\
20.06\end{array}$ & $\begin{array}{c}\mathrm{dx} 1988 \\
13.97 \\
9.77 \\
6.22 \\
9.34\end{array}$ & $\begin{array}{r}2.0 \\
1.6 \\
.5 \\
1.1\end{array}$ & $\begin{array}{l}1.2 \\
2.4 \\
5.6 \\
4.6\end{array}$ & $\begin{array}{l}2.7 \\
6.1 \\
9.2 \\
6.2\end{array}$ & $\begin{array}{l}0.9 \\
1.0 \\
1.3 \\
1.6\end{array}$ & $\begin{array}{r}1.1 \\
.7 \\
.7 \\
1.3\end{array}$ & $\begin{array}{l}4.9 \\
3.9 \\
2.1 \\
3.2\end{array}$ & $\begin{array}{l}7.5 \\
5.5 \\
3.0 \\
3.7\end{array}$ & $\begin{array}{l}3.5 \\
2.5 \\
1.2 \\
2.1\end{array}$ \\
\hline $\begin{array}{l}5 \\
6 \\
7 \\
8\end{array}$ & $\begin{array}{l}1.94 \\
2.71 \\
3.58 \\
3.67\end{array}$ & $\begin{array}{r}66.17 \\
50.57 \\
16.46 \\
9.38\end{array}$ & $\begin{array}{r}133.01 \\
60.58 \\
40.92 \\
37.18\end{array}$ & $\begin{array}{l}13.85 \\
10.32 \\
19.03 \\
11.71\end{array}$ & $\begin{array}{l}3.41 \\
6.36 \\
6.89 \\
4.94\end{array}$ & $\begin{array}{r}9.89 \\
23.47 \\
21.65 \\
21.10\end{array}$ & $\begin{array}{r}4.97 \\
16.10 \\
11.57 \\
40.61\end{array}$ & $\begin{array}{l}1.99 \\
3.58 \\
4.87 \\
7.73\end{array}$ & $\begin{array}{l}.4 \\
.7 \\
1.0 \\
1.1\end{array}$ & $\begin{array}{l}5.4 \\
5.0 \\
3.4 \\
2.3\end{array}$ & $\begin{array}{r}12.3 \\
7.6 \\
7.6 \\
6.7\end{array}$ & $\begin{array}{l}2.1 \\
2.0 \\
3.4 \\
2.3\end{array}$ & $\begin{array}{l}.7 \\
1.5 \\
1.6 \\
1.3\end{array}$ & $\begin{array}{l}1.5 \\
3.8 \\
3.5 \\
3.1\end{array}$ & $\begin{array}{l}1.0 \\
2.5 \\
2.0 \\
5.3\end{array}$ & $\begin{array}{r}.5 \\
.8 \\
1.3 \\
1.7\end{array}$ \\
\hline $\begin{array}{r}9 \\
10 \\
11 \\
12\end{array}$ & $\begin{array}{l}1.68 \\
1.37 \\
3.53 \\
6.78\end{array}$ & $\begin{array}{l}50.18 \\
50.95 \\
48.29 \\
59.30\end{array}$ & $\begin{array}{r}90.36 \\
121.15 \\
110.42 \\
122.76\end{array}$ & $\begin{array}{r}7.70 \\
11.74 \\
11.52 \\
8.02\end{array}$ & $\begin{array}{r}3.84 \\
15.70 \\
2.64 \\
7.68\end{array}$ & $\begin{array}{r}24.07 \\
52.75 \\
3.77 \\
7.49\end{array}$ & $\begin{array}{r}33.26 \\
22.56 \\
5.06 \\
4.44\end{array}$ & $\begin{array}{l}5.26 \\
1.30 \\
4.15 \\
1.10\end{array}$ & $\begin{array}{r}.4 \\
.2 \\
.8 \\
1.0\end{array}$ & $\begin{array}{l}4.5 \\
3.5 \\
4.9 \\
5.8\end{array}$ & $\begin{array}{r}8.9 \\
8.4 \\
11.7 \\
11.3\end{array}$ & $\begin{array}{l}1.4 \\
1.4 \\
2.2 \\
1.8\end{array}$ & $\begin{array}{r}.8 \\
1.8 \\
.7 \\
1.5\end{array}$ & $\begin{array}{l}3.0 \\
5.7 \\
1.1 \\
1.3\end{array}$ & $\begin{array}{r}3.9 \\
2.5 \\
1.3 \\
.9\end{array}$ & $\begin{array}{r}1.0 \\
.3 \\
1.1 \\
.3\end{array}$ \\
\hline $\begin{array}{l}17 \\
18 \\
19 \\
20\end{array}$ & $\begin{array}{l}4.49 \\
2.81 \\
3.17 \\
3.84\end{array}$ & $\begin{array}{l}55.01 \\
13.01 \\
37.51 \\
33.34\end{array}$ & $\begin{array}{r}101.11 \\
31.58 \\
73.82 \\
83.50\end{array}$ & $\begin{array}{l}14.47 \\
23.88 \\
11.33 \\
11.76\end{array}$ & $\begin{array}{l}4.85 \\
7.22 \\
7.36 \\
7.92\end{array}$ & $\begin{array}{r}12.74 \\
14.38 \\
8.47 \\
12.07\end{array}$ & $\begin{array}{l}9.34 \\
8.83 \\
4.30 \\
8.88\end{array}$ & $\begin{array}{l}2.71 \\
5.62 \\
6.99 \\
2.62\end{array}$ & $\begin{array}{r}.6 \\
.9 \\
.9 \\
1.0\end{array}$ & $\begin{array}{l}5.2 \\
2.5 \\
4.8 \\
4.0\end{array}$ & $\begin{array}{r}10.6 \\
5.7 \\
9.0 \\
9.6\end{array}$ & $\begin{array}{l}2.2 \\
4.7 \\
2.4 \\
2.5\end{array}$ & $\begin{array}{l}.9 \\
2.2 \\
1.8 \\
1.7\end{array}$ & $\begin{array}{l}2.1 \\
3.6 \\
2.0 \\
2.3\end{array}$ & $\begin{array}{l}1.6 \\
2.5 \\
1.3 \\
2.0\end{array}$ & $\begin{array}{r}.7 \\
1.7 \\
1.6 \\
.7\end{array}$ \\
\hline $\begin{array}{l}21 \\
22 \\
23 \\
24\end{array}$ & $\begin{array}{l}4.80 \\
2.88 \\
5.38 \\
4.90\end{array}$ & $\begin{array}{l}51.72 \\
69.12 \\
38.14 \\
46.37\end{array}$ & $\begin{array}{r}81.34 \\
116.81 \\
47.86 \\
75.00\end{array}$ & $\begin{array}{l}7.08 \\
2.52 \\
6.22 \\
3.86\end{array}$ & $\begin{array}{l}2.42 \\
1.87 \\
3.22 \\
2.76\end{array}$ & $\begin{array}{r}10.27 \\
7.39 \\
9.60 \\
10.49\end{array}$ & $\begin{array}{r}13.08 \\
6.22 \\
9.07 \\
11.26\end{array}$ & $\begin{array}{l}5.81 \\
2.35 \\
6.65 \\
6.07\end{array}$ & $\begin{array}{r}1.0 \\
.8 \\
1.4 \\
1.3\end{array}$ & $\begin{array}{l}5.8 \\
7.1 \\
5.8 \\
5.7\end{array}$ & $\begin{array}{r}9.7 \\
11.2 \\
7.7 \\
8.4\end{array}$ & $\begin{array}{r}1.4 \\
.7 \\
1.7 \\
1.1\end{array}$ & $\begin{array}{r}.7 \\
.6 \\
1.0 \\
.9\end{array}$ & $\begin{array}{l}1.7 \\
1.5 \\
2.2 \\
2.4\end{array}$ & $\begin{array}{l}2.1 \\
1.4 \\
2.2 \\
2.4\end{array}$ & $\begin{array}{r}1.3 \\
.7 \\
1.8 \\
1.6\end{array}$ \\
\hline $\begin{array}{l}25 \\
26 \\
27 \\
28\end{array}$ & $\begin{array}{l}.41 \\
1.70 \\
4.03 \\
2.62\end{array}$ & $\begin{array}{r}105.74 \\
49.39 \\
35.28 \\
11.50\end{array}$ & $\begin{array}{r}151.27 \\
97.27 \\
76.08 \\
31.42\end{array}$ & $\begin{array}{r}4.82 \\
9.48 \\
11.76 \\
8.71\end{array}$ & $\begin{array}{r}.34 \\
1.75 \\
11.09 \\
14.30\end{array}$ & $\begin{array}{r}.10 \\
6.00 \\
10.63 \\
21.12\end{array}$ & $\begin{array}{r}.19 \\
7.20 \\
7.18 \\
10.10\end{array}$ & $\begin{array}{l}.19 \\
2.06 \\
3.36 \\
2.52\end{array}$ & $\begin{array}{l}.1 \\
.4 \\
1.0 \\
1.0\end{array}$ & $\begin{array}{l}8.8 \\
5.7 \\
4.4 \\
2.1\end{array}$ & $\begin{array}{r}14.2 \\
12.8 \\
9.0 \\
5.4\end{array}$ & $\begin{array}{l}.6 \\
1.7 \\
2.4 \\
2.2\end{array}$ & $\begin{array}{r}.0 \\
.4 \\
2.2 \\
3.6\end{array}$ & $\begin{array}{r}.0 \\
1.1 \\
2.1 \\
5.0\end{array}$ & $\begin{array}{r}.1 \\
1.3 \\
1.7 \\
3.3\end{array}$ & $\begin{array}{r}.0 \\
.5 \\
1.1 \\
1.3\end{array}$ \\
\hline
\end{tabular}


Table 3.--wind-run and wind-curation histogran data, october 1987-Docamber 1989--Continued

\begin{tabular}{|c|c|c|c|c|c|c|c|c|c|c|c|c|c|c|c|c|}
\hline $\begin{array}{l}\text { Day } \\
\text { of } \\
\text { eonth }\end{array}$ & $\begin{array}{l}0 \\
0 \\
\text { to } \\
45\end{array}$ & $\begin{array}{c}\text { ector: } \\
45 \\
\text { to } \\
90\end{array}$ & $\begin{array}{l}\begin{array}{c}\text { Wind ru } \\
\text { are ind }\end{array} \\
90 \\
\text { to } \\
135\end{array}$ & $\begin{array}{c}\begin{array}{c}\text { a per } \\
\text { icated }\end{array} \\
135 \\
\text { to } \\
180\end{array}$ & $\begin{array}{r}\text { ector, } \\
\text { as des } \\
180 \\
\text { to } \\
225\end{array}$ & $\begin{array}{l}n \text { ail } \\
\text { es fx } \\
225 \\
\text { to } \\
270\end{array}$ & $\begin{array}{c}\text { north } \\
270 \\
\text { to } \\
315\end{array}$ & $\begin{array}{r}315 \\
\text { to } \\
360\end{array}$ & $\begin{array}{l}0 \\
t 0 \\
45\end{array}$ & $\begin{array}{l}\text { tors } \\
45 \\
\text { to } \\
90\end{array}$ & $\begin{array}{l}\text { ind du } \\
\text { re in } \\
90 \\
\text { to } \\
135\end{array}$ & $\begin{array}{l}\text { tion } \\
\text { cated } \\
135 \\
\text { to } \\
180\end{array}$ & $\begin{array}{r}\text { sec } \\
\text { deg } \\
180 \\
\text { to } \\
225\end{array}$ & $\begin{array}{r}\frac{r, \text { in }}{\text { es fx }} \\
225 \\
\text { to } \\
270\end{array}$ & $\begin{array}{l}\text { ours } \\
\text { nort } \\
270 \\
\text { to } \\
315\end{array}$ & $\begin{array}{r}315 \\
\text { to } \\
360\end{array}$ \\
\hline \multicolumn{17}{|c|}{ JUII 1988} \\
\hline $\begin{array}{l}1 \\
2 \\
3 \\
4\end{array}$ & $\begin{array}{r}19.10 \\
5.30 \\
6.91 \\
9.46\end{array}$ & $\begin{array}{l}40.61 \\
35.23 \\
50.81 \\
20.81\end{array}$ & $\begin{array}{l}21.24 \\
79.68 \\
77.83 \\
41.83\end{array}$ & $\begin{array}{r}14.16 \\
19.06 \\
5.93 \\
12.31\end{array}$ & $\begin{array}{l}8.28 \\
9.55 \\
2.95 \\
3.98\end{array}$ & $\begin{array}{r}2.11 \\
6.46 \\
10.18 \\
8.81\end{array}$ & $\begin{array}{l}0.67 \\
3.86 \\
9.46 \\
9.05\end{array}$ & $\begin{array}{l}0.70 \\
2.18 \\
5.52 \\
7.61\end{array}$ & $\begin{array}{l}3.8 \\
1.4 \\
1.4 \\
2.3\end{array}$ & $\begin{array}{l}7.8 \\
4.3 \\
6.1 \\
3.7\end{array}$ & $\begin{array}{l}5.4 \\
9.5 \\
8.8 \\
7.3\end{array}$ & $\begin{array}{l}3.8 \\
3.3 \\
1.2 \\
2.8\end{array}$ & $\begin{array}{r}2.1 \\
2.3 \\
.7 \\
1.3\end{array}$ & $\begin{array}{l}0.6 \\
1.5 \\
2.0 \\
2.1\end{array}$ & $\begin{array}{l}0.2 \\
1.0 \\
2.0 \\
2.2\end{array}$ & $\begin{array}{r}0.2 \\
.6 \\
1.5 \\
2.2\end{array}$ \\
\hline $\begin{array}{l}5 \\
6 \\
7 \\
8\end{array}$ & $\begin{array}{l}8.04 \\
3.60 \\
3.24 \\
3.98\end{array}$ & $\begin{array}{l}70.18 \\
31.85 \\
14.54 \\
17.45\end{array}$ & $\begin{array}{r}104.54 \\
55.56 \\
45.24 \\
38.14\end{array}$ & $\begin{array}{r}23.18 \\
24.72 \\
8.52 \\
8.57\end{array}$ & $\begin{array}{r}5.04 \\
3.86 \\
14.74 \\
4.15\end{array}$ & $\begin{array}{r}2.81 \\
8.14 \\
28.63 \\
25.85\end{array}$ & $\begin{array}{r}2.62 \\
8.26 \\
12.24 \\
36.46\end{array}$ & $\begin{array}{l}.65 \\
3.00 \\
4.66 \\
9.46\end{array}$ & $\begin{array}{r}1.1 \\
.9 \\
1.0 \\
1.0\end{array}$ & $\begin{array}{l}7.0 \\
4.8 \\
2.5 \\
2.8\end{array}$ & $\begin{array}{r}10.5 \\
8.3 \\
7.7 \\
6.1\end{array}$ & $\begin{array}{l}3.4 \\
4.3 \\
2.1 \\
1.7\end{array}$ & $\begin{array}{l}1.0 \\
1.0 \\
2.3 \\
1.2\end{array}$ & $\begin{array}{r}.4 \\
1.9 \\
4.4 \\
3.9\end{array}$ & $\begin{array}{r}.3 \\
1.9 \\
2.5 \\
5.1\end{array}$ & $\begin{array}{l}.1 \\
.8 \\
1.3 \\
2.0\end{array}$ \\
\hline $\begin{array}{r}9 \\
10 \\
11 \\
12\end{array}$ & $\begin{array}{l}5.35 \\
1.92 \\
3.55 \\
3.17\end{array}$ & $\begin{array}{r}45.98 \\
95.38 \\
39.53 \\
7.70\end{array}$ & $\begin{array}{r}45.67 \\
126.98 \\
61.92 \\
31.08\end{array}$ & $\begin{array}{r}6.79 \\
13.22 \\
10.15 \\
18.22\end{array}$ & $\begin{array}{r}3.41 \\
3.24 \\
17.78 \\
13.44\end{array}$ & $\begin{array}{r}17.42 \\
4.27 \\
24.46 \\
52.70\end{array}$ & $\begin{array}{r}17.71 \\
2.90 \\
11.78 \\
16.99\end{array}$ & $\begin{array}{r}5.40 \\
.91 \\
4.63 \\
3.84\end{array}$ & $\begin{array}{r}1.1 \\
.3 \\
.8 \\
.9\end{array}$ & $\begin{array}{l}5.3 \\
7.6 \\
4.5 \\
1.7\end{array}$ & $\begin{array}{r}6.6 \\
11.8 \\
7.1 \\
5.6\end{array}$ & $\begin{array}{l}1.6 \\
2.0 \\
1.9 \\
3.1\end{array}$ & $\begin{array}{r}1.2 \\
.6 \\
2.7 \\
2.3\end{array}$ & $\begin{array}{r}3.6 \\
.8 \\
3.5 \\
6.8\end{array}$ & $\begin{array}{r}3.3 \\
.5 \\
2.0 \\
2.4\end{array}$ & $\begin{array}{r}1.3 \\
.2 \\
1.2 \\
1.0\end{array}$ \\
\hline $\begin{array}{l}17 \\
18 \\
19 \\
20\end{array}$ & $\begin{array}{l}3.36 \\
3.98 \\
4.87 \\
4.97\end{array}$ & $\begin{array}{r}7.75 \\
13.18 \\
13.51 \\
24.67\end{array}$ & $\begin{array}{l}43.20 \\
50.38 \\
39.07 \\
58.85\end{array}$ & $\begin{array}{r}10.75 \\
6.55 \\
3.31 \\
7.20\end{array}$ & $\begin{array}{r}12.91 \\
1.39 \\
1.87 \\
2.95\end{array}$ & $\begin{array}{l}35.42 \\
21.12 \\
28.08 \\
16.70\end{array}$ & $\begin{array}{l}29.45 \\
32.95 \\
35.81 \\
16.22\end{array}$ & $\begin{array}{l}8.42 \\
9.17 \\
7.42 \\
6.29\end{array}$ & $\begin{array}{l}.9 \\
1.1 \\
1.2 \\
1.2\end{array}$ & $\begin{array}{l}1.8 \\
3.1 \\
3.0 \\
4.1\end{array}$ & $\begin{array}{l}7.1 \\
8.9 \\
8.1 \\
8.9\end{array}$ & $\begin{array}{r}1.7 \\
1.2 \\
.9 \\
1.8\end{array}$ & $\begin{array}{r}1.9 \\
.5 \\
.5 \\
.9\end{array}$ & $\begin{array}{l}4.7 \\
2.9 \\
3.8 \\
3.1\end{array}$ & $\begin{array}{l}4.0 \\
4.4 \\
4.6 \\
2.6\end{array}$ & $\begin{array}{l}1.8 \\
1.8 \\
1.6 \\
1.3\end{array}$ \\
\hline $\begin{array}{l}21 \\
22 \\
23 \\
24\end{array}$ & $\begin{array}{l}4.70 \\
2.78 \\
5.16 \\
5.81\end{array}$ & $\begin{array}{l}14.76 \\
13.63 \\
14.23 \\
12.19\end{array}$ & $\begin{array}{l}25.78 \\
55.08 \\
47.71 \\
54.07\end{array}$ & $\begin{array}{r}9.89 \\
19.56 \\
21.62 \\
15.89\end{array}$ & $\begin{array}{r}13.13 \\
4.87 \\
3.96 \\
7.94\end{array}$ & $\begin{array}{l}35.28 \\
20.04 \\
15.98 \\
10.63\end{array}$ & $\begin{array}{r}19.75 \\
17.83 \\
17.98 \\
7.78\end{array}$ & $\begin{array}{l}6.43 \\
6.86 \\
5.14 \\
3.89\end{array}$ & $\begin{array}{r}1.3 \\
.7 \\
1.2 \\
1.5\end{array}$ & $\begin{array}{l}3.3 \\
2.7 \\
2.9 \\
3.0\end{array}$ & $\begin{array}{l}5.0 \\
8.7 \\
8.3 \\
9.3\end{array}$ & $\begin{array}{l}2.1 \\
2.9 \\
3.5 \\
3.1\end{array}$ & $\begin{array}{l}2.2 \\
1.2 \\
1.0 \\
1.6\end{array}$ & $\begin{array}{l}4.9 \\
3.4 \\
2.9 \\
2.3\end{array}$ & $\begin{array}{l}3.4 \\
2.9 \\
2.9 \\
1.8\end{array}$ & $\begin{array}{l}1.7 \\
1.4 \\
1.1 \\
1.1\end{array}$ \\
\hline
\end{tabular}


Table 3.--Wind-rm and wind-curation histogren data, Octobor 1987-Decamber 1989--Continued

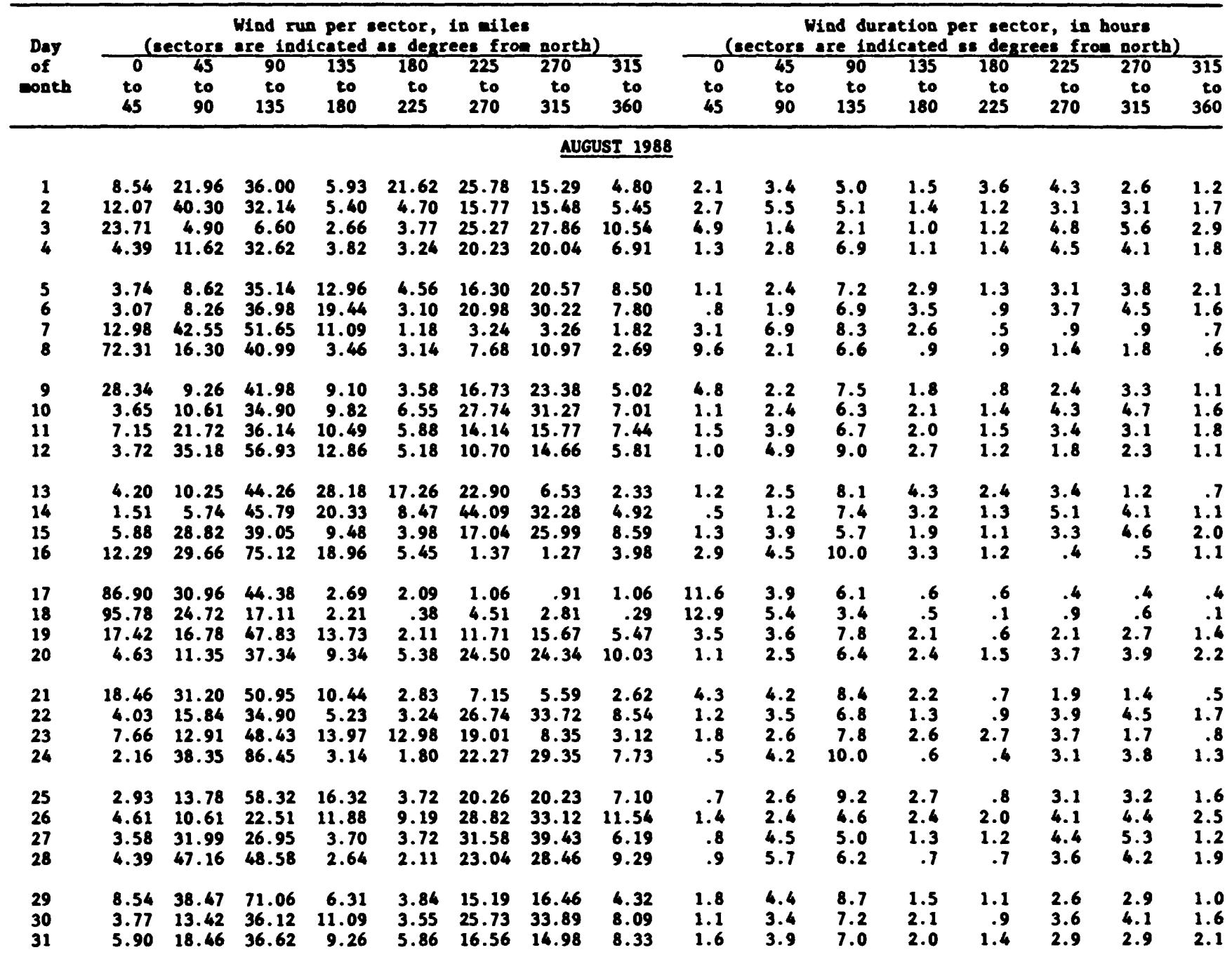


Table 3.--Wind-rum and wind-quration histogran data, October 1987-Decumbr 1989--Continued

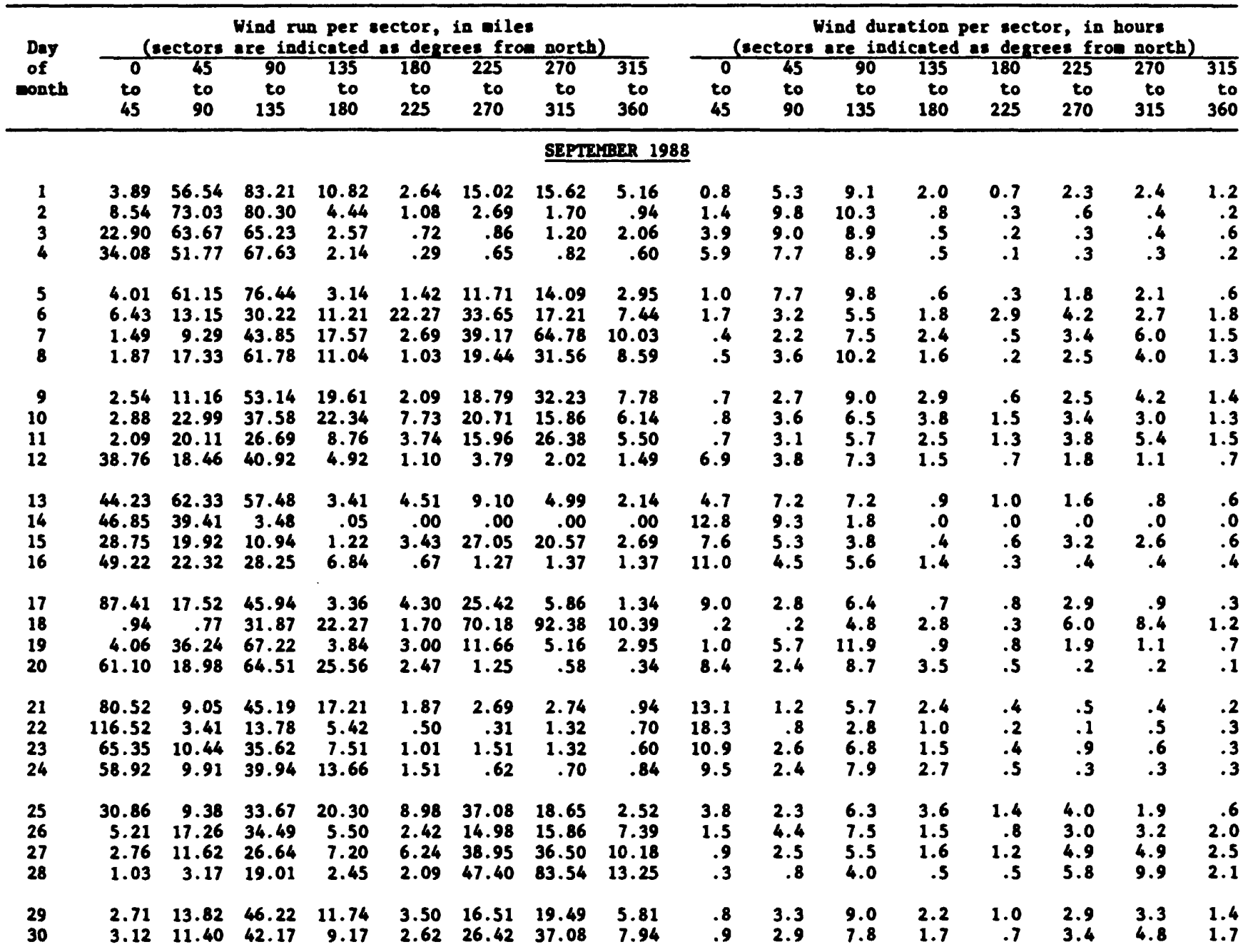


Table 3.--wind-run and wind-duretion histogren data, october 1987-Dece-ber 1989--Continued

\begin{tabular}{|c|c|c|c|c|c|c|c|c|c|c|c|c|c|c|c|c|}
\hline \multirow{2}{*}{$\begin{array}{l}\text { Dey } \\
\text { of } \\
\text { eonth }\end{array}$} & \multicolumn{8}{|c|}{$\begin{array}{l}\text { Wind run per sector, in ailes } \\
\text { sectors ere indiceted as degrees froe north) }\end{array}$} & \multicolumn{8}{|c|}{$\begin{array}{l}\text { Wind duration per sector, in hours } \\
\text { tors are indicsted as degrees from north) }\end{array}$} \\
\hline & $\begin{array}{r}0 \\
20 \\
45\end{array}$ & $\begin{array}{l}45 \\
t 0 \\
90\end{array}$ & $\begin{array}{r}90 \\
t 0 \\
135\end{array}$ & $\begin{array}{r}135 \\
t 0 \\
180\end{array}$ & $\begin{array}{r}180 \\
t 0 \\
225\end{array}$ & $\begin{array}{r}225 \\
t 0 \\
270\end{array}$ & $\begin{array}{r}270 \\
t 0 \\
315\end{array}$ & $\begin{array}{r}315 \\
t 0 \\
360\end{array}$ & $\begin{array}{r}0 \\
t 0 \\
45\end{array}$ & $\begin{array}{l}45 \\
t 0 \\
90\end{array}$ & $\begin{array}{r}90 \\
\text { to } \\
135\end{array}$ & $\begin{array}{r}135 \\
20 \\
180\end{array}$ & $\begin{array}{r}180 \\
t 0 \\
225\end{array}$ & $\begin{array}{r}225 \\
t 0 \\
270\end{array}$ & $\begin{array}{r}270 \\
t 0 \\
315\end{array}$ & $\begin{array}{r}315 \\
t 0 \\
360\end{array}$ \\
\hline \multicolumn{17}{|c|}{ OCTOBBR 1988} \\
\hline $\begin{array}{l}1 \\
2 \\
3 \\
4\end{array}$ & $\begin{array}{r}15.02 \\
10.94 \\
10.49 \\
6.19\end{array}$ & $\begin{array}{l}14.81 \\
13.10 \\
13.66 \\
33.46\end{array}$ & $\begin{array}{l}55.10 \\
34.03 \\
34.97 \\
44.83\end{array}$ & $\begin{array}{r}22.54 \\
6.98 \\
9.65 \\
14.47\end{array}$ & $\begin{array}{l}5.64 \\
7.03 \\
5.40 \\
8.30\end{array}$ & $\begin{array}{l}11.30 \\
20.26 \\
18.29 \\
16.34\end{array}$ & $\begin{array}{r}7.32 \\
22.25 \\
24.86 \\
12.82\end{array}$ & $\begin{array}{l}1.56 \\
7.73 \\
8.78 \\
7.68\end{array}$ & $\begin{array}{l}2.4 \\
2.0 \\
2.0 \\
1.6\end{array}$ & $\begin{array}{l}3.1 \\
2.8 \\
3.0 \\
4.8\end{array}$ & $\begin{array}{l}9.8 \\
6.2 \\
6.5 \\
6.4\end{array}$ & $\begin{array}{l}3.8 \\
1.8 \\
2.1 \\
2.3\end{array}$ & $\begin{array}{l}1.2 \\
1.7 \\
1.2 \\
1.5\end{array}$ & $\begin{array}{l}1.9 \\
3.5 \\
2.9 \\
2.5\end{array}$ & $\begin{array}{l}1.4 \\
4.0 \\
3.9 \\
2.7\end{array}$ & $\begin{array}{l}0.4 \\
1.8 \\
2.1 \\
2.0\end{array}$ \\
\hline $\begin{array}{l}5 \\
6 \\
7 \\
8\end{array}$ & $\begin{array}{r}6.91 \\
39.00 \\
31.61 \\
40.97\end{array}$ & $\begin{array}{l}45.70 \\
16.51 \\
11.88 \\
12.67\end{array}$ & $\begin{array}{l}66.53 \\
37.13 \\
42.89 \\
41.90\end{array}$ & $\begin{array}{r}20.83 \\
7.66 \\
9.17 \\
11.06\end{array}$ & $\begin{array}{l}7.18 \\
2.59 \\
2.54 \\
2.23\end{array}$ & $\begin{array}{l}6.10 \\
4.03 \\
7.94 \\
1.70\end{array}$ & $\begin{array}{l}3.02 \\
3.17 \\
4.03 \\
2.09\end{array}$ & $\begin{array}{l}2.57 \\
3.16 \\
3.26 \\
3.50\end{array}$ & $\begin{array}{l}1.8 \\
7.1 \\
5.8 \\
5.8\end{array}$ & $\begin{array}{l}5.7 \\
3.3 \\
2.8 \\
3.6\end{array}$ & $\begin{array}{l}8.5 \\
7.3 \\
8.6 \\
8.9\end{array}$ & $\begin{array}{l}3.6 \\
1.8 \\
1.9 \\
2.3\end{array}$ & $\begin{array}{r}1.4 \\
.9 \\
.8 \\
.7\end{array}$ & $\begin{array}{r}1.2 \\
1.3 \\
1.9 \\
.6\end{array}$ & $\begin{array}{r}.8 \\
1.2 \\
1.1 \\
.9\end{array}$ & $\begin{array}{r}.8 \\
1.1 \\
1.0 \\
1.2\end{array}$ \\
\hline $\begin{array}{r}9 \\
10 \\
11 \\
12\end{array}$ & $\begin{array}{l}19.49 \\
48.70 \\
43.51 \\
36.62\end{array}$ & $\begin{array}{l}19.49 \\
28.39 \\
11.88 \\
16.75\end{array}$ & $\begin{array}{l}75.43 \\
70.68 \\
32.59 \\
27.74\end{array}$ & $\begin{array}{r}16.34 \\
2.95 \\
7.01 \\
6.17\end{array}$ & $\begin{array}{r}1.61 \\
.74 \\
3.79 \\
3.58\end{array}$ & $\begin{array}{l}2.40 \\
1.46 \\
2.95 \\
8.98\end{array}$ & $\begin{array}{l}2.83 \\
1.51 \\
3.22 \\
8.11\end{array}$ & $\begin{array}{l}2.86 \\
1.70 \\
3.41 \\
4.90\end{array}$ & $\begin{array}{l}4.2 \\
8.8 \\
7.8 \\
6.4\end{array}$ & $\begin{array}{l}3.0 \\
3.5 \\
3.2 \\
3.4\end{array}$ & $\begin{array}{r}11.0 \\
9.8 \\
7.0 \\
5.4\end{array}$ & $\begin{array}{r}2.9 \\
.6 \\
1.7 \\
1.6\end{array}$ & $\begin{array}{r}.5 \\
.2 \\
1.0 \\
1.1\end{array}$ & $\begin{array}{r}.6 \\
.3 \\
.9 \\
2.5\end{array}$ & $\begin{array}{r}.8 \\
.3 \\
1.1 \\
2.0\end{array}$ & $\begin{array}{r}.9 \\
.4 \\
1.2 \\
1.5\end{array}$ \\
\hline $\begin{array}{l}13 \\
14 \\
15 \\
16\end{array}$ & $\begin{array}{r}50.33 \\
25.66 \\
57.46 \\
1.30\end{array}$ & $\begin{array}{l}12.12 \\
14.90 \\
14.50 \\
10.15\end{array}$ & $\begin{array}{l}36.10 \\
37.37 \\
40.66 \\
53.35\end{array}$ & $\begin{array}{l}2.57 \\
9.84 \\
4.63 \\
7.01\end{array}$ & $\begin{array}{l}1.34 \\
5.09 \\
1.73 \\
1.13\end{array}$ & $\begin{array}{r}7.68 \\
10.97 \\
16.15 \\
29.26\end{array}$ & $\begin{array}{r}7.15 \\
3.53 \\
20.71 \\
52.92\end{array}$ & $\begin{array}{r}2.52 \\
.84 \\
2.64 \\
8.40\end{array}$ & $\begin{array}{r}7.8 \\
4.6 \\
5.9 \\
.4\end{array}$ & $\begin{array}{l}3.2 \\
4.1 \\
3.3 \\
2.6\end{array}$ & $\begin{array}{r}7.6 \\
8.1 \\
9.2 \\
10.2\end{array}$ & $\begin{array}{r}.7 \\
2.1 \\
1.0 \\
1.2\end{array}$ & $\begin{array}{r}.4 \\
1.2 \\
.3 \\
.4\end{array}$ & $\begin{array}{l}1.8 \\
2.2 \\
1.5 \\
3.0\end{array}$ & $\begin{array}{l}1.5 \\
1.1 \\
2.2 \\
5.0\end{array}$ & $\begin{array}{r}.8 \\
.4 \\
.5 \\
1.1\end{array}$ \\
\hline $\begin{array}{l}17 \\
18 \\
19 \\
20\end{array}$ & $\begin{array}{l}.74 \\
2.57 \\
2.09 \\
2.71\end{array}$ & $\begin{array}{r}6.89 \\
7.92 \\
20.09 \\
16.27\end{array}$ & $\begin{array}{l}35.95 \\
34.87 \\
66.43 \\
59.71\end{array}$ & $\begin{array}{r}.91 \\
.60 \\
18.55 \\
22.10\end{array}$ & $\begin{array}{l}2.38 \\
1.06 \\
2.11 \\
1.13\end{array}$ & $\begin{array}{r}39.12 \\
27.72 \\
6.24 \\
10.85\end{array}$ & $\begin{array}{r}77.57 \\
46.03 \\
7.73 \\
16.10\end{array}$ & $\begin{array}{r}8.14 \\
10.10 \\
2.83 \\
5.54\end{array}$ & $\begin{array}{l}.3 \\
.7 \\
.6 \\
.8\end{array}$ & $\begin{array}{l}1.8 \\
2.3 \\
4.1 \\
4.2\end{array}$ & $\begin{array}{r}6.7 \\
8.8 \\
12.0 \\
10.4\end{array}$ & $\begin{array}{r}.2 \\
.4 \\
3.4 \\
3.1\end{array}$ & $\begin{array}{l}.6 \\
.4 \\
.6 \\
.4\end{array}$ & $\begin{array}{l}5.2 \\
3.9 \\
1.2 \\
1.5\end{array}$ & $\begin{array}{l}8.0 \\
5.5 \\
1.3 \\
2.4\end{array}$ & $\begin{array}{r}1.1 \\
1.9 \\
.6 \\
1.1\end{array}$ \\
\hline $\begin{array}{l}21 \\
22 \\
23 \\
24\end{array}$ & $\begin{array}{l}49.03 \\
61.94 \\
31.58 \\
21.41\end{array}$ & $\begin{array}{r}14.26 \\
9.65 \\
10.99 \\
17.21\end{array}$ & $\begin{array}{l}68.11 \\
43.15 \\
57.26 \\
69.60\end{array}$ & $\begin{array}{r}18.41 \\
18.48 \\
6.62 \\
11.16\end{array}$ & $\begin{array}{r}.31 \\
.50 \\
1.46 \\
.82\end{array}$ & $\begin{array}{r}.38 \\
5.78 \\
11.90 \\
11.74\end{array}$ & $\begin{array}{r}.53 \\
17.35 \\
15.14 \\
20.83\end{array}$ & $\begin{array}{r}.12 \\
6.50 \\
5.02 \\
2.28\end{array}$ & $\begin{array}{l}8.2 \\
7.4 \\
4.0 \\
3.0\end{array}$ & $\begin{array}{l}2.8 \\
2.3 \\
2.4 \\
3.6\end{array}$ & $\begin{array}{r}10.2 \\
6.8 \\
10.5 \\
11.6\end{array}$ & $\begin{array}{l}2.4 \\
2.1 \\
1.3 \\
1.6\end{array}$ & $\begin{array}{l}.1 \\
.2 \\
.4 \\
.1\end{array}$ & $\begin{array}{r}.1 \\
.9 \\
1.8 \\
1.3\end{array}$ & $\begin{array}{l}.2 \\
2.7 \\
2.2 \\
2.3\end{array}$ & $\begin{array}{r}.0 \\
1.5 \\
1.2 \\
.4\end{array}$ \\
\hline $\begin{array}{l}25 \\
26 \\
27 \\
28\end{array}$ & $\begin{array}{r}22.51 \\
35.47 \\
2.62 \\
2.16\end{array}$ & $\begin{array}{r}12.46 \\
8.09 \\
11.42 \\
8.26\end{array}$ & $\begin{array}{l}66.10 \\
47.11 \\
55.15 \\
43.49\end{array}$ & $\begin{array}{r}26.47 \\
19.68 \\
2.33 \\
25.66\end{array}$ & $\begin{array}{l}2.14 \\
6.14 \\
1.37 \\
7.18\end{array}$ & $\begin{array}{l}13.99 \\
34.06 \\
25.08 \\
31.90\end{array}$ & $\begin{array}{l}15.14 \\
19.78 \\
45.22 \\
28.20\end{array}$ & $\begin{array}{l}2.83 \\
8.76 \\
9.74 \\
5.71\end{array}$ & $\begin{array}{r}3.0 \\
3.5 \\
.6 \\
.7\end{array}$ & $\begin{array}{l}2.7 \\
1.6 \\
2.7 \\
2.0\end{array}$ & $\begin{array}{r}10.3 \\
6.9 \\
9.9 \\
7.3\end{array}$ & $\begin{array}{r}3.2 \\
2.7 \\
.6 \\
3.6\end{array}$ & $\begin{array}{r}.4 \\
1.1 \\
.4 \\
1.4\end{array}$ & $\begin{array}{l}1.7 \\
4.3 \\
3.2 \\
4.3\end{array}$ & $\begin{array}{l}1.9 \\
2.5 \\
4.8 \\
3.2\end{array}$ & $\begin{array}{r}.6 \\
1.6 \\
1.6 \\
1.2\end{array}$ \\
\hline $\begin{array}{l}29 \\
30 \\
31\end{array}$ & $\begin{array}{r}3.48 \\
45.58 \\
23.50\end{array}$ & $\begin{array}{l}28.03 \\
12.43 \\
20.50\end{array}$ & $\begin{array}{l}65.88 \\
33.41 \\
72.60\end{array}$ & $\begin{array}{r}16.25 \\
9.72 \\
10.13\end{array}$ & $\begin{array}{r}3.65 \\
2.35 \\
.58\end{array}$ & $\begin{array}{r}5.42 \\
12.70 \\
.82\end{array}$ & $\begin{array}{r}8.09 \\
14.95 \\
1.30\end{array}$ & $\begin{array}{l}6.22 \\
4.32 \\
1.03\end{array}$ & $\begin{array}{r}.8 \\
6.2 \\
4.9\end{array}$ & $\begin{array}{l}4.4 \\
3.6 \\
4.1\end{array}$ & $\begin{array}{r}10.8 \\
6.4 \\
12.2\end{array}$ & $\begin{array}{l}2.8 \\
1.5 \\
1.5\end{array}$ & $\begin{array}{l}.7 \\
.6 \\
.2\end{array}$ & $\begin{array}{r}1.3 \\
2.2 \\
.2\end{array}$ & $\begin{array}{r}1.7 \\
2.4 \\
.4\end{array}$ & $\begin{array}{r}1.3 \\
1.1 \\
.3\end{array}$ \\
\hline
\end{tabular}


Table 3.--Wind-run and wind-curation histogran data, octobor 1987-Doce-ber 1989--Continued

\begin{tabular}{|c|c|c|c|c|c|c|c|c|c|c|c|c|c|c|c|c|}
\hline $\begin{array}{l}\text { Day } \\
\text { of } \\
\text { conth }\end{array}$ & $\begin{array}{r}0 \\
\text { to } \\
45\end{array}$ & $\begin{array}{l}45 \\
\text { to } \\
90\end{array}$ & $\begin{array}{l}\text { Wind } \\
\text { ore in } \\
90 \\
\text { to } \\
135\end{array}$ & $\begin{array}{l}\text { o per } \\
\text { icated } \\
135 \\
\text { to } \\
180\end{array}$ & $\begin{array}{l}\text { ctor, } \\
\text { s des } \\
180 \\
\text { to } \\
225\end{array}$ & $\begin{array}{l}\text { in aile } \\
\text { ees fro } \\
225 \\
\text { to } \\
270\end{array}$ & $\begin{array}{l}\text { north } \\
270 \\
\text { to } \\
315\end{array}$ & $\begin{array}{r}315 \\
\text { to } \\
360\end{array}$ & $\begin{array}{l}0 \\
\text { to } \\
45\end{array}$ & $\begin{array}{l}\text { ctors } \\
45 \\
\text { to } \\
90\end{array}$ & $\begin{array}{l}\text { nd du } \\
\text { re in } \\
90 \\
\text { to } \\
135\end{array}$ & $\begin{array}{l}\text { tion } \\
\text { cated } \\
135 \\
\text { to } \\
180\end{array}$ & $\begin{array}{r}x \text { sec } \\
\text { des } \\
180 \\
\text { to } \\
225\end{array}$ & $\begin{array}{l}225 \\
t 0 \\
270\end{array}$ & $\begin{array}{r}\text { nours } \\
270 \\
\text { to } \\
315\end{array}$ & $\begin{array}{r}315 \\
\text { to } \\
360\end{array}$ \\
\hline \multicolumn{17}{|c|}{ MOVIRMER 1988} \\
\hline $\begin{array}{l}1 \\
2 \\
3 \\
4\end{array}$ & $\begin{array}{r}42.41 \\
16.06 \\
1.34 \\
1.10\end{array}$ & $\begin{array}{r}12.50 \\
8.09 \\
2.64 \\
.41\end{array}$ & $\begin{array}{r}32.42 \\
35.02 \\
11.93 \\
.17\end{array}$ & $\begin{array}{r}6.84 \\
13.22 \\
3.34 \\
.26\end{array}$ & $\begin{array}{r}6.05 \\
3.70 \\
2.64 \\
.89\end{array}$ & $\begin{array}{l}13.56 \\
20.95 \\
36.60 \\
63.70\end{array}$ & $\begin{array}{r}4.37 \\
23.28 \\
97.97 \\
188.40\end{array}$ & $\begin{array}{r}4.49 \\
5.81 \\
28.08 \\
34.18\end{array}$ & $\begin{array}{r}5.9 \\
2.4 \\
.4 \\
.3\end{array}$ & $\begin{array}{r}3.5 \\
2.4 \\
.9 \\
.1\end{array}$ & $\begin{array}{r}6.8 \\
8.0 \\
3.1 \\
.0\end{array}$ & $\begin{array}{r}1.7 \\
2.7 \\
.8 \\
.1\end{array}$ & $\begin{array}{r}1.3 \\
.9 \\
.4 \\
.1\end{array}$ & $\begin{array}{l}2.2 \\
2.9 \\
4.1 \\
4.8\end{array}$ & $\begin{array}{r}1.1 \\
3.2 \\
10.4 \\
14.7\end{array}$ & $\begin{array}{l}1.4 \\
1.4 \\
3.9 \\
3.6\end{array}$ \\
\hline $\begin{array}{l}5 \\
6 \\
7 \\
8\end{array}$ & $\begin{array}{r}4.27 \\
58.30 \\
13.56 \\
2.47\end{array}$ & $\begin{array}{r}9.53 \\
7.39 \\
12.74 \\
22.20\end{array}$ & $\begin{array}{l}21.79 \\
10.82 \\
27.10 \\
35.21\end{array}$ & $\begin{array}{r}.96 \\
3.50 \\
6.62 \\
11.95\end{array}$ & $\begin{array}{l}4.56 \\
3.55 \\
2.69 \\
3.22\end{array}$ & $\begin{array}{l}32.64 \\
26.30 \\
23.59 \\
18.24\end{array}$ & $\begin{array}{l}54.41 \\
34.01 \\
40.10 \\
18.41\end{array}$ & $\begin{array}{r}20.57 \\
10.87 \\
5.57 \\
2.54\end{array}$ & $\begin{array}{r}1.1 \\
7.1 \\
3.2 \\
.9\end{array}$ & $\begin{array}{l}1.7 \\
2.0 \\
2.5 \\
4.0\end{array}$ & $\begin{array}{l}3.9 \\
2.2 \\
4.6 \\
6.4\end{array}$ & $\begin{array}{r}.3 \\
.8 \\
1.5 \\
2.1\end{array}$ & $\begin{array}{r}.6 \\
.7 \\
.9 \\
1.1\end{array}$ & $\begin{array}{l}4.5 \\
3.9 \\
4.0 \\
4.0\end{array}$ & $\begin{array}{l}7.7 \\
5.0 \\
6.0 \\
4.6\end{array}$ & $\begin{array}{r}4.1 \\
2.1 \\
1.2 \\
.9\end{array}$ \\
\hline $\begin{array}{r}9 \\
10\end{array}$ & $\begin{array}{l}1.39 \\
5.71\end{array}$ & $\begin{array}{l}18.65 \\
15.41\end{array}$ & $\begin{array}{l}21.70 \\
57.26\end{array}$ & $\begin{array}{r}2.30 \\
52.18\end{array}$ & $\begin{array}{l}3.65 \\
1.99\end{array}$ & $\begin{array}{r}27.82 \\
3.36\end{array}$ & $\begin{array}{r}55.63 \\
4.44\end{array}$ & $\begin{array}{r}8.90 \\
11.69\end{array}$ & $\begin{array}{r}.6 \\
1.2\end{array}$ & $\begin{array}{l}3.3 \\
2.5\end{array}$ & $\begin{array}{l}3.7 \\
8.0\end{array}$ & $\begin{array}{r}.7 \\
6.2\end{array}$ & $\begin{array}{l}.7 \\
.7\end{array}$ & $\begin{array}{l}4.0 \\
1.1\end{array}$ & $\begin{array}{l}8.7 \\
1.3\end{array}$ & $\begin{array}{l}2.2 \\
2.7\end{array}$ \\
\hline $\begin{array}{l}11 \\
12\end{array}$ & $\ddot{--}$ & $\ddot{-}$ & $\ddot{--}$ & $=$ & $\ddot{--}$ & 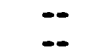 & $=$ & $=$ & $\because$ & 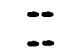 & $=$ & $\ddot{-}$ & $=$ & $=$ & $\because$ & 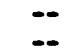 \\
\hline 13 & .- & .. & .. & .- & .. & .. & -. & $\ldots$ & -. & .- & .. & -. & -. & .- & -- & -- \\
\hline 14 & -- & -- & -- & -- & -- & -- & -- & -- & $\cdots$ & - & -- & -- & -- & -- & -- & -- \\
\hline $\begin{array}{l}15 \\
16\end{array}$ & $=$ & $=$ & $=$ & $=$ & $=$ & $=$ & $=$ & $\because$ & $\because$ & $\because$ & $\ddot{--}$ & $=$ & $\because$ & $\because$ & $\ddot{-}$ & $\because$ \\
\hline 17 & -. & -. & -- & -. & -- & -- & -- & -- & -- & -- & -- & -- & -- & -- & .- & -- \\
\hline 18 & -- & -- & -- & -- & -- & -- & -- & - & - & -. & -- & -- & .. & -. & - & -- \\
\hline 19 & $\cdots$ & - & - & -. & - & - & - & $\cdots$ & $\cdots$ & - & -- & -- & - & -- & $\cdots$ & -- \\
\hline 20 & - & - & -- & -- & -- & - & $\cdots$ & -- & -- & - & -- & -- & $\cdots$ & -- & $\cdots$ & -- \\
\hline 21 & -- & -. & -- & -- & -. & -. & -- & -- & $\cdots$ & -- & -- & -- & -. & -. & -- & -- \\
\hline 22 & -- & -- & -- & -- & -- & -- & -- & -- & $=$ & $\cdots$ & -. & -- & - & -- & $\cdots$ & -. \\
\hline 23 & -- & -- & -- & - & -- & -- & -- & $\cdots$ & $\because$ & $\cdots$ & -- & -- & - & -- & - & -- \\
\hline 24 & -- & -- & -- & -- & -- & -- & - & -- & -- & -- & -- & -- & -- & -- & -- & -- \\
\hline 25 & -- & -- & -- & -- & -- & -- & -- & -- & - & -. & -. & -- & -. & -. & - & -- \\
\hline 26 & -- & - & $\cdots$ & -- & -- & -. & - & -- & -- & $\cdots$ & -- & -- & -- & -- & -- & $\cdots$ \\
\hline 27 & -- & -- & -- & -- & -- & $=$ & -- & - & $\because$ & $\cdots$ & - & 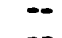 & $\because$ & -- & $\because$ & $-\overline{-}$ \\
\hline 28 & & & & & & & & & & & & & & & & \\
\hline 29 & -- & -- & - & -- & -- & - & -- & -. & - & - & -- & -- & -- & -- & -- & -- \\
\hline 30 & -- & -- & -- & -- & -- & -- & -- & -- & - & - & -- & -- & -- & -- & - & -- \\
\hline
\end{tabular}


Table 3.--Wind-run and wind-duration histogran data, Octobor 1987-Decambor 1989--Continued

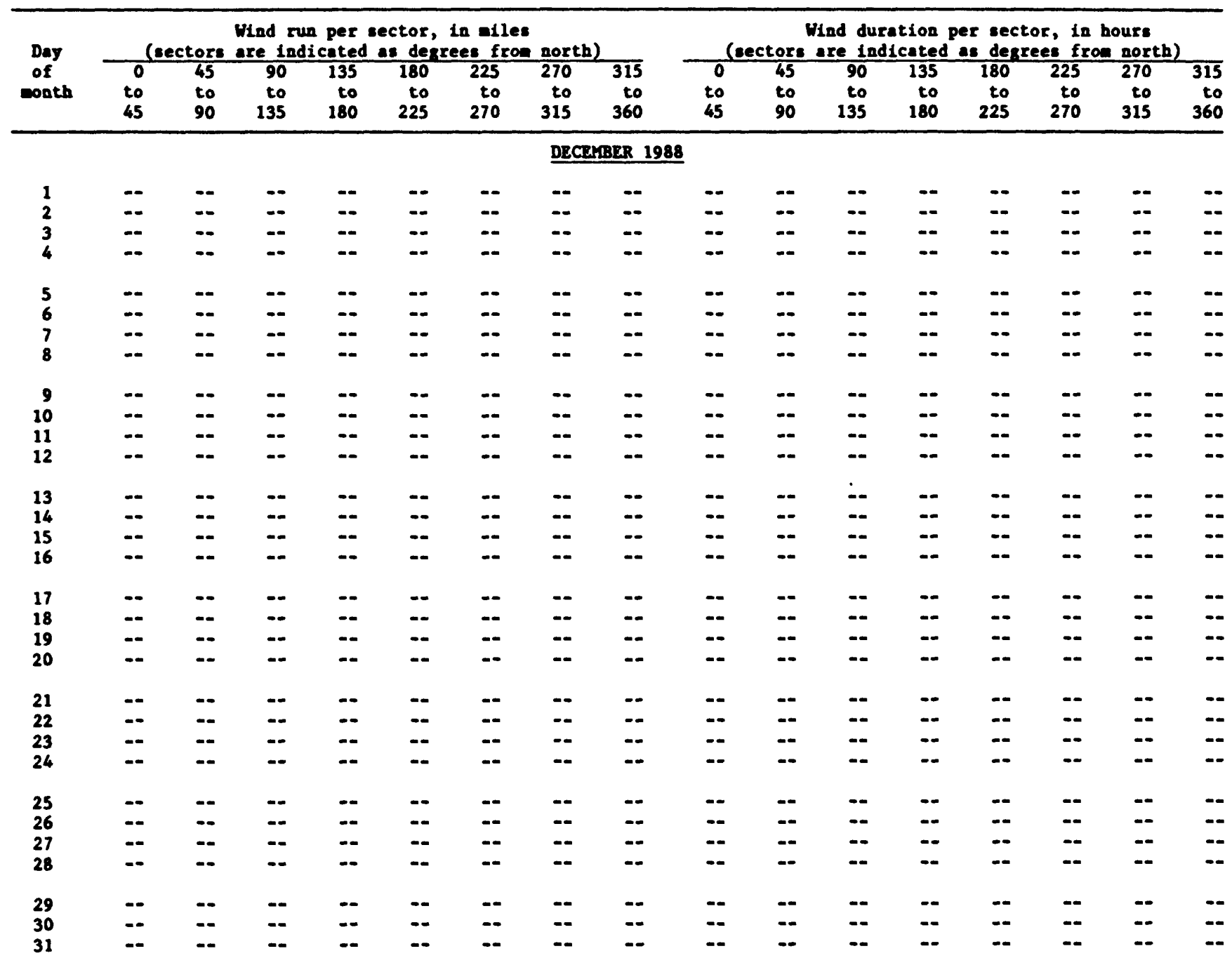


Table 3.--Wind-run and wind-curation histograv data, octobor 1987-December 1989--Continued

\begin{tabular}{|c|c|c|c|c|c|c|c|c|c|c|c|c|c|c|c|c|}
\hline Day & & (sectors & $\begin{array}{l}\text { Wind r } \\
\text { are ind }\end{array}$ & $\begin{array}{l}\text { un per } \\
\text { idicated }\end{array}$ & $\begin{array}{l}\text { sector, } \\
\text { as des }\end{array}$ & $\begin{array}{l}\text {, in afles } \\
\text { grees fron }\end{array}$ & north) & & & (sectors & $\begin{array}{l}\text { Wind dur } \\
\text { are ind }\end{array}$ & $\begin{array}{l}\text { ation } \\
\text { icated }\end{array}$ & $\begin{array}{l}\text { per se } \\
\text { d as de }\end{array}$ & $\begin{array}{l}\text { tor, it } \\
\text { rees f }\end{array}$ & $\begin{array}{l}\text { in hours } \\
\text { fron north) }\end{array}$ & \\
\hline
\end{tabular}

JANUARY 1989

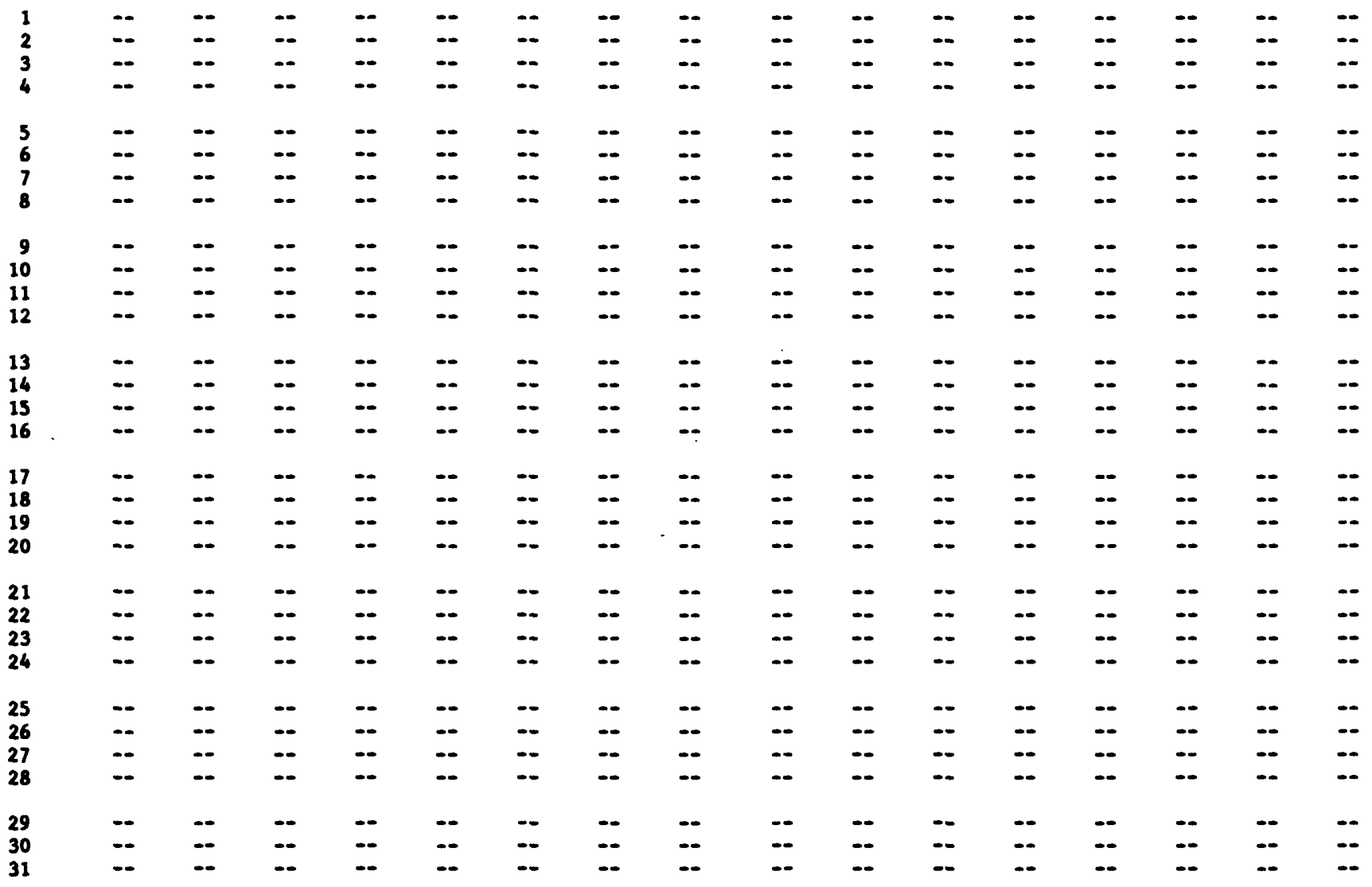


Table 3.--wind-rm and wind-churetion histogra data, october 1987-Decamber 1989--Continued

\begin{tabular}{|c|c|c|c|c|c|c|c|c|c|c|c|c|c|c|c|c|}
\hline $\begin{array}{l}\text { Dey } \\
\text { of } \\
\text { onth }\end{array}$ & $\begin{array}{l}0 \\
0 \\
45\end{array}$ & $\begin{array}{c}\text { ectora } \\
45 \\
\text { to } \\
90\end{array}$ & $\begin{array}{l}\text { Wind ry } \\
\text { are ind } \\
90 \\
\text { to } \\
135\end{array}$ & $\begin{array}{l}\text { a per } \\
\text { icated } \\
135 \\
\text { to } \\
180\end{array}$ & $\begin{array}{l}\text { ector, } \\
\text { es des } \\
180 \\
t 0 \\
225\end{array}$ & $\begin{array}{c}\text { in aile } \\
\text { see fro } \\
225 \\
\text { to } \\
270\end{array}$ & 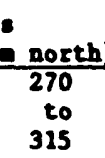 & $\begin{array}{r}315 \\
t 0 \\
360\end{array}$ & $\begin{array}{l}0 \\
t 0 \\
45\end{array}$ & $\begin{array}{l}\text { tors } \\
45 \\
\text { to } \\
90\end{array}$ & $\begin{array}{l}\text { ind du } \\
\text { ere in } \\
90 \\
\text { to } \\
135\end{array}$ & 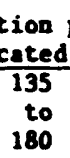 & $\begin{array}{r}\text { per sec } \\
\text { as des } \\
180 \\
\text { to } \\
225\end{array}$ & $\begin{array}{r}\text { or, in } \\
\text { ees fr } \\
225 \\
\text { to } \\
270\end{array}$ & $\begin{array}{l}\text { hours } \\
\text { nortl } \\
270 \\
\text { to } \\
315\end{array}$ & $\begin{array}{r}315 \\
\text { to } \\
360\end{array}$ \\
\hline \multicolumn{17}{|c|}{ FERRUARY 1989} \\
\hline $\begin{array}{l}1 \\
2 \\
3 \\
4\end{array}$ & $\begin{array}{l}2.86 \\
5.50 \\
7.78 \\
4.22\end{array}$ & $\begin{array}{l}3.91 \\
2.59 \\
3.60 \\
2.69\end{array}$ & $\begin{array}{r}8.76 \\
.79 \\
11.64 \\
4.58\end{array}$ & $\begin{array}{r}15.12 \\
1.20 \\
1.80 \\
2.57\end{array}$ & $\begin{array}{r}41.93 \\
16.85 \\
1.03 \\
.74\end{array}$ & $\begin{array}{r}146.14 \\
73.49 \\
9.24 \\
13.03\end{array}$ & $\begin{array}{l}23.47 \\
45.65 \\
27.62 \\
73.92\end{array}$ & $\begin{array}{r}3.58 \\
16.22 \\
16.01 \\
16.56\end{array}$ & $\begin{array}{l}0.7 \\
1.3 \\
2.6 \\
1.3\end{array}$ & $\begin{array}{r}1.0 \\
.6 \\
1.2 \\
.9\end{array}$ & $\begin{array}{r}1.6 \\
.2 \\
1.6 \\
1.1\end{array}$ & $\begin{array}{r}2.1 \\
.4 \\
.6 \\
.6\end{array}$ & $\begin{array}{r}3.8 \\
1.7 \\
.5 \\
.3\end{array}$ & $\begin{array}{r}10.9 \\
7.8 \\
3.2 \\
3.0\end{array}$ & $\begin{array}{r}2.6 \\
8.4 \\
8.4 \\
12.9\end{array}$ & $\begin{array}{l}0.9 \\
3.3 \\
5.3 \\
3.6\end{array}$ \\
\hline $\begin{array}{l}5 \\
6 \\
7 \\
8\end{array}$ & $\begin{array}{l}6.43 \\
6.53 \\
6.38 \\
6.17\end{array}$ & $\begin{array}{l}23.64 \\
36.05 \\
27.74 \\
18.41\end{array}$ & $\begin{array}{l}29.76 \\
31.70 \\
37.92 \\
18.70\end{array}$ & $\begin{array}{r}3.41 \\
11.06 \\
8.42 \\
4.80\end{array}$ & $\begin{array}{l}.34 \\
1.37 \\
2.54 \\
4.08\end{array}$ & $\begin{array}{l}5.93 \\
3.86 \\
8.16 \\
7.10\end{array}$ & $\begin{array}{r}32.06 \\
2.02 \\
2.52 \\
9.43\end{array}$ & $\begin{array}{r}9.89 \\
4.30 \\
3.79 \\
13.39\end{array}$ & $\begin{array}{l}2.0 \\
1.9 \\
2.0 \\
1.8\end{array}$ & $\begin{array}{l}5.9 \\
8.9 \\
6.7 \\
5.5\end{array}$ & $\begin{array}{l}6.0 \\
6.8 \\
7.1 \\
5.0\end{array}$ & $\begin{array}{r}.7 \\
2.0 \\
1.7 \\
1.3\end{array}$ & $\begin{array}{r}.1 \\
.6 \\
1.0 \\
1.3\end{array}$ & $\begin{array}{l}1.2 \\
1.5 \\
3.0 \\
2.5\end{array}$ & $\begin{array}{r}5.6 \\
.8 \\
1.1 \\
2.7\end{array}$ & $\begin{array}{l}2.2 \\
1.1 \\
1.1 \\
3.4\end{array}$ \\
\hline $\begin{array}{r}9 \\
10 \\
11 \\
12\end{array}$ & $\begin{array}{l}6.43 \\
7.49 \\
9.43 \\
5.50\end{array}$ & $\begin{array}{r}29.06 \\
19.54 \\
9.58 \\
9.17\end{array}$ & $\begin{array}{l}64.06 \\
32.06 \\
15.19 \\
17.62\end{array}$ & $\begin{array}{r}19.20 \\
9.60 \\
1.61 \\
4.46\end{array}$ & $\begin{array}{r}2.14 \\
1.51 \\
.62 \\
.89\end{array}$ & $\begin{array}{r}3.79 \\
2.76 \\
6.31 \\
15.10\end{array}$ & $\begin{array}{r}7.08 \\
4.08 \\
18.98 \\
49.20\end{array}$ & $\begin{array}{r}12.29 \\
6.46 \\
7.42 \\
11.88\end{array}$ & $\begin{array}{l}1.7 \\
2.9 \\
3.2 \\
1.7\end{array}$ & $\begin{array}{l}3.8 \\
4.8 \\
4.0 \\
2.2\end{array}$ & $\begin{array}{l}7.8 \\
6.5 \\
4.2 \\
3.0\end{array}$ & $\begin{array}{r}2.9 \\
2.1 \\
.6 \\
.8\end{array}$ & $\begin{array}{l}.8 \\
.8 \\
.6 \\
.4\end{array}$ & $\begin{array}{l}1.3 \\
1.7 \\
2.5 \\
3.2\end{array}$ & $\begin{array}{l}2.0 \\
2.0 \\
5.5 \\
9.4\end{array}$ & $\begin{array}{l}3.0 \\
2.6 \\
2.8 \\
3.0\end{array}$ \\
\hline $\begin{array}{l}13 \\
14 \\
15 \\
16\end{array}$ & $\begin{array}{l}5.93 \\
2.86 \\
2.78 \\
4.87\end{array}$ & $\begin{array}{r}14.98 \\
4.22 \\
48.53 \\
74.54\end{array}$ & $\begin{array}{l}41.52 \\
15.02 \\
39.58 \\
50.30\end{array}$ & $\begin{array}{r}6.00 \\
7.49 \\
25.78 \\
22.49\end{array}$ & $\begin{array}{l}1.37 \\
2.18 \\
1.56 \\
2.35\end{array}$ & $\begin{array}{r}9.12 \\
10.30 \\
1.90 \\
3.05\end{array}$ & $\begin{array}{r}20.04 \\
20.23 \\
1.97 \\
5.38\end{array}$ & $\begin{array}{l}4.18 \\
5.09 \\
1.97 \\
7.49\end{array}$ & $\begin{array}{r}1.9 \\
1.3 \\
.9 \\
1.0\end{array}$ & $\begin{array}{l}3.7 \\
1.9 \\
7.4 \\
6.1\end{array}$ & $\begin{array}{l}9.6 \\
5.6 \\
7.6 \\
8.1\end{array}$ & $\begin{array}{l}1.7 \\
2.3 \\
4.6 \\
3.3\end{array}$ & $\begin{array}{r}.5 \\
1.5 \\
.7 \\
.7\end{array}$ & $\begin{array}{r}1.5 \\
3.4 \\
.9 \\
1.0\end{array}$ & $\begin{array}{r}3.6 \\
5.6 \\
.8 \\
1.6\end{array}$ & $\begin{array}{r}1.2 \\
2.2 \\
.8 \\
1.8\end{array}$ \\
\hline $\begin{array}{l}17 \\
18 \\
19 \\
20\end{array}$ & $\begin{array}{r}4.73 \\
6.05 \\
3.55 \\
.02\end{array}$ & $\begin{array}{r}42.84 \\
62.18 \\
8.81 \\
.00\end{array}$ & $\begin{array}{r}37.32 \\
50.98 \\
13.32 \\
.00\end{array}$ & $\begin{array}{r}14.21 \\
9.05 \\
4.92 \\
.00\end{array}$ & $\begin{array}{r}1.85 \\
1.82 \\
.79 \\
.38\end{array}$ & $\begin{array}{r}5.06 \\
3.72 \\
10.34 \\
48.60\end{array}$ & $\begin{array}{r}3.29 \\
6.22 \\
25.92 \\
257.04\end{array}$ & $\begin{array}{l}3.77 \\
4.70 \\
5.90 \\
8.52\end{array}$ & $\begin{array}{r}1.7 \\
1.9 \\
1.4 \\
.0\end{array}$ & $\begin{array}{r}6.1 \\
7.8 \\
2.7 \\
.0\end{array}$ & $\begin{array}{r}7.2 \\
6.6 \\
2.8 \\
.0\end{array}$ & $\begin{array}{r}2.5 \\
1.6 \\
1.2 \\
.0\end{array}$ & $\begin{array}{l}.8 \\
.6 \\
.6 \\
.0\end{array}$ & $\begin{array}{l}2.6 \\
1.4 \\
4.2 \\
3.7\end{array}$ & $\begin{array}{r}1.4 \\
2.1 \\
8.8 \\
19.2\end{array}$ & $\begin{array}{l}1.4 \\
1.7 \\
2.1 \\
1.0\end{array}$ \\
\hline $\begin{array}{l}21 \\
22 \\
23 \\
24\end{array}$ & $\begin{array}{l}4.34 \\
4.06 \\
6.70 \\
6.00\end{array}$ & $\begin{array}{r}6.43 \\
76.49 \\
22.73 \\
49.61\end{array}$ & $\begin{array}{l}31.97 \\
91.56 \\
60.17 \\
40.42\end{array}$ & $\begin{array}{r}1.78 \\
18.82 \\
15.53 \\
18.60\end{array}$ & $\begin{array}{r}.82 \\
.91 \\
1.37 \\
2.98\end{array}$ & $\begin{array}{r}5.66 \\
.72 \\
1.49 \\
4.32\end{array}$ & $\begin{array}{r}22.10 \\
1.73 \\
2.52 \\
4.99\end{array}$ & $\begin{array}{l}9.74 \\
3.53 \\
5.76 \\
7.49\end{array}$ & $\begin{array}{l}2.2 \\
1.1 \\
1.7 \\
1.5\end{array}$ & $\begin{array}{l}2.0 \\
7.9 \\
5.1 \\
6.7\end{array}$ & $\begin{array}{r}6.3 \\
10.3 \\
10.7 \\
6.9\end{array}$ & $\begin{array}{r}.7 \\
2.2 \\
2.7 \\
2.8\end{array}$ & $\begin{array}{r}.5 \\
.3 \\
.5 \\
1.0\end{array}$ & $\begin{array}{r}2.1 \\
.2 \\
.6 \\
1.4\end{array}$ & $\begin{array}{r}6.4 \\
.6 \\
.8 \\
1.5\end{array}$ & $\begin{array}{l}3.4 \\
1.0 \\
1.5 \\
1.9\end{array}$ \\
\hline $\begin{array}{l}25 \\
26 \\
27 \\
28\end{array}$ & $\begin{array}{l}3.00 \\
3.79 \\
1.13 \\
2.11\end{array}$ & $\begin{array}{r}17.16 \\
7.34 \\
3.62 \\
19.68\end{array}$ & $\begin{array}{l}65.52 \\
16.15 \\
19.58 \\
45.38\end{array}$ & $\begin{array}{r}18.17 \\
4.30 \\
1.44 \\
16.75\end{array}$ & $\begin{array}{r}.86 \\
1.32 \\
.60 \\
1.51\end{array}$ & $\begin{array}{r}1.63 \\
20.47 \\
22.20 \\
7.80\end{array}$ & $\begin{array}{r}3.60 \\
68.54 \\
55.01 \\
15.50\end{array}$ & $\begin{array}{r}3.24 \\
18.48 \\
9.05 \\
4.51\end{array}$ & $\begin{array}{r}1.0 \\
1.4 \\
.4 \\
.7\end{array}$ & $\begin{array}{l}4.2 \\
2.4 \\
1.1 \\
4.1\end{array}$ & $\begin{array}{r}12.3 \\
3.8 \\
5.5 \\
9.3\end{array}$ & $\begin{array}{r}2.9 \\
1.0 \\
.4 \\
3.1\end{array}$ & $\begin{array}{l}.4 \\
.4 \\
.3 \\
.6\end{array}$ & $\begin{array}{r}.7 \\
2.9 \\
4.6 \\
1.9\end{array}$ & $\begin{array}{l}1.2 \\
8.5 \\
9.5 \\
3.0\end{array}$ & $\begin{array}{l}1.1 \\
3.3 \\
2.0 \\
1.1\end{array}$ \\
\hline
\end{tabular}


Table 3.--wind-run and wind-duration histogran data, October 1987-Decamber 1989--Continued

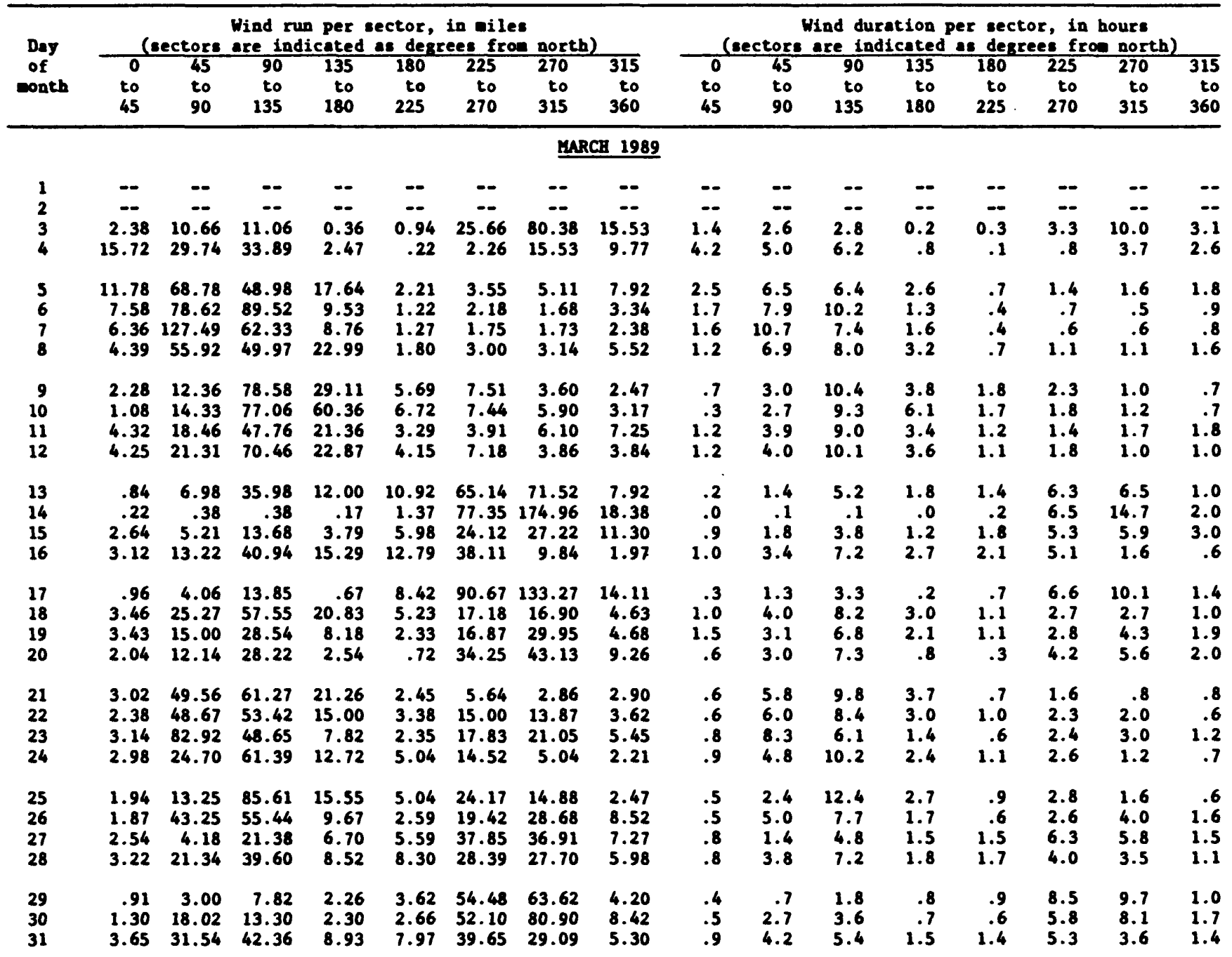


Table 3.--Wind-run and wind-curation histogren date, octobor 1987-Decu-ber 1989--Continued

\begin{tabular}{|c|c|c|c|c|c|c|c|c|c|c|c|c|c|c|c|c|}
\hline $\begin{array}{l}\text { Day } \\
\text { of } \\
\text { nonth }\end{array}$ & $\begin{array}{l}0 \\
\text { to } \\
45\end{array}$ & $\begin{array}{r}\text { ectors } \\
45 \\
\text { to } \\
90\end{array}$ & $\begin{array}{l}\text { Wind } \mathrm{n} \\
\text { are in } \\
90 \\
\text { to } \\
135\end{array}$ & $\begin{array}{c}\text { per } \\
\text { cated } \\
135 \\
\text { to } \\
180\end{array}$ & $\begin{array}{l}\text { ctor, } \\
\text { s degr } \\
180 \\
\text { to } \\
225\end{array}$ & $\begin{array}{l}\text { in alle } \\
\text { ees fro } \\
225 \\
\text { to } \\
270\end{array}$ & 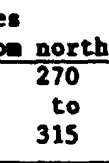 & $\begin{array}{r}315 \\
\text { to } \\
360\end{array}$ & $\begin{array}{l}0 \\
\text { to } \\
45\end{array}$ & $\begin{array}{l}\text { tors } \\
45 \\
\text { to } \\
90\end{array}$ & $\begin{array}{l}\text { ad du } \\
\text { re in } \\
90 \\
\text { to } \\
135\end{array}$ & $\begin{array}{c}\text { tion } \\
\text { cated } \\
135 \\
\text { to } \\
180\end{array}$ & $\begin{array}{r}r \text { sec } \\
180 \\
\text { to } \\
225\end{array}$ & $\begin{array}{r}225 \\
\text { to } \\
270\end{array}$ & $\begin{array}{l}\text { ours } \\
\text { nort } \\
270 \\
\text { to } \\
315\end{array}$ & $\begin{array}{r}315 \\
t 0 \\
360\end{array}$ \\
\hline \multicolumn{17}{|c|}{ APRIL 1989} \\
\hline $\begin{array}{l}1 \\
2 \\
3 \\
4\end{array}$ & $\begin{array}{r}1.87 \\
2.14 \\
.12 \\
.84\end{array}$ & $\begin{array}{r}33.36 \\
1.58 \\
.00 \\
1.27\end{array}$ & $\begin{array}{r}30.02 \\
1.82 \\
.00 \\
3.31\end{array}$ & $\begin{array}{r}6.38 \\
1.39 \\
.12 \\
.48\end{array}$ & $\begin{array}{r}2.09 \\
11.47 \\
4.27 \\
.98\end{array}$ & $\begin{array}{r}8.66 \\
97.82 \\
74.47 \\
78.77\end{array}$ & $\begin{array}{r}7.70 \\
86.81 \\
132.12 \\
180.72\end{array}$ & $\begin{array}{r}1.51 \\
7.56 \\
12.43 \\
17.76\end{array}$ & $\begin{array}{r}0.6 \\
.9 \\
.0 \\
.2\end{array}$ & $\begin{array}{r}6.6 \\
.8 \\
.0 \\
.4\end{array}$ & $\begin{array}{r}8.5 \\
.8 \\
.0 \\
.9\end{array}$ & $\begin{array}{r}2.3 \\
.4 \\
.0 \\
.2\end{array}$ & $\begin{array}{r}0.9 \\
1.7 \\
.6 \\
.2\end{array}$ & $\begin{array}{l}2.2 \\
9.5 \\
8.3 \\
5.6\end{array}$ & $\begin{array}{r}2.0 \\
8.2 \\
13.4 \\
13.9\end{array}$ & $\begin{array}{l}0.6 \\
1.4 \\
1.6 \\
2.3\end{array}$ \\
\hline $\begin{array}{l}5 \\
6 \\
7 \\
8\end{array}$ & $\begin{array}{r}3.98 \\
3.02 \\
1.39 \\
.84\end{array}$ & $\begin{array}{r}5.35 \\
11.95 \\
8.93 \\
.38\end{array}$ & $\begin{array}{r}6.10 \\
20.45 \\
17.02 \\
.19\end{array}$ & $\begin{array}{r}2.14 \\
.48 \\
.58 \\
.05\end{array}$ & $\begin{array}{l}4.97 \\
1.63 \\
3.29 \\
2.42\end{array}$ & $\begin{array}{l}37.85 \\
63.72 \\
56.90 \\
78.26\end{array}$ & $\begin{array}{r}59.14 \\
118.90 \\
110.88 \\
154.80\end{array}$ & $\begin{array}{r}15.14 \\
9.98 \\
14.57 \\
21.48\end{array}$ & $\begin{array}{l}1.4 \\
.9 \\
.5 \\
.2\end{array}$ & $\begin{array}{r}1.9 \\
2.9 \\
2.4 \\
.1\end{array}$ & $\begin{array}{r}2.2 \\
4.6 \\
3.7 \\
.1\end{array}$ & $\begin{array}{l}.8 \\
.2 \\
.3 \\
.0\end{array}$ & $\begin{array}{r}1.3 \\
.3 \\
.7 \\
.3\end{array}$ & $\begin{array}{l}5.4 \\
4.7 \\
5.1 \\
6.5\end{array}$ & $\begin{array}{r}7.7 \\
8.8 \\
9.6 \\
13.7\end{array}$ & $\begin{array}{l}2.9 \\
1.4 \\
1.7 \\
2.9\end{array}$ \\
\hline $\begin{array}{r}9 \\
10 \\
11 \\
12\end{array}$ & $\begin{array}{r}3.84 \\
6.67 \\
4.61 \\
10.08\end{array}$ & $\begin{array}{l}10.27 \\
46.54 \\
34.61 \\
41.33\end{array}$ & $\begin{array}{r}4.56 \\
60.00 \\
48.34 \\
61.22\end{array}$ & $\begin{array}{r}.98 \\
4.03 \\
5.28 \\
11.38\end{array}$ & $\begin{array}{l}2.64 \\
3.26 \\
4.25 \\
3.84\end{array}$ & $\begin{array}{r}70.70 \\
10.99 \\
27.41 \\
2.81\end{array}$ & $\begin{array}{r}112.44 \\
11.09 \\
23.21 \\
4.22\end{array}$ & $\begin{array}{r}14.18 \\
7.18 \\
4.87 \\
3.79\end{array}$ & $\begin{array}{l}1.0 \\
1.6 \\
1.0 \\
1.9\end{array}$ & $\begin{array}{l}1.9 \\
5.9 \\
4.9 \\
6.7\end{array}$ & $\begin{array}{l}1.0 \\
8.5 \\
7.1 \\
9.5\end{array}$ & $\begin{array}{l}.3 \\
1.0 \\
1.0 \\
2.3\end{array}$ & $\begin{array}{l}.5 \\
.9 \\
.9 \\
.9\end{array}$ & $\begin{array}{l}6.4 \\
2.1 \\
4.4 \\
.7\end{array}$ & $\begin{array}{r}10.3 \\
1.9 \\
3.5 \\
.9\end{array}$ & $\begin{array}{r}2.3 \\
1.6 \\
1.0 \\
.9\end{array}$ \\
\hline $\begin{array}{l}17 \\
18 \\
19 \\
20\end{array}$ & $\begin{array}{l}2.35 \\
1.99 \\
4.94 \\
6.19\end{array}$ & $\begin{array}{l}14.54 \\
19.34 \\
33.74 \\
47.64\end{array}$ & $\begin{array}{l}22.39 \\
38.21 \\
49.22 \\
62.09\end{array}$ & $\begin{array}{r}3.22 \\
.38 \\
11.62 \\
11.54\end{array}$ & $\begin{array}{r}5.74 \\
1.70 \\
12.26 \\
3.24\end{array}$ & $\begin{array}{l}64.44 \\
48.17 \\
28.01 \\
10.18\end{array}$ & $\begin{array}{r}88.39 \\
71.66 \\
10.61 \\
8.98\end{array}$ & $\begin{array}{l}8.23 \\
6.89 \\
3.31 \\
5.09\end{array}$ & $\begin{array}{l}.6 \\
.5 \\
1.2 \\
1.5\end{array}$ & $\begin{array}{l}2.6 \\
4.1 \\
5.0 \\
5.9\end{array}$ & $\begin{array}{l}4.4 \\
6.8 \\
7.1 \\
8.8\end{array}$ & $\begin{array}{r}.7 \\
.1 \\
2.0 \\
2.0\end{array}$ & $\begin{array}{r}.8 \\
.2 \\
2.0 \\
.7\end{array}$ & $\begin{array}{l}6.0 \\
4.7 \\
3.9 \\
1.8\end{array}$ & $\begin{array}{l}7.6 \\
6.4 \\
1.9 \\
1.8\end{array}$ & $\begin{array}{r}1.1 \\
1.0 \\
.8 \\
1.2\end{array}$ \\
\hline $\begin{array}{l}21 \\
22 \\
23 \\
24\end{array}$ & $\begin{array}{l}.67 \\
.22 \\
.74 \\
.72\end{array}$ & $\begin{array}{r}8.33 \\
28.58 \\
39.19 \\
34.42\end{array}$ & $\begin{array}{l}74.86 \\
66.89 \\
89.66 \\
87.74\end{array}$ & $\begin{array}{l}19.27 \\
29.47 \\
26.78 \\
22.80\end{array}$ & $\begin{array}{r}9.55 \\
22.51 \\
16.94 \\
13.92\end{array}$ & $\begin{array}{l}40.61 \\
57.17 \\
21.02 \\
17.98\end{array}$ & $\begin{array}{r}21.43 \\
7.06 \\
2.23 \\
1.75\end{array}$ & $\begin{array}{r}1.08 \\
.41 \\
.48 \\
.29\end{array}$ & $\begin{array}{l}.2 \\
.0 \\
.1 \\
.1\end{array}$ & $\begin{array}{l}1.4 \\
3.1 \\
4.0 \\
3.6\end{array}$ & $\begin{array}{r}11.0 \\
9.3 \\
11.7 \\
12.2\end{array}$ & $\begin{array}{l}2.7 \\
3.8 \\
3.5 \\
3.4\end{array}$ & $\begin{array}{l}1.4 \\
2.3 \\
2.0 \\
2.0\end{array}$ & $\begin{array}{l}4.5 \\
4.6 \\
2.1 \\
2.2\end{array}$ & $\begin{array}{r}2.4 \\
.7 \\
.3 \\
.3\end{array}$ & $\begin{array}{l}.2 \\
.1 \\
.1 \\
.0\end{array}$ \\
\hline
\end{tabular}


Table 3.--Wtad-run and wind-duretion histogra data, october 1987-Decumber 1989--Continued

\begin{tabular}{|c|c|c|c|c|c|c|c|c|c|c|c|c|c|c|c|c|}
\hline $\begin{array}{l}\text { Dey } \\
\text { of } \\
\text { onth }\end{array}$ & $\begin{array}{l}0 \\
\text { to } \\
45\end{array}$ & $\begin{array}{c}\text { sectors } \\
45 \\
\text { to } \\
90\end{array}$ & $\begin{array}{l}\text { Wind } \\
\text { are Ind } \\
90 \\
\text { to } \\
135\end{array}$ & $\begin{array}{r}\text { a per } \\
\text { 1cated } \\
135 \\
\text { to } \\
180\end{array}$ & $\begin{array}{l}\text { sector, } \\
\text { e der } \\
180 \\
\text { to } \\
225\end{array}$ & $\begin{array}{l}\text { In alle } \\
\text { ees fro } \\
225 \\
\text { to } \\
270\end{array}$ & $\begin{array}{r}\text { es nortb } \\
270 \\
\text { to } \\
315\end{array}$ & $\begin{array}{r}315 \\
t 0 \\
360\end{array}$ & $\underbrace{0}_{20}$ & $\begin{array}{r}\text { ectors } \\
45 \\
t 0 \\
90\end{array}$ & $\begin{array}{r}\text { Lnd du } \\
\text { are in } \\
90 \\
\text { to } \\
135\end{array}$ & $\begin{array}{l}\text { 10n } \\
\text { sated } \\
135 \\
\text { to } \\
180\end{array}$ & $\begin{array}{r}\text { sec } \\
\text { der } \\
180 \\
\text { to } \\
225\end{array}$ & $\begin{array}{r}r, 10 \\
e s f x \\
225 \\
t 0 \\
270\end{array}$ & $\begin{array}{r}\text { hours } \\
\text { nort } \\
270 \\
\text { to } \\
315\end{array}$ & $\begin{array}{r}315 \\
\text { to } \\
360\end{array}$ \\
\hline \multicolumn{17}{|c|}{ MAI 1989} \\
\hline $\begin{array}{l}1 \\
2 \\
3 \\
4\end{array}$ & $\begin{array}{r}3.34 \\
5.81 \\
.82 \\
3.46\end{array}$ & $\begin{array}{r}15.91 \\
41.62 \\
13.32 \\
2.45\end{array}$ & $\begin{array}{r}18.94 \\
51.14 \\
16.27 \\
5.64\end{array}$ & $\begin{array}{r}6.05 \\
7.44 \\
1.87 \\
.41\end{array}$ & $\begin{array}{l}5.66 \\
7.03 \\
9.48 \\
2.86\end{array}$ & $\begin{array}{l}52.80 \\
27.36 \\
61.15 \\
60.10\end{array}$ & $\begin{array}{r}69.72 \\
25.08 \\
75.10 \\
117.34\end{array}$ & $\begin{array}{r}10.49 \\
6.58 \\
9.94 \\
18.74\end{array}$ & $\begin{array}{r}0.8 \\
1.2 \\
.4 \\
1.2\end{array}$ & $\begin{array}{r}3.0 \\
5.4 \\
1.9 \\
.7\end{array}$ & $\begin{array}{l}3.8 \\
7.0 \\
2.5 \\
1.2\end{array}$ & $\begin{array}{r}1.3 \\
1.6 \\
.6 \\
.1\end{array}$ & $\begin{array}{r}1.1 \\
1.2 \\
1.4 \\
.6\end{array}$ & $\begin{array}{l}5.0 \\
3.1 \\
6.7 \\
5.6\end{array}$ & $\begin{array}{r}6.8 \\
3.0 \\
8.6 \\
10.9\end{array}$ & $\begin{array}{l}1.9 \\
1.3 \\
1.7 \\
3.2\end{array}$ \\
\hline $\begin{array}{l}5 \\
6 \\
7 \\
8\end{array}$ & $\begin{array}{l}3.84 \\
3.29 \\
3.70 \\
2.28\end{array}$ & $\begin{array}{l}20.02 \\
19.75 \\
31.63 \\
70.82\end{array}$ & $\begin{array}{l}33.10 \\
44.14 \\
45.89 \\
33.24\end{array}$ & $\begin{array}{l}.72 \\
5.90 \\
8.86 \\
7.20\end{array}$ & $\begin{array}{l}1.70 \\
1.42 \\
5.06 \\
2.52\end{array}$ & $\begin{array}{l}38.93 \\
29.88 \\
35.86 \\
36.24\end{array}$ & $\begin{array}{l}52.63 \\
40.30 \\
39.02 \\
36.77\end{array}$ & $\begin{array}{l}9.10 \\
7.99 \\
4.06 \\
5.88\end{array}$ & $\begin{array}{l}1.0 \\
1.0 \\
1.0 \\
.6\end{array}$ & $\begin{array}{l}4.5 \\
4.2 \\
4.9 \\
7.3\end{array}$ & $\begin{array}{l}6.4 \\
7.3 \\
6.7 \\
4.9\end{array}$ & $\begin{array}{l}.2 \\
1.0 \\
1.5 \\
1.3\end{array}$ & $\begin{array}{r}.3 \\
.3 \\
1.0 \\
.6\end{array}$ & $\begin{array}{l}4.3 \\
3.5 \\
4.2 \\
4.1\end{array}$ & $\begin{array}{l}5.7 \\
5.0 \\
3.9 \\
3.8\end{array}$ & $\begin{array}{l}1.4 \\
1.4 \\
.7 \\
1.0\end{array}$ \\
\hline $\begin{array}{r}9 \\
10 \\
11 \\
12\end{array}$ & $\begin{array}{r}3.55 \\
.60 \\
4.15 \\
1.85\end{array}$ & $\begin{array}{r}108.96 \\
149.04 \\
114.29 \\
7.20\end{array}$ & $\begin{array}{l}49.08 \\
98.33 \\
48.79 \\
17.62\end{array}$ & $\begin{array}{l}6.79 \\
9.77 \\
8.66 \\
3.46\end{array}$ & $\begin{array}{l}4.54 \\
7.15 \\
7.94 \\
3.31\end{array}$ & $\begin{array}{r}9.74 \\
8.04 \\
18.00 \\
36.29\end{array}$ & $\begin{array}{r}17.45 \\
.46 \\
7.39 \\
42.70\end{array}$ & $\begin{array}{r}8.16 \\
.02 \\
2.47 \\
5.69\end{array}$ & $\begin{array}{l}.8 \\
.0 \\
.9 \\
.7\end{array}$ & $\begin{array}{r}9.8 \\
10.5 \\
9.9 \\
2.5\end{array}$ & $\begin{array}{r}5.2 \\
10.3 \\
5.8 \\
5.0\end{array}$ & $\begin{array}{l}1.1 \\
1.4 \\
1.4 \\
1.1\end{array}$ & $\begin{array}{r}.8 \\
.8 \\
1.2 \\
1.1\end{array}$ & $\begin{array}{r}1.6 \\
.8 \\
2.5 \\
6.2\end{array}$ & $\begin{array}{r}2.7 \\
.0 \\
1.4 \\
6.0\end{array}$ & $\begin{array}{r}1.6 \\
.0 \\
.7 \\
1.2\end{array}$ \\
\hline $\begin{array}{l}13 \\
14 \\
15 \\
16\end{array}$ & $\begin{array}{l}3.38 \\
3.65 \\
2.57 \\
2.64\end{array}$ & $\begin{array}{r}67.01 \\
138.07 \\
102.24 \\
22.99\end{array}$ & $\begin{array}{l}49.01 \\
72.77 \\
45.10 \\
38.95\end{array}$ & $\begin{array}{l}3.58 \\
1.58 \\
5.40 \\
6.38\end{array}$ & $\begin{array}{l}2.76 \\
2.78 \\
2.90 \\
3.65\end{array}$ & $\begin{array}{l}26.59 \\
12.43 \\
13.32 \\
20.98\end{array}$ & $\begin{array}{r}19.13 \\
5.47 \\
13.73 \\
17.88\end{array}$ & $\begin{array}{l}3.41 \\
1.39 \\
3.84 \\
7.51\end{array}$ & $\begin{array}{l}.7 \\
.7 \\
.6 \\
.8\end{array}$ & $\begin{array}{r}7.5 \\
11.4 \\
9.7 \\
4.3\end{array}$ & $\begin{array}{l}6.8 \\
7.1 \\
6.1 \\
7.9\end{array}$ & $\begin{array}{r}.6 \\
.5 \\
1.4 \\
1.5\end{array}$ & $\begin{array}{l}.6 \\
.7 \\
.7 \\
.9\end{array}$ & $\begin{array}{l}3.8 \\
2.2 \\
2.2 \\
3.6\end{array}$ & $\begin{array}{r}3.1 \\
.9 \\
2.2 \\
2.9\end{array}$ & $\begin{array}{r}.7 \\
.3 \\
.9 \\
1.7\end{array}$ \\
\hline $\begin{array}{l}17 \\
18 \\
19 \\
20\end{array}$ & $\begin{array}{r}3.22 \\
5.54 \\
.62 \\
4.30\end{array}$ & $\begin{array}{r}11.62 \\
12.19 \\
5.50 \\
21.60\end{array}$ & $\begin{array}{c}20.88 \\
19.70 \\
16.68 \\
32.83\end{array}$ & $\begin{array}{r}3.34 \\
9.89 \\
.82 \\
3.89\end{array}$ & $\begin{array}{r}2.33 \\
11.93 \\
3.12 \\
4.49\end{array}$ & $\begin{array}{l}31.10 \\
48.70 \\
69.22 \\
29.66\end{array}$ & $\begin{array}{l}35.26 \\
32.54 \\
87.07 \\
24.65\end{array}$ & $\begin{array}{r}7.61 \\
8.11 \\
11.90 \\
8.54\end{array}$ & $\begin{array}{r}1.2 \\
1.6 \\
.2 \\
1.2\end{array}$ & $\begin{array}{l}3.2 \\
3.0 \\
1.3 \\
4.9\end{array}$ & $\begin{array}{l}4.8 \\
4.1 \\
3.2 \\
6.1\end{array}$ & $\begin{array}{r}1.2 \\
2.0 \\
.2 \\
.8\end{array}$ & $\begin{array}{r}.8 \\
2.0 \\
.4 \\
.8\end{array}$ & $\begin{array}{l}5.0 \\
5.4 \\
7.5 \\
4.0\end{array}$ & $\begin{array}{l}5.5 \\
3.8 \\
9.6 \\
3.8\end{array}$ & $\begin{array}{l}2.0 \\
1.9 \\
1.5 \\
2.1\end{array}$ \\
\hline $\begin{array}{l}21 \\
22 \\
23 \\
24\end{array}$ & $\begin{array}{l}4.03 \\
2.52 \\
2.11 \\
2.14\end{array}$ & $\begin{array}{r}21.17 \\
22.49 \\
16.25 \\
.41\end{array}$ & $\begin{array}{r}41.06 \\
45.74 \\
26.11 \\
.46\end{array}$ & $\begin{array}{r}9.26 \\
6.89 \\
11.59 \\
.43\end{array}$ & $\begin{array}{r}11.52 \\
7.54 \\
24.46 \\
3.77\end{array}$ & $\begin{array}{l}40.34 \\
37.13 \\
86.95 \\
70.56\end{array}$ & $\begin{array}{r}22.06 \\
25.73 \\
25.61 \\
114.10\end{array}$ & $\begin{array}{r}5.59 \\
4.54 \\
3.24 \\
17.06\end{array}$ & $\begin{array}{r}1.2 \\
.7 \\
.6 \\
.5\end{array}$ & $\begin{array}{r}4.1 \\
4.2 \\
3.5 \\
.1\end{array}$ & $\begin{array}{r}6.6 \\
7.5 \\
4.5 \\
.2\end{array}$ & $\begin{array}{r}1.7 \\
1.3 \\
1.9 \\
.2\end{array}$ & $\begin{array}{r}1.6 \\
1.4 \\
2.6 \\
.7\end{array}$ & $\begin{array}{l}4.6 \\
4.7 \\
7.7 \\
7.6\end{array}$ & $\begin{array}{r}2.7 \\
3.2 \\
2.4 \\
11.9\end{array}$ & $\begin{array}{r}1.3 \\
.9 \\
.6 \\
2.5\end{array}$ \\
\hline $\begin{array}{l}25 \\
26 \\
27 \\
28\end{array}$ & $\begin{array}{l}3.41 \\
6.34 \\
4.30 \\
1.80\end{array}$ & $\begin{array}{l}15.79 \\
23.02 \\
25.13 \\
16.58\end{array}$ & $\begin{array}{l}19.54 \\
36.91 \\
38.38 \\
52.32\end{array}$ & $\begin{array}{r}2.38 \\
8.18 \\
8.26 \\
44.78\end{array}$ & $\begin{array}{r}2.88 \\
2.30 \\
15.65 \\
23.38\end{array}$ & $\begin{array}{l}51.07 \\
18.05 \\
56.90 \\
54.10\end{array}$ & $\begin{array}{r}60.26 \\
24.02 \\
16.90 \\
7.70\end{array}$ & $\begin{array}{r}10.37 \\
11.42 \\
4.46 \\
.79\end{array}$ & $\begin{array}{r}.8 \\
1.4 \\
1.1 \\
.4\end{array}$ & $\begin{array}{l}2.6 \\
4.2 \\
4.3 \\
2.2\end{array}$ & $\begin{array}{l}3.6 \\
6.6 \\
5.9 \\
6.9\end{array}$ & $\begin{array}{r}.8 \\
1.6 \\
1.4 \\
5.7\end{array}$ & $\begin{array}{r}1.0 \\
.6 \\
2.0 \\
2.8\end{array}$ & $\begin{array}{l}6.0 \\
3.0 \\
5.8 \\
4.8\end{array}$ & $\begin{array}{r}7.0 \\
3.9 \\
2.2 \\
.8\end{array}$ & $\begin{array}{r}1.8 \\
2.3 \\
1.1 \\
.2\end{array}$ \\
\hline $\begin{array}{l}29 \\
30 \\
31\end{array}$ & $\begin{array}{l}1.27 \\
2.50 \\
1.46\end{array}$ & $\begin{array}{r}17.71 \\
8.69 \\
15.17\end{array}$ & $\begin{array}{l}46.56 \\
24.82 \\
23.26\end{array}$ & $\begin{array}{r}19.78 \\
8.83 \\
3.53\end{array}$ & $\begin{array}{r}21.10 \\
18.12 \\
2.47\end{array}$ & $\begin{array}{l}58.73 \\
70.56 \\
52.82\end{array}$ & $\begin{array}{l}12.98 \\
34.15 \\
62.42\end{array}$ & $\begin{array}{r}.43 \\
5.78 \\
6.94\end{array}$ & $\begin{array}{l}.3 \\
.7 \\
.6\end{array}$ & $\begin{array}{l}3.0 \\
2.0 \\
3.2\end{array}$ & $\begin{array}{l}7.9 \\
4.4 \\
4.7\end{array}$ & $\begin{array}{r}3.2 \\
1.6 \\
.9\end{array}$ & $\begin{array}{r}2.7 \\
2.5 \\
.6\end{array}$ & $\begin{array}{l}5.6 \\
7.8 \\
6.0\end{array}$ & $\begin{array}{l}1.2 \\
3.7 \\
6.9\end{array}$ & $\begin{array}{l}.1 \\
1.0 \\
1.1\end{array}$ \\
\hline
\end{tabular}


Table 3.--Wind-run and wind-curetion histogran data, Octobar 1987-Decenbor 1989--Continued

\begin{tabular}{|c|c|c|c|c|c|c|c|c|c|c|c|c|c|c|c|c|}
\hline $\begin{array}{l}\text { Day } \\
\text { of } \\
\text { eonth }\end{array}$ & $\begin{array}{l}0 \\
t 0 \\
45\end{array}$ & $\begin{array}{c}\text { sectors } \\
45 \\
\text { to } \\
90\end{array}$ & $\begin{array}{r}\text { Wind } \\
\text { are in } \\
90 \\
\text { to } \\
135\end{array}$ & $\begin{array}{c}\text { per } \\
\text { icated } \\
135 \\
\text { to } \\
180\end{array}$ & $\begin{array}{c}\text { ctor, } \\
\text { des } \\
180 \\
\text { to } \\
225\end{array}$ & $\begin{array}{r}\text { in ail } \\
\text { sees fr } \\
225 \\
\text { to } \\
270\end{array}$ & 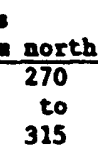 & $\begin{array}{r}315 \\
\text { to } \\
360\end{array}$ & $\begin{array}{l}0 \\
\text { to } \\
45\end{array}$ & $\begin{array}{l}\text { ctor: } \\
45 \\
\text { to } \\
90\end{array}$ & $\begin{array}{l}\text { nd du } \\
\text { ire in } \\
90 \\
\text { to } \\
135\end{array}$ & $\begin{array}{l}\text { tion } \\
\text { cated } \\
135 \\
\text { to } \\
180\end{array}$ & $\begin{array}{r}\text { sec } \\
\text { des: } \\
180 \\
\text { to } \\
225\end{array}$ & $\begin{array}{l}r \text {, in } \\
\text { es fr } \\
225 \\
\text { to } \\
270\end{array}$ & $\begin{array}{l}\text { ours } \\
\text { nort } \\
270 \\
\text { to } \\
315\end{array}$ & $\begin{array}{r}315 \\
\text { to } \\
360\end{array}$ \\
\hline & & & & & & & & IE 1989 & & & & & & & & \\
\hline $\begin{array}{l}1 \\
2 \\
3 \\
4\end{array}$ & $\begin{array}{l}4.68 \\
2.50 \\
3.14 \\
4.20\end{array}$ & $\begin{array}{l}23.11 \\
26.16 \\
69.72 \\
55.97\end{array}$ & $\begin{array}{l}26.38 \\
58.42 \\
33.07 \\
42.84\end{array}$ & $\begin{array}{l}4.32 \\
9.43 \\
3.86 \\
3.24\end{array}$ & $\begin{array}{l}3.46 \\
5.59 \\
4.58 \\
5.83\end{array}$ & $\begin{array}{l}27.79 \\
28.39 \\
24.65 \\
22.87\end{array}$ & $\begin{array}{l}35.26 \\
22.06 \\
20.11 \\
13.56\end{array}$ & $\begin{array}{r}10.66 \\
6.24 \\
3.36 \\
5.83\end{array}$ & $\begin{array}{r}1.1 \\
.6 \\
.6 \\
1.1\end{array}$ & $\begin{array}{l}4.3 \\
3.8 \\
7.8 \\
7.0\end{array}$ & $\begin{array}{l}5.2 \\
8.0 \\
5.1 \\
6.2\end{array}$ & $\begin{array}{r}1.1 \\
1.6 \\
1.2 \\
.7\end{array}$ & $\begin{array}{l}0.9 \\
1.2 \\
1.1 \\
1.2\end{array}$ & $\begin{array}{l}3.8 \\
4.2 \\
4.1 \\
3.7\end{array}$ & $\begin{array}{l}5.0 \\
3.1 \\
3.1 \\
2.4\end{array}$ & $\begin{array}{r}2.2 \\
1.2 \\
.7 \\
1.3\end{array}$ \\
\hline $\begin{array}{l}5 \\
6 \\
7 \\
8\end{array}$ & $\begin{array}{l}4.37 \\
3.79 \\
9.58 \\
1.58\end{array}$ & $\begin{array}{r}39.38 \\
46.03 \\
43.37 \\
121.97\end{array}$ & $\begin{array}{l}47.42 \\
37.78 \\
38.95 \\
67.78\end{array}$ & $\begin{array}{l}6.74 \\
7.25 \\
8.66 \\
3.96\end{array}$ & $\begin{array}{l}5.18 \\
4.03 \\
5.35 \\
2.83\end{array}$ & $\begin{array}{l}23.98 \\
21.91 \\
14.64 \\
11.93\end{array}$ & $\begin{array}{r}17.81 \\
32.52 \\
21.69 \\
5.26\end{array}$ & $\begin{array}{l}6.48 \\
5.95 \\
4.73 \\
2.23\end{array}$ & $\begin{array}{r}1.0 \\
.8 \\
1.6 \\
.3\end{array}$ & $\begin{array}{r}5.6 \\
6.8 \\
6.5 \\
11.0\end{array}$ & $\begin{array}{l}6.9 \\
6.2 \\
6.9 \\
7.9\end{array}$ & $\begin{array}{r}1.4 \\
1.5 \\
1.8 \\
.8\end{array}$ & $\begin{array}{r}1.2 \\
.9 \\
1.2 \\
.6\end{array}$ & $\begin{array}{l}3.6 \\
2.8 \\
2.5 \\
1.9\end{array}$ & $\begin{array}{r}2.7 \\
3.8 \\
2.1 \\
.9\end{array}$ & $\begin{array}{r}1.4 \\
1.0 \\
1.2 \\
.5\end{array}$ \\
\hline $\begin{array}{r}9 \\
10 \\
11 \\
12\end{array}$ & $\begin{array}{l}3.07 \\
4.82 \\
4.87 \\
4.06\end{array}$ & $\begin{array}{l}96.26 \\
76.66 \\
36.70 \\
52.51\end{array}$ & $\begin{array}{l}54.24 \\
44.35 \\
28.51 \\
38.78\end{array}$ & $\begin{array}{l}5.18 \\
5.35 \\
2.74 \\
3.38\end{array}$ & $\begin{array}{l}3.43 \\
3.29 \\
3.46 \\
3.24\end{array}$ & $\begin{array}{l}13.37 \\
10.25 \\
33.10 \\
13.51\end{array}$ & $\begin{array}{l}12.00 \\
12.36 \\
31.56 \\
11.88\end{array}$ & $\begin{array}{l}5.45 \\
6.84 \\
5.83 \\
5.50\end{array}$ & $\begin{array}{l}.7 \\
1.0 \\
1.2 \\
1.0\end{array}$ & $\begin{array}{l}9.3 \\
8.2 \\
5.8 \\
7.5\end{array}$ & $\begin{array}{l}6.6 \\
6.4 \\
5.3 \\
6.6\end{array}$ & $\begin{array}{r}1.2 \\
1.3 \\
.8 \\
1.0\end{array}$ & $\begin{array}{l}.8 \\
.9 \\
.8 \\
.9\end{array}$ & $\begin{array}{l}2.1 \\
2.1 \\
4.6 \\
3.0\end{array}$ & $\begin{array}{l}2.0 \\
2.3 \\
4.1 \\
2.6\end{array}$ & $\begin{array}{l}1.2 \\
1.5 \\
1.2 \\
1.4\end{array}$ \\
\hline $\begin{array}{l}17 \\
18 \\
19 \\
20\end{array}$ & $\begin{array}{r}3.50 \\
4.85 \\
3.41 \\
.72\end{array}$ & $\begin{array}{r}41.11 \\
35.18 \\
37.32 \\
2.64\end{array}$ & $\begin{array}{l}23.42 \\
46.66 \\
36.36 \\
17.90\end{array}$ & $\begin{array}{r}2.76 \\
5.83 \\
12.17 \\
20.18\end{array}$ & $\begin{array}{r}2.74 \\
7.03 \\
16.08 \\
30.29\end{array}$ & $\begin{array}{r}30.74 \\
30.98 \\
47.16 \\
109.75\end{array}$ & $\begin{array}{l}35.88 \\
16.44 \\
17.76 \\
42.70\end{array}$ & $\begin{array}{l}9.07 \\
3.22 \\
6.62 \\
2.76\end{array}$ & $\begin{array}{l}1.0 \\
1.2 \\
.7 \\
.1\end{array}$ & $\begin{array}{l}5.7 \\
6.0 \\
4.2 \\
.6\end{array}$ & $\begin{array}{l}4.0 \\
6.7 \\
4.9 \\
3.3\end{array}$ & $\begin{array}{r}.8 \\
1.2 \\
2.2 \\
3.2\end{array}$ & $\begin{array}{l}.7 \\
1.3 \\
2.4 \\
3.3\end{array}$ & $\begin{array}{l}4.4 \\
4.2 \\
5.3 \\
9.5\end{array}$ & $\begin{array}{l}5.1 \\
2.3 \\
2.3 \\
3.6\end{array}$ & $\begin{array}{r}1.9 \\
.8 \\
1.6 \\
.3\end{array}$ \\
\hline $\begin{array}{l}21 \\
22 \\
23 \\
24\end{array}$ & $\begin{array}{l}3.74 \\
6.14 \\
7.18 \\
1.63\end{array}$ & $\begin{array}{l}25.32 \\
44.64 \\
58.85 \\
22.46\end{array}$ & $\begin{array}{r}6.84 \\
30.38 \\
31.34 \\
28.58\end{array}$ & $\begin{array}{l}4.46 \\
5.09 \\
4.06 \\
2.18\end{array}$ & $\begin{array}{l}4.58 \\
4.08 \\
3.65 \\
2.64\end{array}$ & $\begin{array}{l}53.18 \\
17.69 \\
25.18 \\
40.30\end{array}$ & $\begin{array}{l}68.69 \\
16.63 \\
22.99 \\
49.87\end{array}$ & $\begin{array}{r}11.02 \\
7.94 \\
5.04 \\
10.25\end{array}$ & $\begin{array}{r}.9 \\
1.3 \\
1.5 \\
.5\end{array}$ & $\begin{array}{l}3.1 \\
6.8 \\
7.2 \\
3.5\end{array}$ & $\begin{array}{l}1.2 \\
5.2 \\
4.4 \\
4.7\end{array}$ & $\begin{array}{r}.9 \\
1.2 \\
.8 \\
.4\end{array}$ & $\begin{array}{r}1.1 \\
1.1 \\
.9 \\
.6\end{array}$ & $\begin{array}{l}6.4 \\
3.3 \\
4.3 \\
5.3\end{array}$ & $\begin{array}{l}8.0 \\
3.1 \\
3.6 \\
6.8\end{array}$ & $\begin{array}{l}2.1 \\
1.7 \\
1.1 \\
1.9\end{array}$ \\
\hline
\end{tabular}


Table 3.--Wind-ren and wind-chretion histogran data, October 1987-Decuber 1989--Continued

\begin{tabular}{|c|c|c|c|c|c|c|c|c|c|c|c|c|c|c|c|c|}
\hline $\begin{array}{l}\text { Day } \\
\text { of } \\
\text { nonth }\end{array}$ & $\begin{array}{l}0 \\
\text { to } \\
45\end{array}$ & $\begin{array}{c}\text { ctore } \\
45 \\
\text { to } \\
90\end{array}$ & $\begin{array}{l}\text { Wind } \\
\text { are in } \\
90 \\
\text { to } \\
135\end{array}$ & $\begin{array}{l}\text { per } \\
\text { cated } \\
135 \\
\text { to } \\
180\end{array}$ & $\begin{array}{c}\text { sector, } \\
\text { es degz } \\
180 \\
\text { to } \\
225\end{array}$ & $\begin{array}{r}\text { in } \text { ail } \\
\text { fees } \mathrm{fr} \\
225 \\
\text { to } \\
270\end{array}$ & $\begin{array}{r}\text { nort } \\
270 \\
\text { to } \\
315\end{array}$ & $\begin{array}{r}315 \\
\text { to } \\
360\end{array}$ & $\begin{array}{l}0 \\
\text { to } \\
45\end{array}$ & $\begin{array}{c}\text { ctore } \\
45 \\
\text { to } \\
90\end{array}$ & $\begin{array}{l}\text { re in } \\
90 \\
\text { to } \\
135\end{array}$ & $\begin{array}{r}\text { cerce } \\
135 \\
\text { to } \\
180\end{array}$ & $\begin{array}{l}\text { des: } \\
180 \\
\text { to } \\
225\end{array}$ & $\begin{array}{l}x, \text { in } \\
\frac{e s}{225} \\
\text { to } \\
270\end{array}$ & $\begin{array}{l}\text { oure } \\
\text { nortl } \\
270 \\
\text { to } \\
315\end{array}$ & $\begin{array}{r}315 \\
t 0 \\
360\end{array}$ \\
\hline \multicolumn{17}{|c|}{ JUI 1989} \\
\hline $\begin{array}{l}1 \\
2 \\
3 \\
4\end{array}$ & $\begin{array}{r}1.94 \\
10.06 \\
5.66 \\
4.66\end{array}$ & $\begin{array}{l}13.25 \\
45.24 \\
32.52 \\
55.49\end{array}$ & $\begin{array}{l}49.10 \\
50.23 \\
43.54 \\
32.62\end{array}$ & $\begin{array}{r}19.10 \\
9.82 \\
11.11 \\
4.70\end{array}$ & $\begin{array}{r}10.54 \\
2.83 \\
4.80 \\
6.86\end{array}$ & $\begin{array}{r}49.51 \\
7.27 \\
16.44 \\
31.87\end{array}$ & $\begin{array}{r}24.41 \\
6.10 \\
16.66 \\
19.01\end{array}$ & $\begin{array}{l}5.02 \\
4.80 \\
7.18 \\
4.49\end{array}$ & $\begin{array}{l}0.5 \\
2.0 \\
1.3 \\
1.1\end{array}$ & $\begin{array}{l}2.6 \\
7.6 \\
5.5 \\
6.4\end{array}$ & $\begin{array}{l}7.2 \\
7.6 \\
6.8 \\
4.6\end{array}$ & $\begin{array}{l}2.8 \\
1.8 \\
1.9 \\
1.2\end{array}$ & $\begin{array}{r}1.5 \\
.7 \\
1.1 \\
1.6\end{array}$ & $\begin{array}{l}5.4 \\
1.5 \\
2.9 \\
4.8\end{array}$ & $\begin{array}{l}2.8 \\
1.3 \\
2.8 \\
3.0\end{array}$ & $\begin{array}{l}0.9 \\
1.2 \\
1.5 \\
1.2\end{array}$ \\
\hline $\begin{array}{l}5 \\
6 \\
7 \\
8\end{array}$ & $\begin{array}{l}6.00 \\
4.01 \\
2.28 \\
5.95\end{array}$ & $\begin{array}{l}21.05 \\
44.45 \\
66.96 \\
78.55\end{array}$ & $\begin{array}{l}34.75 \\
37.08 \\
47.64 \\
48.29\end{array}$ & $\begin{array}{r}10.66 \\
6.96 \\
10.49 \\
9.96\end{array}$ & $\begin{array}{l}4.49 \\
3.36 \\
7.54 \\
5.74\end{array}$ & $\begin{array}{r}18.74 \\
19.44 \\
16.66 \\
7.37\end{array}$ & $\begin{array}{r}15.96 \\
19.68 \\
9.91 \\
5.74\end{array}$ & $\begin{array}{l}9.58 \\
9.17 \\
5.64 \\
6.19\end{array}$ & $\begin{array}{l}1.6 \\
1.0 \\
.6 \\
1.1\end{array}$ & $\begin{array}{l}4.2 \\
6.6 \\
6.9 \\
8.7\end{array}$ & $\begin{array}{l}5.6 \\
5.7 \\
6.1 \\
6.6\end{array}$ & $\begin{array}{l}2.0 \\
1.2 \\
1.8 \\
1.9\end{array}$ & $\begin{array}{r}1.2 \\
.8 \\
1.6 \\
1.4\end{array}$ & $\begin{array}{l}3.7 \\
3.3 \\
3.2 \\
1.5\end{array}$ & $\begin{array}{l}3.3 \\
3.3 \\
2.1 \\
1.2\end{array}$ & $\begin{array}{l}2.2 \\
1.8 \\
1.5 \\
1.3\end{array}$ \\
\hline $\begin{array}{r}9 \\
10 \\
11 \\
12\end{array}$ & $\begin{array}{l}4.99 \\
4.73 \\
4.27 \\
2.38\end{array}$ & $\begin{array}{r}115.92 \\
64.82 \\
101.02 \\
99.29\end{array}$ & $\begin{array}{l}67.85 \\
34.92 \\
53.59 \\
63.17\end{array}$ & $\begin{array}{l}3.96 \\
4.75 \\
3.17 \\
4.80\end{array}$ & $\begin{array}{l}4.70 \\
3.48 \\
1.85 \\
2.18\end{array}$ & $\begin{array}{r}10.30 \\
13.97 \\
2.30 \\
6.89\end{array}$ & $\begin{array}{r}5.40 \\
19.56 \\
2.66 \\
4.42\end{array}$ & $\begin{array}{l}2.23 \\
8.62 \\
1.70 \\
1.34\end{array}$ & $\begin{array}{r}.8 \\
1.1 \\
.9 \\
.7\end{array}$ & $\begin{array}{r}11.3 \\
7.4 \\
12.0 \\
10.0\end{array}$ & $\begin{array}{l}7.3 \\
5.9 \\
7.6 \\
8.4\end{array}$ & $\begin{array}{r}.7 \\
1.0 \\
.9 \\
1.1\end{array}$ & $\begin{array}{l}.9 \\
.8 \\
.6 \\
.6\end{array}$ & $\begin{array}{r}1.6 \\
2.5 \\
.6 \\
1.6\end{array}$ & $\begin{array}{r}.9 \\
3.3 \\
.7 \\
.9\end{array}$ & $\begin{array}{r}.4 \\
1.8 \\
.5 \\
.4\end{array}$ \\
\hline $\begin{array}{l}17 \\
18 \\
19 \\
20\end{array}$ & $\begin{array}{l}1.68 \\
4.10 \\
5.47 \\
4.61\end{array}$ & $\begin{array}{r}27.72 \\
35.83 \\
45.50 \\
100.42\end{array}$ & $\begin{array}{l}34.73 \\
33.12 \\
49.30 \\
47.62\end{array}$ & $\begin{array}{l}3.96 \\
6.96 \\
5.74 \\
2.83\end{array}$ & $\begin{array}{l}3.86 \\
4.13 \\
6.19 \\
2.93\end{array}$ & $\begin{array}{l}54.50 \\
18.26 \\
22.63 \\
11.26\end{array}$ & $\begin{array}{r}58.15 \\
12.96 \\
13.97 \\
7.99\end{array}$ & $\begin{array}{l}6.70 \\
5.83 \\
4.75 \\
3.02\end{array}$ & $\begin{array}{l}.5 \\
1.0 \\
1.0 \\
1.0\end{array}$ & $\begin{array}{r}5.3 \\
7.2 \\
6.1 \\
10.9\end{array}$ & $\begin{array}{l}5.9 \\
6.3 \\
6.5 \\
6.1\end{array}$ & $\begin{array}{r}.6 \\
1.4 \\
1.2 \\
.7\end{array}$ & $\begin{array}{r}.5 \\
.9 \\
1.4 \\
.6\end{array}$ & $\begin{array}{l}4.9 \\
3.3 \\
3.7 \\
2.2\end{array}$ & $\begin{array}{l}5.1 \\
2.3 \\
2.7 \\
1.5\end{array}$ & $\begin{array}{r}.9 \\
1.2 \\
1.2 \\
.7\end{array}$ \\
\hline $\begin{array}{l}21 \\
22 \\
23 \\
24\end{array}$ & $\begin{array}{l}6.60 \\
7.27 \\
3.62 \\
4.75\end{array}$ & $\begin{array}{r}117.14 \\
54.60 \\
55.22 \\
42.65\end{array}$ & $\begin{array}{l}55.46 \\
37.73 \\
42.02 \\
40.97\end{array}$ & $\begin{array}{l}3.05 \\
6.31 \\
5.95 \\
5.26\end{array}$ & $\begin{array}{l}2.33 \\
2.71 \\
5.81 \\
3.05\end{array}$ & $\begin{array}{r}8.76 \\
19.49 \\
21.22 \\
8.71\end{array}$ & $\begin{array}{r}6.82 \\
16.94 \\
14.06 \\
8.78\end{array}$ & $\begin{array}{l}2.42 \\
7.70 \\
2.95 \\
4.94\end{array}$ & $\begin{array}{l}1.1 \\
1.3 \\
1.0 \\
1.4\end{array}$ & $\begin{array}{r}11.8 \\
7.2 \\
7.1 \\
6.4\end{array}$ & $\begin{array}{l}6.9 \\
6.0 \\
6.4 \\
7.2\end{array}$ & $\begin{array}{r}.7 \\
1.4 \\
1.5 \\
1.5\end{array}$ & $\begin{array}{r}.5 \\
.7 \\
1.5 \\
1.0\end{array}$ & $\begin{array}{l}1.3 \\
2.8 \\
3.5 \\
2.5\end{array}$ & $\begin{array}{l}1.1 \\
2.8 \\
2.2 \\
2.4\end{array}$ & $\begin{array}{r}.5 \\
1.6 \\
.7 \\
1.4\end{array}$ \\
\hline
\end{tabular}


Table 3.--Wind-run and wind-duration histogra data, october 1987-Documbr 1989--Continued

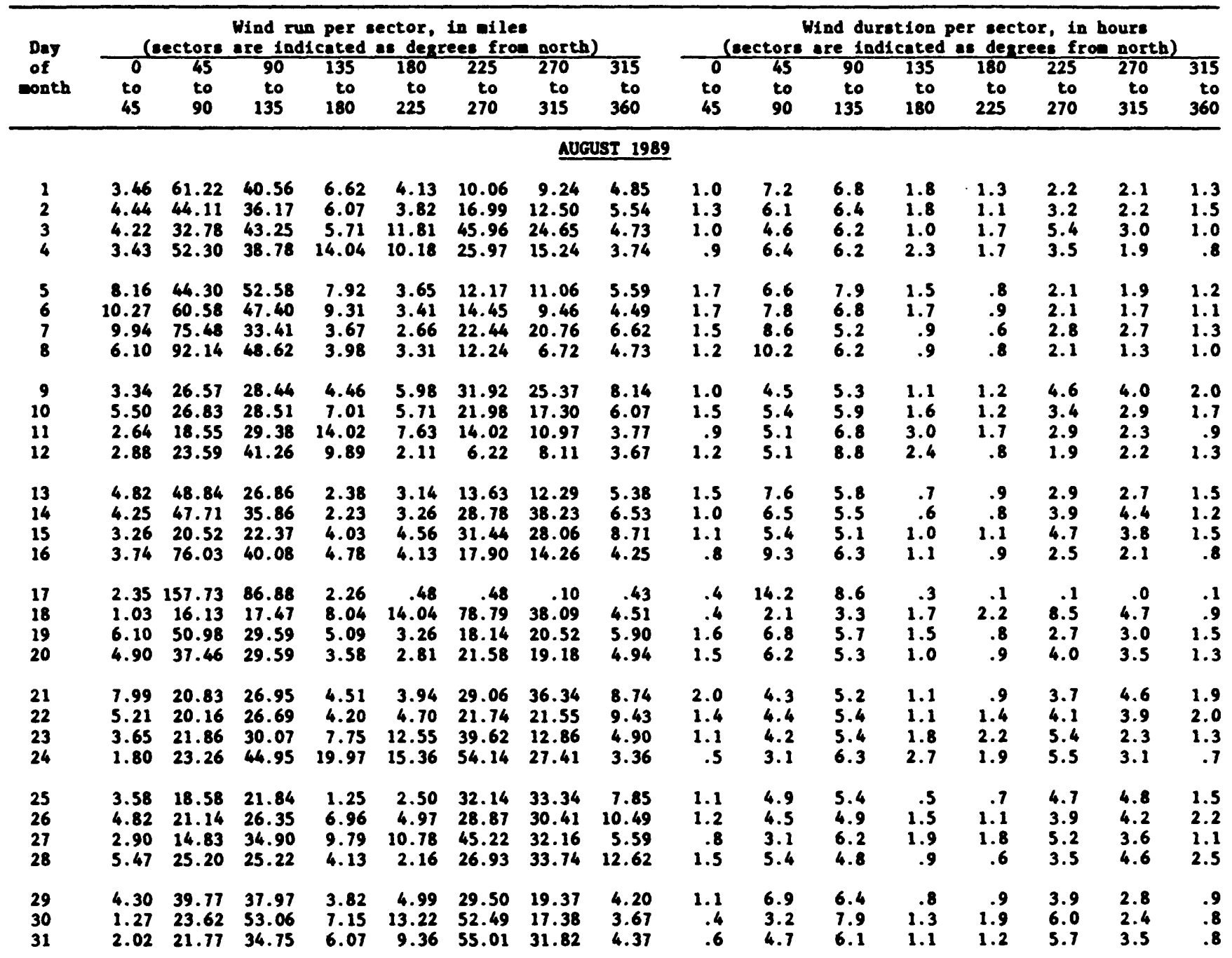


Table 3.--Wind-run and ind-curation histogran data, October 1987-Decanber 1989--Continued

\begin{tabular}{|c|c|c|c|c|c|c|c|c|c|c|c|c|c|c|c|c|}
\hline $\begin{array}{l}\text { Day } \\
\text { of } \\
\text { eonth }\end{array}$ & $\begin{array}{l}0 \\
0 \\
\text { to } \\
45\end{array}$ & $\begin{array}{c}\text { ectors } \\
45 \\
\text { to } \\
90\end{array}$ & $\begin{array}{l}\begin{array}{l}\text { Wind ru } \\
\text { are ind }\end{array} \\
90 \\
\text { to } \\
135\end{array}$ & $\begin{array}{r}\text { on per } \\
\text { licated } \\
135 \\
\text { to } \\
180\end{array}$ & $\begin{array}{l}\text { ector, } \\
\text { as degr } \\
180 \\
\text { to } \\
225\end{array}$ & $\begin{array}{r}\text { in oile } \\
\text { rees fro } \\
225 \\
\text { to } \\
270\end{array}$ & $\begin{array}{l}\text { north } \\
270 \\
\text { to } \\
315\end{array}$ & $\begin{array}{r}315 \\
\text { to } \\
360\end{array}$ & $\begin{array}{r}0 \\
\text { to } \\
45\end{array}$ & $\begin{array}{c}\text { ctors } \\
45 \\
\text { to } \\
90\end{array}$ & $\begin{array}{r}\text { ad du } \\
\text { ire in } \\
90 \\
\text { to } \\
135\end{array}$ & $\begin{array}{r}135 \\
\text { to } \\
180\end{array}$ & $\begin{array}{r}x \text { sec } \\
8 \text { dep } \\
180 \\
\text { to } \\
225\end{array}$ & $\begin{array}{r}r \text { in } \\
\frac{e s \text { fr }}{225} \\
\text { to } \\
270\end{array}$ & $\begin{array}{c}\text { 20urs } \\
\frac{\text { nort }}{270} \\
\text { to } \\
315\end{array}$ & $\begin{array}{r}315 \\
\text { to } \\
360\end{array}$ \\
\hline \multicolumn{17}{|c|}{ SEPTEMRER 1989} \\
\hline $\begin{array}{l}1 \\
2 \\
3 \\
4\end{array}$ & $\begin{array}{l}4.51 \\
2.78 \\
2.74 \\
2.64\end{array}$ & $\begin{array}{l}39.17 \\
17.11 \\
22.56 \\
31.34\end{array}$ & $\begin{array}{l}39.91 \\
32.33 \\
29.90 \\
44.30\end{array}$ & $\begin{array}{r}7.22 \\
11.93 \\
5.69 \\
6.00\end{array}$ & $\begin{array}{r}3.70 \\
11.90 \\
4.70 \\
3.31\end{array}$ & $\begin{array}{l}29.76 \\
61.97 \\
51.41 \\
23.02\end{array}$ & $\begin{array}{l}20.59 \\
37.61 \\
57.10 \\
28.01\end{array}$ & $\begin{array}{l}5.64 \\
2.38 \\
8.40 \\
6.55\end{array}$ & $\begin{array}{r}1.2 \\
.8 \\
.8 \\
.7\end{array}$ & $\begin{array}{l}6.2 \\
3.8 \\
4.3 \\
6.4\end{array}$ & $\begin{array}{l}6.1 \\
5.7 \\
5.5 \\
7.4\end{array}$ & $\begin{array}{l}1.5 \\
2.2 \\
1.2 \\
1.2\end{array}$ & $\begin{array}{r}0.8 \\
1.8 \\
.8 \\
.7\end{array}$ & $\begin{array}{l}3.9 \\
5.9 \\
4.7 \\
2.9\end{array}$ & $\begin{array}{l}2.8 \\
3.2 \\
5.0 \\
3.4\end{array}$ & $\begin{array}{l}1.2 \\
.5 \\
1.4 \\
1.1\end{array}$ \\
\hline $\begin{array}{l}5 \\
6 \\
7 \\
8\end{array}$ & $\begin{array}{r}3.77 \\
.79 \\
.07 \\
3.31\end{array}$ & $\begin{array}{r}17.02 \\
37.61 \\
7.06 \\
14.23\end{array}$ & $\begin{array}{l}49.37 \\
67.70 \\
79.70 \\
26.09\end{array}$ & $\begin{array}{r}13.30 \\
17.81 \\
15.89 \\
9.58\end{array}$ & $\begin{array}{r}10.75 \\
13.30 \\
17.76 \\
6.98\end{array}$ & $\begin{array}{l}33.79 \\
45.24 \\
51.48 \\
33.50\end{array}$ & $\begin{array}{l}12.17 \\
12.58 \\
11.42 \\
23.11\end{array}$ & $\begin{array}{r}3.82 \\
.91 \\
.34 \\
5.88\end{array}$ & $\begin{array}{r}1.2 \\
.1 \\
.0 \\
1.0\end{array}$ & $\begin{array}{l}4.0 \\
3.9 \\
1.1 \\
2.9\end{array}$ & $\begin{array}{r}8.2 \\
9.6 \\
11.8 \\
4.6\end{array}$ & $\begin{array}{l}2.2 \\
2.7 \\
2.2 \\
1.8\end{array}$ & $\begin{array}{l}1.5 \\
1.7 \\
2.2 \\
1.4\end{array}$ & $\begin{array}{l}4.0 \\
4.3 \\
5.4 \\
6.0\end{array}$ & $\begin{array}{l}1.8 \\
1.3 \\
1.2 \\
4.4\end{array}$ & $\begin{array}{r}1.0 \\
.1 \\
.0 \\
1.5\end{array}$ \\
\hline $\begin{array}{r}9 \\
10 \\
11 \\
12\end{array}$ & $\begin{array}{l}3.43 \\
3.07 \\
1.37 \\
2.66\end{array}$ & $\begin{array}{r}8.26 \\
16.70 \\
40.82 \\
60.67\end{array}$ & $\begin{array}{l}12.14 \\
20.30 \\
39.19 \\
34.66\end{array}$ & $\begin{array}{l}3.12 \\
2.78 \\
2.02 \\
3.00\end{array}$ & $\begin{array}{l}4.25 \\
3.05 \\
1.73 \\
1.18\end{array}$ & $\begin{array}{r}27.62 \\
35.45 \\
22.01 \\
6.29\end{array}$ & $\begin{array}{r}36.43 \\
46.06 \\
29.52 \\
9.24\end{array}$ & $\begin{array}{r}10.30 \\
10.68 \\
5.93 \\
4.75\end{array}$ & $\begin{array}{l}.9 \\
.9 \\
.4 \\
.8\end{array}$ & $\begin{array}{l}1.7 \\
3.6 \\
6.2 \\
7.5\end{array}$ & $\begin{array}{l}2.6 \\
4.2 \\
6.6 \\
7.0\end{array}$ & $\begin{array}{r}1.0 \\
.8 \\
.5 \\
1.2\end{array}$ & $\begin{array}{r}1.5 \\
.8 \\
.5 \\
.6\end{array}$ & $\begin{array}{l}5.9 \\
4.9 \\
3.7 \\
2.1\end{array}$ & $\begin{array}{l}7.3 \\
6.5 \\
4.6 \\
2.8\end{array}$ & $\begin{array}{l}2.7 \\
2.0 \\
1.3 \\
1.8\end{array}$ \\
\hline $\begin{array}{l}17 \\
18 \\
19 \\
20\end{array}$ & $\begin{array}{l}3.34 \\
2.04 \\
5.62 \\
3.02\end{array}$ & $\begin{array}{l}27.29 \\
26.30 \\
23.18 \\
47.93\end{array}$ & $\begin{array}{l}46.15 \\
35.52 \\
22.20 \\
22.25\end{array}$ & $\begin{array}{r}16.80 \\
6.82 \\
3.53 \\
2.69\end{array}$ & $\begin{array}{r}14.88 \\
4.99 \\
3.38 \\
3.67\end{array}$ & $\begin{array}{l}41.93 \\
34.51 \\
17.02 \\
32.66\end{array}$ & $\begin{array}{r}7.44 \\
39.38 \\
18.31 \\
45.41\end{array}$ & $\begin{array}{r}1.99 \\
6.91 \\
10.61 \\
10.63\end{array}$ & $\begin{array}{r}.9 \\
.6 \\
1.7 \\
.6\end{array}$ & $\begin{array}{l}4.3 \\
4.8 \\
5.4 \\
5.4\end{array}$ & $\begin{array}{l}7.3 \\
6.8 \\
4.9 \\
3.3\end{array}$ & $\begin{array}{l}2.7 \\
1.3 \\
1.0 \\
.8\end{array}$ & $\begin{array}{r}2.2 \\
.7 \\
1.1 \\
.8\end{array}$ & $\begin{array}{l}4.7 \\
3.9 \\
3.5 \\
4.5\end{array}$ & $\begin{array}{l}1.1 \\
4.7 \\
3.6 \\
6.2\end{array}$ & $\begin{array}{r}.6 \\
1.1 \\
2.5 \\
2.1\end{array}$ \\
\hline $\begin{array}{l}21 \\
22 \\
23 \\
24\end{array}$ & $\begin{array}{r}.94 \\
8.26 \\
3.50 \\
1.63\end{array}$ & $\begin{array}{r}6.58 \\
94.56 \\
60.05 \\
23.42\end{array}$ & $\begin{array}{l}11.14 \\
83.42 \\
35.40 \\
54.86\end{array}$ & $\begin{array}{l}.62 \\
4.44 \\
1.61 \\
9.00\end{array}$ & $\begin{array}{l}2.06 \\
1.06 \\
2.38 \\
2.88\end{array}$ & $\begin{array}{r}32.90 \\
.89 \\
23.14 \\
30.05\end{array}$ & $\begin{array}{r}48.29 \\
1.03 \\
34.20 \\
24.48\end{array}$ & $\begin{array}{r}10.87 \\
1.08 \\
10.03 \\
3.62\end{array}$ & $\begin{array}{r}.4 \\
1.3 \\
.8 \\
.5\end{array}$ & $\begin{array}{r}1.6 \\
10.4 \\
6.9 \\
4.9\end{array}$ & $\begin{array}{r}2.5 \\
10.1 \\
4.9 \\
8.8\end{array}$ & $\begin{array}{r}.3 \\
.7 \\
.4 \\
1.4\end{array}$ & $\begin{array}{l}.6 \\
.3 \\
.5 \\
.6\end{array}$ & $\begin{array}{r}6.2 \\
.3 \\
3.3 \\
3.9\end{array}$ & $\begin{array}{l}9.2 \\
.4 \\
4.8 \\
3.0\end{array}$ & $\begin{array}{r}2.8 \\
.3 \\
2.1 \\
.7\end{array}$ \\
\hline
\end{tabular}


Table 3.--Wind-rm and wind-duration histogran data, october 1987-Decubur 1989--Continued

\begin{tabular}{|c|c|c|c|c|c|c|c|c|c|c|c|c|c|c|c|c|}
\hline $\begin{array}{l}\text { Day } \\
\text { of } \\
\text { conth }\end{array}$ & $\begin{array}{c}\text { (a } \\
0 \\
40 \\
45\end{array}$ & $\begin{array}{c}\text { ectore } \\
45 \\
\text { to } \\
90\end{array}$ & $\begin{array}{r}\text { Wind ro } \\
\text { are Ind } \\
90 \\
\text { to } \\
135\end{array}$ & $\begin{array}{c}\text { per } \\
\text { cated }\end{array}$ & $\begin{array}{l}\text { ector, } \\
\text { as des } \\
180 \\
\text { to } \\
225\end{array}$ & $\begin{array}{r}\text { In ail } \\
\text { ees fre } \\
225 \\
20 \\
270\end{array}$ & $\begin{array}{l}\text { nortb } \\
270 \\
\text { to } \\
315\end{array}$ & $\begin{array}{r}315 \\
t 0 \\
360\end{array}$ & $\begin{array}{l}0 \\
\text { to } \\
45\end{array}$ & $\begin{array}{c}\text { ctors } \\
45 \\
\text { to } \\
90\end{array}$ & $\begin{array}{r}\text { ind du } \\
\text { are in } \\
90 \\
\text { to } \\
135\end{array}$ & $\begin{array}{l}\text { tion } \\
\text { cated } \\
135 \\
\text { to } \\
180\end{array}$ & $\begin{array}{r}r \text { sec } \\
18 \text { des } \\
180 \\
\text { to } \\
225\end{array}$ & $\begin{array}{r}\text { in } \\
\frac{\text { in }}{225} \\
\text { to } \\
270\end{array}$ & $\begin{array}{l}\text { oure } \\
\text { nort } \\
270 \\
\text { to } \\
315\end{array}$ & $\begin{array}{r}315 \\
\text { to } \\
360\end{array}$ \\
\hline \multicolumn{17}{|c|}{ OCTOBER 1989} \\
\hline $\begin{array}{l}1 \\
2 \\
3 \\
4\end{array}$ & $\begin{array}{l}2.26 \\
2.59 \\
1.15 \\
2.40\end{array}$ & $\begin{array}{r}13.87 \\
28.97 \\
19.87 \\
8.28\end{array}$ & $\begin{array}{l}19.78 \\
42.38 \\
48.77 \\
18.36\end{array}$ & $\begin{array}{r}4.49 \\
15.26 \\
37.51 \\
13.06\end{array}$ & $\begin{array}{r}3.60 \\
17.47 \\
22.54 \\
14.62\end{array}$ & $\begin{array}{l}47.52 \\
49.70 \\
36.48 \\
58.85\end{array}$ & $\begin{array}{r}59.78 \\
11.47 \\
5.64 \\
40.03\end{array}$ & $\begin{array}{r}11.30 \\
3.36 \\
.72 \\
8.54\end{array}$ & $\begin{array}{r}0.6 \\
.7 \\
.3 \\
.7\end{array}$ & $\begin{array}{l}2.6 \\
3.7 \\
3.0 \\
2.0\end{array}$ & $\begin{array}{l}4.0 \\
6.4 \\
7.8 \\
3.5\end{array}$ & $\begin{array}{l}1.0 \\
2.7 \\
5.4 \\
1.8\end{array}$ & $\begin{array}{l}0.6 \\
2.5 \\
3.0 \\
1.9\end{array}$ & $\begin{array}{l}5.8 \\
5.4 \\
3.6 \\
6.9\end{array}$ & $\begin{array}{r}7.3 \\
1.7 \\
.7 \\
5.2\end{array}$ & $\begin{array}{r}1.8 \\
.8 \\
.2 \\
1.8\end{array}$ \\
\hline $\begin{array}{l}5 \\
6 \\
7 \\
8\end{array}$ & $\begin{array}{l}2.64 \\
4.42 \\
1.30 \\
2.30\end{array}$ & $\begin{array}{l}31.92 \\
25.97 \\
30.62 \\
26.42\end{array}$ & $\begin{array}{l}42.96 \\
34.25 \\
39.50 \\
43.87\end{array}$ & $\begin{array}{l}3.17 \\
5.42 \\
4.44 \\
5.62\end{array}$ & $\begin{array}{l}1.56 \\
3.07 \\
2.14 \\
1.68\end{array}$ & $\begin{array}{l}19.63 \\
22.13 \\
28.75 \\
21.84\end{array}$ & $\begin{array}{l}20.28 \\
35.45 \\
31.63 \\
24.36\end{array}$ & $\begin{array}{l}5.04 \\
8.83 \\
5.98 \\
8.71\end{array}$ & $\begin{array}{r}.7 \\
1.3 \\
.4 \\
.7\end{array}$ & $\begin{array}{l}6.8 \\
5.5 \\
6.0 \\
5.9\end{array}$ & $\begin{array}{l}8.3 \\
6.0 \\
7.1 \\
7.5\end{array}$ & $\begin{array}{l}.7 \\
1.2 \\
1.0 \\
1.0\end{array}$ & $\begin{array}{l}.5 \\
.8 \\
.5 \\
.4\end{array}$ & $\begin{array}{l}2.9 \\
3.0 \\
3.7 \\
3.1\end{array}$ & $\begin{array}{l}3.1 \\
4.2 \\
4.1 \\
3.5\end{array}$ & $\begin{array}{l}1.0 \\
1.7 \\
1.1 \\
1.8\end{array}$ \\
\hline $\begin{array}{r}9 \\
10 \\
11 \\
12\end{array}$ & $\begin{array}{l}1.44 \\
2.90 \\
1.99 \\
4.22\end{array}$ & $\begin{array}{l}36.98 \\
27.70 \\
26.83 \\
29.81\end{array}$ & $\begin{array}{l}46.15 \\
52.73 \\
38.21 \\
30.14\end{array}$ & $\begin{array}{l}8.30 \\
8.76 \\
1.78 \\
3.02\end{array}$ & $\begin{array}{l}2.11 \\
2.42 \\
3.60 \\
2.06\end{array}$ & $\begin{array}{l}23.52 \\
23.93 \\
42.96 \\
20.83\end{array}$ & $\begin{array}{l}25.25 \\
28.08 \\
60.77 \\
29.50\end{array}$ & $\begin{array}{r}4.42 \\
6.77 \\
8.78 \\
11.50\end{array}$ & $\begin{array}{r}.4 \\
.8 \\
.6 \\
1.2\end{array}$ & $\begin{array}{l}7.4 \\
5.3 \\
5.3 \\
5.9\end{array}$ & $\begin{array}{l}7.4 \\
8.3 \\
6.7 \\
5.7\end{array}$ & $\begin{array}{r}1.2 \\
1.4 \\
.4 \\
.8\end{array}$ & $\begin{array}{l}.5 \\
.6 \\
.5 \\
.6\end{array}$ & $\begin{array}{l}3.0 \\
2.8 \\
3.8 \\
3.0\end{array}$ & $\begin{array}{l}3.2 \\
3.4 \\
5.2 \\
4.2\end{array}$ & $\begin{array}{l}1.3 \\
1.2 \\
2.3\end{array}$ \\
\hline $\begin{array}{l}13 \\
14 \\
15 \\
16\end{array}$ & $\begin{array}{l}5.23 \\
2.88 \\
3.05 \\
2.02\end{array}$ & $\begin{array}{r}26.06 \\
22.34 \\
9.29 \\
18.12\end{array}$ & $\begin{array}{l}33.29 \\
46.49 \\
22.63 \\
13.06\end{array}$ & $\begin{array}{r}7.87 \\
13.06 \\
5.54 \\
2.35\end{array}$ & $\begin{array}{l}2.62 \\
3.31 \\
2.50 \\
2.02\end{array}$ & $\begin{array}{l}19.54 \\
19.70 \\
14.98 \\
17.23\end{array}$ & $\begin{array}{l}30.17 \\
22.22 \\
22.13 \\
28.66\end{array}$ & $\begin{array}{l}8.52 \\
5.40 \\
8.28 \\
6.89\end{array}$ & $\begin{array}{r}1.5 \\
.8 \\
1.2 \\
.8\end{array}$ & $\begin{array}{l}5.7 \\
5.0 \\
3.2 \\
4.0\end{array}$ & $\begin{array}{l}5.8 \\
8.0 \\
5.7 \\
3.0\end{array}$ & $\begin{array}{r}1.4 \\
2.2 \\
1.6 \\
.9\end{array}$ & $\begin{array}{l}.7 \\
.7 \\
.8 \\
.9\end{array}$ & $\begin{array}{l}2.7 \\
2.8 \\
3.8 \\
5.0\end{array}$ & $\begin{array}{l}4.1 \\
3.2 \\
5.0 \\
7.0\end{array}$ & $\begin{array}{l}1.9 \\
1.1 \\
2.4 \\
2.0\end{array}$ \\
\hline $\begin{array}{l}17 \\
18 \\
19 \\
20\end{array}$ & $\begin{array}{l}5.16 \\
4.46 \\
2.23 \\
6.82\end{array}$ & $\begin{array}{l}26.59 \\
26.66 \\
98.16 \\
27.89\end{array}$ & $\begin{array}{l}29.16 \\
38.47 \\
50.40 \\
28.87\end{array}$ & $\begin{array}{l}8.54 \\
6.19 \\
5.11 \\
4.37\end{array}$ & $\begin{array}{l}2.88 \\
4.01 \\
3.41 \\
4.37\end{array}$ & $\begin{array}{r}17.76 \\
10.80 \\
7.66 \\
10.13\end{array}$ & $\begin{array}{r}15.29 \\
9.86 \\
7.42 \\
14.23\end{array}$ & $\begin{array}{l}4.44 \\
5.30 \\
3.05 \\
9.50\end{array}$ & $\begin{array}{r}1.6 \\
1.4 \\
.7 \\
1.8\end{array}$ & $\begin{array}{l}6.9 \\
6.0 \\
9.9 \\
5.5\end{array}$ & $\begin{array}{l}6.4 \\
7.4 \\
6.6 \\
5.7\end{array}$ & $\begin{array}{l}1.8 \\
1.5 \\
1.2 \\
1.2\end{array}$ & $\begin{array}{r}.8 \\
1.2 \\
.9 \\
1.2\end{array}$ & $\begin{array}{l}2.7 \\
2.4 \\
1.8 \\
2.4\end{array}$ & $\begin{array}{l}2.3 \\
2.2 \\
1.7 \\
3.2\end{array}$ & $\begin{array}{r}1.5 \\
.9 \\
2.5\end{array}$ \\
\hline $\begin{array}{l}21 \\
22 \\
23 \\
24\end{array}$ & $\begin{array}{l}5.11 \\
4.25 \\
3.19 \\
4.87\end{array}$ & $\begin{array}{l}19.34 \\
16.49 \\
20.83 \\
25.27\end{array}$ & $\begin{array}{l}29.02 \\
20.54 \\
48.22 \\
51.46\end{array}$ & $\begin{array}{l}6.67 \\
2.52 \\
8.74 \\
6.26\end{array}$ & $\begin{array}{l}9.58 \\
2.28 \\
3.67 \\
6.02\end{array}$ & $\begin{array}{l}27.60 \\
15.77 \\
19.75 \\
21.14\end{array}$ & $\begin{array}{r}8.78 \\
23.26 \\
16.13 \\
13.49\end{array}$ & $\begin{array}{l}2.50 \\
9.67 \\
6.48 \\
7.20\end{array}$ & $\begin{array}{l}1.8 \\
1.7 \\
1.0 \\
1.2\end{array}$ & $\begin{array}{l}5.3 \\
4.4 \\
4.5 \\
4.6\end{array}$ & $\begin{array}{l}6.7 \\
5.0 \\
8.1 \\
8.1\end{array}$ & $\begin{array}{r}1.5 \\
.9 \\
1.5 \\
1.4\end{array}$ & $\begin{array}{r}1.7 \\
.8 \\
1.0 \\
1.3\end{array}$ & $\begin{array}{l}3.9 \\
3.5 \\
3.2 \\
3.3\end{array}$ & $\begin{array}{l}1.8 \\
4.8 \\
2.8 \\
2.3\end{array}$ & $\begin{array}{l}1 . \\
2 . \\
1 . \\
1 .\end{array}$ \\
\hline $\begin{array}{l}25 \\
26 \\
27 \\
28\end{array}$ & $\begin{array}{r}.94 \\
.77 \\
3.41 \\
1.94\end{array}$ & $\begin{array}{r}82.73 \\
3.94 \\
37.92 \\
8.23\end{array}$ & $\begin{array}{l}72.26 \\
12.74 \\
77.86 \\
30.22\end{array}$ & $\begin{array}{r}20.64 \\
6.12 \\
12.50 \\
9.36\end{array}$ & $\begin{array}{r}9.48 \\
10.06 \\
1.44 \\
3.29\end{array}$ & $\begin{array}{r}14.93 \\
48.70 \\
3.34 \\
38.21\end{array}$ & $\begin{array}{r}2.21 \\
48.00 \\
2.11 \\
66.89\end{array}$ & $\begin{array}{r}.46 \\
10.08 \\
1.10 \\
6.50\end{array}$ & $\begin{array}{r}.3 \\
.2 \\
1.2 \\
.6\end{array}$ & $\begin{array}{l}7.8 \\
1.4 \\
6.7 \\
2.1\end{array}$ & $\begin{array}{r}9.1 \\
3.3 \\
11.5 \\
5.8\end{array}$ & $\begin{array}{l}2.8 \\
1.6 \\
2.0 \\
1.7\end{array}$ & $\begin{array}{r}1.4 \\
1.5 \\
.5 \\
.9\end{array}$ & $\begin{array}{l}1.9 \\
6.5 \\
1.0 \\
4.5\end{array}$ & $\begin{array}{r}.4 \\
7.1 \\
.6 \\
7.1\end{array}$ & 2. \\
\hline $\begin{array}{l}29 \\
30 \\
31\end{array}$ & $\begin{array}{r}1.92 \\
.84 \\
1.85\end{array}$ & $\begin{array}{r}3.84 \\
25.18 \\
10.87\end{array}$ & $\begin{array}{l}14.40 \\
29.30 \\
26.64\end{array}$ & $\begin{array}{l}1.73 \\
2.81 \\
8.45\end{array}$ & $\begin{array}{l}2.33 \\
3.31 \\
1.99\end{array}$ & $\begin{array}{l}31.90 \\
18.60 \\
21.74\end{array}$ & $\begin{array}{l}36.29 \\
17.83 \\
23.64\end{array}$ & $\begin{array}{l}5.59 \\
4.87 \\
5.16\end{array}$ & $\begin{array}{l}.8 \\
.3 \\
.7\end{array}$ & $\begin{array}{l}1.6 \\
5.9 \\
3.3\end{array}$ & $\begin{array}{l}5.1 \\
7.7 \\
6.3\end{array}$ & $\begin{array}{l}.9 \\
1.1 \\
1.8\end{array}$ & $\begin{array}{r}1.1 \\
.9 \\
.7\end{array}$ & $\begin{array}{l}6.0 \\
3.8 \\
4.9\end{array}$ & $\begin{array}{l}6.6 \\
3.1 \\
4.6\end{array}$ & 1.2 \\
\hline
\end{tabular}


Table 3.--Wind-run and wind-curation histogren date, octobor 1987-Docamber 1989--Continued

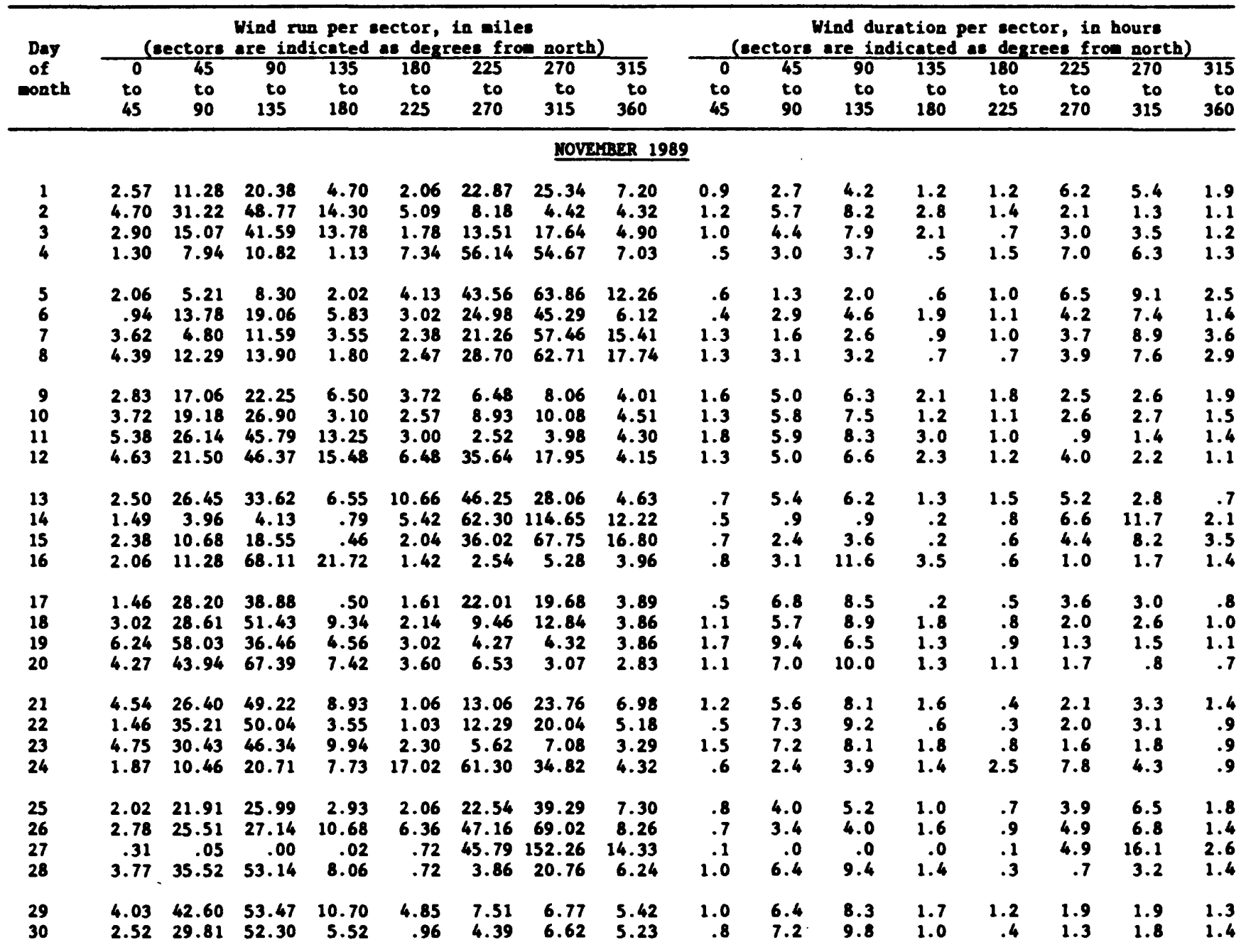


Table 3.--Wind-run and wind-curation histogren data, Octobor 1987-Decamber 1989--Continued

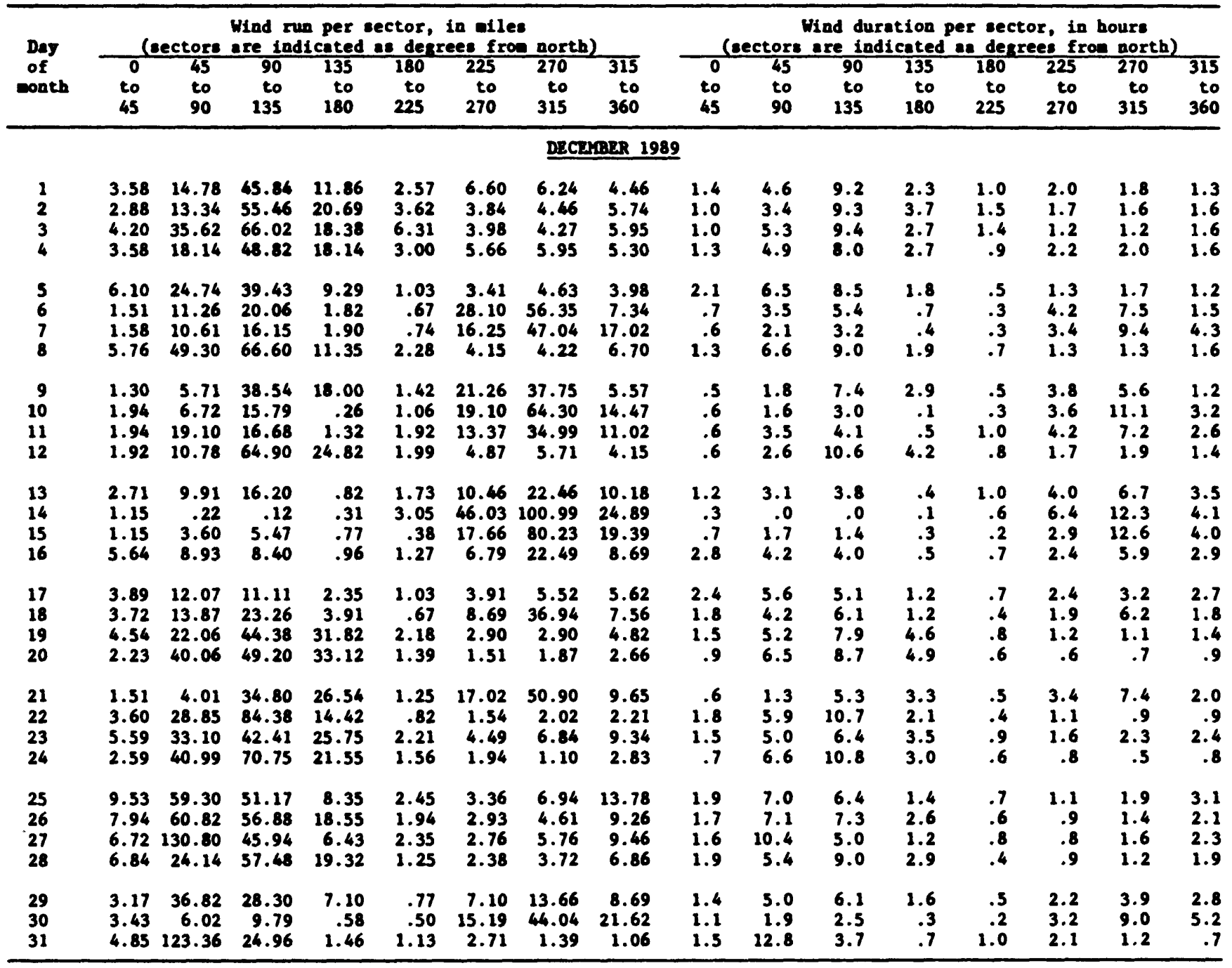


Table 4.--Snow-course information for 1988 and 1989 at the Spring Creek, Cow Camp Creek, and soil-water control sites

[All data are in inches; --, no data]

\begin{tabular}{|c|c|c|c|c|c|c|}
\hline \multirow[b]{2}{*}{ Date } & \multicolumn{2}{|c|}{ Spring Creek } & Cow Camp & \multirow{2}{*}{ 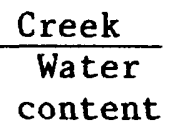 } & \multicolumn{2}{|c|}{ Soil-water control } \\
\hline & $\begin{array}{l}\text { Snow } \\
\text { depth }\end{array}$ & $\begin{array}{c}\text { Water } \\
\text { content }\end{array}$ & $\begin{array}{l}\text { Snow } \\
\text { depth }\end{array}$ & & $\begin{array}{l}\text { Snow } \\
\text { depth }\end{array}$ & $\begin{array}{l}\text { Water } \\
\text { content }\end{array}$ \\
\hline $01-26-88$ & 17.2 & 4.5 & -- & -- & -- & -- \\
\hline $01-27-88$ & -- & -- & 17.9 & 4.4 & -- & -- \\
\hline $01-28-88$ & -- & -- & -- & -- & 17.9 & 4.5 \\
\hline $02-29-88$ & .0 & .0 & 10.8 & 3.2 & -- & -- \\
\hline $03-01-88$ & -- & -- & -- & -- & 12.1 & 4.0 \\
\hline $01-30-89$ & 6.1 & 2.0 & -- & -- & -- & -- \\
\hline $01-31-89$ & -- & -- & 11.9 & 3.0 & 15.4 & 3.4 \\
\hline $03-02-89$ & 5.2 & 1.6 & 14.0 & 4.8 & 18.6 & 5.8 \\
\hline
\end{tabular}


Table 5.--Soil-water content at the Spring Creek site during 1988 and 1989

[Contents in percent by volume; --, no measurement]

\begin{tabular}{|c|c|c|c|c|c|c|c|}
\hline \multirow{2}{*}{$\begin{array}{c}\text { Date of } \\
\text { measurement }\end{array}$} & \multirow{2}{*}{$\begin{array}{l}\text { Depth of } \\
\text { measure- } \\
\text { ment } \\
\text { (feet) }\end{array}$} & \multicolumn{6}{|c|}{ Soil-water access tubes } \\
\hline & & 1 & 2 & 3 & 4 & 5 & 6 \\
\hline $01-26-88$ & 1 & 19.9 & 16.6 & 18.7 & 15.0 & 19.6 & 16.6 \\
\hline $01-26-88$ & 2 & 18.4 & 14.5 & 17.3 & 13.8 & 20.0 & 16.8 \\
\hline $01-26-88$ & 3 & 20.0 & 18.2 & 9.6 & 14.4 & 12.9 & 13.2 \\
\hline $01-26-88$ & 4 & 18.7 & 17.4 & 10.7 & 16.4 & 13.1 & 13.2 \\
\hline $01-26-88$ & 5 & 17.2 & 13.4 & 17.9 & 15.0 & -- & 9.3 \\
\hline $01-26-88$ & 6 & 14.5 & 17.2 & 16.7 & 15.9 & -- & -- \\
\hline $01-26-88$ & 7 & - & 12.3 & 17.7 & 17.6 & -- & -- \\
\hline $01-26-88$ & 8 & -- & 10.3 & 18.8 & 20.9 & -- & -- \\
\hline $01-26-88$ & 9 & -- & -- & -- & 16.0 & -- & -- \\
\hline $02-29-88$ & 1 & 19.8 & 20.0 & 21.0 & 21.4 & 21.5 & 18.3 \\
\hline $02-29-88$ & 2 & 19.9 & 20.0 & 17.8 & 17.0 & 21.3 & 18.1 \\
\hline $02-29-88$ & 3 & 19.5 & 18.2 & 9.7 & 18.7 & 15.1 & 14.8 \\
\hline $02-29-88$ & 4 & 19.4 & 16.5 & 10.1 & 18.3 & 15.7 & 15.8 \\
\hline $02-29-88$ & 5 & 17.5 & 13.1 & 17.3 & 14.6 & - & 11.7 \\
\hline $02-29-88$ & 6 & 15.3 & 16.7 & 16.7 & 15.4 & -- & -- \\
\hline $02-29-88$ & 7 & -- & 12.3 & 17.2 & 17.1 & -- & -- \\
\hline $02-29-88$ & 8 & - & 10.3 & 18.1 & 20.2 & -- & -- \\
\hline $02-29-88$ & 9 & -- & - & - & 15.6 & -- & -- \\
\hline $04-06-88$ & 1 & 20.5 & 21.2 & 20.9 & 21.1 & 21.2 & 17.9 \\
\hline $04-06-88$ & 2 & 20.8 & 21.1 & 18.4 & 17.4 & 21.1 & 18.2 \\
\hline $04-06-88$ & 3 & 20.4 & 20.7 & 10.1 & 19.6 & 14.9 & 15.3 \\
\hline $04-06-88$ & 4 & 19.9 & 18.9 & 10.8 & 19.9 & 14.5 & 16.0 \\
\hline $04-06-88$ & 5 & 18.1 & 13.9 & 18.1 & 15.5 & -- & -- \\
\hline $04-06-88$ & 6 & 15.1 & 17.6 & 17.2 & 15.6 & - & -- \\
\hline $04-06-88$ & 7 & -- & 12.5 & 18.0 & 17.8 & -- & -- \\
\hline $04-06-88$ & 8 & -- & 10.4 & 19.1 & 20.9 & -- & -- \\
\hline $04-06-88$ & 9 & -- & - & - & 16.1 & -- & -- \\
\hline $05-04-88$ & 1 & 20.3 & 20.8 & 20.4 & 20.5 & 20.6 & 17.6 \\
\hline $05-04-88$ & 2 & 20.6 & 20.4 & 17.8 & 16.9 & 20.1 & 18.3 \\
\hline $05-04-88$ & 3 & 19.8 & 20.4 & 10.1 & 19.1 & 14.1 & 15.5 \\
\hline $05-04-88$ & 4 & 19.0 & 19.1 & 10.3 & 19.5 & 14.2 & 16.1 \\
\hline $05-04-88$ & 5 & 17.4 & 14.0 & 17.8 & 15.6 & - & 11.9 \\
\hline $05-04-88$ & 6 & 15.0 & 17.2 & 17.0 & 15.7 & -- & - \\
\hline $05-04-88$ & 7 & -- & 12.5 & 17.8 & 17.3 & -- & -- \\
\hline $05-04-88$ & 8 & -- & 10.2 & 18.6 & 20.1 & -- & -- \\
\hline $05-04-88$ & 9 & -- & -- & -- & 15.7 & -- & -- \\
\hline
\end{tabular}


Table 5.--Soil-water content at the Spring Creek site during 1988 and 1989--Continued

\begin{tabular}{|c|c|c|c|c|c|c|c|}
\hline \multirow{2}{*}{$\begin{array}{c}\text { Date of } \\
\text { measurement }\end{array}$} & \multirow{2}{*}{$\begin{array}{c}\text { Depth of } \\
\text { measure- } \\
\text { ment } \\
\text { (feet) }\end{array}$} & \multicolumn{6}{|c|}{ Soil-water access tubes } \\
\hline & & 1 & 2 & 3 & 4 & 5 & 6 \\
\hline $06-06-88$ & 1 & 20.4 & $21: 2$ & 20.2 & 20.0 & 20.7 & 17.5 \\
\hline $06-06-88$ & 2 & 21.0 & 20.8 & 18.1 & 17.4 & 20.5 & 18.5 \\
\hline $06-06-88$ & 3 & 20.5 & 20.5 & 10.6 & 19.3 & 14.4 & 15.1 \\
\hline $06-06-88$ & 4 & 19.3 & 19.5 & 10.8 & 19.7 & 14.5 & 16.4 \\
\hline $06-06-88$ & 5 & 18.1 & 14.2 & 17.8 & 15.9 & -- & 12.1 \\
\hline $06-06-88$ & 6 & 14.9 & 17.6 & 17.1 & 15.7 & -- & - \\
\hline $06-06-88$ & 7 & - & 12.6 & 17.8 & 17.6 & -- & - \\
\hline $06-06-88$ & 8 & -- & 10.4 & 18.6 & 21.0 & -- & -- \\
\hline $06-06-88$ & 9 & -- & -- & -- & 15.8 & -- & -- \\
\hline $07-12-88$ & 1 & 19.9 & 17.1 & 17.8 & 15.7 & 19.4 & 15.7 \\
\hline $07-12-88$ & 2 & 20.3 & 20.1 & 18.0 & 16.8 & 21.0 & 18.0 \\
\hline $07-12-88$ & 3 & 20.7 & 20.8 & 10.9 & 19.6 & 14.8 & 15.2 \\
\hline $07-12-88$ & 4 & 19.6 & 19.7 & 11.2 & 20.3 & 15.0 & 15.8 \\
\hline $07-12-88$ & 5 & 17.8 & 15.2 & 17.7 & 16.3 & -- & 12.2 \\
\hline $07-12-88$ & 6 & 15.1 & 18.0 & 17.3 & 16.0 & -- & - \\
\hline $07-12-88$ & 7 & - & 12.9 & 18.3 & 17.7 & -- & -- \\
\hline $07-12-88$ & 8 & -- & 10.5 & 18.8 & 21.0 & -- & -- \\
\hline $07-12-88$ & 9 & -- & - & - & 16.0 & -- & -- \\
\hline $07-25-88$ & 1 & 18.5 & 15.3 & 15.8 & 15.0 & 18.3 & 14.4 \\
\hline $07-25-88$ & 2 & 20.9 & 16.3 & 18.1 & 16.8 & 20.8 & 17.8 \\
\hline $07-25-88$ & 3 & 15.0 & 19.7 & 11.4 & 20.7 & 21.7 & 15.1 \\
\hline $07-25-88$ & 4 & 15.2 & 20.4 & 11.3 & 19.8 & 20.0 & 16.5 \\
\hline $07-25-88$ & 5 & -- & 16.8 & 18.3 & 15.3 & -- & 12.5 \\
\hline $07-25-88$ & 6 & -- & 16.2 & 17.6 & 18.5 & -- & -- \\
\hline $07-25-88$ & 7 & -- & 18.1 & 18.8 & 13.2 & -- & -- \\
\hline $07-25-88$ & 8 & -- & 21.9 & 19.6 & 10.9 & -- & -- \\
\hline $07-25-88$ & 9 & -- & - & - & 12.3 & -- & -- \\
\hline $08-29-88$ & 1 & 16.2 & 13.8 & 14.0 & 14.3 & 16.7 & 12.5 \\
\hline $08-29-88$ & 2 & 18.1 & 12.9 & 15.9 & 15.2 & 19.4 & 15.2 \\
\hline $08-29-88$ & 3 & 19.8 & 17.7 & 10.6 & 18.3 & 14.4 & 12.7 \\
\hline $08-29-88$ & 4 & 18.9 & 18.3 & 11.2 & 19.7 & 14.8 & 15.3 \\
\hline $08-29-88$ & 5 & 17.7 & 15.5 & 17.9 & 16.0 & -- & 12.0 \\
\hline $08-29-88$ & 6 & 14.8 & 18.4 & 17.0 & 16.3 & -- & - \\
\hline $08-29-88$ & 7 & -- & 12.6 & 18.5 & 17.6 & -- & -- \\
\hline $08-29-88$ & 8 & -- & 10.5 & 18.9 & 21.0 & -- & -- \\
\hline $08-29-88$ & 9 & -- & -- & - & 16.0 & -- & -- \\
\hline
\end{tabular}


Table 5.--Soil-water content at the spring Creek site during 1988 and 1989--Continued

\begin{tabular}{|c|c|c|c|c|c|c|c|}
\hline \multirow{2}{*}{$\begin{array}{c}\text { Date of } \\
\text { measurement }\end{array}$} & \multirow{2}{*}{$\begin{array}{l}\text { Depth of } \\
\text { measure- } \\
\text { ment } \\
\text { (feet) }\end{array}$} & \multicolumn{6}{|c|}{ Soil-water access tubes } \\
\hline & & 1 & 2 & 3 & 4 & 5 & 6 \\
\hline $10-05-88$ & 1 & 18.0 & 14.6 & 14.9 & 15.0 & 17.1 & 13.8 \\
\hline $10-05-88$ & 2 & 18.8 & 12.7 & 15.0 & 15.1 & 19.6 & 15.0 \\
\hline $10-05-88$ & 3 & 19.8 & 16.6 & 10.0 & 17.9 & 14.5 & 12.8 \\
\hline $10-05-88$ & 4 & 19.0 & 17.8 & 11.3 & 19.4 & 14.6 & 15.1 \\
\hline $10-05-88$ & 5 & 17.6 & 15.0 & 17.3 & 16.2 & -- & 11.5 \\
\hline $10-05-88$ & 6 & 14.6 & 18.1 & 16.6 & 15.8 & -- & -- \\
\hline $10-05-88$ & 7 & -- & 12.6 & 18.0 & 17.1 & -- & -- \\
\hline $10-05-88$ & 8 & -- & 10.2 & 18.2 & 20.7 & -- & -- \\
\hline $10-05-88$ & 9 & -- & -- & -- & 15.8 & -- & -- \\
\hline $11-16-88$ & 1 & 19.5 & 13.6 & 19.4 & 14.8 & 18.9 & 13.5 \\
\hline $11-16-88$ & 2 & 19.5 & 12.7 & 15.0 & 14.6 & 19.6 & 14.7 \\
\hline $11-16-88$ & 3 & 20.0 & 16.2 & 9.9 & 17.0 & 14.1 & 12.2 \\
\hline $11-16-88$ & 4 & 18.8 & 17.3 & 11.0 & 19.2 & 14.2 & 14.4 \\
\hline $11-16-88$ & 5 & 18.0 & 14.3 & 17.3 & 16.0 & -- & 11.2 \\
\hline $11-16-88$ & 6 & 14.8 & 17.8 & 16.2 & 16.0 & -- & -- \\
\hline $11-16-88$ & 7 & -- & 12.1 & 18.0 & 17.0 & -- & -- \\
\hline $11-16-88$ & 8 & -- & 10.1 & 18.6 & 20.6 & -- & -- \\
\hline $11-16-88$ & 9 & -- & -- & -- & 15.5 & -- & -- \\
\hline $12-12-88$ & 1 & 20.0 & 17.2 & 19.6 & 17.8 & 20.0 & 16.4 \\
\hline $12-12-88$ & 2 & 20.3 & 13.0 & 15.4 & 14.9 & 19.8 & 17.1 \\
\hline $12-12-88$ & 3 & 19.7 & 16.3 & 9.8 & 17.6 & 13.9 & 13.6 \\
\hline $12-12-88$ & 4 & 18.5 & 17.1 & 10.9 & 19.2 & 14.4 & 15.6 \\
\hline $12-12-88$ & 5 & 17.5 & 14.5 & 17.5 & 16.1 & -- & 11.9 \\
\hline $12-12-88$ & 6 & 14.7 & 17.6 & 16.6 & 16.5 & -- & -- \\
\hline $12-12-88$ & 7 & -- & 12.4 & 18.1 & 17.2 & -- & -- \\
\hline $12-12-88$ & 8 & -- & 10.2 & 18.4 & 20.4 & -- & -- \\
\hline $12-12-88$ & 9 & -- & -- & -- & 15.5 & -- & -- \\
\hline $01-30-89$ & 1 & 20.4 & 20.0 & 20.6 & 20.3 & 20.9 & 17.1 \\
\hline $01-30-89$ & 2 & 20.4 & 18.3 & 17.6 & 17.5 & 20.1 & 17.8 \\
\hline $01-30-89$ & 3 & 20.1 & 16.7 & 11.2 & 18.9 & 15.4 & 14.6 \\
\hline $01-30-89$ & 4 & 18.8 & 17.1 & 11.8 & 19.2 & 15.3 & 15.9 \\
\hline $01-30-89$ & 5 & 18.0 & 14.2 & 17.6 & 16.4 & -- & 12.1 \\
\hline $01-30-89$ & 6 & 15.0 & 17.9 & 16.7 & 16.8 & -- & -- \\
\hline $01-30-89$ & 7 & -- & 13.1 & 18.2 & 17.3 & -- & -- \\
\hline $01-30-89$ & 8 & -- & 10.7 & 18.8 & 20.7 & -- & -- \\
\hline $01-30-89$ & 9 & -- & -- & -- & 15.9 & -- & -- \\
\hline
\end{tabular}


Table 5.--Soil-water content at the Spring Creek site during 1988 and 1989--Continued

\begin{tabular}{|c|c|c|c|c|c|c|c|}
\hline \multirow{2}{*}{$\begin{array}{c}\text { Date of } \\
\text { measurement }\end{array}$} & \multirow{2}{*}{$\begin{array}{l}\text { Depth of } \\
\text { measure- } \\
\text { ment } \\
\text { (feet) }\end{array}$} & \multicolumn{6}{|c|}{ Soil-water access tubes } \\
\hline & & 1 & 2 & 3 & 4 & 5 & 6 \\
\hline $03-01-89$ & 1 & 20.4 & 20.4 & 20.5 & 21.0 & 21.3 & 17.2 \\
\hline $03-01-89$ & 2 & 20.1 & 20.5 & 18.0 & 18.2 & 21.5 & 17.7 \\
\hline $03-01-89$ & 3 & 20.4 & 19.5 & 12.9 & 19.5 & 15.8 & 14.9 \\
\hline $03-01-89$ & 4 & 18.7 & 17.5 & 12.3 & 20.1 & 16.2 & 16.2 \\
\hline $03-01-89$ & 5 & 18.0 & 14.3 & 17.8 & 18.3 & -- & 12.3 \\
\hline $03-01-89$ & 6 & 15.4 & 17.9 & 16.8 & 16.3 & -- & -- \\
\hline $03-01-89$ & 7 & -- & 12.7 & 19.1 & 17.2 & -- & -- \\
\hline $03-01-89$ & 8 & -- & 10.6 & 19.7 & 20.7 & -- & -- \\
\hline $03-01-89$ & 9 & -- & -- & -- & 15.6 & -- & -- \\
\hline $03-21-89$ & 1 & 20.5 & 20.3 & 21.0 & 20.5 & 21.0 & 16.9 \\
\hline $03-21-89$ & 2 & 20.4 & 20.6 & 18.0 & 18.1 & 20.5 & 17.6 \\
\hline $03-21-89$ & 3 & 20.4 & 20.7 & 13.7 & 19.3 & 15.5 & 14.8 \\
\hline $03-21-89$ & 4 & 18.6 & 19.1 & 13.5 & 20.1 & 15.7 & 16.3 \\
\hline $03-21-89$ & 5 & 17.7 & 18.2 & 17.9 & 19.0 & -- & 12.1 \\
\hline $03-21-89$ & 6 & 14.6 & 20.0 & 17.1 & 17.7 & -- & -- \\
\hline $03-21-89$ & 7 & -- & 14.0 & 19.2 & 17.2 & -- & -- \\
\hline $03-21-89$ & 8 & -- & 11.7 & 19.1 & 20.5 & -- & -- \\
\hline $03-21-89$ & 9 & -- & -- & -- & 15.8 & -- & -- \\
\hline $04-04-89$ & 1 & 20.0 & 20.2 & 20.4 & 20.4 & 20.4 & 17.0 \\
\hline $04-04-89$ & 2 & 19.7 & 20.1 & 17.8 & 17.6 & 20.0 & 17.6 \\
\hline $04-04-89$ & 3 & 20.0 & 20.1 & 13.2 & 19.1 & 15.3 & 14.6 \\
\hline $04-04-89$ & 4 & 18.5 & 19.0 & 12.8 & 19.8 & 15.0 & 15.9 \\
\hline $04-04-89$ & 5 & 17.4 & 18.0 & 17.6 & 18.5 & -- & 11.8 \\
\hline $04-04-89$ & 6 & 14.4 & 19.7 & 16.8 & 17.6 & -- & -- \\
\hline $04-04-89$ & 7 & -- & 13.8 & 18.6 & 16.8 & -- & -- \\
\hline $04-04-89$ & 8 & -- & 11.6 & 19.0 & 20.4 & -- & -- \\
\hline $04-04-89$ & 9 & -- & -- & -- & 15.5 & -- & -- \\
\hline $05-09-89$ & 1 & 19.5 & 19.8 & 19.0 & 19.6 & 20.2 & 15.8 \\
\hline $05-09-89$ & 2 & 20.6 & 20.0 & 17.7 & 17.6 & 20.4 & 17.6 \\
\hline $05-09-89$ & 3 & 20.6 & 20.2 & 13.5 & 19.2 & 15.6 & 14.7 \\
\hline $05-09-89$ & 4 & 18.6 & 19.1 & 13.5 & 20.1 & 15.4 & 14.7 \\
\hline $05-09-89$ & 5 & 17.7 & 18.1 & 18.1 & 18.7 & -- & 12.0 \\
\hline $05-09-89$ & 6 & 14.8 & 19.7 & 17.2 & 18.0 & -- & -- \\
\hline $05-09-89$ & 7 & -- & 13.8 & 18.9 & 17.2 & -- & -- \\
\hline $05-09-89$ & 8 & -- & 11.6 & 19.5 & 21.2 & -- & -- \\
\hline $05-09-89$ & 9 & -- & -- & -- & 15.9 & -- & -- \\
\hline
\end{tabular}


Table 5.--Soil-water content at the Spring Creek site during 1988 and 1989--Continued

\begin{tabular}{|c|c|c|c|c|c|c|c|}
\hline \multirow{2}{*}{$\begin{array}{c}\text { Date of } \\
\text { measurement }\end{array}$} & \multirow{2}{*}{$\begin{array}{l}\text { Depth of } \\
\text { measure- } \\
\text { ment } \\
\text { (feet) }\end{array}$} & \multicolumn{6}{|c|}{ Soil-water access tubes } \\
\hline & & 1 & 2 & 3 & 4 & 5 & 6 \\
\hline $06-07-89$ & 1 & 17.5 & 15.1 & 14.7 & 14.8 & 17.1 & 13.4 \\
\hline $06-07-89$ & 2 & 20.0 & 16.4 & 17.2 & 16.6 & 19.7 & 15.6 \\
\hline $06-07-89$ & 3 & 20.3 & 19.9 & 13.5 & 19.1 & 15.2 & 15.7 \\
\hline $06-07-89$ & 4 & 18.5 & 18.7 & 13.5 & 20.4 & 15.5 & 14.1 \\
\hline $06-07-89$ & 5 & 17.5 & 17.8 & 18.1 & 18.2 & - & 15.8 \\
\hline $06-07-89$ & 6 & 14.4 & 19.5 & 17.2 & 18.2 & -- & -- \\
\hline $06-07-89$ & 7 & - & 13.7 & 19.0 & 17.4 & -- & -- \\
\hline $06-07-89$ & 8 & -- & 11.4 & 19.5 & 21.1 & -- & -- \\
\hline $06-07-89$ & 9 & -- & -- & -- & 15.9 & -- & -- \\
\hline $07-19-89$ & 1 & 15.7 & 12.7 & 13.5 & 13.1 & 14.9 & 11.9 \\
\hline $07-19-89$ & 2 & 17.2 & 12.6 & 14.5 & 15.1 & 16.9 & 13.2 \\
\hline $07-19-89$ & 3 & 19.3 & 15.1 & 12.6 & 17.9 & 14.3 & 11.3 \\
\hline $07-19-89$ & 4 & 17.3 & 17.8 & 13.3 & 19.9 & 15.1 & 14.8 \\
\hline $07-19-89$ & 5 & 17.5 & 16.9 & 17.9 & 18.1 & -- & 11.9 \\
\hline $07-19-89$ & 6 & 14.5 & 19.2 & 16.8 & 18.0 & -- & -- \\
\hline $07-19-89$ & 7 & - & 13.5 & 19.1 & 17.2 & -- & -- \\
\hline $07-19-89$ & 8 & -- & 11.0 & 19.2 & 21.0 & -- & -- \\
\hline $07-19-89$ & 9 & -- & - & - & 15.7 & - & -- \\
\hline $08-31-89$ & 1 & 14.9 & 12.7 & 13.3 & 13.5 & 15.4 & 11.8 \\
\hline $08-31-89$ & 2 & 16.4 & 12.2 & 13.9 & 14.9 & 16.5 & 12.9 \\
\hline $08-31-89$ & 3 & 19.2 & 13.8 & 12.0 & 17.3 & 13.6 & 10.8 \\
\hline $08-31-89$ & 4 & 17.6 & 16.4 & 12.9 & 19.3 & 15.0 & 13.9 \\
\hline $08-31-89$ & 5 & 17.2 & 14.8 & 18.1 & 18.2 & - & 11.7 \\
\hline $08-31-89$ & 6 & 14.6 & 19.0 & 16.6 & 18.4 & -- & - \\
\hline $08-31-89$ & 7 & -- & 13.5 & 19.2 & 17.4 & -- & -- \\
\hline $08-31-89$ & 8 & -- & 11.1 & 19.1 & 21.3 & -- & -- \\
\hline $08-31-89$ & 9 & -- & - & - & 15.7 & -- & -- \\
\hline $10-03-89$ & 1 & 15.5 & 12.5 & 13.7 & 13.4 & 15.4 & 11.9 \\
\hline $10-03-89$ & 2 & 17.4 & 12.2 & 13.5 & 15.3 & 16.1 & 13.3 \\
\hline $10-03-89$ & 3 & 19.8 & 13.8 & 11.2 & 16.6 & 13.2 & 10.7 \\
\hline $10-03-89$ & 4 & 17.7 & 16.0 & 12.8 & 19.3 & 14.8 & 13.3 \\
\hline $10-03-89$ & 5 & 17.4 & 15.0 & 17.6 & 17.6 & - & 11.2 \\
\hline $10-03-89$ & 6 & 14.4 & 18.9 & 16.6 & 18.1 & -- & -- \\
\hline $10-03-89$ & 7 & - & 13.2 & 19.0 & 16.9 & -- & -- \\
\hline $10-03-89$ & 8 & -- & 10.8 & 19.2 & 21.0 & -- & -- \\
\hline $10-03-89$ & 9 & -- & - & - & 16.1 & -- & -- \\
\hline
\end{tabular}


Table 6.--Soil-water content at the Cow Camp Creek site during 1988 and 1989

[Contents in percent by volume; --, no measurement]

\begin{tabular}{|c|c|c|c|c|c|c|}
\hline \multirow{2}{*}{$\begin{array}{c}\text { Date of } \\
\text { measurement }\end{array}$} & \multirow{2}{*}{$\begin{array}{c}\text { Depth of } \\
\text { measure- } \\
\text { ment } \\
\text { (feet) }\end{array}$} & \multicolumn{5}{|c|}{ Soil-water access tubes } \\
\hline & & 1 & 2 & 3 & 4 & 5 \\
\hline $01-07-88$ & 1 & 14.8 & 15.0 & 11.0 & 13.4 & 14.8 \\
\hline $01-07-88$ & 2 & 13.1 & 13.1 & 9.2 & 16.0 & 16.5 \\
\hline $01-07-88$ & 3 & 14.7 & 16.4 & 9.0 & 15.5 & 15.1 \\
\hline $01-07-88$ & 4 & 16.5 & 16.2 & 8.8 & 16.8 & 16.5 \\
\hline $01-07-88$ & 5 & 16.3 & 15.8 & -- & 17.4 & 16.0 \\
\hline $01-07-88$ & 6 & 17.3 & 15.8 & -- & 17.4 & 16.0 \\
\hline $01-07-88$ & 7 & 15.3 & 17.0 & -- & 14.2 & 11.5 \\
\hline $01-07-88$ & 8 & -- & 16.5 & -- & 14.1 & 12.5 \\
\hline $01-27-88$ & 1 & 14.7 & 15.3 & 10.2 & 13.7 & 15.3 \\
\hline $01-27-88$ & 2 & 13.4 & 13.6 & 9.5 & 16.4 & 16.2 \\
\hline $01-27-88$ & 3 & 14.8 & 15.8 & 9.1 & 15.7 & 14.6 \\
\hline $01-27-88$ & 4 & 16.6 & 16.3 & 9.2 & 17.0 & 16.7 \\
\hline $01-27-88$ & 5 & 16.1 & 16.4 & -- & 17.5 & 15.8 \\
\hline $01-27-88$ & 6 & 16.9 & 16.1 & -- & 16.1 & 16.7 \\
\hline $01-27-88$ & 7 & 14.7 & 16.6 & -- & 13.8 & 11.2 \\
\hline $01-27-88$ & 8 & -- & 16.7 & -- & 14.4 & 12.6 \\
\hline $02-29-88$ & 1 & 17.2 & 16.4 & 13.1 & 16.4 & 17.2 \\
\hline $02-29-88$ & 2 & 14.9 & 15.9 & 10.3 & 16.7 & 17.0 \\
\hline $02-29-88$ & 3 & 14.8 & 16.2 & 8.9 & 16.3 & 16.1 \\
\hline $02-29-88$ & 4 & 16.1 & 15.6 & 8.7 & 16.9 & 14.1 \\
\hline $02-29-88$ & 5 & 15.5 & 15.5 & -- & 17.7 & 16.2 \\
\hline $02-29-88$ & 6 & 16.5 & 15.6 & -- & 17.0 & 14.6 \\
\hline $02-29-88$ & 7 & 14.4 & 16.3 & -- & 14.3 & 16.0 \\
\hline $02-29-88$ & 8 & -- & 15.8 & -- & 17.3 & 10.8 \\
\hline $04-12-88$ & 1 & 17.2 & 16.5 & 13.3 & 16.6 & 17.7 \\
\hline $04-12-88$ & 2 & 15.6 & 16.6 & 14.9 & 17.4 & 18.1 \\
\hline $04-12-88$ & 3 & 17.0 & 17.4 & 17.1 & 17.5 & 16.7 \\
\hline $04-12-88$ & 4 & 17.5 & 16.8 & 17.0 & 17.8 & 17.8 \\
\hline $04-12-88$ & 5 & 17.0 & 16.7 & -- & 17.8 & 16.8 \\
\hline $04-12-88$ & 6 & 17.6 & 15.8 & -- & 17.3 & 17.8 \\
\hline $04-12-88$ & 7 & 15.2 & 16.8 & -- & 14.4 & 12.3 \\
\hline $04-12-88$ & 8 & - & 16.4 & -- & 15.3 & 14.6 \\
\hline $05-04-88$ & 1 & 16.5 & 16.3 & 14.3 & 15.9 & 17.5 \\
\hline $05-04-88$ & 2 & 15.3 & 16.0 & 15.1 & 17.1 & 18.1 \\
\hline $05-04-88$ & 3 & 16.7 & 17.2 & 16.8 & 17.6 & 16.7 \\
\hline $05-04-88$ & 4 & 17.8 & 16.5 & 16.7 & 18.1 & 17.9 \\
\hline $05-04-88$ & 5 & 16.9 & 16.4 & -- & 18.2 & 17.0 \\
\hline $05-04-88$ & 6 & 17.4 & 15.9 & -- & 17.2 & 17.6 \\
\hline $05-04-88$ & 7 & 14.9 & 16.6 & -- & 14.1 & 11.9 \\
\hline $05-04-88$ & 8 & - & 16.4 & -- & 14.9 & 13.8 \\
\hline
\end{tabular}


Table 6.--Soil-water content at the Cow Camp Creek site during 1988 and 1989--Continued

\begin{tabular}{|c|c|c|c|c|c|c|}
\hline \multirow{2}{*}{$\begin{array}{c}\text { Date of } \\
\text { measurement }\end{array}$} & \multirow{2}{*}{$\begin{array}{l}\text { Depth of } \\
\text { measure- } \\
\text { ment } \\
(\text { feet) }\end{array}$} & \multicolumn{5}{|c|}{ Soil-water access tubes } \\
\hline & & 1 & 2 & 3 & 4 & 5 \\
\hline $05-04-88$ & 1 & 16.5 & 16.3 & 14.3 & 15.9 & 17.5 \\
\hline $05-04-88$ & 2 & 15.3 & 16.0 & 15.1 & 17.1 & 18.1 \\
\hline $05-04-88$ & 3 & 16.7 & 17.2 & 16.8 & 17.6 & 16.7 \\
\hline $05-04-88$ & 4 & 17.8 & 16.5 & 16.7 & 18.1 & 17.9 \\
\hline $05-04-88$ & 5 & 16.9 & 16.4 & - & 18.2 & 17.0 \\
\hline $05-04-88$ & 6 & 17.4 & 15.9 & -- & 17.2 & 17.6 \\
\hline $05-04-88$ & 7 & 14.9 & 16.6 & -- & 14.1 & 11.9 \\
\hline $05-04-88$ & 8 & -- & 16.4 & -- & 14.9 & 13.8 \\
\hline $06-06-88$ & 1 & 13.4 & 14.9 & 9.2 & 14.3 & 14.4 \\
\hline $06-06-88$ & 2 & 14.9 & 15.8 & 13.2 & 17.3 & 18.2 \\
\hline $06-06-88$ & 3 & 17.0 & 17.0 & 15.9 & 17.4 & 16.6 \\
\hline $06-06-88$ & 4 & 18.1 & 17.1 & 16.7 & 18.1 & 18.2 \\
\hline $06-06-88$ & 5 & 17.4 & 16.9 & -- & 18.1 & 17.0 \\
\hline $06-06-88$ & 6 & 17.6 & 16.1 & -- & 17.5 & 17.8 \\
\hline $06-06-88$ & 7 & 15.4 & 16.8 & -- & 14.5 & 11.8 \\
\hline $06-06-88$ & 8 & - & 16.4 & -- & 15.5 & 13.6 \\
\hline $07-12-88$ & 1 & 13.2 & 12.8 & 8.7 & 12.5 & 13.2 \\
\hline $07-12-88$ & 2 & 14.4 & 14.9 & 10.6 & 17.3 & 17.3 \\
\hline $07-12-88$ & 3 & 16.6 & 17.0 & 12.1 & 17.1 & 16.2 \\
\hline $07-12-88$ & 4 & 17.9 & 17.2 & 13.3 & 18.1 & 17.8 \\
\hline $07-12-88$ & 5 & 17.5 & 17.1 & -- & 18.5 & 17.1 \\
\hline $07-12-88$ & 6 & 17.7 & 16.5 & -- & 17.5 & 17.6 \\
\hline $07-12-88$ & 7 & 15.4 & 17.1 & -- & 14.6 & 11.8 \\
\hline $07-12-88$ & 8 & -- & 16.8 & -- & 15.8 & 14.0 \\
\hline $07-25-88$ & 1 & 11.9 & 12.0 & 7.6 & 11.6 & 11.6 \\
\hline $07-25-88$ & 2 & 14.1 & 14.1 & 9.7 & 16.5 & 16.6 \\
\hline $07-25-88$ & 3 & 16.1 & 16.8 & 10.9 & 17.0 & 16.0 \\
\hline $07-25-88$ & 4 & 18.0 & 17.2 & 11.3 & 18.1 & 18.0 \\
\hline $07-25-88$ & 5 & 17.0 & 17.0 & - & 18.2 & 17.4 \\
\hline $07-25-88$ & 6 & 18.0 & 16.4 & -- & 17.3 & 18.0 \\
\hline $07-25-88$ & 7 & 15.3 & 17.3 & - & 14.6 & 11.9 \\
\hline $07-25-88$ & 8 & -- & 16.9 & -- & 15.5 & 14.0 \\
\hline $08-29-88$ & 1 & 11.8 & 12.0 & 7.2 & 11.3 & 11.8 \\
\hline $08-29-88$ & 2 & 13.6 & 13.7 & 9.1 & 16.4 & 16.0 \\
\hline $08-29-88$ & 3 & 15.6 & 15.6 & 10.0 & 16.6 & 14.4 \\
\hline $08-29-88$ & 4 & 17.3 & 16.9 & 9.8 & 17.5 & 17.5 \\
\hline $08-29-88$ & 5 & 16.6 & 16.7 & -- & 17.9 & 16.5 \\
\hline $08-29-88$ & 6 & 17.6 & 16.3 & -- & 17.4 & 17.6 \\
\hline $08-29-88$ & 7 & 15.4 & 16.8 & -- & 14.3 & 11.5 \\
\hline $08-29-88$ & 8 & - & 16.6 & -- & 15.4 & 13.9 \\
\hline
\end{tabular}


Table 6.--Soil-water content at the Cow Camp Creek site during 1988 and 1989--Continued

\begin{tabular}{|c|c|c|c|c|c|c|}
\hline \multirow{2}{*}{$\begin{array}{c}\text { Date of } \\
\text { measurement }\end{array}$} & \multirow{2}{*}{$\begin{array}{c}\text { Depth of } \\
\text { measure- } \\
\text { ment } \\
\text { (feet) }\end{array}$} & \multicolumn{5}{|c|}{ Soil-water access tubes } \\
\hline & & 1 & 2 & 3 & 4 & 5 \\
\hline $10-05-88$ & 1 & 11.9 & 12.3 & 9.7 & 11.1 & 13.8 \\
\hline $10-05-88$ & 2 & 13.0 & 12.9 & 10.6 & 15.8 & 15.8 \\
\hline $10-05-88$ & 3 & 15.2 & 15.3 & 9.8 & 15.9 & 14.0 \\
\hline $10-05-88$ & 4 & 17.0 & 16.0 & 9.5 & 17.2 & 17.0 \\
\hline $10-05-88$ & 5 & 16.5 & 16.1 & - & 17.2 & 15.3 \\
\hline $10-05-88$ & 6 & 17.2 & 16.0 & -- & 17.2 & 16.8 \\
\hline $10-05-88$ & 7 & 15.0 & 16.5 & -- & 13.7 & 11.5 \\
\hline $10-05-88$ & 8 & -- & 16.4 & -- & 15.0 & 13.9 \\
\hline $11-14-88$ & 1 & 11.9 & 13.0 & 10.9 & 12.2 & 15.6 \\
\hline $11-14-88$ & 2 & 13.2 & 13.1 & 9.4 & 15.7 & 16.5 \\
\hline $11-14-88$ & 3 & 15.0 & 15.3 & 10.0 & 15.7 & 14.5 \\
\hline $11-14-88$ & 4 & 16.9 & 16.5 & 9.6 & 17.2 & 17.4 \\
\hline $11-14-88$ & 5 & 16.4 & 16.3 & - & 17.5 & 15.9 \\
\hline $11-14-88$ & 6 & 17.6 & 15.9 & -- & 17.1 & 17.1 \\
\hline $11-14-88$ & 7 & 15.3 & 16.4 & -- & 13.9 & 11.9 \\
\hline $11-14-88$ & 8 & - & 16.6 & -- & 14.8 & 13.9 \\
\hline $12-13-88$ & 1 & 13.7 & 15.3 & 14.7 & 15.3 & 16.6 \\
\hline $12-13-88$ & 2 & 13.8 & 13.7 & 14.4 & 16.4 & 16.5 \\
\hline $12-13-88$ & 3 & 15.4 & 15.7 & 10.3 & 15.9 & 14.0 \\
\hline $12-13-88$ & 4 & 17.5 & 16.4 & 10.1 & 17.2 & 17.3 \\
\hline $12-13-88$ & 5 & 16.5 & 16.6 & - & 18.1 & 15.9 \\
\hline $12-13-88$ & 6 & 17.9 & 16.5 & -- & 17.6 & 17.0 \\
\hline $12-13-88$ & 7 & 15.5 & 17.3 & -- & 14.0 & 11.6 \\
\hline $12-13-88$ & 8 & - & 16.8 & -- & 14.9 & 13.7 \\
\hline $01-31-89$ & 1 & 13.9 & 15.1 & 14.2 & 16.2 & 16.3 \\
\hline $01-31-89$ & 2 & 13.3 & 13.3 & 13.5 & 16.2 & 15.9 \\
\hline $01-31-89$ & 3 & 14.6 & 15.0 & 10.7 & 15.6 & 14.0 \\
\hline $01-31-89$ & 4 & 16.0 & 16.1 & 9.6 & 16.6 & 16.4 \\
\hline $01-31-89$ & 5 & 15.9 & 15.9 & -- & 17.1 & 15.0 \\
\hline $01-31-89$ & 6 & 17.0 & 15.6 & -- & 16.6 & 16.2 \\
\hline $01-31-89$ & 7 & 14.7 & 16.1 & -- & 13.5 & 11.0 \\
\hline $01-31-89$ & 8 & - & 16.0 & - & 14.2 & 13.0 \\
\hline $03-01-89$ & 1 & 13.8 & 15.2 & 14.3 & 16.6 & 16.2 \\
\hline $03-01-89$ & 2 & 13.1 & 13.5 & 13.5 & 17.0 & 15.8 \\
\hline $03-01-89$ & 3 & 14.6 & 15.1 & 11.2 & 16.3 & 13.8 \\
\hline $03-01-89$ & 4 & 16.0 & 16.0 & 9.7 & 16.4 & 16.4 \\
\hline $03-01-89$ & 5 & 15.7 & 15.8 & - & 16.8 & 14.7 \\
\hline $03-01-89$ & 6 & 16.6 & 15.8 & -- & 16.6 & 16.2 \\
\hline $03-01-89$ & 7 & 14.6 & 15.8 & -- & 13.4 & 10.9 \\
\hline $03-01-89$ & 8 & - & 16.3 & -- & 13.9 & 12.7 \\
\hline
\end{tabular}


Table 6.--Soil-water content at the Cow Camp Creek site during 1988 and 1989--Continued

\begin{tabular}{|c|c|c|c|c|c|c|}
\hline \multirow{2}{*}{$\begin{array}{c}\text { Date of } \\
\text { measurement }\end{array}$} & \multirow{2}{*}{$\begin{array}{c}\text { Depth of } \\
\text { measure- } \\
\text { ment } \\
(\text { feet) }\end{array}$} & \multicolumn{5}{|c|}{ Soil-water access tubes } \\
\hline & & 1 & 2 & 3 & 4 & 5 \\
\hline $03-22-89$ & 1 & 17.1 & 17.0 & 15.4 & 17.4 & 18.6 \\
\hline $03-22-89$ & 2 & 15.2 & 17.0 & 15.7 & 17.7 & 17.8 \\
\hline $03-22-89$ & 3 & 17.4 & 16.4 & 17.1 & 17.6 & 16.6 \\
\hline $03-22-89$ & 4 & 17.7 & 16.8 & 16.4 & 18.2 & 18.1 \\
\hline $03-22-89$ & 5 & 16.7 & 16.4 & -- & 18.1 & 16.5 \\
\hline $03-22-89$ & 6 & 17.4 & 16.0 & -- & 17.2 & 17.7 \\
\hline $03-22-89$ & 7 & 15.3 & 16.2 & -- & 14.5 & 12.0 \\
\hline $03-22-89$ & 8 & -- & 16.2 & -- & -- & 14.6 \\
\hline $04-05-89$ & 1 & 16.9 & 16.8 & 14.5 & 17.3 & 18.3 \\
\hline $04-05-89$ & 2 & 15.0 & 17.2 & 15.4 & 17.4 & 17.8 \\
\hline $04-05-89$ & 3 & 17.5 & 16.3 & 16.2 & 17.6 & 16.4 \\
\hline $04-05-89$ & 4 & 17.6 & 16.6 & 16.1 & 18.1 & 18.0 \\
\hline $04-05-89$ & 5 & 16.5 & 16.0 & - & 18.3 & 16.7 \\
\hline $04-05-89$ & 6 & 17.3 & 16.0 & -- & 17.1 & 17.3 \\
\hline $04-05-89$ & 7 & 15.2 & 16.1 & -- & 14.0 & 11.9 \\
\hline $04-05-89$ & 8 & -- & 16.3 & -- & 16.2 & 14.4 \\
\hline $05-09-89$ & 1 & 15.9 & 15.6 & 10.9 & 15.8 & 16.0 \\
\hline $05-09-89$ & 2 & 14.9 & 16.0 & 13.8 & 17.3 & 17.4 \\
\hline $05-09-89$ & 3 & 17.2 & 16.4 & 15.5 & 17.6 & 16.0 \\
\hline $05-09-89$ & 4 & 17.9 & 16.8 & 15.5 & 18.0 & 18.0 \\
\hline $05-09-89$ & 5 & 17.3 & 16.1 & -- & 17.8 & 16.0 \\
\hline $05-09-89$ & 6 & 17.4 & 16.3 & -- & 17.3 & 17.7 \\
\hline $05-09-89$ & 7 & 15.1 & 16.2 & -- & 14.0 & 11.7 \\
\hline $05-09-89$ & 8 & -- & 16.3 & -- & 15.4 & 14.0 \\
\hline $06-07-89$ & 1 & 13.3 & 13.5 & 8.8 & 13.4 & 12.9 \\
\hline $06-07-89$ & 2 & 14.5 & 15.3 & 11.1 & 17.1 & 16.2 \\
\hline $06-07-89$ & 3 & 16.8 & 16.4 & 12.7 & 17.5 & 15.5 \\
\hline $06-07-89$ & 4 & 17.7 & 16.9 & 14.4 & 18.2 & 17.8 \\
\hline $06-07-89$ & 5 & 17.0 & 16.3 & -- & 17.7 & 16.2 \\
\hline $06-07-89$ & 6 & 17.2 & 16.4 & -- & 17.2 & 17.2 \\
\hline $06-07-89$ & 7 & 15.0 & 16.2 & -- & 13.8 & 11.4 \\
\hline $06-07-89$ & 8 & -- & 16.5 & -- & 15.4 & 13.8 \\
\hline $07-19-89$ & 1 & 12.7 & 11.9 & 9.4 & 12.3 & 13.1 \\
\hline $07-19-89$ & 2 & 13.4 & 13.1 & 10.8 & 16.2 & 15.0 \\
\hline $07-19-89$ & 3 & 16.1 & 15.0 & 10.0 & 16.8 & 14.0 \\
\hline $07-19-89$ & 4 & 17.4 & 16.3 & 10.3 & 17.4 & 17.6 \\
\hline $07-19-89$ & 5 & 16.7 & 16.5 & -- & 17.6 & 15.6 \\
\hline $07-19-89$ & 6 & 17.4 & 16.4 & -- & 17.0 & 16.8 \\
\hline $07-19-89$ & 7 & 14.9 & 16.1 & -- & 13.9 & 11.1 \\
\hline $07-19-89$ & 8 & -- & 16.4 & -- & -- & 13.4 \\
\hline
\end{tabular}


Table 6.--Soil-water content at the Cow Camp Creek site during 1988 and 1989--Continued

\begin{tabular}{|c|c|c|c|c|c|c|}
\hline \multirow{2}{*}{$\begin{array}{c}\text { Date of } \\
\text { measurement }\end{array}$} & \multirow{2}{*}{$\begin{array}{c}\text { Depth of } \\
\text { measure- } \\
\text { ment } \\
(\text { feet) }\end{array}$} & \multicolumn{5}{|c|}{ Soil-water access tubes } \\
\hline & & 1 & 2 & 3 & 4 & 5 \\
\hline $08-31-89$ & 1 & 12.6 & 12.0 & 7.8 & 12.5 & 12.4 \\
\hline $08-31-89$ & 2 & 13.5 & 12.8 & 8.8 & 16.0 & 14.7 \\
\hline $08-31-89$ & 3 & 15.7 & 14.0 & 9.6 & 16.0 & 13.1 \\
\hline $08-31-89$ & 4 & 17.4 & 16.3 & 9.4 & 16.8 & 16.8 \\
\hline $08-31-89$ & 5 & 16.7 & 16.2 & -- & 17.3 & 14.9 \\
\hline $08-31-89$ & 6 & 16.9 & 16.1 & -- & 17.1 & 16.8 \\
\hline $08-31-89$ & 7 & 15.1 & 16.1 & -- & 13.9 & 11.4 \\
\hline $08-31-89$ & 8 & -- & 16.4 & -- & 14.9 & 13.4 \\
\hline $10-03-89$ & 1 & 12.9 & 12.5 & 8.3 & 12.7 & 12.9 \\
\hline $10-03-89$ & 2 & 13.2 & 12.7 & 8.9 & 15.9 & 14.0 \\
\hline $10-03-89$ & 3 & 15.2 & 14.0 & 9.5 & 16.0 & 12.5 \\
\hline $10-03-89$ & 4 & 16.9 & 15.9 & 9.2 & 16.8 & 16.5 \\
\hline $10-03-89$ & 5 & 16.3 & 15.9 & -- & 16.9 & 14.6 \\
\hline $10-03-89$ & 6 & 16.9 & 16.2 & -- & 17.1 & 16.6 \\
\hline $10-03-89$ & 7 & 15.4 & 15.8 & -- & 13.3 & 11.1 \\
\hline $10-03-89$ & 8 & -- & 15.9 & -- & -- & 13.4 \\
\hline
\end{tabular}


Table 7.--Soil-water content at the soil-water control site during 1988 and 1989

[Contents in percent by volume; --, no measurement]

\begin{tabular}{|c|c|c|c|c|c|c|c|c|c|}
\hline \multirow{2}{*}{$\begin{array}{c}\text { Date of } \\
\text { measurement }\end{array}$} & \multirow{2}{*}{$\begin{array}{l}\text { Depth of } \\
\text { measure- } \\
\text { ment } \\
\text { (feet) }\end{array}$} & \multicolumn{8}{|c|}{ Soil-water access } \\
\hline & & 1 & 2 & 3 & 4 & 5 & 6 & 7 & 8 \\
\hline $01-28-88$ & 1 & 16.2 & 18.0 & 19.3 & 17.4 & 13.1 & 12.7 & 12.2 & 12.2 \\
\hline $01-28-88$ & 2 & 12.7 & 13.9 & 17.2 & 14.2 & 12.7 & 12.2 & 12.0 & 11.6 \\
\hline $01-28-88$ & 3 & 13.7 & 13.2 & 16.0 & 13.8 & 14.5 & 12.5 & 12.2 & 12.7 \\
\hline $01-28-88$ & 4 & 15.1 & 16.1 & 16.8 & 14.6 & 14.5 & 14.6 & 13.1 & 15.8 \\
\hline $01-28-88$ & 5 & 16.8 & 17.2 & 17.4 & 16.4 & 17.0 & 17.8 & -- & 17.0 \\
\hline $01-28-88$ & 6 & 16.6 & 16.6 & 17.2 & 17.0 & 17.3 & 16.7 & -- & -- \\
\hline $01-28-88$ & 7 & 17.0 & 17.2 & 17.1 & 17.2 & 17.2 & -- & -- & -- \\
\hline $03-01-88$ & 1 & 20.9 & -- & 19.2 & 19.9 & 20.4 & 20.4 & 20.8 & 20.5 \\
\hline $03-01-88$ & 2 & 19.9 & -- & 16.9 & 17.7 & 18.8 & 18.2 & 20.0 & 19.4 \\
\hline $03-01-88$ & 3 & 16.8 & -- & 15.8 & 13.7 & 15.7 & 14.7 & 17.9 & 15.5 \\
\hline $03-01-88$ & 4 & 15.5 & -- & 17.0 & 14.4 & 14.6 & 14.8 & 15.2 & 16.1 \\
\hline $03-01-88$ & 5 & 16.6 & -- & 17.6 & 16.4 & 17.0 & 17.7 & -- & 16.7 \\
\hline $03-01-88$ & 6 & 16.7 & -- & 16.8 & 16.7 & 17.2 & 16.8 & -- & -- \\
\hline $03-01-88$ & 7 & 17.1 & -- & 17.7 & 17.3 & 17.4 & -- & -- & -- \\
\hline $04-13-88$ & 1 & 22.1 & 21.3 & 21.0 & 21.3 & 21.5 & 21.6 & 21.8 & 21.7 \\
\hline $04-13-88$ & 2 & 21.9 & 21.6 & 21.5 & 21.6 & 21.5 & 21.8 & 21.3 & 21.3 \\
\hline $04-13-88$ & 3 & 20.5 & 20.4 & 20.4 & 20.0 & 21.7 & 20.6 & 19.7 & 20.1 \\
\hline $04-13-88$ & 4 & 18.1 & 18.7 & 18.5 & -- & 17.9 & 18.7 & 17.8 & 19.1 \\
\hline $04-13-88$ & 5 & 17.6 & 18.6 & 17.6 & -- & 18.6 & 18.6 & -- & 17.5 \\
\hline $04-13-88$ & 6 & 17.3 & 17.2 & 17.5 & -- & 18.2 & 17.1 & -- & -- \\
\hline $04-13-88$ & 7 & 17.8 & 17.5 & 17.6 & -- & 18.0 & -- & -- & -- \\
\hline $05-04-88$ & 1 & 20.8 & 20.2 & 20.6 & 20.5 & 20.3 & 20.6 & 20.7 & 20.5 \\
\hline $05-04-88$ & 2 & 21.3 & 20.9 & 20.7 & 20.7 & 20.9 & 20.9 & 21.2 & 21.1 \\
\hline $05-04-88$ & 3 & 20.2 & 19.7 & 20.0 & 19.7 & 21.2 & 19.7 & 19.0 & 20.0 \\
\hline $05-04-88$ & 4 & 17.9 & 19.0 & 18.5 & 17.2 & 17.4 & 18.3 & 17.1 & 18.4 \\
\hline $05-04-88$ & 5 & 17.3 & 18.1 & 17.5 & -- & 18.3 & 18.5 & -- & 17.3 \\
\hline $05-04-88$ & 6 & 16.9 & 17.1 & 17.3 & - & 17.9 & 17.3 & -- & $\ldots$ \\
\hline $05-04-88$ & 7 & 17.5 & 17.4 & 17.6 & -- & 17.8 & -- & -- & - \\
\hline $06-07-88$ & 1 & 14.3 & 15.9 & 15.7 & 14.8 & 14.9 & 14.5 & 14.3 & 14.4 \\
\hline $06-07-88$ & 2 & 17.6 & 17.5 & 18.7 & 16.0 & 18.6 & 17.9 & 16.8 & 19.1 \\
\hline $06-07-88$ & 3 & 19.4 & 18.6 & 19.7 & 17.8 & 21.2 & 19.4 & 16.8 & 19.3 \\
\hline $06-07-88$ & 4 & 17.9 & 19.0 & 18.7 & 17.0 & 17.7 & 18.5 & 17.0 & 18.4 \\
\hline $06-07-88$ & 5 & 17.6 & 18.1 & 18.0 & -- & 18.5 & 18.8 & -- & 17.5 \\
\hline $06-07-88$ & 6 & 17.4 & 17.4 & 17.5 & -- & 18.1 & 17.2 & -- & -- \\
\hline $06-07-88$ & 7 & 17.4 & 17.4 & 17.7 & -- & 18.3 & -- & -- & -- \\
\hline
\end{tabular}


Table 7.--Soil-water content at the soil-water control site during 1988 and 1989--Continued

\begin{tabular}{|c|c|c|c|c|c|c|c|c|c|}
\hline \multirow{2}{*}{$\begin{array}{c}\text { Date of } \\
\text { measurement }\end{array}$} & \multirow{2}{*}{$\begin{array}{c}\text { Depth of } \\
\text { measure- } \\
\text { ment } \\
\text { (feet) }\end{array}$} & \multicolumn{4}{|c|}{ Soil-water } & \multirow{2}{*}{$\frac{\operatorname{access}}{5}$} & \multicolumn{2}{|l|}{ tubes } & \multirow[b]{2}{*}{8} \\
\hline & & 1 & 2 & 3 & 4 & & 6 & 7 & \\
\hline $07-12-88$ & 1 & 13.2 & 14.7 & 15.6 & 14.0 & 13.3 & 13.2 & 12.2 & 13.4 \\
\hline $07-12-88$ & 2 & 14.2 & 14.2 & 15.7 & 14.2 & 14.3 & 14.5 & 12.9 & 15.1 \\
\hline $07-12-88$ & 3 & 16.6 & 14.4 & 17.6 & 14.4 & 17.1 & 15.8 & 13.6 & 17.0 \\
\hline $07-12-88$ & 4 & 17.6 & 18.5 & 18.6 & 15.2 & 17.1 & 17.8 & 14.9 & 18.2 \\
\hline $07-12-88$ & 5 & 17.7 & 18.3 & 17.9 & 16.8 & 18.6 & 19.2 & -- & 17.6 \\
\hline $07-12-88$ & 6 & 17.2 & 17.5 & 17.7 & 17.5 & 18.1 & 17.4 & -- & -- \\
\hline $07-12-88$ & 7 & 17.9 & 17.5 & 17.9 & 18.2 & 18.0 & -- & -- & -- \\
\hline $07-26-88$ & 1 & 11.5 & 13.1 & 12.9 & 12.7 & 12.2 & 12.4 & 10.4 & 11.3 \\
\hline $07-26-88$ & 2 & 12.1 & 13.3 & 13.4 & 13.1 & 13.0 & 12.8 & 11.5 & 12.9 \\
\hline $07-26-88$ & 3 & 14.2 & 13.8 & 15.5 & 13.8 & 15.4 & 13.8 & 12.3 & 14.6 \\
\hline $07-26-88$ & 4 & 16.7 & 17.5 & 17.7 & 14.6 & 16.4 & 16.8 & 13.8 & 17.2 \\
\hline $07-26-88$ & 5 & 17.4 & 17.5 & 18.0 & 16.6 & 18.0 & 18.8 & -- & 17.2 \\
\hline $07-26-88$ & 6 & 17.1 & 17.2 & 17.0 & 17.1 & 17.7 & 16.9 & -- & -- \\
\hline $07-26-88$ & 7 & 17.2 & 17.6 & 17.4 & 17.7 & 17.9 & -- & -- & -- \\
\hline $08-29-88$ & 1 & 11.2 & 13.2 & 12.0 & 12.7 & 11.9 & 12.2 & 9.9 & 11.0 \\
\hline $08-29-88$ & 2 & 12.1 & 13.5 & 12.5 & 13.3 & 12.8 & 12.4 & 11.2 & 12.4 \\
\hline $08-29-88$ & 3 & 13.3 & 13.5 & 14.3 & 13.5 & 14.9 & 12.9 & 11.8 & 13.8 \\
\hline $08-29-88$ & 4 & 15.6 & 16.9 & 17.6 & 14.9 & 15.8 & 15.6 & 13.6 & 16.5 \\
\hline $08-29-88$ & 5 & 17.5 & 17.4 & 18.0 & 16.7 & 18.2 & 18.9 & - & 17.5 \\
\hline $08-29-88$ & 6 & 17.2 & 17.1 & 17.6 & 17.3 & 17.8 & 16.9 & - & - \\
\hline $08-29-88$ & 7 & 17.6 & 17.6 & 17.9 & 17.6 & 18.2 & -- & -- & - \\
\hline $10-05-88$ & 1 & 13.3 & 15.5 & 17.2 & 14.7 & 13.7 & 14.6 & 13.9 & 15.8 \\
\hline $10-05-88$ & 2 & 13.1 & 14.0 & 13.8 & 14.3 & 13.2 & 13.9 & 12.5 & 14.8 \\
\hline $10-05-88$ & 3 & 13.6 & 13.8 & 14.2 & 14.0 & 14.8 & 13.0 & 12.6 & 14.4 \\
\hline $10-05-88$ & 4 & 15.6 & 16.5 & 17.5 & 14.7 & 15.8 & 15.1 & 13.5 & 16.6 \\
\hline $10-05-88$ & 5 & 17.3 & 17.2 & 17.7 & 16.1 & 17.2 & 18.1 & -- & 17.2 \\
\hline $10-05-88$ & 6 & 16.9 & 16.9 & 17.5 & 17.2 & 17.3 & 16.9 & -- & - \\
\hline $10-05-88$ & 7 & 17.4 & 17.2 & 17.5 & 17.3 & 17.9 & -- & -- & -- \\
\hline $11-17-88$ & 1 & 12.3 & 15.0 & 13.8 & 14.0 & 13.2 & 15.0 & 14.9 & 17.9 \\
\hline $11-17-88$ & 2 & 12.3 & 13.6 & 12.7 & 13.7 & 13.1 & 12.9 & 12.1 & 15.4 \\
\hline $11-17-88$ & 3 & 13.8 & 13.3 & 14.0 & 13.7 & 14.7 & 12.6 & 12.3 & 13.5 \\
\hline $11-17-88$ & 4 & 14.9 & 16.2 & 16.7 & 14.5 & 15.2 & 14.8 & 13.3 & 15.9 \\
\hline $11-17-88$ & 5 & 16.9 & 16.6 & 17.2 & 16.1 & 17.0 & 17.8 & -- & 16.5 \\
\hline $11-17-88$ & 6 & 16.6 & 16.8 & 17.2 & 16.5 & 17.2 & 16.4 & -- & - \\
\hline $11-17-88$ & 7 & 17.0 & 17.2 & 17.1 & 17.0 & 17.4 & - & - & -- \\
\hline
\end{tabular}


Table 7.--Soil-water content at the soil-water control site during 1988 and 1989--Continued

\begin{tabular}{|c|c|c|c|c|c|c|c|c|c|}
\hline \multirow{2}{*}{$\begin{array}{c}\text { Date of } \\
\text { measurement }\end{array}$} & \multirow{2}{*}{$\begin{array}{c}\text { Depth of } \\
\text { measure- } \\
\text { ment } \\
\text { (feet) }\end{array}$} & \multicolumn{8}{|c|}{ Soil-water access tubes } \\
\hline & & 1 & 2 & 3 & 4 & 5 & 6 & 7 & 8 \\
\hline $12-13-88$ & 1 & 13.3 & 16.9 & 16.6 & 15.6 & 15.1 & 17.0 & 16.9 & 19.2 \\
\hline $12-13-88$ & 2 & 13.5 & 14.6 & 13.6 & 14.8 & 13.8 & 13.8 & 13.3 & 16.6 \\
\hline $12-13-88$ & 3 & 14.5 & 14.5 & 15.1 & 14.9 & 16.1 & 13.6 & 13.1 & 14.4 \\
\hline $12-13-88$ & 4 & 15.8 & 17.0 & 17.8 & 15.1 & 16.3 & 16.0 & 14.2 & 16.5 \\
\hline $12-13-88$ & 5 & 17.5 & 18.0 & 18.4 & 16.8 & 18.2 & 18.8 & -- & 17.4 \\
\hline $12-13-88$ & 6 & 17.4 & 17.4 & 18.3 & 17.3 & 18.1 & 17.3 & - & -- \\
\hline $12-13-88$ & 7 & 18.1 & 17.9 & 18.4 & 18.1 & 18.2 & -- & -- & -- \\
\hline $01-31-89$ & 1 & 13.2 & 15.8 & 15.9 & 14.8 & 14.6 & 15.5 & 15.6 & 17.8 \\
\hline $01-31-89$ & 2 & 13.2 & 14.1 & 13.2 & 14.3 & 13.9 & 13.5 & 13.0 & 15.0 \\
\hline $01-31-89$ & 3 & 14.0 & 13.8 & 14.2 & 14.4 & 15.5 & 13.3 & 13.0 & 14.2 \\
\hline $01-31-89$ & 4 & 15.4 & 16.4 & 17.0 & 14.6 & 15.4 & 15.4 & 13.7 & 15.8 \\
\hline $01-31-89$ & 5 & 16.7 & 16.8 & 17.3 & 16.1 & 17.0 & 17.9 & -- & 16.6 \\
\hline $01-31-89$ & 6 & 16.6 & 16.6 & 17.6 & 16.5 & 17.3 & 16.4 & -- & -- \\
\hline $01-31-89$ & 7 & 17.1 & 17.0 & 17.6 & 17.0 & 17.9 & -- & -- & -- \\
\hline $03-02-89$ & 1 & 13.9 & 15.6 & 20.7 & 15.7 & 18.1 & 20.3 & 18.4 & 20.2 \\
\hline $03-02-89$ & 2 & 13.4 & 14.4 & 15.5 & 14.4 & 14.0 & 14.8 & 13.4 & 15.4 \\
\hline $03-02-89$ & 3 & 14.2 & 14.1 & 14.4 & 14.6 & 15.5 & 13.0 & 13.1 & 14.0 \\
\hline $03-02-89$ & 4 & 15.3 & 16.4 & 17.2 & 14.7 & $15 \cdot 3$ & 14.9 & 13.6 & 15.9 \\
\hline $03-02-89$ & 5 & 16.7 & 17.0 & 17.0 & 16.3 & 16.9 & 17.5 & -- & 16.6 \\
\hline $03-02-89$ & 6 & 16.5 & 16.5 & 17.0 & 16.1 & 17.2 & 16.3 & -- & -- \\
\hline $03-02-89$ & 7 & 16.8 & 17.1 & 17.5 & 17.0 & 17.8 & -- & -- & -- \\
\hline $03-22-89$ & 1 & 22.3 & 21.4 & 21.8 & 21.8 & 22.2 & 21.6 & 22.4 & 22.2 \\
\hline $03-22-89$ & 2 & 21.5 & 21.6 & 21.1 & 21.3 & 20.9 & 21.1 & 21.2 & 21.2 \\
\hline $03-22-89$ & 3 & 19.6 & 19.8 & 20.2 & 20.1 & 21.1 & 20.1 & 19.1 & 19.6 \\
\hline $03-22-89$ & 4 & 16.9 & 18.7 & 18.4 & -- & 18.1 & 17.8 & 15.6 & 17.8 \\
\hline $03-22-89$ & 5 & 17.1 & 16.9 & 17.4 & -- & 17.4 & 19.1 & -- & 17.5 \\
\hline $03-22-89$ & 6 & 16.7 & 16.8 & 17.0 & -- & 17.0 & 16.8 & -- & -- \\
\hline $03-22-89$ & 7 & 17.5 & 17.2 & 17.4 & -- & 17.7 & -- & -- & -- \\
\hline $04-04-89$ & 1 & 21.4 & 21.0 & 21.2 & 20.8 & 21.1 & 21.2 & 21.3 & 21.2 \\
\hline $04-04-89$ & 2 & 21.2 & 20.8 & 20.9 & 20.9 & 20.6 & 20.4 & 20.9 & 20.5 \\
\hline $04-04-89$ & 3 & 19.3 & 19.2 & 19.7 & 19.7 & 21.0 & 19.7 & 18.2 & 19.4 \\
\hline $04-04-89$ & 4 & 16.4 & 18.3 & 18.2 & -- & 17.5 & 17.2 & 15.3 & 17.2 \\
\hline $04-04-89$ & 5 & 16.6 & 16.9 & 17.0 & -- & 17.4 & 18.6 & - & 17.2 \\
\hline $04-04-89$ & 6 & 16.2 & 16.6 & 16.8 & -- & 17.0 & 16.2 & -- & -- \\
\hline $04-04-89$ & 7 & 16.6 & 16.7 & 17.1 & -- & 17.2 & -- & -- & -- \\
\hline
\end{tabular}


Table 7.--Soil-water content at the soil-water control site during 1988 and 1989--Continued

\begin{tabular}{|c|c|c|c|c|c|c|c|c|c|}
\hline \multirow{2}{*}{$\begin{array}{c}\text { Date of } \\
\text { measurement }\end{array}$} & \multirow{2}{*}{$\begin{array}{c}\text { Depth of } \\
\text { measure- } \\
\text { ment } \\
\text { (feet) }\end{array}$} & \multicolumn{8}{|c|}{ Soil-water access tubes } \\
\hline & & 1 & 2 & 3 & 4 & 5 & 6 & 7 & 8 \\
\hline $05-09-89$ & 1 & 18.9 & 18.9 & 18.9 & 18.5 & 18.2 & 18.0 & 19.2 & 18.2 \\
\hline $05-09-89$ & 2 & 20.8 & 20.4 & 20.3 & 19.9 & 20.6 & 20.6 & 20.2 & 20.4 \\
\hline $05-09-89$ & 3 & 20.2 & 19.3 & 20.1 & 19.9 & 21.1 & 19.6 & 18.4 & 19.6 \\
\hline $05-09-89$ & 4 & 17.6 & 18.6 & 18.5 & -- & 17.8 & 17.8 & 15.7 & 17.4 \\
\hline $05-09-89$ & 5 & 17.1 & 17.3 & 17.6 & -- & 17.9 & 18.8 & -- & 17.4 \\
\hline $05-09-89$ & 6 & 17.1 & 17.0 & 17.4 & -- & 17.3 & 17.1 & -- & -- \\
\hline $05-09-89$ & 7 & 17.3 & 17.1 & 17.7 & -- & 17.8 & -- & -- & -- \\
\hline $06-07-89$ & 1 & 13.6 & 14.8 & 14.8 & 14.4 & 13.5 & 13.5 & 13.3 & 12.6 \\
\hline $06-07-89$ & 2 & 16.4 & 15.3 & 17.0 & 14.7 & 16.2 & 15.4 & 14.5 & 15.8 \\
\hline $06-07-89$ & 3 & 18.4 & 16.8 & 19.5 & 15.7 & 20.2 & 17.8 & 14.4 & 18.6 \\
\hline $06-07-89$ & 4 & 17.3 & 18.4 & 19.0 & 16.2 & 17.4 & 17.2 & 15.0 & 17.4 \\
\hline $06-07-89$ & 5 & 17.4 & 17.1 & 17.5 & 16.4 & 17.6 & 18.8 & -- & 16.9 \\
\hline $06-07-89$ & 6 & 16.7 & 16.9 & 17.2 & 16.6 & 17.4 & 16.7 & -- & -- \\
\hline $06-07-89$ & 7 & 17.1 & 17.0 & 17.2 & 17.2 & 17.8 & -- & -- & -- \\
\hline $07-19-89$ & 1 & 11.9 & 14.0 & 13.6 & 13.0 & 11.9 & 12.3 & 11.5 & 10.7 \\
\hline $07-19-89$ & 2 & 12.6 & 13.7 & 13.6 & 13.4 & 12.8 & 12.6 & 11.8 & 12.4 \\
\hline $07-19-89$ & 3 & 13.8 & 13.8 & 15.6 & 13.5 & 14.9 & 13.7 & 12.1 & 14.0 \\
\hline $07-19-89$ & 4 & 16.0 & 16.8 & 18.4 & 14.3 & 16.1 & 16.0 & 13.6 & 16.1 \\
\hline $07-19-89$ & 5 & 17.0 & 16.9 & 17.7 & 16.2 & 17.2 & 18.6 & -- & 16.8 \\
\hline $07-19-89$ & 6 & 16.5 & 16.8 & 17.4 & 16.3 & 17.1 & 16.3 & -- & -- \\
\hline $07-19-89$ & 7 & 17.1 & 17.3 & 17.6 & 17.3 & 17.3 & -- & -- & -- \\
\hline $08-31-89$ & 1 & 11.7 & 13.5 & 12.2 & 13.1 & 11.8 & 12.2 & 11.0 & 11.1 \\
\hline $08-31-89$ & 2 & 12.3 & 13.5 & 12.6 & 13.3 & 12.7 & 12.4 & 11.5 & 12.9 \\
\hline $08-31-89$ & 3 & 13.9 & 14.0 & 14.4 & 13.8 & 14.8 & 12.8 & 11.9 & 13.7 \\
\hline $08-31-89$ & 4 & 15.7 & 16.5 & 17.7 & 14.8 & 15.8 & 15.2 & 13.4 & 15.6 \\
\hline $08-31-89$ & 5 & 16.9 & 16.4 & 17.6 & 16.1 & 17.2 & 18.1 & -- & 16.5 \\
\hline $08-31-89$ & 6 & 16.6 & 16.5 & 17.4 & 16.3 & 17.4 & 16.4 & -- & -- \\
\hline $08-31-89$ & 7 & 16.9 & 16.8 & 17.3 & 16.7 & 17.5 & -- & -- & -- \\
\hline $10-03-89$ & 1 & 11.6 & 13.7 & 13.1 & 13.3 & 12.0 & 12.6 & 11.1 & 11.8 \\
\hline $10-03-89$ & 2 & 12.3 & 13.6 & 12.4 & 13.2 & 12.8 & 13.0 & 11.6 & 12.6 \\
\hline $10-03-89$ & 3 & 13.6 & 13.8 & 14.0 & 13.9 & 15.1 & 12.6 & 12.0 & 13.9 \\
\hline $10-03-89$ & 4 & 15.4 & 16.2 & 17.5 & 14.8 & 15.5 & 14.8 & 13.4 & 15.3 \\
\hline $10-03-89$ & 5 & 16.6 & 16.7 & 17.5 & 15.9 & 16.8 & 18.2 & - & 16.2 \\
\hline $10-03-89$ & 6 & 16.8 & 16.5 & 17.3 & 16.2 & 16.8 & 16.3 & -- & -- \\
\hline $10-03-89$ & 7 & 17.1 & 17.1 & 17.4 & 16.5 & 17.6 & -- & -- & -- \\
\hline
\end{tabular}


Table 8.--Soil dry density at the Spring Creek, Cow Camp Creek, and soil-water control sites, May 11, 1989

[Densities in grams per cubic centimeter; --, no measurement]

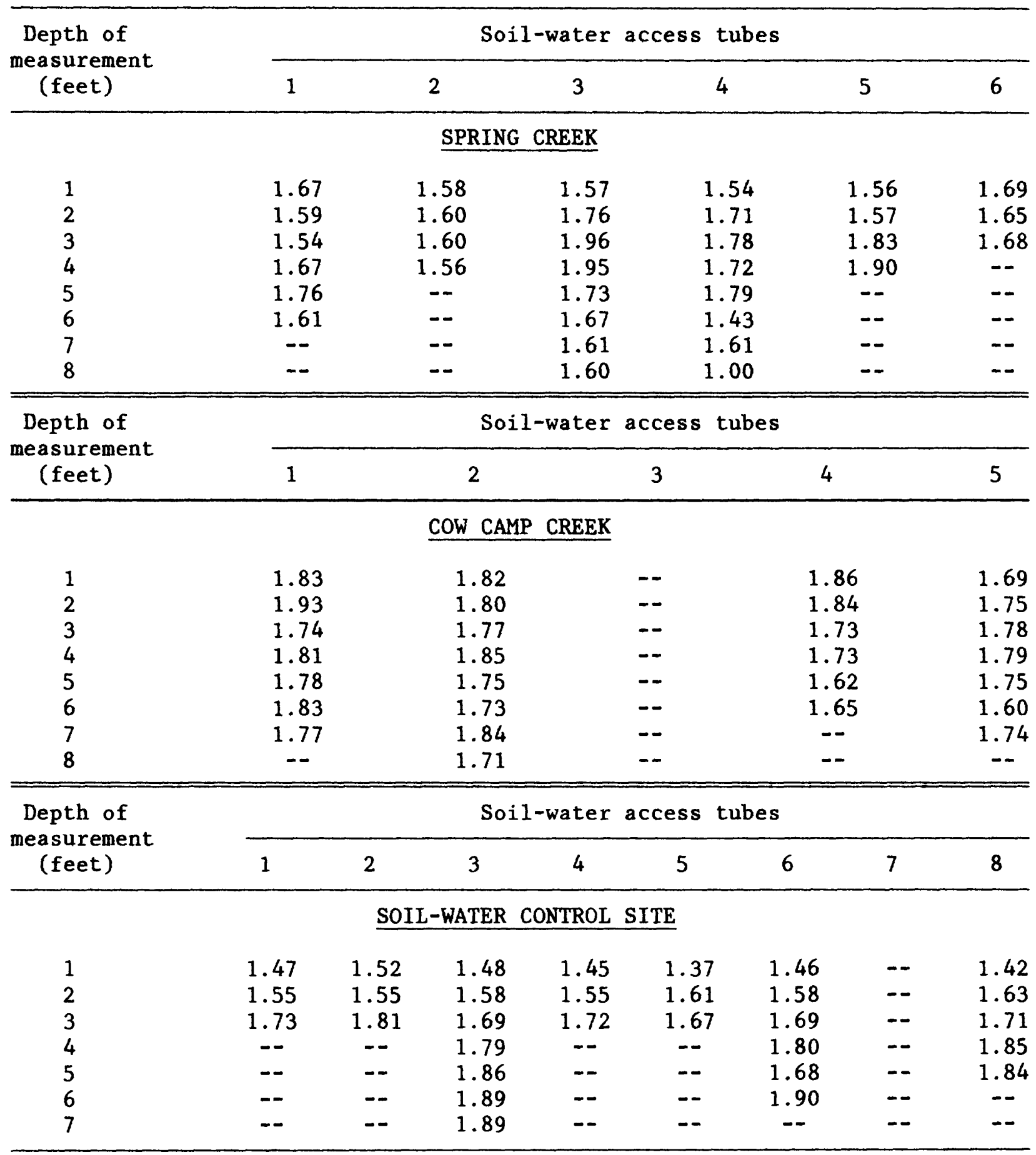


Table 9.--Well information

[feet above NGVD, feet above National Geodetic Vertical Datum of 1929;

Interburden, bedrock zone between Lennox coal and Wadge coal; Underburden, bedrock zone below Wadge coal; Rec spoil, reclaimed spoil]

\begin{tabular}{|c|c|c|c|c|c|c|}
\hline Site & $\begin{array}{l}\text { Well } \\
\text { identifi- } \\
\text { cation }\end{array}$ & $\begin{array}{l}\text { Well } \\
\text { name }^{2}\end{array}$ & $\begin{array}{c}\text { Land-surface } \\
\text { elevation } \\
\text { (feet above } \\
\text { NGVD) }\end{array}$ & $\begin{array}{l}\text { Well } \\
\text { depth } \\
\text { (feet) }\end{array}$ & $\begin{array}{c}\text { Interval of } \\
\text { completion } \\
\text { (feet below } \\
\text { land surface) }\end{array}$ & $\begin{array}{l}\text { Zone of } \\
\text { completion }\end{array}$ \\
\hline Spring Creek & SSL287 & SL2 & 6,861 & 58 & $48-56$ & Lennox coal \\
\hline Spring Creek & SSI 287 & SI2 & 6,860 & 80 & $56-80$ & Interburden \\
\hline Spring Creek & SSW287 & SW2 & 6,860 & 98 & 91-98 & Wadge coal \\
\hline Spring Creek & SSU487 & SU4 & 6,861 & 105 & $95-105$ & Underburden \\
\hline Spring Creek & SSU287 & SU2 & 6,862 & 127 & $94-127$ & Underburden \\
\hline Spring Creek & SSD487 & SD4 & 6,860 & 194 & $179-193$ & Underburden \\
\hline Spring Creek & SSS487-59 & S59 & 6,899 & 33 & $6-33$ & Rec spoil \\
\hline Spring Creek & $\operatorname{SSS} 487-60$ & S60 & 6,893 & 23 & $3-23$ & Rec spoil \\
\hline Spring Creek & SSS487-61 & S61 & 6,892 & 26 & $7-26$ & Rec spoil \\
\hline Cow Camp & SCL287 & CL2 & 6,957 & 30 & $24-30$ & Lennox coal \\
\hline Cow Camp & SCI 287 & $\mathrm{CI} 2$ & 6,957 & 70 & $40-70$ & Interburden \\
\hline Cow Camp & SCW287 & CW2 & 6,957 & 90 & $79-90$ & Wadge coal \\
\hline Cow Camp & SCU287 & $\mathrm{CU} 2$ & 6,957 & 122 & $103-122$ & Underburden \\
\hline Cow Camp & $\operatorname{scs} 487-62$ & $\mathrm{C} 62$ & 6,976 & 52 & 4-52 & Rec spoil \\
\hline Cow Camp & $\operatorname{scs} 487-63$ & $\mathrm{C} 63$ & 6,982 & 38 & $7-38$ & Rec spoil \\
\hline Cow Camp & $\operatorname{scS} 487-64$ & $\mathrm{C} 64$ & 6,980 & 37 & $7-37$ & Rec spoil \\
\hline Cow Camp & $\operatorname{scs} 487-65$ & $\mathrm{C} 65$ & 6,997 & 25 & $7-25$ & Rec spoil \\
\hline Zuli & SZL287 & ZI2 & 6,822 & 182 & $176-182$ & Lennox coal \\
\hline $\mathrm{Zuli}$ & SZI287 & $\mathrm{ZI2}$ & 6,819 & 222 & $190-222$ & Interburden \\
\hline Zuli & SZW287 & $\mathrm{ZW} 2$ & 6,823 & 228 & $220-228$ & Wadge coal \\
\hline $\mathrm{Zuli}$ & SZU287 & ZU2 & 6,820 & 265 & $251-265$ & Underburden \\
\hline Bond Creek & SBL287 & BL2 & 7,032 & 98 & $92-98$ & Lennox coal \\
\hline Bond Creek & SBI 287 & $\mathrm{BI} 2$ & 7,031 & 150 & $113-150$ & Interburden \\
\hline Bond Creek & SBW287 & BW2 & 7,032 & 164 & $154-164$ & Wadge coal \\
\hline Bond Creek & SBU287 & BU2 & 7,030 & 175 & $167-175$ & Underburden \\
\hline
\end{tabular}

${ }^{1}$ Well identifications were assigned as follows: The first letter (S), designates the Seneca II Coal Mine. The second letter (S, C, Z, or B) designates the site location as Spring Creek, Cow Camp Creek, Zuli, or Bond Creek. The third letter (, , I, W, U, D, or S) designates a specific zoné of completion. These zones are the Lennox coal, Interburden, Wadge coal, Underburden, Deep underburden, or reclaimed Spoil. The number 2 or 4 designates the well-casing diameter, in inches, and $8 \overrightarrow{7}$ is the year the wells were drilled (1987). Wells completed in reclaimed spoil were assigned an additional well number by the Seneca II Coal Mine operators; these are indicated by a hyphen followed by the assigned well number.

${ }^{2}$ Well names were assigned to each well. For all wells, the first letter designates the site location. For bedrock wells, the second letter designates a specific zone of completion, and the number 2 or 4 designates casing diameter, in inches. For wells completed in reclaimed spoil, the last two digits indicate the well number assigned by the Seneca II Coal Mine operators. 
Table 10.--Summary of geophysical and Iithologic logs

[C, caliper; NG, natural gamma; SP, spontaneous potential; $R$, resistivity; GG, gamma-gamma; $N$, neutron; $X$, data; -, no data;]

\begin{tabular}{|c|c|c|c|c|c|c|c|c|}
\hline \multirow{2}{*}{$\begin{array}{l}\text { Well } \\
\text { name }\end{array}$} & \multicolumn{2}{|c|}{ Lithologic logs } & \multicolumn{6}{|c|}{ Geophysical logs } \\
\hline & Core & Cuttings & $\overline{\mathrm{C}}$ & NG & $\mathrm{SP}$ & $\bar{R}$ & GG & $\bar{N}$ \\
\hline SL2 & - & $\mathrm{X}$ & $\mathrm{X}$ & $\mathrm{X}$ & - & $\mathrm{X}$ & $\mathrm{X}$ & - \\
\hline SI2 & - & $\mathrm{X}$ & $\mathrm{X}$ & $\mathrm{X}$ & - & $\mathrm{X}$ & $\mathrm{x}$ & - \\
\hline SW2 & - & $\mathrm{X}$ & $\mathrm{X}$ & $\mathrm{X}$ & - & $\mathrm{X}$ & $\mathrm{X}$ & - \\
\hline SU4 & $X$ & - & $X$ & $X$ & $X$ & - & $X$ & $X$ \\
\hline SU2 & - & $\mathrm{X}$ & - & - & - & - & - & - \\
\hline $\mathrm{SD} 4$ & - & $\mathrm{X}$ & $\mathrm{X}$ & $\mathrm{X}$ & $\mathrm{X}$ & $\mathrm{X}$ & $\mathrm{X}$ & - \\
\hline CL2 & - & $\mathrm{X}$ & - & - & - & - & - & - \\
\hline $\mathrm{CI} 2$ & - & $\mathrm{X}$ & $\mathrm{X}$ & $\mathrm{X}$ & $\mathrm{X}$ & $\mathrm{X}$ & $\mathrm{X}$ & - \\
\hline $\mathrm{CW} 2$ & - & $\mathrm{X}$ & $\mathrm{X}$ & $\mathrm{X}$ & $\mathrm{X}$ & $\mathrm{X}$ & $\mathrm{X}$ & - \\
\hline $\mathrm{CU} 2$ & $X$ & - & $X$ & $X$ & $x$ & $X$ & $\mathrm{X}$ & - \\
\hline $\mathrm{ZL2}$ & - & $X$ & $\mathrm{X}$ & $\mathrm{X}$ & $X$ & $\mathrm{X}$ & $X$ & - \\
\hline ZI2 & - & $X$ & $\mathrm{X}$ & $\mathrm{X}$ & $\mathrm{X}$ & $\mathrm{X}$ & $\mathrm{X}$ & - \\
\hline ZW2 & - & $X$ & - & - & - & - & - & - \\
\hline $\mathrm{ZU} 2$ & $X$ & - & $\mathrm{X}$ & $\mathrm{X}$ & $X$ & $\mathrm{X}$ & $X$ & - \\
\hline BL2 & - & $X$ & $X$ & $\mathrm{X}$ & $\mathrm{X}$ & $\mathrm{X}$ & $X$ & - \\
\hline BI2 & - & $X$ & $X$ & $\mathrm{X}$ & $\mathrm{X}$ & $\mathrm{X}$ & $\mathrm{X}$ & - \\
\hline BW2 & - & $X$ & $X$ & $X$ & $X$ & $\mathrm{X}$ & $\mathrm{X}$ & - \\
\hline BU2 & $\mathrm{X}$ & - & $X$ & $\mathrm{X}$ & $\mathrm{X}$ & $\mathrm{X}$ & $X$ & - \\
\hline
\end{tabular}

${ }^{1}$ Well names were assigned to each well. For all wells the first letter (S, C, Z, or B) designates the site location as Spring Creek, Cow Camp Creek, Zuli, or Bond Creek. For bedrock wells, the second letter ( $,{ }^{-} \mathrm{I}, \mathrm{W}, \mathrm{U}$, and D) designates a specific zone of completion. These zones are the Lennox coal, Interburden, Wadge coal, Underburden, and Deep underburden. The number 2 or $\overline{4}$ designates the well-casing diameter, in inches. 
Table 11.--Hydraulic-head data for bedrock wells

[Well ID corresponds to well identifications listed in table 9; --, no data collected]

\begin{tabular}{cccc}
\hline $\begin{array}{c}\text { Date of } \\
\text { measure- } \\
\text { ment }\end{array}$ & $\begin{array}{c}\text { Hydraulic head } \\
\text { (feet above } \\
\text { land surface) }\end{array}$ & $\begin{array}{c}\text { Hydraulic head } \\
\text { (feet above } \\
\text { land surface) }\end{array}$ & $\begin{array}{c}\text { Hydraulic head } \\
\text { (feet above } \\
\text { land surface) }\end{array}$ \\
\hline
\end{tabular}

SPRING CREEK SITE

\begin{tabular}{|c|c|c|c|}
\hline & $\frac{\text { WELL ID }}{\text { SSL287 }}$ & $\frac{\text { WELL ID }}{\text { SSI287 }}$ & $\frac{\text { WELL ID }}{\text { SSW287 }}$ \\
\hline $01-26-88$ & $\frac{4.96}{4.9}$ & 4.60 & 10.39 \\
\hline $03-04-88$ & 6.00 & 7.17 & 14.83 \\
\hline $03-22-88$ & 5.97 & 7.56 & 14.71 \\
\hline $04-04-88$ & 6.21 & 7.14 & 10.62 \\
\hline $05-04-88$ & 6.92 & 9.85 & 9.05 \\
\hline $06-06-88$ & 6.67 & 7.86 & 12.15 \\
\hline $07-12-88$ & 7.32 & 8.25 & 13.46 \\
\hline $07-26-88$ & 7.18 & 8.14 & 13.30 \\
\hline $08-29-88$ & 6.95 & 7.98 & 10.53 \\
\hline $10-04-88$ & 7.41 & 8.09 & 12.91 \\
\hline $10-26-88$ & 7.64 & 7.98 & 13.07 \\
\hline $11-14-88$ & 9.97 & 10.88 & 12.20 \\
\hline \multirow[t]{3}{*}{$12-13-88$} & 9.51 & 10.19 & 12.20 \\
\hline & WELL ID & WELL ID & WELL ID \\
\hline & $\overline{\text { SSL287 }}$ & SSI287 & SSW287 \\
\hline $01-30-89$ & 9.28 & 9.49 & 8.73 \\
\hline $02-28-89$ & 11.13 & 11.34 & 14.04 \\
\hline $04-04-89$ & 12.70 & 19.00 & 14.70 \\
\hline $04-19-89$ & 9.17 & 10.32 & 16.89 \\
\hline $05-08-89$ & 8.48 & 9.58 & 14.47 \\
\hline $06-05-89$ & 8.01 & 8.91 & 13.33 \\
\hline $06-26-89$ & 7.88 & 8.68 & 13.13 \\
\hline $07-25-89$ & 7.97 & 8.63 & 13.15 \\
\hline $09-11-89$ & 7.88 & 8.45 & 12.87 \\
\hline \multirow[t]{3}{*}{$10-03-89$} & 7.90 & 8.86 & 12.85 \\
\hline & WELL ID & WELL ID & WELL ID \\
\hline & $\overline{\text { SSU487 }}$ & SSU287 & SSD487 \\
\hline $01-26-88$ & 9.69 & 4.43 & 69.60 \\
\hline $03-04-88$ & 14.18 & 6.44 & 68.20 \\
\hline $03-22-88$ & 13.94 & 7.20 & 68.89 \\
\hline $04-04-88$ & 14.11 & -- & 71.00 \\
\hline $05-04-88$ & 13.62 & 8.02 & 73.07 \\
\hline $06-06-88$ & 12.10 & 7.12 & 73.77 \\
\hline $07-12-88$ & 12.74 & 7.95 & 74.00 \\
\hline $07-26-88$ & 12.67 & 7.75 & 74.69 \\
\hline $08-29-88$ & 12.30 & 7.49 & 74.46 \\
\hline $10-04-88$ & 12.30 & 7.93 & 74.00 \\
\hline $10-26-88$ & 12.40 & 8.05 & 74.20 \\
\hline $11-14-88$ & 14.25 & 8.52 & 76.16 \\
\hline $12-13-88$ & 14.02 & 8.29 & -- \\
\hline
\end{tabular}


Table 11.--Hydraulic-head data for bedrock wells--Continued

\begin{tabular}{cccc}
\hline $\begin{array}{c}\text { Date of } \\
\text { measure- } \\
\text { ment }\end{array}$ & $\begin{array}{c}\text { Hydraulic head } \\
\text { (feet above } \\
\text { land surface) }\end{array}$ & $\begin{array}{c}\text { Hydraulic head } \\
\text { (feet above } \\
\text { land surface) }\end{array}$ & $\begin{array}{c}\text { Hydraulic head } \\
\text { (feet above } \\
\text { land surface) }\end{array}$ \\
\hline
\end{tabular}

\section{SPRING CREEK SITE--Continued}

$\begin{array}{lrrr} & \text { WELL ID } & \text { WELL ID } & \frac{\text { WELL ID }}{\text { SSU }} \\ 01-30-89 & \frac{\text { SSU287 }}{12.40} & 6.28 & \frac{73.00}{73} \\ 02-28-89 & 15.87 & 9.51 & 75.08 \\ 04-04-89 & 21.87 & 12.06 & 76.00 \\ 04-19-89 & 16.37 & 10.22 & 74.08 \\ 05-08-89 & 10.27 & 8.01 & 74.31 \\ 06-05-89 & 12.81 & 8.58 & 74.31 \\ 06-26-89 & 12.65 & 8.38 & 74.54 \\ 07-25-89 & 12.63 & 8.40 & 74.77 \\ 09-11-89 & 12.33 & 8.61 & 74.77 \\ 10-03-89 & 12.24 & 8.52 & 74.77\end{array}$

COW CAMP CREEK SITE

WELL ID

$\frac{\text { SCL287 }}{3.22}$

3.22

3.15

3.07

3.56

3.82

1.70

1.71

1.71

1.71

1.71

1.71

3.97

3.97

$12-13-88$

01-31-89

03-02-89

$03-16-89$

04-05-89

04-19-89

05-08-89

06-05-89

06-26-89

07-25-89

09-11-89

10-03-89
WELL ID

$\underline{\text { SCL287 }}$

4.20

4.43

4.66

4.66

2.17

1.71

1.71

1.71

1.71

1.71

1.71
WELL ID

$\underline{\mathrm{SCI} 287}$

4.67

3.26

4.07

3.26

3.72

2.55

2.55

2.30

2.30

2.00

2.00

3.97

4.20

WELL ID

SCI287

4.20

4.43

5.35

3.28

2.55

2.00

2.00

2.00

2.00

2.00

2.00 
Table 11.--Hydraulic-head data for bedrock wells--Continued

\begin{tabular}{ccc}
\hline $\begin{array}{c}\text { Date of } \\
\text { measure- } \\
\text { ment }\end{array}$ & Hydraulic head & Hydraulic head \\
(feet above & (feet above \\
land surface) & land surface) \\
\hline
\end{tabular}

COW CAMP CREEK SITE--Continued

$\begin{array}{lcc} & \text { WELL ID } & \frac{\text { WELL ID }}{\text { SCW287 }} \\ 01-27-88 & \frac{\text { SCU287 }}{10.35} & 20.01 \\ 03-07-88 & 5.80 & 20.18 \\ 03-22-88 & 4.62 & 22.79 \\ 04-04-88 & 4.64 & 18.17 \\ 05-04-88 & 4.85 & 15.53 \\ 06-06-88 & 2.85 & 14.40 \\ 07-12-88 & 2.75 & 13.89 \\ 07-26-88 & 2.59 & 13.48 \\ 08-29-88 & 2.56 & 13.49 \\ 10-04-88 & 2.55 & 13.65 \\ 10-25-88 & 2.58 & 15.57 \\ 11-14-88 & 4.66 & 15.34 \\ 12-13-88 & 4.20 & \\ 01-38 & \text { WELL ID } \\ 01-31-89 & & \text { SCU287 } \\ 03-02-89 & \text { WELL ID } & 15.80 \\ 03-16-89 & \text { SCW287 } & 18.34 \\ 04-05-89 & 4.43 & 26.20 \\ 04-19-89 & 5.58 & 24.81 \\ 05-08-89 & 5.82 & 22.29 \\ 06-05-89 & 4.66 & 19.40 \\ 06-26-89 & 2.72 & 17.53 \\ 07-25-89 & 2.00 & 16.63 \\ 09-11-89 & 2.00 & 15.98 \\ 10-03-89 & 2.00 & 15.59 \\ 0 & 2.00 & 15.45\end{array}$

\section{ZULI SITE}

WELL ID

SZL287

20.04

$01-28-88$

03-04-88

03-23-88

04-04-88

05-04-88

06-06-88

$07-12-88$

07-26-88

08-29-88

10-03-88

10-26-88

$11-15-88$

$12-13-88$
13.36

18.77

18.56

16.88

19.35

19.62

20.64

21.22

20.39

25.27

29.66
WELL ID

SZI287

9.69

32.33

33.11

34.29

33.64

33.94

35.42

35.63

36.12

35.61

33.14

36.38

36.84 
Table 11.--Hydraulic-head data for bedrock wells--Continued

\begin{tabular}{ccc}
\hline $\begin{array}{c}\text { Date of } \\
\text { measure- } \\
\text { ment }\end{array}$ & $\begin{array}{c}\text { Hydraulic head } \\
\text { (feet above } \\
\text { land surface) }\end{array}$ & $\begin{array}{c}\text { Hydraulic head } \\
\text { (feet above } \\
\text { land surface) }\end{array}$ \\
\hline
\end{tabular}

\section{ZULI SITE--Continued}

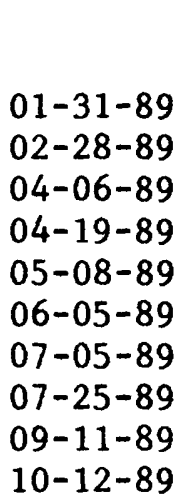

01-28-88

03-04-88

03-23-88

06-06-88

$07-12-88$

07-26-88

08-29-88

10-03-88

10-26-88

11-15-88

12-13-88

01-31-89

02-28-88

04-06-89

04-19-88

05-08-89

06-05-89

07-05-89

07-25-89

09-11-89

10-12-89
WELL ID

SZL287

31.04

35.43

31.51

22.72

23.53

23.39

23.53

24.00

23.88

24.18

WELL ID

SZW287

--

$--$

$--$

$--$

--

--

$--$

$--$

27.12

29.67

33.36

WELL ID

SZW287

34.06

37.06

37.52

32.64

33.68

33.48

33.94

33.01

32.99

32.67
WELL ID

SZI287

35.22

38.68

39.84

38.33

38.56

37.64

36.86

36.95

36.72

36.62

WELL ID

SZU287

2.61

4.92

24.23

50.66

54.35

56.66

55.74

54.82

--

$--$

$-$

WELL ID

SZU287

56.78

$--$

63.94

52.97

52.51

52.28

52.97

52.51

52.28 
Table 11.--Hydraulic-head data for bedrock wells--Continued

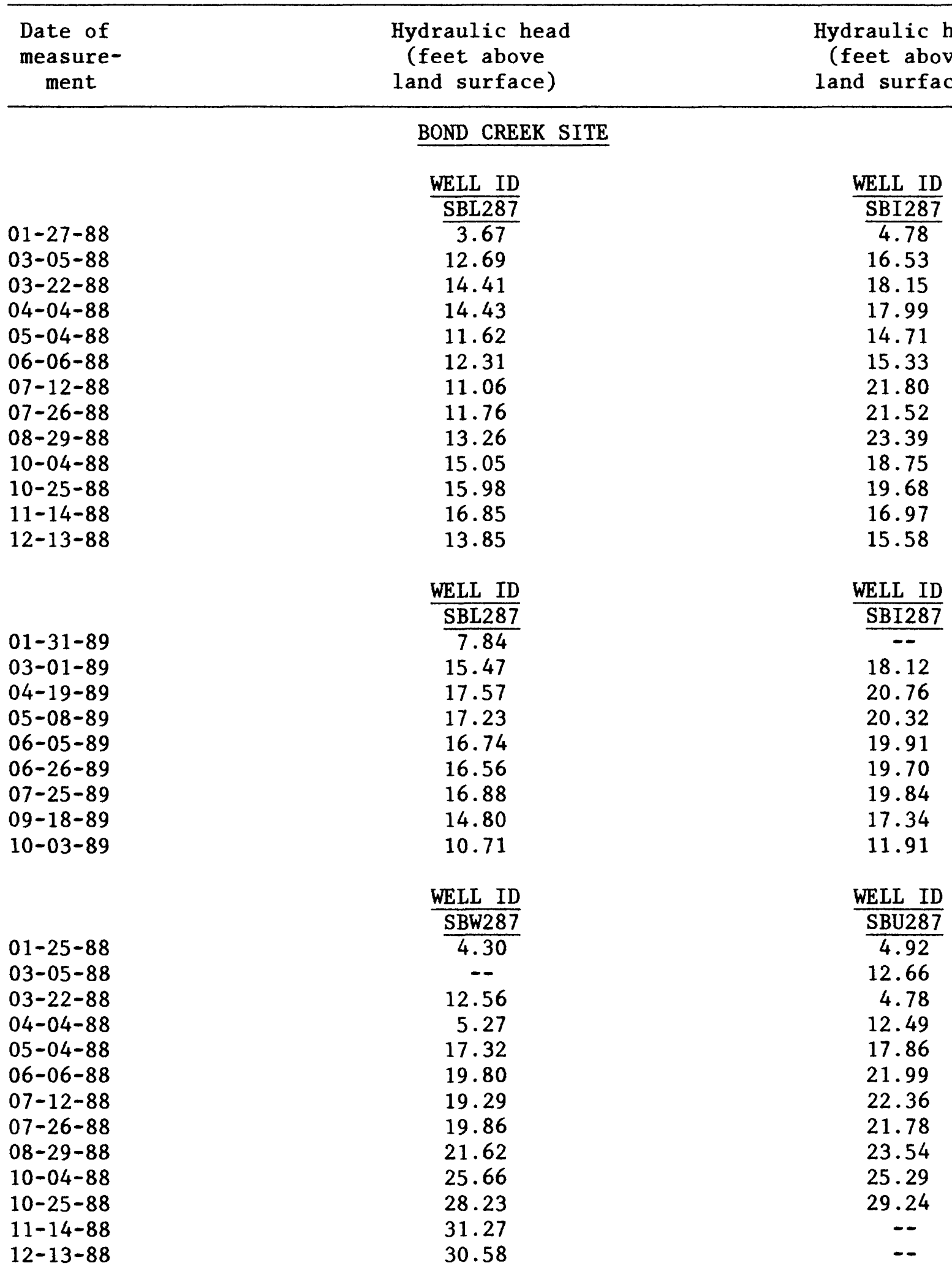


Table 11.--Hydraulic-head data for bedrock wells--Continued

\begin{tabular}{ccc}
\hline $\begin{array}{c}\text { Date of } \\
\text { measure- } \\
\text { ment }\end{array}$ & $\begin{array}{c}\text { Hydraulic head } \\
\text { (feet above } \\
\text { land surface) }\end{array}$ & $\begin{array}{c}\text { Hydraulic head } \\
\text { (feet above } \\
\text { land surface) }\end{array}$ \\
\hline BOND CREEK SITE--Continued & \\
$01-31-89$ & $\frac{\text { WELL ID }}{\text { SBW287 }}$ & WELL ID \\
$03-01-89$ & $\frac{\text { SBU287 }}{04.42}$ & -- \\
$05-19-89$ & -- & -- \\
$06-08-89$ & 19.33 & 18.01 \\
$06-26-89$ & 18.99 & 18.36 \\
$07-25-89$ & 18.59 & 16.51 \\
$09-18-89$ & 19.12 & 16.70 \\
$10-03-89$ & 18.53 & 16.74 \\
\hline
\end{tabular}


Table 12.--Water-level data for reclaimed spoil wells

[Well ID corresponds to well identifications listed in table 9]

\begin{tabular}{cccc}
\hline $\begin{array}{c}\text { Date of } \\
\text { measure- } \\
\text { ment }\end{array}$ & $\begin{array}{c}\text { Water level } \\
\text { (feet below } \\
\text { land surface) }\end{array}$ & $\begin{array}{l}\text { Water level } \\
\text { (feet below } \\
\text { land surface) }\end{array}$ & $\begin{array}{c}\text { Water level } \\
\text { (feet below } \\
\text { land surface) }\end{array}$ \\
\hline
\end{tabular}

SPRING CREEK SITE

$\begin{array}{lccc} & \frac{\text { WELL ID }}{\text { SSS487-59 }} & \frac{\text { WELL ID }}{\text { SSS487-60 }} & \text { WELL ID } \\ 12-07-87 & 25.27 & 19.52 & 17.68 \\ 01-26-88 & 25.36 & 19.43 & 17.81 \\ 03-07-88 & 18.88 & 14.01 & 12.35 \\ 03-23-88 & 20.76 & 14.88 & 13.21 \\ 04-05-88 & 19.65 & 13.78 & 12.10 \\ 05-10-88 & 22.18 & 16.30 & 14.66 \\ 06-07-88 & 23.62 & 17.73 & 16.10 \\ 07-11-88 & 22.54 & 16.63 & 15.00 \\ 07-25-88 & 22.67 & 16.76 & 15.13 \\ 08-30-88 & 23.06 & 17.15 & 15.51 \\ 10-05-88 & 23.07 & 17.16 & 15.52 \\ 11-14-88 & 23.08 & 17.17 & 15.53 \\ 12-13-88 & 23.15 & 17.24 & 15.59 \\ & & & \\ 01-30-89 & \text { WELL ID } & \text { WELL ID } & \text { WELL ID } \\ 02-28-89 & \text { SSS487-59 } & \text { SSS487-60 } & 13 \text { SS487-61 } \\ 04-04-89 & 23.20 & 17.26 & 15.65 \\ 04-19-89 & 21.01 & 15.11 & 6.10 \\ 05-08-89 & 13.57 & 7.72 & 11.72 \\ 06-05-89 & 18.21 & 12.36 & 13.94 \\ 06-26-89 & 21.46 & 15.57 & 15.26 \\ 07-25-89 & 22.78 & 16.89 & 15.53 \\ 09-11-89 & 23.05 & 17.13 & 15.85 \\ 10-03-89 & 23.41 & 17.47 & 16.10 \\ & 23.65 & 17.71 & 16.16\end{array}$


Table 12.-Water-level data for reclaimed spoil wells--Continued

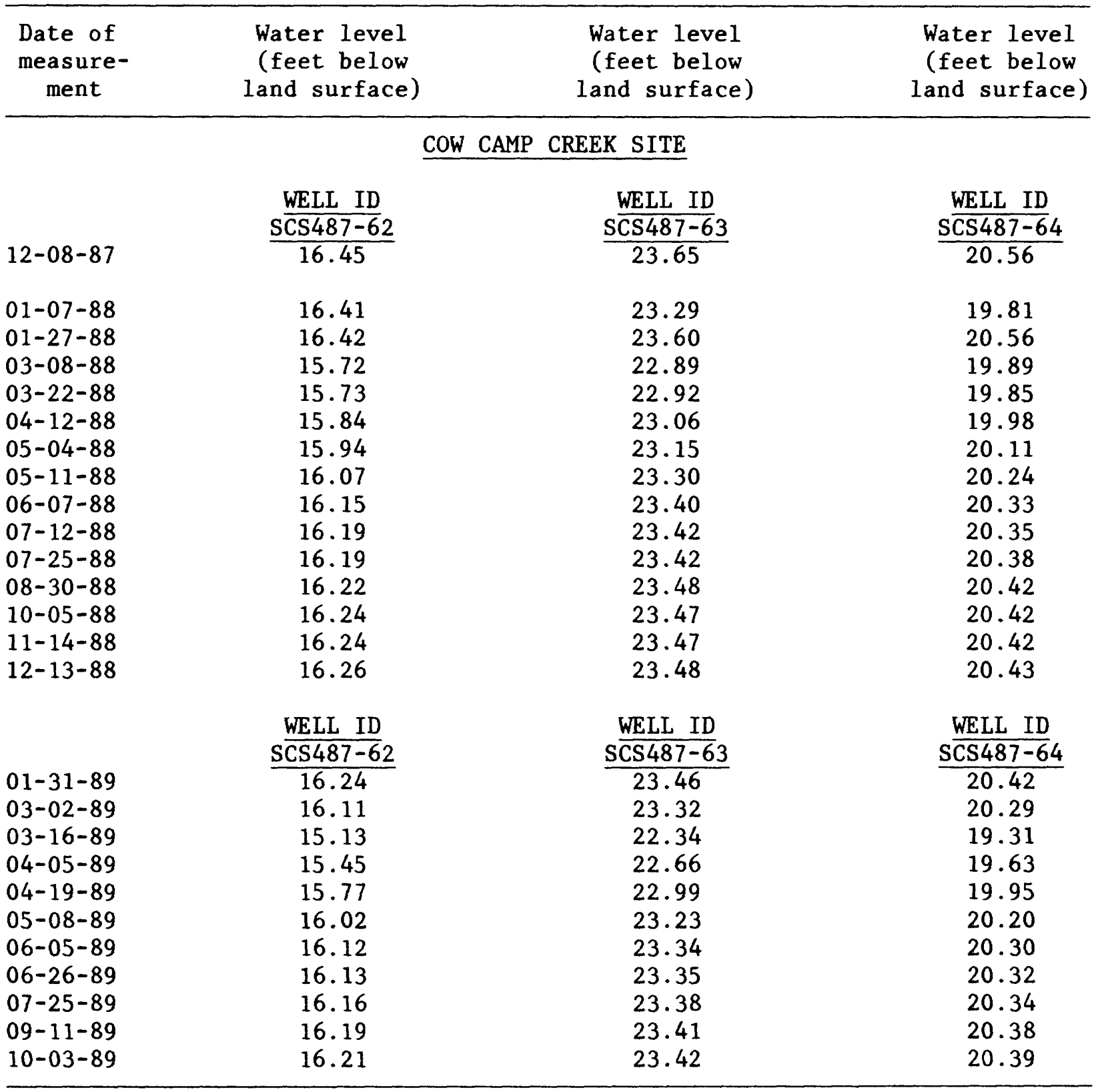


Table 13.--Mean daily water levels for reclaimed spoil well SSS487-60 at the Spring Creek site [--, no data; MEAN, mean water level; MAX, maximum water level; MIN, minimum water level; WL, water level] MEAN VALUES OF DEPTH OF WATER LEVEL BELOW LAND SURFACE, IN FEET, WATER YEAR 1989

\begin{tabular}{|c|c|c|c|c|c|c|c|c|c|c|c|c|}
\hline DAY & $\mathrm{OCT}$ & NOV & DEC & JAN & FEB & MAR & APR & MAY & JUNE & JULY & AUG & SEPT \\
\hline $\begin{array}{l}1 \\
2 \\
3 \\
4 \\
5\end{array}$ & $\begin{array}{l}-- \\
-- \\
-- \\
-- \\
--\end{array}$ & $\begin{array}{l}-- \\
-- \\
-- \\
-- \\
--\end{array}$ & $\begin{array}{l}17.17 \\
17.17 \\
17.17 \\
17.18 \\
17.19\end{array}$ & $\begin{array}{l}17.40 \\
17.41 \\
17.43 \\
17.44 \\
17.43\end{array}$ & $\begin{array}{l}17.18 \\
17.10 \\
16.99 \\
16.90 \\
16.87\end{array}$ & $\begin{array}{c}14.93 \\
-- \\
-- \\
-- \\
--\end{array}$ & $\begin{array}{l}-- \\
7.16 \\
7.45 \\
7.83 \\
8.21\end{array}$ & $\begin{array}{l}14.80 \\
14.93 \\
15.04 \\
15.16 \\
15.27\end{array}$ & $\begin{array}{l}16.75 \\
16.78 \\
16.80 \\
16.82 \\
16.85\end{array}$ & $\begin{array}{l}17.16 \\
17.18 \\
17.19 \\
17.20 \\
17.22\end{array}$ & $\begin{array}{l}17.43 \\
17.43 \\
17.43 \\
17.44 \\
17.44\end{array}$ & $\begin{array}{l}17.58 \\
17.59 \\
17.60 \\
17.61 \\
17.62\end{array}$ \\
\hline $\begin{array}{r}6 \\
7 \\
8 \\
9 \\
10\end{array}$ & $\begin{array}{l}-- \\
-- \\
-- \\
-- \\
--\end{array}$ & $\begin{array}{l}-- \\
-- \\
-- \\
-- \\
--\end{array}$ & $\begin{array}{l}17.18 \\
17.17 \\
17.18 \\
17.19 \\
17.19\end{array}$ & $\begin{array}{l}17.42 \\
17.44 \\
17.46 \\
17.47 \\
17.47\end{array}$ & $\begin{array}{l}16.86 \\
16.86 \\
16.87 \\
16.88 \\
16.88\end{array}$ & $\begin{array}{l}-- \\
-- \\
-- \\
-- \\
--\end{array}$ & $\begin{array}{l}8.60 \\
8.96 \\
9.29 \\
9.63 \\
9.95\end{array}$ & $\begin{array}{l}15.37 \\
15.47 \\
15.55 \\
15.63 \\
15.70\end{array}$ & $\begin{array}{l}16.86 \\
16.88 \\
16.90 \\
16.91 \\
16.93\end{array}$ & $\begin{array}{l}17.23 \\
17.25 \\
17.26 \\
17.26 \\
17.28\end{array}$ & $\begin{array}{l}17.44 \\
17.45 \\
17.45 \\
17.45 \\
17.45\end{array}$ & $\begin{array}{l}17.62 \\
17.63 \\
17.64 \\
17.65 \\
17.65\end{array}$ \\
\hline $\begin{array}{l}11 \\
12 \\
13 \\
14 \\
15\end{array}$ & $\begin{array}{l}-- \\
-- \\
-- \\
-- \\
--\end{array}$ & $\begin{array}{l}-- \\
-- \\
-- \\
-- \\
--\end{array}$ & $\begin{array}{l}17.20 \\
17.22 \\
17.22 \\
17.22 \\
17.24\end{array}$ & $\begin{array}{l}17.48 \\
17.14 \\
17.51 \\
17.50 \\
17.52\end{array}$ & $\begin{array}{l}16.89 \\
16.88 \\
16.88 \\
16.89 \\
16.91\end{array}$ & $\begin{array}{l}-- \\
-- \\
-- \\
-- \\
--\end{array}$ & $\begin{array}{l}10.21 \\
10.48 \\
10.75 \\
11.01 \\
11.27\end{array}$ & $\begin{array}{l}15.76 \\
15.83 \\
15.89 \\
15.95 \\
16.01\end{array}$ & $\begin{array}{l}16.95 \\
16.97 \\
16.98 \\
16.99 \\
17.00\end{array}$ & $\begin{array}{l}17.30 \\
17.32 \\
17.33 \\
17.34 \\
17.35\end{array}$ & $\begin{array}{l}17.45 \\
17.45 \\
17.46 \\
17.46 \\
17.47\end{array}$ & $\begin{array}{l}17.66 \\
17.67 \\
17.68 \\
17.69 \\
17.69\end{array}$ \\
\hline $\begin{array}{l}16 \\
17 \\
18 \\
19 \\
20\end{array}$ & $\begin{array}{l}-- \\
-- \\
-- \\
-- \\
--\end{array}$ & $\begin{array}{c}-- \\
-- \\
17.09 \\
17.09 \\
17.09\end{array}$ & $\begin{array}{l}17.27 \\
17.28 \\
17.28 \\
17.26 \\
17.28\end{array}$ & $\begin{array}{l}17.53 \\
17.53 \\
17.54 \\
17.54 \\
17.54\end{array}$ & $\begin{array}{l}16.93 \\
16.93 \\
16.92 \\
16.88 \\
16.88\end{array}$ & $\begin{array}{l}-- \\
7.59 \\
7.30 \\
6.97 \\
6.74\end{array}$ & $\begin{array}{l}11.54 \\
11.83 \\
12.10 \\
12.35 \\
12.59\end{array}$ & $\begin{array}{l}16.07 \\
16.13 \\
16.18 \\
16.24 \\
16.29\end{array}$ & $\begin{array}{l}17.00 \\
17.03 \\
17.04 \\
17.04 \\
17.05\end{array}$ & $\begin{array}{l}17.35 \\
17.37 \\
17.38 \\
17.39 \\
17.39\end{array}$ & $\begin{array}{l}17.46 \\
17.47 \\
17.47 \\
17.48 \\
17.48\end{array}$ & $\begin{array}{l}17.69 \\
17.69 \\
17.69 \\
17.69 \\
17.69\end{array}$ \\
\hline $\begin{array}{l}21 \\
22 \\
23 \\
24 \\
25\end{array}$ & $\begin{array}{l}-- \\
-- \\
-- \\
-- \\
--\end{array}$ & $\begin{array}{l}17.08 \\
17.08 \\
17.07 \\
17.07 \\
17.08\end{array}$ & $\begin{array}{l}17.29 \\
17.30 \\
17.31 \\
17.32 \\
17.32\end{array}$ & $\begin{array}{l}17.51 \\
17.49 \\
17.47 \\
17.44 \\
17.41\end{array}$ & $\begin{array}{l}16.89 \\
16.89 \\
16.84 \\
16.79 \\
16.59\end{array}$ & $\begin{array}{l}6.64 \\
6.47 \\
6.34 \\
6.27 \\
6.25\end{array}$ & $\begin{array}{l}12.82 \\
13.05 \\
13.26 \\
13.48 \\
13.70\end{array}$ & $\begin{array}{l}16.33 \\
16.37 \\
16.41 \\
16.46 \\
16.50\end{array}$ & $\begin{array}{l}17.07 \\
17.07 \\
17.07 \\
17.09 \\
17.09\end{array}$ & $\begin{array}{l}17.40 \\
17.41 \\
17.42 \\
17.43 \\
17.43\end{array}$ & $\begin{array}{l}17.48 \\
17.49 \\
17.50 \\
17.50 \\
17.51\end{array}$ & $\begin{array}{l}17.71 \\
17.72 \\
17.72 \\
17.72 \\
17.72\end{array}$ \\
\hline $\begin{array}{l}26 \\
27 \\
28 \\
29 \\
30 \\
31\end{array}$ & $\begin{array}{l}-- \\
-- \\
-- \\
-- \\
-- \\
--\end{array}$ & $\begin{array}{c}17.07 \\
17.10 \\
17.12 \\
17.14 \\
17.15 \\
=-\end{array}$ & $\begin{array}{l}17.33 \\
17.36 \\
17.36 \\
17.37 \\
17.38 \\
17.38\end{array}$ & $\begin{array}{l}17.38 \\
17.33 \\
17.30 \\
17.29 \\
17.27 \\
17.24\end{array}$ & $\begin{array}{c}16.09 \\
15.44 \\
15.12 \\
-- \\
-- \\
--\end{array}$ & $\begin{array}{l}6.28 \\
6.36 \\
6.45 \\
6.55 \\
6.68 \\
--\end{array}$ & $\begin{array}{c}13.91 \\
14.11 \\
14.31 \\
14.49 \\
14.66 \\
--\end{array}$ & $\begin{array}{l}16.55 \\
16.58 \\
16.61 \\
16.64 \\
16.67 \\
16.72\end{array}$ & $\begin{array}{c}17.11 \\
17.12 \\
17.13 \\
17.14 \\
17.15 \\
--\end{array}$ & $\begin{array}{l}17.44 \\
17.44 \\
17.44 \\
17.44 \\
17.44 \\
17.44\end{array}$ & $\begin{array}{l}17.52 \\
17.53 \\
17.54 \\
17.55 \\
17.55 \\
17.57\end{array}$ & $\begin{array}{c}17.72 \\
17.73 \\
17.73 \\
17.73 \\
17.73 \\
--\end{array}$ \\
\hline $\begin{array}{l}\text { MEAN WL } \\
\text { MAX WL } \\
\text { MIN WL }\end{array}$ & $\begin{array}{l}-- \\
-- \\
--\end{array}$ & $\begin{array}{l}-- \\
-- \\
--\end{array}$ & $\begin{array}{l}17.26 \\
17.38 \\
17.17\end{array}$ & $\begin{array}{l}17.43 \\
17.54 \\
17.14\end{array}$ & $\begin{array}{l}16.75 \\
17.18 \\
15.12\end{array}$ & $\begin{array}{l}-- \\
-- \\
--\end{array}$ & $\begin{array}{l}-- \\
-- \\
--\end{array}$ & $\begin{array}{l}15.97 \\
16.72 \\
14.80\end{array}$ & $\begin{array}{l}16.99 \\
17.15 \\
16.75\end{array}$ & $\begin{array}{l}17.34 \\
17.44 \\
17.16\end{array}$ & $\begin{array}{l}17.48 \\
17.57 \\
17.43\end{array}$ & $\begin{array}{l}17.68 \\
17.73 \\
17.58\end{array}$ \\
\hline
\end{tabular}


Table 14.--Nean daily water levels for reclaimed spoil well scs487-63 at the Cow Camp Creek site

[--, no data; MEAN, mean water level; MAX, maximum water level; MIN, minimum water level; WL, water level]

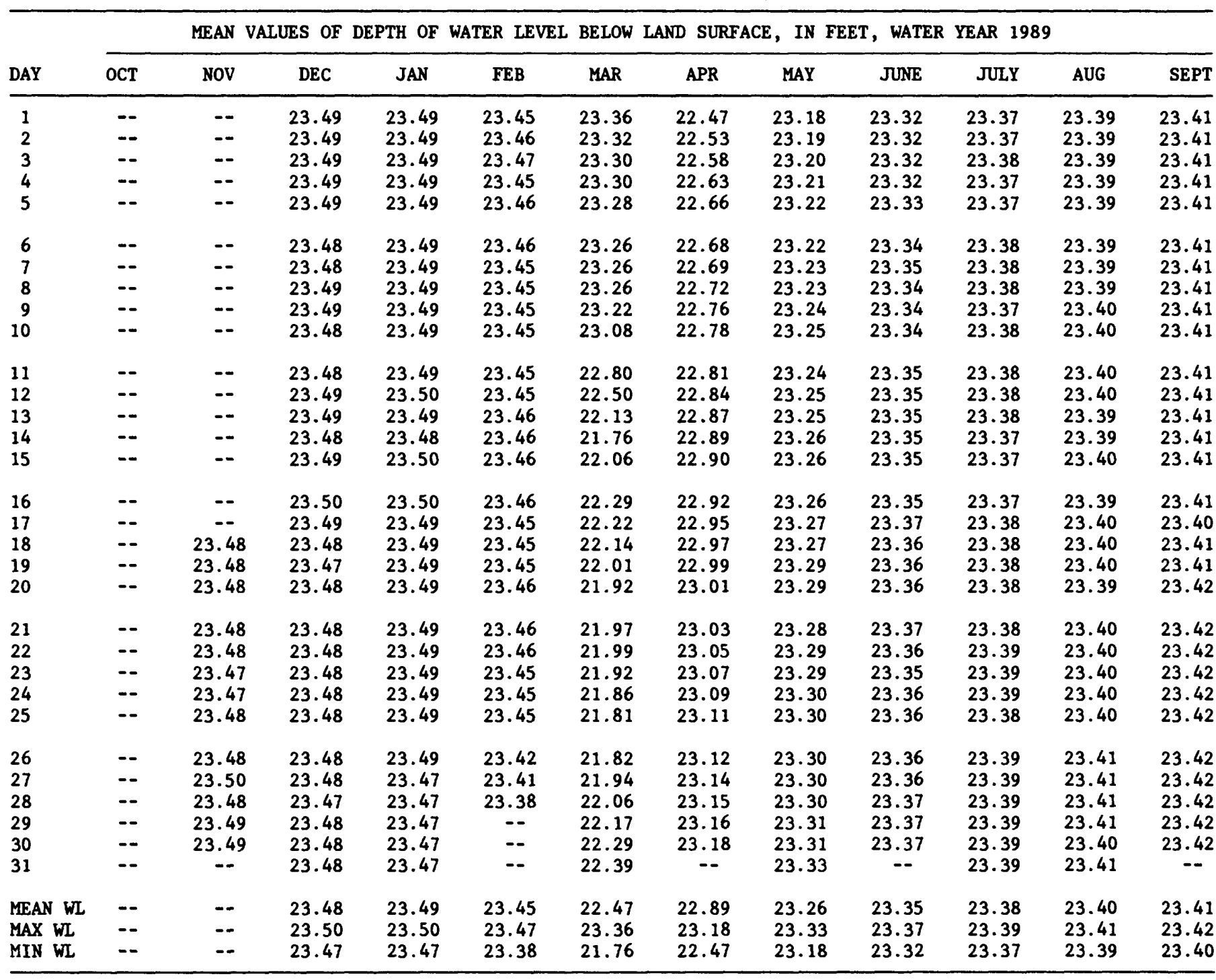


Table 15.--Porosity, grain density, and permeability of selected core samples from the spring Creek site core hole

[Ka, permeability to air; $\mathrm{Kl}, \mathrm{Kl}$ inkenberg permeability to gas equivalent to that of a non reactive liquid; $\mathrm{Kw}$, permeability to water; $\mathrm{g} / \mathrm{cm}^{3}$, grams per cubic centimeter; $\mathrm{mD}$, millidarcies; --, no measurement; <, less than]

\begin{tabular}{|c|c|c|c|c|c|c|c|}
\hline \multirow{2}{*}{$\begin{array}{c}\text { Sample } \\
\text { identi- } \\
\text { fication } \\
\quad(\text { see } \\
\text { fig. 22) }\end{array}$} & \multirow{2}{*}{$\begin{array}{l}\text { Depth } \\
\text { (feet) }\end{array}$} & \multirow[t]{2}{*}{ Lithology } & \multirow{2}{*}{$\begin{array}{l}\text { Porosity } \\
\text { (percent) }\end{array}$} & \multirow{2}{*}{$\begin{array}{c}\text { Grain } \\
\text { density } \\
\left(\mathrm{g} / \mathrm{cm}^{3}\right)\end{array}$} & \multicolumn{3}{|c|}{$\begin{array}{c}\text { Permeability } \\
(\mathrm{mD})\end{array}$} \\
\hline & & & & & $\mathrm{Ka}$ & $\mathrm{Kl}$ & $\mathrm{Kw}$ \\
\hline SIV & $35.1-35.4$ & Shale & 3.6 & 2.50 & - & - & - \\
\hline $\mathrm{S} 2 \mathrm{~V}$ & $37.1-37.7$ & Sandstone & 9.6 & 2.67 & 11.1 & 9.35 & -- \\
\hline $\mathrm{S} 3 \mathrm{H}$ & $41.1-41.6$ & Lennox coal & 7.1 & 1.35 & 2.37 & - & $<0.001$ \\
\hline $\mathrm{S} 4 \mathrm{~V}$ & $48.5-49.0$ & Shale & 5.6 & 2.64 & 0.016 & $<0.01$ & - \\
\hline S5H & $60.6-61.1$ & Sands tone & 16.7 & 2.67 & 2.02 & 1.29 & .429 \\
\hline S5V & $60.6-61.1$ & Sandstone & 17.7 & 2.65 & 5.84 & 4.68 & -- \\
\hline $\mathrm{S} 6 \mathrm{H}$ & $75.0-75.5$ & Sandstone & 15.2 & 2.66 & 7.32 & 5.64 & -- \\
\hline $\mathrm{S} 8 \mathrm{H}$ & $86.5-86.9$ & Wadge coal & 6.7 & 1.34 & - & - & - \\
\hline S10V & $94.4-94.7$ & Shale & 6.2 & 2.40 & $<.01$ & $<.01$ & -- \\
\hline $\mathrm{S} 11 \mathrm{H}$ & $100.0-100.5$ & Sandstone & 10.8 & 2.67 & .149 & .046 & .009 \\
\hline $\mathrm{S} 12 \mathrm{H}$ & $102.1-102.6$ & Sandstone & 8.1 & 2.64 & .011 & $<.01$ & $<.001$ \\
\hline
\end{tabular}

${ }^{1}$ Sample indentifications were designated as follows: The first letter of the identification corresponds to the site location, $S$ indicating the Spring Creek site. The numeral represents the relative depth of the sample at each site, 1 indicates the shallowest and 12 the deepest. The last letter in the identification indicates the orientation of the sample; $V$ indicates vertically oriented, and $\mathrm{H}$ indicates horizontally oriented. 
Table 16.--Porosity, grain density, and permeability of selected core samples from the Cow Camp Creek site core hole

[Ka, permeability to air; $\mathrm{K} 1$, Klinkenberg permeability to gas equivalent to that of a non reactive liquid; $\mathrm{Kw}$, permeability to water; $\mathrm{g} / \mathrm{cm}^{3}$, grams per cubic centimeter; $\mathrm{mD}$, millidarcies; --, no measurement; <, less than]

\begin{tabular}{|c|c|c|c|c|c|c|c|}
\hline \multirow{2}{*}{$\begin{array}{c}\text { Sample } \\
\text { identi- } \\
\text { fication } \\
\text { (see } \\
\text { fig. } 23)^{1}\end{array}$} & \multirow{2}{*}{$\begin{array}{l}\text { Depth } \\
\text { (feet) }\end{array}$} & \multirow[t]{2}{*}{ Lithology } & \multirow{2}{*}{$\begin{array}{l}\text { Porosity } \\
\text { (percent) }\end{array}$} & \multirow{2}{*}{$\begin{array}{c}\text { Grain } \\
\text { density } \\
\left(\mathrm{g} / \mathrm{cm}^{3}\right)\end{array}$} & \multicolumn{3}{|c|}{$\begin{array}{c}\begin{array}{c}\text { Permeability } \\
(\mathrm{mD})\end{array} \\
\end{array}$} \\
\hline & & & & & $\mathrm{Ka}$ & $\mathrm{Kl}$ & $\mathrm{Kw}$ \\
\hline $\mathrm{C} 1 \mathrm{~V}$ & $18.4-19.0$ & Sandstone & 9.8 & 2.63 & 0.105 & 0.030 & -- \\
\hline $\mathrm{C} 2 \mathrm{H}$ & $22.3-22.9$ & Lennox coal & 6.2 & 1.38 & 1.76 & -- & -- \\
\hline $\mathrm{C} 3 \mathrm{~V}$ & $27.7-28.2$ & Sandstone & 11.2 & 2.67 & .027 & .010 & -- \\
\hline $\mathrm{C} 4 \mathrm{H}$ & $29.8-30.2$ & Sandstone & 9.2 & 2.64 & 46.5 & 43.9 & -- \\
\hline $\mathrm{C} 6 \mathrm{H}$ & $44.8-45.3$ & Sandstone & 16.7 & 2.66 & 4.59 & 3.35 & 1.28 \\
\hline $\mathrm{C} 7 \mathrm{H}$ & $55.0-55.5$ & Sandstone & 15.4 & 2.66 & 1.75 & 1.22 & -- \\
\hline $\mathrm{C} 7 \mathrm{~V}$ & $55.0-55.5$ & Sandstone & 15.4 & 2.64 & .839 & .500 & -- \\
\hline $\mathrm{C} 9 \mathrm{H}$ & $66.0-66.5$ & Sandstone & 15.7 & 2.66 & 7.71 & 5.97 & 1.57 \\
\hline $\mathrm{CgV}$ & $66.0-66.5$ & Sandstone & 16.0 & 2.65 & 1.96 & 1.35 & -- \\
\hline $\mathrm{C} 10 \mathrm{~V}$ & $73.5-73.7$ & Shale & 3.4 & 2.57 & -- & -- & -- \\
\hline $\mathrm{C} 11 \mathrm{H}$ & $76.0-76.6$ & Wadge coal & 2.6 & 1.32 & -- & -- & -- \\
\hline $\mathrm{C} 12 \mathrm{~V}$ & $86.6-87.1$ & Sandstone & 14.8 & 2.63 & .247 & .082 & .009 \\
\hline $\mathrm{C} 13 \mathrm{H}$ & $90.2-91.2$ & Shale & 5.0 & 2.56 & .0013 & .0007 & -- \\
\hline C13V & $90.2-91.2$ & Shale & 5.4 & 2.59 & .0016 & .0004 & -- \\
\hline $\mathrm{C} 15 \mathrm{H}$ & $106.2-106.7$ & Sandstone & 10.5 & 2.68 & .178 & .059 & .020 \\
\hline $\mathrm{C} 15 \mathrm{~V}$ & $106.2-106.7$ & Sandstone & 10.6 & 2.68 & .067 & .020 & -- \\
\hline $\mathrm{C} 16 \mathrm{H}$ & $114.5-114.9$ & Sandstone & 4.5 & 2.66 & -- & -- & -- \\
\hline $\mathrm{C} 17 \mathrm{~V}$ & $118.8-119.2$ & Shale & 1.4 & 2.47 & -- & -- & -- \\
\hline
\end{tabular}

${ }^{1}$ Sample indentifications were designated as follows: The first letter of the identification corresponds to the site location, C indicating the Cow Camp Creek site. The numeral represents the relative depth of the sample at each site; 1 indicates the shallowest and 20 the deepest. The last letter in the identification indicates the orientation of the sample; $V$ indicates vertically oriented, and $\mathrm{H}$ indicates horizontally oriented. 
Table 17.--Porosity, grain density, and permeability of selected core samples from the Zuli site core hole

[Ka, permeability to air; $\mathrm{Kl}$, Klinkenberg permeability to gas equivalent to that of a non reactive liquid; $\mathrm{Kw}$, permeability to water; $\mathrm{g} / \mathrm{cm}^{3}$, grams per cubic centimeter; $\mathrm{mD}$, millidarcies; --, no measurement; <, less than]

\begin{tabular}{|c|c|c|c|c|c|c|c|}
\hline \multirow{2}{*}{$\begin{array}{c}\text { Sample } \\
\text { identi- } \\
\text { fication } \\
\quad(\text { see } \\
\text { fig. 24) }\end{array}$} & \multirow{2}{*}{$\begin{array}{l}\text { Depth } \\
\text { (feet) }\end{array}$} & \multirow[t]{2}{*}{ Lithology } & \multirow{2}{*}{$\begin{array}{r}\text { Porosity } \\
\text { (percent) }\end{array}$} & \multirow{2}{*}{$\begin{array}{c}\text { Grain } \\
\text { density } \\
\left(\mathrm{g} / \mathrm{cm}^{3}\right)\end{array}$} & \multicolumn{3}{|c|}{$\begin{array}{c}\text { Permeability } \\
(\mathrm{mD})\end{array}$} \\
\hline & & & & & $\mathrm{Ka}$ & $\mathrm{Kl}$ & $\mathrm{Kw}$ \\
\hline $\mathrm{Z1V}$ & $165.8-166.1$ & Shale & 5.2 & 2.90 & 0.039 & 0.032 & -- \\
\hline $\mathrm{Z} 2 \mathrm{~V}$ & $176.0-176.5$ & Sands tone & 6.0 & 2.64 & .030 & .010 & -- \\
\hline $\mathrm{Z} 3 \mathrm{H}$ & $181.2-181.6$ & Lennox coal & 2.8 & 1.30 & .858 & -- & 0.001 \\
\hline $\mathrm{Z4V}$ & $184.8-185.3$ & Shale & 3.8 & 2.32 & -- & -- & -- \\
\hline $\mathrm{Z6H}$ & $193.7-194.2$ & Sandstone & 16.1 & 2.64 & 2.07 & 1.83 & -- \\
\hline $\mathrm{Z} 7 \mathrm{H}$ & $208.5-209.0$ & Sandstone & 18.5 & 2.65 & 22.7 & 19.2 & 7.97 \\
\hline $\mathrm{Z} 8 \mathrm{~V}$ & $220.0-220.5$ & Sands tone & 18.4 & 2.66 & 13.8 & 11.4 & 3.53 \\
\hline $\mathrm{Z9H}$ & $230.8-231.3$ & Wadge coal & 3.5 & 1.40 & .152 & -- & $<.001$ \\
\hline $\mathrm{Z10V}$ & $237.1-237.7$ & Sandstone & 10.7 & 2.65 & .022 & $<.01$ & -- \\
\hline $\mathrm{Z14H}$ & $250.5-251.0$ & Sandstone & 12.7 & 2.69 & 1.31 & .754 & -- \\
\hline $\mathrm{Z15H}$ & $258.4-258.9$ & Sandstone & 6.1 & 2.70 & $<.01$ & $<.01$ & $<.001$ \\
\hline
\end{tabular}

${ }^{1}$ Sample indentifications were designated as follows: The first letter of the identification corresponds to the site location, $Z$ indicating the Zuli site. The numeral represents the relative depth of the sample at each site; 1 indicates the shallowest and 15 the deepest. The last letter in the identification indicates the orientation of the sample; $V$ indicates vertically oriented, and $\mathrm{H}$ indicates horizontally oriented. 
Table 18.--Porosity, grain density, and permeability of selected core samples from the Bond Creek site core hole

[Ka, permeability to air; K1, K1inkenberg permeability to gas equivalent to that of a non reactive liquid; $\mathrm{Kw}$, permeability to water; $\mathrm{g} / \mathrm{cm}^{3}$, grams per cubic centimeter; $\mathrm{mD}$, millidarcies; --, no measurement; <, less than]

\begin{tabular}{|c|c|c|c|c|c|c|c|}
\hline \multirow{2}{*}{$\begin{array}{c}\text { Sample } \\
\text { identi- } \\
\text { fication } \\
\text { (see } \\
\text { fig. 25) }\end{array}$} & \multirow{2}{*}{$\begin{array}{l}\text { Depth } \\
\text { (feet) }\end{array}$} & \multirow[t]{2}{*}{ Lithology } & \multirow{2}{*}{$\begin{array}{l}\text { Porosity } \\
\text { (percent) }\end{array}$} & \multirow{2}{*}{$\begin{array}{c}\text { Grain } \\
\text { density } \\
\left(\mathrm{g} / \mathrm{cm}^{3}\right)\end{array}$} & \multicolumn{3}{|c|}{$\begin{array}{c}\text { Permeability } \\
(\mathrm{mD})\end{array}$} \\
\hline & & & & & $\mathrm{Ka}$ & $\mathrm{K} 1$ & $\mathrm{Kw}$ \\
\hline B3V & $55.2-55.6$ & Shale & 3.6 & 2.55 & -- & -- & -- \\
\hline $\mathrm{B} 4 \mathrm{~V}$ & $70.8-71.5$ & Shale & 5.5 & 2.64 & -- & -- & -- \\
\hline $\mathrm{B} 6 \mathrm{~V}$ & $86.5-87.0$ & Sandstone & 9.9 & 2.66 & 0.249 & 0.109 & -- \\
\hline $\mathrm{B} 7 \mathrm{H}$ & $95.4-95.9$ & Lennox coal & 3.9 & 1.31 & .118 & - & $<.001$ \\
\hline $\mathrm{B} 8 \mathrm{~V}$ & $103.7-104.2$ & Sandstone & 9.3 & 2.63 & .011 & $<.01$ & -- \\
\hline $\mathrm{B} 10 \mathrm{H}$ & $119.1-119.6$ & Sandstone & 15.5 & 2.66 & 3.62 & 2.54 & 0.849 \\
\hline $\mathrm{B} 10 \mathrm{~V}$ & $119.1-119.6$ & Sandstone & 15.6 & 2.66 & 2.08 & 1.46 & -- \\
\hline B11H & $128.5-129.0$ & Sandstone & 15.5 & 2.65 & 2.83 & 2.12 & -- \\
\hline $\mathrm{B} 11 \mathrm{~V}$ & $128.5-129.0$ & Sandstone & 16.4 & 2.66 & 3.45 & 2.57 & -- \\
\hline $\mathrm{B} 13 \mathrm{H}$ & $145.0-145.4$ & Sands tone & 13.0 & 2.67 & .951 & .501 & .146 \\
\hline $\mathrm{B} 14 \mathrm{~V}$ & $152.7-153.2$ & Shale & 4.3 & 2.59 & -- & -- & -- \\
\hline $\mathrm{B} 15 \mathrm{H}$ & $159.9-160.5$ & Wadge coal & 4.1 & 1.30 & -- & -- & -- \\
\hline $\mathrm{B} 16 \mathrm{~V}$ & $168.6-169.0$ & Shale & 5.8 & 2.47 & -- & -- & -- \\
\hline $\mathrm{B} 17 \mathrm{H}$ & $171.3-171.8$ & Sands tone & 13.5 & 2.67 & 1.47 & .891 & .313 \\
\hline
\end{tabular}

${ }^{1}$ Sample indentifications were designated as follows: The first letter of the identification corresponds to the site location, B indicating the Bond Creek site. The numeral represents the relative depth of the sample at each site; 3 indicates the shallowest and 17 the deepest. The last letter in the identification indicates the orientation of the sample; $V$ indicates vertically oriented, and $\mathrm{H}$ indicates horizontally oriented. 
Table 19.--Nineral composition of core samples from selected lithologic units at the Cow Camp Creek site

[Mineralogy in percent by weight; <, less than; $\mu m$, micrometer; --, none detected; tr, less than 1 percent]

\begin{tabular}{|c|c|c|c|c|c|c|c|c|c|c|c|c|c|}
\hline \multirow{2}{*}{$\begin{array}{l}\text { Sample } \\
\text { identifi- } \\
\text { cation } \\
\text { (see } \\
\text { fig. 28) }\end{array}$} & \multirow{2}{*}{$\begin{array}{l}\text { Litho- } \\
\text { logic } \\
\text { type }\end{array}$} & \multicolumn{12}{|c|}{ Bulk rock mineralogy } \\
\hline & & Quartz & $\begin{array}{r}\text { Clay } \\
<2 \mathrm{\mu m}\end{array}$ & $\begin{array}{l}\text { Cal- } \\
\text { cite }\end{array}$ & $\begin{array}{l}\text { Dolomite/ } \\
\text { ankerite }\end{array}$ & $\begin{array}{c}\text { Plagio- } \\
\text { clase } \\
\text { feldspar }\end{array}$ & $\begin{array}{r}\text { Potassiun } \\
\text { feldspar }\end{array}$ & Pyrite & $\begin{array}{l}\text { Sider- } \\
\text { ite }\end{array}$ & Illite & $\begin{array}{l}\text { Mixed } \\
\text { layer } \\
\text { clays }\end{array}$ & $\begin{array}{l}\text { Kao- } \\
\text { linite }\end{array}$ & $\begin{array}{c}\text { Chlor- } \\
\text { ite }\end{array}$ \\
\hline SSOI & Sandstone & 66 & 20 & $\ldots$ & $7 /=$ & 3 & 2 & 2 & $\ldots$ & 5 & 23 & 70 & 2 \\
\hline $\mathrm{SSO} 3$ & Sandstone & 65 & 18 & -- & $10 /=$ & 2 & 2 & tr & 3 & 17 & 37 & 44 & 2 \\
\hline SH04 & Shale & 33 & 54 & 1 & $71-$ & 2 & 2 & 1 & - & 24 & 42 & 34 & 2 \\
\hline SS05 & Sandstone & 73 & 10 & tr & $7 /--$ & 4 & 4 & 2 & -- & 14 & 20 & 64 & 2 \\
\hline SS06 & Sandstone & 71 & 9 & tr & $8 /=-$ & 3 & 7 & 2 & -- & 22 & 24 & 52 & 2 \\
\hline SH07 & Shale & 50 & 31 & 2 & $11 /=$ & 3 & 2 & 1 & -- & 21 & 37 & 38 & 2 \\
\hline SS08 & Sandstone & 70 & 11 & tr & $8 / 3$ & 4 & 4 & -- & -- & 14 & 18 & 63 & 5 \\
\hline SH09 & Shale & 30 & 56 & -- & $7 /--$ & 2 & 2 & 3 & -- & 23 & 50 & 25 & 2 \\
\hline SS12 & Sands tone & 72 & 16 & -- & $1 /--$ & 6 & 5 & $t r$ & -- & 21 & 19 & 58 & 2 \\
\hline SS13 & Sandstone & 60 & 15 & -- & $5 / 4$ & 6 & 6 & tr & 3 & 9 & 19 & 70 & 2 \\
\hline SH 14 & Shale & 63 & 19 & -- & $3 / 1$ & 5 & 4 & -- & 5 & 17 & 26 & 47 & 2 \\
\hline SH16 & Shale & 75 & 14 & -- & $1 /--$ & 5 & 4 & 1 & -- & 16 & 20 & 62 & 2 \\
\hline SS17 & Sandstone & 54 & 18 & 3 & $8 / 3$ & 5 & 7 & -- & 4 & 20 & 15 & 63 & 2 \\
\hline SH 18 & Shale & 30 & 58 & 2 & $4 /--$ & 2 & 1 & 2 & 1 & 17 & 56 & 25 & 2 \\
\hline
\end{tabular}

\section{COAL SAMPLES}

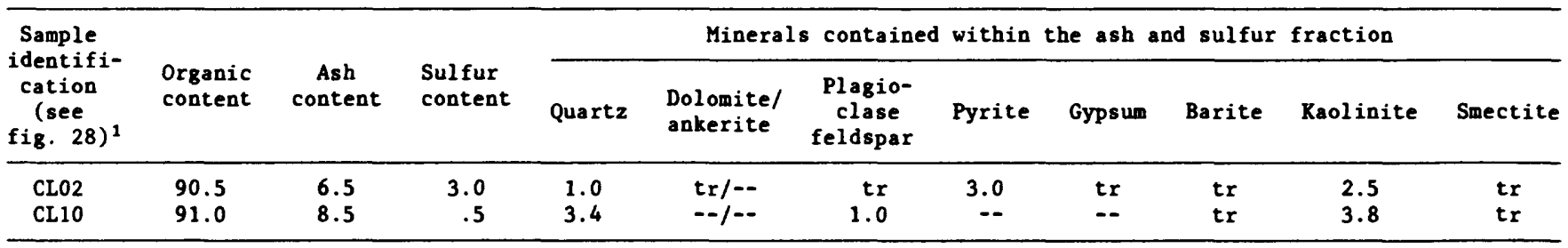

Sample indentifications were designated as follows: The first letter of the identification corresponds to the site

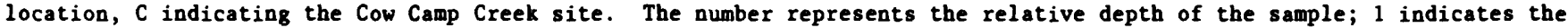
shallowest, and 20 the deepest. 
Table 20.--Spring and lysimeter information

[NGVD, National Geodetic Vertical Datum of 1929]

\begin{tabular}{llc}
\hline Site & $\begin{array}{c}\text { Station } \\
\text { identification }\end{array}$ & $\begin{array}{c}\text { Land-surface elevation } \\
\text { (feet above NGVD) }\end{array}$ \\
\hline Spring Creek & SSSS87-SPRING & 6,875 \\
Spring Creek & SSLYS-LYSIMETER & 6,955 \\
Cow Camp Creek & SCSS87-SPRING & 6,960 \\
Cow Camp Creek & SCLYS-LYSIMETER & 7,020 \\
\hline
\end{tabular}

${ }^{1}$ Station identifications were assigned as follows: The first letter (S), designates the Seneca II Coal Mine. The second letter ( $S$ or $C$ ) designates the site location as Spring Creek or Cow Camp Creek. The remaining alphanumeric characters designate the station as a spring or a lysimeter. 
Table 21.--Mean daily discharge from the spring at the Spring Creek site during water year 1988

[---, no data; TOTAL, sum of the discharges; MEAN, mean discharge; MAX, maximum discharge; MIN, minimum discharge; ACRE-FT, acre-foot; CAL YR, calender year; WTR YR, water year]

\begin{tabular}{|c|c|c|c|c|c|c|c|c|c|c|c|c|}
\hline & MEAN & $\checkmark$ VALUES & OF D & ISCHARGE, & , IN Cl & JBIC FEET & I PER & SECOND, & WATER & YEAR 1988 & & \\
\hline & 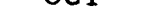 & 40 & & & 2.2 & & 80 & $11 \mathrm{nI}$ & 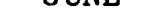 & 5021 & AOU & $\operatorname{ser} 1$ \\
\hline 1 & 0.04 & 0.03 & 0.03 & 0.04 & 0.03 & 0.11 & 0.11 & 0.08 & 0.06 & 0.04 & 0.04 & 0.04 \\
\hline 2 & .04 & .04 & .03 & .04 & .03 & .10 & .11 & .08 & .06 & .04 & .03 & .03 \\
\hline 3 & .05 & .04 & .03 & .04 & .04 & .11 & .11 & .07 & .06 & .04 & .03 & .03 \\
\hline 4 & .05 & .04 & .03 & .04 & .04 & .13 & .11 & .07 & .07 & .04 & .03 & .03 \\
\hline 5 & .05 & .03 & .03 & .04 & .04 & .17 & .11 & .07 & .06 & .04 & .03 & .03 \\
\hline 6 & .04 & .03 & .03 & .04 & .04 & .20 & .12 & .06 & .06 & .04 & .03 & .03 \\
\hline 7 & .04 & .03 & .03 & .04 & .04 & .21 & .12 & .06 & .05 & .04 & .04 & .03 \\
\hline 8 & .04 & .03 & .03 & .04 & .04 & .18 & .12 & .06 & .05 & .04 & .03 & .03 \\
\hline 9 & .03 & .03 & .03 & .04 & .05 & .09 & .11 & .07 & .05 & .04 & .03 & .03 \\
\hline 10 & .03 & .03 & .03 & .04 & .05 & .09 & .11 & .06 & .05 & .04 & .03 & .03 \\
\hline 11 & .04 & .03 & .03 & .04 & .05 & .09 & .12 & .09 & .05 & .04 & .03 & .05 \\
\hline 12 & .06 & .03 & .03 & .04 & .05 & .09 & .18 & .11 & .06 & .05 & .03 & .14 \\
\hline 13 & .08 & .03 & .03 & .04 & .05 & .09 & .14 & .12 & .08 & .04 & .04 & .07 \\
\hline 14 & .06 & .03 & .03 & .04 & .04 & .09 & .13 & .11 & .08 & .04 & .04 & .05 \\
\hline 15 & .04 & .03 & .04 & .04 & .04 & .09 & .13 & .10 & .04 & .04 & .04 & .04 \\
\hline 16 & .04 & .03 & .04 & .04 & .04 & .09 & .13 & .09 & .04 & .04 & .05 & .03 \\
\hline 17 & .04 & .03 & .04 & .04 & .04 & .09 & .13 & .07 & .04 & .04 & .04 & .03 \\
\hline 18 & .04 & .03 & .04 & .04 & .04 & .09 & .13 & .06 & .04 & .04 & .04 & .03 \\
\hline 19 & .05 & .03 & .04 & .04 & .04 & .09 & .13 & .06 & .04 & .04 & .04 & .02 \\
\hline 20 & .04 & .03 & .03 & .04 & .04 & .09 & .13 & .06 & .04 & .04 & .04 & .02 \\
\hline 21 & .04 & .03 & .04 & .04 & .07 & .09 & .13 & .06 & .04 & .04 & .04 & .04 \\
\hline 22 & .04 & .03 & .04 & .04 & .05 & .10 & .13 & .06 & .05 & .05 & .04 & .04 \\
\hline 23 & .06 & .03 & .04 & .04 & .07 & .10 & .13 & .05 & .04 & .05 & .04 & .03 \\
\hline 24 & .06 & .03 & .04 & .03 & .11 & .10 & .13 & .06 & .05 & .06 & .04 & .03 \\
\hline 25 & .04 & .03 & .04 & .03 & .08 & .10 & .12 & .06 & .05 & .05 & .04 & .03 \\
\hline 26 & .04 & .03 & .04 & .03 & .07 & .10 & .11 & .05 & .04 & .04 & .04 & .03 \\
\hline 27 & .04 & .03 & .04 & .04 & .07 & .10 & .11 & .05 & .03 & .03 & .04 & .05 \\
\hline 28 & .03 & .03 & .04 & .04 & .06 & .10 & .10 & .05 & .08 & .03 & .04 & .05 \\
\hline 29 & .04 & .03 & .04 & .03 & .08 & .11 & .10 & .05 & .06 & .03 & .04 & .04 \\
\hline 30 & .03 & .03 & .04 & .03 & -- & .10 & .08 & .06 & .04 & .03 & .04 & .04 \\
\hline 31 & .03 & -- & .04 & .03 & -- & .10 & -- & .06 & --- & .03 & .04 & --- \\
\hline TOTAL & 1.35 & 0.93 & 1.09 & 1.18 & 1.49 & 3.39 & 3.62 & 2.16 & 1.56 & 1.25 & 1.15 & 1.17 \\
\hline MEAN & .044 & .031 & .035 & .038 & .051 & .11 & .12 & .070 & .052 & .040 & .037 & .039 \\
\hline MAX & .08 & .04 & .04 & .04 & .11 & .21 & .18 & .12 & .08 & .06 & .05 & .14 \\
\hline MIN & .03 & .03 & .03 & .03 & .03 & .09 & .08 & .05 & .03 & .03 & .03 & .02 \\
\hline ACRE-FT & 2.7 & 1.8 & 2.2 & 2.3 & 3.0 & 6.7 & 7.2 & 4.3 & 3.1 & 2.5 & 2.3 & 2.3 \\
\hline \multicolumn{2}{|c|}{ WTR YR 1988} & OTAL 20 & .34 & MEAN 0.05 & & 0.21 & MIN & 0.02 & CRE-FT & 40 & & \\
\hline
\end{tabular}


Table 22.--Mean daily discharge from the spring at the spring Creek site during water year 1989

[---, no data; TOTAL, sum of the discharges; MEAN, mean discharge; MAX, maximum discharge;

MIN, minimum discharge; ACRE-FT, acre-foot; CAL YR, calender year; WTR YR, water year]

\begin{tabular}{|c|c|c|c|c|c|c|c|c|c|c|c|c|}
\hline DAY & $\begin{array}{l}\text { MEAN } \\
\text { OCT }\end{array}$ & $\begin{array}{l}\text { N VALUES } \\
\text { NOV }\end{array}$ & $\begin{array}{l}\text { OF DI } \\
\text { DEC }\end{array}$ & $\begin{array}{c}\text { ISCHARGE, } \\
\text { JAN }\end{array}$ & , ${ }_{\mathrm{FEB}}^{\mathrm{IN}} \mathrm{Cl}$ & $\begin{array}{l}\text { JBIC FEET } \\
\text { MAR }\end{array}$ & $\begin{array}{l}\text { PER } \\
\text { APR }\end{array}$ & $\begin{array}{c}\text { SECOND, } \\
\text { MAY }\end{array}$ & $\begin{array}{l}\text { WATER } \\
\text { JUNE }\end{array}$ & $\begin{array}{l}\text { YEAR } 1988 \\
\text { JULY }\end{array}$ & 8 AUG & SEPT \\
\hline 1 & 0.04 & 0.03 & 0.03 & 0.04 & 0.03 & 0.13 & 0.18 & 0.07 & 0.05 & 0.04 & 0.04 & 0.03 \\
\hline 2 & .04 & .03 & .04 & .04 & .03 & .07 & .17 & .07 & .04 & .04 & .04 & .03 \\
\hline 3 & .04 & .03 & .04 & .04 & .03 & .07 & .17 & .06 & .04 & .04 & .04 & .03 \\
\hline 4 & .04 & .03 & .03 & .03 & .03 & .07 & .17 & .06 & .04 & .04 & .04 & .03 \\
\hline 5 & .03 & .03 & .03 & .03 & .03 & .07 & .16 & .06 & .04 & .04 & .04 & .03 \\
\hline 6 & .03 & .03 & .03 & .03 & .03 & .07 & .16 & .06 & .04 & .04 & .04 & .03 \\
\hline 7 & .03 & .03 & .03 & .03 & .03 & .08 & .16 & .06 & .04 & .04 & .04 & .03 \\
\hline 8 & .03 & .03 & .03 & .03 & .03 & .13 & .16 & .06 & .04 & .04 & .04 & .03 \\
\hline 9 & .03 & .03 & .03 & .03 & .03 & .13 & .16 & .06 & .04 & .04 & .04 & .03 \\
\hline 10 & .03 & .03 & .03 & .03 & .03 & .13 & .14 & .07 & .04 & .04 & .04 & .03 \\
\hline 11 & .03 & .03 & .03 & .03 & .03 & .12 & .13 & .07 & .04 & .04 & .04 & .03 \\
\hline 12 & .03 & .03 & .03 & .03 & .03 & .12 & .13 & .06 & .05 & .04 & .04 & .03 \\
\hline 13 & .03 & .03 & .03 & .03 & .03 & .14 & .13 & .06 & .05 & .04 & .04 & .03 \\
\hline 14 & .03 & .03 & .03 & .03 & .04 & .16 & .12 & .06 & .05 & .04 & .04 & .03 \\
\hline 15 & .03 & .03 & .03 & .03 & .04 & .16 & .12 & .06 & .05 & .04 & .04 & .03 \\
\hline 16 & .03 & .03 & .03 & .03 & .04 & .16 & .12 & .06 & .04 & .04 & .04 & .03 \\
\hline 17 & .03 & .03 & .03 & .03 & .04 & .18 & .12 & .06 & .04 & .04 & .04 & .03 \\
\hline 18 & .03 & .03 & .03 & .03 & .04 & .18 & .11 & .06 & .04 & .04 & .03 & .03 \\
\hline 19 & .03 & .03 & .03 & .03 & .04 & .19 & .11 & .06 & .04 & .04 & .03 & .03 \\
\hline 20 & .03 & .03 & .03 & .03 & .04 & .19 & .11 & .05 & .04 & .04 & .03 & .03 \\
\hline 21 & .03 & .03 & .03 & .03 & .04 & .20 & .09 & .05 & .04 & .04 & .03 & .03 \\
\hline 22 & .03 & .03 & .03 & .03 & .04 & .20 & .09 & .05 & .05 & .04 & .03 & .04 \\
\hline 23 & .03 & .03 & .03 & .03 & .09 & .21 & .09 & .05 & .05 & .04 & .03 & .04 \\
\hline 24 & .03 & .04 & .03 & .03 & .10 & .22 & .09 & .06 & .05 & .04 & .03 & .04 \\
\hline 25 & .03 & .04 & .03 & .03 & .10 & .23 & .09 & .06 & .05 & .04 & .03 & .04 \\
\hline 26 & .03 & .04 & .03 & .03 & .09 & .23 & .09 & .05 & .05 & .04 & .03 & .04 \\
\hline 27 & .03 & .03 & .03 & .03 & .09 & .22 & .09 & .05 & .04 & .04 & .03 & .03 \\
\hline 28 & .03 & .03 & .03 & .03 & .14 & .21 & .08 & .05 & .04 & .04 & .03 & .03 \\
\hline 29 & .03 & .03 & .03 & .03 & $\cdots$ & .20 & .08 & .05 & .04 & .04 & .03 & .03 \\
\hline 30 & .03 & .03 & .03 & .03 & -- & .19 & .07 & .05 & .04 & .04 & .03 & .03 \\
\hline 31 & .03 & -- & .04 & .03 & $\cdots$ & .18 & --- & .05 & -- & .04 & .03 & --- \\
\hline TOTAL & 0.97 & 0.93 & 0.96 & 0.96 & 1.36 & 4.84 & 3.69 & 1.80 & 1.30 & 1.24 & 1.10 & 0.95 \\
\hline MEAN & .031 & .031 & .031 & .031 & .049 & .16 & .12 & .058 & .043 & .040 & .035 & .032 \\
\hline MAX & .04 & .04 & .04 & .04 & .14 & .23 & .18 & .07 & .05 & .04 & .04 & .04 \\
\hline MIN & .03 & .03 & .03 & .03 & .03 & .07 & .07 & .05 & .04 & .04 & .03 & .03 \\
\hline ACRE-FT & 1.9 & 1.8 & 1.9 & 1.9 & 2.7 & 9.6 & 7.3 & 3.6 & 2.6 & 2.5 & 2.2 & 1.9 \\
\hline CAL YR & 1988 & \multirow{2}{*}{\multicolumn{2}{|c|}{$\begin{array}{ll}\text { TOTAL } & 19.83 \\
\text { TOTAL } & 20.10\end{array}$}} & MEAN 0 . & .054 & $\operatorname{MAX} 0.21$ & & IN 0.02 & \multicolumn{2}{|c|}{ ACRE-FT 39} & & \\
\hline WTR YR & 1989 & & & MEAN 0 . & .055 & MAX 0.23 & & IN 0.03 & \multicolumn{2}{|c|}{ ACRE-FT 40} & & \\
\hline
\end{tabular}


Table 23.--Mean daily discharge from the spring at the Cow Camp Creek site during water year 1988

--, no data; TOTAL, sum of the discharges; MEAN, mean discharge; MAX, maximum discharge; MIN, minimum discharge; ACRE-FT, acre-foot; CAL YR, calender year; WTR YR, water year]

\begin{tabular}{|c|c|c|c|c|c|c|c|c|c|c|c|c|}
\hline AY & $\begin{array}{l}\text { MEAN } \\
\text { OCT }\end{array}$ & $\begin{array}{l}\text { N VALUES } \\
\text { NOV }\end{array}$ & $\begin{array}{l}S \text { OF DI } \\
\text { DEC }\end{array}$ & $\begin{array}{c}\text { SCHARGE } \\
\text { JAN }\end{array}$ & $\begin{array}{l}\text { IN } \mathrm{Cl} \\
\mathrm{FEB}\end{array}$ & $\begin{array}{l}\text { JBIC FEET } \\
\text { MAR }\end{array}$ & $\begin{array}{l}\text { T PER } \\
\text { APR }\end{array}$ & $\begin{array}{c}\text { SECOND, } \\
\text { MAY }\end{array}$ & $\begin{array}{r}\text { WATER } \\
\text { JUNE }\end{array}$ & $\begin{array}{l}\text { YEAR } 1988 \\
\text { JULY }\end{array}$ & AUG & SEPT \\
\hline 1 & 0.02 & 0.02 & 0.03 & 0.02 & 0.02 & 0.20 & 0.54 & 0.22 & 0.13 & 0.10 & 0.08 & 0.05 \\
\hline 2 & .02 & .03 & .02 & .02 & .02 & .25 & .61 & .22 & .12 & .10 & .08 & .05 \\
\hline 3 & .02 & .03 & .02 & .02 & .02 & .31 & .63 & .23 & .12 & .10 & .08 & .05 \\
\hline 4 & .02 & .03 & .02 & .02 & .02 & .45 & .60 & .21 & .13 & .10 & .08 & .05 \\
\hline 5 & .02 & .03 & .02 & .02 & .03 & .43 & .50 & .16 & .13 & .09 & .09 & .05 \\
\hline 6 & .02 & .03 & .02 & .02 & .03 & .43 & .50 & .15 & .12 & .09 & .08 & .05 \\
\hline 7 & .02 & .02 & .02 & .02 & .03 & .53 & .46 & .15 & .12 & .08 & .08 & .05 \\
\hline 8 & .02 & .02 & .02 & .02 & .03 & .46 & .42 & .16 & .12 & .08 & .09 & .06 \\
\hline 9 & .02 & .02 & .02 & .02 & .03 & .41 & .38 & .16 & .12 & .08 & .09 & .06 \\
\hline 0 & .02 & .02 & .02 & .02 & .03 & .41 & .36 & .16 & .11 & .08 & .08 & .05 \\
\hline 1 & .02 & .02 & .02 & .03 & .02 & .37 & .34 & .15 & .11 & .08 & .07 & .06 \\
\hline 2 & .02 & .02 & .02 & .02 & .03 & .30 & .31 & .14 & .11 & .08 & .06 & .08 \\
\hline 3 & .03 & .03 & .02 & .02 & .03 & .27 & .32 & .14 & .11 & .08 & .07 & .09 \\
\hline 4 & .03 & .03 & .02 & .02 & .03 & .24 & .31 & .15 & .11 & .08 & .06 & .09 \\
\hline 5 & .03 & .03 & .02 & .02 & .03 & .24 & .32 & .14 & .11 & .07 & .07 & .07 \\
\hline 6 & .03 & .03 & .02 & .02 & .03 & .22 & .30 & .14 & .11 & .07 & .07 & .07 \\
\hline 7 & .03 & .03 & .02 & .02 & .03 & .19 & .33 & .13 & .10 & .07 & .07 & .06 \\
\hline 8 & .03 & .03 & .02 & .02 & .03 & .19 & .33 & .13 & .10 & .08 & .07 & .05 \\
\hline 9 & .03 & .02 & .02 & .02 & .03 & .19 & .30 & .14 & .10 & .07 & .07 & .05 \\
\hline 0 & .03 & .03 & .02 & .02 & .03 & .20 & .31 & .14 & .10 & .07 & .07 & .05 \\
\hline 1 & .03 & .03 & .02 & .02 & .03 & .31 & .29 & .14 & .11 & .07 & .07 & .05 \\
\hline 2 & .03 & .03 & .02 & .02 & .03 & .47 & .28 & .14 & .13 & .07 & .07 & .03 \\
\hline 3 & .03 & .03 & .03 & .02 & .04 & .49 & .32 & .14 & .13 & .07 & .07 & .04 \\
\hline 4 & .03 & .03 & .03 & .02 & .04 & .47 & .34 & .12 & .12 & .07 & .07 & .05 \\
\hline$j$ & .03 & .03 & .03 & .02 & .05 & .41 & .34 & .12 & .09 & .07 & .08 & .05 \\
\hline 5 & .03 & .03 & .03 & .02 & .08 & .44 & .33 & .12 & .09 & .07 & .07 & .05 \\
\hline 7 & .03 & .03 & .03 & .02 & .13 & .64 & .30 & .13 & .09 & .07 & .06 & .05 \\
\hline 3 & .02 & .04 & .02 & .02 & .20 & .66 & .26 & .14 & .09 & .07 & .05 & .05 \\
\hline$\exists$ & .02 & .03 & .02 & .02 & .19 & .59 & .24 & .14 & .12 & .07 & .06 & .05 \\
\hline ) & .03 & .03 & .02 & .02 & -- & .56 & .25 & .14 & .10 & .08 & .06 & .05 \\
\hline 1 & .02 & $\cdots$ & .02 & .02 & -- & .53 & -- & .15 & -- & .08 & .05 & --- \\
\hline ГAL & 0.78 & 0.83 & 0.68 & 0.63 & 1.34 & 11.86 & 11.12 & 4.70 & 3.35 & 2.44 & 2.22 & 1.66 \\
\hline $\mathbb{A N}$ & .025 & .028 & .022 & .020 & .046 & .38 & .37 & .15 & .11 & .079 & .072 & .055 \\
\hline$x$ & .03 & .04 & .03 & .03 & .20 & .66 & .63 & .23 & .13 & .10 & .09 & .09 \\
\hline$v$ & .02 & .02 & .02 & .02 & .02 & .19 & .24 & .12 & .09 & .07 & .05 & .03 \\
\hline $3 \mathrm{E}-\mathrm{FT}$ & 1.5 & 1.6 & 1.3 & 1.2 & 2.7 & 24 & 22 & 9.3 & 6.6 & 4.8 & 4.4 & 3.3 \\
\hline
\end{tabular}

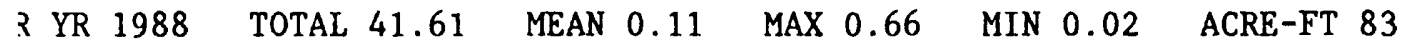


Table 24.--Mean daily discharge from the spring at the Cow Camp Creek site during water year 1989

[---, no data; TOTAL, sum of the discharges; MEAN, mean discharge; MAX, maximum discharge; MIN, minimum discharge; ACRE-FT, acre-foot; CAL YR, calender year; WTR YR, water year]

\begin{tabular}{|c|c|c|c|c|c|c|c|c|c|c|c|c|}
\hline DAY & $\begin{array}{l}\text { MEAN } \\
\text { OCT }\end{array}$ & $\begin{array}{l}\text { N VALUES } \\
\text { NOV }\end{array}$ & $\begin{array}{l}\text { OF DI } \\
\text { DEC }\end{array}$ & $\begin{array}{l}\text { ISCHARGE, } \\
\text { JAN }\end{array}$ & $\begin{array}{l}\text { IN } \mathrm{Cl} \\
\mathrm{FEB}\end{array}$ & $\begin{array}{l}\text { UBIC FEET } \\
\text { MAR }\end{array}$ & $\begin{array}{l}\text { T PER } \\
\text { APR }\end{array}$ & $\begin{array}{c}\text { SECOND, } \\
\text { MAY }\end{array}$ & $\begin{array}{l}\text { WATER } \\
\text { JUNE }\end{array}$ & $\begin{array}{l}\text { YEAR } 1988 \\
\text { JULY }\end{array}$ & AUG & SEPT \\
\hline 1 & 0.05 & 0.05 & 0.04 & 0.04 & 0.06 & 0.08 & 0.57 & 0.18 & 0.10 & 0.09 & 0.09 & 0.07 \\
\hline 2 & .05 & .06 & .05 & .04 & .05 & .10 & .54 & .17 & .11 & .09 & .09 & .07 \\
\hline 3 & .05 & .06 & .05 & .04 & .05 & .11 & .52 & .16 & .11 & .09 & .09 & .07 \\
\hline 4 & .05 & .05 & .04 & .04 & .05 & .11 & .51 & .16 & .11 & .09 & .08 & .07 \\
\hline 5 & .05 & .05 & .05 & .04 & .05 & .11 & .50 & .15 & .11 & .09 & .08 & .07 \\
\hline 6 & .05 & .06 & .05 & .04 & .06 & .12 & .50 & .15 & .10 & .09 & .08 & .07 \\
\hline 7 & .05 & .06 & .05 & .05 & .06 & .12 & .49 & .15 & .10 & .09 & .08 & .07 \\
\hline 8 & .05 & .05 & .04 & .04 & .06 & .12 & .47 & .15 & .10 & .09 & .08 & .07 \\
\hline 9 & .05 & .05 & .04 & .04 & .06 & .13 & .44 & .15 & .10 & .09 & .08 & .07 \\
\hline 10 & .05 & .05 & .05 & .05 & .06 & .20 & .41 & .14 & .10 & .09 & .08 & .07 \\
\hline 11 & .05 & .05 & .04 & .05 & .06 & .32 & .41 & .14 & .10 & .09 & .08 & .07 \\
\hline 12 & .05 & .05 & .04 & .05 & .06 & .48 & .38 & .14 & .10 & .10 & .08 & .07 \\
\hline 13 & .05 & .05 & .05 & .05 & .06 & .66 & .36 & .14 & .10 & .10 & .08 & .07 \\
\hline 14 & .05 & .05 & .05 & .05 & .06 & .82 & .35 & .14 & .10 & .10 & .08 & .07 \\
\hline 15 & .05 & .05 & .04 & .04 & .05 & .74 & .35 & .14 & .10 & .10 & .08 & .07 \\
\hline 16 & .05 & .05 & .04 & .05 & .06 & .65 & .33 & .14 & .10 & .10 & .08 & .07 \\
\hline 17 & .05 & .05 & .04 & .05 & .06 & .67 & .31 & .13 & .09 & .10 & .08 & .08 \\
\hline 18 & .05 & .05 & .05 & .05 & .06 & .69 & .29 & .13 & .09 & .09 & .08 & .08 \\
\hline 19 & .05 & .05 & .05 & .05 & .06 & .75 & .28 & .12 & .10 & .09 & .07 & .07 \\
\hline 20 & .05 & .05 & .04 & .05 & .06 & .79 & .28 & .12 & .09 & .09 & .07 & .07 \\
\hline 21 & .05 & .05 & .05 & .05 & .05 & .77 & .26 & .12 & .09 & .09 & .07 & .07 \\
\hline 22 & .05 & .05 & .04 & .05 & .05 & .77 & .24 & .12 & .09 & .09 & .07 & .07 \\
\hline 23 & .05 & .05 & .04 & .05 & .06 & .79 & .24 & .12 & .09 & .09 & .07 & .07 \\
\hline 24 & .05 & .05 & .04 & .05 & .06 & .79 & .23 & .12 & .09 & .09 & .07 & .07 \\
\hline 25 & .04 & .05 & .05 & .05 & .06 & .82 & .21 & .12 & .10 & .09 & .07 & .07 \\
\hline 26 & .05 & .05 & .04 & .05 & .07 & .82 & .21 & .12 & .10 & .09 & .07 & .07 \\
\hline 27 & .05 & .04 & .04 & .05 & .07 & .79 & .19 & .12 & .09 & .09 & .07 & .07 \\
\hline 28 & .05 & .05 & .05 & .05 & .08 & .71 & .19 & .12 & .09 & .09 & .07 & .07 \\
\hline 29 & .05 & .04 & .04 & .05 & -- & .67 & .18 & .11 & .09 & .09 & .07 & .07 \\
\hline 30 & .05 & .04 & .04 & .05 & $-\cdots$ & .63 & .18 & .11 & .09 & .09 & .07 & .08 \\
\hline 31 & .05 & -- & .04 & .06 & -- & .59 & --- & .10 & $\cdots$ & .08 & .07 & -- \\
\hline TOTAL & 1.54 & 1.51 & 1.37 & 1.47 & 1.65 & 15.92 & 10.42 & 4.18 & 2.93 & 2.84 & 2.38 & 2.13 \\
\hline MEAN & .050 & .050 & .044 & .047 & .059 & .51 & .35 & .13 & .098 & .092 & .077 & .071 \\
\hline MAX & .05 & .06 & .05 & .06 & .08 & .82 & .57 & .18 & .11 & .10 & .09 & .08 \\
\hline MIN & .04 & .04 & .04 & .04 & .05 & .08 & .18 & .10 & .09 & .08 & .07 & .07 \\
\hline ACRE-FT & 3.1 & 3.0 & 2.7 & 2.9 & 3.3 & 32 & 21 & 8.3 & 5.8 & 5.6 & 4.7 & 4.2 \\
\hline \multirow{2}{*}{\multicolumn{2}{|c|}{$\begin{array}{ll}\text { CAL YR } 1988 \\
\text { WTR YR } 1989\end{array}$}} & \multirow{2}{*}{\multicolumn{2}{|c|}{$\begin{array}{l}\text { TOTAL } 43.74 \\
\text { TOTAL } 48.34\end{array}$}} & \multirow{2}{*}{$\begin{array}{ll}\text { MEAN } & 0.12 \\
\text { MEAN } & 0.13\end{array}$} & \multirow{2}{*}{\multicolumn{2}{|c|}{$\begin{array}{ll}\operatorname{MAX} & 0.66 \\
\operatorname{MAX} & 0.82\end{array}$}} & \multirow{2}{*}{\multicolumn{2}{|c|}{$\begin{array}{ll}\text { MIN } & 0.02 \\
\text { MIN } & 0.04\end{array}$}} & \multirow{2}{*}{\multicolumn{2}{|c|}{$\begin{array}{l}\text { ACRE-FT } 87 \\
\text { ACRE-FT } 96\end{array}$}} & & \\
\hline & & & & & & & & & & & & \\
\hline
\end{tabular}


[Well identification corresponds to wells listed in table 9 ; $\mu S / C M$, microsiemens per centimeter at 25 degrees Celsius; ${ }^{\circ} \mathrm{C}$, degrees Celsius; MG/L, milligrams per liter; NONCARB, noncarbonate; WH, whole; WAT, water; TOT, total; FLD, field; --, no data collected; <, less than; LAB, laboratory; DEG. C, degrees Celsius; $\mu$ G/L, micrograms per liter; $C_{-}$, carbon isotope; $H_{-}$, hydrogen isotope; $0-$, oxygen isotope; $S_{-}$, sulfur isotope; per mil, parts per thousand; PCI/L, picocuries per liter]

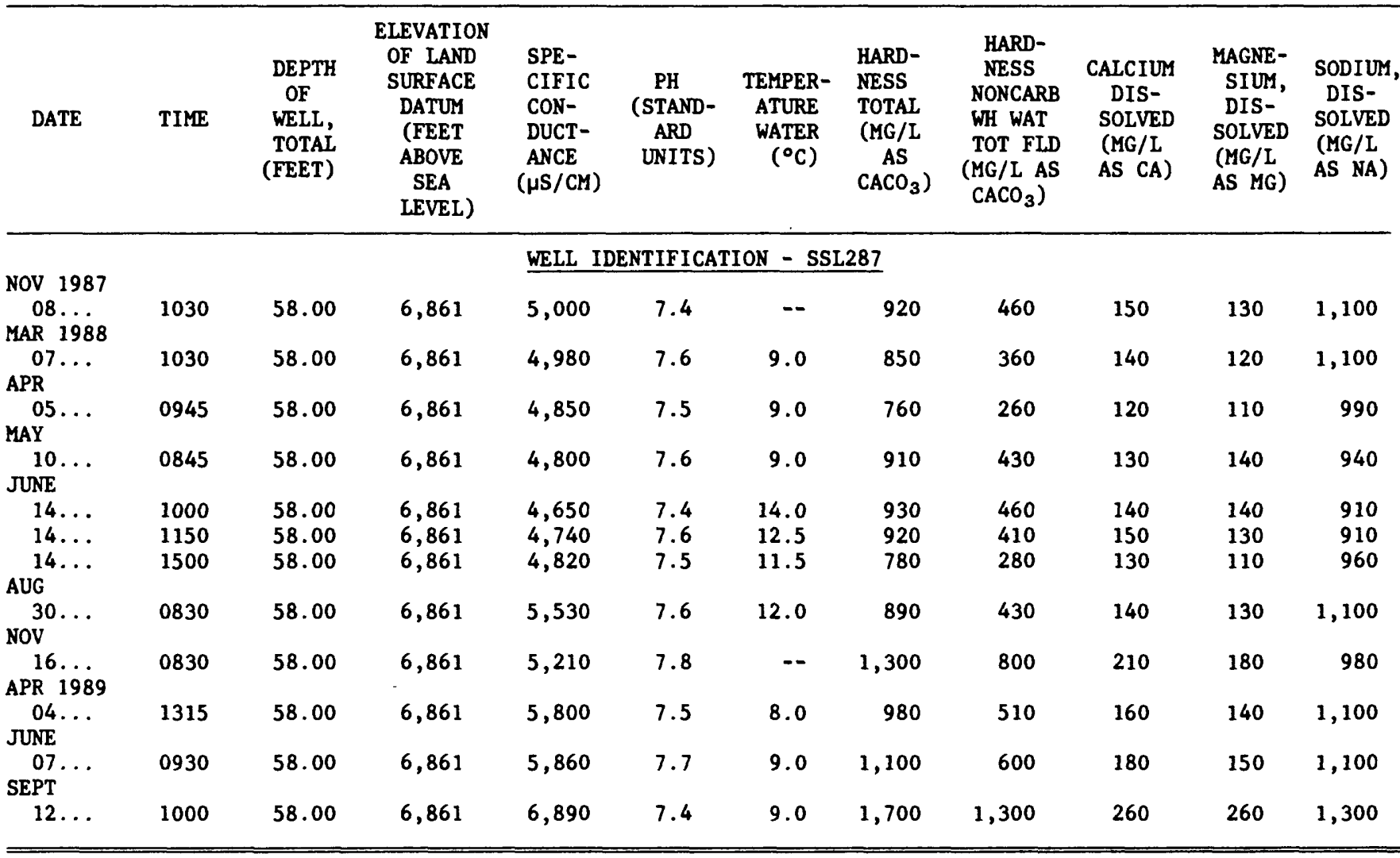

\begin{tabular}{|c|c|c|c|c|c|c|c|c|}
\hline IE & $\begin{array}{l}\text { POTAS- } \\
\text { SIUM, } \\
\text { DIS- } \\
\text { SOLVED } \\
\text { (MG/L } \\
\text { AS K) }\end{array}$ & $\begin{array}{c}\text { ALKA- } \\
\text { LINITY } \\
\text { LAB } \\
(\mathrm{MG} / \mathrm{L} \\
\text { AS } \\
\left.\mathrm{CACO}_{3}\right)\end{array}$ & $\begin{array}{c}\text { SULFATE } \\
\text { DIS- } \\
\text { SOLVED } \\
(\mathrm{MG} / \mathrm{L} \\
\text { AS SO } 4)\end{array}$ & $\begin{array}{l}\text { CHLO- } \\
\text { RIDE, } \\
\text { DIS- } \\
\text { SOLVED } \\
\text { (MG/L } \\
\text { AS CL) }\end{array}$ & $\begin{array}{r}\text { FLUO- } \\
\text { RIDE, } \\
\text { DIS- } \\
\text { SOLVED } \\
\text { (MG/L } \\
\text { AS F) }\end{array}$ & $\begin{array}{l}\text { SILICA, } \\
\text { DIS- } \\
\text { SOLVED } \\
\text { (MG/L } \\
\text { AS } \\
\left.\mathrm{SIO}_{2}\right)\end{array}$ & $\begin{array}{l}\text { SOLIDS, } \\
\text { RESIDUE } \\
\text { AT } 180 \\
\text { DEG. C } \\
\text { DIS- } \\
\text { SOLVED } \\
\text { (MG/L) }\end{array}$ & $\begin{array}{c}\text { SOLIDS, } \\
\text { SUM OF } \\
\text { CONSTIT- } \\
\text { UENTS, } \\
\text { DIS- } \\
\text { SOLVED } \\
\text { (MG/L) }\end{array}$ \\
\hline
\end{tabular}

\begin{tabular}{|c|c|c|c|c|c|c|c|c|}
\hline $\begin{array}{c}\text { NOV } 1987 \\
08 \ldots \\
\text { MAR } 1988\end{array}$ & 7.4 & 454 & 2,900 & 17 & 0.3 & 9.2 & 4,690 & 4,600 \\
\hline $\begin{array}{c}07 \ldots \\
\mathrm{APR}\end{array}$ & 9.4 & 496 & 2,400 & 22 & 1.1 & 10 & 4,070 & 4,110 \\
\hline$\underset{\mathrm{MAY}}{05 \ldots}$ & 7.6 & 502 & 2,400 & 17 & 1.1 & 8.8 & 3,950 & 3,960 \\
\hline $\begin{array}{l}10 \ldots \\
\text { JUNE }\end{array}$ & 8.5 & 478 & 2,500 & 23 & 1.2 & 8.7 & 4,090 & 4,050 \\
\hline $\begin{array}{l}14 \ldots \\
14 \ldots \\
14 \ldots\end{array}$ & $\begin{array}{l}8.7 \\
8.4 \\
8.1\end{array}$ & $\begin{array}{l}471 \\
504 \\
505\end{array}$ & $\begin{array}{l}2,500 \\
2,400 \\
2,400\end{array}$ & $\begin{array}{l}25 \\
22 \\
20\end{array}$ & $\begin{array}{l}1.1 \\
1.1 \\
1.0\end{array}$ & $\begin{array}{l}8.3 \\
9.3 \\
8.6\end{array}$ & $\begin{array}{l}4,100 \\
4,000 \\
4,050\end{array}$ & $\begin{array}{l}4,030 \\
3,950 \\
3,950\end{array}$ \\
\hline $\begin{array}{l}\text { AUG } \\
30 \ldots \\
\text { Nov }\end{array}$ & 8.5 & 465 & 2,800 & 19 & 1.1 & 8.7 & 4,380 & 4,500 \\
\hline $\begin{array}{r}16 \ldots \\
\text { APR } 1989\end{array}$ & 10 & 472 & 3,000 & 21 & .9 & 9.3 & 4,680 & 4,710 \\
\hline $\begin{array}{l}04 \ldots \\
\text { JUNE }\end{array}$ & 10 & 478 & 3,000 & 16 & 1.1 & 8.4 & 4,750 & 4,730 \\
\hline$\underset{\text { SEPT }}{07 \ldots}$ & 10 & 476 & 2,900 & 20 & 1.0 & 8.9 & 4,630 & 4,660 \\
\hline $12 \ldots$ & 12 & 452 & 4,000 & 15 & .7 & 8.8 & 6,070 & 6,140 \\
\hline
\end{tabular}


Table 25.--Water-quality analyses for wells--Continued

\begin{tabular}{|c|c|c|c|c|c|c|c|c|c|c|}
\hline DATE & & $\begin{array}{l}\text { NITRO- } \\
\text { GEN, } \\
\text { NITRITE } \\
\text { DIS- } \\
\text { SOLVED } \\
\text { (MG/L } \\
\text { AS N) }\end{array}$ & $\begin{array}{c}\text { NITRO- } \\
\text { GEN, } \\
\text { NITRITE } \\
\text { DIS- } \\
\text { SOLVED } \\
\text { (MG/L } \\
\text { AS } \mathrm{NO}_{2} \text { ) }\end{array}$ & $\begin{array}{c}\text { NITRO- } \\
\text { GEN, } \\
\mathrm{NO}_{2}+\mathrm{NO}_{3} \\
\text { DIS- } \\
\text { SOLVED } \\
\text { (MG/L } \\
\text { AS N) }\end{array}$ & $\begin{array}{l}\text { NITRO- } \\
\text { GEN, } \\
\text { AMMONIA } \\
\text { DIS- } \\
\text { SOLVED } \\
\text { (MG/L } \\
\text { AS N) }\end{array}$ & $\begin{array}{l}\text { NITRO- } \\
\text { GEN, } \\
\text { AMONIA } \\
\text { DIS- } \\
\text { SOLVED } \\
\text { (MG/L } \\
\text { AS NH4) }\end{array}$ & $\begin{array}{l}\text { NITRO- } \\
\text { GEN, } \\
\text { ORGANIC } \\
\text { DIS- } \\
\text { SOLVED } \\
\text { (MG/L } \\
\text { AS N) }\end{array}$ & $\begin{array}{l}\text { NITRO- } \\
\text { GEN,AM- } \\
\text { MONIA + } \\
\text { ORGANIC } \\
\text { DISSOLVED } \\
\text { (MG/L } \\
\text { AS N) }\end{array}$ & $\begin{array}{l}\text { PHOS- } \\
\text { PHORUS } \\
\text { DIS- } \\
\text { SOLVED } \\
\text { (MG/L } \\
\text { AS P) }\end{array}$ & $\begin{array}{l}\text { PHOS- } \\
\text { PHORUS } \\
\text { ORTHO, } \\
\text { DIS- } \\
\text { SOLVED } \\
\text { (MG/L } \\
\text { AS P) }\end{array}$ \\
\hline \multicolumn{11}{|c|}{ WELL IDENTIFICATION - SSL287--Continued } \\
\hline $\begin{array}{c}\text { NOV } 1987 \\
\text { O8... } \\
\text { MAR } 1988\end{array}$ & & $<0.01$ & $\cdot--$ & $<0.10$ & 4.80 & 6.2 & 0.5 & 5.3 & 0.02 & 0.02 \\
\hline $\begin{array}{c}07 \ldots \\
\text { APR }\end{array}$ & & $<.01$ & $\sim$ & $<.10$ & 4.40 & 5.7 & .1 & 4.5 & .05 & $<.01$ \\
\hline$\underset{\text { MAY }}{05 \ldots}$ & & $<.01$ & -- & $<.10$ & 1.00 & 1.3 & 3.0 & 4.0 & .04 & .04 \\
\hline${ }_{\text {JUNE }}^{10}$ & & $<.01$ & -- & $<.10$ & 4.40 & 5.7 & .4 & 4.8 & .03 & .02 \\
\hline $\begin{array}{l}14 \ldots \\
14 \ldots \\
14 \ldots\end{array}$ & & $\begin{array}{l}.01 \\
.01 \\
.02\end{array}$ & $\begin{array}{r}0.03 \\
.03 \\
.07\end{array}$ & $\begin{array}{l}<.10 \\
<.10 \\
<.10\end{array}$ & $\begin{array}{l}4.60 \\
4.20 \\
3.60\end{array}$ & $\begin{array}{l}5.9 \\
5.4 \\
4.6\end{array}$ & $\begin{array}{l}.0 \\
.0 \\
.3\end{array}$ & $\begin{array}{l}4.5 \\
3.8 \\
3.9\end{array}$ & $\begin{array}{l}.01 \\
.03 \\
.02\end{array}$ & $\begin{array}{l}.01 \\
.04 \\
.02\end{array}$ \\
\hline $\begin{array}{l}\text { AUG } \\
30 \ldots \\
\text { NOV }\end{array}$ & & $<.01$ & $\sim$ & $<.10$ & 5.50 & 7.1 & .0 & 4.5 & .03 & .02 \\
\hline $\begin{array}{l}16 \ldots \\
\text { APR } 1989\end{array}$ & & $<.01$ & - & $<.10$ & 5.60 & 7.2 & .0 & 4.9 & .03 & .02 \\
\hline $\begin{array}{l}04 \ldots \\
\text { JUNE }\end{array}$ & & - & - & - & -- & - & - & -- & -- & - \\
\hline $\begin{array}{l}07 \\
\text { SEPT }\end{array}$ & & -- & - & - & -- & -- & - & -- & -- & - \\
\hline $12 \ldots$ & & $\sim$ & -- & $\sim$ & - & -- & - & -- & -- & 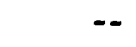 \\
\hline DATE & 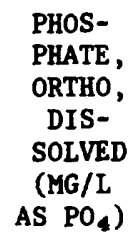 & $\begin{array}{l}\text { PHOS- } \\
\text { PHORUS } \\
\text { ORGANIC } \\
\text { DIS- } \\
\text { SOLVED } \\
\text { (MG/L } \\
\text { AS P) }\end{array}$ & $\begin{array}{l}\text { ALU- } \\
\text { MINUM, } \\
\text { DIS- } \\
\text { SOLVED } \\
\text { ( } \mu G / L \\
\text { AS AL) }\end{array}$ & $\begin{array}{l}\text { ARSENIC } \\
\text { DIS- } \\
\text { SOLVED } \\
(\mu G / L \\
\text { AS AS) }\end{array}$ & $\begin{array}{l}\text { BARIUM, } \\
\text { DIS- } \\
\text { SOLVED } \\
\text { ( } \mu G / L \\
\text { AS BA) }\end{array}$ & $\begin{array}{l}\text { BERYL- } \\
\text { LIUM, } \\
\text { DIS- } \\
\text { SOLVED } \\
(\mu G / L \\
\text { AS BE) }\end{array}$ & $\begin{array}{l}\text { BORON } \\
\text { DIS- } \\
\text { SOLVED } \\
\text { ( } \mu G / L \\
\text { AS B) }\end{array}$ & $\begin{array}{l}\text { CADMIUM } \\
\text { DIS- } \\
\text { SOLVED } \\
(\mu G / L \\
\text { AS CD) }\end{array}$ & $\begin{array}{l}\text { CHRO- } \\
\text { MIUM, } \\
\text { DIS- } \\
\text { SOLVED } \\
(\mu G / L \\
\text { AS CR) }\end{array}$ & $\begin{array}{l}\text { COBALT, } \\
\text { DIS- } \\
\text { SOLVED } \\
(\mu G / L \\
\text { AS CO) }\end{array}$ \\
\hline NOV 1987 & & & & & & & & & & \\
\hline $\begin{array}{l}08 \ldots \\
\text { MAR } 1988\end{array}$ & 0.06 & 0.0 & $<10$ & 1 & 20 & $<1$ & 340 & $<2$ & $<10$ & $<6$ \\
\hline $\begin{array}{l}07 \ldots \\
\text { APR }\end{array}$ & $\sim$ & .05 & 20 & $<1$ & 26 & $<2$ & 320 & $<3$ & $<20$ & $<9$ \\
\hline$\underset{M A Y}{05}$ & .12 & .0 & $<10$ & $<1$ & 20 & $<2$ & 320 & $<3$ & $<5$ & $<9$ \\
\hline$\underset{\text { JUNE }}{10 \ldots}$ & .06 & .01 & $<10$ & $<1$ & 16 & $<2$ & 320 & $<3$ & $<20$ & $<9$ \\
\hline $14 \ldots$ & .03 & .0 & $<10$ & $<1$ & 21 & $<.5$ & 350 & $<1$ & $<5$ & $<3$ \\
\hline $14 \ldots$ & .12 & .0 & $<10$ & $<1$ & 22 & $<.5$ & 310 & $<1$ & $<5$ & $<3$ \\
\hline${ }_{A U G}^{14 \ldots}$ & .06 & .0 & $<10$ & $<1$ & 17 & $<.5$ & 320 & $<1$ & $<5$ & $<3$ \\
\hline $\begin{array}{l}30 \ldots \\
\text { Nov }\end{array}$ & .06 & .01 & $<10$ & $<1$ & 15 & $<.5$ & 310 & 3 & $<5$ & $<3$ \\
\hline $\begin{array}{l}16 \ldots \\
\text { APR } 1989\end{array}$ & .06 & .01 & $<10$ & $<1$ & 14 & $<.5$ & 330 & $<1$ & $<5$ & $<3$ \\
\hline $\begin{array}{l}04 \ldots \\
\text { JUNE }\end{array}$ & -- & - & 10 & $<1$ & 18 & $<3$ & 300 & $<5$ & $<30$ & $<20$ \\
\hline$\underset{\text { SEPT }}{07}$ & $\sim$ & $\sim$ & $<10$ & $<1$ & 24 & $<2$ & 330 & $<3$ & $<20$ & $<9$ \\
\hline $12 \ldots$ & -- & -- & 20 & $<1$ & 14 & $<2$ & 310 & $<3$ & $<20$ & $<9$ \\
\hline
\end{tabular}


Table 25.--Water-quality analyses for wells--Continued

\begin{tabular}{|c|c|c|c|c|c|c|c|c|c|c|}
\hline DATE & $\begin{array}{l}\text { COPPER, } \\
\text { DIS- } \\
\text { SOLVED } \\
\text { ( } \mu G / L \\
\text { AS CU) }\end{array}$ & $\begin{array}{l}\text { IRON, } \\
\text { DIS- } \\
\text { SOLVED } \\
\text { ( } \mu G / L \\
\text { AS FE) }\end{array}$ & $\begin{array}{l}\text { LEAD, } \\
\text { DIS- } \\
\text { SOLVED } \\
\text { ( } \mu G / L \\
\text { AS PB) }\end{array}$ & $\begin{array}{l}\text { MANGA- } \\
\text { NESE, } \\
\text { DIS- } \\
\text { SOLVED } \\
\text { ( } \mu G / L \\
\text { AS MN) }\end{array}$ & $\begin{array}{l}\text { MOLYB- } \\
\text { DENUM, } \\
\text { DIS- } \\
\text { SOLVED } \\
(\mu G / L \\
\text { AS MO) }\end{array}$ & $\begin{array}{l}\text { NICKEL, } \\
\text { DIS- } \\
\text { SOLVED } \\
(\mu G / L \\
\text { AS NI) }\end{array}$ & $\begin{array}{l}\text { SILVER, } \\
\text { DIS- } \\
\text { SOLVED } \\
(\mu G / L \\
\text { AS AG) }\end{array}$ & $\begin{array}{c}\text { STRON- } \\
\text { TIUM, } \\
\text { DIS- } \\
\text { SOLVED } \\
(\mu G / L \\
\text { AS SR) }\end{array}$ & $\begin{array}{l}\text { VANA- } \\
\text { DIUM, } \\
\text { DIS- } \\
\text { SOLVED } \\
(\mu G / L \\
\text { AS V) }\end{array}$ & $\begin{array}{l}\text { ZINC, } \\
\text { DIS- } \\
\text { SOLVED } \\
\text { ( } \mu G / L \\
\text { AS ZN) }\end{array}$ \\
\hline \multicolumn{11}{|c|}{ WELL IDENTIFICATION - SSL287--Continued } \\
\hline NOV 1987 & & & & & & & & & & \\
\hline $\begin{array}{l}08 \ldots \\
\text { MAR } 1988\end{array}$ & $<20$ & 110 & $<20$ & 490 & $<20$ & $<20$ & $<1.0$ & 6,200 & $<12$ & $<6$ \\
\hline $\begin{array}{c}07 \ldots \\
\text { APR }\end{array}$ & $<30$ & 68 & $<30$ & 460 & $<30$ & $<30$ & $<3.0$ & 6,000 & $<20$ & 10 \\
\hline MAY 05 & $<30$ & 80 & $<30$ & 370 & $<30$ & $<10$ & $<3.0$ & 5,300 & $<18$ & 12 \\
\hline$\frac{10 \ldots}{J_{N E}}$ & $<30$ & 34 & $<30$ & 240 & $<30$ & $<30$ & $<3.0$ & 6,500 & $<18$ & $<9$ \\
\hline $14 \ldots$ & $<10$ & 610 & $<10$ & 490 & $<10$ & $<10$ & 1.0 & 5,900 & $<6$ & 970 \\
\hline $14 \ldots$ & $<10$ & 170 & $<10$ & 690 & $<10$ & $<10$ & $<1.0$ & 6,000 & $<6$ & 20 \\
\hline $14 \ldots$ & $<10$ & 120 & $<10$ & 280 & $<10$ & $<10$ & $<1.0$ & 2,600 & $<6$ & 6 \\
\hline \multicolumn{11}{|l|}{ AUG } \\
\hline $\begin{array}{l}30 \ldots \\
\text { NOV }\end{array}$ & $<10$ & 74 & $<10$ & 200 & $<10$ & $<10$ & 9.0 & 6,200 & $<6$ & 8 \\
\hline $\begin{array}{l}16 \ldots \\
\text { APR } 1989\end{array}$ & $<10$ & 92 & $<10$ & 330 & $<10$ & $<10$ & $<1.0$ & 7,600 & $<6$ & 7 \\
\hline$\underset{\text { JNE }}{04 \ldots}$ & $<50$ & 210 & 50 & 350 & $<50$ & $<50$ & $<5.0$ & 6,600 & $<30$ & 25 \\
\hline$\underset{\text { SEPT }}{07} \cdots$ & $<30$ & 69 & $<30$ & 340 & $<30$ & $<30$ & $<3.0$ & 7,100 & $<18$ & 11 \\
\hline $12 \ldots$ & $<30$ & 600 & $<30$ & 560 & $<30$ & $<30$ & $<3.0$ & 10,000 & $<18$ & 30 \\
\hline DATE & TIME & $\begin{array}{l}\text { DEPTH } \\
\text { OF } \\
\text { WELL, } \\
\text { TOTAL } \\
\text { (FEET) }\end{array}$ & $\begin{array}{l}\text { ELEVATION } \\
\text { OF LAND } \\
\text { SURFACE } \\
\text { DATUM } \\
\text { (FEET } \\
\text { ABOVE } \\
\text { SEA } \\
\text { LEVEL) }\end{array}$ & $\begin{array}{l}\text { SPE- } \\
\text { CIFIC } \\
\text { CON- } \\
\text { DUCT- } \\
\text { ANCE } \\
(\mu S / C M)\end{array}$ & $\begin{array}{c}\text { PH } \\
\text { (STAND- } \\
\text { ARD } \\
\text { UNITS) }\end{array}$ & $\begin{array}{l}\text { TEMPER- } \\
\text { ATURE } \\
\text { WATER } \\
\left({ }^{\circ} \mathrm{C}\right)\end{array}$ & $\begin{array}{l}\text { HARD- } \\
\text { NESS } \\
\text { TOTAL } \\
(\mathrm{MG} / \mathrm{L} \\
\text { AS } \\
\left.\mathrm{CACO}_{3}\right)\end{array}$ & $\begin{array}{l}\text { HARD- } \\
\text { NESS } \\
\text { NONCARB } \\
\text { WH WAT } \\
\text { TOT FLD } \\
(\mathrm{MG} / \mathrm{L} \text { AS } \\
\left.\mathrm{CACO}_{3}\right)\end{array}$ & $\begin{array}{l}\text { CALCIUM } \\
\text { DIS- } \\
\text { SOLVED } \\
\text { (MG/L } \\
\text { AS CA) }\end{array}$ & $\begin{array}{l}\text { MAGNE- } \\
\text { SIUM, } \\
\text { DIS- } \\
\text { SOLVED } \\
\text { (MG/L } \\
\text { AS MG) }\end{array}$ \\
\hline \multicolumn{11}{|c|}{ WELL IDENTIFICATION - SSI287 } \\
\hline NOV 1987 & & & & & & & & & & \\
\hline $\begin{array}{l}08 \ldots \\
\text { MAR } 1988\end{array}$ & 1000 & 79.80 & 6,860 & 2,280 & 8.0 & -- & 92 & 0 & 20 & 9.7 \\
\hline$\underset{A P R}{04 \ldots}$ & 1230 & 79.80 & 6,860 & 2,910 & 7.9 & 7.5 & 170 & 0 & 35 & 19 \\
\hline$\underset{\mathrm{MAY}}{05}$ & 1430 & 79.80 & 6,860 & 2,850 & 7.9 & 9.0 & 180 & 0 & 37 & 20 \\
\hline AUG $^{10 \ldots}$ & 0945 & 79.80 & 6,860 & 3,250 & 7.8 & 9.0 & 240 & 0 & 51 & 27 \\
\hline $\begin{array}{c}30 \ldots \\
\text { NOV }\end{array}$ & 0945 & 79.80 & 6,860 & 3,100 & 8.1 & 11.0 & 200 & 0 & 40 & 23 \\
\hline $\begin{array}{c}16 \ldots \\
\text { APR } 1989\end{array}$ & 0930 & 79.80 & 6,860 & 3,000 & 8.1 & -- & 220 & 0 & 46 & 24 \\
\hline$\underset{\text { JUNE }}{04 \ldots}$ & 1300 & 79.80 & 6,860 & 3,030 & 7.9 & 9.5 & 170 & 0 & 36 & 19 \\
\hline$\underset{\text { SEPT }}{07} \cdots$ & 0900 & 79.80 & 6,860 & 3,110 & 8.0 & 10.5 & 170 & 0 & 37 & 19 \\
\hline $12 \ldots$ & 1100 & 79.80 & 6,860 & 3,140 & 7.9 & 10.0 & 190 & 0 & 39 & 22 \\
\hline
\end{tabular}


Table 25.--Water-quality analyses for wells--Continued

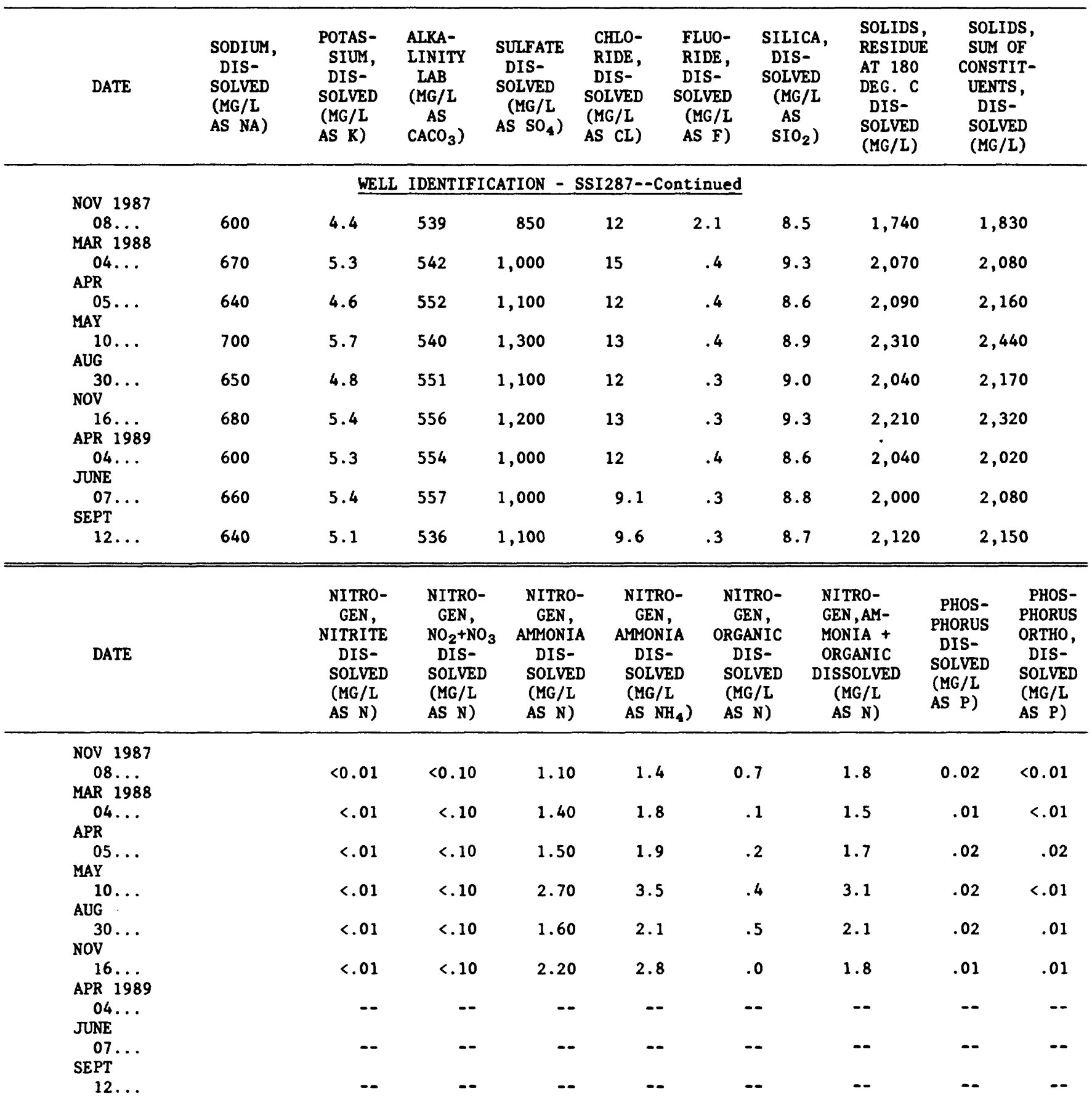




\begin{tabular}{|c|c|c|c|c|c|c|c|c|c|c|}
\hline DATE & $\begin{array}{l}\text { PHOS- } \\
\text { PHATE, } \\
\text { ORTHO, } \\
\text { DIS- } \\
\text { SOLVED } \\
(\mathrm{MG} / \mathrm{L} \\
\text { AS PO4) }\end{array}$ & $\begin{array}{l}\text { PHOS- } \\
\text { PHORUS } \\
\text { ORGANIC } \\
\text { DIS- } \\
\text { SOLVED } \\
\text { (MG/L } \\
\text { AS P) }\end{array}$ & $\begin{array}{l}\text { ALU- } \\
\text { MINUM, } \\
\text { DIS- } \\
\text { SOLVED } \\
\text { ( } \mu G / L \\
\text { AS AL) }\end{array}$ & $\begin{array}{l}\text { ARSENIC } \\
\text { DIS- } \\
\text { SOLVED } \\
(\mu G / L \\
\text { AS AS) }\end{array}$ & $\begin{array}{l}\text { BARIUM, } \\
\text { DIS- } \\
\text { SOLVED } \\
\text { ( } \mu \mathrm{G} / \mathrm{L} \\
\text { AS BA) }\end{array}$ & $\begin{array}{l}\text { BERYL- } \\
\text { LIUM, } \\
\text { DIS- } \\
\text { SOLVED } \\
(\mu G / L \\
\text { AS BE) }\end{array}$ & $\begin{array}{l}\text { BORON } \\
\text { DIS- } \\
\text { SOLVED } \\
(\mu G / L \\
\text { AS B) }\end{array}$ & $\begin{array}{c}\text { CADMIUM } \\
\text { DIS- } \\
\text { SOLVED } \\
(\mu G / L \\
\text { AS CD) }\end{array}$ & $\begin{array}{l}\text { CHRO- } \\
\text { MIUM, } \\
\text { DIS- } \\
\text { SOLVED } \\
\text { ( } \mu G / L \\
\text { AS CR) }\end{array}$ & $\begin{array}{l}\text { COBALT, } \\
\text { DIS- } \\
\text { SOLVED } \\
(\mu G / L \\
\text { AS CO) }\end{array}$ \\
\hline \multicolumn{11}{|c|}{ WELL IDENTIFICATION - SSI287--Cont inued } \\
\hline $\begin{array}{c}\text { NOV } 1987 \\
08 \ldots \\
\text { MAR } 1988\end{array}$ & -- & 0.02 & $<10$ & $<1$ & 32 & $<2$ & 130 & $<3$ & $<5$ & $<9$ \\
\hline $\begin{array}{c}04 \ldots \\
\text { APR }\end{array}$ & -- & .01 & $<10$ & $<1$ & 37 & $<2$ & 130 & 7 & $<20$ & $<9$ \\
\hline MAY & 0.06 & .0 & $<10$ & $<1$ & 30 & $<2$ & 130 & $<3$ & $<20$ & $<9$ \\
\hline${ }_{\text {AUG }}^{10} \ldots$ & -- & .02 & $<10$ & $<1$ & 36 & $<2$ & 140 & $<3$ & $<20$ & $<9$ \\
\hline $\begin{array}{l}30 \ldots \\
\text { Nov }\end{array}$ & .03 & .01 & $<10$ & $<1$ & 27 & $<.5$ & 130 & $<1$ & $<5$ & $<3$ \\
\hline $\begin{array}{c}16 \ldots \\
\text { APR } 1989\end{array}$ & .03 & .0 & $<10$ & $<1$ & 28 & $<.5$ & 140 & $<1$ & $<5$ & $<3$ \\
\hline JNEE & -- & -- & $<10$ & $<1$ & 21 & $<.5$ & 140 & $<1$ & $<5$ & $<3$ \\
\hline$\underset{\text { SEPT }}{07}$ & -- & -- & $<10$ & $<1$ & 23 & $<2$ & 140 & $<3$ & $<20$ & $<9$ \\
\hline $12 \ldots$ & -- & -- & 10 & $<1$ & 20 & $<1$ & 130 & $<2$ & $<10$ & $<6$ \\
\hline DATE & $\begin{array}{l}\text { COPPER, } \\
\text { DIS- } \\
\text { SOLVED } \\
(\mu G / L \\
\text { AS CU) }\end{array}$ & $\begin{array}{c}\text { IRON, } \\
\text { DIS- } \\
\text { SOLVED } \\
(\mu G / L \\
\text { AS FE })\end{array}$ & $\begin{array}{l}\text { LEAD, } \\
\text { DIS- } \\
\text { SOLVED } \\
(\mu G / L \\
\text { AS PB) }\end{array}$ & $\begin{array}{l}\text { MANGA- } \\
\text { NESE, } \\
\text { DIS- } \\
\text { SOLVED } \\
(\mu G / L \\
\text { AS MN) }\end{array}$ & $\begin{array}{l}\text { MOLYB- } \\
\text { DENUM, } \\
\text { DIS- } \\
\text { SOLVED } \\
(\mu G / L \\
\text { AS MO) }\end{array}$ & $\begin{array}{l}\text { NICKEI, } \\
\text { DIS- } \\
\text { SOLVED } \\
(\mu G / L \\
\text { AS NI) }\end{array}$ & $\begin{array}{l}\text { SILVER, } \\
\text { DIS- } \\
\text { SOLVED } \\
(\mu G / L \\
\text { AS AG) }\end{array}$ & $\begin{array}{c}\text { STRON- } \\
\text { TIUM, } \\
\text { DIS- } \\
\text { SOLVED } \\
(\mu G / L \\
\text { AS SR) }\end{array}$ & $\begin{array}{l}\text { VANA- } \\
\text { DIUM, } \\
\text { DIS- } \\
\text { SOLVED } \\
(\mu G / L \\
\text { AS V) }\end{array}$ & $\begin{array}{l}\text { ZINC, } \\
\text { DIS- } \\
\text { SOLVED } \\
(\mu G / L \\
\text { AS ZN) }\end{array}$ \\
\hline NOV 1987 & & & & & & & & & & \\
\hline $\begin{array}{l}08 \ldots \\
\text { MAR } 1988\end{array}$ & $<30$ & 51 & $<30$ & 11 & $<30$ & $<30$ & $<1.0$ & 1,500 & $<18$ & $<9$ \\
\hline $\begin{array}{l}04 \ldots \\
\text { APR }\end{array}$ & $<30$ & 160 & $<30$ & 22 & $<30$ & $<10$ & $<3.0$ & 2,300 & $<18$ & $<9$ \\
\hline$\underset{\text { MAY }}{05}$ & $<30$ & 170 & $<30$ & 20 & $<30$ & $<30$ & $<3.0$ & 2,300 & $<18$ & $<9$ \\
\hline${ }_{\text {AUG }}^{10} \ldots$ & $<30$ & 160 & $<30$ & 23 & $<30$ & $<30$ & 3.0 & 3,300 & $<18$ & $<9$ \\
\hline${ }_{\text {Nov }}^{30 \ldots}$ & $<10$ & 200 & $<10$ & 22 & $<10$ & $<10$ & $<1.0$ & 2,500 & $<6$ & $<3$ \\
\hline $\begin{array}{c}16 \ldots \\
\text { APR } 1989\end{array}$ & $<10$ & 220 & $<10$ & 20 & $<10$ & $<10$ & $<1.0$ & 2,800 & $<6$ & 6 \\
\hline$\underset{\text { JUNE }}{04 \ldots}$ & $<10$ & 180 & $<10$ & 19 & $<10$ & $<10$ & $<1.0$ & 2,200 & $<6$ & 7 \\
\hline$\underset{\text { SEPT }}{07}$ & $<30$ & 160 & $<30$ & 20 & $<30$ & $<30$ & $<3.0$ & 2,400 & $<18$ & $<9$ \\
\hline $12 \ldots$ & $<20$ & 210 & $<20$ & 23 & $<20$ & $<20$ & $<2.0$ & 2,400 & $<12$ & $<6$ \\
\hline
\end{tabular}


Table 25.--Water-quality analyses for wells--Continued

\begin{tabular}{|c|c|c|c|c|c|c|c|c|c|c|c|}
\hline DATE & TIME & $\begin{array}{c}\text { DEPTH } \\
\text { OF } \\
\text { WELL, } \\
\text { TOTAL } \\
\text { (FEET) }\end{array}$ & $\begin{array}{l}\text { ELEVATION } \\
\text { OF LAND } \\
\text { SURFACE } \\
\text { DATUM } \\
\text { (FEET } \\
\text { ABOVE } \\
\text { SEA } \\
\text { LEVEL) }\end{array}$ & $\begin{array}{l}\text { SPE- } \\
\text { CIFIC } \\
\text { CON- } \\
\text { DUCT- } \\
\text { ANCE } \\
(\mu S / C M)\end{array}$ & $\begin{array}{c}\text { PH } \\
\text { (STAND- } \\
\text { ARD } \\
\text { UNITS) }\end{array}$ & $\begin{array}{c}\text { TEMPER- } \\
\text { ATURE } \\
\text { WATER } \\
\left({ }^{\circ} \mathrm{C}\right)\end{array}$ & $\begin{array}{l}\text { HARD- } \\
\text { NESS } \\
\text { TOTAL } \\
\text { (MG/L } \\
\mathrm{AS}^{-} \\
\mathrm{CACO}_{3} \text { ) }\end{array}$ & $\begin{array}{l}\text { HARD- } \\
\text { NESS } \\
\text { NONCARB } \\
\text { WH WAT } \\
\text { TOT FLD } \\
(\mathrm{MG} / \mathrm{L} \text { AS } \\
\left.\text { CACO }_{3}\right)\end{array}$ & $\begin{array}{l}\text { CALCIUM } \\
\text { DIS- } \\
\text { SOLVED } \\
\text { (MG/L } \\
\text { AS CA) }\end{array}$ & $\begin{array}{l}\text { MAGNE- } \\
\text { SIUM, } \\
\text { DIS- } \\
\text { SOLVED } \\
\text { (MG/L } \\
\text { AS MG) }\end{array}$ & $\begin{array}{l}\text { SODIUM, } \\
\text { DIS- } \\
\text { SOLVED } \\
\text { (MG/L } \\
\text { AS NA) }\end{array}$ \\
\hline
\end{tabular}

\begin{tabular}{|c|c|c|c|c|c|c|c|c|c|c|c|}
\hline & & & & WELL & DENTIFIC & TION - S & SW287 & & & & \\
\hline NOV 1987 & & & & & & & & & & & \\
\hline $\begin{array}{l}08 \ldots \\
\text { MAR } 1988\end{array}$ & 1015 & 98.50 & 6,860 & 1,710 & 8.0 & -- & 36 & 0 & 8.8 & 3.2 & 470 \\
\hline$\underset{A P R}{07 \ldots}$ & 0945 & 98.50 & 6,860 & 1,775 & 8.2 & 6.0 & 31 & 0 & 6.7 & 3.3 & 460 \\
\hline${ }_{M A Y}^{05} \ldots$ & 1015 & 98.50 & 6,860 & 1,810 & 8.2 & 10.0 & 18 & 5 & 5.7 & -- & 440 \\
\hline${ }_{A U G}^{10}$ & 0900 & 98.50 & 6,860 & 2,210 & 8.3 & 9.5 & 35 & 0 & 8.8 & 3.0 & 510 \\
\hline $\begin{array}{l}30 \ldots \\
\text { Nov }\end{array}$ & 0900 & 98.50 & 6,860 & 2,150 & 8.6 & 11.0 & 28 & 0 & 6.8 & 2.5 & 480 \\
\hline $\begin{array}{c}16 \ldots \\
\text { APR } 1989\end{array}$ & 0900 & 98.50 & 6,860 & 3,060 & 8.5 & -- & 63 & 0 & 16 & 5.3 & 710 \\
\hline$\underset{\text { JNE }}{04 \ldots}$ & 1400 & 98.50 & 6,860 & 1,930 & 8.3 & 9.0 & 22 & 0 & 5.4 & 1.9 & 440 \\
\hline$\underset{\text { SEPT }}{07}$ & 0915 & 98.50 & 6,860 & 2,820 & 8.4 & 10.0 & 49 & 0 & 13 & 3.8 & 630 \\
\hline $12 \ldots$ & 1015 & 98.50 & 6,860 & 2,270 & 8.3 & 9.5 & 32 & 0 & 8.2 & 2.6 & 530 \\
\hline & & $\begin{array}{l}\text { POTAS- } \\
\text { SIUM, } \\
\text { DIS- } \\
\text { SOLVED } \\
\text { (MG/L } \\
\text { AS K) }\end{array}$ & $\begin{array}{c}\text { ALKA- } \\
\text { LINITY } \\
\text { LAB } \\
(\mathrm{MG} / \mathrm{L} \\
\mathrm{AS} \\
\left.\mathrm{CACO}_{3}\right)\end{array}$ & $\begin{array}{c}\text { SULFATE } \\
\text { DIS- } \\
\text { SOLVED } \\
(\mathrm{MG} / \mathrm{L} \\
\left.\text { AS } \mathrm{SO}_{4}\right)\end{array}$ & $\begin{array}{l}\text { CHLO- } \\
\text { RIDE, } \\
\text { DIS- } \\
\text { SOLVED } \\
\text { (MG/L } \\
\text { AS CL) }\end{array}$ & $\begin{array}{l}\text { FLUO- } \\
\text { RIDE, } \\
\text { DIS- } \\
\text { SOLVED } \\
\text { (MG/L } \\
\text { AS F) }\end{array}$ & $\begin{array}{l}\text { SILICA, } \\
\text { DIS- } \\
\text { SOLVED } \\
\text { (MG/L } \\
\mathrm{AS} \\
\left.\mathrm{SIO}_{2}\right)\end{array}$ & $\begin{array}{l}\text { SOLIDS, } \\
\text { RESIDUE } \\
\text { AT } 180 \\
\text { DEG. C } \\
\text { DIS- } \\
\text { SOLVED } \\
(\mathrm{MG} / \mathrm{L})\end{array}$ & $\begin{array}{c}\text { SOLIDS, } \\
\text { SUM OF } \\
\text { CONSTIT- } \\
\text { UENTS, } \\
\text { DIS- } \\
\text { SOLVED } \\
\text { (MG/L) }\end{array}$ & & \\
\hline NO & 987 & & & & & & & & & & \\
\hline MAI & $\ddot{988}$ & 3.2 & 622 & 400 & 22 & 0.9 & 11 & 1,300 & 1,290 & & \\
\hline API & & 3.4 & 670 & 320 & 15 & 2.2 & 9.8 & 1,220 & 1,230 & & \\
\hline MA & & 2.6 & -- & 300 & 14 & 2.3 & 9.8 & 1,160 & -- & & \\
\hline $\mathrm{AUC}$ & & 3.3 & 641 & 530 & 19 & 2.1 & 7.5 & 1,470 & 1,470 & & \\
\hline No & & 2.8 & 643 & 440 & 18 & 1.9 & 7.5 & 1,330 & 1,350 & & \\
\hline API & $\ddot{989}$ & 4.4 & 544 & 1,000 & 34 & 1.5 & 8.0 & 2,140 & 2,110 & & \\
\hline ת! & & 2.8 & 658 & 360 & 17 & 2.3 & 7.7 & 1,240 & 1,230 & & \\
\hline SE & & 3.6 & 580 & 800 & 28 & 1.7 & 7.8 & 1,810 & 1,840 & & \\
\hline & & 3.1 & 631 & 540 & 22 & 2.0 & 8.1 & 1,490 & 1,500 & & \\
\hline
\end{tabular}


Table 25.--Water-quality analyses for wells--Continued

\begin{tabular}{|c|c|c|c|c|c|c|c|c|c|c|c|}
\hline DATE & $\begin{array}{l}\text { NITRO- } \\
\text { GEN, } \\
\text { NITRITE } \\
\text { DIS- } \\
\text { SOLVED } \\
\text { (MG/L } \\
\text { AS N) }\end{array}$ & $\begin{array}{l}\text { NITRO- } \\
\text { GEN, } \\
\text { NITRITE } \\
\text { DIS- } \\
\text { SOLVED } \\
(\mathrm{MG} / \mathrm{L} \\
\left.\text { AS } \mathrm{NO}_{2}\right)\end{array}$ & $\begin{array}{l}\text { NITRO- } \\
\text { GEN, } \\
\mathrm{NO}_{2}+\mathrm{NO}_{3} \\
\text { DIS- } \\
\text { SOLVED } \\
\text { (MG/L } \\
\text { AS N) }\end{array}$ & $\begin{array}{l}\text { NITRO- } \\
\text { GEN, } \\
\text { AMMONIA } \\
\text { DIS- } \\
\text { SOLVED } \\
\text { (MG/L } \\
\text { AS N) }\end{array}$ & $\begin{array}{l}\text { NITRO- } \\
\text { GEN, } \\
\text { AMMONIA } \\
\text { DIS- } \\
\text { SOLVED } \\
(\mathrm{MG} / \mathrm{L} \\
\left.\text { AS } \mathrm{NH}_{4}\right)\end{array}$ & $\begin{array}{l}\text { NITRO- } \\
\text { GEN, } \\
\text { ORGANIC } \\
\text { DIS- } \\
\text { SOLVED } \\
\text { (MG/L } \\
\text { AS N) }\end{array}$ & $\begin{array}{l}\text { NITRO- } \\
\text { GEN, AM- } \\
\text { MONIA + } \\
\text { ORGANIC } \\
\text { DISSOLVED } \\
\text { (MG/L } \\
\text { AS N) }\end{array}$ & $\begin{array}{l}\text { PHOS- } \\
\text { PHORUS } \\
\text { DIS- } \\
\text { SOLVED } \\
\text { (MG/L } \\
\text { AS P) }\end{array}$ & $\begin{array}{l}\text { PHOS- } \\
\text { PHORUS } \\
\text { ORTHO, } \\
\text { DIS- } \\
\text { SOLVED } \\
\text { (MG/L } \\
\text { AS P) }\end{array}$ & $\begin{array}{l}\text { PHOS- } \\
\text { PHATE, } \\
\text { ORTHO, } \\
\text { DIS- } \\
\text { SOLVED } \\
\text { (MG/L } \\
\text { AS P04) }\end{array}$ & $\begin{array}{l}\text { PHOS- } \\
\text { PHORUS } \\
\text { ORGANIC } \\
\text { DIS- } \\
\text { SOLVED } \\
\text { (MG/L } \\
\text { AS P) }\end{array}$ \\
\hline \multicolumn{12}{|c|}{ WELL IDENTIFICATION - SSW287--Continued } \\
\hline NOV 1987 & & & & & & & & & & & \\
\hline $\begin{array}{l}08 \ldots \\
\text { MAR } 1988\end{array}$ & -- & -- & -- & -- & -- & -- & 1.6 & 0.07 & -- & -- & 0.07 \\
\hline $\begin{array}{c}07 \ldots \\
\text { APR }\end{array}$ & $<0.01$ & -- & $<0.10$ & 0.13 & 0.17 & 1.5 & 1.6 & .12 & $<0.09$ & -- & .12 \\
\hline$\underset{\mathrm{MAY}}{05}$ & .01 & 0.03 & $<.10$ & .90 & 1.2 & .3 & 1.2 & .07 & .06 & 0.18 & .01 \\
\hline${ }_{A U G}^{10 \ldots}$ & $<.01$ & -- & $<.10$ & .62 & .8 & .78 & 1.4 & .04 & .03 & .09 & .01 \\
\hline $\begin{array}{l}30 \ldots \\
\text { NoV }\end{array}$ & $<.01$ & -- & $<.10$ & .45 & .58 & .75 & 1.2 & .05 & .05 & .15 & .0 \\
\hline $\begin{array}{c}16 \ldots \\
\text { APR } 1989\end{array}$ & $<.01$ & -- & $<.10$ & 1.10 & 1.4 & .0 & 1.0 & .02 & .02 & .06 & .0 \\
\hline JUNE & -- & - & -- & -- & -- & -- & -- & - & -- & -- & -- \\
\hline $\begin{array}{c}07 \\
\text { SEPT }\end{array}$ & -- & -- & -- & -- & -- & -- & -- & -- & -- & -- & -- \\
\hline $12 \ldots$ & -- & -- & -- & -- & -- & -- & -- & -- & -- & -- & -- \\
\hline DATE & $\begin{array}{c}\text { ALU- } \\
\text { MINUM, } \\
\text { DIS- } \\
\text { SOLVED } \\
(\mu G / L \\
\text { AS AL) }\end{array}$ & $\begin{array}{l}\text { ARSENIC } \\
\text { DIS- } \\
\text { SOLVED } \\
(\mu G / L \\
\text { AS AS) }\end{array}$ & $\begin{array}{l}\text { BARIUM, } \\
\text { DIS- } \\
\text { SOLVED } \\
(\mu G / L \\
\text { AS BA) }\end{array}$ & $\begin{array}{l}\text { BERYL- } \\
\text { LIUM, } \\
\text { DIS- } \\
\text { SOLVED } \\
(\mu G / L \\
\text { AS BE) }\end{array}$ & $\begin{array}{l}\text { BORON, } \\
\text { DIS- } \\
\text { SOLVED } \\
(\mu G / L \\
\text { AS B) }\end{array}$ & $\begin{array}{l}\text { CADMIUM } \\
\text { DIS- } \\
\text { SOLVED } \\
(\mu G / L \\
\text { AS CD) }\end{array}$ & $\begin{array}{l}\text { CHRO- } \\
\text { MIUM, } \\
\text { DIS- } \\
\text { SOLVED } \\
(\mu G / L \\
\text { AS CR) }\end{array}$ & $\begin{array}{l}\text { COBALT, } \\
\text { DIS- } \\
\text { SOLVED } \\
(\mu G / L \\
\text { AS CO) }\end{array}$ & $\begin{array}{l}\text { COPPER, } \\
\text { DIS- } \\
\text { SOLVED } \\
(\mu G / L \\
\text { AS CU) }\end{array}$ & $\begin{array}{l}\text { IRON, } \\
\text { DIS- } \\
\text { SOLVED } \\
(\mu G / L \\
\text { AS FE) }\end{array}$ & $\begin{array}{l}\text { LEAD, } \\
\text { DIS- } \\
\text { SOLVED } \\
(\mu G / L \\
\text { AS PB) }\end{array}$ \\
\hline NOV 1987 & & & & & & & & & & & \\
\hline $\begin{array}{l}08 \ldots \\
\text { MAR } 1988\end{array}$ & 300 & 2 & 75 & $<1$ & 130 & $<2$ & $<10$ & $<6$ & $<20$ & 190 & $<20$ \\
\hline $\begin{array}{l}07 \ldots \\
\text { APR }\end{array}$ & 5,000 & $<1$ & 62 & $<.5$ & 120 & $<3$ & $<5$ & $<3$ & $<10$ & 510 & $<10$ \\
\hline$\underset{M A Y}{05 \ldots}$ & 450 & 1 & 62 & $<2$ & 120 & 3 & $<20$ & $<9$ & $<30$ & 340 & 50 \\
\hline $\begin{array}{l}10 \ldots \\
\text { AUG }\end{array}$ & $<10$ & $<1$ & 99 & $<2$ & 120 & $<3$ & $<20$ & $<9$ & $<30$ & 74 & $<30$ \\
\hline $\begin{array}{c}30 \ldots \\
\text { NOV }\end{array}$ & $<10$ & $<1$ & 84 & 1 & 110 & 1 & $<5$ & $<3$ & $<10$ & 100 & 20 \\
\hline $\begin{array}{l}16 \ldots \\
\text { APR } 1989\end{array}$ & 10 & $<1$ & 130 & $<.5$ & 120 & 2 & $<5$ & $<3$ & $<10$ & 140 & $<10$ \\
\hline $\begin{array}{l}04 \ldots \\
\text { JNE }\end{array}$ & 130 & 1 & 61 & $<.5$ & 110 & $<1$ & $<5$ & $<3$ & $<10$ & 190 & $<10$ \\
\hline$\underset{\text { SEPT }}{07} \ldots$ & 30 & $<1$ & 110 & $<2$ & 110 & $<3$ & $<20$ & $<9$ & $<30$ & 210 & $<30$ \\
\hline $12 \ldots$ & 30 & 1 & 80 & $<1$ & 110 & $<2$ & $<10$ & $<6$ & $<20$ & 140 & $<20$ \\
\hline
\end{tabular}


Table 25.--Water-quality analyses for wells--Continued

\begin{tabular}{|c|c|c|c|c|c|c|c|c|c|c|c|c|}
\hline DATE & $\begin{array}{l}\text { MANGA- } \\
\text { NESE, } \\
\text { DIS- } \\
\text { SOLVED } \\
(\mu G / L \\
\text { AS MN) }\end{array}$ & $\begin{array}{l}\text { MOLYB- } \\
\text { DENUM, } \\
\text { DIS- } \\
\text { SOLVED } \\
(\mu G / L \\
\text { AS MO) }\end{array}$ & $\begin{array}{l}\text { NICKEL, } \\
\text { DIS- } \\
\text { SOLVED' } \\
(\mu G / L \\
\text { AS NI) }\end{array}$ & $\begin{array}{l}\text { SILVER, } \\
\text { DIS- } \\
\text { SOLVED } \\
(\mu G / L \\
\text { AS AG) }\end{array}$ & $\begin{array}{c}\text { STRON- } \\
\text { TIUM, } \\
\text { DIS- } \\
\text { SOLVED } \\
\text { ( } \mu G / L \\
\text { AS SR) }\end{array}$ & $\begin{array}{l}\text { VANA- } \\
\text { DIUM, } \\
\text { DIS- } \\
\text { SOLVED } \\
(\mu G / L \\
\text { AS V) }\end{array}$ & $\begin{array}{l}\text { ZINC, } \\
\text { DIS- } \\
\text { SOLVED } \\
(\mu G / L \\
\text { AS ZN) }\end{array}$ & $\begin{array}{c}\text { C-13/ } \\
\text { C-12 } \\
\text { STABIE } \\
\text { ISOTOPE } \\
\text { RATIO } \\
\text { PER } \\
\text { MIL }\end{array}$ & $\begin{array}{l}\text { H-2/ } \\
\text { H-1 } \\
\text { STABLE } \\
\text { ISOTOPE } \\
\text { RATIO } \\
\text { PER } \\
\text { MIL }\end{array}$ & $\begin{array}{c}0-18 / \\
0-16 \\
\text { STABLE } \\
\text { ISOTOPE } \\
\text { RATIO } \\
\text { PER } \\
\text { MIL }\end{array}$ & $\begin{array}{c}\text { S-34/ } \\
\text { S-32 } \\
\text { STABLE } \\
\text { ISOTOPE } \\
\text { RATIO } \\
\text { PER } \\
\text { MIL }\end{array}$ & $\begin{array}{l}\text { TRIT- } \\
\text { IUM } \\
\text { TOTAL } \\
(\mathrm{PCI} / \mathrm{L})\end{array}$ \\
\hline
\end{tabular}

NOV 1987

$08 \ldots$

MAR 1988

$07 \ldots$

APR

05... $\quad 180<30<30$

MAY

$10 \ldots<310<30<30$

AUG

$30 \ldots \quad 340<10 \quad 20$

NOV

16.. $140<10<10$

APR 1989

$04 \ldots<180<10<10$

JNE

$07 \ldots \quad 190<30<30$

SEPT

12... 210

(2)

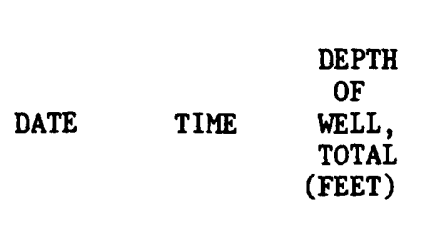

DEPTH
OF
WELL,
TOTAL
(FEET)

NOV 1987
$08 \ldots$

MAR 1988

$04 \ldots$
APR
$05 \ldots$

$05 \ldots$
MAY
10.

UNE

$15 \ldots$

$15 \ldots$

$15 \ldots$

AUG

$30 .$.

NOV

$16 \ldots$

$04 \ldots$

תNE

$07 \ldots$

SEPT

$12 .$.

$\begin{array}{ll}0940 & 105.30 \\ 1035 & 105.30 \\ 1100 & 105.30 \\ 1015 & 105.30 \\ 0900 & 105.30 \\ 1115 & 105 \cdot 30 \\ 1300 & 105.30 \\ 1015 & 105.30 \\ 1030 & 105.30 \\ 1115 & 105.30 \\ 0845 & 105.30 \\ 1215 & 105.30\end{array}$

6,861

$6,861 \quad 3,320$

$6,861 \quad 3,950$

8.1

9.5

7.9

$11.0 \quad 360$

$\begin{array}{lll}7.9 & 10.0 \quad 480\end{array}$

$6,861 \quad 4,150$

$\begin{array}{lll}7.9 & 11.0 & 430\end{array}$

$6,861 \quad 3,970$

$8.0 \quad 10.5 \quad 130$

$\begin{array}{ll}6,861 & 3,590 \\ 6,861 & 3,800\end{array}$

$\begin{array}{lll}7.9 & 11.0 & 170\end{array}$

$6,861 \quad 3,950$

$8.3 \quad 10.5 \quad 240$

$6,861 \quad 3,940$

$8.4 \quad--250$

$6,861 \quad 3,880$

$8.1 \quad 10.0 \quad 170$

$6,861 \quad 4,100$

$8.2 \quad 10.0 \quad 200$

$6,861 \quad 3,880$

$8.210 .0 \quad 150$

$1215 \quad 105.30$

\section{tinued}

$<2.0 \quad 300<12<6 \quad--$

$$
12 . .215,105.30,6,861
$$

8.2 .10 .0 .150 .00


Table 25.--Water-quality analyses for wells--Continued

\begin{tabular}{|c|c|c|c|c|c|c|c|c|c|c|c|}
\hline \multicolumn{2}{|c|}{ DATE } & $\begin{array}{l}\text { POTAS- } \\
\text { SIUM, } \\
\text { DIS- } \\
\text { SOLVED } \\
\text { (MG/L } \\
\text { AS K) }\end{array}$ & $\begin{array}{l}\text { ALKA- } \\
\text { IINITY } \\
\text { LAB } \\
(\mathrm{MG} / \mathrm{L} \\
\mathrm{AS} \\
\left.\mathrm{CACO}_{3}\right)\end{array}$ & $\begin{array}{l}\text { SULFATE } \\
\text { DIS- } \\
\text { SOLVED } \\
(\mathrm{MG} / \mathrm{L} \\
\left.\mathrm{AS} \mathrm{SO} \mathrm{S}_{4}\right)\end{array}$ & $\begin{array}{l}\text { CHLO- } \\
\text { RIDE, } \\
\text { DIS- } \\
\text { SOLVED } \\
\text { (MG/L } \\
\text { AS CL) }\end{array}$ & $\begin{array}{l}\text { FLUO- } \\
\text { RIDE, } \\
\text { DIS- } \\
\text { SOLVED } \\
\text { (MG/L } \\
\text { AS F) }\end{array}$ & $\begin{array}{l}\text { SILICA, } \\
\text { DIS- } \\
\text { SOLVED } \\
\text { (MG/L } \\
\text { AS } \\
\left.\mathrm{SIO}_{2}\right)\end{array}$ & $\begin{array}{l}\text { SOLIDS, } \\
\text { RESIDUE } \\
\text { AT } 180 \\
\text { DEG. C } \\
\text { DIS- } \\
\text { SOLVED } \\
\text { (MG/L) }\end{array}$ & $\begin{array}{l}\text { SOLIDS, } \\
\text { SUM OF } \\
\text { CONSTIT- } \\
\text { UENTS, } \\
\text { DIS- } \\
\text { SOLVED } \\
\text { (MG/L) }\end{array}$ & & \\
\hline \multicolumn{12}{|c|}{ WELL IDENTIFICATION - SSU487--Continued } \\
\hline \multicolumn{2}{|c|}{$\begin{array}{c}\text { NOV } 1987 \\
08 \ldots \\
\text { MAR } 1988\end{array}$} & 5.1 & 475 & 1,400 & 40 & 1.2 & 7.9 & 2,560 & 2,610 & & \\
\hline \multicolumn{2}{|c|}{$\begin{array}{c}04 \ldots \\
\mathrm{APR}\end{array}$} & 5.7 & 504 & 1,200 & 32 & 1.3 & 9.0 & 2,380 & 2,400 & & \\
\hline \multicolumn{2}{|c|}{ MAY } & 8.8 & 472 & 1,800 & 39 & .9 & 8.3 & 3,100 & 3,150 & & \\
\hline \multicolumn{2}{|c|}{$\frac{10}{\text { JNE }}$} & 7.5 & 466 & 1,900 & 42 & .8 & 8.5 & 3,180 & 3,280 & & \\
\hline \multicolumn{2}{|c|}{$\begin{array}{r}15 \ldots \\
15 \ldots \\
15 \ldots \\
\text { AUG }\end{array}$} & $\begin{array}{l}7.4 \\
5.3 \\
5.8\end{array}$ & $\begin{array}{l}457 \\
484 \\
472\end{array}$ & $\begin{array}{l}1,800 \\
1,400 \\
1,500\end{array}$ & $\begin{array}{l}48 \\
40 \\
44\end{array}$ & $\begin{array}{r}.7 \\
1.1 \\
.8\end{array}$ & $\begin{array}{l}9.3 \\
7.8 \\
7.9\end{array}$ & $\begin{array}{l}3,140 \\
2,570 \\
2,740\end{array}$ & $\begin{array}{l}3,140 \\
2,590 \\
2,740\end{array}$ & & \\
\hline \multicolumn{2}{|l|}{ AUG } & 6.6 & 477 & 1,600 & 46 & .9 & 8.0 & 2,750 & 2,860 & & \\
\hline \multicolumn{2}{|c|}{$\begin{array}{l}16 \ldots \\
\text { APR } 1989\end{array}$} & 6.9 & 483 & 1,800 & 54 & .8 & 8.3 & 3,030 & 3,160 & & \\
\hline \multicolumn{2}{|c|}{$\begin{array}{l}04 \ldots \\
\text { JNE }\end{array}$} & 6.4 & 487 & 1,500 & 49 & 1.3 & 8.0 & 2,740 & 2,750 & & \\
\hline \multicolumn{2}{|c|}{$07 \ldots$} & 6.6 & 478 & 1,600 & 50 & 1.0 & 8.2 & 2,820 & 2,920 & & \\
\hline \multicolumn{2}{|c|}{$\begin{array}{r}\text { SEPI } \\
12 \ldots\end{array}$} & 5.9 & 488 & 1,500 & 46 & 1.2 & 7.9 & 2,650 & 2,740 & & \\
\hline DATE & $\begin{array}{l}\text { NITRO- } \\
\text { GEN, } \\
\text { NITRITE } \\
\text { DIS- } \\
\text { SOLVED } \\
\text { (MG/L } \\
\text { AS N) }\end{array}$ & $\begin{array}{c}\text { NITRO- } \\
\text { GEN, } \\
\text { NITRITE } \\
\text { DIS- } \\
\text { SOLVED } \\
(\mathrm{MG} / \mathrm{L} \\
\left.\text { AS } \mathrm{NO}_{2}\right)\end{array}$ & $\begin{array}{c}\text { NITRO- } \\
\text { GEN, } \\
\mathrm{NO}_{2}+\mathrm{NO}_{3} \\
\text { DIS- } \\
\text { SOLVED } \\
\text { (MG/L } \\
\text { AS N) }\end{array}$ & $\begin{array}{l}\text { NITRO- } \\
\text { GEN, } \\
\text { AMMONIA } \\
\text { DIS- } \\
\text { SOLVED } \\
\text { (MG/L } \\
\text { AS N) }\end{array}$ & $\begin{array}{c}\text { NITRO- } \\
\text { GEN, } \\
\text { AMMONIA } \\
\text { DIS- } \\
\text { SOLVED } \\
(\mathrm{MG} / \mathrm{L} \\
\text { AS NH } \\
\text { AS }\end{array}$ & $\begin{array}{l}\text { NITRO- } \\
\text { GEN, } \\
\text { ORGANIC } \\
\text { DIS- } \\
\text { SOLVED } \\
\text { (MG/L } \\
\text { AS N) }\end{array}$ & $\begin{array}{l}\text { NITRO- } \\
\text { GEN, AM- } \\
\text { MONIA + } \\
\text { ORGANIC } \\
\text { DISSOLVED } \\
(M G / L \\
\text { AS N) }\end{array}$ & $\begin{array}{c}\text { PHOS- } \\
\text { PHORUS } \\
\text { DIS- } \\
\text { SOLVED } \\
\text { (MG/L } \\
\text { AS P) }\end{array}$ & $\begin{array}{l}\text { PHOS- } \\
\text { PHORUS } \\
\text { ORTHO, } \\
\text { DIS- } \\
\text { SOLVED } \\
\text { (MG/L } \\
\text { AS P) }\end{array}$ & $\begin{array}{l}\text { PHOS- } \\
\text { PHATE, } \\
\text { ORTHO, } \\
\text { DIS- } \\
\text { SOLVED } \\
(\mathrm{MG} / \mathrm{L} \\
\text { AS PO } \\
\text { AS) }\end{array}$ & $\begin{array}{l}\text { PHOS- } \\
\text { PHORUS } \\
\text { ORGANIC } \\
\text { DIS- } \\
\text { SOLVED } \\
\text { (MG/L } \\
\text { AS P) }\end{array}$ \\
\hline \multicolumn{12}{|l|}{ NOV 1987} \\
\hline $\begin{array}{l}08 \ldots \\
\text { MAR } 1988\end{array}$ & -- & -- & -- & -- & -- & -- & 1.7 & 0.01 & -- & -- & 0.01 \\
\hline $\begin{array}{l}04 \ldots \\
\text { APR }\end{array}$ & $<0.01$ & -- & $<0.10$ & 1.20 & 1.5 & 0.8 & 2.0 & .01 & $<0.01$ & -- & .01 \\
\hline$\underset{M A Y}{05}$ & $<.01$ & -- & $<.10$ & 2.10 & 2.7 & .4 & 2.5 & $<.01$ & .01 & 0.03 & -- \\
\hline${ }_{\text {JNE }}^{10} \ldots$ & $<.01$ & -- & $<.10$ & 2.20 & 2.8 & .6 & 2.8 & $<.01$ & $<.01$ & -- & -- \\
\hline $15 \ldots$ & .01 & 0.03 & $<.10$ & 2.00 & 2.6 & .1 & 2.1 & $<.01$ & $<.01$ & -- & -- \\
\hline $15 \ldots$ & .01 & .03 & $<.10$ & .94 & 1.2 & .76 & 1.7 & $<.01$ & .01 & .03 & -- \\
\hline $15 \ldots$ & .01 & .03 & $<.10$ & 1.70 & 2.2 & .2 & 1.9 & $<.01$ & $<.01$ & -- & -- \\
\hline Nov $30 \ldots$ & $<.01$ & -- & $<.10$ & 1.60 & 2.1 & .6 & 2.2 & .01 & $<.01$ & -- & .01 \\
\hline $\begin{array}{l}16 \ldots \\
\text { APR } 1989\end{array}$ & $<.01$ & -- & $<.10$ & 2.50 & 3.2 & .0 & 1.9 & .01 & $<.01$ & -- & .01 \\
\hline$\underset{\text { JUNE }}{04 \ldots}$ & -- & -- & -- & -- & -- & -- & -- & -- & -- & -- & -- \\
\hline$\underset{\text { SEPT }}{07} \ldots$ & -- & -- & -- & -- & -- & -- & -- & -- & -- & -- & -- \\
\hline $12 \ldots$ & -- & -- & -- & -- & -- & -- & -- & -- & -- & -- & -- \\
\hline
\end{tabular}


Table 25.--Water-quality analyses for wells--Continued

\begin{tabular}{|c|c|c|c|c|c|c|c|c|c|c|c|c|}
\hline DAT & & $\begin{array}{l}\text { ALU- } \\
\text { MINUM, } \\
\text { DIS- } \\
\text { SOLVED } \\
\text { ( } \mu G / L \\
\text { AS AL) }\end{array}$ & $\begin{array}{l}\text { ARSENIC } \\
\text { DIS- } \\
\text { SOLVED } \\
(\mu G / L \\
\text { AS AS) }\end{array}$ & $\begin{array}{l}\text { BARIUM, } \\
\text { DIS- } \\
\text { SOLVED } \\
(\mu G / L \\
\text { AS BA) }\end{array}$ & $\begin{array}{l}\text { BERYL- } \\
\text { LIUM, } \\
\text { DIS- } \\
\text { SOLVED } \\
\text { ( } \mu G / L \\
\text { AS BE) }\end{array}$ & $\begin{array}{l}\text { BORON, } \\
\text { DIS- } \\
\text { SOLVED } \\
(\mu G / L \\
\text { AS B) }\end{array}$ & $\begin{array}{l}\text { CADMIUM } \\
\text { DIS- } \\
\text { SOLVED } \\
(\mu G / L \\
\text { AS CD) }\end{array}$ & $\begin{array}{l}\text { CHRO- } \\
\text { MIUM, } \\
\text { DIS- } \\
\text { SOLVED } \\
\text { ( } \mu \text { G/L } \\
\text { AS CR) }\end{array}$ & $\begin{array}{l}\text { COBALT, } \\
\text { DIS- } \\
\text { SOLVED } \\
(\mu G / L \\
\text { AS CO) }\end{array}$ & $\begin{array}{l}\text { COPPER, } \\
\text { DIS- } \\
\text { SOLVED } \\
\text { ( } \mu G / L \\
\text { AS CU) }\end{array}$ & $\begin{array}{l}\text { IRON, } \\
\text { DIS- } \\
\text { SOLVED } \\
\text { ( } \mu \text { G/L } \\
\text { AS FE) }\end{array}$ & $\begin{array}{l}\text { LEAD, } \\
\text { DIS- } \\
\text { SOIVED } \\
\text { ( } \mu G / L \\
\text { AS PB) }\end{array}$ \\
\hline & & & & WELL & IDENTIFIC & SATION - & SSU487-- & Sontinued & & & & \\
\hline NOV 1 & & & & & & & & & & & & \\
\hline $\begin{array}{r}08 . \\
\text { MAR } 1\end{array}$ & & $<10$ & $<1$ & 48 & $<2$ & 140 & $<3$ & $<20$ & $<9$ & $<30$ & 71 & $<30$ \\
\hline $\begin{array}{l}04 . \\
\text { APR }\end{array}$ & & $<200$ & $<1$ & 36 & $<2$ & 130 & 7 & $<20$ & $<9$ & $<30$ & 120 & $<30$ \\
\hline $\begin{array}{l}05 . \\
\text { MAY }\end{array}$ & & $<10$ & $<1$ & 38 & $<2$ & 140 & $<3$ & $<20$ & $<9$ & $<10$ & 160 & $<30$ \\
\hline JUNE & & 10 & $<1$ & 32 & $<2$ & 150 & $<3$ & $<20$ & $<9$ & $<30$ & 230 & $<30$ \\
\hline 15. & & $<10$ & $<1$ & 43 & $<.5$ & 160 & $<1$ & $<5$ & $<3$ & $<10$ & 10 & $<10$ \\
\hline 15. & & $<10$ & $<1$ & 26 & $<2$ & 140 & $<3$ & $<20$ & $<9$ & $<30$ & 44 & $<30$ \\
\hline 15. & & $<10$ & $<1$ & 29 & $<.5$ & 160 & $<1$ & $<5$ & $<3$ & $<10$ & 63 & $<10$ \\
\hline AUG & & & & & & & & & & & & \\
\hline $\begin{array}{l}30 . \\
\text { NOV }\end{array}$ & & $<10$ & $<1$ & 32 & $<.5$ & 130 & $<1$ & $<5$ & $<3$ & $<10$ & 80 & $<10$ \\
\hline $\begin{array}{l}16 . \\
\text { APR } 1\end{array}$ & & $<10$ & $<1$ & 31 & $<.5$ & 130 & $<1$ & $<5$ & $<3$ & $<10$ & 67 & $<10$ \\
\hline $\begin{array}{l}04 . \\
\text { JUNE }\end{array}$ & & 10 & $<1$ & 30 & $<2$ & 130 & 4 & $<20$ & $<9$ & $<30$ & 51 & $<30$ \\
\hline $\begin{array}{l}07 . \\
\text { SEPT }\end{array}$ & & 10 & $<1$ & 36 & $<2$ & 130 & $<3$ & $<20$ & $<9$ & $<30$ & 50 & $<30$ \\
\hline 12. & & 20 & $<1$ & 26 & $<1$ & 120 & $<2$ & $<10$ & $<6$ & $<20$ & 28 & $<20$ \\
\hline DATE & $\begin{array}{l}\text { MANGA- } \\
\text { NESE, } \\
\text { DIS- } \\
\text { SOLVED } \\
(\mu G / L \\
\text { AS MN) }\end{array}$ & $\begin{array}{c}\text { MOLYB- } \\
\text { DENUM, } \\
\text { DIS- } \\
\text { SOLVED } \\
(\mu G / L \\
\text { AS MO) }\end{array}$ & $\begin{array}{l}\text { NICKEL, } \\
\text { DUS- } \\
\text { SOLVED } \\
(\mu G / L \\
\text { AS NI }\end{array}$ & $\begin{array}{c}\text { SILVER, } \\
\text { DIS- } \\
\text { SOLVED } \\
\text { ( } \mu G / L \\
\text { AS AG) }\end{array}$ & $\begin{array}{c}\text { STRON- } \\
\text { TIUM, } \\
\text { DIS- } \\
\text { SOLVED } \\
\text { ( } \mu G / L \\
\text { AS SR) }\end{array}$ & $\begin{array}{l}\text { VANA- } \\
\text { DIUM, } \\
\text { DIS- } \\
\text { SOLVED } \\
(\mu G / L \\
\text { AS V) }\end{array}$ & $\begin{array}{l}\text { ZINC, } \\
\text { DIS- } \\
\text { SOLVED } \\
\text { ( } \mu G / L \\
\text { AS ZN) }\end{array}$ & $\begin{array}{c}\text { C-13/ } \\
\text { C-12 } \\
\text { STABLE } \\
\text { ISOTOPE } \\
\text { RATIO } \\
\text { PER } \\
\text { MIL }\end{array}$ & $\begin{array}{l}\text { H-2/ } \\
\text { H-1 } \\
\text { STABLE } \\
\text { ISOTOPE } \\
\text { RATIO } \\
\text { PER } \\
\text { MIL }\end{array}$ & $\begin{array}{c}0-18 / \\
0-16 \\
\text { STABLE } \\
\text { ISOTOPE } \\
\text { RATIO } \\
\text { PER } \\
\text { MIL }\end{array}$ & $\begin{array}{l}\text { S-34/ } \\
\text { S-32 } \\
\text { STABLE } \\
\text { ISOTOPE } \\
\text { RATIO } \\
\text { PER } \\
\text { MIL }\end{array}$ & $\begin{array}{l}\text { TRIT- } \\
\text { IUM } \\
\text { TOTAL } \\
\text { (PCI/L) }\end{array}$ \\
\hline NOV 1987 & & & & & & & & & & & & \\
\hline $\begin{array}{l}08 \ldots \\
\text { MAR } 1988\end{array}$ & 7 & $<30$ & $<30$ & $<3.0$ & 1,400 & $<6$ & $<9$ & -- & -- & -- & -- & -- \\
\hline $\begin{array}{l}04 \ldots \\
\text { APR }\end{array}$ & 12 & $<30$ & $<30$ & $<3.0$ & 1,100 & $<18$ & $<9$ & -- & -- & -- & -- & -- \\
\hline MAY 05 & 47 & $<10$ & $<10$ & $<3.0$ & 3,300 & $<18$ & $<9$ & -9.0 & -142 & -18.2 & 1.30 & 26 \\
\hline $\begin{array}{l}10 \ldots \\
\text { JNE }\end{array}$ & 73 & $<30$ & $<30$ & $<3.0$ & 4,000 & $<18$ & 13 & -- & -- & -- & -- & -- \\
\hline $15 \ldots$ & 120 & $<10$ & $<10$ & $<1.0$ & 3,600 & $<6$ & $<3$ & -- & -- & -- & -- & -- \\
\hline $15 \ldots$ & 14 & $<30$ & $<30$ & $<3.0$ & 1,500 & $<18$ & 12 & -- & -- & -- & -- & -- \\
\hline $15 \ldots$ & 17 & $<10$ & $<10$ & 2.0 & 1,900 & $<6$ & 17 & -- & -- & -- & -- & -- \\
\hline AUG & & & & & & & & & & & & \\
\hline${ }_{\text {NOV }}^{30} \ldots$ & 24 & $<10$ & 10 & $<1.0$ & 2,500 & $<6$ & 9 & -9.2 & -140 & -18.2 & 1.70 & 24 \\
\hline $\begin{array}{l}16 \ldots \\
\text { APR } 1989\end{array}$ & 23 & $<10$ & $<10$ & $<1.0$ & 2,700 & $<6$ & $<3$ & -- & -- & -- & -- & -- \\
\hline $\begin{array}{l}04 \ldots \\
\text { JUNE }\end{array}$ & 15 & $<30$ & $<30$ & $<3.0$ & 2,000 & $<18$ & $<9$ & -- & -- & -- & -- & -- \\
\hline $\begin{array}{l}07 \ldots \\
\text { SEPT }\end{array}$ & 21 & $<30$ & $<30$ & $<3.0$ & 2,300 & $<18$ & $<9$ & -- & -- & -- & -- & -- \\
\hline $12 \ldots$ & 11 & $<20$ & $<20$ & $<2.0$ & 170 & $<12$ & $<6$ & -- & -- & -- & -- & -- \\
\hline
\end{tabular}


Table 25.--Water-quality analyses for wells--Continued

\begin{tabular}{|c|c|c|c|c|c|c|c|c|c|c|c|}
\hline DATE & TIME & $\begin{array}{l}\text { DEPTH } \\
\text { OF } \\
\text { WELL, } \\
\text { TOTAL } \\
\text { (FEET) }\end{array}$ & $\begin{array}{c}\text { ELEVATIOI } \\
\text { OF LAND } \\
\text { SURFACE } \\
\text { DATUM } \\
\text { (FEET } \\
\text { ABOVE } \\
\text { SEA } \\
\text { LEVEL) }\end{array}$ & $\begin{array}{ll} & \text { SPE- } \\
\text { CIFIC } \\
\text { CON- } \\
\text { DUCT- } \\
\text { ANCE } \\
(\mu S / C M)\end{array}$ & $\begin{array}{c}\text { PH } \\
\text { (STAND- } \\
\text { ARD } \\
\text { UNITS) }\end{array}$ & $\begin{array}{l}\text { TEMPER- } \\
\text { ATURE } \\
\text { WATER } \\
\left({ }^{\circ} \mathrm{C}\right)\end{array}$ & $\begin{array}{l}\text { HARD- } \\
\text { NESS } \\
\text { TOTAL } \\
(\mathrm{MG} / \mathrm{L} \\
\mathrm{AS} \\
\left.\mathrm{CACO}_{3}\right)\end{array}$ & $\begin{array}{l}\text { HARD- } \\
\text { NESS } \\
\text { NONCARB } \\
\text { WH WAT } \\
\text { TOT FLD } \\
\left(\mathrm{MG} / \mathrm{L} \text { AS }_{\left.\mathrm{CACO}_{3}\right)}\right.\end{array}$ & $\begin{array}{l}\text { CALCIUM } \\
\text { DIS- } \\
\text { SOLVED } \\
\text { (MG/L } \\
\text { AS CA) }\end{array}$ & $\begin{array}{l}\text { MAGNE- } \\
\text { SIUM, } \\
\text { DIS- } \\
\text { SOLVED } \\
\text { (MG/L } \\
\text { AS MG) }\end{array}$ & $\begin{array}{l}\text { SODIUM, } \\
\text { DIS- } \\
\text { SOLVED } \\
\text { (MG/L } \\
\text { AS NA) }\end{array}$ \\
\hline \multicolumn{12}{|c|}{ WELI IDENTIFICATION - SSU287 } \\
\hline NOV 1987 & & & & & & & & & & & \\
\hline $\begin{array}{l}08 \ldots \\
\text { MAR } 1988\end{array}$ & 0915 & 127.00 & 6,861 & 1,150 & 8.4 & -- & 11 & 0 & 3.1 & 0.83 & 300 \\
\hline $\begin{array}{c}04 \ldots \\
\text { APR }\end{array}$ & 1100 & 127.00 & 6,861 & 1,520 & 8.6 & 10.0 & 15 & 0 & 4.2 & 1.1 & 380 \\
\hline MAY & 1330 & 127.00 & 6,861 & 1,620 & 8.7 & 10.5 & 56 & 0 & 12 & 6.1 & 370 \\
\hline $10 \ldots$ & 1050 & 127.00 & 6,861 & 1,730 & 8.6 & 10.5 & 79 & 0 & 16 & 9.4 & 360 \\
\hline $30 \ldots$ & 1115 & 127.00 & 6,861 & 1,600 & 8.9 & 11.0 & 28 & 0 & 6.6 & 2.7 & 340 \\
\hline $\begin{array}{l}16 \ldots \\
\text { APR } 1989\end{array}$ & 1130 & 127.00 & 6,861 & 1,320 & 9.1 & -- & 12 & 0 & 3.3 & .86 & 320 \\
\hline$\underset{\mathrm{J} N \mathrm{NE}}{04 \cdots}$ & 1100 & 127.00 & 6,861 & 1,375 & 8.8 & 10.5 & 13 & 0 & 3.4 & .98 & 310 \\
\hline $\begin{array}{l}07 \\
\text { SEPT }\end{array}$ & 0800 & 127.00 & 6,861 & 1,360 & 8.9 & 10.5 & 12 & 0 & 3.2 & .81 & 330 \\
\hline $12 \ldots$ & 1045 & 127.00 & 6,861 & 1,280 & 8.8 & 10.5 & 11 & 0 & 3.0 & .79 & 300 \\
\hline DATE & & $\begin{array}{l}\text { POTAS- } \\
\text { SIUM, } \\
\text { DIS- } \\
\text { SOLVED } \\
(\text { MG/L } \\
\text { AS K) }\end{array}$ & $\begin{array}{c}\text { ALKA- } \\
\text { LINITY } \\
\text { LAB } \\
(\mathrm{MG} / \mathrm{L} \\
\mathrm{AS} \\
\left.\mathrm{CACO}_{3}\right)\end{array}$ & $\begin{array}{l}\text { SULFATE } \\
\text { DIS- } \\
\text { SOLVED } \\
(\mathrm{MG} / \mathrm{L} \\
\left.\text { AS } \mathrm{SO}_{4}\right)\end{array}$ & $\begin{array}{l}\text { CHLO- } \\
\text { RIDE, } \\
\text { DIS- } \\
\text { SOLVED } \\
\text { (MG/L } \\
\text { AS CL) }\end{array}$ & $\begin{array}{l}\text { FLUO- } \\
\text { RIDE, } \\
\text { DIS- } \\
\text { SOLVED } \\
\text { (MG/L } \\
\text { AS F) }\end{array}$ & $\begin{array}{l}\text { SILICA, } \\
\text { DIS- } \\
\text { SOLVED } \\
\text { (MG/L } \\
\text { AS } \\
\left.\mathrm{SIO}_{2}\right)\end{array}$ & $\begin{array}{l}\text { SOLIDS, } \\
\text { RESIDUE } \\
\text { AT 180 } \\
\text { DEG. C } \\
\text { DIS- } \\
\text { SOLVED } \\
\text { (MG/L) }\end{array}$ & $\begin{array}{l}\text { SOLIDS, } \\
\text { SUM OF } \\
\text { CONSTIT- } \\
\text { UENTS, } \\
\text { DIS- } \\
\text { SOLVED } \\
\text { (MG/L) }\end{array}$ & & \\
\hline
\end{tabular}

NOV 1987

$08 \ldots$

MAR 1988

$04 \ldots$

APR

$05 .$.

MAY

$10 .$.

AUG

30 ...

NOV

16 ...

APR 1989

$04 \ldots$

JUNE

$07 . .$.

SEPT

$12 .$.

1.9425

$2.7 \quad 438$

$3.3 \quad 443$

$2.5 \quad 444$

$2.1 \quad 448$

$2.0 \quad 451$

$2.0 \quad 447$

$1.9 \quad 445$

$1.9 \quad 452$

\section{WELL IDENTIFICATI}

230

340

430

450

7.0

0.8

7.4

818

807

12

1.0

7.3

977

7.5

7.4

7.3

7.6

7.3

7.4

7.2

210

$250 \quad 10$

1.0

1.1

$9.9 \quad 1.0$

$9.9 \quad 1.1$

$1,090 \quad 1,110$

$1,120 \quad 1,130$

$950 \quad 971$

$870 \quad 866$

$849 \quad 863$

$857 \quad 871$

$795 \quad 805$ 
Table 25.--Water-quality analyses for wells--Continued

\begin{tabular}{|c|c|c|c|c|c|c|c|c|c|c|c|}
\hline DATE & $\begin{array}{l}\text { NITRO- } \\
\text { GEN, } \\
\text { NITRITE } \\
\text { DIS- } \\
\text { SOLVED } \\
\text { (MG/L } \\
\text { AS N) }\end{array}$ & $\begin{array}{c}\text { NITRO- } \\
\text { GEN, } \\
\mathrm{NO}_{2}+\mathrm{NO}_{3} \\
\text { DIS- } \\
\text { SOLVED } \\
(\mathrm{MG} / \mathrm{L} \\
\text { AS N) }\end{array}$ & $\begin{array}{l}\text { NITRO- } \\
\text { GEN, } \\
\text { AMMONIA } \\
\text { DIS- } \\
\text { SOLVED } \\
\text { (MG/L } \\
\text { AS N) }\end{array}$ & $\begin{array}{l}\text { NITRO- } \\
\text { GEN, } \\
\text { AMMONIA } \\
\text { DIS- } \\
\text { SOLVED } \\
(\mathrm{MG} / \mathrm{L} \\
\left.\text { AS } \mathrm{NH}_{4}\right)\end{array}$ & $\begin{array}{l}\text { NITRO- } \\
\text { GEN, } \\
\text { ORGANIC } \\
\text { DIS- } \\
\text { SOLVED } \\
\text { (MG/L } \\
\text { AS N) }\end{array}$ & $\begin{array}{l}\text { NITRO- } \\
\text { GEN, AM- } \\
\text { MONIA + } \\
\text { ORGANIC } \\
\text { DISSOLVED } \\
\text { (MG/L } \\
\text { AS N) }\end{array}$ & $\begin{array}{l}\text { PHOS- } \\
\text { PHORUS } \\
\text { DIS- } \\
\text { SOLVED } \\
\text { (MG/L } \\
\text { AS P) }\end{array}$ & $\begin{array}{l}\text { PHOS- } \\
\text { PHORUS } \\
\text { ORTHO, } \\
\text { DIS- } \\
\text { SOLVED } \\
\text { (MG/L } \\
\text { AS P) }\end{array}$ & $\begin{array}{l}\text { PHOS- } \\
\text { PHATE, } \\
\text { ORTHO, } \\
\text { DIS- } \\
\text { SOLVED } \\
(\mathrm{MG} / \mathrm{L} \\
\left.\text { AS } \mathrm{PO}_{4}\right)\end{array}$ & $\begin{array}{c}\text { PHOS- } \\
\text { PHORUS } \\
\text { ORGANIC } \\
\text { DIS- } \\
\text { SOLVED } \\
\text { (MG/L } \\
\text { AS P) }\end{array}$ & $\begin{array}{l}\text { ALU- } \\
\text { MINUM, } \\
\text { DIS- } \\
\text { SOLVED } \\
\text { (MG/L } \\
\text { AS AL) }\end{array}$ \\
\hline \multicolumn{12}{|c|}{ WELL IDENTIFICATION - SSU287--Continued } \\
\hline NOV 1987 & & & & & & & & & & & \\
\hline $\begin{array}{l}08 \ldots \\
\text { MAR } 1988\end{array}$ & $<0.01$ & $<0.10$ & 0.43 & 0.55 & 0.37 & 0.8 & 0.03 & 0.01 & 0.03 & 0.02 & 10 \\
\hline $\begin{array}{c}04 \ldots \\
\text { APR }\end{array}$ & $<.01$ & $<.10$ & .08 & .1 & .42 & .5 & .03 & $<.01$ & -- & .03 & $<10$ \\
\hline MAY 05 & $<.01$ & .10 & .60 & .77 & .2 & .8 & .03 & .02 & .06 & .01 & $<10$ \\
\hline${ }_{A U G}^{10 \ldots}$ & $<.01$ & $<.10$ & .45 & .58 & .35 & .8 & .02 & .02 & .06 & .0 & $<10$ \\
\hline$\stackrel{30 \ldots}{\text { Nov }}$ & $<.01$ & $<.10$ & .16 & .21 & .64 & .8 & .03 & .02 & .06 & .01 & $<10$ \\
\hline $\begin{array}{l}16 \ldots \\
\text { APR } 1989\end{array}$ & $<.01$ & $<.10$ & .11 & .14 & -- & $<.2$ & .03 & .02 & .06 & .01 & $<10$ \\
\hline$\underset{\text { JNE }}{04 \ldots}$ & -- & -- & -- & -- & -- & -- & -- & -- & -- & -- & $<10$ \\
\hline$\underset{\text { SEPT }}{07} \cdots$ & -- & -- & -- & -- & -- & -- & -- & -- & -- & -- & 10 \\
\hline $12 \ldots$ & -- & -- & - & -- & -- & -- & -- & -- & -- & -- & 10 \\
\hline DATE & $\begin{array}{l}\text { ARSENIC } \\
\text { DIS- } \\
\text { SOLVED } \\
\text { ( } \mu \text { G/L } \\
\text { AS AS) }\end{array}$ & $\begin{array}{l}\text { BARIUM, } \\
\text { DIS- } \\
\text { SOLVED } \\
(\mu G / L \\
\text { AS BA) }\end{array}$ & $\begin{array}{c}\text { BERYL- } \\
\text { LIUM, } \\
\text { DIS- } \\
\text { SOLVED } \\
(\mu G / L \\
\text { AS BE) }\end{array}$ & $\begin{array}{l}\text { BORON, } \\
\text { DIS- } \\
\text { SOLVED } \\
\text { ( } \mu G / L \\
\text { AS B) }\end{array}$ & $\begin{array}{c}\text { CADMIUM } \\
\text { DIS- } \\
\text { SOLVED } \\
\text { ( } \mu G / L \\
\text { AS CD) }\end{array}$ & $\begin{array}{l}\text { CHRO- } \\
\text { MIUM, } \\
\text { DIS- } \\
\text { SOLVED } \\
(\mu G / L \\
\text { AS CR) }\end{array}$ & $\begin{array}{l}\text { COBALT, } \\
\text { DIS- } \\
\text { SOLVED } \\
(\mu G / L \\
\text { AS CO) }\end{array}$ & $\begin{array}{l}\text { COPPER, } \\
\text { DIS- } \\
\text { SOLVED } \\
(\mu G / L \\
\text { AS CU) }\end{array}$ & $\begin{array}{l}\text { IRON, } \\
\text { DIS- } \\
\text { SOLVED } \\
\text { ( } \mu \mathrm{G} / \mathrm{L} \\
\text { AS FE) }\end{array}$ & $\begin{array}{l}\text { IEAD, } \\
\text { DIS- } \\
\text { SOLVED } \\
(\mu G / L \\
\text { AS PB) }\end{array}$ & $\begin{array}{c}\text { MANGA- } \\
\text { NESE, } \\
\text { DIS- } \\
\text { SOLVED } \\
\text { ( } \mu G / L \\
\text { AS MN) }\end{array}$ \\
\hline \multicolumn{12}{|l|}{ NOV 1987} \\
\hline $\begin{array}{l}08 \ldots \\
\text { MAR } 1988\end{array}$ & $<1$ & 120 & $<0.5$ & 50 & $<1$ & $<5$ & $<3$ & $<10$ & 19 & $<10$ & 2 \\
\hline$\underset{A P R}{04 \ldots}$ & $<1$ & 91 & $<.5$ & 50 & $<2$ & $<5$ & $<3$ & $<10$ & 20 & $<10$ & 3 \\
\hline $\operatorname{MAY}_{\mathrm{MA}}^{05}$ & $<1$ & 100 & $<.5$ & 50 & 1 & $<5$ & $<3$ & $<10$ & 63 & $<10$ & 14 \\
\hline${ }_{A U G}^{10} \cdots$ & $<1$ & 92 & $<.5$ & 50 & $<1$ & $<5$ & $<3$ & $<10$ & 61 & $<10$ & 21 \\
\hline $\begin{array}{c}30 \ldots \\
\text { Nov }\end{array}$ & $<1$ & 82 & $<.5$ & 50 & $<1$ & $<5$ & $<3$ & $<10$ & 31 & $<10$ & 6 \\
\hline $\begin{array}{l}16 \\
\text { APR } 1989\end{array}$ & $<1$ & 73 & $<.5$ & 50 & $<1$ & $<5$ & $<3$ & $<10$ & 19 & $<10$ & 3 \\
\hline$\underset{\text { JUNE }}{04 \ldots}$ & $<1$ & 74 & $<.5$ & 40 & $<1$ & $<5$ & $<3$ & $<10$ & 19 & $<10$ & 3 \\
\hline$\underset{\text { SEPT }}{07} \cdots$ & $<1$ & 70 & $<.5$ & 40 & $<1$ & $<5$ & $<3$ & $<10$ & 23 & $<10$ & 3 \\
\hline $12 \ldots$ & $<1$ & 62 & $<.5$ & 40 & $<1$ & $<5$ & $<3$ & $<10$ & 17 & $<10$ & 4 \\
\hline
\end{tabular}


Table 25.--Water-quality analyses for wells--Continued

\begin{tabular}{|c|c|c|c|c|c|c|c|c|c|c|c|}
\hline DATE & $\begin{array}{l}\text { MOLYB- } \\
\text { DENUM, } \\
\text { DIS- } \\
\text { SOLVED } \\
(\mu G / L \\
\text { AS MO) }\end{array}$ & $\begin{array}{l}\text { NICKEL, } \\
\text { DIS- } \\
\text { SOLVED } \\
(\mu G / L \\
\text { AS NI) }\end{array}$ & $\begin{array}{l}\text { SILVER } \\
\text { DIS- } \\
\text { SOLVED } \\
(\mu G / L \\
\text { AS AG) }\end{array}$ & $\begin{array}{c}\text { STRON- } \\
\text { TIUM, } \\
\text { DIS- } \\
\text { SOLVED } \\
\text { ( } \mu G / L \\
\text { AS SR) }\end{array}$ & $\begin{array}{l}\text { VANA- } \\
\text { DIUM, } \\
\text { DIS- } \\
\text { SOLVED } \\
(\mu G / L \\
\text { AS V) }\end{array}$ & $\begin{array}{l}\text { ZINC, } \\
\text { DIS- } \\
\text { SOLVED } \\
(\mu G / L \\
\text { AS ZN) }\end{array}$ & $\begin{array}{l}\text { C-13/ } \\
\text { C-12 } \\
\text { STABLE } \\
\text { ISOTOPE } \\
\text { RATIO } \\
\text { PER } \\
\text { MIL }\end{array}$ & $\begin{array}{l}\text { H-2/ } \\
\text { H-1 } \\
\text { STABLE } \\
\text { ISOTOPE } \\
\text { RATIO } \\
\text { PER } \\
\text { MIL }\end{array}$ & $\begin{array}{l}0-18 / \\
0-16 \\
\text { STABLE } \\
\text { ISOTOPE } \\
\text { RATIO } \\
\text { PER } \\
\text { MIL }\end{array}$ & $\begin{array}{c}\text { S-34/ } \\
\text { S-32 } \\
\text { STABLE } \\
\text { ISOTOPE } \\
\text { RATIO } \\
\text { PER } \\
\text { MIL }\end{array}$ & $\begin{array}{c}\text { TRIT- } \\
\text { IUM } \\
\text { TOTAL } \\
\text { (PCI/L) }\end{array}$ \\
\hline
\end{tabular}

\begin{tabular}{|c|c|c|c|c|c|c|c|c|c|c|c|}
\hline NOV 1987 & & & & & & & & & & & \\
\hline $\begin{array}{c}08 \ldots \\
\text { MAR } 1988\end{array}$ & $<10$ & $<10$ & $<1.0$ & 160 & $<6$ & $<3$ & -- & -- & -- & - & - \\
\hline $\begin{array}{c}04 \ldots \\
\text { APR }\end{array}$ & $<10$ & $<10$ & $<1.0$ & 230 & $<6$ & $<3$ & $\sim$ & - & -- & -- & - \\
\hline$\underset{\mathrm{MAY}}{05}$ & $<10$ & $<10$ & $<1.0$ & 450 & $<6$ & $<3$ & -10.9 & -144 & -18.9 & 2.60 & $<5.7$ \\
\hline${ }_{\text {AUG }}^{10 \ldots}$ & $<10$ & $<10$ & $<1.0$ & 510 & $<6$ & $<3$ & $\sim$ & - & - & - & - \\
\hline $\begin{array}{l}30 \ldots \\
\text { NOV }\end{array}$ & $<10$ & 20 & $<1.0$ & 310 & $<6$ & 9 & -11.1 & -143 & -18.8 & 2.70 & $<5.7$ \\
\hline $\begin{array}{l}16 \ldots \\
\text { APR } 1989\end{array}$ & $<10$ & $<10$ & $<1.0$ & 180 & $<6$ & 6 & -- & - & -- & -- & -- \\
\hline $\begin{array}{c}04 \ldots \\
\text { JUNE }\end{array}$ & $<10$ & $<10$ & $<1.0$ & 190 & $<6$ & $<3$ & -- & $\cdots$ & 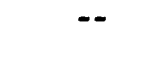 & $\cdots$ & $\cdots$ \\
\hline $\begin{array}{c}07 \ldots \\
\text { SEPT }\end{array}$ & $<10$ & $<10$ & $<1.0$ & 170 & $<6$ & $<3$ & -- & -- & $\cdots$ & $\sim$ & - \\
\hline $12 \ldots$ & $<10$ & $<10$ & $<1.0$ & 160 & $<6$ & $<3$ & -- & -- & -- & -- & -- \\
\hline DATE & TIME & $\begin{array}{c}\text { DEPTH } \\
\text { OF } \\
\text { WELL, } \\
\text { TOTAL } \\
\text { (FEET) }\end{array}$ & $\begin{array}{c}\text { ELEVATION } \\
\text { OF LAND } \\
\text { SURFACE } \\
\text { DATUM } \\
\text { (FEET } \\
\text { ABOVE } \\
\text { SEA } \\
\text { LEVEL) }\end{array}$ & $\begin{array}{l}\text { SPE- } \\
\text { CIFIC } \\
\text { CON- } \\
\text { DUCT- } \\
\text { ANCE } \\
(\mu S / C M)\end{array}$ & $\begin{array}{c}\text { PH } \\
\text { (STAND- } \\
\text { ARD } \\
\text { UNITS) }\end{array}$ & $\begin{array}{l}\text { TEMPER- } \\
\text { ATURE } \\
\text { WATER } \\
\left({ }^{\circ} \mathrm{C}\right)\end{array}$ & $\begin{array}{l}\text { HARD- } \\
\text { NESS } \\
\text { TOTAL } \\
(\mathrm{MG} / \mathrm{L} \\
\mathrm{AS}^{-} \\
\left.\mathrm{CACO}_{3}\right)\end{array}$ & $\begin{array}{l}\text { HARD- } \\
\text { NESS } \\
\text { NONCARB } \\
\text { WH WAT } \\
\text { TOT FLD } \\
(\mathrm{MG} / \mathrm{L} \text { AS } \\
\left.\mathrm{CACO}_{3}\right)\end{array}$ & $\begin{array}{l}\text { CALCIUM } \\
\text { DIS- } \\
\text { SOLVED } \\
\text { (MG/L } \\
\text { AS CA) }\end{array}$ & $\begin{array}{l}\text { MAGNE- } \\
\text { SIUM, } \\
\text { DIS- } \\
\text { SOLVED } \\
\text { (MG/L } \\
\text { AS MG) }\end{array}$ & $\begin{array}{l}\text { SODIUM, } \\
\text { DIS- } \\
\text { SOLVED } \\
\text { (MG/L } \\
\text { AS NA) }\end{array}$ \\
\hline & & & & WELL II & ENTIF ICAT & ION - SSD & 487 & & & & \\
\hline NOV 1987 & & & & & & & & & & & \\
\hline $\begin{array}{l}08 \ldots \\
\text { MAR } 1988\end{array}$ & 1045 & 194.00 & 6,860 & 680 & 8.7 & - & 6 & 0 & 1.5 & 0.49 & 180 \\
\hline $\begin{array}{l}07 \cdots \\
\text { APR }\end{array}$ & 1140 & 194.00 & 6,860 & 740 & 9.0 & 9.5 & 6 & 0 & 1.5 & .53 & 190 \\
\hline$\underset{M A Y}{05 \ldots}$ & 1600 & 194.00 & 6,860 & 760 & 9.0 & 11.0 & 5 & 0 & 1.4 & .38 & 190 \\
\hline${ }_{A U G}^{10} \ldots$ & 0830 & 194.00 & 6,860 & 790 & 9.0 & 10.5 & 6 & 0 & 1.6 & .52 & 180 \\
\hline $\begin{array}{l}30 \ldots \\
\text { Nov }\end{array}$ & 1140 & 194.00 & 6,860 & 870 & 9.2 & 11.5 & 6 & 0 & 1.5 & .48 & 180 \\
\hline $\begin{array}{l}16 \ldots \\
\text { APR } 1989\end{array}$ & 1115 & 194.00 & 6,860 & 750 & 9.4 & -- & 5 & 0 & 1.3 & .37 & 180 \\
\hline$\underset{\text { JUNE }}{04 \ldots}$ & 1030 & 194.00 & 6,860 & 793 & 9.0 & 10.5 & 5 & 0 & 1.3 & .43 & 170 \\
\hline$\underset{\text { SEPT }}{07}$ & 0830 & 194.00 & 6,860 & 760 & 9.1 & 10.5 & 6 & 0 & 1.6 & .45 & 190 \\
\hline $12 \ldots$ & 1200 & 194.00 & 6,860 & 765 & 9.1 & 11.0 & 5 & 0 & 1.4 & .36 & 180 \\
\hline
\end{tabular}


Table 25.--Water-quality analyses for wells--Continued

\begin{tabular}{|c|c|c|c|c|c|c|c|c|c|c|c|}
\hline \multicolumn{2}{|c|}{ DATE } & $\begin{array}{l}\text { POTAS- } \\
\text { SIUM, } \\
\text { DIS- } \\
\text { SOLVED } \\
\text { (MG/L } \\
\text { AS K) }\end{array}$ & $\begin{array}{l}\text { ALKA- } \\
\text { LINITY } \\
\text { LAB } \\
(\mathrm{MG} / \mathrm{L} \\
\mathrm{AS} \\
\left.\mathrm{CACO}_{3}\right)\end{array}$ & $\begin{array}{l}\text { SULFATE } \\
\text { DIS- } \\
\text { SOLVED } \\
(\mathrm{MG} / \mathrm{L}) \\
\left.\text { AS } \mathrm{SO}_{4}\right)\end{array}$ & $\begin{array}{l}\text { CHLO- } \\
\text { RIDE, } \\
\text { DIS- } \\
\text { SOLVED } \\
\text { (MG/L } \\
\text { AS CL) }\end{array}$ & $\begin{array}{l}\text { FLUO- } \\
\text { RIDE, } \\
\text { DIS- } \\
\text { SOLVED } \\
\text { (MG/L } \\
\text { AS F) }\end{array}$ & $\begin{array}{l}\text { SILICA, } \\
\text { DIS- } \\
\text { SOLVED } \\
\text { ( } \mathrm{MG} / \mathrm{L} \\
\mathrm{AS} \\
\mathrm{SIO}_{2} \text { ) }\end{array}$ & $\begin{array}{l}\text { SOLIDS, } \\
\text { RESIDUE } \\
\text { AT 180 } \\
\text { DEG. C } \\
\text { DIS- } \\
\text { SOLVED } \\
\text { (MG/L) }\end{array}$ & $\begin{array}{l}\text { SOLIDS, } \\
\text { SUM OF } \\
\text { CONSTIT- } \\
\text { UENTS, } \\
\text { DIS- } \\
\text { SOLVED } \\
\text { (MG/L) }\end{array}$ & & \\
\hline \multicolumn{12}{|c|}{ WELL IDENTIFICATION - SSD487--Continued } \\
\hline \multicolumn{2}{|c|}{$\begin{array}{c}\text { NOV } 1987 \\
08 \ldots \\
\text { MAR } 1988\end{array}$} & 1.3 & 384 & 44 & 1.8 & 0.4 & 7.5 & 474 & 468 & & \\
\hline \multicolumn{2}{|c|}{$\begin{array}{c}07 \cdots \\
\text { APR }\end{array}$} & 1.4 & 384 & 42 & 2.6 & .1 & 7.3 & 471 & 477 & & \\
\hline \multicolumn{2}{|c|}{$\underset{\text { MAY }}{05 \ldots}$} & 1.7 & 383 & 34 & 2.3 & .2 & 7.3 & 464 & 467 & & \\
\hline \multicolumn{2}{|c|}{$10 \ldots$} & 1.2 & 382 & 38 & 2.9 & .2 & 7.2 & 456 & 462 & & \\
\hline \multicolumn{2}{|c|}{$\begin{array}{l}\text { AUG } \\
30 . . \\
\text { NOV }\end{array}$} & 1.1 & 382 & 42 & 2.2 & .1 & 7.1 & 470 & 464 & & \\
\hline \multicolumn{2}{|c|}{$\begin{array}{l}16 \ldots \\
\text { APR } 1989\end{array}$} & 1.2 & 383 & 35 & 2.1 & .2 & 7.5 & 460 & 458 & & \\
\hline \multicolumn{2}{|c|}{$\begin{array}{c}04 \ldots \\
\text { JUNE }\end{array}$} & 1.2 & 383 & 33 & 2.1 & .1 & 7.3 & 458 & 445 & & \\
\hline \multicolumn{2}{|c|}{$\begin{array}{c}07 \ldots \\
\text { SEPT }\end{array}$} & 1.2 & 381 & 33 & 2.1 & .1 & 7.4 & 474 & 464 & & \\
\hline \multicolumn{2}{|c|}{$\begin{array}{r}\text { SEPT } \\
12 \ldots\end{array}$} & 1.2 & 379 & 34 & 2.2 & .1 & 7.0 & 464 & 454 & & \\
\hline DATE & $\begin{array}{l}\text { NITRO- } \\
\text { GEN, } \\
\text { NITRITE } \\
\text { DIS- } \\
\text { SOLVED } \\
\text { (MG/L } \\
\text { AS N) }\end{array}$ & $\begin{array}{c}\text { NITRO- } \\
\text { GEN, } \\
\mathrm{NO}_{2}+\mathrm{NO}_{3} \\
\text { DIS- } \\
\text { SOLVED } \\
\text { (MG/L } \\
\text { AS N) }\end{array}$ & $\begin{array}{l}\text { NITRO- } \\
\text { GEN, } \\
\text { AMMONIA } \\
\text { DIS- } \\
\text { SOLVED } \\
\text { (MG/L } \\
\text { AS N) }\end{array}$ & $\begin{array}{l}\text { NITRO- } \\
\text { GEN, } \\
\text { AMMONIA } \\
\text { DIS- } \\
\text { SOLVED } \\
\text { (MG/L } \\
\text { AS NH } 4)\end{array}$ & $\begin{array}{l}\text { NITRO- } \\
\text { GEN, } \\
\text { ORGANIC } \\
\text { DIS- } \\
\text { SOLVED } \\
\text { (MG/L } \\
\text { AS N) }\end{array}$ & $\begin{array}{l}\text { NITRO- } \\
\text { GEN, AM- } \\
\text { MONIA + } \\
\text { ORGANIC } \\
\text { DISSOLVED } \\
\text { (MG/L } \\
\text { AS N) }\end{array}$ & $\begin{array}{l}\text { PHOS- } \\
\text { PHORUS } \\
\text { DIS- } \\
\text { SOLVED } \\
\text { (MG/L } \\
\text { AS P) }\end{array}$ & $\begin{array}{l}\text { PHOS- } \\
\text { PHORUS } \\
\text { ORTHO, } \\
\text { DIS- } \\
\text { SOLVED } \\
\text { (MG/L } \\
\text { AS P) }\end{array}$ & $\begin{array}{c}\text { PHOS- } \\
\text { PHATE, } \\
\text { ORTHO, } \\
\text { DIS- } \\
\text { SOLVED } \\
(\mathrm{MG} / \mathrm{L} \\
\left.\text { AS PO }{ }_{4}\right)\end{array}$ & $\begin{array}{l}\text { PHOS- } \\
\text { PHORUS } \\
\text { ORGANIC } \\
\text { DIS- } \\
\text { SOLVED } \\
\text { (MG/L } \\
\text { AS P) }\end{array}$ & $\begin{array}{l}\text { ALU- } \\
\text { MINUM, } \\
\text { DIS- } \\
\text { SOLVED } \\
\text { (MG/L } \\
\text { AS AL) }\end{array}$ \\
\hline \multicolumn{12}{|l|}{ NOV 1987} \\
\hline $\begin{array}{c}08 \ldots \\
\text { MAR } 1988\end{array}$ & $<0.01$ & $<0.10$ & 0.25 & 0.32 & -- & $<0.2$ & 0.03 & 0.02 & 0.06 & 0.01 & 30 \\
\hline $\begin{array}{c}07 \ldots \\
\text { APR }\end{array}$ & $<.01$ & .10 & .03 & .04 & 0.37 & .4 & .03 & .01 & .03 & .02 & 210 \\
\hline$\underset{\text { MAY }}{05 \ldots}$ & $<.01$ & $<.10$ & .16 & .21 & .14 & .3 & .03 & .03 & .09 & .0 & $<10$ \\
\hline AUG $10 .$. & $<.01$ & .12 & .12 & .15 & -- & $<.2$ & .03 & .03 & .09 & .0 & $<10$ \\
\hline $\begin{array}{l}30 . . . \\
\text { Nov }\end{array}$ & $<.01$ & $<.10$ & .10 & .13 & .4 & .5 & .04 & .03 & .09 & .01 & $<10$ \\
\hline $\begin{array}{c}16 \ldots \\
\text { APR } 1989\end{array}$ & $<.01$ & $<.10$ & .09 & .12 & -- & $<.2$ & .03 & .02 & .06 & .01 & $<10$ \\
\hline JUNE & -- & -- & -- & -- & -- & -- & -- & -- & -- & -- & $<10$ \\
\hline $\begin{array}{c}07: \ldots \\
\text { SEPT }\end{array}$ & -- & - & -- & -- & -- & -- & -- & -- & -- & -- & $<10$ \\
\hline $12 \ldots$ & -- & -- & -- & -- & -- & -- & -- & -- & -- & -- & 10 \\
\hline
\end{tabular}


Table 25.--Water-quality analyses for wells--Continued

\begin{tabular}{|c|c|c|c|c|c|c|c|c|c|c|c|}
\hline DATE & $\begin{array}{l}\text { ARSENIC } \\
\text { DIS- } \\
\text { SOLVED } \\
\text { ( } \mu G / L \\
\text { AS AS) }\end{array}$ & $\begin{array}{l}\text { BARIUM, } \\
\text { DIS- } \\
\text { SOLVED } \\
(\mu G / L \\
\text { AS BA) }\end{array}$ & $\begin{array}{l}\text { BERYL- } \\
\text { LIUM, } \\
\text { DIS- } \\
\text { SOLVED } \\
(\mu G / L \\
\text { AS BE) }\end{array}$ & $\begin{array}{l}\text { BORON, } \\
\text { DIS- } \\
\text { SOLVED } \\
(\mu G / L \\
\text { AS B) }\end{array}$ & $\begin{array}{c}\text { CADMIUM } \\
\text { DIS- } \\
\text { SOLVED } \\
(\mu G / L \\
\text { AS CD) }\end{array}$ & $\begin{array}{l}\text { CHRO- } \\
\text { MIUM, } \\
\text { DIS- } \\
\text { SOLVED } \\
(\mu G / L \\
\text { AS CR) }\end{array}$ & $\begin{array}{l}\text { COBALT, } \\
\text { DIS- } \\
\text { SOLVED } \\
(\mu G / L \\
\text { AS CO) }\end{array}$ & $\begin{array}{l}\text { COPPER, } \\
\text { DIS- } \\
\text { SOLVED } \\
\text { ( } \mu \mathrm{G} / \mathrm{L} \\
\text { AS CU) }\end{array}$ & $\begin{array}{l}\text { IRON, } \\
\text { DIS- } \\
\text { SOLVED } \\
\text { ( } \mu G / L \\
\text { AS FE) }\end{array}$ & $\begin{array}{c}\text { LEAD, } \\
\text { DIS- } \\
\text { SOLVED } \\
\text { ( } \mu G / L \\
\text { AS PB) }\end{array}$ & $\begin{array}{l}\text { MANGA- } \\
\text { NESE, } \\
\text { DIS- } \\
\text { SOLVED } \\
\text { ( } \mu G / L \\
\text { AS MN) }\end{array}$ \\
\hline
\end{tabular}

WELL IDENTIFICATION - SSD487--Continued

NOV 1987

$08 \ldots$

$07 \ldots$

APR

05... $<1$

MAY

$10 \ldots<1$

AUG

$30 . .<<1$

NOV

$16 . .$.

APR 1989

$04 \ldots$

JNE

$07 \ldots$

SEPT

$12 \ldots$

$<1$
$<1$
$<1$
$<1$
$<1$
$<1$
$<1$
$<1$
$<1$

34
41
47
46
45
44
43
43
43

$<0.5 \quad 50$

$<1<5$

$<5<3$

$<.5 \quad 50$

$<1<5$

$<3<10$

$<.5 \quad 40$

$2<5$

$<3<10$

$27<10 \quad 2$

12. .

\begin{tabular}{|c|c|c|c|c|c|c|c|c|c|c|c|}
\hline DATE & $\begin{array}{l}\text { MOLYB- } \\
\text { DENUM, } \\
\text { DIS- } \\
\text { SOLVED } \\
(\mu G / L \\
\text { AS MO) }\end{array}$ & $\begin{array}{l}\text { NICKEL, } \\
\text { DIS- } \\
\text { SOLVED } \\
(\mu G / L \\
\text { AS NI) }\end{array}$ & $\begin{array}{l}\text { SILVER, } \\
\text { DIS- } \\
\text { SOLVED } \\
(\mu G / L \\
(\mu G / L\end{array}$ & $\begin{array}{l}\text { STRON- } \\
\text { TIUM, } \\
\text { DIS- } \\
\text { SOLVED } \\
\text { ( } \mu G / L \\
\text { AS SR) }\end{array}$ & $\begin{array}{l}\text { VANA- } \\
\text { DIUM; } \\
\text { DIS- } \\
\text { SOLVED } \\
(\mu G / L \\
\text { AS V) }\end{array}$ & $\begin{array}{l}\text { ZINC, } \\
\text { DIS- } \\
\text { SOLVED } \\
(\mu G / L \\
\text { AS ZN) }\end{array}$ & $\begin{array}{l}\text { C-13/ } \\
\text { C-12 } \\
\text { STABLE } \\
\text { ISOTOPE } \\
\text { RATIO } \\
\text { PER } \\
\text { MIL }\end{array}$ & $\begin{array}{l}\text { H-2/ } \\
\text { H-1 } \\
\text { STABLE } \\
\text { ISOTOPE } \\
\text { RATIO } \\
\text { PER } \\
\text { MIL }\end{array}$ & $\begin{array}{l}0-18 / \\
0-16 \\
\text { STABLE } \\
\text { ISOTOPE } \\
\text { RATIO } \\
\text { PER } \\
\text { MIL }\end{array}$ & $\begin{array}{l}\text { S-34/ } \\
\text { S-32 } \\
\text { STABLE } \\
\text { ISOTOPE } \\
\text { RATIO } \\
\text { PER } \\
\text { MIL }\end{array}$ & $\begin{array}{c}\text { TRIT- } \\
\text { IUM } \\
\text { TOTAL } \\
(P C I / L)\end{array}$ \\
\hline
\end{tabular}

\section{NOV 1987}

$08 \ldots$

MAR 1988

$07 \ldots$

APR

05...

MAY

$10 \ldots$

AUG

30 ...

NoV

$16 \ldots$

APR 1989

04...

JUNE

$07 \ldots$

SEPT

$12 \ldots$

$\begin{array}{ll}<10 & <10 \\ <10 & <10 \\ <10 & <10 \\ <10 & <10 \\ <10 & <10 \\ <10 & <10 \\ <10 & <10 \\ <10 & <10 \\ <10 & <10\end{array}$

$\begin{array}{rr}1.0 & 61 \\ <1.0 & 64 \\ <1.0 & 66\end{array}$

$<6$

$<1.0 \quad 66<6$

$<3$

$\begin{array}{lll}-12.2 & -142 & -18.7\end{array}$

3.10

$<5.7$

$<1.0 \quad 67$

$<1.0 \quad 65$

$<6$

$<1.0 \quad 62$

$<1.0 \quad 64$

$<1.0 \quad 68$

$<1.0 \quad 65$

$<6$

$<3$

$\begin{array}{lll}-12.2 & -141 & -18.7\end{array}$

2.90

$<5.7$

$<6$

$<3$

$<6$

$<3$

$<6$

$<6$

$<3$
3


Table 25.--Water-quality analyses for wells--Continued

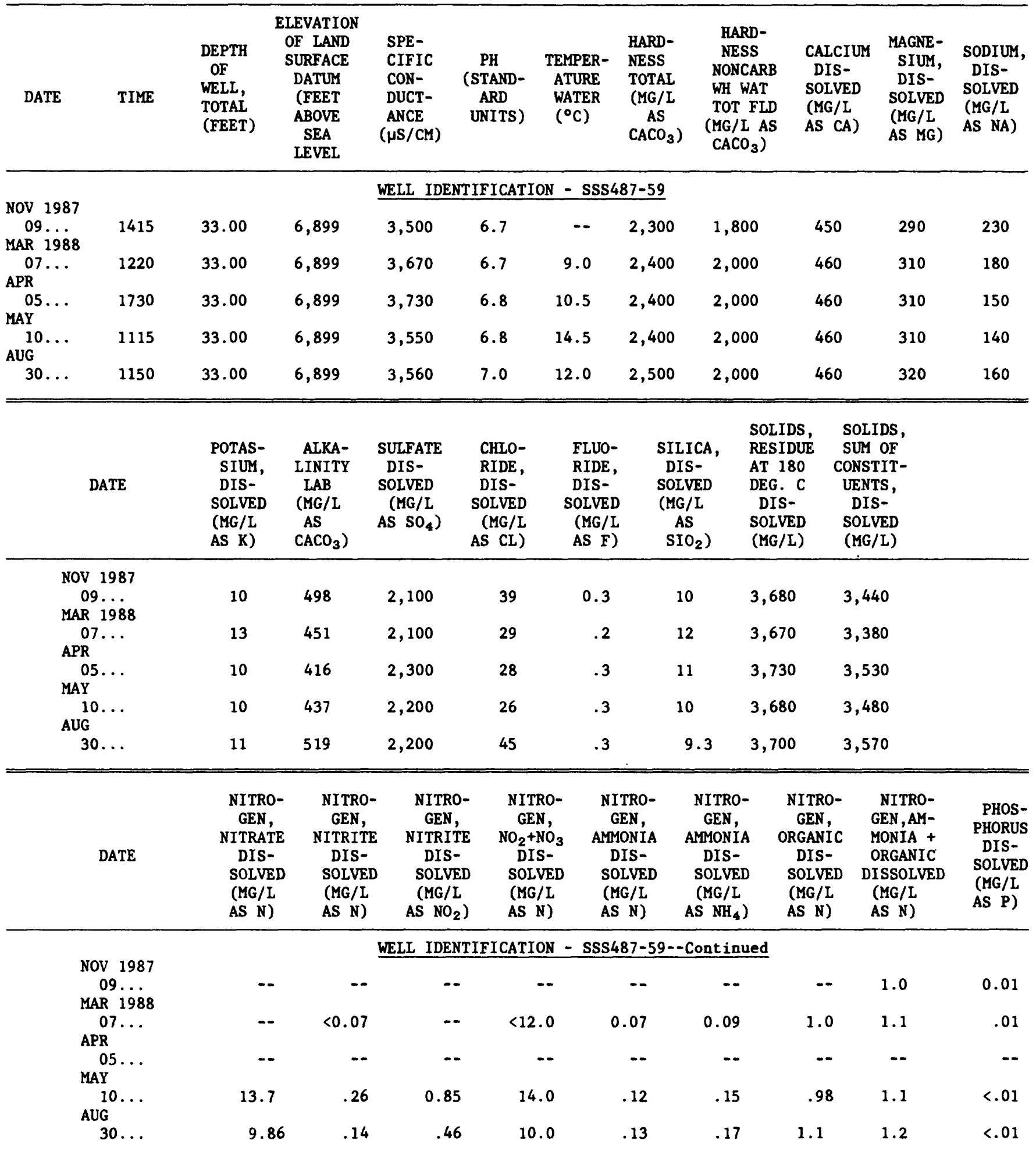


Table 25.--Water-quality analyses for wells--Continued

\begin{tabular}{|c|c|c|c|c|c|c|c|c|c|c|}
\hline DATE & $\begin{array}{l}\text { PHOS- } \\
\text { PHORUS } \\
\text { ORTHO, } \\
\text { DIS- } \\
\text { SOLVED } \\
\text { (MG/L } \\
\text { AS P) }\end{array}$ & $\begin{array}{l}\text { PHOS- } \\
\text { PHORUS } \\
\text { ORGANIC } \\
\text { DIS- } \\
\text { SOLVED } \\
\text { (MG/L } \\
\text { AS P) }\end{array}$ & $\begin{array}{l}\text { ALU- } \\
\text { MINUM, } \\
\text { DIS- } \\
\text { SOLVED } \\
\text { ( } \mu G / L \\
\text { AS AL) }\end{array}$ & $\begin{array}{l}\text { ARSENIC } \\
\text { DIS- } \\
\text { SOLVED } \\
(\mu G / L \\
\text { AS AS) }\end{array}$ & $\begin{array}{l}\text { BARIUM, } \\
\text { DIS- } \\
\text { SOLVED } \\
(\mu \mathrm{G} / \mathrm{L} \\
\text { AS BA) }\end{array}$ & $\begin{array}{l}\text { BERYL- } \\
\text { LIUM, } \\
\text { DIS- } \\
\text { SOLVED } \\
(\mu G / L \\
\text { AS BE) }\end{array}$ & $\begin{array}{l}\text { BORON, } \\
\text { DIS- } \\
\text { SOLVED } \\
(\mu G / L \\
\text { AS B) }\end{array}$ & $\begin{array}{l}\text { CADMIUM } \\
\text { DIS- } \\
\text { SOLVED } \\
\text { ( } \mu G / L \\
\text { AS CD) }\end{array}$ & $\begin{array}{l}\text { CHRO- } \\
\text { MIUM, } \\
\text { DIS- } \\
\text { SOLVED } \\
(\mu G / L \\
\text { AS CR) }\end{array}$ & $\begin{array}{l}\text { COBALT } \\
\text { DIS- } \\
\text { SOLVED } \\
(\mu G / L \\
\text { AS CO) }\end{array}$ \\
\hline \multicolumn{11}{|c|}{$\cdot$ WELL IDENTIFICATION - SSS487-59--Continued } \\
\hline $\begin{array}{c}\text { NOV } 1987 \\
\text { O9... } \\
\text { MAR } 1988\end{array}$ & -- & 0.01 & 20 & $<1$ & 38 & $<2$ & 440 & $<3$ & $<20$ & 10 \\
\hline $\begin{array}{c}07 \ldots \\
\text { APR }\end{array}$ & $<0.01$ & .01 & $<10$ & $<1$ & 26 & $<2$ & 500 & 6 & $<20$ & $<10$ \\
\hline$\underset{M A Y}{05}$ & -- & -- & $<10$ & $<1$ & 27 & $<2$ & 450 & $<3$ & $<20$ & $<9$ \\
\hline AUG $10 \ldots$ & $<.01$ & - & $<10$ & $<1$ & 27 & $<2$ & 440 & $<3$ & $<20$ & $<9$ \\
\hline $30 \ldots$ & $<.01$ & -- & $<10$ & $<1$ & 22 & $<.5$ & 430 & 6 & $<5$ & 5 \\
\hline DATE & $\begin{array}{l}\text { COPPER, } \\
\text { DIS- } \\
\text { SOLVED } \\
(\mu G / L \\
\text { AS CU) }\end{array}$ & $\begin{array}{c}\text { IRON, } \\
\text { DIS- } \\
\text { SOLVED } \\
(\mu G / L \\
\text { AS FE) }\end{array}$ & $\begin{array}{l}\text { LEAD, } \\
\text { DIS- } \\
\text { SOLVED } \\
(\mu G / L \\
\text { AS PB) }\end{array}$ & $\begin{array}{l}\text { MANGA- } \\
\text { NESE, } \\
\text { DIS- } \\
\text { SOLVED } \\
(\mu G / L \\
\text { AS MN })\end{array}$ & $\begin{array}{l}\text { MOLYB- } \\
\text { DENUM, } \\
\text { DIS- } \\
\text { SOLVED } \\
(\mu G / L \\
\text { AS MO) }\end{array}$ & $\begin{array}{l}\text { NICKEL, } \\
\text { DIS- } \\
\text { SOLVED } \\
(\mu G / L \\
\text { AS NI) }\end{array}$ & $\begin{array}{l}\text { SILVER, } \\
\text { DIS- } \\
\text { SOLVED } \\
(\mu G / L \\
\text { AS AG) }\end{array}$ & $\begin{array}{l}\text { STRON- } \\
\text { TIUM, } \\
\text { DIS- } \\
\text { SOLVED } \\
\text { ( } \mu G / L \\
\text { AS SR) }\end{array}$ & $\begin{array}{l}\text { VANA- } \\
\text { DIUM, } \\
\text { DIS- } \\
\text { SOLVED } \\
(\mu G / L \\
\text { AS V) }\end{array}$ & $\begin{array}{l}\text { ZINC, } \\
\text { DIS- } \\
\text { SOLVED } \\
(\mu G / L \\
\text { AS ZN) }\end{array}$ \\
\hline NOV 1987 & & & & & & & & & & \\
\hline $\begin{array}{c}09 \ldots \\
\text { MAR } 1988\end{array}$ & $<30$ & 19 & $<30$ & 1,900 & $<30$ & 40 & $<3.0$ & 7,300 & $<18$ & 24 \\
\hline $\begin{array}{c}07 \ldots \\
\text { APR }\end{array}$ & $<40$ & 15 & $<30$ & 1,000 & $<30$ & $<30$ & $<3.0$ & 7,100 & $<20$ & $<40$ \\
\hline $\begin{array}{l}05 \ldots \\
\text { MAY }\end{array}$ & $<30$ & 18 & $<30$ & 560 & $<30$ & $<30$ & $<3.0$ & 7,100 & $<18$ & 26 \\
\hline${ }_{A U G}^{10} \ldots$ & $<30$ & 10 & $<30$ & 910 & $<30$ & $<30$ & $<3.0$ & 7 & $<18$ & $<9$ \\
\hline $30 \ldots$ & $<10$ & 4 & $<10$ & 1,400 & $<10$ & $<10$ & $<1.0$ & 8,000 & $<6$ & 40 \\
\hline
\end{tabular}


Table 25.--Water-quality analyses for wells--Continued

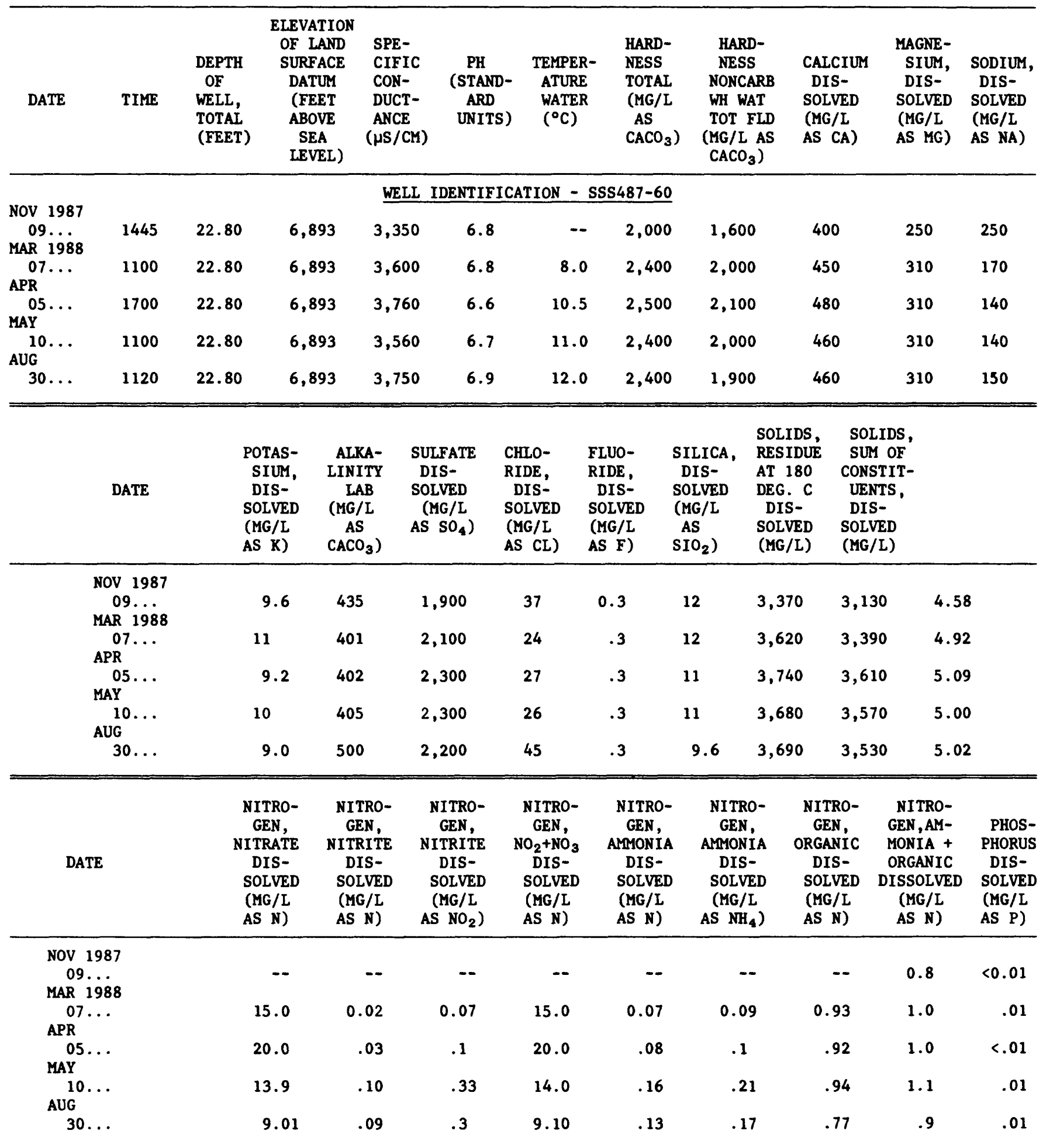


Table 25.--Water-quality analyses for wells--Continued

\begin{tabular}{|c|c|c|c|c|c|c|c|c|c|c|}
\hline DATE & $\begin{array}{l}\text { PHOS- } \\
\text { PHORUS } \\
\text { ORTHO, } \\
\text { DIS- } \\
\text { SOLVED } \\
\text { (MG/L } \\
\text { AS P) }\end{array}$ & $\begin{array}{l}\text { PHOS- } \\
\text { PHORUS } \\
\text { ORGANIC } \\
\text { DIS- } \\
\text { SOLVED } \\
\text { (MG/L } \\
\text { AS P) }\end{array}$ & $\begin{array}{c}\text { ALU- } \\
\text { MINUM, } \\
\text { DIS- } \\
\text { SOLVED } \\
(\mu G / L \\
\text { AS AL) }\end{array}$ & $\begin{array}{c}\text { ARSENIC } \\
\text { DIS- } \\
\text { SOLVED } \\
(\mu G / L \\
\text { AS AS) }\end{array}$ & $\begin{array}{l}\text { BARIUM, } \\
\text { DIS- } \\
\text { SOLVED } \\
(\mu G / L \\
\text { AS BA) }\end{array}$ & $\begin{array}{l}\text { BERYL- } \\
\text { LIUM, } \\
\text { DIS- } \\
\text { SOLVED } \\
(\mu G / L \\
\text { AS BE) }\end{array}$ & $\begin{array}{l}\text { BORON, } \\
\text { DIS- } \\
\text { SOLVED } \\
(\mu G / L \\
\text { AS B) }\end{array}$ & $\begin{array}{l}\text { CADMIUM } \\
\text { DIS- } \\
\text { SOLVED } \\
\text { ( } \mu G / L \\
\text { AS CD) }\end{array}$ & $\begin{array}{l}\text { CHRO- } \\
\text { MIUM, } \\
\text { DIS- } \\
\text { SOLVED } \\
\text { ( } \mu G / L \\
\text { AS CR) }\end{array}$ & $\begin{array}{l}\text { COBALT, } \\
\text { DIS- } \\
\text { SOLVED } \\
(\mu G / L \\
\text { AS CO) }\end{array}$ \\
\hline \multicolumn{11}{|c|}{ WELL IDENTIFICATION - SSS487-60--Continued } \\
\hline NOV 1987 & & & & & & & & & & \\
\hline $\begin{array}{l}09 \\
\text { MAR } 1988\end{array}$ & $\cdots$ & - & 10 & $<1$ & 45 & $<2$ & 460 & $<3$ & $<20$ & $<9$ \\
\hline $\begin{array}{l}07 \ldots \\
\text { APR }\end{array}$ & $<0.01$ & 0.01 & 10 & $<1$ & 28 & $<2$ & 480 & 7 & $<20$ & $<9$ \\
\hline $05 \ldots$ & $<.01$ & -- & 10 & $<1$ & 26 & $<2$ & 440 & $<3$ & $<20$ & $<9$ \\
\hline${ }_{A U G}^{10 \ldots}$ & $<.01$ & .01 & 10 & $<1$ & 26 & $<2$ & 450 & $<4$ & $<5$ & $<10$ \\
\hline $30 \ldots$ & $<.01$ & .01 & $<10$ & $<1$ & 25 & $<.5$ & 440 & $<1$ & $<5$ & $<3$ \\
\hline DATE & $\begin{array}{l}\text { COPPER, } \\
\text { DIS- } \\
\text { SOLVED } \\
(\mu G / L \\
\text { AS CU) }\end{array}$ & $\begin{array}{l}\text { IRON, } \\
\text { DIS- } \\
\text { SOLVED } \\
\text { ( } \mu G / L \\
\text { AS FE) }\end{array}$ & $\begin{array}{l}\text { IEAD, } \\
\text { DIS- } \\
\text { SOLVED } \\
\text { ( } \mu G / L \\
\text { AS PB) }\end{array}$ & $\begin{array}{l}\text { MANGA- } \\
\text { NESE, } \\
\text { DIS- } \\
\text { SOLVED } \\
\text { ( } \mu G / L \\
\text { AS MN) }\end{array}$ & $\begin{array}{l}\text { MOLYB- } \\
\text { DENUM, } \\
\text { DIS- } \\
\text { SOLVED } \\
(\mu G / L \\
\text { AS MO) }\end{array}$ & $\begin{array}{l}\text { NICKEL, } \\
\text { DIS- } \\
\text { SOLVED } \\
(\mu G / L \\
\text { AS NI) }\end{array}$ & $\begin{array}{l}\text { SILVER, } \\
\text { DIS- } \\
\text { SOLVED } \\
\text { ( } \mu G / L \\
\text { AS AG) }\end{array}$ & $\begin{array}{c}\text { STRON- } \\
\text { TIUM, } \\
\text { DIS- } \\
\text { SOLVED } \\
(\mu G / L \\
\text { AS SR) }\end{array}$ & $\begin{array}{l}\text { VANA- } \\
\text { DIUM, } \\
\text { DIS- } \\
\text { SOLVED } \\
(\mu G / L \\
\text { AS V) }\end{array}$ & $\begin{array}{l}\text { ZINC, } \\
\text { DIS- } \\
\text { SOLVED } \\
(\mu G / L \\
\text { AS ZN) }\end{array}$ \\
\hline \multicolumn{11}{|l|}{ Nov 1987} \\
\hline $\begin{array}{l}09 \\
\text { MAR } 1988\end{array}$ & $<30$ & 25 & $<30$ & 1,100 & $<30$ & $<30$ & $<3.0$ & 5,900 & $<18$ & 29 \\
\hline$\underset{\text { APR }}{07 \ldots}$ & 50 & 36 & $<30$ & 450 & $<30$ & $<30$ & $<3.0$ & 6,700 & $<18$ & 53 \\
\hline${ }_{\text {MAY }}^{05} \ldots$ & $<30$ & $<9$ & $<30$ & 290 & $<30$ & $<30$ & $<3.0$ & 7,000 & $<18$ & 28 \\
\hline${ }_{\text {AUG }}^{10 \ldots}$ & $<40$ & $<12$ & $<40$ & 370 & $<40$ & $<40$ & $<4.0$ & 6,800 & $<24$ & 18 \\
\hline $30 \ldots$ & $<10$ & 23 & 10 & 760 & $<10$ & $<10$ & $<1.0$ & 7,700 & $<6$ & $<3$ \\
\hline
\end{tabular}


Table 25.--Water-quality analyses for wells--Continued

\begin{tabular}{|c|c|c|c|c|c|c|c|c|c|c|c|}
\hline DATE & TIME & $\begin{array}{c}\text { DEPTH } \\
\text { OF } \\
\text { WELL, } \\
\text { TOTAL } \\
\text { (FEET) }\end{array}$ & $\begin{array}{c}\text { ELEVATION } \\
\text { OF LAND } \\
\text { SURFACE } \\
\text { DATUM } \\
\text { (FEET } \\
\text { ABOVE } \\
\text { SEA } \\
\text { LEVEL) }\end{array}$ & $\begin{array}{l}\text { SPE- } \\
\text { CIFIC } \\
\text { CON- } \\
\text { DUCT- } \\
\text { ANCE } \\
(\mu S / C M)\end{array}$ & $\begin{array}{c}\text { PH } \\
\text { (STAND- } \\
\text { ARD } \\
\text { UNITS) }\end{array}$ & $\begin{array}{l}\text { TEMPER- } \\
\text { ATURE } \\
\text { WATER } \\
\left({ }^{\circ} \mathrm{C}\right)\end{array}$ & $\begin{array}{l}\text { HARD- } \\
\text { NESS } \\
\text { TOTAL } \\
(\mathrm{MG} / \mathrm{L} \\
\mathrm{AS} \\
\left.\mathrm{CACO}_{3}\right)\end{array}$ & $\begin{array}{l}\text { HARD- } \\
\text { NESS } \\
\text { NONCARB } \\
\text { WH WAT } \\
\text { TOT FLD } \\
(\mathrm{MG} / \mathrm{L} \text { AS } \\
\left.\mathrm{CACO}_{3}\right)\end{array}$ & $\begin{array}{l}\text { CALCIUM } \\
\text { DIS- } \\
\text { SOLVED } \\
\text { (MG/L } \\
\text { AS CA) }\end{array}$ & $\begin{array}{c}\text { MAGNE- } \\
\text { SIUM, } \\
\text { DIS- } \\
\text { SOLVED } \\
\text { (MG/L } \\
\text { AS MG) }\end{array}$ & $\begin{array}{l}\text { SODIUM, } \\
\text { DIS- } \\
\text { SOLVED } \\
\text { (MG/L } \\
\text { AS NA) }\end{array}$ \\
\hline \multicolumn{12}{|c|}{ WELL IDENTIFICATION - SSS487-61 } \\
\hline NOV 1987 & & & & & & & & & & & \\
\hline $\begin{array}{l}09 \ldots \\
\text { MAR } 1988\end{array}$ & 1520 & 26.00 & 6,892 & 3,650 & 6.7 & -- & 2,500 & 2,000 & 490 & 310 & 180 \\
\hline $\begin{array}{c}07 \ldots \\
\text { APR }\end{array}$ & 1145 & 26.00 & 6,892 & 3,640 & 6.8 & 9.0 & 2,500 & 2,100 & 460 & 320 & 180 \\
\hline$\underset{M A Y}{05} \ldots$ & 1545 & 26.00 & 6,892 & 3,720 & 6.7 & 11.5 & 2,500 & 2,100 & 480 & 310 & 150 \\
\hline $\begin{array}{c}10 \\
\text { JUNE }\end{array}$ & 1030 & 26.00 & 6,892 & 3,500 & 6.8 & 12.0 & 2,400 & 2,100 & 460 & 310 & 140 \\
\hline $09 \ldots$ & $\begin{array}{l}1130 \\
1140\end{array}$ & $\begin{array}{l}26.00 \\
26.00\end{array}$ & $\begin{array}{l}6,892 \\
6,892\end{array}$ & $\begin{array}{l}3,600 \\
3,680\end{array}$ & $\begin{array}{l}6.6 \\
6.6\end{array}$ & $\begin{array}{l}13.0 \\
12.0\end{array}$ & $\begin{array}{l}2,300 \\
2,300\end{array}$ & $\begin{array}{l}1,800 \\
1,800\end{array}$ & $\begin{array}{l}420 \\
420\end{array}$ & $\begin{array}{l}300 \\
300\end{array}$ & $\begin{array}{l}140 \\
140\end{array}$ \\
\hline$\underset{\text { AUG }}{09 \ldots}$ & 1150 & 26.00 & 6,892 & 3,680 & 6.6 & 12.0 & 2,300 & 1,800 & 410 & 300 & 150 \\
\hline $\begin{array}{c}30 \ldots \\
\text { NOV }\end{array}$ & 1045 & 26.00 & 6,892 & 3,560 & 6.9 & 11.5 & 2,400 & 1,900 & 440 & 320 & 160 \\
\hline $\begin{array}{l}16 \ldots \\
\text { APR } 1989\end{array}$ & 1200 & 26.00 & 6,892 & 3,550 & 7.0 & -- & 2,400 & 1,900 & 450 & 310 & 180 \\
\hline${ }_{\text {UNE }}^{04 \ldots}$ & 1430 & 26.00 & 6,892 & 3,890 & 6.6 & 9.0 & 2,500 & 2,100 & 480 & 320 & 140 \\
\hline$\underset{\text { SEPT }}{07}$ & 1000 & 26.00 & 6,892 & 3,960 & 7.0 & 11.0 & 2,400 & 1,900 & 450 & 300 & 150 \\
\hline $12 \ldots$ & 1115 & 26.00 & 6,892 & 3,860 & 6.8 & 10.5 & 2,300 & 1,800 & 450 & 290 & 160 \\
\hline DATE & & $\begin{array}{l}\text { POTAS- } \\
\text { SIUM, } \\
\text { DIS- } \\
\text { SOLVED } \\
\text { (MG/L } \\
\text { AS K) }\end{array}$ & $\begin{array}{c}\text { ALKA- } \\
\text { LINITY } \\
\text { LAB } \\
(\mathrm{MG} / \mathrm{L} \\
\mathrm{AS} \\
\left.\mathrm{CACO}_{3}\right)\end{array}$ & $\begin{array}{l}\text { SULFATE } \\
\text { DIS- } \\
\text { SOLVED } \\
(\mathrm{MG} / \mathrm{L} \\
\left.\text { AS } \mathrm{SO}_{4}\right)\end{array}$ & $\begin{array}{l}\text { CHLO- } \\
\text { RIDE, } \\
\text { DIS- } \\
\text { SOLVED } \\
\text { (MG/L } \\
\text { AS CL) }\end{array}$ & $\begin{array}{l}\text { FLUO- } \\
\text { RIDE, } \\
\text { DIS- } \\
\text { SOLVED } \\
\text { (MG/L } \\
\text { AS F) }\end{array}$ & $\begin{array}{l}\text { SILICA, } \\
\text { DIS- } \\
\text { SOLVED } \\
\text { (MG/L } \\
\text { AS } \\
\mathrm{SIO}_{2} \text { ) }\end{array}$ & $\begin{array}{l}\text { SOLIDS, } \\
\text { RESIDUE } \\
\text { AT 180 } \\
\text { DEG. C } \\
\text { DIS- } \\
\text { SOLVED } \\
\text { (MG/L) }\end{array}$ & $\begin{array}{l}\text { SOLIDS, } \\
\text { SUM OF } \\
\text { CONSTIT- } \\
\text { UENTS, } \\
\text { DIS- } \\
\text { SOLVED } \\
\text { (MG/L) }\end{array}$ & & \\
\hline NOV 1987 & & & & & & & & & & & \\
\hline $\begin{array}{l}09 \ldots \\
\text { MAR } 1988\end{array}$ & & 9.6 & 494 & 2,100 & 35 & 1.1 & 13 & 3,710 & 3,440 & & \\
\hline$\underset{\text { APR }}{07 \ldots}$ & & 11 & 416 & 2,200 & 24 & .2 & 13 & 3,690 & 3,530 & & \\
\hline${ }_{\text {MAY }}^{05 \ldots}$ & & 10 & 405 & 2,300 & 26 & .3 & 11 & 3,730 & 3,630 & & \\
\hline${ }_{J N E}^{10} \cdots$ & & 10 & 346 & 2,200 & 26 & .3 & 11 & 3,670 & 3,430 & & \\
\hline $\begin{array}{l}09 \ldots \\
09 \ldots \\
09 \ldots\end{array}$ & & $\begin{array}{l}10 \\
10 \\
10\end{array}$ & $\begin{array}{l}446 \\
446 \\
445\end{array}$ & $\begin{array}{l}2,200 \\
2,200 \\
2,200\end{array}$ & $\begin{array}{l}44 \\
44 \\
44\end{array}$ & $\begin{array}{l}.4 \\
.4 \\
.4\end{array}$ & $\begin{array}{c}10 \\
9.9 \\
10\end{array}$ & $\begin{array}{l}3,660 \\
3,660 \\
3,670\end{array}$ & $\begin{array}{l}3,460 \\
3,460 \\
3,400\end{array}$ & & \\
\hline $\begin{array}{l}\text { AUG } \\
30 \ldots\end{array}$ & & 10 & 504 & 2,200 & 45 & .3 & 9.8 & 3,670 & 3,530 & & \\
\hline $\begin{array}{l}\text { NOV } \\
16 \ldots \\
\text { APR } 1989\end{array}$ & & 9.0 & 494 & 2,200 & 51 & .3 & 11 & 3,710 & 3,550 & & \\
\hline JUNE & & 10 & 403 & 2,300 & 37 & .3 & 11 & 3,650 & 3,660 & & \\
\hline$\underset{\text { SEPT }}{07}$ & & 10 & 458 & 2,200 & 40 & .3 & 10 & 3,660 & 3,440 & & \\
\hline 12. & & 11 & 496 & 2,200 & 48 & .3 & 11 & 3,500 & 3,480 & & \\
\hline
\end{tabular}


Table 25.--Water-quality analyses for wells--Continued

\begin{tabular}{|c|c|c|c|c|c|c|c|c|c|c|c|}
\hline DATE & $\begin{array}{l}\text { NITRO- } \\
\text { GEN, } \\
\text { NITRATE } \\
\text { DIS- } \\
\text { SOLVED } \\
\text { (MG/L } \\
\text { AS N) }\end{array}$ & $\begin{array}{l}\text { NITRO- } \\
\text { GEN, } \\
\text { NITRITE } \\
\text { DIS- } \\
\text { SOLVED } \\
\text { (MG/L } \\
\text { AS N) }\end{array}$ & $\begin{array}{l}\text { NITRO- } \\
\text { GEN, } \\
\text { NITRITE } \\
\text { DIS- } \\
\text { SOLVED } \\
(\mathrm{MG} / \mathrm{L} \\
\left.\text { AS } \mathrm{NO}_{2}\right)\end{array}$ & $\begin{array}{c}\text { NITRO- } \\
\text { GEN, } \\
\mathrm{NO}_{2}+\mathrm{NO}_{3} \\
\text { DIS- } \\
\text { SOLVED } \\
(\mathrm{MG} / \mathrm{L} \\
\text { AS N) }\end{array}$ & $\begin{array}{l}\text { NITRO- } \\
\text { GEN, } \\
\text { AMMONIA } \\
\text { DIS- } \\
\text { SOLVED } \\
\text { (MG/L } \\
\text { AS N) }\end{array}$ & $\begin{array}{l}\text { NITRO- } \\
\text { GEN, } \\
\text { AMMONIA } \\
\text { DIS- } \\
\text { SOLVED } \\
(\mathrm{MG} / \mathrm{L} \\
\left.\text { AS } \mathrm{NH}_{4}\right)\end{array}$ & $\begin{array}{l}\text { NITRO- } \\
\text { GEN, } \\
\text { ORGANIC } \\
\text { DIS- } \\
\text { SOLVED } \\
\text { (MG/L } \\
\text { AS N) }\end{array}$ & $\begin{array}{c}\text { NITRO- } \\
\text { GEN,AM- } \\
\text { MONIA + } \\
\text { ORGANIC } \\
\text { DISSOLVED } \\
\text { (MG/L } \\
\text { AS N) }\end{array}$ & $\begin{array}{l}\text { PHOS- } \\
\text { PHORUS } \\
\text { DIS- } \\
\text { SOLVED } \\
\text { (MG/L } \\
\text { AS P) }\end{array}$ & $\begin{array}{l}\text { PHOS- } \\
\text { PHORUS } \\
\text { ORTHO, } \\
\text { DIS- } \\
\text { SOLVED } \\
\text { (MG/L } \\
\text { AS P) }\end{array}$ & $\begin{array}{l}\text { PHOS- } \\
\text { PHORUS } \\
\text { ORGANIC } \\
\text { DIS- } \\
\text { SOLVED } \\
\text { (MG/L } \\
\text { AS P) }\end{array}$ \\
\hline
\end{tabular}

NOV 1987

WELL IDENTIFICATION - SSS487-61--Continued

$09 \ldots$

MAR 1988

$07 \ldots$

$14.0 \quad 0.04$

0.13

14.0

0.09

$0.12 \quad 1.2$

.13

$20.0 \quad .11$

.14

$14.0 \quad .14$

.18

$13.0 \quad .10$

.36

09 ...

$13.9 \quad .13$

.43

12.9

13.9

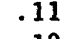

$09 .$.

AUG

$30 \ldots$

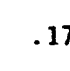

.17

- 12

.39

$14.0 \quad .11$

.43

NOV

$16 \ldots$

APR 1989

$04 \ldots$

$8.40 \quad .10$

.33

$8.30 \quad .13$

$.13 \quad 1.2$

.14

$8.50 \quad .13$

.17

.17

$25.0 \quad .07$

.09

1.0

$\begin{array}{lll}24.9 & .08 \quad .26\end{array}$

JUNE

SEPT

12 ... $\begin{array}{ccc}24.9 & .08 & .26 \\ \ldots & \ldots & \ldots\end{array}$
.97

$\begin{array}{llll}0.8 & 0.01 & - & 0\end{array}$

$\begin{array}{llll}1.3 & .01 & <0.01 & .01\end{array}$

.89

1.0

.01

$<.01$

.01

1.5

1.6

$<.01$

$<.01$

1.3

1.4

$<.01$

$<.01$

$<.01$

$<.01$

$<.01$

$.6 \quad .01$

$<.01$

.01

1.1

.01

$<.01$

.01

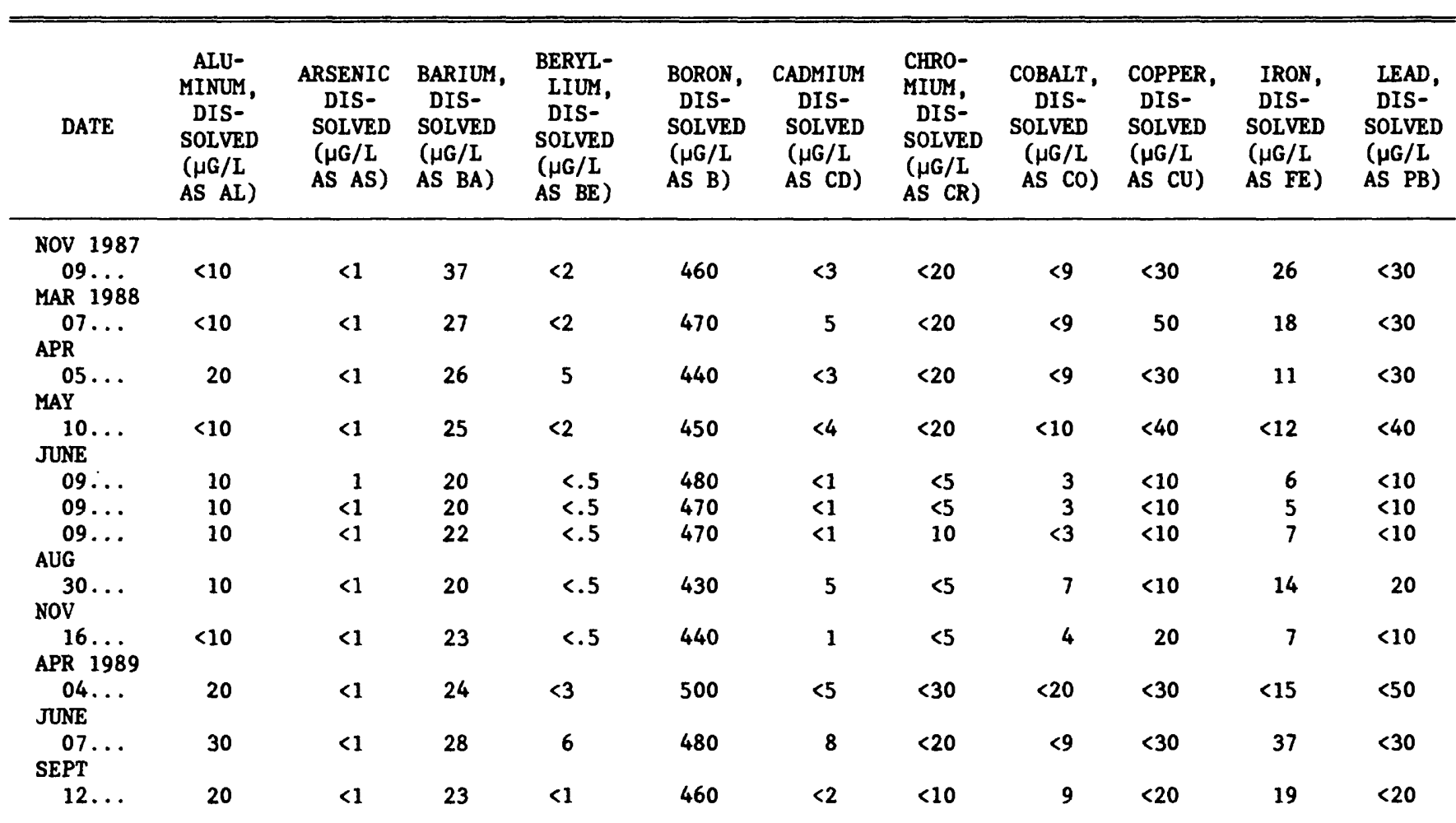


Table 25.--Water-quality analyses for wells--Continued

\begin{tabular}{|c|c|c|c|c|c|c|c|c|c|c|c|c|}
\hline DATE & $\begin{array}{c}\text { MANGA- } \\
\text { NESE, } \\
\text { DIS- } \\
\text { SOLVED } \\
(\mu G / L \\
\text { AS MN) }\end{array}$ & $\begin{array}{l}\text { MOLYB- } \\
\text { DENUM, } \\
\text { DIS- } \\
\text { SOLVED } \\
(\mu G / L \\
\text { AS MO) }\end{array}$ & $\begin{array}{l}\text { NICKEL, } \\
\text { DIS- } \\
\text { SOLVED } \\
(\mu G / L \\
\text { AS NI) }\end{array}$ & $\begin{array}{l}\text { SILVER, } \\
\text { DIS- } \\
\text { SOLVED } \\
(\mu G / I \\
\text { AS AG) }\end{array}$ & $\begin{array}{l}\text { STRON- } \\
\text { TIUM, } \\
\text { DIS- } \\
\text { SOLVED } \\
\text { ( } \mu G / L \\
\text { AS SR) }\end{array}$ & $\begin{array}{l}\text { VANA- } \\
\text { DIUM, } \\
\text { DIS- } \\
\text { SOLVED } \\
(\mu G / L \\
\text { AS V) }\end{array}$ & $\begin{array}{l}\text { ZINC, } \\
\text { DIS- } \\
\text { SOLVED } \\
(\mu G / L \\
\text { AS ZN) }\end{array}$ & $\begin{array}{l}\text { C-13/ } \\
\text { C-12 } \\
\text { STABLE } \\
\text { ISOTOPE } \\
\text { RATIO } \\
\text { PER } \\
\text { MIL }\end{array}$ & $\begin{array}{l}\text { H-2/ } \\
\text { H-1 } \\
\text { STABLE } \\
\text { ISOTOPE } \\
\text { RATIO } \\
\text { PER } \\
\text { MIL }\end{array}$ & $\begin{array}{l}0-18 / \\
0-16 \\
\text { STABLE } \\
\text { ISOTOPE } \\
\text { RATIO } \\
\text { PER } \\
\text { MIL }\end{array}$ & $\begin{array}{l}\text { S-34/ } \\
\text { S-32 } \\
\text { STABLE } \\
\text { ISOTOPE } \\
\text { RATIO } \\
\text { PER } \\
\text { MIL }\end{array}$ & $\begin{array}{l}\text { TRIT- } \\
\text { IUM } \\
\text { TOTAL } \\
\text { (PCI/L) }\end{array}$ \\
\hline
\end{tabular}

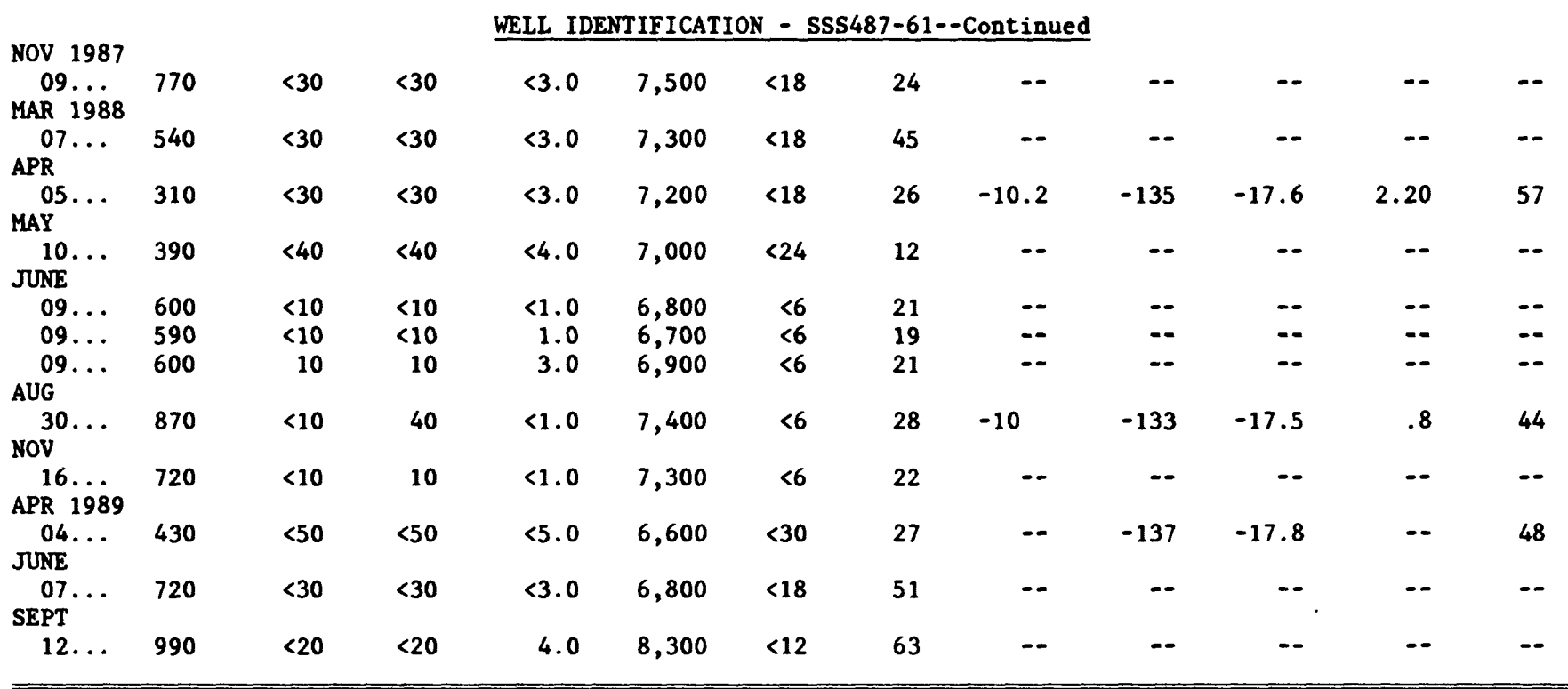

\begin{tabular}{|c|c|c|c|c|c|c|c|c|c|c|}
\hline DATE & TIME & $\begin{array}{l}\text { DEPTH } \\
\text { OF } \\
\text { WELL, } \\
\text { TOTAL } \\
\text { (FEET) }\end{array}$ & $\begin{array}{l}\text { ELEVATION } \\
\text { OF LAND } \\
\text { SURFACE } \\
\text { DATUM } \\
\text { (FEET } \\
\text { ABOVE } \\
\text { SEA } \\
\text { LEVEL) }\end{array}$ & $\begin{array}{l}\text { SPE- } \\
\text { CIFIC } \\
\text { CON- } \\
\text { DUCT- } \\
\text { ANCE } \\
(\mu S / C M)\end{array}$ & $\begin{array}{c}\text { PH } \\
\text { (STAND- } \\
\text { ARD } \\
\text { UNITS) }\end{array}$ & $\begin{array}{l}\text { TEMPER- } \\
\text { ATURE } \\
\text { WATER } \\
\left({ }^{\circ} \mathrm{C}\right)\end{array}$ & $\begin{array}{c}\text { HARD- } \\
\text { NESS } \\
\text { TOTAL } \\
(\mathrm{MG} / \mathrm{L} \\
\text { AS } \\
\left.\mathrm{CACO}_{3}\right)\end{array}$ & $\begin{array}{l}\text { HARD- } \\
\text { NESS } \\
\text { NONCARB } \\
\text { WH WAT } \\
\text { TOT FLD } \\
(\mathrm{MG} / \mathrm{L} \mathrm{AS} \\
\left.\mathrm{CACO}_{3}\right)\end{array}$ & $\begin{array}{c}\text { CALCIUM } \\
\text { DIS- } \\
\text { SOLVED } \\
\text { (MG/I } \\
\text { AS CA) }\end{array}$ & $\begin{array}{c}\text { MAGNE- } \\
\text { SIUM, } \\
\text { DIS- } \\
\text { SOLVED } \\
\text { (MG/L } \\
\text { AS MG) }\end{array}$ \\
\hline \multicolumn{11}{|c|}{ WELL IDENTIFICATION - SCL287 } \\
\hline NOV 1987 & & & & & & & & & & \\
\hline $\begin{array}{r}07 \ldots \\
\text { MAR } 1988\end{array}$ & 1635 & 30.00 & 6,957 & 2,400 & 7.1 & -- & 1,300 & 880 & 270 & 150 \\
\hline $\begin{array}{c}08 \cdots \\
A P R\end{array}$ & 0930 & 30.00 & 6,957 & 2,430 & 7.1 & 6.0 & 1,400 & 1,100 & 260 & 180 \\
\hline${ }_{\mathrm{MAY}}^{12 \ldots}$ & 1145 & 30.00 & 6,957 & 2,810 & 7.0 & 9.0 & 1,800 & 1,500 & 350 & 220 \\
\hline${ }_{\text {AUG }}^{11}$ & 0915 & 30.00 & 6,957 & 2,800 & 7.1 & 9.0 & 1,600 & 1,300 & 310 & 200 \\
\hline$\underset{\text { Nov }}{31 \ldots}$ & 1015 & 30.00 & 6,957 & 2,450 & 7.0 & 8.0 & 1,600 & 1,200 & 300 & 200 \\
\hline $\begin{array}{r}15 \ldots \\
\text { APR } 1989\end{array}$ & 1500 & 30.00 & 6,957 & 2,550 & 7.0 & -- & 1,400 & 1,000 & 280 & 180 \\
\hline $\begin{array}{c}05 \\
\text { JNE }\end{array}$ & 1000 & 30.00 & 6,957 & 3,170 & 6.9 & 9.0 & 1,900 & 1,600 & 380 & 240 \\
\hline $\begin{array}{l}06 \ldots \\
\text { SEPT }\end{array}$ & 1000 & 30.00 & 6,957 & 3,100 & 6.9 & 9.0 & 1,800 & 1,400 & 370 & 220 \\
\hline $18 \ldots$ & 1600 & 30.00 & 6,957 & 2,770 & 7.0 & 11.0 & 1,700 & 1,300 & 330 & 210 \\
\hline
\end{tabular}


Table 25.--Water-quality analyses for wells--Continued

\begin{tabular}{|c|c|c|c|c|c|c|c|c|c|}
\hline DATE & $\begin{array}{l}\text { SODIUM, } \\
\text { DIS- } \\
\text { SOLVED } \\
\text { (MG/L } \\
\text { AS NA) }\end{array}$ & $\begin{array}{l}\text { POTAS- } \\
\text { SIUM, } \\
\text { DIS- } \\
\text { SOLVED } \\
\text { (MG/L } \\
\text { AS K) }\end{array}$ & $\begin{array}{c}\text { ALKA- } \\
\text { LINITY } \\
\text { LAB } \\
(\mathrm{MG} / \mathrm{L} \\
\mathrm{AS} \\
\left.\mathrm{CACO}_{3}\right)\end{array}$ & $\begin{array}{l}\text { SULFATE } \\
\text { DIS- } \\
\text { SOLVED } \\
(\mathrm{MG} / \mathrm{L} \\
\left.\mathrm{AS} \mathrm{SO}_{4}\right)\end{array}$ & $\begin{array}{l}\text { CHLO- } \\
\text { RIDE, } \\
\text { DIS- } \\
\text { SOLVED } \\
\text { (MG/L } \\
\text { AS CL) }\end{array}$ & $\begin{array}{l}\text { FLUO- } \\
\text { RIDE, } \\
\text { DIS- } \\
\text { SOLVED } \\
\text { (MG/L } \\
\text { AS F) }\end{array}$ & $\begin{array}{l}\text { SILICA, } \\
\text { DIS- } \\
\text { SOLVED } \\
\text { (MG/L } \\
\text { AS } \\
\left.\mathrm{SIO}_{2}\right)\end{array}$ & $\begin{array}{l}\text { SOLIDS, } \\
\text { RESIDUE } \\
\text { AT 180 } \\
\text { DEG. C } \\
\text { DIS- } \\
\text { SOLVED } \\
\text { (MG/L) }\end{array}$ & $\begin{array}{l}\text { SOLIDS, } \\
\text { SUM OF } \\
\text { CONSTIT- } \\
\text { UENTS, } \\
\text { DIS- } \\
\text { SOLVED } \\
\text { (MG/L) }\end{array}$ \\
\hline \multicolumn{10}{|c|}{ WELL IDENTIFICATION - SCL287--Continued } \\
\hline NOV 1987 & & & & & & & & \multirow[b]{2}{*}{2,060} & \\
\hline $\begin{array}{l}07 \ldots \\
\text { MAR } 1988\end{array}$ & 150 & 5.9 & 419 & 1,200 & 14 & 0.3 & 12 & & 2,060 \\
\hline $\begin{array}{l}08 \ldots \\
\text { APR }\end{array}$ & 140 & 6.8 & 312 & 1,300 & 17 & .4 & 11 & 2,330 & 2,110 \\
\hline $12 \ldots$ & 120 & 6.1 & 325 & 1,600 & 14 & .4 & 10 & 2,670 & 2,530 \\
\hline${ }_{A U G}^{11 \ldots}$ & 150 & 6.2 & 344 & 1,600 & 13 & .5 & 11 & 2,610 & 2,500 \\
\hline $\mathrm{NOV}^{31} \ldots$ & 130 & 7.0 & 388 & 1,400 & 14 & .4 & 11 & 2,360 & 2,300 \\
\hline $\begin{array}{l}15 \ldots \\
\text { APR } 1989\end{array}$ & 140 & 6.3 & 411 & 1,300 & 16 & .4 & 11 & 2,270 & 2,190 \\
\hline JNE & 95 & 7.0 & 387 & 1,800 & 16 & .4 & 11 & 2,790 & 2,790 \\
\hline $\begin{array}{c}06 \ldots \\
\text { SEPT }\end{array}$ & 120 & 6.8 & 412 & 1,700 & 14 & .3 & 11 & 2,810 & 2,690 \\
\hline $18 \ldots$ & 130 & 7.1 & 434 & 1,500 & 13 & .4 & 11 & 2,490 & 2,470 \\
\hline DATE & & $\begin{array}{l}\text { NITRO- } \\
\text { GEN, } \\
\text { NITRITE } \\
\text { DIS- } \\
\text { SOLVED } \\
\text { (MG/L } \\
\text { AS N) }\end{array}$ & $\begin{array}{c}\text { NITRO- } \\
\text { GEN, } \\
\mathrm{NO}_{2}+\mathrm{NO}_{3} \\
\text { DIS- } \\
\text { SOLVED } \\
(\mathrm{MG} / \mathrm{L} \\
\mathrm{AS} \mathrm{N})\end{array}$ & $\begin{array}{l}\text { NITRO- } \\
\text { GEN, } \\
\text { AMMONIA } \\
\text { DIS- } \\
\text { SOLVED } \\
\text { (MG/L } \\
\text { AS N) }\end{array}$ & $\begin{array}{c}\text { NITRO- } \\
\text { GEN, } \\
\text { AMMONIA } \\
\text { DIS- } \\
\text { SOLVED } \\
(\mathrm{MG} / \mathrm{L} \\
\text { AS NH4 })\end{array}$ & $\begin{array}{l}\text { NITRO- } \\
\text { GEN, } \\
\text { ORGANIC } \\
\text { DIS- } \\
\text { SOLVED } \\
\text { (MG/L } \\
\text { AS N) }\end{array}$ & $\begin{array}{l}\text { NITRO- } \\
\text { GEN,AM- } \\
\text { MONIA + } \\
\text { ORGANIC } \\
\text { DISSOLVED } \\
\text { (MG/L } \\
\text { AS N) }\end{array}$ & $\begin{array}{l}\text { PHOS- } \\
\text { PHORUS } \\
\text { DIS- } \\
\text { SOLVED } \\
\text { (MG/L } \\
\text { AS P) }\end{array}$ & $\begin{array}{l}\text { PHOS- } \\
\text { PHORUS } \\
\text { ORTHO, } \\
\text { DIS- } \\
\text { SOLVED } \\
\text { (MG/L } \\
\text { AS P) }\end{array}$ \\
\hline \multicolumn{10}{|l|}{ NOV 1987} \\
\hline $\begin{array}{l}07 \ldots \\
\text { MAR } 1988\end{array}$ & & $<0.01$ & $<0.10$ & 0.64 & 0.82 & 0.16 & 0.8 & $<0.01$ & $<0.01$ \\
\hline $\begin{array}{c}08 \ldots \\
\text { APR }\end{array}$ & & $<.01$ & .13 & .44 & .57 & .36 & .8 & .01 & .01 \\
\hline${ }_{\mathrm{MAY}}^{12 \ldots}$ & & $<.01$ & 3.00 & .15 & .19 & .35 & .5 & $<.01$ & $<.01$ \\
\hline${ }_{A U G}^{11 \ldots}$ & & $<.01$ & .43 & .45 & .58 & .75 & 1.2 & $<.01$ & $<.01$ \\
\hline$\underset{\text { NOV }}{31 \ldots}$ & & $<.01$ & $<.10$ & .39 & .5 & .21 & .6 & $<.01$ & $<.01$ \\
\hline $\begin{array}{l}15 \ldots \\
\text { APR } 1989\end{array}$ & & $<.01$ & $<.10$ & .52 & .67 & .18 & .7 & $<.01$ & $<.01$ \\
\hline$\underset{\text { JNE }}{05}$ & & -- & -- & -- & -- & -- & -- & -- & -- \\
\hline $\begin{array}{l}06 \ldots \\
\text { SEPT }\end{array}$ & & -- & -- & -- & -- & -- & -- & -- & -- \\
\hline $18 \ldots$ & & -- & -- & -- & -- & -- & -- & -- & -- \\
\hline
\end{tabular}


Table 25.--Water-quality analyses for wells--Continued

\begin{tabular}{|c|c|c|c|c|c|c|c|c|c|c|}
\hline DATE & $\begin{array}{l}\text { PHOS- } \\
\text { PHATE, } \\
\text { ORTHO, OI } \\
\text { DIS- } \\
\text { SOLVED } \\
(\mathrm{MG} / \mathrm{L} \\
\text { AS PO })\end{array}$ & $\begin{array}{l}\text { PHOS- } \\
\text { PHORUS } \\
\text { ORGANIC } \\
\text { DIS- } \\
\text { SOLVED } \\
\text { (MG/L } \\
\text { AS P) }\end{array}$ & $\begin{array}{l}\text { ALU- } \\
\text { MINUM, } \\
\text { DIS- } \\
\text { SOLVED } \\
\text { ( } \mu \mathrm{G} / \mathrm{L} \\
\text { AS AI) }\end{array}$ & $\begin{array}{l}\text { ARSENIC } \\
\text { DIS- } \\
\text { SOLVED } \\
\text { ( } \mu \mathrm{G} / \mathrm{L} \\
\text { AS AS) }\end{array}$ & $\begin{array}{l}\text { BARIUM, } \\
\text { DIS- } \\
\text { SOLVED } \\
\text { ( } \mu G / L \\
\text { AS BA) }\end{array}$ & $\begin{array}{l}\text { BERYL- } \\
\text { LIUM, } \\
\text { DIS- } \\
\text { SOLVED } \\
\text { ( } \mu \mathrm{G} / \mathrm{L} \\
\text { AS BE) }\end{array}$ & $\begin{array}{l}\text { BORON, } \\
\text { DIS- } \\
\text { SOLVED } \\
(\mu G / L \\
\text { AS B) }\end{array}$ & $\begin{array}{l}\text { CADMIUM } \\
\text { DIS- } \\
\text { SOLVED } \\
(\mu G / L \\
\text { AS CD) }\end{array}$ & $\begin{array}{l}\text { CHRO- } \\
\text { MIUM, } \\
\text { DIS- } \\
\text { SOLVED } \\
\text { ( } \mu G / L \\
\text { AS CR) }\end{array}$ & $\begin{array}{l}\text { COBALT, } \\
\text { DIS- } \\
\text { SOLVED } \\
(\mu G / L \\
\text { AS CO) }\end{array}$ \\
\hline \multicolumn{11}{|c|}{ WELL IDENTIFICATION - SCL287--Continued } \\
\hline NOV 1987 & & & & & & & & & & \\
\hline $\begin{array}{l}07 \ldots \\
\text { MAR } 1988\end{array}$ & -- & -- & $<10$ & $<1$ & 49 & $<2$ & 190 & $<3$ & $<20$ & $<9$ \\
\hline $\begin{array}{l}08 \ldots \\
\text { APR }\end{array}$ & 0.03 & 0.0 & $<10$ & $<1$ & 45 & $<2$ & 180 & 3 & $<20$ & 10 \\
\hline${ }_{\mathrm{MAY}}^{12 \ldots}$ & -- & -- & $<10$ & $<1$ & 44 & $<2$ & 190 & $<3$ & $<20$ & 10 \\
\hline${ }_{A U G}^{11 \ldots}$ & -- & -- & $<10$ & $<1$ & 50 & $<2$ & 190 & $<3$ & $<20$ & $<9$ \\
\hline $31 \ldots$ & -- & -- & $<10$ & $<1$ & 39 & $<.5$ & 180 & 5 & $<5$ & $<3$ \\
\hline $\begin{array}{c}15 \ldots \\
\text { APR } 1989\end{array}$ & -- & -- & $<10$ & $<1$ & 37 & $<.5$ & 200 & $<1$ & $<5$ & 5 \\
\hline $\begin{array}{l}05 \ldots \\
\text { JUNE }\end{array}$ & -- & -- & $<10$ & $<1$ & 38 & $<2$ & 210 & $<3$ & $<20$ & $<9$ \\
\hline $\begin{array}{l}06 . . \\
\text { SEPT }\end{array}$ & -- & -- & $<10$ & $<1$ & 42 & $<2$ & 200 & $<3$ & $<20$ & 10 \\
\hline $18 \ldots$ & -- & -- & $<10$ & 2 & 35 & $<1$ & 190 & $<2$ & $<10$ & $<6$ \\
\hline DATE & $\begin{array}{l}\text { COPPER, } \\
\text { DIS- } \\
\text { SOLVED } \\
(\mu G / L \\
\text { AS CU) }\end{array}$ & $\begin{array}{l}\text { IRON, } \\
\text { DIS- } \\
\text { SOLVED } \\
(\mu G / L \\
\text { AS FE) }\end{array}$ & $\begin{array}{l}\text { LEAD, } \\
\text { DIS- } \\
\text { SOLVED } \\
(\mu G / L \\
\text { AS PB) }\end{array}$ & $\begin{array}{l}\text { MANGA- } \\
\text { NESE, } \\
\text { DIS- } \\
\text { SOLVED } \\
\text { ( } \mu G / L \\
\text { AS MN) }\end{array}$ & $\begin{array}{l}\text { MOLYB- } \\
\text { DENUM, } \\
\text { DIS- } \\
\text { SOLVED } \\
(\mu G / L \\
\text { AS MO) }\end{array}$ & $\begin{array}{l}\text { NICKEL, } \\
\text { DIS- } \\
\text { SOLVED } \\
(\mu G / L \\
\text { AS NI) }\end{array}$ & $\begin{array}{l}\text { SILVER, } \\
\text { DIS- } \\
\text { SOLVED } \\
(\mu G / L \\
\text { AS AG) }\end{array}$ & $\begin{array}{c}\text { STRON- } \\
\text { TIUM, } \\
\text { DIS- } \\
\text { SOLVED } \\
(\mu G / L \\
\text { AS SR) }\end{array}$ & $\begin{array}{l}\text { VANA- } \\
\text { DIUM, } \\
\text { DIS- } \\
\text { SOLVED } \\
(\mu G / L \\
\text { AS V) }\end{array}$ & $\begin{array}{l}\text { ZINC, } \\
\text { DIS- } \\
\text { SOLVED } \\
(\mu G / L \\
\text { AS ZN) }\end{array}$ \\
\hline \multicolumn{11}{|l|}{ NOV 1987} \\
\hline $\begin{array}{c}07 \ldots \\
\text { MAR } 1988\end{array}$ & $<30$ & 590 & $<30$ & 670 & $<30$ & $<30$ & $<3.0$ & 3,500 & $<18$ & 12 \\
\hline$\underset{\text { APR }}{08 \ldots}$ & $<30$ & 31 & $<30$ & 830 & $<30$ & $<30$ & 3.0 & 4,000 & $<18$ & 39 \\
\hline $\operatorname{MAY}^{12 \ldots}$ & $<30$ & 22 & $<30$ & 590 & $<30$ & $<30$ & $<3.0$ & 4,100 & $<18$ & 38 \\
\hline AUG $^{11 \ldots}$ & $<30$ & 86 & $<30$ & 570 & $<30$ & $<30$ & $<3.0$ & 3,900 & $<18$ & 14 \\
\hline${ }_{\text {NOV }}^{31 \ldots}$ & $<10$ & 520 & $<10$ & 610 & $<10$ & $<10$ & 6.0 & 3,700 & $<6$ & 27 \\
\hline $\begin{array}{c}15 \ldots \\
\text { APR } 1989\end{array}$ & $<10$ & 250 & $<10$ & 600 & $<10$ & 10 & $<1.0$ & 3,500 & $<6$ & 29 \\
\hline$\underset{\text { JUNE }}{05 \cdots}$ & $<30$ & 31 & $<30$ & 480 & $<30$ & 40 & $<3.0$ & 4,200 & $<18$ & 45 \\
\hline$\underset{\text { SEPT }}{06 \ldots}$ & $<30$ & 45 & $<30$ & 560 & $<30$ & $<30$ & $<3.0$ & 4,400 & $<18$ & 43 \\
\hline $18 \ldots$ & $<20$ & 530 & $<20$ & 610 & $<20$ & $<20$ & $<2.0$ & 4,100 & $<12$ & 45 \\
\hline
\end{tabular}


Table 25.--Water-quality analyses for wells--Continued

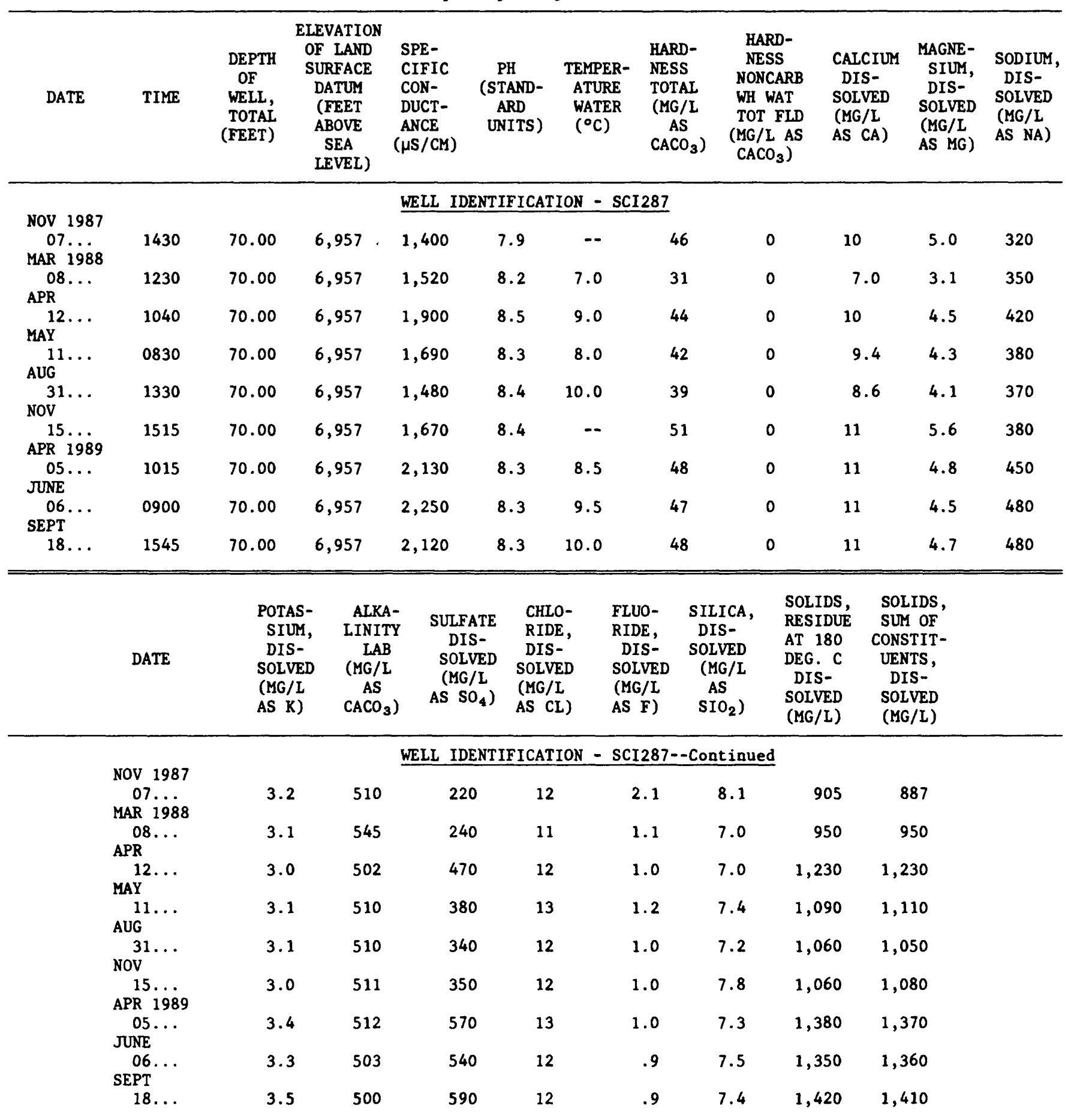


Table 25.--Water-quality analyses for wells--Continued

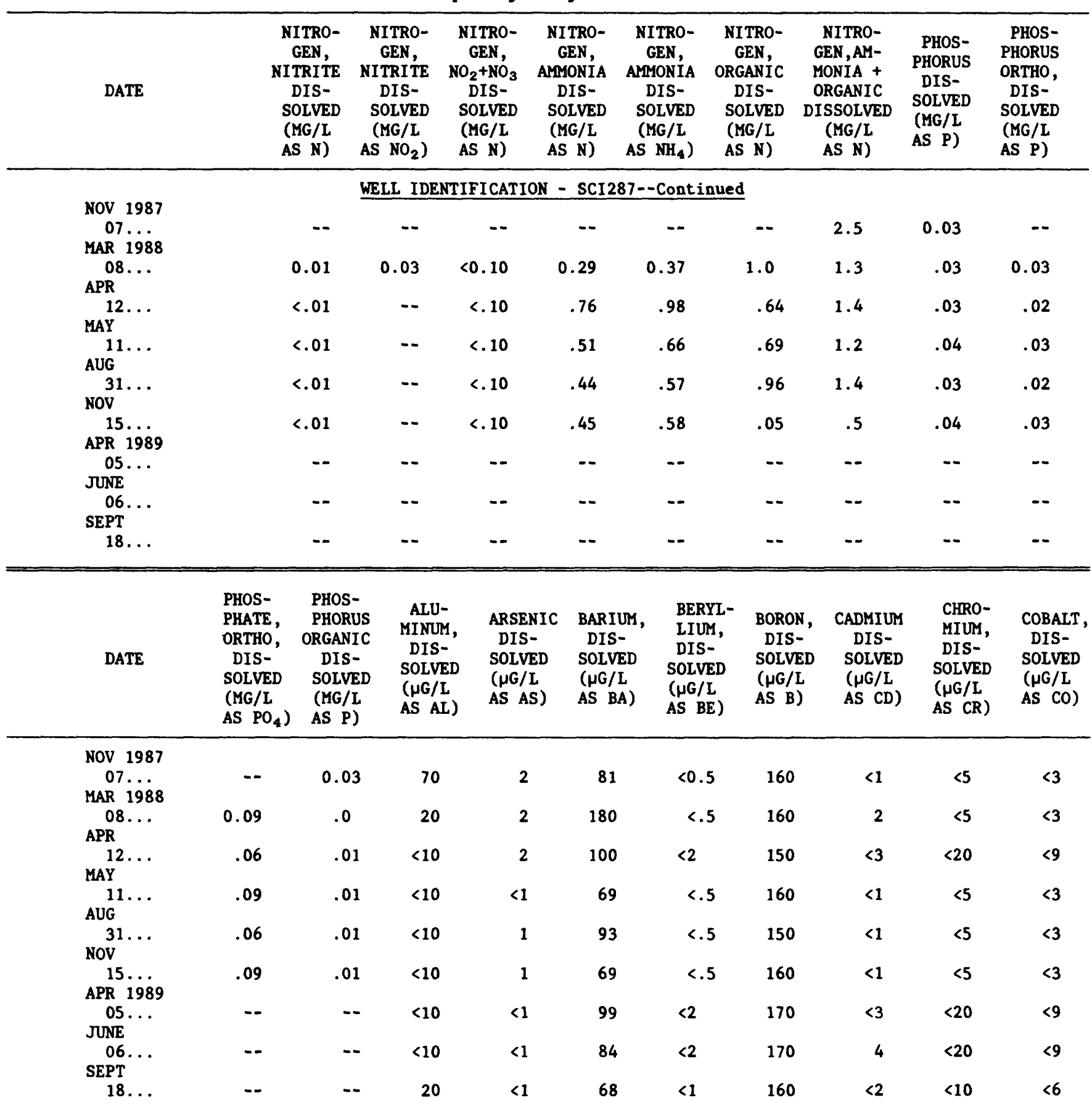


Table 25.--Water-quality analyses for wells--Continued

\begin{tabular}{|c|c|c|c|c|c|c|c|c|c|c|c|}
\hline \multicolumn{2}{|l|}{ DATE } & $\begin{array}{l}\text { COPPER, } \\
\text { DIS- } \\
\text { SOLVED } \\
(\mu G / L \\
\text { AS CU) }\end{array}$ & $\begin{array}{l}\text { IRON, } \\
\text { DIS - } \\
\text { SOLVED } \\
\text { ( } \mu G / L \\
\text { AS FE) }\end{array}$ & $\begin{array}{l}\text { IEAD, } \\
\text { DIS- } \\
\text { SOLVED } \\
(\mu G / L \\
\text { AS PB) }\end{array}$ & $\begin{array}{l}\text { MANGA- } \\
\text { NESE, } \\
\text { DIS- } \\
\text { SOLVED } \\
\text { ( } \mu G / L \\
\text { AS MN) }\end{array}$ & $\begin{array}{l}\text { MOLYB- } \\
\text { DENUM, } \\
\text { DIS- } \\
\text { SOLVED } \\
(\mu G / L \\
\text { AS MO) }\end{array}$ & $\begin{array}{l}\text { NICKEL, } \\
\text { DIS- } \\
\text { SOLVED } \\
(\mu G / L \\
\text { AS NI) }\end{array}$ & $\begin{array}{l}\text { SILVER, } \\
\text { DIS- } \\
\text { SOLVED } \\
(\mu G / L \\
\text { AS AG) }\end{array}$ & $\begin{array}{c}\text { STRON- } \\
\text { TIUM, } \\
\text { DIS- } \\
\text { SOLVED } \\
\text { ( } \mu G / L \\
\text { AS SR) }\end{array}$ & $\begin{array}{l}\text { VANA- } \\
\text { DIUM, } \\
\text { DIS- } \\
\text { SOLVED } \\
(\mu G / L \\
\text { AS V) }\end{array}$ & $\begin{array}{l}\text { ZINC, } \\
\text { DIS- } \\
\text { SOLVED } \\
\text { ( } \mu G / L \\
\text { AS ZN) }\end{array}$ \\
\hline \multicolumn{12}{|c|}{ WELL IDENTIFICATION - SCI287--Continued } \\
\hline \multicolumn{2}{|l|}{$\begin{array}{c}\text { NOV } 1987 \\
07 \ldots \\
\text { MAR } 1988\end{array}$} & $<10$ & 39 & $<10$ & 66 & $<10$ & $<10$ & $<1.0$ & 320 & $<6$ & $<3$ \\
\hline \multicolumn{2}{|l|}{$\begin{array}{c}08 \ldots \\
\mathrm{APR}\end{array}$} & $<10$ & 18 & $<10$ & 30 & $<10$ & $<10$ & 2.0 & 360 & $<6$ & $<3$ \\
\hline \multicolumn{2}{|l|}{$12 \ldots$} & $<30$ & 23 & $<30$ & 31 & $<30$ & $<10$ & $<3.0$ & 550 & $<18$ & $<9$ \\
\hline \multicolumn{2}{|l|}{${ }_{\text {AUG }}^{11 \ldots}$} & $<10$ & 38 & $<10$ & 50 & $<10$ & $<10$ & $<1.0$ & 450 & $<6$ & $<3$ \\
\hline \multicolumn{2}{|l|}{$\begin{array}{l}31 \ldots \\
\text { Nov }\end{array}$} & $<10$ & 40 & $<10$ & 36 & $<10$ & $<10$ & $<1.0$ & 460 & $<6$ & $<3$ \\
\hline \multicolumn{2}{|l|}{$\begin{array}{l}15 \ldots \\
\text { APR } 1989\end{array}$} & $<10$ & 66 & $<10$ & 58 & $<10$ & $<10$ & $<1.0$ & 490 & $<6$ & 35 \\
\hline \multicolumn{2}{|l|}{$\begin{array}{c}05 . \cdots \\
\text { JUNE }\end{array}$} & $<30$ & 25 & $<30$ & 19 & $<30$ & 40 & $<3.0$ & 750 & $<18$ & 10 \\
\hline \multicolumn{2}{|l|}{ SEPT } & $<30$ & 18 & $<30$ & 30 & $<30$ & $<30$ & $<3.0$ & 690 & $<18$ & $<9$ \\
\hline \multicolumn{2}{|l|}{$18 \ldots$} & $<20$ & 17 & $<20$ & 28 & $<20$ & $<20$ & $<2.0$ & 710 & $<12$ & $<6$ \\
\hline \multirow[t]{2}{*}{ DATE } & TIME & $\begin{array}{c}\text { DEPTH } \\
\text { OF } \\
\text { WELL, } \\
\text { TOTAL } \\
\text { (FEET) }\end{array}$ & $\begin{array}{c}\text { ELEVATION } \\
\text { OF LAND } \\
\text { SURFACE } \\
\text { DATUM } \\
\text { (FEET } \\
\text { ABOVE } \\
\text { SEA } \\
\text { LEVEL) }\end{array}$ & $\begin{array}{l}\text { SPE- } \\
\text { CIFIC } \\
\text { CON- } \\
\text { DUCT- } \\
\text { ANCE } \\
(\mu S / C M)\end{array}$ & $\begin{array}{c}\text { PH } \\
\text { (STAND- } \\
\text { ARD } \\
\text { UNITS) }\end{array}$ & $\begin{array}{c}\text { TEMPER- } \\
\text { ATURE } \\
\text { WATER } \\
\left({ }^{\circ} \mathrm{C}\right)\end{array}$ & $\begin{array}{l}\text { HARD- } \\
\text { NESS } \\
\text { TOTAL } \\
\text { ( } \mathrm{MG} / \mathrm{L} \\
\text { AS } \\
\left.\mathrm{CACO}_{3}\right)\end{array}$ & $\begin{array}{l}\text { HARD- } \\
\text { NESS } \\
\text { NONCARB } \\
\text { WH WAT } \\
\text { TOT FLD } \\
(\mathrm{MG} / \mathrm{L} \text { AS } \\
\left.\mathrm{CACO}_{3}\right)\end{array}$ & $\begin{array}{c}\text { CALCIUM } \\
\text { DIS- } \\
\text { SOLVED } \\
\text { (MG/L } \\
\text { AS CA) }\end{array}$ & $\begin{array}{l}\text { MAGNE- } \\
\text { SIUM, } \\
\text { DIS- } \\
\text { SOLVED } \\
\text { (MG/L } \\
\text { AS MG) }\end{array}$ & $\begin{array}{c}\text { SODIUM, } \\
\text { DIS- } \\
\text { SOLVED } \\
\text { (MG/L } \\
\text { AS NA) }\end{array}$ \\
\hline \multirow{2}{*}{\multicolumn{12}{|c|}{ WELL IDENTIFICATION - SCW287 }} \\
\hline & & & & & & & & & & & \\
\hline $\begin{array}{l}07 \ldots \\
\text { MAR } 1988\end{array}$ & 1515 & 90.00 & 6,957 & 1,250 & 8.3 & -- & 59 & 0 & 11 & 7.4 & 300 \\
\hline $\begin{array}{c}08 \ldots \\
\text { APR }\end{array}$ & 1130 & 90.00 & 6,957 & 1,470 & 8.2 & 9.0 & 58 & 0 & 8.7 & 8.5 & 330 \\
\hline $12 \ldots$ & 1115 & 90.00 & 6,957 & 1,480 & 8.6 & 9.5 & 57 & 0 & 8.4 & 8.5 & 340 \\
\hline $11 \ldots$ & 0900 & 90.00 & 6,957 & 1,570 & 8.5 & 9.5 & 57 & 0 & 8.6 & 8.4 & 350 \\
\hline${ }_{\text {Nov }}^{31 \ldots}$ & 1400 & 90.00 & 6,957 & 1,580 & 8.5 & 10.5 & 71 & 0 & 10 & 11 & 370 \\
\hline $\begin{array}{l}15 \ldots \\
\text { APR } 1989\end{array}$ & 1530 & 90.00 & 6,957 & 1,890 & 8.5 & -- & 79 & 0 & 13 & 11 & 400 \\
\hline$\underset{\text { JUNE }}{05}$ & 1030 & 90.00 & 6,957 & 2,170 & 8.4 & 9.0 & 96 & 0 & 15 & 14 & 440 \\
\hline$\underset{\text { SEPT }}{06 . . .}$ & 0945 & 90.00 & 6,957 & 2,180 & 8.3 & 9.5 & 100 & 0 & 16 & 15 & 470 \\
\hline $18 \ldots$ & 1630 & 90.00 & 6,957 & 2,230 & 8.3 & 10.0 & 110 & 0 & 16 & 16 & 480 \\
\hline
\end{tabular}


Table 25.--Water-quality analyses for wells--Continued

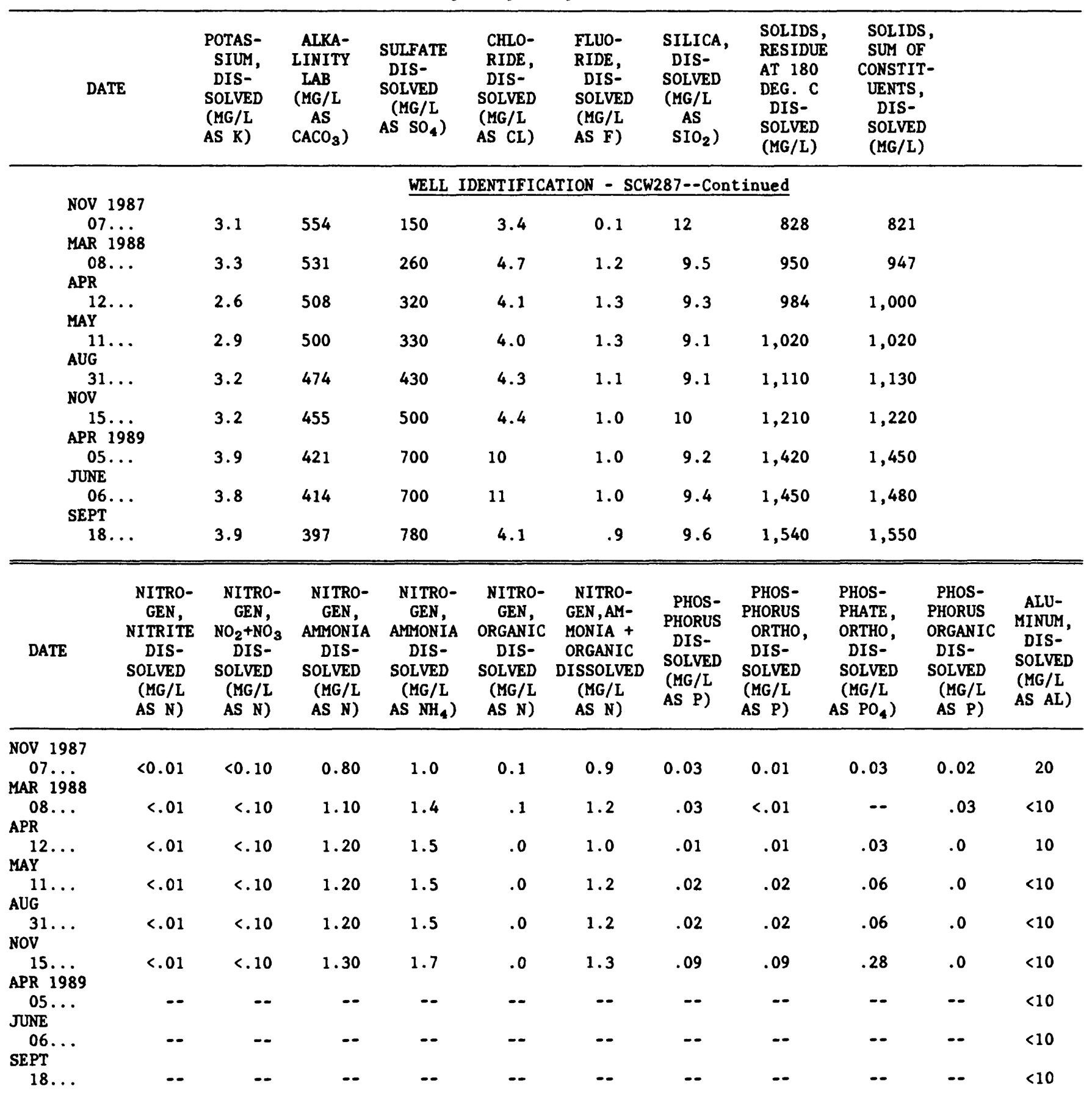


Table 25.--Water-quality analyses for wells--Continued

\begin{tabular}{|c|c|c|c|c|c|c|c|c|c|c|c|}
\hline DATE & $\begin{array}{l}\text { ARSENIC } \\
\text { DIS- } \\
\text { SOLVED } \\
(\mu G / L \\
\text { AS AS) }\end{array}$ & $\begin{array}{l}\text { BARIUM, } \\
\text { DIS- } \\
\text { SOLVED } \\
(\mu G / L \\
\text { AS BA) }\end{array}$ & $\begin{array}{l}\text { BERYL- } \\
\text { LIUM, } \\
\text { DIS- } \\
\text { SOLVED } \\
(\mu G / L \\
\text { AS BE) }\end{array}$ & $\begin{array}{l}\text { BORON, } \\
\text { DIS- } \\
\text { SOLVED } \\
(\mu G / L \\
\text { AS B) }\end{array}$ & $\begin{array}{c}\text { CADMIUM } \\
\text { DIS- } \\
\text { SOLVED } \\
(\mu G / L \\
\text { AS CD) }\end{array}$ & $\begin{array}{l}\text { CHRO- } \\
\text { MIUM, } \\
\text { DIS- } \\
\text { SOLVED } \\
(\mu G / L \\
\text { AS CR) }\end{array}$ & $\begin{array}{l}\text { COBALT, } \\
\text { DIS- } \\
\text { SOLVED } \\
(\mu G / L \\
\text { AS CO) }\end{array}$ & $\begin{array}{l}\text { COPPER, } \\
\text { DIS- } \\
\text { SOLVED } \\
(\mu G / L \\
\text { AS CU) }\end{array}$ & $\begin{array}{l}\text { IRON, } \\
\text { DIS- } \\
\text { SOLVED } \\
(\mu G / L \\
\text { AS FE) }\end{array}$ & $\begin{array}{l}\text { LEAD, } \\
\text { DIS- } \\
\text { SOLVED } \\
\text { ( } \mu G / L \\
\text { AS PB) }\end{array}$ & $\begin{array}{c}\text { MANGA- } \\
\text { NESE, } \\
\text { DIS- } \\
\text { SOLVED } \\
\text { ( } \mu G / L \\
\text { AS MN) }\end{array}$ \\
\hline \multicolumn{12}{|c|}{ WELL IDENTIFICATION - SCW287--Continued } \\
\hline NOV 1987 & & & & & & & & & & & \\
\hline $\begin{array}{l}07 \ldots \\
\text { MAR } 1988\end{array}$ & $<1$ & 130 & $<0.5$ & 160 & $<1$ & $<5$ & $<3$ & $<10$ & 46 & 10 & 55 \\
\hline$\underset{\text { APR }}{08 \ldots}$ & $<1$ & 130 & $<.5$ & 150 & $<1$ & $<5$ & $<3$ & $<10$ & 11 & $<10$ & 14 \\
\hline $\operatorname{MAY}^{12 \ldots}$ & $<1$ & 94 & $<.5$ & 150 & $<1$ & $<5$ & $<3$ & $<10$ & 7 & $<10$ & 8 \\
\hline${ }_{A U G}^{11 \ldots}$ & $<1$ & 74 & $<.5$ & 150 & $<1$ & $<5$ & $<3$ & $<10$ & 7 & $<10$ & 17 \\
\hline $\operatorname{Nov}^{31 \ldots}$ & $<1$ & 74 & $<.5$ & 140 & $<1$ & $<5$ & $<3$ & $<10$ & 10 & $<10$ & 15 \\
\hline $\begin{array}{l}15 \ldots \\
\text { APR } 1989\end{array}$ & 1 & 71 & $<.5$ & 150 & $<1$ & $<5$ & $<3$ & $<10$ & 13 & $<10$ & 59 \\
\hline JUNE & $<1$ & 67 & $<2$ & 150 & $<3$ & $<20$ & $<9$ & $<30$ & 13 & 40 & 18 \\
\hline$\underset{\text { SEPT }}{06 \ldots}$ & $<1$ & 67 & $<2$ & 150 & $<3$ & $<20$ & $<9$ & $<30$ & 18 & $<30$ & 19 \\
\hline $18 \ldots$ & $<1$ & 63 & $<.5$ & 140 & $<1$ & $<5$ & $<3$ & $<10$ & 14 & $<10$ & 23 \\
\hline DATE & $\begin{array}{l}\text { MOLYB- } \\
\text { DENUM, } \\
\text { DIS- } \\
\text { SOLVED } \\
\text { ( } \mu \text { G/L } \\
\text { AS MO) }\end{array}$ & $\begin{array}{l}\text { NICKEL, } \\
\text { DIS- } \\
\text { SOLVED } \\
(\mu G / L \\
\text { AS NI) }\end{array}$ & $\begin{array}{l}\text { SILVER, } \\
\text { DIS- } \\
\text { SOLVED } \\
(\mu G / L \\
\text { AS AG) }\end{array}$ & $\begin{array}{l}\text { STRON- } \\
\text { TIUM, } \\
\text { DIS- } \\
\text { SOLVED } \\
\text { ( } \mu \text { G/L } \\
\text { AS SR) }\end{array}$ & $\begin{array}{l}\text { VANA- } \\
\text { DIUM, } \\
\text { DIS- } \\
\text { SOLVED } \\
(\mu G / L \\
\text { AS V) }\end{array}$ & $\begin{array}{c}\text { ZINC, } \\
\text { DIS- } \\
\text { SOLVED } \\
(\mu G / L \\
\text { AS ZN) }\end{array}$ & $\begin{array}{c}\text { C-13/ } \\
\text { C-12 } \\
\text { STABLE } \\
\text { ISOTOPE } \\
\text { RATIO } \\
\text { PER } \\
\text { MIL }\end{array}$ & $\begin{array}{l}\text { H-2/ } \\
\text { H-1 } \\
\text { STABLE } \\
\text { ISOTOPE } \\
\text { RATIO } \\
\text { PER } \\
\text { MIL }\end{array}$ & $\begin{array}{l}0-18 / \\
0-16 \\
\text { STABLE } \\
\text { ISOTOPE } \\
\text { RATIO } \\
\text { PER } \\
\text { MIL }\end{array}$ & $\begin{array}{c}\text { S-34/ } \\
\text { S-32 } \\
\text { STABLE } \\
\text { ISOTOPE } \\
\text { RATIO } \\
\text { PER } \\
\text { MIL }\end{array}$ & $\begin{array}{l}\text { TRIT- } \\
\text { IUM } \\
\text { TOTAL } \\
(\mathrm{PCI} / \mathrm{L})\end{array}$ \\
\hline \multicolumn{12}{|l|}{ NOV 1987} \\
\hline $\begin{array}{l}07 \ldots \\
\text { MAR } 1988\end{array}$ & $<10$ & $<10$ & $<1.0$ & 420 & $<6$ & $<3$ & -- & -- & -- & - & $\cdots$ \\
\hline $\begin{array}{l}08 \ldots \\
\text { APR }\end{array}$ & $<10$ & $<10$ & $<1.0$ & 600 & $<6$ & $<3$ & -- & - & - & - & - \\
\hline${ }_{\mathrm{MAY}}^{12 \ldots}$ & $<10$ & $<10$ & $<1.0$ & 630 & $<6$ & $<3$ & -21.6 & -142 & -18.8 & -4.7 & -- \\
\hline${ }_{\text {AUG }}^{11 \ldots}$ & $<10$ & $<10$ & 2.0 & 630 & $<6$ & $<3$ & -- & -- & -- & -- & -- \\
\hline$\underset{\text { Nov }}{31 \ldots}$ & $<10$ & $<10$ & 2.0 & 730 & $<6$ & 5 & -21.8 & -142 & -18.8 & 11.30 & 26 \\
\hline $\begin{array}{c}15 \ldots \\
\text { APR } 1989\end{array}$ & $<10$ & $<10$ & $<1.0$ & 680 & $<6$ & $<3$ & -- & -- & -- & -- & -- \\
\hline$\underset{\text { JUNE }}{05 \ldots}$ & $<30$ & $<30$ & $<3.0$ & 1,000 & $<18$ & $<9$ & -- & -- & -- & -- & -- \\
\hline $\begin{array}{l}06 \ldots \\
\text { SEPT }\end{array}$ & $<30$ & $<30$ & $<3.0$ & 1,100 & $<18$ & $<9$ & $\cdots$ & -- & -- & -- & -- \\
\hline $18 \ldots$ & $<10$ & $<10$ & $<1.0$ & 1,100 & $<6$ & $<3$ & -- & -- & -- & -- & -- \\
\hline
\end{tabular}


Table 25.--Water-quality analyses for wells--Continued

\begin{tabular}{|c|c|c|c|c|c|c|c|c|c|c|c|}
\hline DATE & TIME & $\begin{array}{l}\text { DEPTH } \\
\text { OF } \\
\text { WELL, } \\
\text { TOTAL } \\
\text { (FEET) }\end{array}$ & $\begin{array}{l}\text { ELEVATION } \\
\text { OF LAND } \\
\text { SURFACE } \\
\text { DATUM } \\
\text { (FEET } \\
\text { ABOVE } \\
\text { SEA } \\
\text { LEVEL) }\end{array}$ & $\begin{array}{l}\text { SPE- } \\
\text { CIFIC } \\
\text { CON- } \\
\text { DUCT- } \\
\text { ANCE } \\
(\mu S / C M)\end{array}$ & $\begin{array}{c}\text { PH } \\
\text { (STAND- } \\
\text { ARD } \\
\text { UNITS) }\end{array}$ & $\begin{array}{l}\text { TEMPER- } \\
\text { ATURE } \\
\text { WATER } \\
\left({ }^{\circ} \mathrm{C}\right)\end{array}$ & $\begin{array}{l}\text { HARD- } \\
\text { NESS } \\
\text { TOTAL } \\
(\mathrm{MG} / \mathrm{L} \\
\mathrm{AS} \\
\left.\mathrm{CACO}_{3}\right)\end{array}$ & $\begin{array}{l}\text { HARD- } \\
\text { NESS } \\
\text { NONCARB } \\
\text { WH WAT } \\
\text { TOT FLD } \\
(\mathrm{MG} / \mathrm{L} \text { AS } \\
\left.\mathrm{CACO}_{3}\right)\end{array}$ & $\begin{array}{l}\text { CALCIUM } \\
\text { DIS- } \\
\text { SOLVED } \\
\text { (MG/L } \\
\text { AS CA) }\end{array}$ & $\begin{array}{l}\text { MAGNE- } \\
\text { SIUM, } \\
\text { DIS- } \\
\text { SOLVED } \\
\text { (MG/L } \\
\text { AS MG) }\end{array}$ & $\begin{array}{l}\text { SODIUM, } \\
\text { DIS- } \\
\text { SOLVED } \\
\text { (MG/L } \\
\text { AS NA) }\end{array}$ \\
\hline \multicolumn{12}{|c|}{ WELL IDENTIFICATION - SCU287 } \\
\hline $\begin{array}{c}\text { NOV } 1987 \\
\text { O7 . } \\
\text { MAR } 1988\end{array}$ & 1430 & 122.00 & 6,957 & 1,010 & 8.2 & -- & 9 & 0 & 2.4 & 0.63 & 240 \\
\hline $\begin{array}{c}08 \ldots \\
\text { APR }\end{array}$ & 0920 & 122.00 & 6,957 & 1,010 & 8.3 & 9.5 & 11 & 0 & 3.1 & .85 & 290 \\
\hline $12 \ldots$ & 1230 & 122.00 & 6,957 & 1,490 & 8.4 & 10.5 & 21 & 0 & 5.7 & 1.6 & 360 \\
\hline JUNE & 0845 & 122.00 & 6,957 & 1,430 & 8.5 & 10.5 & 18 & 0 & 4.9 & 1.3 & 340 \\
\hline $\begin{array}{l}16 \ldots \\
16 \ldots \\
\text { AUG }\end{array}$ & $\begin{array}{l}1040 \\
1050\end{array}$ & $\begin{array}{l}122.00 \\
122.00\end{array}$ & $\begin{array}{l}6,957 \\
6,957\end{array}$ & $\begin{array}{l}1,510 \\
1,560\end{array}$ & $\begin{array}{l}8.4 \\
8.6\end{array}$ & $\begin{array}{l}17.5 \\
11.0\end{array}$ & $\begin{array}{l}15 \\
14\end{array}$ & $\begin{array}{l}0 \\
0\end{array}$ & $\begin{array}{l}4.1 \\
3.9\end{array}$ & $\begin{array}{l}1.1 \\
1.0\end{array}$ & $\begin{array}{l}300 \\
290\end{array}$ \\
\hline NOV & 1030 & 122.00 & 6,957 & 1,130 & 8.6 & 10.5 & 12 & 0 & 3.2 & .85 & 280 \\
\hline $\begin{array}{l}15 \ldots \\
\text { APR } 1989\end{array}$ & 1400 & 122.00 & 6,957 & 1,220 & 8.7 & - & 12 & 0 & 3.2 & .86 & 280 \\
\hline JUNE & 0930 & 122.00 & 6,957 & 1,150 & 8.7 & 10.0 & 11 & 0 & 2.9 & .83 & 250 \\
\hline$\underset{\text { SEPT }}{06 . .}$ & 0930 & 122.00 & 6,957 & 1,160 & 8.7 & 10.5 & 10 & 0 & 2.8 & .71 & 270 \\
\hline $18 \ldots$ & 1515 & 122.00 & 6,957 & 1,080 & 8.7 & 10.5 & 10 & 0 & 2.9 & .74 & 270 \\
\hline DATE & & $\begin{array}{l}\text { POTAS- } \\
\text { SIUM, } \\
\text { DIS- } \\
\text { SOLVED } \\
\text { (MG/L } \\
\text { AS K) }\end{array}$ & $\begin{array}{c}\text { ALKA- } \\
\text { LINITY } \\
\text { LAB } \\
(\mathrm{MG} / \mathrm{L} \\
\mathrm{AS} \\
\left.\mathrm{CACO}_{3}\right)\end{array}$ & $\begin{array}{l}\text { SULFATE } \\
\text { DIS- } \\
\text { SOLVED } \\
(\mathrm{MG} / \mathrm{L} \\
\left.\text { AS } \mathrm{SO}_{4}\right)\end{array}$ & $\begin{array}{l}\text { CHLO- } \\
\text { RIDE, } \\
\text { DIS- } \\
\text { SOLVED } \\
\text { (MG/L } \\
\text { AS CL) }\end{array}$ & $\begin{array}{l}\text { FLUO- } \\
\text { RIDE, } \\
\text { DIS- } \\
\text { SOLVED } \\
\text { (MG/L } \\
\text { AS F) }\end{array}$ & $\begin{array}{l}\text { SILICA, } \\
\text { DIS- } \\
\text { SOLVED } \\
\text { (MG/L } \\
\text { AS } \\
\mathrm{SIO}_{2} \text { ) }\end{array}$ & $\begin{array}{l}\text { SOLIDS, } \\
\text { RESIDUE } \\
\text { AT 180 C } \\
\text { DEG. C } \\
\text { DIS- } \\
\text { SOLVED } \\
\text { (MG/L) }\end{array}$ & $\begin{array}{l}\text { SOLIDS, } \\
\text { SUM OF } \\
\text { CONSTIT- } \\
\text { UENTS, } \\
\text { DIS- } \\
\text { SOLVED } \\
\text { (MG/L) }\end{array}$ & & \\
\hline \multicolumn{12}{|l|}{ NOV 1987} \\
\hline $\begin{array}{l}07 \\
\text { MAR } 1988\end{array}$ & & 1.8 & 475 & 98 & 3.3 & 1.0 & 7.5 & 656 & 640 & & \\
\hline$\underset{\text { APR }}{08 \ldots}$ & & 2.3 & 472 & 140 & 5.0 & .8 & 8.0 & 724 & 733 & & \\
\hline${ }_{\mathrm{MAY}}^{12 \ldots}$ & & 2.5 & 513 & 300 & 7.3 & .6 & 9.8 & 989 & 996 & & \\
\hline$\frac{11 \ldots}{\text { JUNE }}$ & & 2.5 & 503 & 250 & 6.9 & .6 & 9.5 & 909 & 918 & & \\
\hline $\begin{array}{l}16 \ldots \\
16 \ldots \\
\text { AUG }\end{array}$ & & $\begin{array}{l}2.1 \\
2.1\end{array}$ & $\begin{array}{l}588 \\
463\end{array}$ & $\begin{array}{l}110 \\
220\end{array}$ & $\begin{array}{l}4.9 \\
6.5\end{array}$ & $\begin{array}{r}2.2 \\
.8\end{array}$ & $\begin{array}{l}8.4 \\
7.9\end{array}$ & $\begin{array}{l}811 \\
825\end{array}$ & $\begin{array}{l}786 \\
811\end{array}$ & & \\
\hline${ }_{\mathrm{NOV}}^{31 \ldots}$ & & 2.1 & 467 & 180 & 6.8 & .8 & 7.7 & 758 & 762 & & \\
\hline $\begin{array}{l}15 \\
A P R \\
1989\end{array}$ & & 1.9 & $465^{\circ}$ & 140 & 7.4 & 1.0 & 7.9 & 733 & 722 & & \\
\hline JUNE & & 1.9 & 473 & 130 & 6.3 & 1.2 & 7.4 & 702 & 684 & & \\
\hline $\begin{array}{c}06 \ldots \\
\text { SEPT }\end{array}$ & & 1.8 & 471 & 120 & 6.5 & 1.1 & 7.4 & 688 & 693 & & \\
\hline $18 \ldots$ & & 1.9 & 462 & 120 & 6.4 & 1.2 & 7.3 & 682 & 688 & & \\
\hline
\end{tabular}


Table 25.--Water-quality analyses for wells--Continued

\begin{tabular}{|c|c|c|c|c|c|c|c|c|c|c|c|}
\hline DATE & $\begin{array}{l}\text { NITRO- } \\
\text { GEN, } \\
\text { NITRITE } \\
\text { DIS- } \\
\text { SOLVED } \\
\text { (MG/L } \\
\text { AS N) }\end{array}$ & $\begin{array}{c}\text { NITRO- } \\
\text { GEN, } \\
\text { NITRITE } \\
\text { DIS- } \\
\text { SOLVED } \\
(\mathrm{MG} / \mathrm{L} \\
\left.\text { AS } \mathrm{NO}_{2}\right)\end{array}$ & $\begin{array}{c}\text { NITRO- } \\
\text { GEN, } \\
\mathrm{NO}_{2}+\mathrm{NO}_{3} \\
\text { DIS- } \\
\text { SOLVED } \\
\text { (MG/L } \\
\text { AS N) }\end{array}$ & $\begin{array}{l}\text { NITRO- } \\
\text { GEN, } \\
\text { AMMONIA } \\
\text { DIS- } \\
\text { SOLVED } \\
\text { (MG/L } \\
\text { AS N) }\end{array}$ & $\begin{array}{c}\text { NITRO- } \\
\text { GEN, } \\
\text { AMONIA } \\
\text { DIS- } \\
\text { SOLVED } \\
(\mathrm{MG} / \mathrm{L} \\
\text { AS NH4 })\end{array}$ & $\begin{array}{l}\text { NITRO- } \\
\text { GEN, } \\
\text { ORGANIC } \\
\text { DIS- } \\
\text { SOLVED D } \\
\text { (MG/L } \\
\text { AS N) }\end{array}$ & $\begin{array}{l}\text { NITRO- } \\
\text { GEN,AM- } \\
\text { MONIA + } \\
\text { ORGANIC } \\
\text { DISSOLVED } \\
\text { (MG/L } \\
\text { AS N) }\end{array}$ & $\begin{array}{l}\text { PHOS- } \\
\text { PHORUS } \\
\text { DIS- } \\
\text { SOLVED } \\
\text { (MG/L } \\
\text { AS P) }\end{array}$ & $\begin{array}{l}\text { PHOS- } \\
\text { PHORUS } \\
\text { ORTHO, } \\
\text { DIS- } \\
\text { SOLVED } \\
\text { (MG/L } \\
\text { AS P) }\end{array}$ & $\begin{array}{l}\text { PHOS- } \\
\text { PHATE, } \\
\text { ORTHO, } \\
\text { DIS- } \\
\text { SOLVED } \\
\text { (MG/L } \\
\text { AS PO4) }\end{array}$ & $\begin{array}{l}\text { PHOS- } \\
\text { PHORUS } \\
\text { ORGANIC } \\
\text { DIS- } \\
\text { SOLVED } \\
\text { (MG/L } \\
\text { AS P) }\end{array}$ \\
\hline \multicolumn{12}{|c|}{ WELL IDENTIFICATION - SCU287--Continued } \\
\hline NOV 1987 & & & & & & & & & & & \\
\hline $\begin{array}{l}07 \ldots \\
\text { MAR } 1988\end{array}$ & 0.01 & 0.03 & $<0.10$ & 0.29 & 0.37 & 0.21 & 0.5 & 0.03 & 0.04 & 0.12 & 0.0 \\
\hline $\begin{array}{l}08 \ldots \\
\text { APR }\end{array}$ & $<.01$ & -- & $<.10$ & .03 & .04 & .77 & .8 & .02 & .03 & .09 & .0 \\
\hline $\mathrm{MAY}^{12 \ldots}$ & $<.01$ & -- & $<.10$ & .37 & .48 & .53 & .9 & .02 & .02 & .06 & .0 \\
\hline JUNE & $<.01$ & -- & $<.10$ & .21 & .27 & .79 & 1.0 & .02 & .02 & .06 & .0 \\
\hline $\begin{array}{l}16 \ldots \\
16 \ldots\end{array}$ & $\begin{array}{l}.01 \\
.01\end{array}$ & $\begin{array}{l}.03 \\
.03\end{array}$ & $\begin{array}{l}<.10 \\
<.10\end{array}$ & $\begin{array}{l}.12 \\
.15\end{array}$ & $\begin{array}{l}.15 \\
.19\end{array}$ & $\begin{array}{l}.18 \\
--\end{array}$ & $\begin{array}{r}.3 \\
<.2\end{array}$ & $\begin{array}{l}.03 \\
.02\end{array}$ & $\begin{array}{l}.04 \\
.04\end{array}$ & $\begin{array}{l}.12 \\
.12\end{array}$ & $\begin{array}{l}.0 \\
.0\end{array}$ \\
\hline AUG & & & & & & & & & & & \\
\hline $\begin{array}{l}31 \ldots \\
\text { NOV }\end{array}$ & $<.01$ & -- & $<.10$ & .17 & .22 & .73 & .9 & .03 & .03 & .09 & .0 \\
\hline $\begin{array}{c}15 \ldots \\
\text { APR } 1989\end{array}$ & $<.01$ & -- & $<.10$ & .09 & .12 & -- & $<.2$ & .03 & .03 & .09 & .0 \\
\hline JUNE & -- & -- & -- & -- & -- & -- & -- & -- & -- & -- & -- \\
\hline $\begin{array}{c}06 \ldots \\
\text { SEPT }\end{array}$ & -- & -- & -- & -- & -- & -- & -- & -- & -- & -- & -- \\
\hline $18 \ldots$ & -- & -- & -- & -- & -- & -- & -- & -- & -- & -- & -- \\
\hline DATE & $\begin{array}{c}\text { ALU- } \\
\text { MINUM, } \\
\text { DIS- } \\
\text { SOLVED } \\
(\mu G / L \\
\text { AS AL) }\end{array}$ & $\begin{array}{l}\text { ARSENIC } \\
\text { DIS- } \\
\text { SOLVED } \\
(\mu G / L \\
\text { AS AS) }\end{array}$ & $\begin{array}{l}\text { BARIUM, } \\
\text { DIS- } \\
\text { SOLVED } \\
(\mu G / L \\
\text { AS BA) }\end{array}$ & $\begin{array}{l}\text { BERYL- } \\
\text { LIUM, } \\
\text { DIS- } \\
\text { SOLVED } \\
\text { ( } \mu \mathrm{G} / \mathrm{L} \\
\text { AS BE) }\end{array}$ & $\begin{array}{l}\text { BORON, } \\
\text { DIS- } \\
\text { SOLVED } \\
(\mu G / L \\
\text { AS B) }\end{array}$ & $\begin{array}{l}\text { CADMIUM } \\
\text { DIS- } \\
\text { SOLVED } \\
(\mu G / L \\
\text { AS CD) }\end{array}$ & $\begin{array}{l}\text { CHRO- } \\
\text { MIUM, } \\
\text { DIS- } \\
\text { SOLVED } \\
(\mu G / L \\
\text { AS CR) }\end{array}$ & $\begin{array}{l}\text { COBALT, } \\
\text { DIS- } \\
\text { SOLVED } \\
(\mu G / L \\
\text { AS CO })\end{array}$ & $\begin{array}{l}\text { COPPER, } \\
\text { DIS- } \\
\text { SOLVED } \\
(\mu G / L \\
\text { AS CU) }\end{array}$ & $\begin{array}{l}\text { IRON, } \\
\text { DIS- } \\
\text { SOLVED } \\
(\mu G / L \\
\text { AS FE) }\end{array}$ & $\begin{array}{l}\text { LEAD, } \\
\text { DIS- } \\
\text { SOLVED } \\
(\mu G / L \\
\text { AS PB) }\end{array}$ \\
\hline \multicolumn{12}{|l|}{ NOV 1987} \\
\hline $\begin{array}{l}07 \ldots \\
\text { MAR } 1988\end{array}$ & $<10$ & $<1$ & 66 & $<0.5$ & 50 & 1 & $<5$ & $<3$ & $<10$ & 14 & $<10$ \\
\hline APR 08 & $\ldots<10$ & $<1$ & 98 & $<.5$ & 40 & 2 & $<5$ & $<3$ & $<10$ & 23 & $<10$ \\
\hline${ }_{\mathrm{MAY}}^{12 \ldots}$ & $<10$ & $<1$ & 190 & $<.5$ & 60 & 3 & $<5$ & $<3$ & $<10$ & 36 & $<10$ \\
\hline${ }_{\text {JNNE }}^{11}$ & $<10$ & $<1$ & 160 & $<.5$ & 60 & $<1$ & $<5$ & $<3$ & $<10$ & 29 & $<10$ \\
\hline $16 \ldots$ & $<10$ & $<1$ & 110 & $<.5$ & 90 & $<1$ & $<5$ & $<3$ & $<10$ & 51 & $<10$ \\
\hline AUG $16 \ldots$ & $<10$ & $<1$ & 120 & $<.5$ & 60 & $<1$ & $<5$ & $<3$ & $<10$ & 26 & $<10$ \\
\hline $\begin{array}{l}31 \ldots \\
\text { NOV }\end{array}$ & $<10$ & $<1$ & 98 & $<.5$ & 40 & $<1$ & $<5$ & $<3$ & $<10$ & 18 & $<10$ \\
\hline $\begin{array}{c}15 \ldots \\
\text { APR } 1989\end{array}$ & $<10$ & $<1$ & 94 & $<.5$ & 40 & $<1$ & $<5$ & $<3$ & $<10$ & 21 & $<10$ \\
\hline$\underset{\text { JUNE }}{05 \ldots}$ & $<10$ & $<1$ & 80 & $<.5$ & 40 & $<1$ & $<5$ & $<3$ & $<10$ & 16 & $<10$ \\
\hline $\begin{array}{c}06 \\
\text { SEPT }\end{array}$ & $<10$ & $<1$ & 82 & $<.5$ & 50 & $<1$ & $<5$ & $<3$ & $<10$ & 18 & $<10$ \\
\hline $18 \ldots$ & $<10$ & 2 & 78 & $<.5$ & 40 & $<1$ & $<5$ & $<3$ & $<10$ & 19 & $<10$ \\
\hline
\end{tabular}


Table 25.--Water-quality analyses for wells--Continued

\begin{tabular}{|c|c|c|c|c|c|c|c|c|c|c|c|c|}
\hline DATE & $\begin{array}{l}\text { MANGA- } \\
\text { NESE, } \\
\text { DIS- } \\
\text { SOLVED } \\
\text { ( } \mu G / L \\
\text { AS MN) }\end{array}$ & $\begin{array}{l}\text { MOLYB- } \\
\text { DENUM, } \\
\text { DIS- } \\
\text { SOLVED } \\
(\mu G / L \\
\text { AS MO) }\end{array}$ & $\begin{array}{l}\text { NICKEL, } \\
\text { DIS- } \\
\text { SOLVED } \\
\text { ( } \mu G / L \\
\text { AS NI) }\end{array}$ & $\begin{array}{l}\text { SILVER, } \\
\text { DIS- } \\
\text { SOLVED } \\
(\mu G / L \\
\text { AS AG) }\end{array}$ & $\begin{array}{c}\text { STRON- } \\
\text { TIUM, } \\
\text { DIS- } \\
\text { SOLVED } \\
\text { ( } \mu \text { G/L } \\
\text { AS SR) }\end{array}$ & $\begin{array}{l}\text { VANA- } \\
\text { DIUM, } \\
\text { DIS- } \\
\text { SOLVED } \\
(\mu G / L \\
\text { AS V) }\end{array}$ & $\begin{array}{l}\text { ZINC, } \\
\text { DIS- } \\
\text { SOLVED } \\
\text { ( } \mu G / L \\
\text { AS ZN) }\end{array}$ & $\begin{array}{l}\text { C-13/ } \\
\text { C-12 } \\
\text { STABLE } \\
\text { ISOTOPE } \\
\text { RATIO } \\
\text { PER } \\
\text { MIL }\end{array}$ & $\begin{array}{l}\text { H-2/ } \\
\text { H-1 } \\
\text { STABLE } \\
\text { ISOTOPE } \\
\text { RATIO } \\
\text { PER } \\
\text { MIL }\end{array}$ & $\begin{array}{l}0-18 / \\
0-16 \\
\text { STABLE } \\
\text { ISOTOPE } \\
\text { RATIO } \\
\text { PER } \\
\text { MIL }\end{array}$ & $\begin{array}{c}\text { S-34/ } \\
\text { S-32 } \\
\text { STABLE } \\
\text { ISOTOPE } \\
\text { RATIO } \\
\text { PER } \\
\text { MIL }\end{array}$ & $\begin{array}{c}\text { TRIT- } \\
\text { IUM } \\
\text { TOTAL } \\
(\mathrm{PCI} / \mathrm{L})\end{array}$ \\
\hline
\end{tabular}

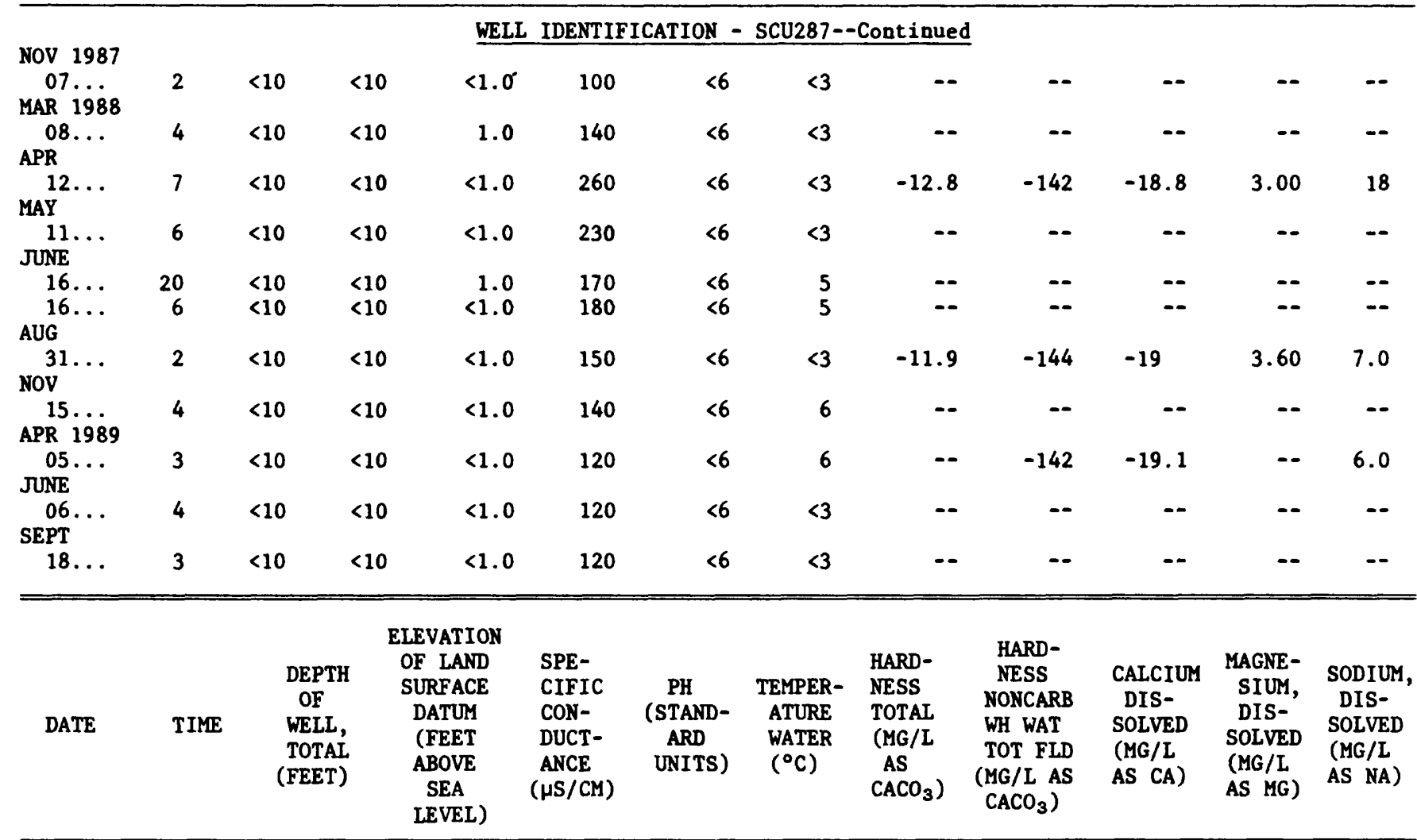

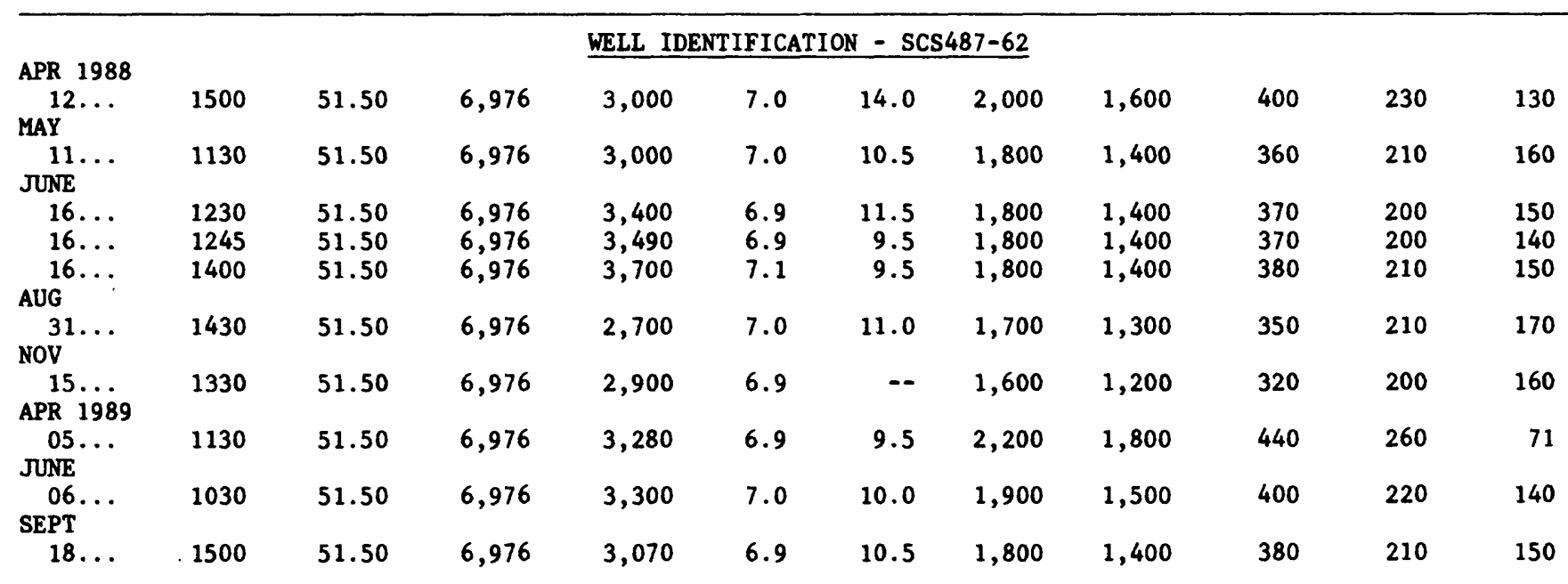


Table 25.--Water-quality analyses for wells--Continued

\begin{tabular}{|c|c|c|c|c|c|c|c|c|c|c|c|}
\hline DATE & & $\begin{array}{l}\text { POTAS- } \\
\text { SIUM, } \\
\text { DIS- } \\
\text { SOLVED } \\
\text { (MG/L } \\
\text { AS K) }\end{array}$ & $\begin{array}{c}\text { ALKA- } \\
\text { LINITY } \\
\text { LAB } \\
(\mathrm{MG} / \mathrm{L} \\
\mathrm{AS} \\
\left.\mathrm{CACO}_{3}\right)\end{array}$ & $\begin{array}{l}\text { SULFATE } \\
\text { DIS- } \\
\text { SOLVED } \\
(\mathrm{MG} / \mathrm{L} \\
\left.\text { AS } \mathrm{SO}_{4}\right)\end{array}$ & $\begin{array}{l}\text { CHLO- } \\
\text { RIDE, } \\
\text { DIS- } \\
\text { SOLVED } \\
\text { (MG/L } \\
\text { AS CL) }\end{array}$ & $\begin{array}{l}\text { FLUO- } \\
\text { RIDE, } \\
\text { DIS- } \\
\text { SOLVED } \\
\text { (MG/L } \\
\text { AS F) }\end{array}$ & $\begin{array}{l}\text { SILICA, } \\
\text { DIS- } \\
\text { SOLVED } \\
\text { (MG/L } \\
\text { AS } \\
\mathrm{SIO}_{2} \text { ) }\end{array}$ & $\begin{array}{l}\text { SOLIDS, } \\
\text { RESIDUE } \\
\text { AT } 180 \\
\text { DEG. C } \\
\text { DIS- } \\
\text { SOLVED } \\
\text { (MG/L) }\end{array}$ & $\begin{array}{l}\text { SOLIDS, } \\
\text { SUM OF } \\
\text { CONSTIT- } \\
\text { UENTS, } \\
\text { DIS- } \\
\text { SOLVED } \\
\text { (MG/L) }\end{array}$ & & \\
\hline \multicolumn{12}{|c|}{ WELL IDENTIFICATION - SCS487-62--Continued } \\
\hline $\begin{array}{c}\text { APR } 1988 \\
12 \ldots \\
\text { MAY }\end{array}$ & & 7.5 & 364 & 1,800 & 13 & 0.2 & 11 & 2,930 & 2,850 & & \\
\hline$\underset{\text { JUNE }}{11 \ldots}$ & & 7.5 & 363 & 1,800 & 14 & .3 & 12 & 2,890 & 2,810 & & \\
\hline $\begin{array}{l}16 \ldots \\
16 \ldots \\
16 \ldots \\
\text { AUG }\end{array}$ & & $\begin{array}{l}7.5 \\
7.8 \\
7.4\end{array}$ & $\begin{array}{l}391 \\
399 \\
392\end{array}$ & $\begin{array}{l}1,600 \\
1,600 \\
1,700\end{array}$ & $\begin{array}{l}15 \\
16 \\
15\end{array}$ & $\begin{array}{l}.3 \\
.2 \\
.3\end{array}$ & $\begin{array}{l}13 \\
13 \\
13\end{array}$ & $\begin{array}{l}2,780 \\
2,740 \\
2,870\end{array}$ & $\begin{array}{l}2,610 \\
2,600 \\
2,730\end{array}$ & & \\
\hline $\begin{array}{l}31 \ldots \\
\text { Nov }\end{array}$ & & 7.7 & 413 & 1,600 & 11 & .2 & 13 & 2,750 & 2,620 & & \\
\hline $\begin{array}{c}15 \\
\text { APR } 1989\end{array}$ & & 7.7 & 415 & 1,600 & 16 & .2 & 13 & 2,710 & 2,570 & & \\
\hline$\underset{\text { JUNE }}{05 \ldots}$ & & 7.8 & 362 & 1,900 & 15 & .2 & 10 & 2,930 & 2,990 & & \\
\hline $\begin{array}{c}06 \ldots \\
\text { SEPT }\end{array}$ & & 7.6 & 407 & 1,800 & 16 & .2 & 13 & 3,010 & 2,850 & & \\
\hline $18 \ldots$ & & 8.4 & 421 & 1,800 & 16 & .2 & 13 & 2,790 & 2,830 & & \\
\hline DATE & $\begin{array}{c}\text { NITRO- } \\
\text { GEN, } \\
\text { NITRATE } \\
\text { DIS- } \\
\text { SOLVED } \\
\text { (MG/L } \\
\text { AS N) }\end{array}$ & $\begin{array}{l}\text { NITRO- } \\
\text { GEN, } \\
\text { NITRITE } \\
\text { DIS- } \\
\text { SOLVED } \\
\text { (MG/L } \\
\text { AS N) }\end{array}$ & $\begin{array}{c}\text { NITRO- } \\
\text { GEN, } \\
\text { NITRITE } \\
\text { DIS- } \\
\text { SOLVED } \\
(\mathrm{MG} / \mathrm{L} \\
\left.\text { AS } \mathrm{NO}_{2}\right)\end{array}$ & $\begin{array}{c}\text { NITRO- } \\
\text { GEN, } \\
\mathrm{NO}_{2}+\mathrm{NO}_{3} \\
\text { DIS- } \\
\text { SOLVED } \\
(\mathrm{MG} / \mathrm{L} \\
\mathrm{AS} \mathrm{N})\end{array}$ & $\begin{array}{c}\text { NITRO- } \\
\text { GEN, } \\
\text { AMMONIA } \\
\text { DIS- } \\
\text { SOLVED } \\
\text { (MG/L } \\
\text { AS N) }\end{array}$ & $\begin{array}{l}\text { NITRO- } \\
\text { GEN, } \\
\text { AMMONIA } \\
\text { DIS- } \\
\text { SOLVED } \\
(\mathrm{MG} / \mathrm{L} \\
\text { AS NH } \\
\text { N })\end{array}$ & $\begin{array}{l}\text { NITRO- } \\
\text { GEN, } \\
\text { ORGANIC } \\
\text { DIS- } \\
\text { SOLVED } \\
\text { (MG/L } \\
\text { AS N) }\end{array}$ & $\begin{array}{l}\text { NITRO- } \\
\text { GEN,AM- } \\
\text { MONIA + } \\
\text { ORGANIC } \\
\text { DISSOLVED } \\
\text { (MG/L } \\
\text { AS N) }\end{array}$ & $\begin{array}{l}\text { PHOS- } \\
\text { PHORUS } \\
\text { DIS- } \\
\text { SOIVED } \\
\text { (MG/L } \\
\text { AS P) }\end{array}$ & $\begin{array}{l}\text { PHOS- } \\
\text { PHORUS } \\
\text { ORTHO, } \\
\text { DIS- } \\
\text { SOLVED } \\
\text { (MG/L } \\
\text { AS P) }\end{array}$ & $\begin{array}{l}\text { PHOS- } \\
\text { PHORUS } \\
\text { ORGANIC } \\
\text { DIS- } \\
\text { SOLVED } \\
\text { (MG/L } \\
\text { AS P) }\end{array}$ \\
\hline APR 1988 & & & & & & & & & & & \\
\hline${ }_{M A Y}^{12 \ldots}$ & -- & $<0.01$ & - & 8.30 & 0.29 & 0.37 & 0.61 & 0.9 & $<0.01$ & $<0.01$ & $=-$ \\
\hline${ }_{\text {JNE }}^{11 \ldots}$ & -- & $<.01$ & -- & 5.30 & .18 & .23 & .62 & .8 & $<.01$ & $<.01$ & -- \\
\hline $\begin{array}{l}16 \ldots \\
16 \ldots \\
16 \ldots \\
\text { AUG }\end{array}$ & $\begin{array}{l}2.28 \\
1.99 \\
2.58\end{array}$ & $\begin{array}{l}.02 \\
.01 \\
.02\end{array}$ & $\begin{array}{r}0.07 \\
.03 \\
.07\end{array}$ & $\begin{array}{l}2.30 \\
2.00 \\
2.60\end{array}$ & $\begin{array}{l}.29 \\
.53 \\
.24\end{array}$ & $\begin{array}{l}.37 \\
.68 \\
.31\end{array}$ & $\begin{array}{l}.81 \\
.77 \\
.76\end{array}$ & $\begin{array}{l}1.1 \\
1.3 \\
1.0\end{array}$ & $\begin{array}{l}<.01 \\
<.01 \\
<.01\end{array}$ & $\begin{array}{l}<.01 \\
<.01 \\
<.01\end{array}$ & $\begin{array}{l}-- \\
-- \\
--\end{array}$ \\
\hline $\mathrm{NOV}^{31 \ldots}$ & -- & $<.01$ & -- & .87 & .24 & .31 & .46 & .7 & $<.01$ & $<.01$ & -- \\
\hline $\begin{array}{l}15 \ldots \\
\text { APR } 1989\end{array}$ & -- & $<.01$ & -- & .71 & .26 & .33 & .34 & .6 & .03 & $<.01$ & 0.03 \\
\hline JUNE & -- & $<.01$ & -- & 14.0 & .07 & .09 & .53 & .6 & $<.01$ & $<.01$ & -- \\
\hline $\begin{array}{l}06 \ldots \\
\text { SEPT }\end{array}$ & -- & -- & -- & -- & -- & -- & -- & -- & -- & -- & -- \\
\hline $18 \ldots$ & -- & -- & -- & -- & -- & -- & -- & -- & -- & -- & -- \\
\hline
\end{tabular}


Table 25.--Water-quality analyses for wells--Continued

\begin{tabular}{|c|c|c|c|c|c|c|c|c|c|c|c|c|}
\hline \multicolumn{2}{|c|}{ DATE } & $\begin{array}{l}\text { ALU- } \\
\text { IINUM, } \\
\text { DIS- } \\
\text { SOLVED } \\
(\mu G / L \\
\text { AS AL) }\end{array}$ & $\begin{array}{l}\text { ARSENIC } \\
\text { DIS- } \\
\text { SOLVED } \\
(\mu G / L \\
\text { AS AS) }\end{array}$ & $\begin{array}{l}\text { BARIUM, } \\
\text { DIS- } \\
\text { SOLVED } \\
\text { ( } \mu G / L \\
\text { AS BA) }\end{array}$ & $\begin{array}{l}\text { BERYL- } \\
\text { LIUM, } \\
\text { DIS- } \\
\text { SOLVED } \\
\text { ( } \mu G / L \\
\text { AS BE) }\end{array}$ & $\begin{array}{l}\text { BORON, } \\
\text { DIS- } \\
\text { SOLVED } \\
(\mu G / L \\
\text { AS B) }\end{array}$ & $\begin{array}{l}\text { CADMIUM } \\
\text { DIS- } \\
\text { SOLVED } \\
\text { ( } \mu G / L \\
\text { AS CD) }\end{array}$ & $\begin{array}{l}\text { CHRO- } \\
\text { MIUM, } \\
\text { DIS- } \\
\text { SOLVED } \\
\text { ( } \mu G / L \\
\text { AS CR) }\end{array}$ & $\begin{array}{l}\text { COBALT, } \\
\text { DIS- } \\
\text { SOLVED } \\
\text { ( } \mu G / L \\
\text { AS CO) }\end{array}$ & $\begin{array}{l}\text { COPPER, } \\
\text { DIS- } \\
\text { SOLVED } \\
(\mu G / L \\
\text { AS CU) }\end{array}$ & $\begin{array}{l}\text { IRON, } \\
\text { DIS- } \\
\text { SOLVED } \\
\text { ( } \mu \text { G/L } \\
\text { AS FE) }\end{array}$ & $\begin{array}{l}\text { LEAD, } \\
\text { DIS- } \\
\text { SOLVED } \\
(\mu G / L \\
\text { AS PB) }\end{array}$ \\
\hline \multicolumn{13}{|c|}{ WELL IDENTIFICATION - SCS487-62--Cont inued } \\
\hline \multicolumn{4}{|c|}{ APR 1988} & & & & & & & & & \\
\hline \multicolumn{2}{|c|}{$12 \ldots$} & 20 & $<1$ & 50 & 2 & 210 & 4 & $<20$ & $<9$ & $<30$ & 15 & $<30$ \\
\hline \multicolumn{2}{|c|}{$\underset{\text { JUNE }}{11}$} & $<10$ & $<1$ & 45 & $<2$ & 210 & $<3$ & $<20$ & $<9$ & $<30$ & 22 & $<30$ \\
\hline \multirow{2}{*}{\multicolumn{2}{|c|}{$\begin{array}{l}16 . . \\
16 .\end{array}$}} & $<10$ & $<1$ & 49 & $<2$ & 210 & $<3$ & $<20$ & $<9$ & $<30$ & 14 & $<30$ \\
\hline & & $<10$ & $<1$ & 53 & $<2$ & 220 & $<3$ & $<20$ & $<9$ & $<30$ & 19 & $<30$ \\
\hline \multirow{2}{*}{\multicolumn{2}{|c|}{${ }_{\text {AUG }}^{16} \ldots$}} & $<10$ & $<1$ & 45 & $<2$ & 210 & $<3$ & $<20$ & $<3$ & $<30$ & 14 & $<30$ \\
\hline & & & & & & & & & & & & \\
\hline \multicolumn{2}{|c|}{$\mathrm{NOV}^{31 \ldots}$} & $<10$ & $<1$ & 47 & $<.5$ & 210 & 7 & 10 & $<3$ & $<10$ & 15 & $<10$ \\
\hline \multicolumn{2}{|c|}{$\begin{array}{l}15 \ldots \\
\text { APR } 1989\end{array}$} & $<10$ & $<1$ & 46 & .6 & 210 & $<1$ & $<5$ & $<3$ & 20 & 20 & $<10$ \\
\hline \multicolumn{2}{|c|}{ JNNE } & 10 & $<1$ & 37 & $<2$ & 260 & 5 & $<20$ & $<9$ & $<30$ & 13 & $<30$ \\
\hline \multicolumn{2}{|c|}{ SEPT } & $<10$ & $<1$ & 40 & $<2$ & 230 & $<3$ & $<20$ & $<9$ & $<30$ & 26 & $<30$ \\
\hline \multicolumn{2}{|c|}{$18 \ldots$} & 10 & $<1$ & 37 & $<1$ & 230 & $<2$ & $<10$ & $<6$ & $<20$ & 19 & $<20$ \\
\hline DATE & $\begin{array}{l}\text { MANGA- } \\
\text { NESE, } \\
\text { DIS- } \\
\text { SOLVED } \\
(\mu G / L \\
\text { AS MN) }\end{array}$ & $\begin{array}{c}\text { MOLYB- } \\
\text { DENUM, } \\
\text { DIS- } \\
\text { SOLVED } \\
(\mu G / L \\
\text { AS MO) }\end{array}$ & $\begin{array}{c}\text { NICKEL, } \\
\text { DIS- } \\
\text { SOLVED } \\
(\mu G / L \\
\text { AS NI) }\end{array}$ & $\begin{array}{c}\text { SILVER, } \\
\text { DIS- } \\
\text { SOLVED } \\
\text { ( } \mu G / L \\
\text { AS AG) }\end{array}$ & $\begin{array}{c}\text { STRON- } \\
\text { TIUM, } \\
\text { DIS- } \\
\text { SOLVED } \\
\text { ( } \mu G / L \\
\text { AS SR) }\end{array}$ & $\begin{array}{l}\text { VANA- } \\
\text { DIUM, } \\
\text { DIS- } \\
\text { SOLVED } \\
(\mu G / L \\
\text { AS V) }\end{array}$ & $\begin{array}{l}\text { ZINC, } \\
\text { DIS- } \\
\text { SOLVED } \\
(\mu G / L \\
\text { AS ZN) }\end{array}$ & $\begin{array}{c}\text { C-13/ } \\
\text { C-12 } \\
\text { STABLE } \\
\text { ISOTOPE } \\
\text { RATIO } \\
\text { PER } \\
\text { MIL }\end{array}$ & $\begin{array}{l}\text { H-2/ } \\
\text { H-1 } \\
\text { STABLE } \\
\text { ISOTOPE } \\
\text { RATIO } \\
\text { PER } \\
\text { MIL }\end{array}$ & $\begin{array}{l}\text { 0-18/ } \\
0-16 \\
\text { STABLE } \\
\text { ISOTOPE } \\
\text { RATIO } \\
\text { PER } \\
\text { MIL }\end{array}$ & $\begin{array}{c}\text { S-34/ } \\
\text { S-32 } \\
\text { STABLE } \\
\text { ISOTOPE } \\
\text { RATIO } \\
\text { PER } \\
\text { MIL }\end{array}$ & $\begin{array}{c}\text { TRIT- } \\
\text { IUM } \\
\text { TOTAL } \\
\text { (PCI/L) }\end{array}$ \\
\hline \multicolumn{13}{|l|}{ APR 1988} \\
\hline $12 \ldots$ & 550 & $<30$ & $<30$ & $<3.0$ & 4,800 & $<18$ & 31 & -8.8 & -135 & -17.7 & 2.70 & 45 \\
\hline${ }_{\text {JNE }}^{11} \ldots$ & 170 & $<30$ & $<30$ & $<3.0$ & 3,700 & $<18$ & 12 & - & - & -- & -- & -- \\
\hline \multirow[t]{2}{*}{$\begin{array}{l}16 \ldots \\
16 \ldots \\
16 \ldots\end{array}$} & $\begin{array}{l}230 \\
480 \\
170\end{array}$ & $\begin{array}{l}<30 \\
<30 \\
<30\end{array}$ & $\begin{array}{l}<30 \\
<30 \\
<30\end{array}$ & $\begin{array}{l}<3.0 \\
<3.0 \\
<3.0\end{array}$ & $\begin{array}{l}3,800 \\
3,900 \\
3,800\end{array}$ & $\begin{array}{r}<18 \\
<18 \\
<6\end{array}$ & $\begin{array}{l}28 \\
24 \\
27\end{array}$ & $\begin{array}{l}\ldots \\
- \\
-\end{array}$ & $\begin{array}{l}-- \\
-- \\
--\end{array}$ & $\begin{array}{l}-- \\
--\end{array}$ & $\begin{array}{l}-- \\
-- \\
--\end{array}$ & $\begin{array}{l}-- \\
-- \\
--\end{array}$ \\
\hline & \multicolumn{12}{|c|}{ AUG } \\
\hline \multicolumn{13}{|l|}{ NOV } \\
\hline $\begin{array}{r}15 \ldots \\
\text { APR } 1989\end{array}$ & 240 & $<10$ & $<10$ & 1.0 & 3,800 & $<6$ & 19 & -- & $-\cdot$ & -- & -- & -- \\
\hline JUNE & 110 & $<30$ & $<30$ & $<3.0$ & 4,800 & $<18$ & 42 & -- & -138 & -18.1 & -- & 43 \\
\hline \multicolumn{13}{|l|}{$\begin{array}{l}06 . . \\
\text { SEPT }\end{array}$} \\
\hline $18 \ldots$ & 240 & $<20$ & $<20$ & 5.0 & 4,100 & $<12$ & 40 & -- & -- & -- & -- & -- \\
\hline
\end{tabular}


Table 25.--Water-quality analyses for wells--Continued

\begin{tabular}{|c|c|c|c|c|c|c|c|c|c|c|c|}
\hline DATE & TIME & $\begin{array}{l}\text { DEPTH } \\
\text { OF } \\
\text { WELL, } \\
\text { TOTAL } \\
\text { (FEET) }\end{array}$ & $\begin{array}{c}\text { ELEVATION } \\
\text { OF LAND } \\
\text { SURFACE } \\
\text { DATUM } \\
\text { (FEET } \\
\text { ABOVE } \\
\text { SEA } \\
\text { LEVEL) }\end{array}$ & $\begin{array}{l}\text { SPE- } \\
\text { CIFIC } \\
\text { CON- } \\
\text { DUCT- } \\
\text { ANCE } \\
(\mu S / C M)\end{array}$ & $\begin{array}{c}\text { PH } \\
\text { (STAND- } \\
\text { ARD } \\
\text { UNITS) }\end{array}$ & $\begin{array}{l}\text { TEMPER- } \\
\text { ATURE } \\
\text { WATER } \\
\left({ }^{\circ} \mathrm{C}\right)\end{array}$ & $\begin{array}{l}\text { HARD- } \\
\text { NESS } \\
\text { TOTAL } \\
\text { (MG/L } \\
\text { AS } \\
\left.\mathrm{CACO}_{3}\right)\end{array}$ & $\begin{array}{l}\text { HARD- } \\
\text { NESS } \\
\text { NONCARB } \\
\text { WH WAT } \\
\text { TOT FLD } \\
(\mathrm{MG} / \mathrm{L} \text { AS } \\
\left.\mathrm{CACO}_{3}\right)\end{array}$ & $\begin{array}{c}\text { CALCIUM } \\
\text { DIS- } \\
\text { SOLVED } \\
\text { (MG/L } \\
\text { AS CA) }\end{array}$ & $\begin{array}{l}\text { MAGNE- } \\
\text { SIUM, } \\
\text { DIS- } \\
\text { SOLVED } \\
\text { (MG/L } \\
\text { AS MG) }\end{array}$ & $\begin{array}{c}\text { SODIUM, } \\
\text { DIS- } \\
\text { SOLVED } \\
\text { (MG/L } \\
\text { AS NA) }\end{array}$ \\
\hline
\end{tabular}

\begin{tabular}{|c|c|c|c|c|c|c|c|c|c|c|c|}
\hline \multirow{2}{*}{\multicolumn{12}{|c|}{$\operatorname{scs} 487-63$}} \\
\hline & & & & & & & & & & & \\
\hline $\begin{array}{l}08 \cdots \\
\mathrm{APR}\end{array}$ & 1000 & 37.50 & 6,980 & 2,510 & 6.8 & 9.5 & 1,600 & 1,200 & 340 & 180 & 130 \\
\hline $12 \ldots$ & 1330 & 37.50 & 6,980 & 2,820 & 6.9 & 10.5 & 1,900 & 1,600 & 390 & 230 & 90 \\
\hline AUG & 1100 & 37.50 & 6,980 & 3,000 & 7.0 & 10.5 & 1,800 & 1,500 & 370 & 220 & 92 \\
\hline $30 \ldots$ & 1600 & 37.50 & 6,980 & 2,750 & 7.0 & 10.5 & 1,700 & 1,200 & 330 & 200 & 100 \\
\hline
\end{tabular}

\begin{tabular}{|c|c|c|c|c|c|c|c|c|c|}
\hline DATE & $\begin{array}{l}\text { POTAS- } \\
\text { SIUM, } \\
\text { DIS- } \\
\text { SOLVED } \\
\text { (MG/L } \\
\text { AS K) }\end{array}$ & $\begin{array}{l}\text { ALKA- } \\
\text { LINITY } \\
\text { LAB } \\
(\mathrm{MG} / \mathrm{L} \\
\mathrm{AS} \\
\left.\mathrm{CACO}_{3}\right)\end{array}$ & 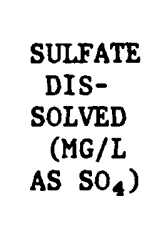 & $\begin{array}{l}\text { CHIO- } \\
\text { RIDE, } \\
\text { DIS- } \\
\text { SOLVED } \\
\text { (MG/L } \\
\text { AS CL) }\end{array}$ & $\begin{array}{l}\text { FLUO- } \\
\text { RIDE, } \\
\text { DIS- } \\
\text { SOLVED } \\
\text { (MG/L } \\
\text { AS F) }\end{array}$ & $\begin{array}{l}\text { SILICA, } \\
\text { DIS- } \\
\text { SOLVED } \\
(\mathrm{MG} / \mathrm{L} \\
\mathrm{AS} \\
\left.\mathrm{SIO}_{2}\right)\end{array}$ & $\begin{array}{l}\text { SOLIDS, } \\
\text { RESIDUE } \\
\text { AT } 180 \\
\text { DEG. C } \\
\text { DIS- } \\
\text { SOLVED } \\
\text { (MG/L) }\end{array}$ & $\begin{array}{l}\text { SOLIDS, } \\
\text { SUM OF } \\
\text { CONSTIT- } \\
\text { UENTS, } \\
\text { DIS- } \\
\text { SOLVED } \\
\text { (MG/L) }\end{array}$ & \\
\hline \multicolumn{10}{|l|}{ MAR 1988} \\
\hline \multicolumn{3}{|l|}{ APR } & 1,300 & 17 & 0.2 & 12 & 2,370 & 2,250 & \\
\hline \multicolumn{9}{|l|}{ MAY } & \\
\hline${ }_{A U G}^{11 \ldots}$ & 8.1 & 375 & 1,600 & 16 & .3 & 11 & 2,680 & 2,560 & \\
\hline \multicolumn{10}{|l|}{$30 \ldots$} \\
\hline DATE & $\begin{array}{l}\text { NITRO- } \\
\text { GEN, } \\
\text { NITRATE } \\
\text { DIS- } \\
\text { SOLVED } \\
\text { (MG/L } \\
\text { AS N) }\end{array}$ & $\begin{array}{l}\text { NITRO- } \\
\text { GEN, } \\
\text { NITRITE } \\
\text { DIS- } \\
\text { SOLVED } \\
\text { (MG/L } \\
\text { AS N) }\end{array}$ & $\begin{array}{c}\text { NITRO- } \\
\text { GEN, } \\
\text { NITRITE } \\
\text { DIS- } \\
\text { SOLVED } \\
(\mathrm{MG} / \mathrm{L} \\
\left.\text { AS } \mathrm{NO}_{2}\right)\end{array}$ & $\begin{array}{c}\text { NITRO- } \\
\text { GEN, } \\
\mathrm{NO}_{2}+\mathrm{NO}_{3} \\
\text { DIS- } \\
\text { SOLVED } \\
\text { (MG/L } \\
\text { AS N) }\end{array}$ & $\begin{array}{l}\text { NITRO- } \\
\text { GEN, } \\
\text { AMMONIA } \\
\text { DIS- } \\
\text { SOLVED } \\
\text { (MG/L } \\
\text { AS N) }\end{array}$ & $\begin{array}{l}\text { NITRO- } \\
\text { GEN, } \\
\text { AMMONIA } \\
\text { DIS- } \\
\text { SOLVED } \\
\left(\mathrm{MG} / L^{\prime}\right. \\
\left.\text { AS } \mathrm{NH}_{4}\right)\end{array}$ & $\begin{array}{l}\text { NITRO- } \\
\text { GEN, } \\
\text { ORGANIC } \\
\text { DIS- } \\
\text { SOLVED } \\
\text { (MG/L } \\
\text { AS N) }\end{array}$ & $\begin{array}{c}\text { NITRO- } \\
\text { GEN, AM- } \\
\text { MONIA + } \\
\text { ORGANIC } \\
\text { DISSOLVED } \\
\text { (MG/L } \\
\text { AS N) }\end{array}$ & $\begin{array}{l}\text { PHOS- } \\
\text { PHORUS } \\
\text { DIS- } \\
\text { SOLVED } \\
\text { (MG/L } \\
\text { AS P) }\end{array}$ \\
\hline \multicolumn{10}{|l|}{ MAR 1988} \\
\hline $\begin{array}{c}08 \ldots \\
\text { APR }\end{array}$ & 0.15 & 0.03 & 0.1 & 0.18 & 0.32 & 0.41 & 0.38 & 0.7 & 0.01 \\
\hline $\operatorname{MAY}_{12 \ldots}$ & -- & $<.01$ & -- & 3.40 & .12 & .15 & .68 & .8 & $<.01$ \\
\hline AUG $^{11 \ldots}$ & 1.97 & .03 & .1 & 2.00 & .11 & .14 & 1.1 & 1.2 & $<.01$ \\
\hline $30 \ldots$ & -- & $<.01$ & -- & $<.10$ & .12 & .15 & .68 & .8 & $<.01$ \\
\hline
\end{tabular}

\begin{tabular}{|c|c|c|c|c|c|c|c|c|c|c|}
\hline ATE & $\begin{array}{l}\text { PHOS- } \\
\text { PHORUS } \\
\text { ORTHO, } \\
\text { DIS- } \\
\text { SOLVED } \\
\text { (MG/L } \\
\text { AS P) }\end{array}$ & $\begin{array}{c}\text { PHOS- } \\
\text { PHORUS } \\
\text { ORGANIC } \\
\text { DIS- } \\
\text { SOLVED } \\
\text { (MG/I } \\
\text { AS P) }\end{array}$ & $\begin{array}{l}\text { ALU- } \\
\text { MINUM, } \\
\text { DIS- } \\
\text { SOLVED } \\
\text { ( } \mu G / L \\
\text { AS AL) }\end{array}$ & $\begin{array}{c}\text { ARSENIC } \\
\text { DIS- } \\
\text { SOLVED } \\
(\mu G / L \\
\text { AS AS) }\end{array}$ & $\begin{array}{l}\text { BARIUM, } \\
\text { DIS- } \\
\text { SOLVED } \\
(\mu G / L \\
\text { AS BA) }\end{array}$ & $\begin{array}{l}\text { BERYL- } \\
\text { LIUM, } \\
\text { DIS- } \\
\text { SOLVED } \\
\text { ( } \mu G / L \\
\text { AS BE) }\end{array}$ & $\begin{array}{l}\text { BORON, } \\
\text { DIS- } \\
\text { SOLVED } \\
(\mu G / L \\
\text { AS B) }\end{array}$ & $\begin{array}{l}\text { CADMIUM } \\
\text { DIS- } \\
\text { SOLVED } \\
(\mu G / L \\
\text { AS CD) }\end{array}$ & $\begin{array}{l}\text { CHRO- } \\
\text { MIUM, } \\
\text { DIS- } \\
\text { SOLVED } \\
\text { ( } \mu G / L \\
\text { AS CR) }\end{array}$ & $\begin{array}{l}\text { COBALT, } \\
\text { DIS- } \\
\text { SOLVED } \\
(\mu G / L \\
\text { AS CO })\end{array}$ \\
\hline
\end{tabular}

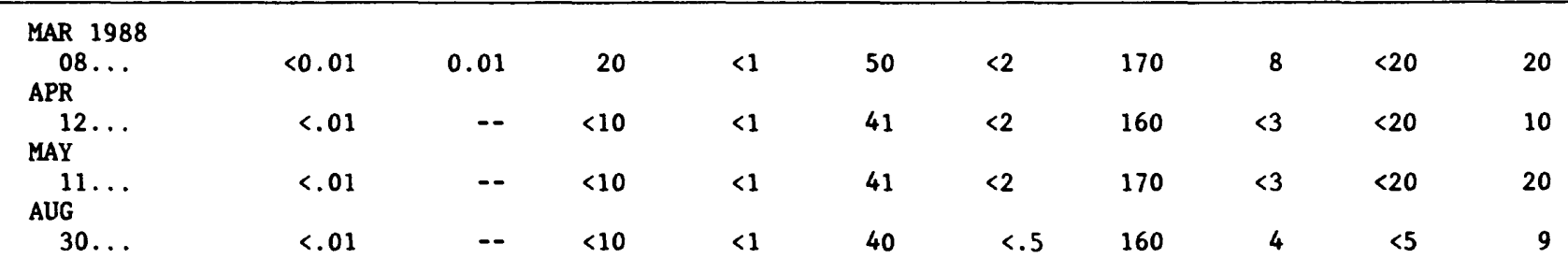


Table 25.--Water-quality analyses for wells--Continued

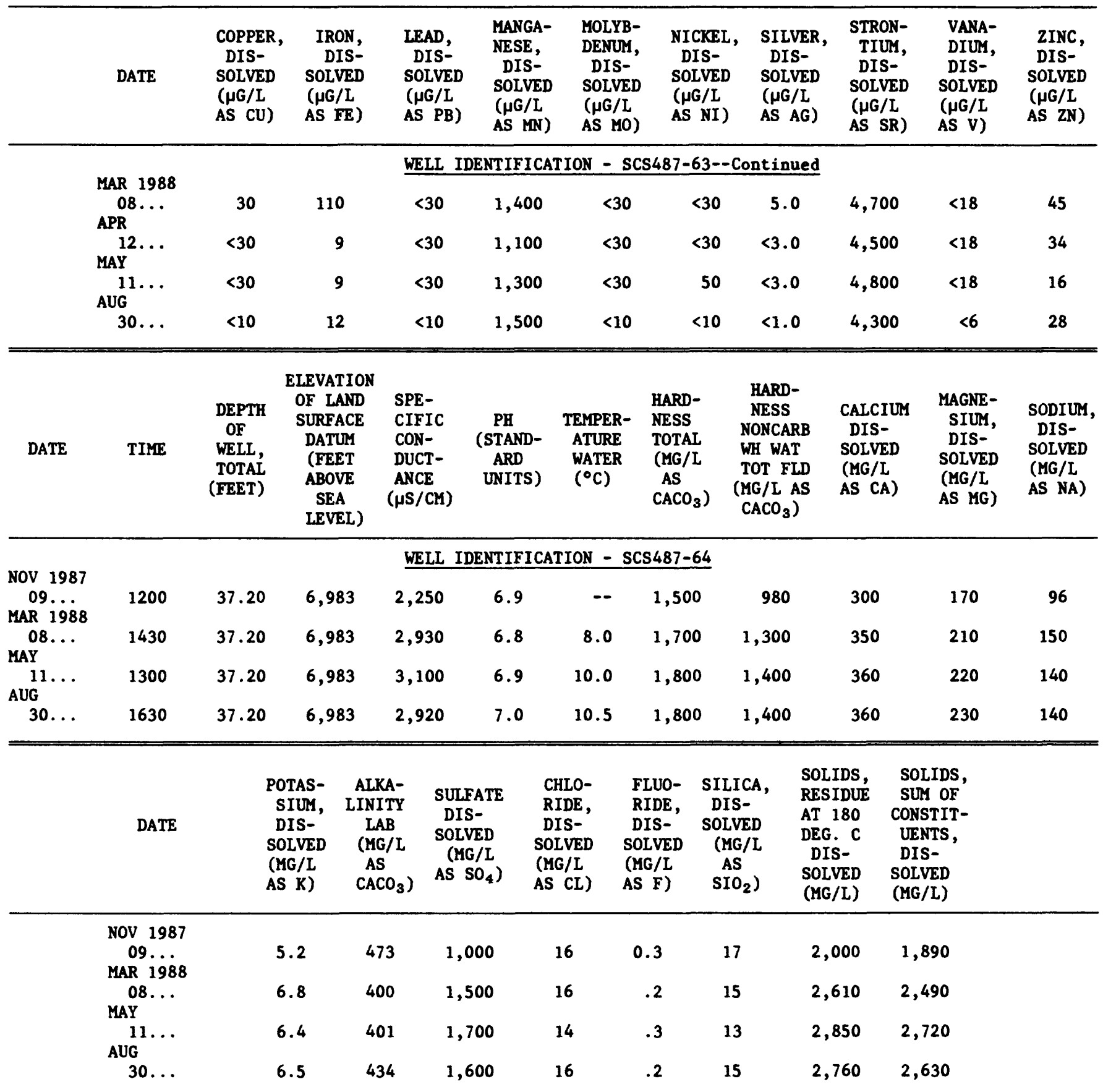


Table 25.--Water-quality analyses for wells--Continued

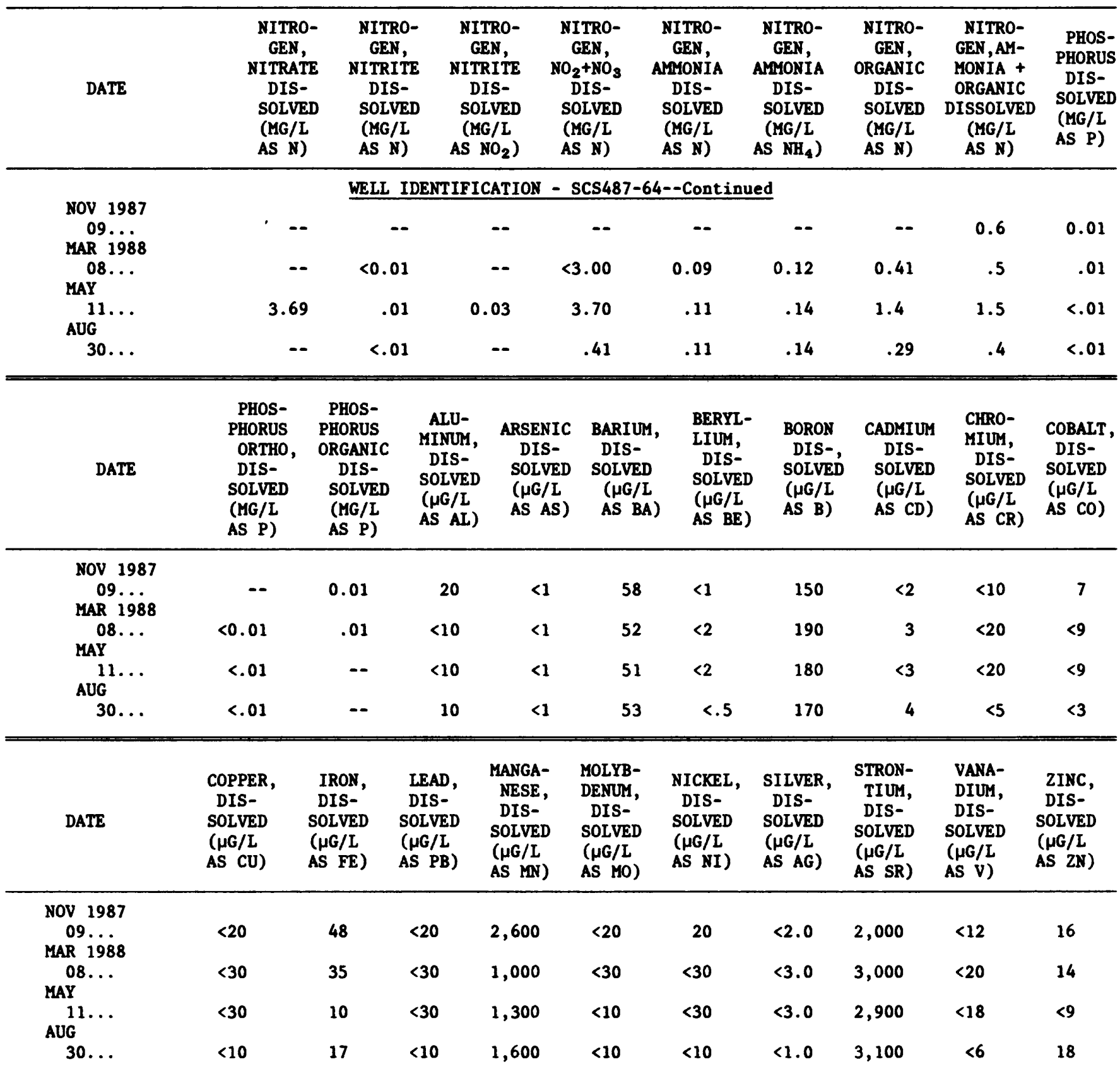


Table 25.--Water-quality analyses for wells--Continued

\begin{tabular}{|c|c|c|c|c|c|c|c|c|c|c|c|c|c|c|}
\hline DATE & TIME & $\begin{array}{l}\text { DEPTH } \\
\text { OF } \\
\text { WELL, } \\
\text { TOTAL } \\
\text { (FEET) }\end{array}$ & \multicolumn{2}{|c|}{$\begin{array}{l}\text { ELEVATION } \\
\text { OF LAND } \\
\text { SURFACE } \\
\text { DATUM } \\
\text { (FEET } \\
\text { ABOVE } \\
\text { SEA } \\
\text { LEVEL) }\end{array}$} & \multicolumn{2}{|c|}{$\begin{array}{l}\text { SPE- } \\
\text { CIFIC } \\
\text { CON- } \\
\text { DUCT- } \\
\text { ANCE } \\
(\mu S / C M)\end{array}$} & $\begin{array}{c}\text { PH } \\
\text { (STAND- } \\
\text { ARD } \\
\text { UNITS) }\end{array}$ & $\begin{array}{c}\text { TEMPER- } \\
\text { ATURE } \\
\text { WATER } \\
\text { ( } \\
\left({ }^{\circ} \mathrm{C}\right)\end{array}$ & $\begin{array}{l}\text { HARD- } \\
\text { NESS } \\
\text { TOTAL } \\
(\mathrm{MG} / \mathrm{L} \\
\text { AS } \\
\left.\mathrm{CACO}_{3}\right)\end{array}$ & $\begin{array}{c}\text { HAR } \\
\text { NES } \\
\text { NONC } \\
\text { WH } W \\
\text { TOT } \\
\text { (MG } / L \\
\text { CACO }\end{array}$ & $\begin{array}{l}\text { D- } \\
\text { S } \\
\text { ARB } \\
\text { AT } \\
\text { FLD } \\
\text { AS } \\
\text { 3) }\end{array}$ & \multicolumn{2}{|c|}{$\begin{array}{l}\text { CALCIUM } \\
\text { DIS- } \\
\text { SOLVED } \\
\text { (MG/L } \\
\text { AS CA) }\end{array}$} & $\begin{array}{l}\text { MAGNE- } \\
\text { SIUM, } \\
\text { DIS- } \\
\text { SOLVED } \\
\text { (MG/L } \\
\text { AS MG) }\end{array}$ \\
\hline \multicolumn{15}{|c|}{ WELL IDENTIFICATION - SZL287 } \\
\hline $\begin{array}{c}\text { NOV } 1987 \\
08 \ldots \\
\text { MAR } 1988\end{array}$ & 1415 & 181.00 & \multicolumn{2}{|c|}{6,822} & \multicolumn{2}{|c|}{1,820} & 8.1 & -- & 36 & \multicolumn{2}{|c|}{0} & \multicolumn{2}{|c|}{7.6} & 4.1 \\
\hline $\begin{array}{l}06 \ldots \\
\text { APR }\end{array}$ & 1640 & 181.00 & \multicolumn{2}{|c|}{6,822} & \multicolumn{2}{|c|}{1,495} & 8.2 & 9.0 & 23 & \multicolumn{2}{|c|}{0} & \multicolumn{2}{|c|}{5.3} & 2.4 \\
\hline${ }_{\mathrm{MAY}}^{11} \cdots$ & 1245 & 181.00 & \multicolumn{2}{|c|}{6,822} & \multicolumn{2}{|c|}{1,970} & 8.3 & 10.0 & 21 & \multicolumn{2}{|c|}{0} & \multicolumn{2}{|c|}{4.7} & 2.2 \\
\hline $\begin{array}{l}09 \ldots \\
\text { SEPT }\end{array}$ & 1245 & 181.00 & \multicolumn{2}{|c|}{6,822} & \multicolumn{2}{|c|}{2,000} & 8.3 & 10.5 & 19 & 0 & & 4.5 & & 1.9 \\
\hline Nov & 0900 & 181.00 & 6, & & 1, & & 8.3 & 11.0 & 19 & 0 & & 4. & & 1.9 \\
\hline $\begin{array}{c}16 \ldots \\
\text { APR } 1989\end{array}$ & 1045 & 181.00 & 6, & & 2, & & 8.6 & -- & 18 & 0 & & 4. & & 1.8 \\
\hline $\begin{array}{l}06 . . \\
\text { JUNE }\end{array}$ & 1345 & 181.00 & 6,8 & & 2, & & 8.3 & 10.5 & 18 & 0 & & 4. & & 1.8 \\
\hline $\begin{array}{c}06 . . . \\
\text { SEPT }\end{array}$ & 1445 & 181.00 & 6,8 & & 2, & & 8.2 & 10.0 & 18 & 0 & & 4. & & 1.7 \\
\hline $12 \ldots$ & 1430 & 181.00 & 6, & & 2, & & 8.3 & 10.0 & 18 & 0 & & 4. & & 1.7 \\
\hline DATE & & $\begin{array}{l}\text { SODIUM, } \\
\text { DIS- } \\
\text { SOLVED } \\
\text { (MG/L } \\
\text { AS NA) }\end{array}$ & $\begin{array}{l}\text { POTAS- } \\
\text { SIUM, } \\
\text { DIS- } \\
\text { SOLVED } \\
\text { (MG/L } \\
\text { AS K) }\end{array}$ & $\begin{array}{r}A \\
\mathrm{LI} \\
\mathrm{L} \\
\mathrm{C} \\
\mathrm{A} \\
\mathrm{CA}\end{array}$ & $\begin{array}{l}\text { KA- } \\
\text { ITY } \\
\text { B } \\
/ \mathrm{I} \\
\left.\mathrm{O}_{3}\right)\end{array}$ & $\begin{array}{r}\text { SUL } \\
\text { DI } \\
\text { SOL } \\
\text { (M } \\
\text { AS }\end{array}$ & $\begin{array}{l}\text { FATE } \\
\mathrm{S}- \\
\mathrm{VED} \\
\mathrm{IG} / \mathrm{L} \\
\mathrm{SO}_{4} \text { ) }\end{array}$ & $\begin{array}{l}\text { CHLO- } \\
\text { RIDE, } \\
\text { DIS- } \\
\text { SOLVED } \\
\text { (MG/L } \\
\text { AS CL) }\end{array}$ & $\begin{array}{l}\text { FLUO- } \\
\text { RIDE, } \\
\text { DIS- } \\
\text { SOLVED } \\
\text { (MG/L } \\
\text { AS F) }\end{array}$ & $\begin{array}{l}\text { SILICA, } \\
\text { DIS- } \\
\text { SOLVED } \\
(\mathrm{MG} / \mathrm{L} \\
\mathrm{AS} \\
\left.\mathrm{SIO}_{2}\right)\end{array}$ & $\begin{array}{l}\text { SOL } \\
\text { RES } \\
\text { AT } \\
\text { DEG } \\
\text { DI } \\
\text { SOL } \\
\text { (MG }\end{array}$ & $\begin{array}{l}\text { DS, } \\
\text { DUE } \\
80 \\
\text { C } \\
- \\
\text { ED } \\
\text { L) }\end{array}$ & $\begin{array}{l}\text { SOI } \\
\text { SUI } \\
\text { CONS } \\
\text { UE? } \\
\text { DIs } \\
\text { SOLI } \\
\text { (MG }\end{array}$ & $\begin{array}{l}\text { DS, } \\
\text { OF } \\
\text { [IT- } \\
\text { [S, } \\
\text { D } \\
\text { ) }\end{array}$ \\
\hline NOV 1987 & & & & & & & & & & & & & & \\
\hline $\begin{array}{l}08 \ldots \\
\text { MAR } 1988\end{array}$ & & 510 & 3.1 & 1, & & 4 & 4 & 9.0 & 0.2 & 9.6 & 1,3 & & 1,28 & \\
\hline$\underset{A P R}{06 \ldots}$ & & 550 & 2.9 & 1, & & 2 & 7 & 8.6 & 2.4 & 9.6 & 1,3 & & 1,32 & \\
\hline${ }_{\mathrm{MAY}}^{11} \ldots$ & & 530 & 2.0 & 1, & & 1 & 7 & 8.4 & 3.2 & 8.3 & 1,3 & & 1,29 & \\
\hline $\begin{array}{l}09 . . \\
\text { SEPT }\end{array}$ & & 520 & 2.3 & 1, & & 1 & 5 & 9.8 & 3.2 & 8.0 & 1,3 & & 1,2 & \\
\hline$\underset{\text { Nov }}{01}$ & & 530 & 2.1 & 1, & & 1 & 2 & 8.6 & 2.8 & 8.4 & 1,2 & & 1,28 & \\
\hline $\begin{array}{c}16 \ldots \\
\text { APR } 1989\end{array}$ & & 520 & 2.3 & 1, & & 1 & 6 & 9.0 & 2.8 & 8.4 & 1,3 & & 1,28 & \\
\hline JUNE & & 510 & 2.3 & 1, & & 1 & 3 & 8.3 & 2.9 & 8.2 & 1,2 & & 1,2 & \\
\hline $\begin{array}{c}06 \ldots \\
\text { SEPT }\end{array}$ & & 540 & 2.1 & 1, & 60 & 2 & 1 & 8.1 & 3.0 & 8.0 & 1,2 & & 1,28 & \\
\hline $12 \ldots$ & & 520 & 2.2 & 1, & 50 & 2 & 5 & 8.8 & 3.0 & 8.0 & 1,2 & & 1,2 & \\
\hline
\end{tabular}


Table 25.--Water-quality analyses for wells--Continued

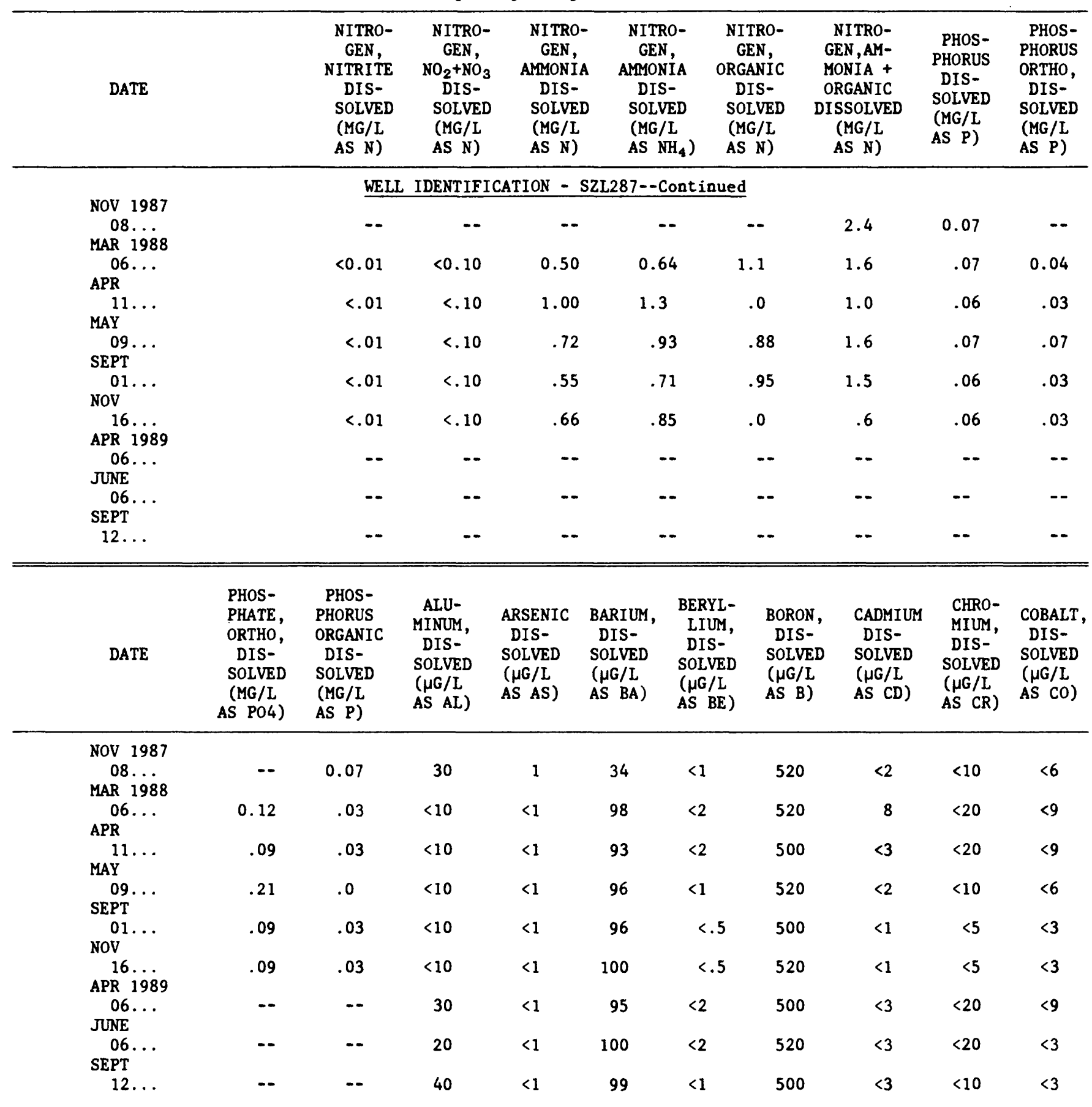


Table 25.--Water-quality analyses for wells--Continued

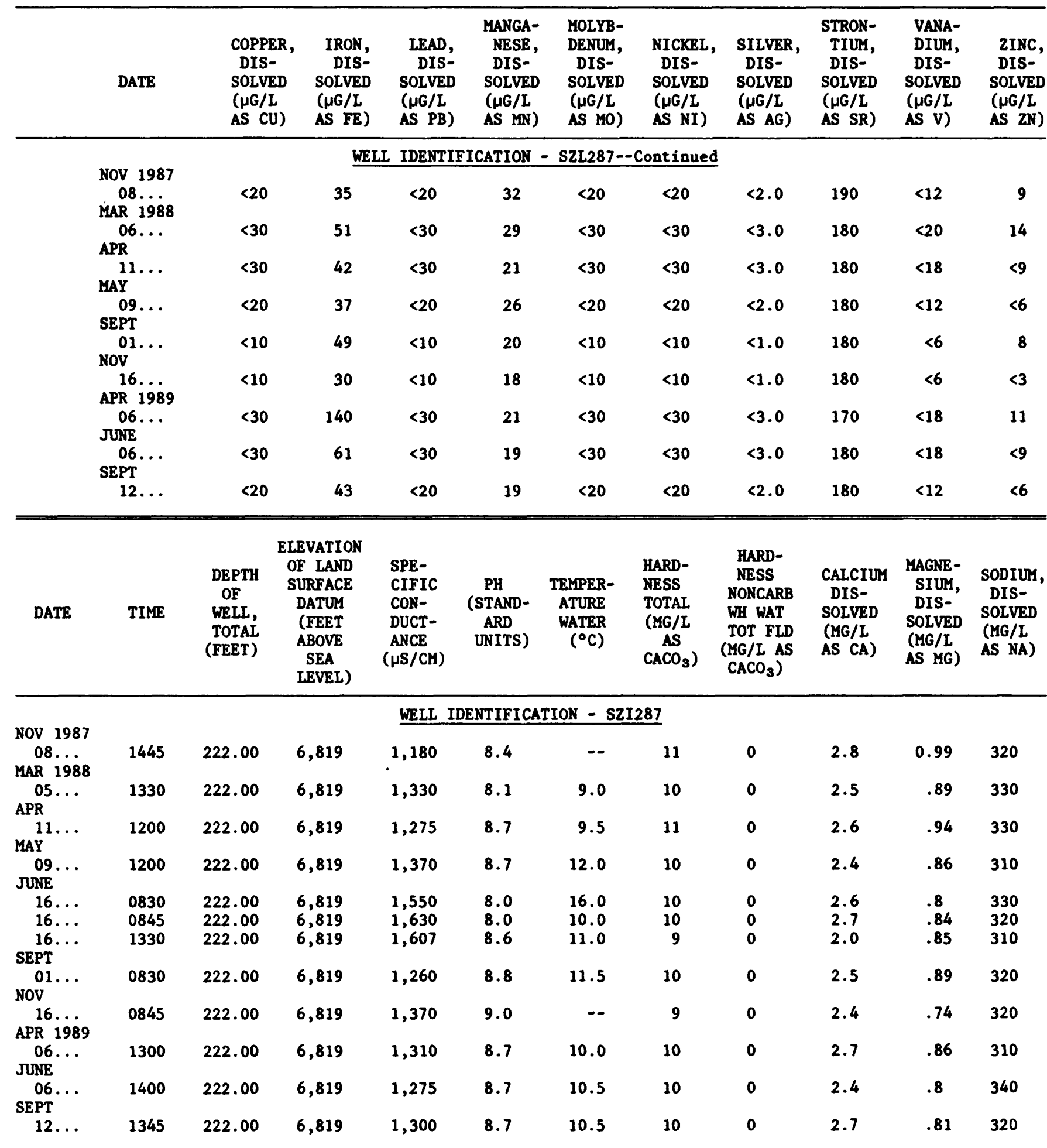


Table 25.--Water-quality analyses for wells--Continued

\begin{tabular}{|c|c|c|c|c|c|c|c|c|}
\hline ATE & $\begin{array}{l}\text { POTAS- } \\
\text { SIUM, } \\
\text { DIS- } \\
\text { SOLVED } \\
\text { (MG/L } \\
\text { AS K) }\end{array}$ & $\begin{array}{c}\text { ALKA- } \\
\text { LINITY } \\
\text { LAB } \\
(\mathrm{MG} / \mathrm{L} \\
\text { AS } \\
\left.\mathrm{CACO}_{3}\right)\end{array}$ & $\begin{array}{c}\text { SULFATE } \\
\text { DIS- } \\
\text { SOLVED } \\
(\mathrm{MG} / \mathrm{L} \\
\left.\text { AS } \mathrm{SO}_{4}\right)\end{array}$ & $\begin{array}{l}\text { CHLO- } \\
\text { RIDE, } \\
\text { DIS- } \\
\text { SOLVED } \\
\text { (MG/L } \\
\text { AS CL) }\end{array}$ & $\begin{array}{l}\text { FLUO- } \\
\text { RIDE, } \\
\text { DIS- } \\
\text { SOLVED } \\
\text { (MG/L } \\
\text { AS F) }\end{array}$ & $\begin{array}{c}\text { SILICA, } \\
\text { DIS- } \\
\text { SOLVED } \\
\text { (MG/L } \\
\text { AS } \\
\left.\mathrm{SIO}_{2}\right)\end{array}$ & $\begin{array}{l}\text { SOLIDS, } \\
\text { RESIDUE } \\
\text { AT } 180 \\
\text { DEG. C } \\
\text { DIS- } \\
\text { SOLVED } \\
\text { (MG/L) }\end{array}$ & $\begin{array}{c}\text { SOLIDS, } \\
\text { SUM OF } \\
\text { CONSTIT- } \\
\text { UENTS, } \\
\text { DIS- } \\
\text { SOLVED } \\
\text { (MG/L) }\end{array}$ \\
\hline
\end{tabular}

NOV 1987

$08 \ldots$

MAR 1988

$05 . .$.

APR

$11 \ldots$

MAY

09.

JNE

16 ...

$16 . .$.

$16 .$.

SEPT

$01 . .$.

NOV

$16 \ldots$

APR 1989

$06 \ldots$

JUNE

$06 . .$.

SEPT

$12 .$.

$2.0 \quad 655$

WELL IDENTIFICATION - S2I287--Continued

2.2650

69

3.3

1.0

7.9

806

801

$650 \quad 75$

4.1

2.0

7.7

813

815

$1.8 \quad 660$

87

3.7

2.1

7.6

834

833

$1.9 \quad 652$

3.8

2.1

7.5

834

808

1.9646

86

3.8

1.8

7.7

800

823

1.9647

3.7

1.9

7.5

830

820

73

3.9

1.7

815

790

$1.6 \quad 651$

3.8

2.0

7.7

814

809

$1.9 \quad 651$

3.9

1.9

$7.9 \quad 810$

797

$1.9 \quad 655$

4.1

2.1

$7.9 \quad 825$

803

$1.8 \quad 651$

3.9

2.0

$\begin{array}{ll}7.8 & 800\end{array}$

812

$1.9 \quad 649$

4.1

.2

7.5805

804

\begin{tabular}{|c|c|c|c|c|c|c|c|c|c|}
\hline DATE & $\begin{array}{l}\text { NITRO- } \\
\text { GEN, } \\
\text { NITRITE } \\
\text { DIS- } \\
\text { SOLVED } \\
\text { (MG/L } \\
\text { AS N) }\end{array}$ & $\begin{array}{l}\text { NITRO- } \\
\text { GEN, } \\
\text { NITRITE } \\
\text { DIS- } \\
\text { SOLVED } \\
(\mathrm{MG} / \mathrm{L} \\
\left.\text { AS } \mathrm{NO}_{2}\right)\end{array}$ & $\begin{array}{c}\text { NITRO- } \\
\text { GEN, } \\
\mathrm{NO}_{2}+\mathrm{NO}_{3} \\
\text { DIS- } \\
\text { SOLVED } \\
\text { (MG/L } \\
\text { AS N) }\end{array}$ & $\begin{array}{l}\text { NITRO- } \\
\text { GEN, } \\
\text { AMMONIA } \\
\text { DIS- } \\
\text { SOLVED } \\
\text { (MG/L } \\
\text { AS N) }\end{array}$ & $\begin{array}{l}\text { NITRO- } \\
\text { GEN, } \\
\text { AMMONIA } \\
\text { DIS- } \\
\text { SOLVED } \\
\text { (MG/L } \\
\text { AS NH4) }\end{array}$ & $\begin{array}{l}\text { NITRO- } \\
\text { GEN, } \\
\text { ORGANIC } \\
\text { DIS- } \\
\text { SOLVED } \\
\text { (MG/L } \\
\text { AS N) }\end{array}$ & $\begin{array}{c}\text { NITRO- } \\
\text { GEN,AM- } \\
\text { MONIA + } \\
\text { ORGANIC } \\
\text { DISSOLVED } \\
\text { (MG/L } \\
\text { AS N) }\end{array}$ & $\begin{array}{l}\text { PHOS- } \\
\text { PHORUS } \\
\text { DIS - } \\
\text { SOLVED } \\
\text { (MG/L } \\
\text { AS P) }\end{array}$ & $\begin{array}{l}\text { PHOS- } \\
\text { PHORUS } \\
\text { ORTHO, } \\
\text { DIS- } \\
\text { SOLVED } \\
\text { (MG/L } \\
\text { AS P) }\end{array}$ \\
\hline \multicolumn{10}{|l|}{ NOV 1987} \\
\hline $\begin{array}{l}08 \ldots \\
\text { MAR } 1988\end{array}$ & $<0.01$ & -- & $<0.10$ & 0.32 & 0.41 & 0.68 & 1.0 & 0.03 & 0.02 \\
\hline $\begin{array}{c}05 \ldots \\
\text { APR }\end{array}$ & $<.01$ & -- & $<.10$ & .10 & .13 & .9 & 1.0 & .04 & .03 \\
\hline $\operatorname{MAY}^{11 \ldots}$ & $<.01$ & -- & $<.10$ & .44 & .57 & .46 & .9 & .03 & .03 \\
\hline$\underset{\text { JUNE }}{09 . .}$ & $<.01$ & - & $<.10$ & .15 & .19 & .75 & .9 & .03 & .03 \\
\hline $\begin{array}{l}16 \ldots \\
16 \ldots \\
16 \ldots\end{array}$ & $\begin{array}{l}.01 \\
.04 \\
.01\end{array}$ & $\begin{array}{r}0.03 \\
.13 \\
.03\end{array}$ & $\begin{array}{l}<.10 \\
<.10 \\
<.10\end{array}$ & $\begin{array}{l}.27 \\
.21 \\
.19\end{array}$ & $\begin{array}{l}.35 \\
.27 \\
.24\end{array}$ & $\begin{array}{l}.33 \\
.0 \\
-.\end{array}$ & $\begin{array}{r}.6 \\
.2 \\
<.2\end{array}$ & $\begin{array}{l}.03 \\
.03 \\
.02\end{array}$ & $\begin{array}{l}.02 \\
.04 \\
.04\end{array}$ \\
\hline SEPT & & & & & & & & & \\
\hline$\underset{\text { Nov }}{01 \ldots}$ & $<.01$ & -- & $<.10$ & .20 & .26 & .6 & .8 & .04 & .03 \\
\hline $\begin{array}{l}16 \ldots \\
\text { APR } 1989\end{array}$ & $<.01$ & $-\infty$ & $<.10$ & .17 & .22 & - & $<.2$ & .04 & .04 \\
\hline $\begin{array}{l}06 \ldots \\
\text { JUNE }\end{array}$ & -- & -- & - & - & - & - & -- & -- & -- \\
\hline $\begin{array}{l}\text { 06... } \\
\text { SEPT }\end{array}$ & - & -- & -- & -- & -- & - & -- & -- & - \\
\hline $12 \ldots$ & - & - & -- & -- & -- & -- & -- & -- & -- \\
\hline
\end{tabular}


Table 25.--Water-quality analyses for wells--Continued

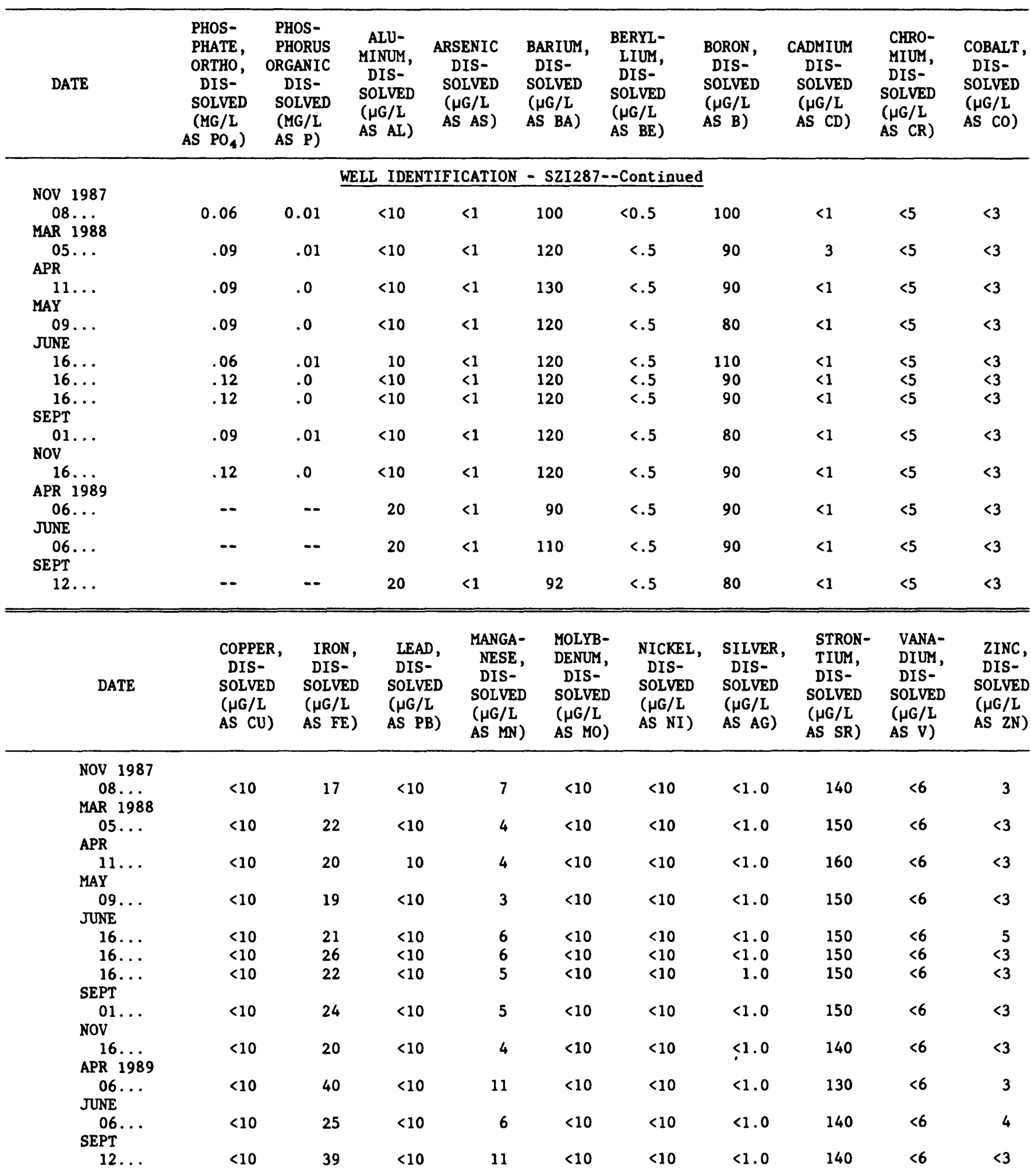


Table 25.--Water-quality analyses for wells--Continued

\begin{tabular}{|c|c|c|c|c|c|c|c|c|c|c|c|}
\hline DATE & TIME & $\begin{array}{l}\text { DEPTH } \\
\text { OF } \\
\text { WELL, } \\
\text { TOTAL } \\
\text { (FEET) }\end{array}$ & $\begin{array}{l}\text { ELEVATION } \\
\text { OF LAND } \\
\text { SURFACE } \\
\text { DATUM } \\
\text { (FEET } \\
\text { ABOVE } \\
\text { SEA } \\
\text { LEVEL) }\end{array}$ & $\begin{array}{l}\text { SPE- } \\
\text { CIFIC } \\
\text { CON- } \\
\text { DUCT- } \\
\text { ANCE } \\
(\mu S / C M)\end{array}$ & $\begin{array}{c}\text { PH } \\
\text { (STAND- } \\
\text { ARD } \\
\text { UNITS) }\end{array}$ & $\begin{array}{c}\text { TEMPER- } \\
\text { ATURE } \\
\text { WATER } \\
\left({ }^{\circ} \mathrm{C}\right)\end{array}$ & $\begin{array}{l}\text { HARD- } \\
\text { NESS } \\
\text { TOTAL } \\
(\mathrm{MG} / \mathrm{L} \\
\mathrm{AS} \\
\left.\mathrm{CACO}_{3}\right)\end{array}$ & $\begin{array}{l}\text { HARD- } \\
\text { NESS } \\
\text { NONCARB } \\
\text { WH WAT } \\
\text { TOT FLD } \\
(\mathrm{MG} / \mathrm{L} \text { AS } \\
\left.\mathrm{CACO}_{3}\right)\end{array}$ & $\begin{array}{l}\text { CALCIUM } \\
\text { DIS- } \\
\text { SOLVED } \\
\text { (MG/L } \\
\text { AS CA) }\end{array}$ & $\begin{array}{l}\text { MAGNE- } \\
\text { SIUM, } \\
\text { DIS- } \\
\text { SOLVED } \\
\text { (MG/L } \\
\text { AS MG) }\end{array}$ & $\begin{array}{l}\text { SODIUM, } \\
\text { DIS- } \\
\text { SOLVED } \\
\text { (MG/L } \\
\text { AS NA) }\end{array}$ \\
\hline
\end{tabular}

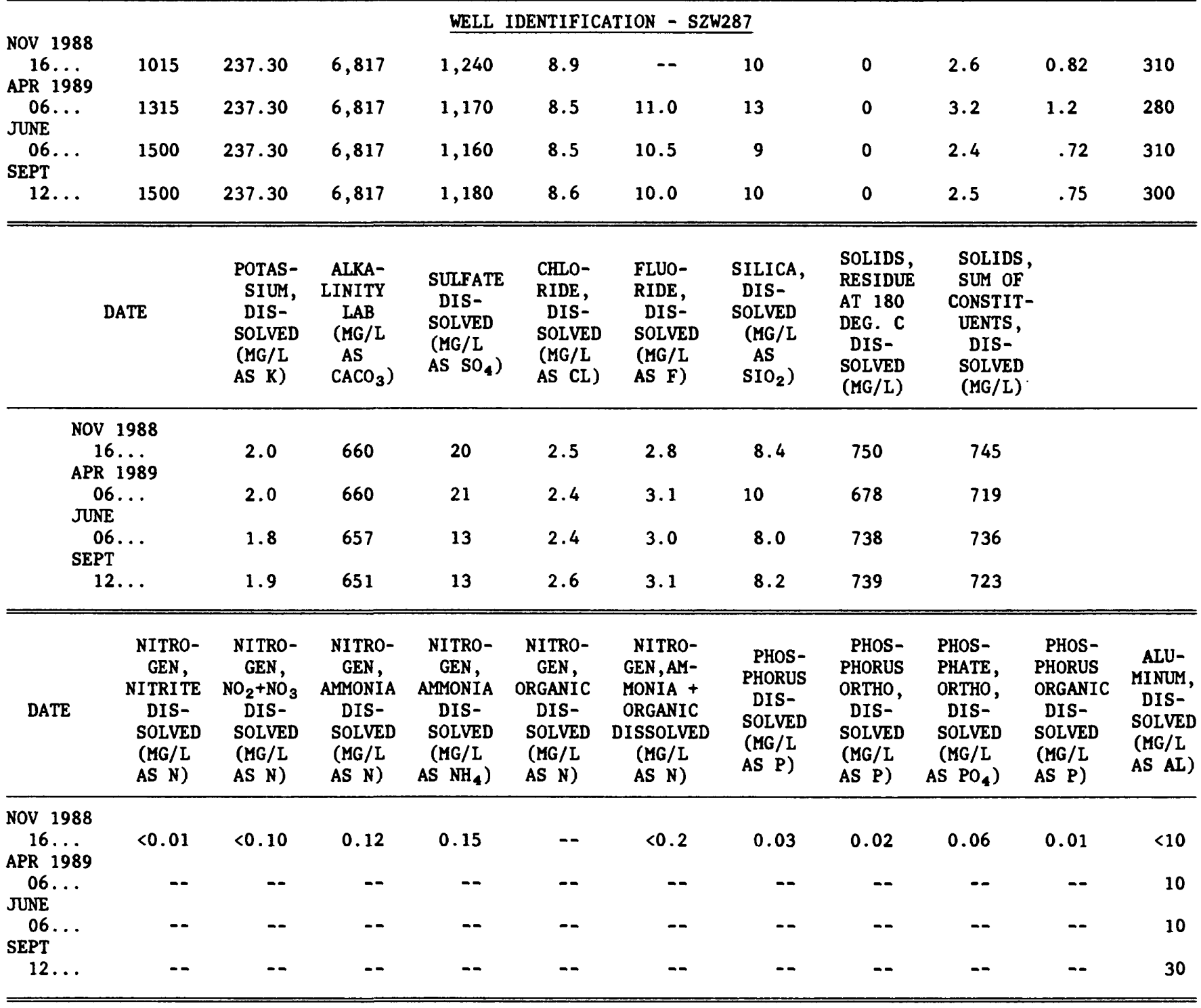

\begin{tabular}{|c|c|c|c|c|c|c|c|c|c|c|c|}
\hline DATE & $\begin{array}{c}\text { ARSENIC } \\
\text { DIS- } \\
\text { SOLVED } \\
(\mu G / L \\
\text { AS AS) }\end{array}$ & $\begin{array}{l}\text { BARIUM, } \\
\text { DIS- } \\
\text { SOLVED } \\
(\mu G / I \\
\text { AS BA) }\end{array}$ & $\begin{array}{l}\text { BERYL- } \\
\text { LIUM, } \\
\text { DIS- } \\
\text { SOLVED } \\
\text { ( } \mu \mathrm{G} / \mathrm{L} \\
\text { AS BE) }\end{array}$ & $\begin{array}{l}\text { BORON, } \\
\text { DIS- } \\
\text { SOLVED } \\
(\mu G / L \\
\text { AS B) }\end{array}$ & $\begin{array}{c}\text { CADMIUM } \\
\text { DIS- } \\
\text { SOLVED } \\
(\mu \mathrm{G} / \mathrm{L} \\
\text { AS CD) }\end{array}$ & $\begin{array}{l}\text { CHRO- } \\
\text { MIUM, } \\
\text { DIS- } \\
\text { SOLVED } \\
\text { ( } \mu G / L \\
\text { AS CR) }\end{array}$ & $\begin{array}{l}\text { COBALT, } \\
\text { DIS- } \\
\text { SOLVED } \\
(\mu G / L \\
\text { AS CO) }\end{array}$ & $\begin{array}{l}\text { COPPER, } \\
\text { DIS- } \\
\text { SOLVED } \\
(\mu G / L \\
\text { AS CU) }\end{array}$ & $\begin{array}{l}\text { IRON, } \\
\text { DIS- } \\
\text { SOLVED } \\
(\mu \mathrm{G} / \mathrm{L} \\
\text { AS FE) }\end{array}$ & $\begin{array}{l}\text { LEAD, } \\
\text { DIS- } \\
\text { SOLVED } \\
(\mu G / I \\
\text { AS PB) }\end{array}$ & $\begin{array}{l}\text { MANGA- } \\
\text { NESE, } \\
\text { DIS- } \\
\text { SOLVED } \\
\text { ( } \mu G / L \\
\text { AS MN) }\end{array}$ \\
\hline
\end{tabular}

AS P) AS P)

\section{.}


Table 25.--Water-quality analyses for wells--Continued

\begin{tabular}{|c|c|c|c|c|c|c|c|c|c|c|c|}
\hline DATE & $\begin{array}{l}\text { MOLYB- } \\
\text { DENUM, } \\
\text { DIS- } \\
\text { SOLVED } \\
(\mu G / L \\
\text { AS MO) }\end{array}$ & $\begin{array}{l}\text { NICKEL, } \\
\text { DIS- } \\
\text { SOLVED } \\
\text { ( } \mu G / L \\
\text { AS NI) }\end{array}$ & $\begin{array}{l}\text { SILVER, } \\
\text { DIS- } \\
\text { SOLVED } \\
(\mu G / L \\
\text { AS AG) }\end{array}$ & $\begin{array}{c}\text { STRON- } \\
\text { TIUM, } \\
\text { DIS- } \\
\text { SOLVED } \\
\text { ( } \mu G / L \\
\text { AS SR) }\end{array}$ & $\begin{array}{l}\text { VANA- } \\
\text { DIUM, } \\
\text { DIS- } \\
\text { SOLVED } \\
(\mu G / L \\
\text { AS V) }\end{array}$ & $\begin{array}{l}\text { ZINC, } \\
\text { DIS- } \\
\text { SOLVED } \\
(\mu G / L \\
\text { AS ZN) }\end{array}$ & $\begin{array}{l}\text { C-13/ } \\
\text { C-12 } \\
\text { STABLE } \\
\text { ISOTOPE } \\
\text { RATIO } \\
\text { PER } \\
\text { MIL }\end{array}$ & $\begin{array}{l}\text { H-2/ } \\
\text { H-1 } \\
\text { STABLE } \\
\text { ISOTOPE } \\
\text { RATIO } \\
\text { PER } \\
\text { MIL }\end{array}$ & $\begin{array}{c}0-18 / \\
0-16 \\
\text { STABLE } \\
\text { ISOTOPE } \\
\text { RATIO } \\
\text { PER } \\
\text { MIL }\end{array}$ & $\begin{array}{l}\text { S-34/ } \\
\text { S-32 } \\
\text { STABLE } \\
\text { ISOTOPE } \\
\text { RATIO } \\
\text { PER } \\
\text { MIL }\end{array}$ & $\begin{array}{c}\text { TRIT- } \\
\text { IUM } \\
\text { TOTAL } \\
\text { (PCI/L) }\end{array}$ \\
\hline
\end{tabular}

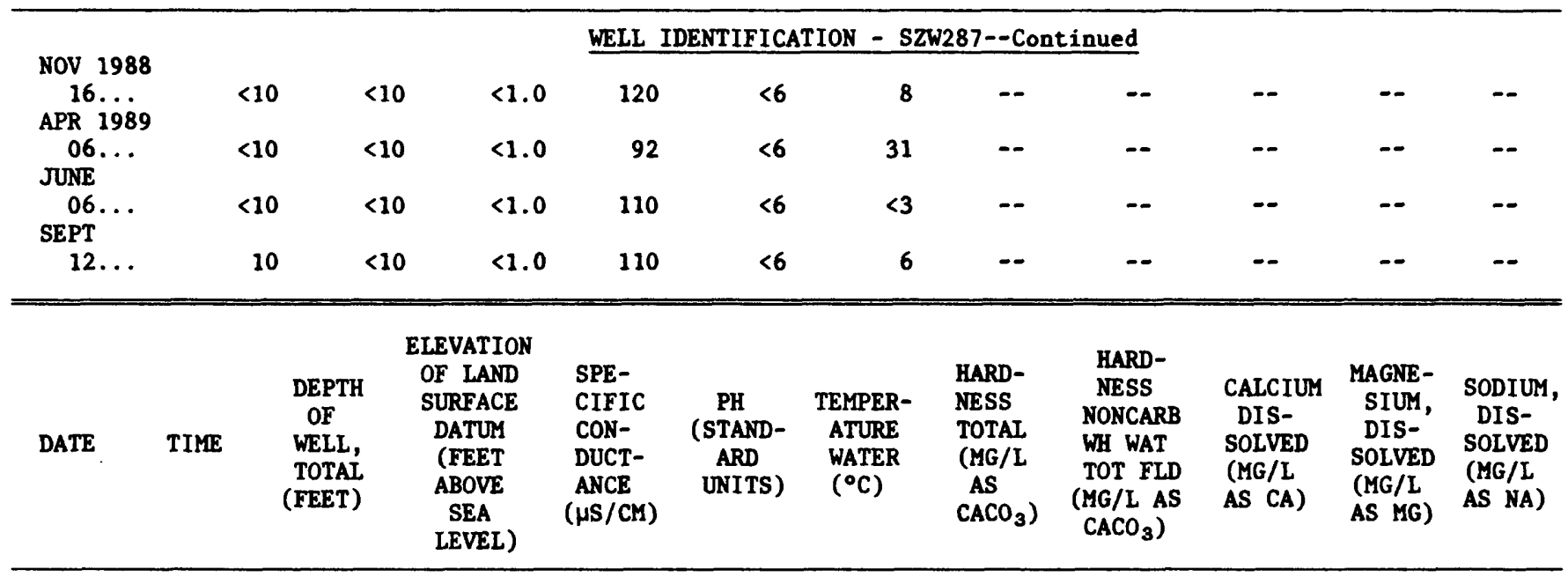

\section{MAR 1988}

06.

$11 \ldots .1215$

MAY

$09 . . \quad 1230$

SEPT

$01 \ldots 0930$

NOV

$16 \ldots 1000$

APR 1989

$06 \ldots 1400$

JUNE

06... 1430

SEPT
$1530 \quad 264.80$

264.80

264.80

264.80

264.80

264.80

264.80

264.80
6,820

6,820

6,820

6,820

6,820

6,820

6,820

6,820

WELL IDENTIFICATION - SZU287

$\begin{array}{llll}1,195 & 8.3 & 10.0 & 36\end{array}$

1,220

1,235

1,100

1,220

1,150

1,130

1,130
8.6

$\begin{array}{lll}8.6 & 10.5 & 35\end{array}$

$8.4 \quad 10.0 \quad 31$

$\begin{array}{lll}8.5 & 10.5 & 24\end{array}$

$\begin{array}{lll}8.5 & 10.0 & 22\end{array}$

8.520 $\begin{array}{lll}8.4 & 11.0 & 28\end{array}$

$8.7 \quad-2 \quad 27$

$\begin{array}{llll}0 & 6.6 & 4.6 & 380 \\ 22 & 6.0 & 4.7 & 290 \\ 0 & 5.5 & 4.2 & 290 \\ 0 & 4.9 & 3.9 & 280 \\ 0 & 4.8 & 3.6 & 290 \\ 0 & 4.3 & 3.1 & 280 \\ 0 & 4.0 & 2.8 & 300 \\ 0 & 3.7 & 2.6 & 280\end{array}$

CHLO- FLUO- SILICA, SOLIDS, SOLIDS, RIDE, RIDE, DIS- RESIDUE SUM OF DIS- DIS- SOLVED AT 180 CONSTITSOLVED SOLVED DEG. C UENTS, (MG/L $\quad$ (MG/L $\quad$ AS DIS- DISAS CL) AS F) $\quad \mathrm{SIO}_{2}$ ) SOLVED

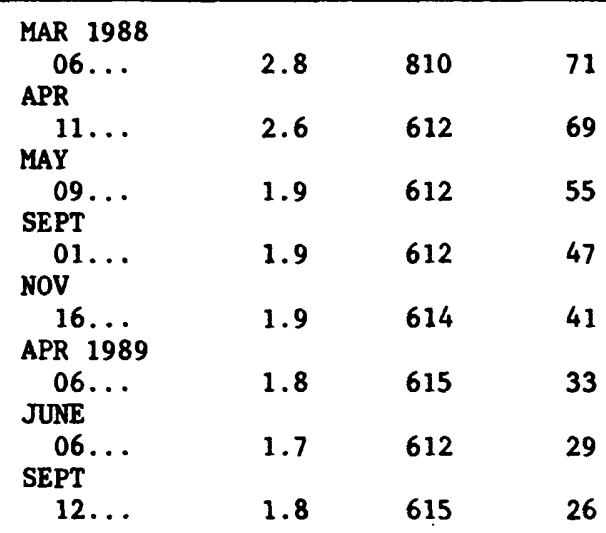

6.0

3.0

2.7

2.3

2.4

2.2

2.1

2.1
2.4

8.6

979

968

3.6

8.4

763

758

3.8

8.2

746

739

4.1

8.2

733

720

3.3

8.6

717

725

.1

8. 3

711

702

3.9

8.3

702

719

4.3

7.7

696

691 
Table 25.--Water-quality analyses for wells--Continued

\begin{tabular}{|c|c|c|c|c|c|c|c|c|c|c|c|}
\hline DATE & $\begin{array}{l}\text { NITRO- } \\
\text { GEN, } \\
\text { NITRITE } \\
\text { DIS- } \\
\text { SOLVED } \\
\text { (MG/L } \\
\text { AS N) }\end{array}$ & $\begin{array}{c}\mathrm{NITRO} \\
\text { GEN, } \\
\mathrm{NO}_{2}+\mathrm{NO}_{3} \\
\text { DIS- } \\
\text { SOLVED } \\
\text { SOLV } \\
\text { (MG/L } \\
\text { AS N) } \\
\mathbf{A}\end{array}$ & $\begin{array}{l}\text { NITRO- } \\
\text { GEN, } \\
\text { MMONIA AY } \\
\text { DIS- } \\
\text { SOLVED } \\
\text { (MG/L } \\
\text { AS N) }\end{array}$ & $\begin{array}{l}\text { NITRO- } \\
\text { GEN, } \\
\text { IMMONIA } \\
\text { DIS- } \\
\text { SOLVED } \\
(\mathrm{MG} / \mathrm{L} \\
\left.\text { IS } \mathrm{NH}_{4}\right)\end{array}$ & $\begin{array}{lr}\text { NITRO- } \\
\text { GEN, } \\
\text { RGANIC } \\
\text { DIS- } \\
\text { SOLVED } \\
\text { (MG/L } \\
\text { AS N) } \\
\end{array}$ & $\begin{array}{l}\text { NITRO- } \\
\text { GEN,AM- } \\
\text { MONIA + } \\
\text { ORGANIC } \\
\text { ISSOLVED } \\
\text { (MG/L } \\
\text { AS N) }\end{array}$ & $\begin{array}{l}\text { PHOS- } \\
\text { PHORUS } \\
\text { DIS- } \\
\text { SOLVED } \\
\text { (MG/L } \\
\text { AS P) }\end{array}$ & $\begin{array}{l}\text { PHOS- } \\
\text { PHORUS } \\
\text { ORTHO, } \\
\text { DIS- } \\
\text { SOLVED } \\
\text { (MG/L } \\
\text { AS P) }\end{array}$ & $\begin{array}{l}\text { PHOS- } \\
\text { PHATE, } \\
\text { ORTHO, } \\
\text { DIS- } \\
\text { SOLVED } \\
(\text { MG/L } \\
\text { AS PO } 4)\end{array}$ & $\begin{array}{c}\text { PHOS- } \\
\text { PHORUS } \\
\text { ORGANIC } \\
\text { DIS- } \\
\text { SOLVED } \\
\text { (MG/L } \\
\text { AS P) }\end{array}$ & $\begin{array}{l}\text { ALU- } \\
\text { MINUM, } \\
\text { DIS- } \\
\text { SOLVED } \\
\text { (MG/L } \\
\text { AS AL) }\end{array}$ \\
\hline \multicolumn{12}{|c|}{ WELL IDENTIFICATION - SZU287--Continued } \\
\hline $\begin{array}{c}\text { MAR } 1988 \\
06 \ldots \\
\text { APR }\end{array}$ & $<0.01$ & $<0.10$ & 0.08 & 0.1 & 1.1 & 1.2 & 0.05 & 0.03 & 0.09 & 0.02 & $<10$ \\
\hline${ }_{\mathrm{MAY}}^{11} \ldots$ & $<.01$ & $<.10$ & .06 & .08 & .54 & .6 & .04 & .03 & .09 & .01 & $<10$ \\
\hline$\underset{\text { SEPT }}{09}$ & $<.01$ & $<.10$ & .06 & .08 & .54 & .6 & .03 & .02 & .06 & .01 & $<10$ \\
\hline Nov & $<.01$ & $<.10$ & .38 & .49 & .32 & .7 & .03 & .02 & .06 & .01 & 20 \\
\hline $\begin{array}{l}16 \\
\text { APR } 1989\end{array}$ & $<.01$ & $<.10$ & .44 & .57 & .26 & .7 & .03 & .03 & .09 & .0 & 30 \\
\hline JUNE & -- & -- & -- & -- & -- & -- & -- & -- & -- & -- & 40 \\
\hline$\underset{\text { SEPT }}{06 \ldots}$ & -- & -- & -- & -- & -- & -- & -- & -- & -- & -- & 30 \\
\hline $12 \ldots$ & -- & -- & -- & - & -- & - & -- & $-\infty$ & -- & -- & 40 \\
\hline DATE & $\begin{array}{l}\text { ARSENIC } \\
\text { DIS- } \\
\text { SOLVED } \\
(\mu G / L \\
\text { AS AS) }\end{array}$ & $\begin{array}{l}\text { BARIUM, } \\
\text { DIS- } \\
\text { SOLVED } \\
\text { ( } \mu G / L \\
\text { AS BA) }\end{array}$ & $\begin{array}{l}\text { BERYL- } \\
\text { LIUM, } \\
\text { DIS- } \\
\text { SOLVED } \\
(\mu \mathrm{G} / \mathrm{L} \\
\mathrm{AS} \mathrm{BE})\end{array}$ & $\begin{array}{l}\text { BORON, } \\
\text { DIS- } \\
\text { SOLVED } \\
(\mu G / L \\
\text { AS B) }\end{array}$ & $\begin{array}{l}\text { CADMIUM } \\
\text { DIS- } \\
\text { SOLVED } \\
(\mu G / L \\
\text { AS CD) }\end{array}$ & $\begin{array}{l}\text { CHRO- } \\
\text { MIUM, } \\
\text { DIS- } \\
\text { SOLVED } \\
\text { ( } \mu G / L \\
\text { AS CR) }\end{array}$ & $\begin{array}{l}\text { COBALT, } \\
\text { DIS- } \\
\text { SOLVED } \\
(\mu G / L \\
\text { AS CO) }\end{array}$ & $\begin{array}{l}\text { COPPER, } \\
\text { DIS- } \\
\text { SOLVED } \\
(\mu G / L \\
\text { AS CU) }\end{array}$ & $\begin{array}{l}\text { IRON, } \\
\text { DIS- } \\
\text { SOLVED } \\
\text { ( } \mu G / L \\
\text { AS FE) }\end{array}$ & $\begin{array}{l}\text { IEAD, } \\
\text { DIS- } \\
\text { SOLVED } \\
(\mu G / L \\
\text { AS PB) }\end{array}$ & $\begin{array}{c}\text { MANGA- } \\
\text { NESE, } \\
\text { DIS- } \\
\text { SOLVED } \\
\text { ( } \mu G / L \\
\text { AS MN) }\end{array}$ \\
\hline $\begin{array}{c}\text { MAR } 1988 \\
\text { 06... } \\
\text { APR }\end{array}$ & $<1$ & 49 & $<0.5$ & 230 & 3 & $<5$ & $<3$ & $<10$ & 30 & $<10$ & 31 \\
\hline $\mathrm{MAY} 11 \ldots$ & 2 & 27 & $<.5$ & 70 & $<1$ & $<5$ & $<3$ & $<10$ & 18 & $<10$ & 30 \\
\hline SEPT $09 .$. & 2 & 28 & $<.5$ & 60 & $<1$ & $<5$ & $<3$ & $<10$ & 40 & $<10$ & 3 \\
\hline Nov $01 \ldots$ & 1 & 28 & $<.5$ & 70 & $<1$ & $<5$ & $<3$ & $<10$ & 41 & $<10$ & 26 \\
\hline $\begin{array}{ll} & 16 \ldots \\
\text { APR } & 1989\end{array}$ & 1 & 25 & $<.5$ & 60 & $<1$ & $<5$ & $<3$ & $<10$ & 38 & $<10$ & 30 \\
\hline JUNE & 1 & 29 & $<.5$ & 70 & $<1$ & 6 & $<3$ & $<10$ & 64 & 10 & 31 \\
\hline SEPT $^{06 \ldots}$ & 1 & 23 & $<.5$ & 70 & $<1$ & $<5$ & $<3$ & $<10$ & 60 & $<10$ & 31 \\
\hline $12 \ldots$ & $<1$ & 24 & $<.5$ & 50 & $<1$ & $<5$ & $<3$ & $<10$ & 42 & $<10$ & 29 \\
\hline
\end{tabular}


Table 25.--Water-quality analyses for wells--Continued

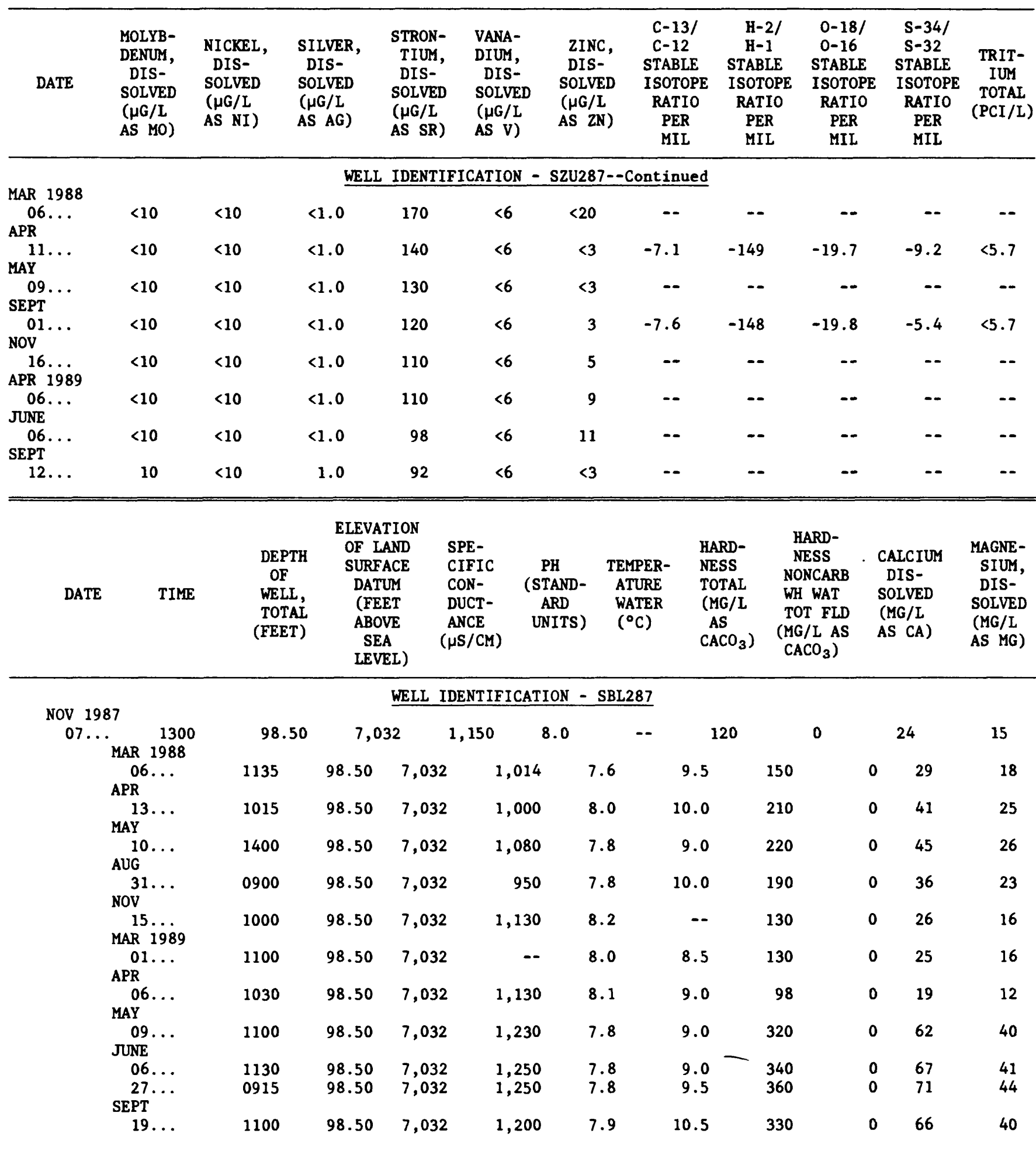


Table 25.--Water-quality analyses for wells--Continued

\begin{tabular}{|c|c|c|c|c|c|c|c|c|c|}
\hline DATE & $\begin{array}{l}\text { SODIUM, } \\
\text { DIS- } \\
\text { SOLVED } \\
\text { (MG/L } \\
\text { AS NA) }\end{array}$ & $\begin{array}{l}\text { POTAS- } \\
\text { SIUM, } \\
\text { DIS- } \\
\text { SOLVED } \\
\text { (MG/L } \\
\text { AS K) }\end{array}$ & $\begin{array}{c}\text { ALKA- } \\
\text { LINITY } \\
\text { LAB } \\
(\mathrm{MG} / \mathrm{L} \\
\mathrm{AS} \\
\left.\mathrm{CACO}_{3}\right)\end{array}$ & $\begin{array}{l}\text { SULFATE } \\
\text { DIS- } \\
\text { SOLVED } \\
(\mathrm{MG} / \mathrm{L} \\
\left.\mathrm{AS} \mathrm{SO}_{4}\right)\end{array}$ & $\begin{array}{l}\text { CHLO- } \\
\text { RIDE, } \\
\text { DIS- } \\
\text { SOLVED } \\
\text { (MG/L } \\
\text { AS CL) }\end{array}$ & $\begin{array}{l}\text { FLUO- } \\
\text { RIDE, } \\
\text { DIS- } \\
\text { SOLVED } \\
\text { (MG/L } \\
\text { AS F) }\end{array}$ & $\begin{array}{l}\text { SILICA, } \\
\text { DIS- } \\
\text { SOLVED } \\
(\mathrm{MG} / \mathrm{L} \\
\mathrm{AS} \\
\left.\mathrm{SIO}_{2}\right)\end{array}$ & $\begin{array}{l}\text { SOLIDS, } \\
\text { RESIDUE } \\
\text { AT } 180 \\
\text { DEG. C } \\
\text { DIS- } \\
\text { SOLVED } \\
\text { (MG/L) }\end{array}$ & $\begin{array}{l}\text { SOLIDS, } \\
\text { SUM OF } \\
\text { CONSTIT- } \\
\text { UENTS, } \\
\text { DIS- } \\
\text { SOLVED } \\
\text { (MG/L) }\end{array}$ \\
\hline
\end{tabular}

Nov 1987

WELL IDENTIFICATION - SBL287--Continued

\begin{tabular}{|c|c|c|c|c|c|c|c|c|c|}
\hline $\begin{array}{l}07 \ldots \\
\text { MAR } 1988\end{array}$ & 230 & 3.0 & 543 & 86 & 4.2 & 0.3 & 12 & 697 & 701 \\
\hline $\begin{array}{c}06 \ldots \\
\text { APR }\end{array}$ & 210 & 2.9 & 480 & 68 & 4.9 & .7 & 13 & 656 & 637 \\
\hline $\operatorname{MAY}^{13} \cdots$ & 180 & 2.5 & 499 & 92 & 5.5 & .6 & 14 & 642 & 663 \\
\hline${ }_{A U G}^{10 \ldots}$ & 160 & 2.7 & 492 & 100 & 5.6 & .7 & 15 & 636 & 653 \\
\hline $\begin{array}{l}31 \ldots \\
\text { NOV }\end{array}$ & 190 & 2.7 & 504 & 82 & 5.2 & .7 & 13 & 647 & 658 \\
\hline $\begin{array}{l}15 \ldots \\
\text { MAR } 1989\end{array}$ & 220 & 2.5 & 545 & 70 & 5.2 & .8 & 12 & 678 & 682 \\
\hline $\begin{array}{c}01 \cdots \\
\text { APR }\end{array}$ & 230 & 2.5 & 558 & 64 & 5.1 & .8 & 12 & 688 & 693 \\
\hline${ }_{M A Y}^{06 \ldots}$ & 230 & 2.4 & 569 & 67 & 5.0 & .9 & 11 & 695 & 690 \\
\hline $\begin{array}{l}09 . \cdots \\
\text { JUNE }\end{array}$ & 190 & 2.9 & 492 & 190 & 5.4 & .6 & 14 & 786 & 804 \\
\hline $\begin{array}{l}06 \ldots \\
27 \ldots\end{array}$ & $\begin{array}{l}170 \\
160\end{array}$ & $\begin{array}{l}3.0 \\
3.3\end{array}$ & $\begin{array}{l}481 \\
445\end{array}$ & $\begin{array}{l}240 \\
240\end{array}$ & $\begin{array}{l}5.5 \\
5.8\end{array}$ & $\begin{array}{l}.5 \\
.5\end{array}$ & $\begin{array}{l}13 \\
13\end{array}$ & $\begin{array}{l}814 \\
812\end{array}$ & $\begin{array}{l}831 \\
807\end{array}$ \\
\hline 19. & 160 & 3.2 & 432 & 220 & 5.2 & .6 & 13 & 692 & 769 \\
\hline
\end{tabular}

$19 \ldots$

$\begin{array}{lll}3.2 & 432 & 220\end{array}$

\begin{tabular}{|c|c|c|c|c|c|c|c|}
\hline $\begin{array}{l}\text { NITRO- } \\
\text { GEN, } \\
\text { NITRITE } \\
\text { DIS- } \\
\text { SOLVED } \\
\text { (MG/L } \\
\text { AS N) }\end{array}$ & $\begin{array}{c}\text { NITRO- } \\
\text { GEN , } \\
\mathrm{NO}_{2}+\mathrm{NO}_{3} \\
\text { DIS- } \\
\text { SOLVED } \\
\text { (MG/I } \\
\text { AS N) }\end{array}$ & $\begin{array}{l}\text { NITRO- } \\
\text { GEN, } \\
\text { AMMONIA } \\
\text { DIS- } \\
\text { SOIVED } \\
\text { (MG/I } \\
\text { AS N) }\end{array}$ & $\begin{array}{l}\text { NITRO- } \\
\text { GEN, } \\
\text { AMMONIA } \\
\text { DIS- } \\
\text { SOLVED } \\
(\mathrm{MG} / \mathrm{L} \\
\left.\text { AS } \mathrm{NH}_{4}\right)\end{array}$ & $\begin{array}{c}\text { NITRO- } \\
\text { GEN, } \\
\text { ORGANIC } \\
\text { DIS- } \\
\text { SOLVED } \\
\text { (MG/L } \\
\text { AS N) }\end{array}$ & $\begin{array}{c}\text { NITRO- } \\
\text { GEN,AM- } \\
\text { MONIA + } \\
\text { ORGANIC } \\
\text { DISSOLVED } \\
\text { (MG/L } \\
\text { AS N) }\end{array}$ & $\begin{array}{l}\text { PHOS- } \\
\text { PHORUS } \\
\text { DIS- } \\
\text { SOLVED } \\
\text { (MG/L } \\
\text { AS P) }\end{array}$ & $\begin{array}{l}\text { PHOS- } \\
\text { PHORUS } \\
\text { ORTHO, } \\
\text { DIS- } \\
\text { SOLVED } \\
\text { (MG/L } \\
\text { AS P) }\end{array}$ \\
\hline
\end{tabular}

NOV 1987

WELI IDENTIFICATION - SBL287--Continued

\begin{tabular}{|c|c|c|c|c|c|c|c|c|}
\hline $\begin{array}{l}07 \ldots \\
\text { MAR } 1988\end{array}$ & -- & - & - & -- & -- & 2.0 & 0.02 & -- \\
\hline $\begin{array}{c}06 \ldots \\
\text { APR }\end{array}$ & $<0.01$ & $<0.10$ & 0.77 & 0.99 & 0.73 & 1.5 & .01 & 0.01 \\
\hline $\operatorname{MAY}_{13}^{13}$ & $<.01$ & $<.10$ & 1.60 & 2.1 & .0 & 1.5 & .01 & $<.01$ \\
\hline${ }_{\text {AUG }}^{10 \ldots}$ & $<.01$ & $<.10$ & 1.20 & 1.5 & .6 & 1.8 & $<.01$ & $<.01$ \\
\hline$\underset{\text { Nov }}{31 \ldots}$ & $<.01$ & .16 & .92 & 1.2 & .08 & 1.0 & .01 & $<.01$ \\
\hline $\begin{array}{l}15 \ldots \\
\text { MAR } 1989\end{array}$ & $<.01$ & $<.10$ & .79 & 1.0 & .01 & .8 & .03 & .01 \\
\hline$\underset{\text { APR }}{01 \ldots}$ & $<.01$ & $<.10$ & .98 & 1.3 & .02 & 1.0 & .03 & .03 \\
\hline MAY & -- & -- & -- & -- & -- & -- & -- & -- \\
\hline$\underset{\text { JUNE }}{09} \ldots$ & $<.01$ & $<.10$ & 1.70 & 2.2 & .4 & 2.1 & .01 & $<.01$ \\
\hline $06 \ldots$ & -- & -- & -- & -- & -- & -- & -- & -- \\
\hline SEPT & 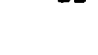 & 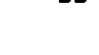 & - & $\cdots$ & -0 & 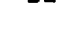 & 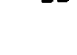 & \\
\hline $19 \ldots$ & -- & -- & -- & -- & -- & -- & - & \\
\hline
\end{tabular}


Table 25.--Water-quality analyses for wells--Continued

\begin{tabular}{|c|c|c|c|c|c|c|c|c|c|c|}
\hline DATE & $\begin{array}{c}\text { PHOS- } \\
\text { PHATE, } \\
\text { ORTHO, } \\
\text { DIS- } \\
\text { SOLVED } \\
(\mathrm{MG} / \mathrm{L} \\
\text { AS PO4) }\end{array}$ & $\begin{array}{c}\text { PHOS- } \\
\text { PHORUS } \\
\text { ORGANIC } \\
\text { DIS- } \\
\text { SOLVED } \\
\text { (MG/L } \\
\text { AS P) }\end{array}$ & $\begin{array}{l}\text { ALU- } \\
\text { MINUM, } \\
\text { DIS- } \\
\text { SOLVED } \\
\text { ( } \mu G / L \\
\text { AS AL) }\end{array}$ & $\begin{array}{l}\text { ARSENIC } \\
\text { DIS } \\
\text { SOLVED } \\
\text { ( } \mu \text { G/L } \\
\text { AS AS) }\end{array}$ & $\begin{array}{l}\text { BARIUM, } \\
\text { DIS- } \\
\text { SOLVED } \\
(\mu G / L \\
\text { AS BA) }\end{array}$ & $\begin{array}{l}\text { BERYL- } \\
\text { LIUM, } \\
\text { DIS- } \\
\text { SOLVED } \\
(\mu G / L \\
\text { AS BE) }\end{array}$ & $\begin{array}{l}\text { BORON, } \\
\text { DIS- } \\
\text { SOLVED } \\
\text { ( } \mu G / L \\
\text { AS B) }\end{array}$ & $\begin{array}{l}\text { CADMIUM } \\
\text { DIS- } \\
\text { SOLVED } \\
(\mu G / L \\
\text { AS CD) }\end{array}$ & $\begin{array}{l}\text { CHRO- } \\
\text { MIUM, } \\
\text { DIS- } \\
\text { SOLVED } \\
\text { ( } \mu G / L \\
\text { AS CR) }\end{array}$ & $\begin{array}{c}\text { COBALT, } \\
\text { DIS- } \\
\text { SOLVED } \\
\text { ( } \mu G / L) \\
\text { AS CO) }\end{array}$ \\
\hline \multicolumn{11}{|c|}{ WELL IDENTIFICATION - SBL287--Continued } \\
\hline NOV 1987 & & & & & & & & & & \\
\hline $\begin{array}{l}07 \ldots \\
\text { MAR } 1988\end{array}$ & -- & 0.02 & 40 & $<1$ & 45 & $<0.5$ & 310 & $<1$ & $<5$ & $<3$ \\
\hline $\begin{array}{c}06 \ldots \\
\text { APR }\end{array}$ & 0.03 & .0 & $<10$ & $<1$ & 110 & $<.5$ & 300 & 2 & $<5$ & $<3$ \\
\hline${ }_{\mathrm{MAY}}^{13} \ldots$ & -- & .01 & $<10$ & $<1$ & 120 & $<.5$ & 270 & 2 & $<5$ & $<3$ \\
\hline AUG $10 \ldots$ & -- & -- & $<10$ & $<1$ & 110 & $<.5$ & 260 & $<1$ & $<5$ & $<3$ \\
\hline${ }_{\text {NOV }}^{31 \ldots}$ & -- & .01 & $<10$ & $<1$ & 110 & $<.5$ & 260 & $<1$ & $<5$ & $<3$ \\
\hline $\begin{array}{l}15 \ldots \\
\text { MAR } 1989\end{array}$ & .03 & .02 & $<10$ & $<1$ & 93 & .6 & 300 & $<1$ & $<5$ & $<3$ \\
\hline$\underset{\text { APR }}{01 \ldots}$ & .09 & .0 & $<10$ & $<1$ & 96 & $<.5$ & 300 & $<1$ & $<5$ & $<3$ \\
\hline${ }_{\operatorname{MAY}}^{06} .$. & - & -- & $<10$ & 1 & 87 & $<.5$ & 320 & $<1$ & $<5$ & $<3$ \\
\hline$\underset{\text { JUNE }}{09 . .}$ & -- & .01 & 10 & 1 & 180 & $<2$ & 270 & 23 & $<20$ & $<9$ \\
\hline $06 \ldots$ & -- & -- & $<10$ & $<1$ & 160 & $<.5$ & 280 & $<1$ & $<5$ & $<3$ \\
\hline$\stackrel{27 \ldots}{\text { SEPT }}$ & -- & -- & $<10$ & 4 & 150 & $<.5$ & 270 & $<1$ & $<5$ & $<3$ \\
\hline $19 \ldots$ & -- & -- & $<10$ & 1 & 140 & $<.5$ & 260 & $<1$ & $<5$ & $<3$ \\
\hline DATE & $\begin{array}{l}\text { COPPER, } \\
\text { DIS- } \\
\text { SOLVED } \\
(\mu G / L \\
\text { AS CU) }\end{array}$ & $\begin{array}{l}\text { IRON, } \\
\text { DIS- } \\
\text { SOLVED } \\
(\mu G / L \\
\text { AS FE) }\end{array}$ & $\begin{array}{l}\text { LEAD, } \\
\text { DIS- } \\
\text { SOLVED } \\
\text { ( } \mu G / L \\
\text { AS PB) }\end{array}$ & $\begin{array}{l}\text { MANGA- } \\
\text { NESE, } \\
\text { DIS- } \\
\text { SOLVED } \\
\text { ( } \mu \text { G/L } \\
\text { AS MN) }\end{array}$ & $\begin{array}{l}\text { MOLYB- } \\
\text { DENUM, } \\
\text { DIS- } \\
\text { SOLVED } \\
\text { ( } \mu G / L \\
\text { AS MO) }\end{array}$ & $\begin{array}{l}\text { NICKEL, } \\
\text { DIS- } \\
\text { SOLVED } \\
(\mu G / L \\
\text { AS NI) }\end{array}$ & $\begin{array}{c}\text { SILVER, } \\
\text { DIS- } \\
\text { SOLVED } \\
\text { ( } \mu G / L \\
\text { AS AG) }\end{array}$ & $\begin{array}{c}\text { STRON- } \\
\text { TIUM, } \\
\text { DIS- } \\
\text { SOLVED } \\
\text { ( } \mu G / L \\
\text { AS SR) }\end{array}$ & $\begin{array}{l}\text { VANA- } \\
\text { DIUM, } \\
\text { DIS- } \\
\text { SOLVED } \\
(\mu G / L \\
\text { AS V) }\end{array}$ & $\begin{array}{l}\text { ZINC, } \\
\text { DIS- } \\
\text { SOLVED } \\
(\mu G / L \\
\text { AS ZN) }\end{array}$ \\
\hline NOV 1987 & & & & & & & & & & \\
\hline $\begin{array}{l}07 . \ldots \\
\text { MAR } 1988\end{array}$ & $<10$ & 55 & $<10$ & 120 & $<10$ & $<10$ & $<1.0$ & 610 & $<6$ & $<3$ \\
\hline $\begin{array}{c}06 \ldots \\
\text { APR }\end{array}$ & $<10$ & 15 & $<10$ & 42 & $<10$ & $<10$ & $<1.0$ & 770 & $<6$ & 3 \\
\hline $\mathrm{MAY}^{13} \cdot$ & $<10$ & 100 & 10 & 33 & $<10$ & $<10$ & 2.0 & 1,000 & $<6$ & 13 \\
\hline $10 \ldots$ & $<10$ & 170 & $<10$ & 31 & $<10$ & $<10$ & $<1.0$ & 1,000 & $<6$ & $<3$ \\
\hline$\underset{\mathrm{NOV}}{31 \ldots}$ & $<10$ & 130 & $<10$ & 35 & $<10$ & $<10$ & 3.0 & 880 & $<6$ & 4 \\
\hline $\begin{array}{l}15 \ldots \\
\text { MAR } 1989\end{array}$ & $<10$ & 150 & $<10$ & 61 & $<10$ & $<10$ & $<1.0$ & 680 & $<6$ & $<3$ \\
\hline $\begin{array}{c}01 \ldots \\
\text { APR }\end{array}$ & $<10$ & 120 & $<10$ & 67 & $<10$ & $<10$ & $<1.0$ & 680 & $<6$ & $<3$ \\
\hline$\underset{\text { MAY }}{06 \ldots}$ & $<10$ & 130 & $<10$ & 74 & $<10$ & $<10$ & $<1.0$ & 560 & $<6$ & 6 \\
\hline$\underset{\text { JUNE }}{09 . .}$ & $<30$ & $<9$ & $<30$ & 74 & $<30$ & $<30$ & $<30$ & 1,600 & $<18$ & 23 \\
\hline $\begin{array}{l}06 \ldots \\
27 \ldots\end{array}$ & $\begin{array}{l}<10 \\
<10\end{array}$ & $\begin{array}{l}280 \\
240\end{array}$ & $\begin{array}{l}<10 \\
<10\end{array}$ & $\begin{array}{l}78 \\
73\end{array}$ & $\begin{array}{l}<10 \\
<10\end{array}$ & $\begin{array}{l}<10 \\
<10\end{array}$ & $\begin{array}{l}<1.0 \\
<1.0\end{array}$ & $\begin{array}{l}1,600 \\
1,600\end{array}$ & $\begin{array}{l}<6 \\
<6\end{array}$ & $\begin{array}{l}<3 \\
15\end{array}$ \\
\hline $\begin{array}{c}\text { SEPT } \\
19 \ldots\end{array}$ & $<10$ & 370 & $<10$ & 94 & $<10$ & $<10$ & $<1.0$ & 1,500 & $<6$ & 8 \\
\hline
\end{tabular}


Table 25.--Water-quality analyses for wells--Continued

\begin{tabular}{|c|c|c|c|c|c|c|c|c|c|c|c|}
\hline DATE & TIME & $\begin{array}{c}\text { DEPTH } \\
\text { OF } \\
\text { WELL, } \\
\text { TOTAL } \\
\text { (FEET) }\end{array}$ & $\begin{array}{l}\text { ELEVATION } \\
\text { OF LAND } \\
\text { SURFACE } \\
\text { DATUI } \\
\text { (FEET } \\
\text { ABOVE } \\
\text { SEA } \\
\text { LEVEL) }\end{array}$ & $\begin{array}{l}\text { SPE- } \\
\text { CIFIC } \\
\text { CON- } \\
\text { DUCT- } \\
\text { ANCE } \\
(\mu S / C M)\end{array}$ & $\begin{array}{c}\text { PH } \\
\text { (STAND- } \\
\text { ARD } \\
\text { UNITS) }\end{array}$ & $\begin{array}{l}\text { TEMPER- } \\
\text { ATURE } \\
\text { WATER } \\
\left({ }^{\circ} \mathrm{C}\right)\end{array}$ & $\begin{array}{l}\text { HARD- } \\
\text { NESS } \\
\text { TOTAL } \\
(\mathrm{MG} / \mathrm{L} \\
\mathrm{AS}^{-} \\
\left.\mathrm{CACO}_{3}\right)\end{array}$ & $\begin{array}{l}\text { HARD- } \\
\text { NESS } \\
\text { NONCARB } \\
\text { WH WAT } \\
\text { TOT FLD } \\
(\mathrm{MG} / \mathrm{L} \text { AS } \\
\left.\mathrm{CACO}_{3}\right)\end{array}$ & $\begin{array}{l}\text { CALCIUM } \\
\text { DIS- } \\
\text { SOLVED } \\
\text { (MG/L } \\
\text { AS CA) }\end{array}$ & $\begin{array}{l}\text { MAGNE- } \\
\text { SIUM, } \\
\text { DIS- } \\
\text { SOLVED } \\
\text { (MG/L } \\
\text { AS MG) }\end{array}$ & $\begin{array}{c}\text { SODIUM, } \\
\text { DIS- } \\
\text { SOLVED } \\
\text { (MG/L } \\
\text { AS NA) }\end{array}$ \\
\hline \multicolumn{12}{|c|}{ WELL IDENTIFICATION - SBI287 } \\
\hline NOV 1987 & & & & & & & & & & & \\
\hline $\begin{array}{l}07 \\
\text { MAR } 1988\end{array}$ & 0930 & 150.00 & 7,031 & 910 & 7.4 & -- & 390 & 0 & 79 & 47 & 54 \\
\hline $\begin{array}{l}05 \cdots \\
\text { APR }\end{array}$ & 1120 & 150.00 & 7,031 & 875 & 7.4 & 9.0 & 430 & 0 & 87 & 50 & 49 \\
\hline $\mathrm{MAY}^{13} \ldots$ & 0915 & 150.00 & 7,031 & 980 & 7.3 & 10.0 & 530 & 99 & 110 & 62 & 34 \\
\hline${ }_{\text {JUNE }}^{10} \ldots$ & 1300 & 150.00 & 7,031 & 930 & 7.1 & 12.5 & 460 & 30 & 95 & 53 & 38 \\
\hline $\begin{array}{r}15 \ldots \\
15 \ldots \\
15 \ldots \\
\text { AUG }\end{array}$ & $\begin{array}{l}1330 \\
1340 \\
1350\end{array}$ & $\begin{array}{l}150.00 \\
150.00 \\
150.00\end{array}$ & $\begin{array}{l}7,031 \\
7,031 \\
7,031\end{array}$ & $\begin{array}{l}733 \\
927 \\
918\end{array}$ & $\begin{array}{l}7.2 \\
7.1 \\
7.1\end{array}$ & $\begin{array}{l}10.0 \\
10.0 \\
10.0\end{array}$ & $\begin{array}{l}280 \\
410 \\
410\end{array}$ & $\begin{array}{l}0 \\
0 \\
0\end{array}$ & $\begin{array}{l}53 \\
82 \\
82\end{array}$ & $\begin{array}{l}35 \\
50 \\
50\end{array}$ & $\begin{array}{l}88 \\
54 \\
53\end{array}$ \\
\hline $\begin{array}{c}31 . . \\
\text { Nov }\end{array}$ & 0830 & 150.00 & 7,031 & 790 & 7.3 & 10.0 & 380 & 0 & 72 & 47 & 61 \\
\hline $\begin{array}{l}15 \ldots \\
\text { MAR } 1989\end{array}$ & 1030 & 150.00 & 7,031 & 940 & 7.4 & -- & 370 & 0 & 73 & 46 & 70 \\
\hline $\begin{array}{l}01 \ldots \\
\text { APR }\end{array}$ & 1030 & 150.00 & 7,031 & -- & 7.4 & 8.5 & 390 & 0 & 74 & 49 & 70 \\
\hline MAY $06 .$. & 0930 & 150.00 & 7,031 & 1,040 & 7.3 & 9.5 & 440 & 0 & 86 & 53 & 62 \\
\hline$\underset{\text { JUNE }}{09}$ & 1030 & 150.00 & 7,031 & 1,125 & 7.3 & 9.5 & 550 & 110 & 110 & 65 & 50 \\
\hline $\begin{array}{r}06 \ldots \\
27 \ldots \\
\text { SEPT }\end{array}$ & $\begin{array}{l}1115 \\
0845\end{array}$ & $\begin{array}{l}150.00 \\
150.00\end{array}$ & $\begin{array}{l}7,031 \\
7,031\end{array}$ & $\begin{array}{l}1,230 \\
1,200\end{array}$ & $\begin{array}{l}7.3 \\
7.1\end{array}$ & $\begin{array}{l}9.5 \\
9.5\end{array}$ & $\begin{array}{l}600 \\
580\end{array}$ & $\begin{array}{l}160 \\
170\end{array}$ & $\begin{array}{l}120 \\
120\end{array}$ & $\begin{array}{l}71 \\
68\end{array}$ & $\begin{array}{l}52 \\
48\end{array}$ \\
\hline $19 \ldots$ & 1000 & 150.00 & 7,031 & 1,180 & 7.3 & 9.5 & 540 & 240 & 110 & 63 & 53 \\
\hline
\end{tabular}


Table 25.--Water-quality analyses for wells--Continued

\begin{tabular}{|c|c|c|c|c|c|c|c|c|c|}
\hline DATE & $\begin{array}{l}\text { POTAS- } \\
\text { SIUM, } \\
\text { DIS- } \\
\text { SOLVED } \\
\text { (MG/L } \\
\text { AS K) }\end{array}$ & $\begin{array}{c}\text { ALKA- } \\
\text { LINITY } \\
\text { LAB } \\
(\mathrm{MG} / \mathrm{L} \\
\mathrm{AS} \\
\left.\mathrm{CACO}_{3}\right)\end{array}$ & $\begin{array}{l}\text { SULFATE } \\
\text { DIS- } \\
\text { SOLVED } \\
(\mathrm{MG} / \mathrm{L} \\
\left.\text { AS } \mathrm{SO}_{4}\right)\end{array}$ & $\begin{array}{l}\text { CHLO- } \\
\text { RIDE, } \\
\text { DIS- } \\
\text { SOLVED } \\
\text { (MG/L } \\
\text { AS CL) }\end{array}$ & $\begin{array}{l}\text { FLUO- } \\
\text { RIDE, } \\
\text { DIS- } \\
\text { SOLVED } \\
\text { (MG/L } \\
\text { AS F) }\end{array}$ & $\begin{array}{l}\text { SILICA, } \\
\text { DIS- } \\
\text { SOLVED } \\
(\mathrm{MG} / \mathrm{L} \\
\text { AS } \\
\left.\mathrm{SIO}_{2}\right)\end{array}$ & $\begin{array}{l}\text { SOLIDS, } \\
\text { RESIDUE } \\
\text { AT } 180 \\
\text { DEG. C } \\
\text { DIS- } \\
\text { SOLVED } \\
(\mathrm{MG} / \mathrm{L})\end{array}$ & $\begin{array}{l}\text { SOLIDS, } \\
\text { SUM OF } \\
\text { CONSTIT- } \\
\text { UENTS, } \\
\text { DIS- } \\
\text { SOLVED } \\
\text { (MG/L) }\end{array}$ & \\
\hline \multicolumn{10}{|c|}{ WELL IDENTIFICATION - SBI 287--Continued } \\
\hline NOV 1987 & & & & & & & & & \\
\hline $\begin{array}{l}07 \\
\text { MAR } 1988\end{array}$ & 3.0 & 419 & 84 & 3.5 & 0.9 & 18 & 526 & 545 & \\
\hline $\begin{array}{c}05 \ldots \\
\text { APR }\end{array}$ & 3.0 & 436 & 87 & 5.7 & .3 & 18 & 546 & 565 & \\
\hline${ }_{\mathrm{MAY}}^{13 \ldots}$ & 2.2 & 433 & 160 & 6.4 & .3 & 18 & 639 & 655 & \\
\hline${ }_{\mathrm{JNE}}^{10 \ldots}$ & 2.6 & 428 & 110 & 5.7 & .3 & 18 & 554 & 582 & \\
\hline $\begin{array}{l}15 \ldots \\
15 \ldots \\
15 \ldots\end{array}$ & $\begin{array}{l}2.9 \\
2.7 \\
2.7\end{array}$ & $\begin{array}{l}411 \\
433 \\
431\end{array}$ & $\begin{array}{l}63 \\
91 \\
84\end{array}$ & $\begin{array}{l}4.1 \\
5.4 \\
5.4\end{array}$ & $\begin{array}{l}.4 \\
.2 \\
.3\end{array}$ & $\begin{array}{l}15 \\
17 \\
17\end{array}$ & $\begin{array}{l}493 \\
532 \\
527\end{array}$ & $\begin{array}{l}512 \\
566 \\
556\end{array}$ & \\
\hline AUG & & & & & & & & & \\
\hline$\underset{\text { Nov }}{31 \ldots}$ & 2.9 & 422 & 63 & 4.4 & .2 & 17 & 491 & 643 & \\
\hline $\begin{array}{l}15 \ldots \\
\text { MAR } 1989\end{array}$ & 2.9 & 430 & 61 & 4.5 & .2 & 17 & 516 & 537 & \\
\hline$\underset{\text { APR }}{01 \ldots}$ & 3.2 & 436 & 87 & 4.6 & .2 & 16 & 553 & 579 & \\
\hline $\begin{array}{c}06 \ldots \\
\text { MAY }\end{array}$ & 3.5 & 448 & 140 & 4.4 & .2 & 14 & 628 & 634 & \\
\hline $\begin{array}{c}09 . \\
\text { JUNE }\end{array}$ & 3.6 & 437 & 230 & 4.8 & .2 & 14 & 769 & 745 & \\
\hline $\begin{array}{r}06 \ldots \\
27 \ldots \\
\text { SEPT }\end{array}$ & $\begin{array}{l}3.6 \\
3.9\end{array}$ & $\begin{array}{l}435 \\
416\end{array}$ & $\begin{array}{l}280 \\
280\end{array}$ & $\begin{array}{l}4.8 \\
5.2\end{array}$ & $\begin{array}{l}.2 \\
.2\end{array}$ & $\begin{array}{l}15 \\
14\end{array}$ & $\begin{array}{l}742 \\
788\end{array}$ & $\begin{array}{l}811 \\
792\end{array}$ & \\
\hline $19 \ldots$ & 3.4 & 297 & 260 & 5.6 & .2 & 14 & 641 & 690 & \\
\hline DATE & $\begin{array}{l}\text { NITRO- } \\
\text { GEN, } \\
\text { NITRATE } \\
\text { DIS- } \\
\text { SOLVED } \\
\text { (MG/L } \\
\text { AS N) }\end{array}$ & $\begin{array}{l}\text { NITRO- } \\
\text { GEN, } \\
\text { NITRITE } \\
\text { DIS- } \\
\text { SOLVED } \\
\text { (MG/L } \\
\text { AS N) }\end{array}$ & $\begin{array}{l}\text { NITRO- } \\
\text { GEN, } \\
\text { NITRITE } \\
\text { DIS- } \\
\text { SOLVED } \\
(\mathrm{MG} / \mathrm{L} \\
\left.\text { AS } \mathrm{NO}_{2}\right)\end{array}$ & $\begin{array}{c}\text { NITRO- } \\
\text { GEN, } \\
\mathrm{NO}_{2}+\mathrm{NO}_{3} \\
\text { DIS- } \\
\text { SOLVED } \\
\text { (MG/L } \\
\text { AS N) }\end{array}$ & $\begin{array}{l}\text { NITRO- } \\
\text { GEN, } \\
\text { AMMONIA } \\
\text { DIS- } \\
\text { SOLVED } \\
\text { (MG/L } \\
\text { AS N) }\end{array}$ & $\begin{array}{l}\text { NITRO- } \\
\text { GEN, } \\
\text { AMMONIA } \\
\text { DIS- } \\
\text { SOLVED } \\
(\mathrm{MG} / \mathrm{L} \\
\left.\text { AS } \mathrm{NH}_{4}\right)\end{array}$ & $\begin{array}{l}\text { NITRO- } \\
\text { GEN, } \\
\text { ORGANIC } \\
\text { DIS- } \\
\text { SOLVED } \\
\text { (MG/L } \\
\text { AS N) }\end{array}$ & $\begin{array}{l}\text { NITRO- } \\
\text { GEN,AM- } \\
\text { MONIA + } \\
\text { ORGANIC } \\
\text { DISSOLVED } \\
\text { (MG/L } \\
\text { AS N) }\end{array}$ & $\begin{array}{l}\text { PHOS- } \\
\text { PHORUS } \\
\text { DIS- } \\
\text { SOLVED } \\
\text { (MG/L } \\
\text { AS P) }\end{array}$ \\
\hline NOV 1987 & & & & & & & & & \\
\hline $\begin{array}{l}07 \ldots \\
\text { MAR } 1988\end{array}$ & $\cdots$ & $<0.01$ & -- & $<0.10$ & 1.10 & 1.4 & 0.0 & 1.0 & 0.01 \\
\hline $\begin{array}{c}05 \ldots \\
\text { APR }\end{array}$ & $\cdots$ & $<.01$ & -- & $<.10$ & .91 & 1.2 & .09 & 1.0 & .01 \\
\hline$\underset{M A Y}{13 \ldots}$ & - & $<.01$ & -- & $<.10$ & .63 & .81 & .0 & .6 & $<.01$ \\
\hline JUNE & -- & $<.01$ & -- & $<.10$ & .79 & 1.0 & .11 & .9 & $<.01$ \\
\hline $15 \ldots$ & -- & .01 & .03 & $<.10$ & 1.20 & 1.5 & .4 & 1.6 & .01 \\
\hline $15 \ldots$ & - & .01 & .03 & $<.10$ & 1.00 & 1.3 & .2 & 1.2 & $<.01$ \\
\hline $15 \ldots$ & -- & $<.01$ & - & $<.10$ & .99 & 1.3 & .21 & 1.2 & $<.01$ \\
\hline AUG & & & & & & & & & \\
\hline $\begin{array}{l}31 \ldots \\
\text { NOV }\end{array}$ & 27.0 & .02 & .07 & 27.0 & .93 & 1.2 & 1.2 & 2.1 & $<.01$ \\
\hline $\begin{array}{l}15 \ldots \\
\text { MAR } 1989\end{array}$ & $\cdots$ & $<.01$ & -- & .17 & 1.10 & 1.4 & .1 & 1.2 & $<.01$ \\
\hline $\begin{array}{l}01 \ldots \\
\text { APR }\end{array}$ & 2.18 & .02 & .07 & 2.20 & 1.20 & 1.5 & .3 & 1.5 & $<.01$ \\
\hline$\underset{\text { MAY }}{06 \ldots}$ & -- & - & -- & -- & $\cdots$ & -- & -- & - & -- \\
\hline$\underset{\text { JUNE }}{09 .}$ & .28 & .02 & .07 & .30 & 1.30 & 1.7 & .3 & 1.6 & $<.01$ \\
\hline $06 \ldots$ & - & -- & -- & -- & -- & -- & -- & -- & -- \\
\hline$\underset{\text { SEPT }}{27 \ldots}$ & -- & -- & -- & -- & -- & -- & $\cdots$ & -- & -- \\
\hline $19 \ldots$ & -- & $\cdots$ & -- & -- & -- & -- & -- & -- & -- \\
\hline
\end{tabular}


Table 25.--Water-quality analyses for wells--Continued

\begin{tabular}{|c|c|c|c|c|c|c|c|c|c|c|}
\hline DATE & $\begin{array}{l}\text { PHOS- } \\
\text { PHORUS } \\
\text { ORTHO, } \\
\text { DIS- } \\
\text { SOLVED } \\
\text { (MG/L } \\
\text { AS P) }\end{array}$ & $\begin{array}{c}\text { PHOS- } \\
\text { PHORUS } \\
\text { ORGANIC } \\
\text { DIS- } \\
\text { SOLVED } \\
\text { (MG/L } \\
\text { AS P) }\end{array}$ & $\begin{array}{l}\text { ALU- } \\
\text { MINUM, } \\
\text { DIS- } \\
\text { SOLVED } \\
(\mu G / L \\
\text { AS AL) }\end{array}$ & $\begin{array}{c}\text { ARSENIC } \\
\text { DIS- } \\
\text { SOLVED } \\
\text { ( } \mu \mathrm{G} / \mathrm{L} \\
\text { AS AS) }\end{array}$ & $\begin{array}{l}\text { BARIUM, } \\
\text { DIS- } \\
\text { SOLVED } \\
(\mu G / L \\
\text { AS BA) }\end{array}$ & $\begin{array}{l}\text { BERYL- } \\
\text { LIUM, } \\
\text { DIS- } \\
\text { SOLVED } \\
(\mu G / L \\
\text { AS BE) }\end{array}$ & $\begin{array}{l}\text { BORON } \\
\text { DIS-, } \\
\text { SOLVED } \\
(\mu G / L \\
\text { AS B) }\end{array}$ & $\begin{array}{c}\text { CADMIUM } \\
\text { DIS- } \\
\text { SOLVED } \\
\text { ( } \mu \mathrm{G} / \mathrm{L} \\
\text { AS CD) }\end{array}$ & $\begin{array}{l}\text { CHRO- } \\
\text { MIUM, } \\
\text { DIS- } \\
\text { SOLVED } \\
(\mu G / L \\
\text { AS CR) }\end{array}$ & $\begin{array}{c}\text { COBALT, } \\
\text { DIS- } \\
\text { SOLVED } \\
(\mu G / L \\
\text { AS CO) }\end{array}$ \\
\hline
\end{tabular}

NOV 1987

$07 \ldots$

MAR 1988

$05 \ldots$

APR

13...

MAY

$10 \ldots$

JUNE

$15 \ldots$

$15 \ldots$

$15 \ldots$

AUG

31...

NOV

$15 \ldots$

MAR 1989

01 ...

APR

06...

MAY

$09 .$.

JUNE

06...

$27 \ldots$

SEPT

$19 .$.
$<0.01$

$$
<.01
$$

$<.01$

$<.01$

$<.01$

$<.01$

$<.01$

$<.01$

$<.01$

$<.01$

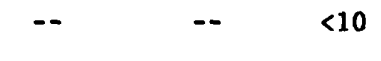

$<.01$

$\begin{array}{lll}-- & -- & <10 \\ -- & -- & <10\end{array}$

$<10$
$<1$

$<1$

$<1 \quad 180$

$<1 \quad 200$

$<1 \quad 300$

$<1 \quad 240$

$<1 \quad 240$

$<1 \quad 250$

$<1 \quad 280$

$<1 \quad 240$

$<1 \quad 170$

$<1 \quad 130$

<1 120

$1 \quad 94$

$<1$
$<0.5 \quad 80$

$<.5 \quad 70$

$<.5 \quad 60$

$<.5 \quad 60$

$<.5 \quad 120$

$<.5 \quad 90$

$<.5 \quad 90$

$<.5 \quad 80$

$<.5 \quad 90$

$<.5 \quad 90$

$<.590$

$<.5 \quad 90$

$<.5 \quad 80$

\begin{tabular}{l}
$<.5 \quad 80$ \\
\hline
\end{tabular}

91

$<.5 \quad 80$
MANGA- MOLYB-

NESE, DENUM, NICKEL, SILVER,

DIS- DIS-

DATE

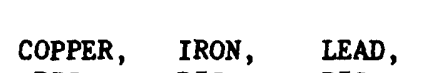

COPPER, IRON,

SOLVED SOLVED SOLVED

( $\mu \mathrm{G} / \mathrm{L}$

( $\mu \mathrm{G} / \mathrm{L}$ AS FE) AS PB)
SOLVED SOLVED

$(\mu G / L \quad(\mu G / L$

AS MN) AS MO)
NOV 1987

$07 \ldots$

MAR 1988

05...

APR

$13 \ldots$

MAY

$10 .$.

JUNE

$15 . .$.

$15 \ldots$

$15 \ldots$

AUG

31...

NOV

$15 \ldots$

MAR 1989

01...

APR

06...

MAY

$09 .$.

JUNE

06...

$27 \ldots$

SEPT

$19 .$.
$<10 \quad 220$

$<10$

$<10$

$<10$

$<10$

$<10$

$<10$

$<10$

$<10$

$<10$

$<10$

$<10$

$<10$

$<10$

$<10$

63

$220<10$

76

$<10$

$<10$

85

$<10$

$<10$

78

$<10$

$<10$

85

$<10$

$<10$

60

94

$<10$

$<10$

$<10$

$<10$

81

$<10$

$<10$

$84<10<10$

$93<10<10$

$48<10$

$10<10$

$5<10$

$55<10$

$78<10$

$<10$

$92<10<10$

$110<10<10$

$120<10<10$

120

100

$<10$

$<10$

$<10$ 
Table 25.--Water-quality analyses for wells--Continued

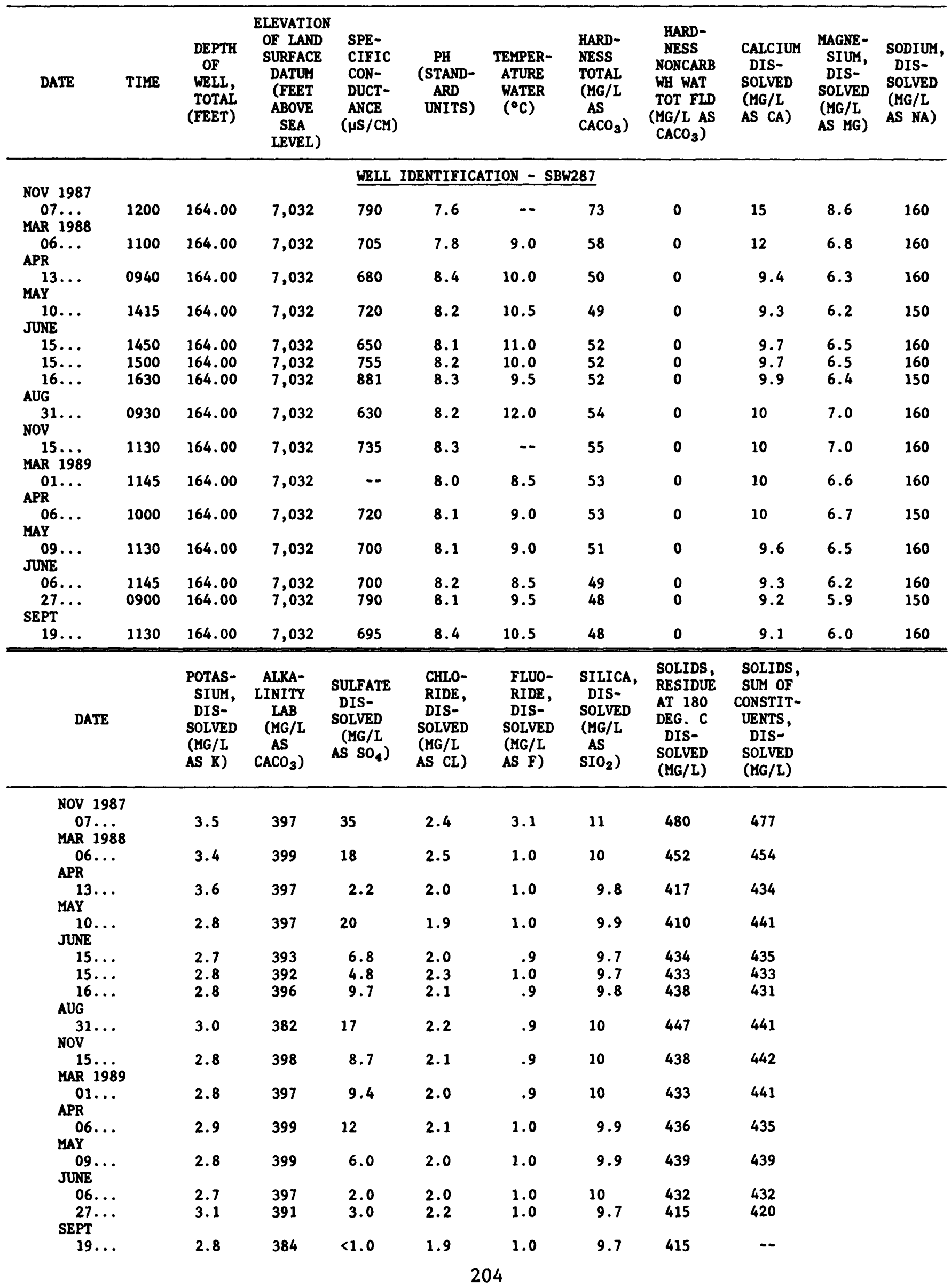


Table 25.--Water-quality analyses for wells--Continued

\begin{tabular}{|c|c|c|c|c|c|c|c|c|c|c|c|}
\hline DATE & $\begin{array}{l}\text { NITRO- } \\
\text { GEN, } \\
\text { NITRITE } \\
\text { DIS- } \\
\text { SOLVED } \\
\text { (HG/L } \\
\text { AS N) }\end{array}$ & $\begin{array}{l}\text { NITRO- } \\
\text { GEN, } \\
\text { NITRITE } \\
\text { DIS- } \\
\text { SOLVED } \\
(\mathrm{MG} / \mathrm{L} \\
\left.\text { AS } \mathrm{NO}_{2}\right)\end{array}$ & $\begin{array}{c}\text { NITRO- } \\
\text { GEN, } \\
\mathrm{NO}_{2}+\mathrm{NO}_{3} \\
\text { DIS- } \\
\text { SOLVED } \\
\text { (MG/L } \\
\text { AS N) }\end{array}$ & $\begin{array}{l}\text { NITRO- } \\
\text { GEN, } \\
\text { AMMONIA } \\
\text { DIS- } \\
\text { SOLVED } \\
\text { (MG/L } \\
\text { AS N) }\end{array}$ & $\begin{array}{l}\text { NITRO- } \\
\text { GEN, } \\
\text { AMYONIA } \\
\text { DIS- } \\
\text { SOLVED } \\
\text { (MG/L } \\
\text { AS NH4) }\end{array}$ & $\begin{array}{l}\text { NITRO- } \\
\text { GEN, } \\
\text { ORGANIC } \\
\text { DIS- } \\
\text { SOLVED } \\
\text { (MG/L } \\
\text { AS N) }\end{array}$ & $\begin{array}{c}\text { NITRO- } \\
\text { GEN,AM- } \\
\text { MONIA + } \\
\text { ORGANIC } \\
\text { DISSOLVED } \\
\text { (MG/L } \\
\text { AS N) }\end{array}$ & $\begin{array}{l}\text { PHOS- } \\
\text { PHORUS } \\
\text { DIS- } \\
\text { SOLVED } \\
\text { (MG/L } \\
\text { AS P) }\end{array}$ & $\begin{array}{l}\text { PHOS- } \\
\text { PHORUS } \\
\text { ORTHO, } \\
\text { DIS- } \\
\text { SOLVED } \\
\text { (MG/L } \\
\text { AS P) }\end{array}$ & $\begin{array}{l}\text { PHOS- } \\
\text { PHATE, } \\
\text { ORTHO, } \\
\text { DIS- } \\
\text { SOLVED } \\
\left(\mathrm{MG}^{-} \mathrm{L}\right. \\
\text { AS PO }\end{array}$ & $\begin{array}{l}\text { PHOS- } \\
\text { PHORUS } \\
\text { ORGANIC } \\
\text { DIS- } \\
\text { SOLVED } \\
\text { (MG/L } \\
\text { AS P) }\end{array}$ \\
\hline
\end{tabular}

WELL IDENTIFICATION - SBW287--Continued

NOV 1987

$07 \ldots$

MAR 1988

06...

APR

13.

$<0.01$
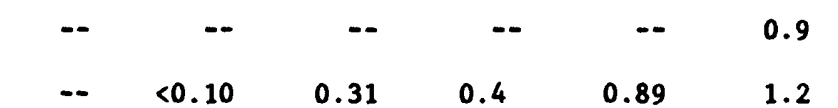

0.04

0.31

0.4

0.89

1.2

.05

$<0.04$

$-$

0.04

MAY

10 . .

$<.01$

$-\quad<.10$

.49

.63

.41

.9

.02

.02

0.06

.05

JUNE

$15 \ldots<.01$

$--\quad<10$

.51

.66

.39

.9

.02

.02

.06

.0

$15 \ldots<.01$

$-\quad<.10$

.34

.44

.16

.5

.03

.01

.03

.0

$16 \ldots$

0.03

$<.10$
$<.10$

.37
.41

.48
.53

.53
.0

.9
.3

.02

$<.01$
.03

.09

.02

AUG

$31 \ldots<.01$

$-2 \quad<.10$

.50

.64

.6

1.1

.03

.03

.09

.0

NOV 15

$<.01$

$<.10$

.47

.61

.03

.5

.03

.02

.06

.01

MAR 1989

$<.01$

$<.10$

.41

.53

.0

.4

.02

.01

.03

.01

APR
$06 \ldots$

MAY

$09 \ldots$

$<.01$

$<.10$

.39

5

51

9

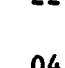

.03

.09

.01

JUNE
$06 \ldots$

$27 .$.

SEPT

19 ...

$-$

$\begin{array}{ll}-- & - \\ -- & --\end{array}$

$--$

$\because$

$+\quad-\infty$

$\begin{array}{cccc}\text { ALU- } & \text { ARSENIC } & \text { BARIUM, } & \text { BERYL- } \\ \text { MINUM, } & \text { DIS- } & \text { DIS- } & \text { LIUM, }\end{array}$

DATE
$\begin{array}{ccc}\text { DIS- } & \text { DIS- } & \text { DIS- } \\ \text { SOLVED } & \text { SOLVED } & \text { SOLVED }\end{array}$ $(\mu G / L \quad(\mu G / L \quad(\mu G / L$ AS AL) AS AS) AS BA)
BERYL-

LIUM, BORON, CADMIUM

DIS- DIS- DIS-

SOLVED SOLVED SOLVED

( $\mu G / L$ AS BE) $(\mu G / L \quad(\mu G / L$

$\begin{array}{ll}\text { AS B) } & \text { AS CD })\end{array}$
CHRO- COBALT, COPPER, IRON, LEAD, DIS- DIS- DIS- DIS- DISSOLVED SOLVED SOLVED SOLVED SOLVED

$\begin{array}{lllll}(\mu G / L & (\mu G / L & (\mu G / L & (\mu G / L & (\mu G / L \\ \text { AS CO) } & \text { AS CU) } & \text { AS FE }) & \text { AS PB })\end{array}$ AS CR)

AS (O)

AS FE

AS PB)
Nov 1987

$07 \ldots$

MAR 1988

$06 . .$.

APR .

$13 . .<<10$

MAY

$10 . .<<10$

JUNE

$15 \ldots<<10$

$15 \ldots<10$

$16 \ldots \quad 30$

AUG

$31 \ldots<10$

NoV

15 ...

MAR 1989

$01 \ldots$

$<10$

APR

06 ...

MAY

$09 . .<<10$

JUNE

06... 10

$27 \ldots<10$

SEPT

$19 . .<10$ 
Table 25.--Water-quality analyses for wells--Continued

\begin{tabular}{|c|c|c|c|c|c|c|c|c|c|c|c|c|}
\hline DATE & $\begin{array}{l}\text { MANGA- } \\
\text { NESE, } \\
\text { DIS- } \\
\text { SOLVED } \\
\text { ( } \mu G / L \\
\text { AS MN) }\end{array}$ & $\begin{array}{l}\text { MOLYB- } \\
\text { DENUM, } \\
\text { DIS- } \\
\text { SOLVED } \\
(\mu G / L \\
\text { AS MO) }\end{array}$ & $\begin{array}{l}\text { NICKEL, } \\
\text { DIS- } \\
\text { SOLVED } \\
(\mu G / L \\
\text { AS NI) }\end{array}$ & $\begin{array}{l}\text { SILVER, } \\
\text { DIS- } \\
\text { SOLVED } \\
(\mu G / L \\
\text { AS AG) }\end{array}$ & $\begin{array}{c}\text { STRON- } \\
\text { TIUM, } \\
\text { DIS- } \\
\text { SOLVED } \\
(\mu G / L \\
\text { AS SR) }\end{array}$ & $\begin{array}{l}\text { VANA- } \\
\text { DIUM, } \\
\text { DIS- } \\
\text { SOLVED } \\
\text { ( } \mu G / L \\
\text { AS V) }\end{array}$ & $\begin{array}{c}\text { ZINC, } \\
\text { DIS- } \\
\text { SOLVED } \\
(\mu \mathrm{G} / \mathrm{L} \\
\text { AS ZN) }\end{array}$ & $\begin{array}{c}\text { C-13/ } \\
\text { C-12 } \\
\text { STABLE } \\
\text { ISOTOPE } \\
\text { RATIO } \\
\text { PER } \\
\text { MIL }\end{array}$ & $\begin{array}{c}\text { H-2/ } \\
\text { H-1 } \\
\text { STABLE } \\
\text { ISOTOPE } \\
\text { RATIO } \\
\text { PER } \\
\text { MIL }\end{array}$ & $\begin{array}{c}\text { 0-18/ } \\
\text { O-16 } \\
\text { STABIE } \\
\text { ISOTOPE } \\
\text { RATIO } \\
\text { PER } \\
\text { MIL }\end{array}$ & $\begin{array}{c}\text { S-34/ } \\
\text { S-32 } \\
\text { STABLE } \\
\text { ISOTOPE } \\
\text { RATIO } \\
\text { PER } \\
\text { MIL }\end{array}$ & $\begin{array}{l}\text { TRIT- } \\
\text { IUM } \\
\text { TOTAL } \\
\text { (PCI/L) }\end{array}$ \\
\hline
\end{tabular}

\begin{tabular}{|c|c|c|c|c|c|c|c|c|c|c|c|c|}
\hline \multicolumn{13}{|l|}{ NOV 1987} \\
\hline MAR 1988 & 82 & $<10$ & $<10$ & $<1.0$ & 330 & $<6$ & 12 & -- & -- & -- & -- & - \\
\hline $\begin{array}{l}06 \ldots \\
\text { APR }\end{array}$ & 81 & $<10$ & $<10$ & $<1.0$ & 370 & $<6$ & 10 & -- & -- & -- & -- & $\cdots$ \\
\hline${ }_{\mathrm{MAY}}^{13 \ldots}$ & 48 & $<10$ & $<10$ & $<1.0$ & 480 & $<6$ & $<3$ & -1.5 & -151 & -20.3 & -- & $<5.7$ \\
\hline$\frac{10 \ldots}{\text { JUNE }}$ & 45 & $<10$ & $<10$ & $<1.0$ & 480 & $<6$ & $<3$ & -- & $-\infty$ & -- & -- & -. \\
\hline $15 \ldots$ & 50 & $<10$ & $<10$ & $<1.0$ & 480 & $<6$ & 8 & -- & -- & -- & -- & - \\
\hline $15 \ldots$ & 45 & $<10$ & $<10$ & 1.0 & 500 & $<6$ & 13 & -- & -- & -- & -- & $\cdots$ \\
\hline $16 \ldots$ & 50 & $<10$ & $<10$ & $<1.0$ & 490 & $<6$ & $<3$ & -- & -- & -- & -- & - \\
\hline AUG & & & & & & & & & & & & \\
\hline$\underset{\text { Nov }}{31 \ldots}$ & 62 & $<10$ & $<10$ & $<1.0$ & 500 & $<6$ & 4 & -2.2 & -152 & -20.1 & -4.0 & $<5.7$ \\
\hline $\begin{array}{l}15 \ldots \\
\text { MAR } 1989\end{array}$ & 57 & $<10$ & $<10$ & $<1.0$ & 520 & $<6$ & 4 & -- & -- & -- & -- & - \\
\hline $\begin{array}{l}01 \ldots \\
\text { APR }\end{array}$ & 58 & $<10$ & $<10$ & $<1.0$ & 510 & $<6$ & 7 & -- & -- & -- & - & - \\
\hline MAY 06. & 56 & $<10$ & $<10$ & $<1.0$ & 490 & $<6$ & 8 & -- & -- & -- & -- & -- \\
\hline $\begin{array}{c}09 . \ldots \\
\text { JUNE }\end{array}$ & 62 & $<10$ & $<10$ & $<1.0$ & 510 & $<6$ & $<3$ & -- & $:-$ & -- & -- & -- \\
\hline $06 \ldots$ & 59 & $<10$ & $<10$ & $<1.0$ & 510 & $<6$ & $<3$ & -- & -- & -- & -- & - \\
\hline$\underset{\text { SEPT }}{27 \ldots}$ & 59 & $<10$ & $<10$ & $<1.0$ & 480 & $<6$ & 7 & -- & -- & -- & -- & - \\
\hline $19 .$. & 58 & $<10$ & $<10$ & $<1.0$ & 530 & $<6$ & 3 & -- & $\cdots$ & -- & -- & -- \\
\hline
\end{tabular}

\begin{tabular}{|c|c|c|c|c|c|c|c|c|c|c|c|}
\hline DATE & TIME & $\begin{array}{l}\text { DEPTH } \\
\text { OF } \\
\text { WELL, } \\
\text { TOTAL } \\
\text { (FEET) }\end{array}$ & $\begin{array}{l}\text { ELEVATION } \\
\text { OF LAND } \\
\text { SURFACE } \\
\text { DATUM } \\
\text { (FEET } \\
\text { ABOVE } \\
\text { SEA } \\
\text { LEVEL) }\end{array}$ & $\begin{array}{l}\text { SPE- } \\
\text { CIFIC } \\
\text { CON- } \\
\text { DUCT- } \\
\text { ANCE } \\
(\mu S / C M)\end{array}$ & $\begin{array}{c}\text { PH } \\
\text { (STAND- } \\
\text { ARD } \\
\text { UNITS) }\end{array}$ & $\begin{array}{l}\text { TEMPER- } \\
\text { ATURE } \\
\text { WATER } \\
\left({ }^{\circ} \mathrm{C}\right)\end{array}$ & $\begin{array}{l}\text { HARD- } \\
\text { NESS } \\
\text { TOTAL } \\
(\mathrm{MG} / \mathrm{L} \\
\text { AS } \\
\left.\mathrm{CACO}_{3}\right)\end{array}$ & $\begin{array}{l}\text { HARD- } \\
\text { NESS } \\
\text { NONCARB } \\
\text { WH WAT } \\
\text { TOT FLD } \\
(\mathrm{MG} / \mathrm{L} \text { AS } \\
\left.\mathrm{CACO}_{3}\right)\end{array}$ & $\begin{array}{l}\text { CALCIUM } \\
\text { DIS- } \\
\text { SOLVED } \\
\text { (MG/L } \\
\text { AS CA) }\end{array}$ & $\begin{array}{l}\text { MAGNE- } \\
\text { SIUM, } \\
\text { DIS- } \\
\text { SOLVED } \\
\text { (MG/L } \\
\text { AS MG) }\end{array}$ & $\begin{array}{l}\text { SODIUM, } \\
\text { DIS- } \\
\text { SOLVED } \\
\text { (MG/L } \\
\text { AS NA) }\end{array}$ \\
\hline
\end{tabular}

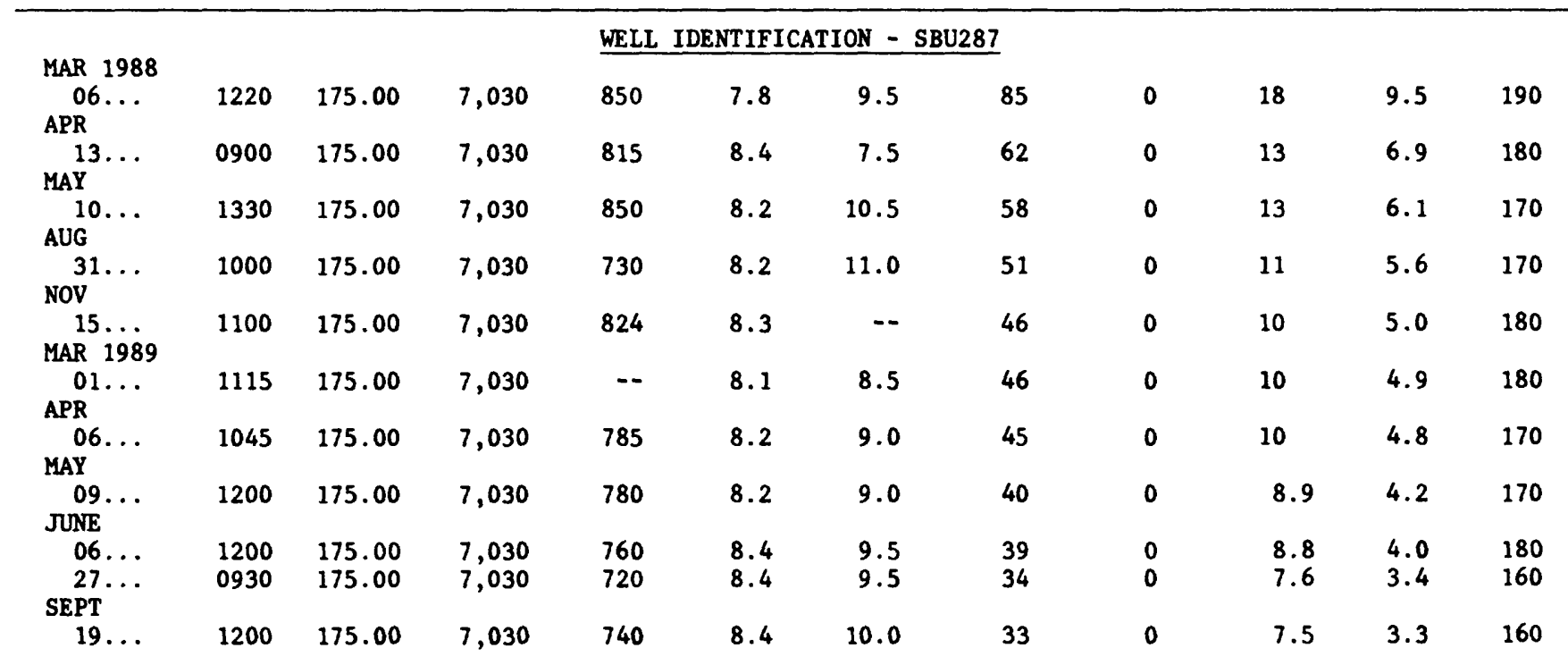


Table 25.--Water-quality analyses for wells--Continued

\begin{tabular}{|c|c|c|c|c|c|c|c|c|}
\hline DATE & $\begin{array}{l}\text { POTAS- } \\
\text { SIUM, } \\
\text { DIS- } \\
\text { SOLVED } \\
(M G / L \\
\text { AS K) }\end{array}$ & $\begin{array}{c}\text { ALKA- } \\
\text { LINITY } \\
\text { LAB } \\
(\mathrm{MG} / \mathrm{L} \\
\mathrm{AS} \\
\left.\mathrm{CACO}_{3}\right)\end{array}$ & $\begin{array}{l}\text { SULFATE } \\
\text { DIS- } \\
\text { SOLVED } \\
(\mathrm{MG} / \mathrm{L} \\
\left.\text { AS } \mathrm{SO}_{4}\right)\end{array}$ & $\begin{array}{l}\text { CHLO- } \\
\text { RIDE, } \\
\text { DIS- } \\
\text { SOLVED } \\
\text { (MG/L } \\
\text { AS CL) }\end{array}$ & $\begin{array}{l}\text { FLUO- } \\
\text { RIDE, } \\
\text { DIS- } \\
\text { SOLVED } \\
\text { (MG/L } \\
\text { AS F) }\end{array}$ & $\begin{array}{l}\text { SILICA, } \\
\text { DIS- } \\
\text { SOLVED } \\
\text { (MG/L } \\
\text { AS } \\
\left.\mathrm{SIO}_{2}\right)\end{array}$ & $\begin{array}{l}\text { SOLIDS, } \\
\text { RESIDUE } \\
\text { AT } 180 \\
\text { DEG. C } \\
\text { DIS- } \\
\text { SOLVED } \\
\text { (MG/L) }\end{array}$ & $\begin{array}{l}\text { SOLIDS, } \\
\text { SUM OF } \\
\text { CONSTIT- } \\
\text { UENTS, } \\
\text { DIS- } \\
\text { SOLVED } \\
\text { (MG/L) }\end{array}$ \\
\hline
\end{tabular}

WELL IDENTIFICATION - SBU287--Continued

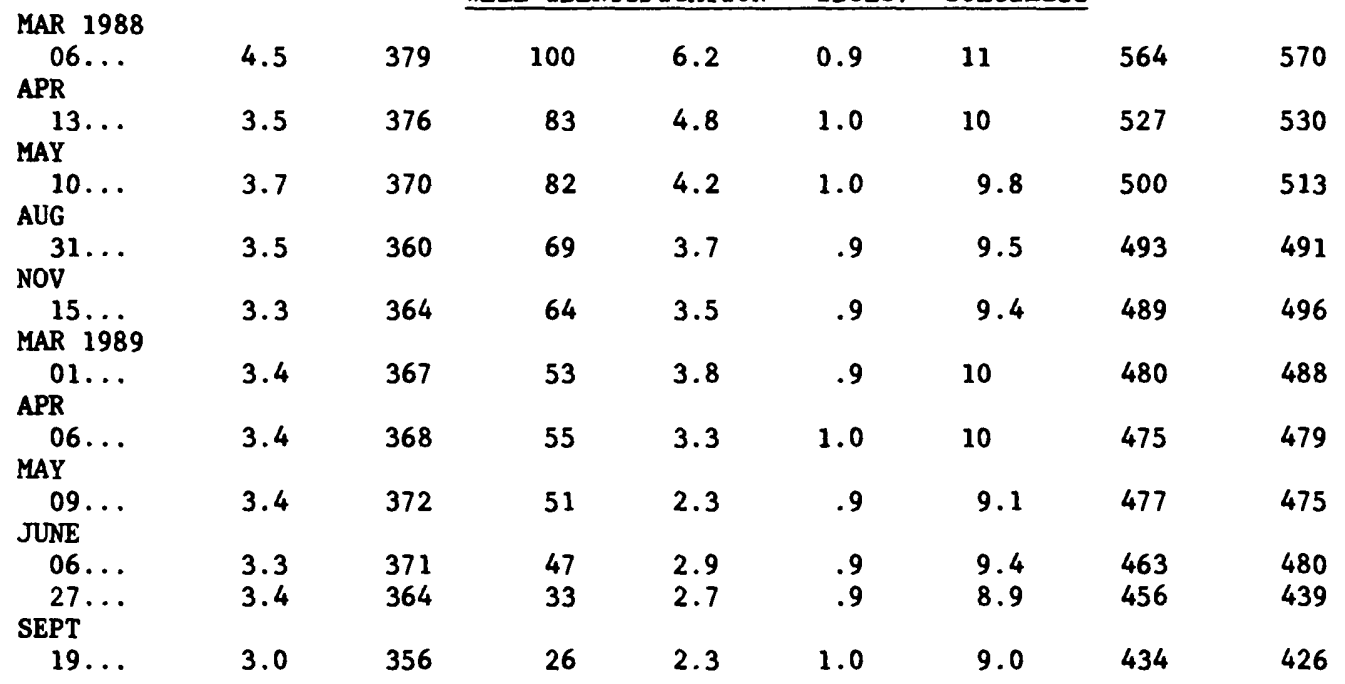

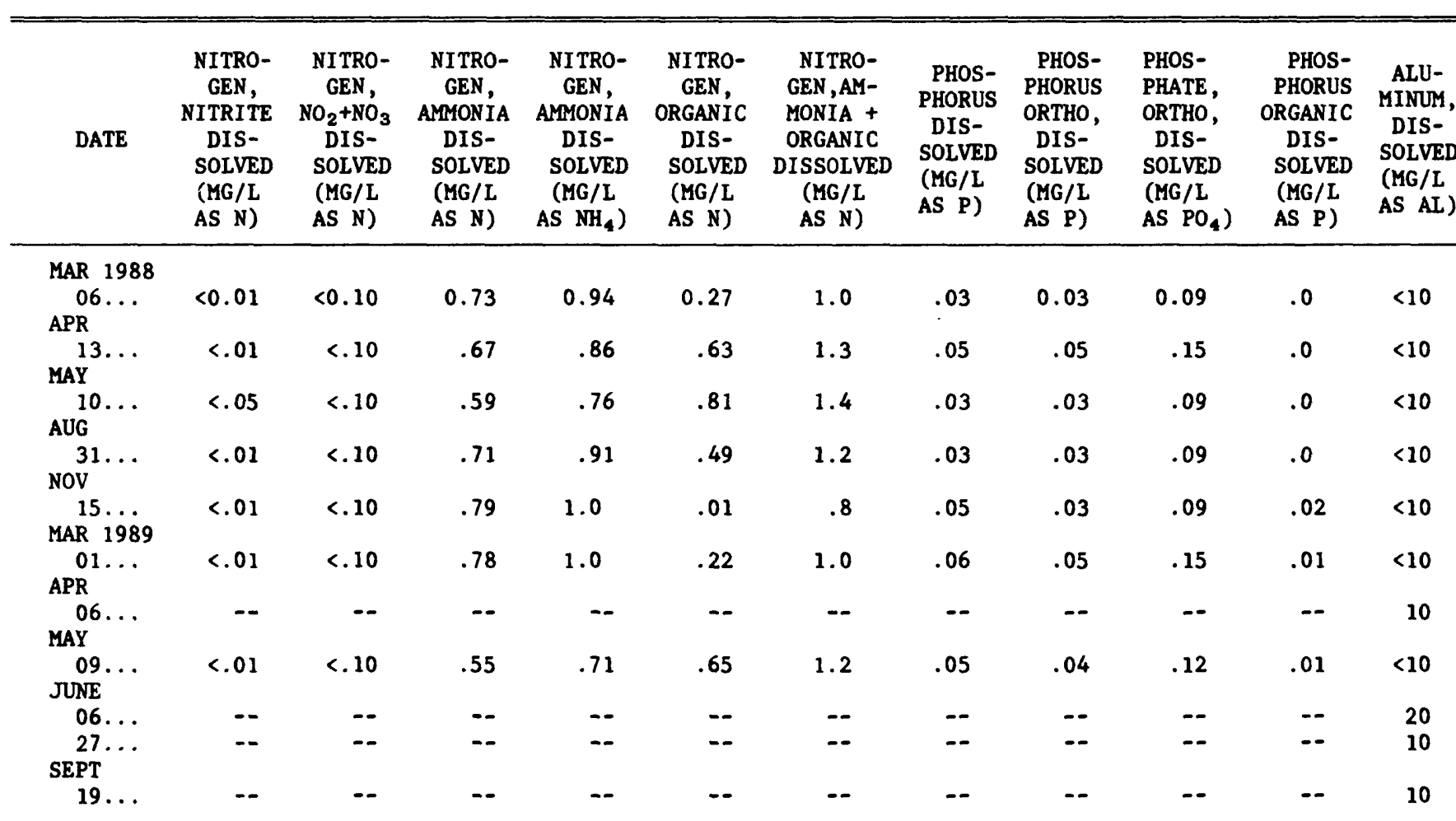


Table 25.--Water-quality analyses for wells--Continued

\begin{tabular}{|c|c|c|c|c|c|c|c|c|c|c|c|}
\hline DATE & $\begin{array}{c}\text { ARSENIC } \\
\text { DIS- } \\
\text { SOLVED } \\
\text { ( } \mu \text { G/L } \\
\text { AS AS) }\end{array}$ & $\begin{array}{l}\text { BARIUM, } \\
\text { DIS- } \\
\text { SOLVED } \\
(\mu G / L \\
\text { AS BA) }\end{array}$ & $\begin{array}{l}\text { BERYL- } \\
\text { IIUM, } \\
\text { DIS- } \\
\text { SOLVED } \\
\text { ( } \mu G / L \\
\text { AS BE) }\end{array}$ & $\begin{array}{l}\text { BORON, } \\
\text { DIS- } \\
\text { SOLVED } \\
\text { ( } \mu G / L \\
\text { AS B) }\end{array}$ & $\begin{array}{c}\text { CADMIUM } \\
\text { DIS- } \\
\text { SOLVED } \\
\text { ( } \mu G / L \\
\text { AS CD) }\end{array}$ & $\begin{array}{l}\text { CHRO- } \\
\text { MIUM, } \\
\text { DIS- } \\
\text { SOLVED } \\
\text { ( } \mu G / L \\
\text { AS CR) }\end{array}$ & $\begin{array}{l}\text { COBALT, } \\
\text { DIS- } \\
\text { SOLVED } \\
(\mu G / L \\
\text { AS CO) }\end{array}$ & $\begin{array}{l}\text { COPPER, } \\
\text { DIS- } \\
\text { SOLVED } \\
\text { ( } \mu G / L \\
\text { AS CU) }\end{array}$ & $\begin{array}{l}\text { IRON, } \\
\text { DIS- } \\
\text { SOLVED } \\
\text { ( } \mu \text { G/L } \\
\text { AS FE) }\end{array}$ & $\begin{array}{l}\text { LEAD, } \\
\text { DIS- } \\
\text { SOLVED } \\
\text { ( } \mu G / L \\
\text { AS PB) }\end{array}$ & $\begin{array}{l}\text { MANGA- } \\
\text { NESE, } \\
\text { DIS- } \\
\text { SOLVED } \\
\text { ( } \mu \text { G/L } \\
\text { AS INN) }\end{array}$ \\
\hline \multirow{2}{*}{\multicolumn{12}{|c|}{ WELL IDENTIFICATION - SBU287--Continued }} \\
\hline & & & & & & & & & & & \\
\hline \multicolumn{12}{|l|}{ APR } \\
\hline MAY $13 .$. & 2 & 140 & $<.5$ & 230 & $<1$ & $<5$ & $<3$ & $<10$ & 60 & 10 & 110 \\
\hline${ }_{\text {AUG }}^{10} \ldots$ & 3 & 130 & $<.5$ & 240 & $<1$ & $<5$ & $<3$ & $<10$ & 12 & $<10$ & 87 \\
\hline $\mathrm{NOV}_{\mathrm{N}}^{31 \ldots}$ & $<1$ & 130 & $<.5$ & 220 & $<1$ & $<5$ & $<3$ & $<10$ & 40 & $<10$ & 77 \\
\hline $\begin{array}{l}15 \ldots \\
\text { MAR } 1989\end{array}$ & 1 & 130 & $<.5$ & 240 & $<1$ & $<5$ & $<3$ & $<10$ & 34 & 10 & 60 \\
\hline$\underset{A P R}{01 \cdots}$ & 3 & 160 & $<.5$ & 230 & $<1$ & $<5$ & $<3$ & $<10$ & 23 & $<10$ & 63 \\
\hline MAY & 3 & 150 & $<.5$ & 240 & 1 & $<5$ & $<3$ & $<10$ & 25 & $<10$ & 64 \\
\hline JUNE & 2 & 160 & $<.5$ & 230 & $<1$ & $<5$ & $<3$ & $<10$ & $<3$ & 10 & 46 \\
\hline $06 \ldots$ & 2 & 150 & $<.5$ & 240 & $<1$ & $<5$ & $<3$ & $<10$ & 43 & $<10$ & 40 \\
\hline $\begin{array}{l}27 \ldots \\
\text { SEPT }\end{array}$ & 1 & 150 & $<.5$ & 240 & $<1$ & $<5$ & $<3$ & $<10$ & 29 & 10 & 30 \\
\hline $19 \ldots$ & 2 & 170 & $<.5$ & 220 & $<1$ & $<5$ & $<3$ & $<10$ & 27 & $<10$ & 27 \\
\hline DATE & $\begin{array}{l}\text { MOLYB- } \\
\text { DENUM, } \\
\text { DIS- } \\
\text { SOLVED } \\
\text { ( } \mu G / L \\
\text { AS MO) }\end{array}$ & $\begin{array}{l}\text { NICKEL, } \\
\text { DIS- } \\
\text { SOLVED } \\
\text { ( } \mu G / L \\
\text { AS NI) }\end{array}$ & $\begin{array}{l}\text { SILVER, } \\
\text { DIS- } \\
\text { SOLVED } \\
\text { ( } \mu G / L \\
\text { AS AG) }\end{array}$ & $\begin{array}{l}\text { STRON- } \\
\text { TIUM, } \\
\text { DIS- } \\
\text { SOLVED } \\
\text { ( } \mu G / L \\
\text { AS SR) }\end{array}$ & $\begin{array}{l}\text { VANA- } \\
\text { DIUM, } \\
\text { DIS- } \\
\text { SOLVED } \\
(\mu G / L \\
\text { AS V) }\end{array}$ & $\begin{array}{l}\text { ZINC, } \\
\text { DIS- } \\
\text { SOLVED } \\
(\mu G / L \\
\text { AS ZN) }\end{array}$ & $\begin{array}{c}\text { C-13/ } \\
\text { C-12 } \\
\text { STABLE } \\
\text { ISOTOPE } \\
\text { RATIO } \\
\text { PER } \\
\text { MIL }\end{array}$ & $\begin{array}{l}\text { H-2/ } \\
\text { H-1 } \\
\text { STABLE } \\
\text { ISOTOPE } \\
\text { RATIO } \\
\text { PER } \\
\text { MIL }\end{array}$ & $\begin{array}{c}\text { 0-18/ } \\
0-16 \\
\text { STABLE } \\
\text { ISOTOPE } \\
\text { RATIO } \\
\text { PER } \\
\text { MIL }\end{array}$ & $\begin{array}{c}\text { S-34/ } \\
\text { S-32 } \\
\text { STABLE } \\
\text { ISOTOPE } \\
\text { RATIO } \\
\text { PER } \\
\text { MIL }\end{array}$ & $\begin{array}{c}\text { TRIT- } \\
\text { IUM } \\
\text { TOTAL } \\
\text { (PCI/L) }\end{array}$ \\
\hline MAR 1988 & & & & & & & & & & & \\
\hline $\begin{array}{c}06 \ldots \\
A P R\end{array}$ & $<10$ & $<10$ & $<1.0$ & 620 & $<6$ & $<10$ & -- & - & -- & -- & -- \\
\hline${ }_{\mathrm{MAY}}^{13}$ & 10 & $<10$ & $<1.0$ & 540 & $<6$ & 22 & -9.6 & -152 & -20 & 9.80 & $<5.7$ \\
\hline${ }_{A U G}^{10}$ & $<10$ & $<10$ & $<1.0$ & 500 & $<6$ & 6 & -- & -- & $=-$ & $=-$ & -- \\
\hline$\underset{\mathrm{NOV}}{31 \ldots}$ & $<10$ & $<10$ & 2.0 & 460 & $<6$ & 7 & -9.5 & -151 & -20 & -7.6 & $<5.7$ \\
\hline $\begin{array}{l}15 \ldots \\
\text { MAR } 1989\end{array}$ & 10 & $<10$ & 1.0 & 460 & $<6$ & 5 & - & - & - & -- & -- \\
\hline $\begin{array}{c}01 \cdots \\
\text { APR }\end{array}$ & $<10$ & $<10$ & 2.0 & 470 & $<6$ & $<3$ & -- & -- & -- & -- & -- \\
\hline MAY & $<10$ & $<10$ & $<1.0$ & 450 & $<6$ & 6 & -- & - & -- & -- & -- \\
\hline $\begin{array}{c}09 . \\
\text { JNE }\end{array}$ & $<10$ & $<10$ & $<1.0$ & 460 & $<6$ & $<3$ & -- & -- & -- & -- & - \\
\hline $06 \ldots$ & $<10$ & $<10$ & $<1.0$ & 430 & $<6$ & 28 & -- & -- & -- & -- & -- \\
\hline $\begin{array}{l}27 \ldots \\
\text { SEPT }\end{array}$ & $<10$ & $<10$ & $<1.0$ & 380 & $<6$ & $<3$ & -- & -- & -- & -- & -- \\
\hline $19 \ldots$ & $<10$ & $<10$ & $<1.0$ & 390 & $<6$ & $<3$ & $\ldots$ & -- & -- & -- & -- \\
\hline
\end{tabular}


Table 26.--Water-quality analyses for springs and lysimeters

[Site identification corresponds to sites listed in table 20; CFS, cubic feet per second; $\mu$ S/CM, microsiemens per centimeter at 25 degrees Celsius; ${ }^{\circ} \mathrm{C}$, degrees Celsius; MG/L, milligrams per liter; NONCARB, noncarbonate; WH, whole; WAT, water; TOT, total; FLD, field; --, no data collected; DEG. C, degrees Celsius; AC-FT, acrefeet; <, less than; $\mu G / L$, micrograms per liter; C-, carbon isotope; H-, hydrogen isotope; 0-, oxygen isotope; S-, sulfur isotope; per mil, parts per thousand; $P C I / L$, picocuries per liter]

\begin{tabular}{|c|c|c|c|c|c|c|c|c|c|c|c|c|}
\hline DATE & TIME & $\begin{array}{l}\text { SPRING- } \\
\text { FLOW } \\
\text { INSTAN- } \\
\text { TANEOUS } \\
\text { (CFS) }\end{array}$ & $\begin{array}{l}\text { SPE- } \\
\text { CIFIC } \\
\text { CON- } \\
\text { DUCT- } \\
\text { ANCE } \\
(\mu S / C M)\end{array}$ & $\begin{array}{c}\text { PH } \\
\text { (STAND- } \\
\text { ARD } \\
\text { UNITS) }\end{array}$ & $\begin{array}{l}\text { TEMPER- } \\
\text { ATURE } \\
\text { WATER } \\
\left({ }^{\circ} \mathrm{C}\right)\end{array}$ & $\begin{array}{l}\text { HARD- } \\
\text { NESS } \\
\text { TOTAL } \\
(\mathrm{MG} / \mathrm{L} \\
\text { AS } \\
\left.\mathrm{CACO}_{3}\right)\end{array}$ & $\begin{array}{l}\text { HARD- } \\
\text { NESS } \\
\text { NONCARB } \\
\text { WH WAT } \\
\text { TOT FLD } \\
(\mathrm{MG} / \mathrm{L} \text { AS } \\
\left.\mathrm{CACO}_{3}\right)\end{array}$ & $\begin{array}{c}\text { CALCIUM } \\
\text { DIS- } \\
\text { SOLVED } \\
\text { (MG/L } \\
\text { AS CA) }\end{array}$ & $\begin{array}{l}\text { MAGNE- } \\
\text { SIUM, } \\
\text { DIS- } \\
\text { SOLVED } \\
\text { (MG/L } \\
\text { AS MG) }\end{array}$ & $\begin{array}{l}\text { SODIUA, } \\
\text { DIS- } \\
\text { SOLVED } \\
\text { (MG/L } \\
\text { AS NA) }\end{array}$ & $\begin{array}{c}\text { SODIUM } \\
\text { AD- } \\
\text { SORP- } \\
\text { TION } \\
\text { RATIO } \\
\text { AS K) }\end{array}$ & $\begin{array}{l}\text { POTAS- } \\
\text { SIUM, } \\
\text { DIS- } \\
\text { SOLVED } \\
\text { (MG/L } \\
\text { AS K) }\end{array}$ \\
\hline
\end{tabular}

NOV 1987

$08 \ldots$

MAR 1988

1200

APR

$1400.0 .122-4,010$

05... $\quad 1745 \quad .133 \quad 4,010$

MAY

$09 \ldots \quad 1530 \quad .066 \quad 3,800$

JNE

$08 \ldots \quad 1100 \quad \ldots \quad 3,880$

AU

No

$\begin{array}{llll}30 \ldots & 1300 \quad .036 \quad 3,780\end{array}$

$16 \ldots \quad 1230 \quad .034 \quad 3,580$

APR 1989

$04 \ldots \quad 1200 \quad .166 \quad 4,060$

JUNE

$\begin{array}{rlll}07 \ldots & 1030 \quad \text { 4, } 050\end{array}$

\section{SITE IDENTIFICATION - SSSS87-SPRING}

$\begin{array}{rrrrrrrrr}6.7 & -- & 2,500 & 2,000 & 470 & 310 & 180 & 2 & 10 \\ 6.9 & 12.0 & 2,700 & 2,200 & 470 & 360 & 220 & 2 & 14 \\ 6.7 & 11.0 & 2,400 & 2,000 & 460 & 310 & 150 & 1 & 10 \\ 6.9 & 10.5 & 2,500 & 2,100 & 470 & 320 & 160 & 1 & 13 \\ 6.8 & 11.0 & 2,400 & 1,900 & 430 & 320 & 160 & 1 & 3 \\ 7.0 & 11.0 & 2,500 & 2,000 & 460 & 330 & 170 & 2 & 10 \\ 7.1 & -- & 2,400 & 1,900 & 440 & 310 & 180 & 2 & 10 \\ 6.8 & 10.5 & 2,600 & 2,200 & 480 & 340 & 150 & 1 & 11 \\ 7.0 & 10.5 & 2,500 & 2,000 & 470 & 310 & 150 & 1 & 10 \\ 6.9 & 9.0 & 2,400 & 1,900 & 450 & 300 & 170 & 2 & 11\end{array}$

\begin{tabular}{|c|c|c|c|c|c|c|c|c|c|c|c|}
\hline DATE & $\begin{array}{l}\text { ALKA- } \\
\text { LINITY } \\
(\mathrm{MG} / \mathrm{L} \\
\mathrm{AS} \\
\left.\mathrm{CACO}_{3}\right)\end{array}$ & 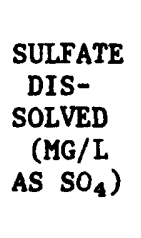 & $\begin{array}{l}\text { CHLO- } \\
\text { RIDE, } \\
\text { DIS- } \\
\text { SOLVED } \\
\text { (MG/L } \\
\text { AS CL) }\end{array}$ & $\begin{array}{l}\text { FLUO- } \\
\text { RIDE, } \\
\text { DIS- } \\
\text { SOLVED } \\
\text { (MG/L } \\
\text { AS F) }\end{array}$ & $\begin{array}{l}\text { SILICA, } \\
\text { DIS- } \\
\text { SOLVED } \\
(\mathrm{MG} / \mathrm{L} \\
\text { AS } \\
\left.\mathrm{SIO}_{2}\right)\end{array}$ & $\begin{array}{l}\text { SOLIDS, } \\
\text { RESIDUE } \\
\text { AT } 180 \\
\text { DEG. C } \\
\text { DIS- } \\
\text { SOLVED } \\
\text { (MG/L) }\end{array}$ & $\begin{array}{l}\text { SOLIDS, } \\
\text { SUM OF } \\
\text { CONSTIT- } \\
\text { UENTS, } \\
\text { DIS- } \\
\text { SOLVED } \\
\text { (MG/L) }\end{array}$ & $\begin{array}{l}\text { SOLIDS, } \\
\text { DIS- } \\
\text { SOLVED } \\
\text { (TONS } \\
\text { PER } \\
\text { AC-FT) }\end{array}$ & $\begin{array}{l}\text { SOLIDS, } \\
\text { DIS- } \\
\text { SOLVED } \\
\text { (TONS } \\
\text { PER } \\
\text { DAY) }\end{array}$ & $\begin{array}{l}\text { NITRO- } \\
\text { GEN, } \\
\text { NITRATE } \\
\text { DIS- } \\
\text { SOLVED } \\
\text { (MG/L } \\
\text { AS N) }\end{array}$ & $\begin{array}{c}\text { NITRO- } \\
\text { GEN, } \\
\text { NITRITE } \\
\text { DIS- } \\
\text { SOLVED } \\
\text { (MG/L } \\
\text { AS N) }\end{array}$ \\
\hline \multicolumn{12}{|l|}{ NOV 1987} \\
\hline $\begin{array}{l}08 \ldots \\
\text { MAR } 1988\end{array}$ & 503 & 2,300 & 36 & 1.2 & 12 & 3,760 & 3,660 & 5.11 & $\cdots$ & 5.92 & 0.08 \\
\hline $\begin{array}{c}03 \ldots \\
\text { APR }\end{array}$ & 459 & 2,500 & 29 & .2 & 11 & 4,100 & 3,890 & 5.58 & 1.28 & -- & $<.04$ \\
\hline$\underset{\mathrm{MAY}}{05 \ldots}$ & 416 & 2,300 & 28 & .3 & 11 & 3,730 & 3,620 & 5.07 & 1.30 & 21.9 & .10 \\
\hline$\underset{\text { JUNE }}{09 . .}$ & 434 & 2,300 & 27 & .3 & 10 & 3,780 & 3,630 & 5.14 & .65 & 12.9 & .07 \\
\hline $\begin{array}{l}08 . . \\
\text { AUG }\end{array}$ & 462 & 2,300 & 38 & .4 & 9.7 & 3,920 & 3,570 & 5.33 & -- & 4.27 & .03 \\
\hline $\operatorname{NOV}^{30} \ldots$ & 490 & 2,300 & 49 & .2 & 11 & 3,830 & 3,660 & 5.21 & .36 & 6.93 & .07 \\
\hline $\begin{array}{l}16 \ldots \\
\text { APR } 1989\end{array}$ & 503 & 2,200 & 53 & .3 & 10 & 3,800 & 3,530 & 5.17 & .32 & 4.34 & .06 \\
\hline$\underset{\text { JUNE }}{04 \ldots}$ & 432 & 2,500 & 38 & .3 & 11 & 3,800 & 3,890 & 5.17 & 1.74 & 22.0 & .04 \\
\hline $\begin{array}{c}07 . . \\
\text { SEPT }\end{array}$ & 465 & 2,300 & 36 & .3 & 10 & 3,780 & 3,570 & 5.14 & -- & -- & -- \\
\hline $12 \ldots$ & 482 & 2,300 & 54 & .3 & 10 & 3,620 & 3,590 & 4.92 & -- & -- & -- \\
\hline
\end{tabular}


Table 26.--Water-quality analyses for springs and lysimeters--Continued

\begin{tabular}{|c|c|c|c|c|c|c|c|c|c|c|c|}
\hline DATE & $\begin{array}{c}\text { NITRO- } \\
\text { GEN, } \\
\text { NITRITE } \\
\text { DIS- } \\
\text { SOLVED } \\
(\mathrm{MG} / \mathrm{L} \\
\left.\text { AS } \mathrm{NO}_{2}\right)\end{array}$ & $\begin{array}{l}\text { NITRO- } \\
\text { GEN, } \\
\mathrm{NO}_{2}+\mathrm{NO}_{3} \\
\text { DIS- } \\
\text { SOLVED } \\
\text { (MG/L } \\
\text { AS N) }\end{array}$ & $\begin{array}{l}\text { NITRO- } \\
\text { GEN, } \\
\text { AMMONIA } \\
\text { DIS- } \\
\text { SOLVED } \\
\text { (MG/L } \\
\text { AS N) }\end{array}$ & $\begin{array}{l}\text { NITRO- } \\
\text { GEN, } \\
\text { AMMONIA } \\
\text { DIS- } \\
\text { SOLVED } \\
(\mathrm{MG} / \mathrm{L} \\
\text { AS NH4) }\end{array}$ & $\begin{array}{l}\text { NITRO- } \\
\text { GEN, } \\
\text { ORGANIC } \\
\text { DIS- } \\
\text { SOLVED } \\
\text { (MG/L } \\
\text { AS N) }\end{array}$ & $\begin{array}{l}\text { NITRO- } \\
\text { GEN,AM- } \\
\text { MONIA + } \\
\text { ORGANIC } \\
\text { DISSOLVED } \\
\text { (MG/L } \\
\text { AS N) }\end{array}$ & $\begin{array}{l}\text { PHOS- } \\
\text { PHORUS } \\
\text { DIS- } \\
\text { SOLVED } \\
\text { (MG/L } \\
\text { AS P) }\end{array}$ & $\begin{array}{l}\text { PHOS- } \\
\text { PHORUS } \\
\text { ORTHO, } \\
\text { DIS- } \\
\text { SOLVED } \\
\text { (MG/L } \\
\text { AS P) }\end{array}$ & $\begin{array}{l}\text { PHOS- } \\
\text { PHATE, } \\
\text { ORTHO, } \\
\text { DIS- } \\
\text { SOLVED } \\
\text { (MG/L } \\
\text { AS PO } 4 \text { ) }\end{array}$ & $\begin{array}{l}\text { PHOS- } \\
\text { PHORUS } \\
\text { ORGANIC } \\
\text { DIS- } \\
\text { SOLVED } \\
\text { (MG/L } \\
\text { AS P) }\end{array}$ & $\begin{array}{c}\text { ALU- } \\
\text { MINUM, } \\
\text { DIS- } \\
\text { SOLVED } \\
\text { (MG/L } \\
\text { AS AL) }\end{array}$ \\
\hline \multicolumn{12}{|c|}{ SITE IDENTIFICATION - SSSS87-SPRING--Continued } \\
\hline $\begin{array}{c}\text { NOV } 1987 \\
08 \ldots \\
\text { MAR } 1988\end{array}$ & 0.26 & 6.00 & 0.11 & 0.14 & 0.89 & 1.0 & $<0.01$ & $<0.01$ & -- & - & $<10$ \\
\hline $\begin{array}{l}03 \ldots \\
\text { APR }\end{array}$ & - & $<11.0$ & .08 & .1 & 1.1 & 1.2 & .01 & $<.01$ & -- & 0.01 & $<10$ \\
\hline$\underset{\text { MAY }}{05}$ & .33 & 22.0 & .09 & .12 & 1.0 & 1.1 & $<.01$ & $<.01$ & -- & -- & $<10$ \\
\hline$\underset{\text { JUNE }}{09}$ & .23 & 13.0 & .14 & .18 & .76 & .9 & .01 & $<.01$ & -- & .01 & $<10$ \\
\hline $\begin{array}{l}08 \ldots \\
\text { AUG }\end{array}$ & .1 & 4.30 & .10 & .13 & 1.0 & 1.1 & .03 & .02 & 0.06 & .01 & 10 \\
\hline $\begin{array}{l}30 \ldots \\
\text { NoV }\end{array}$ & .23 & 7.00 & .13 & .17 & .57 & .7 & $<.01$ & $<.01$ & -- & -- & 20 \\
\hline $\begin{array}{l}16 \ldots \\
\text { APR } 1989\end{array}$ & .2 & 4.40 & .15 & .19 & 1.1 & 1.3 & .01 & $<.01$ & -- & .01 & 10 \\
\hline $\begin{array}{l}04 \ldots \\
\text { JUNE }\end{array}$ & .13 & 22.0 & .08 & .1 & 1.0 & 1.1 & $<.01$ & $<.01$ & -- & 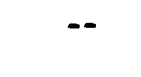 & $<10$ \\
\hline $\begin{array}{l}07 . . \\
\text { SEPT }\end{array}$ & $\ldots$ & -- & -- & - & -- & - & $\cdots$ & -- & -- & -- & $<10$ \\
\hline $12 \ldots$ & -- & -- & -- & -- & -- & -- & -- & -- & -- & -- & $<10$ \\
\hline DATE & $\begin{array}{l}\text { ARSENIC } \\
\text { DIS- } \\
\text { SOLVED } \\
(\mu G / L \\
\text { AS AS) }\end{array}$ & $\begin{array}{l}\text { BARIUM, } \\
\text { DIS- } \\
\text { SOLVED } \\
(\mu \mathrm{G} / \mathrm{L} \\
\text { AS BA) }\end{array}$ & $\begin{array}{l}\text { BERYL- } \\
\text { LIUM, } \\
\text { DIS- } \\
\text { SOLVED } \\
(\mu G / L \\
\text { AS BE) }\end{array}$ & $\begin{array}{l}\text { BORON, } \\
\text { DIS- } \\
\text { SOLVED } \\
(\mu G / L \\
\text { AS B) }\end{array}$ & $\begin{array}{l}\text { CADMIUM } \\
\text { DIS- } \\
\text { SOIVED } \\
(\mu G / L \\
\text { AS CD) }\end{array}$ & $\begin{array}{l}\text { CHRO- } \\
\text { MIUM, } \\
\text { DIS- } \\
\text { SOLVED } \\
(\mu G / L \\
\text { AS CR) }\end{array}$ & $\begin{array}{l}\text { COBALT, } \\
\text { DIS- } \\
\text { SOLVED } \\
(\mu G / L \\
\text { AS CO) }\end{array}$ & $\begin{array}{l}\text { COPPER, } \\
\text { DIS- } \\
\text { SOLVED } \\
\text { ( } \mu G / L \\
\text { AS CU) }\end{array}$ & $\begin{array}{l}\text { IRON, } \\
\text { DIS- } \\
\text { SOLVED } \\
\text { ( } \mu \mathrm{G} / \mathrm{L} \\
\text { AS FE) }\end{array}$ & $\begin{array}{l}\text { LEAD, } \\
\text { DIS- } \\
\text { SOLVED } \\
(\mu G / L \\
\text { AS PB) }\end{array}$ & $\begin{array}{l}\text { MANGA- } \\
\text { NESE, } \\
\text { DIS- } \\
\text { SOLVED } \\
(\mu G / L \\
\text { AS MN) }\end{array}$ \\
\hline NOV 1987 & & & & & & & & & & & \\
\hline $\begin{array}{l}08 \ldots \\
\text { MAR } 1988\end{array}$ & $<1$ & 28 & $<2$ & 460 & $<3$ & $<20$ & $<9$ & $<30$ & 21 & $<30$ & 770 \\
\hline $\begin{array}{l}03 \ldots \\
\text { APR }\end{array}$ & $<1$ & 38 & $<2$ & 430 & $<3$ & $<20$ & $<9$ & $<30$ & 12 & $<30$ & 850 \\
\hline$\underset{\text { MAY }}{05}$ & $<1$ & 27 & $<2$ & 450 & $<3$ & $<20$ & $<9$ & $<30$ & 18 & $<30$ & 560 \\
\hline $\begin{array}{l}09 . \cdots \\
\text { JUNE }\end{array}$ & $<1$ & 24 & $<2$ & 430 & 4 & $<20$ & $<10$ & $<40$ & 14 & $<40$ & 630 \\
\hline $\begin{array}{l}08 \ldots \\
\text { AUG }\end{array}$ & $<1$ & 21 & $<.5$ & 420 & $<1$ & $<5$ & 5 & $<10$ & 6 & $<10$ & 720 \\
\hline $\begin{array}{l}30 \ldots \\
\text { Nov }\end{array}$ & $<1$ & 24 & $<.5$ & 420 & 9 & 10 & 4 & $<10$ & 16 & 10 & 790 \\
\hline $\begin{array}{l}16 \\
\text { APR } 1989\end{array}$ & $<1$ & 24 & $<.5$ & 430 & $<1$ & $<5$ & 4 & $<10$ & 9 & $<10$ & 780 \\
\hline $\begin{array}{l}04 \ldots \\
\text { JNE }\end{array}$ & $<1$ & 23 & $<3$ & 490 & $<5$ & $<20$ & $<20$ & $<50$ & $<15$ & $<50$ & 680 \\
\hline $\begin{array}{c}07 . . \\
\text { SEPT }\end{array}$ & $<1$ & 26 & 3 & 440 & $<3$ & $<20$ & $<9$ & $<30$ & 25 & $<30$ & 760 \\
\hline $12 \ldots$ & $<1$ & 23 & $<2$ & 430 & $<3$ & $<20$ & $<9$ & $<30$ & 9 & $<30$ & 770 \\
\hline
\end{tabular}


Table 26.--Water-quality analyses for springs and lysimeters--Continued

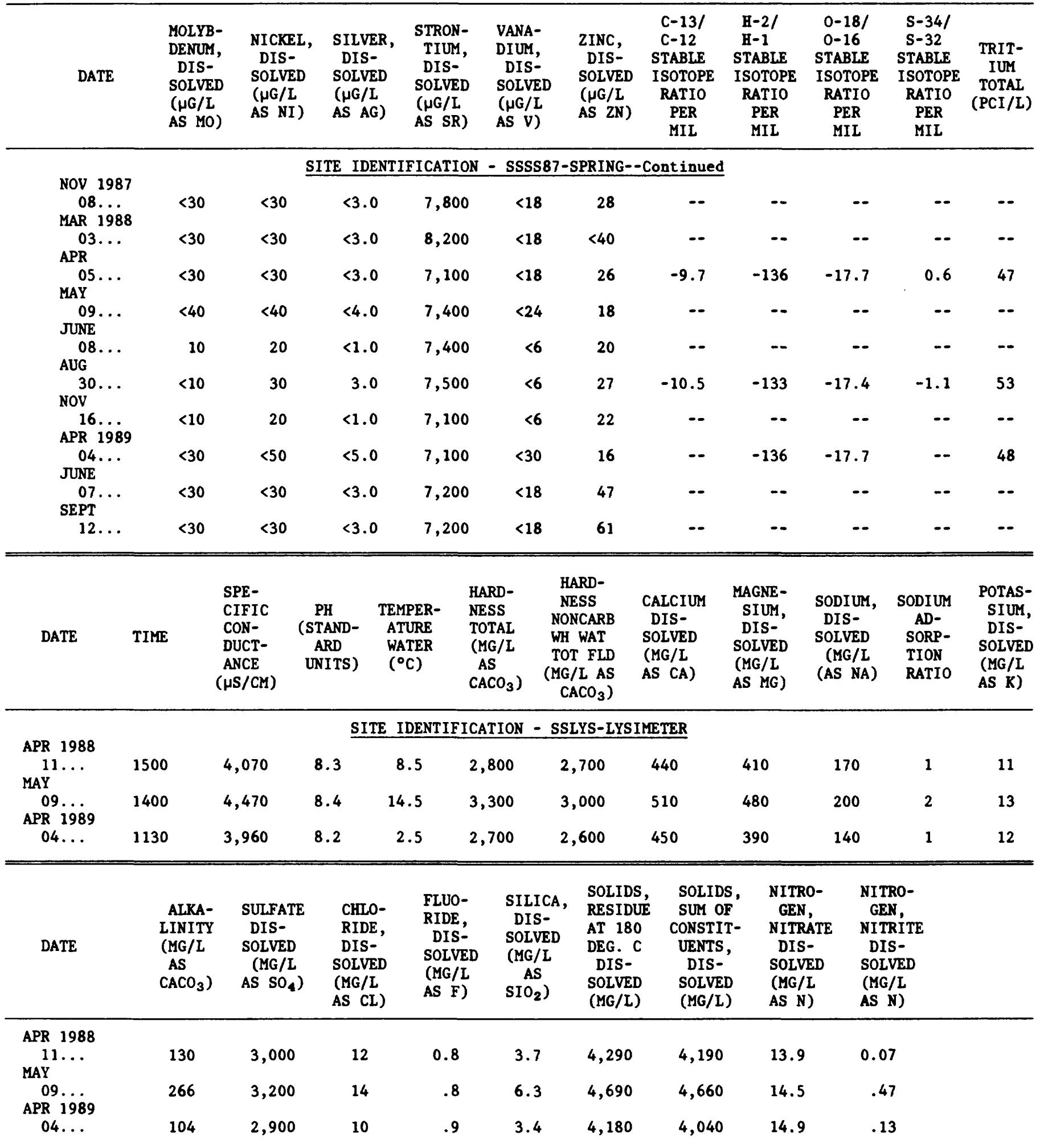


Table 26.--Water-quality analyses for springs and lysimeters--Continued

\begin{tabular}{|c|c|c|c|c|c|c|c|c|c|c|c|}
\hline DATE & $\begin{array}{l}\text { NITRO- } \\
\text { GEN, } \\
\text { NITRITE } \\
\text { DIS- } \\
\text { SOLVED } \\
\text { (MG/L } \\
\text { AS } \mathrm{NO}_{2} \text { ) }\end{array}$ & $\begin{array}{c}\text { NITRO- } \\
\text { GEN, } \\
\mathrm{NO}_{2}+\mathrm{NO}_{3} \\
\text { DIS- } \\
\text { SOLVED } \\
(\mathrm{MG} / \mathrm{L} \\
\text { AS N) }\end{array}$ & $\begin{array}{l}\text { NITRO- } \\
\text { GEN, } \\
\text { AMYONIA } \\
\text { DIS- } \\
\text { SOLVED } \\
\text { (MG/L } \\
\text { AS N) }\end{array}$ & $\begin{array}{c}\text { NITRO- } \\
\text { GEN, } \\
\text { AMYONIA } \\
\text { DIS- } \\
\text { SOLVED } \\
(\mathrm{MG} / \mathrm{L} \\
\left.\text { AS } \mathrm{NH}_{4}\right)\end{array}$ & $\begin{array}{l}\text { NITRO- } \\
\text { GEN, } \\
\text { ORGANIC } \\
\text { DIS- } \\
\text { SOLVED } \\
\text { (MG/L } \\
\text { AS N) }\end{array}$ & $\begin{array}{l}\text { NITRO- } \\
\text { GEN,AM- } \\
\text { MONIA + } \\
\text { ORGANIC } \\
\text { DISSOLVED } \\
\text { (MG/L } \\
\text { AS N) }\end{array}$ & $\begin{array}{l}\text { PHOS- } \\
\text { PHORUS } \\
\text { DIS- } \\
\text { SOLVED } \\
\text { (MG/L } \\
\text { AS P) }\end{array}$ & $\begin{array}{l}\text { PHOS- } \\
\text { PHORUS } \\
\text { ORTHO, } \\
\text { DIS- } \\
\text { SOLVED } \\
\text { (MG/L } \\
\text { AS P) }\end{array}$ & $\begin{array}{l}\text { PHOS- } \\
\text { PHATE, } \\
\text { ORTHO, } \\
\text { DIS- } \\
\text { SOLVED } \\
(\mathrm{MG} / \mathrm{L} \\
\text { AS PO4) }\end{array}$ & $\begin{array}{l}\text { PHOS- } \\
\text { PHORUS } \\
\text { ORGANIC } \\
\text { DIS- } \\
\text { SOLVED } \\
\text { (MG/L } \\
\text { AS P) }\end{array}$ & $\begin{array}{l}\text { ALU- } \\
\text { MINUM, } \\
\text { DIS- } \\
\text { SOLVED } \\
\text { ( } \mu \mathrm{G} / L \\
\text { AS AI) }\end{array}$ \\
\hline \multicolumn{12}{|c|}{ SITE IDENTIFICATION - SSLYS-LYSIMETER--Continued } \\
\hline $\begin{array}{c}\text { APR } 1988 \\
11 \ldots \\
\operatorname{MAY}\end{array}$ & 0.23 & 14.0 & 0.13 & 0.17 & 1.5 & 1.6 & 0.02 & $<0.01$ & -- & 0.02 & 20 \\
\hline $\begin{array}{l}09 \\
\text { APR } 1989\end{array}$ & 1.5 & 15.0 & .43 & .55 & 2.1 & 2.5 & .01 & $<.01$ & - & .01 & 20 \\
\hline $04 \ldots$ & .43 & 15.0 & .09 & .12 & .91 & 1.0 & .02 & .09 & 0.28 & .0 & $<10$ \\
\hline DATE & $\begin{array}{l}\text { ARSENIC } \\
\text { DIS- } \\
\text { SOLVED } \\
(\mu G / L \\
\text { AS AS) }\end{array}$ & $\begin{array}{l}\text { BARIUM, } \\
\text { DIS- } \\
\text { SOLVED } \\
\text { ( } \mu \text { G/L } \\
\text { AS BA) }\end{array}$ & $\begin{array}{l}\text { BERYL- } \\
\text { LIUM, } \\
\text { DIS- } \\
\text { SOLVED } \\
(\mu G / L \\
\text { AS BE) }\end{array}$ & $\begin{array}{l}\text { BORON, } \\
\text { DIS- } \\
\text { SOLVED } \\
(\mu G / L \\
\text { AS B) }\end{array}$ & $\begin{array}{c}\text { CADMIUM } \\
\text { DIS- } \\
\text { SOLVED } \\
\text { ( } \mu G / L \\
\text { AS CD) }\end{array}$ & $\begin{array}{l}\text { CHRO- } \\
\text { MIUI, } \\
\text { DIS- } \\
\text { SOLVED } \\
\text { ( } \mu G / L \\
\text { AS CR) }\end{array}$ & $\begin{array}{l}\text { COBALT, } \\
\text { DIS- } \\
\text { SOLVED } \\
\text { ( } \mu G / L \\
\text { AS CO) }\end{array}$ & $\begin{array}{l}\text { COPPER, } \\
\text { DIS- } \\
\text { SOLVED } \\
(\mu G / L \\
\text { AS CU) }\end{array}$ & $\begin{array}{l}\text { IRON, } \\
\text { DIS- } \\
\text { SOLVED } \\
(\mu G / L \\
\text { AS FE) }\end{array}$ & $\begin{array}{l}\text { IEAD, } \\
\text { DIS- } \\
\text { SOIVED } \\
\text { ( } \mu G / L \\
\text { AS PB) }\end{array}$ & $\begin{array}{l}\text { MANGA- } \\
\text { NESE, } \\
\text { DIS- } \\
\text { SOLVED } \\
\text { ( } \mu \text { G /L } \\
\text { AS MN) }\end{array}$ \\
\hline $\begin{array}{c}\text { APR } 1988 \\
11 \ldots \\
\text { MAY }\end{array}$ & $<1$ & 28 & $<2$ & 100 & $<3$ & $<20$ & $<9$ & $<30$ & 9 & $<30$ & 130 \\
\hline $\begin{array}{c}09 \\
\text { APR } 1989\end{array}$ & 2 & 35 & $<3$ & 170 & $<5$ & $<30$ & $<20$ & $<50$ & 22 & $<50$ & 270 \\
\hline $04 \ldots$ & $<1$ & 25 & $<3$ & 100 & $<5$ & $<20$ & $<20$ & $<50$ & $<15$ & $<50$ & 30 \\
\hline DATE & $\begin{array}{l}\text { MOLYB- } \\
\text { DENUM, } \\
\text { DIS- } \\
\text { SOLVED } \\
(\mu G / L \\
\text { AS MO) }\end{array}$ & $\begin{array}{l}\text { NICKEL, } \\
\text { DIS- } \\
\text { SOLVED } \\
\text { ( } \mu G / L \\
\text { AS NI) }\end{array}$ & $\begin{array}{l}\text { SILVER, } \\
\text { DIS- } \\
\text { SOLVED } \\
(\mu G / L \\
\text { AS AG) }\end{array}$ & $\begin{array}{l}\text { STRON- } \\
\text { TIUM, } \\
\text { DIS- } \\
\text { SOLVED } \\
\text { ( } \mu G / L \\
\text { AS SR) }\end{array}$ & $\begin{array}{l}\text { VANA- } \\
\text { DIUM, } \\
\text { DIS- } \\
\text { SOLVED } \\
(\mu G / L \\
\text { AS V) }\end{array}$ & $\begin{array}{l}\text { ZINC, } \\
\text { DIS- } \\
\text { SOLVED } \\
(\mu G / L \\
\text { AS ZN) }\end{array}$ & $\begin{array}{l}\text { C-13/ } \\
\text { C-12 } \\
\text { STABLE } \\
\text { ISOTOPE } \\
\text { RATIO } \\
\text { PER } \\
\text { MIL }\end{array}$ & $\begin{array}{l}\text { H-2/ } \\
\text { H-1 } \\
\text { STABLE } \\
\text { ISOTOPE } \\
\text { RATIO } \\
\text { PER } \\
\text { MIL }\end{array}$ & $\begin{array}{c}0-18 / \\
0-16 \\
\text { STABLE } \\
\text { ISOTOPE } \\
\text { RATIO } \\
\text { PER } \\
\text { MIL }\end{array}$ & $\begin{array}{c}\text { S-34/ } \\
\text { S-32 } \\
\text { STABLE } \\
\text { ISOTOPE } \\
\text { RATIO } \\
\text { PER } \\
\text { MIL }\end{array}$ & $\begin{array}{l}\text { TRIT- } \\
\text { IUM } \\
\text { TOTAL } \\
\text { (PCI/L) }\end{array}$ \\
\hline \multicolumn{12}{|l|}{ APR 1988} \\
\hline${ }_{\mathrm{MAY}}^{11 \ldots}$ & $<30$ & $<30$ & $<3.0$ & 5,100 & $<18$ & 30 & -- & -129 & -17.2 & -2.9 & 59 \\
\hline $\begin{array}{c}09 . . \\
\text { APR } 1989\end{array}$ & $<50$ & $<50$ & $<5.0$ & 5,700 & $<30$ & 69 & -1.6 & -123 & -15.9 & -2.7 & 63 \\
\hline $04 \ldots$ & $<50$ & $<50$ & $<5.0$ & 5,000 & $<30$ & 27 & -- & -127 & -16.8 & -- & 52 \\
\hline
\end{tabular}




\begin{tabular}{|c|c|c|c|c|c|c|c|c|c|c|c|c|}
\hline DATE & TIME & $\begin{array}{l}\text { SPRING- } \\
\text { FLOW } \\
\text { INSTAN- } \\
\text { TANEOUS } \\
\text { (CFS) }\end{array}$ & $\begin{array}{l}\text { SPE- } \\
\text { CIFIC } \\
\text { CON- } \\
\text { DUCT- } \\
\text { ANCE } \\
(\mu S / C M)\end{array}$ & $\begin{array}{c}\text { PH } \\
\text { (STAND- } \\
\text { ARD } \\
\text { UNITS) }\end{array}$ & $\begin{array}{c}\text { TEMPER- } \\
\text { ATURE } \\
\text { WATER } \\
\left({ }^{\circ} \mathrm{C}\right)\end{array}$ & $\begin{array}{l}\text { HARD- } \\
\text { NESS } \\
\text { TOTAL } \\
\text { (MG/L } \\
\text { AS } \\
\left.\mathrm{CACO}_{3}\right)\end{array}$ & $\begin{array}{l}\text { HARD- } \\
\text { NESS } \\
\text { NONCARB } \\
\text { WH WAT } \\
\text { TOT FID } \\
(\mathrm{MG} / \mathrm{L} \text { AS } \\
\left.\mathrm{CACO}_{3}\right)\end{array}$ & $\begin{array}{l}\text { CALCIUM } \\
\text { DIS- } \\
\text { SOLVED } \\
\text { (MG/L } \\
\text { AS CA) }\end{array}$ & $\begin{array}{l}\text { MAGNE- } \\
\text { SIUA, } \\
\text { DIS- } \\
\text { SOLVED } \\
\text { (MG/L } \\
\text { AS ISG) }\end{array}$ & $\begin{array}{l}\text { SODIUM, } \\
\text { DIS- } \\
\text { SOLVED } \\
\text { (MG/I } \\
\text { AS NA) }\end{array}$ & $\begin{array}{c}\text { SODIUM } \\
\text { AD- } \\
\text { SORP- } \\
\text { TION } \\
\text { RATIO }\end{array}$ & $\begin{array}{l}\text { POTAS- } \\
\text { SIUM, } \\
\text { DIS- } \\
\text { SOLVED } \\
\text { (MG/L } \\
\text { AS K) }\end{array}$ \\
\hline \multicolumn{13}{|c|}{ SITE IDENTIFICATION - SCSS87-SPRING } \\
\hline $\begin{array}{c}\text { MAR } 1988 \\
07 \ldots \\
\text { APR }\end{array}$ & 1430 & -- & 2,610 & 6.9 & 9.0 & 1,700 & 1,300 & 340 & 200 & 140 & 2 & 7.7 \\
\hline${ }_{\operatorname{MAY}}^{12} \cdots$ & 0845 & .281 & 2,850 & 7.0 & 7.0 & 1,800 & 1,500 & 370 & 220 & 140 & 1 & 6.5 \\
\hline$\underset{\pi N E}{11 . . .}$ & 1015 & .153 & 3,050 & 7.1 & 9.0 & 1,800 & 1,500 & 370 & 220 & 150 & 2 & 7.3 \\
\hline AUG & 1300 & -- & 2,970 & 7.1 & 15.0 & 1,700 & 1,400 & 350 & 210 & 140 & 1 & 7.3 \\
\hline Nov $31 .$. & 1130 & .053 & 2,590 & 7.0 & 12.0 & 1,700 & 1,300 & 350 & 210 & 160 & 2 & 8.4 \\
\hline $\begin{array}{l}15 \ldots \\
\text { APR } 1989\end{array}$ & 1430 & .050 & 2,820 & 7.3 & -- & 1,600 & 1,200 & 320 & 200 & 150 & 2 & 7.4 \\
\hline$\underset{\text { NNE }}{05 \ldots}$ & 0900 & .479 & 3,260 & 7.0 & 7.0 & 2,000 & 1,600 & 400 & 240 & 94 & .9 & 7.7 \\
\hline $\begin{array}{c}06 \ldots \\
\text { SEPT }\end{array}$ & 1015 & .103 & 3,250 & 7.1 & 12.0 & 2,000 & 1,600 & 400 & 240 & 110 & 1 & 6.1 \\
\hline $18 \ldots$ & 1445 & -- & 2,870 & 7.4 & 16.0 & 1,800 & 1,500 & 340 & 240 & 110 & 1 & 9.0 \\
\hline DATE & & $\begin{array}{l}\text { ALKA- } \\
\text { LINITY } \\
\text { (MG/L } \\
\text { AS } \\
\left.\mathrm{CACO}_{3}\right)\end{array}$ & $\begin{array}{l}\text { SULFATE } \\
\text { DIS- } \\
\text { SOLVED } \\
(\mathrm{MG} / \mathrm{L}) \\
\left.\mathrm{AS} \mathrm{SO}_{4}\right)\end{array}$ & $\begin{array}{l}\text { CHLO- } \\
\text { RIDE, } \\
\text { DIS- } \\
\text { SOLVED } \\
\text { (MG/L } \\
\text { AS CL) }\end{array}$ & $\begin{array}{l}\text { FLUO- } \\
\text { RIDE, } \\
\text { DIS- } \\
\text { SOLVED } \\
\text { (MG/L } \\
\text { AS F) }\end{array}$ & $\begin{array}{l}\text { SILICA, } \\
\text { DIS- } \\
\text { SOLVED } \\
\text { (MG/L } \\
\mathrm{AS}^{-} \\
\left.\mathrm{SIO}_{2}\right)\end{array}$ & $\begin{array}{l}\text { SOLIDS, } \\
\text { RESIDUE } \\
\text { AT 180 } \\
\text { DEG. C } \\
\text { DIS- } \\
\text { SOLVED } \\
\text { (MG/L) }\end{array}$ & $\begin{array}{c}\text { SOLIDS, } \\
\text { SUA OF } \\
\text { CONSTIT- } \\
\text { UENTS, } \\
\text { DIS- } \\
\text { SOLVED } \\
\text { (MG/L) }\end{array}$ & $\begin{array}{l}\text { SOLIDS, } \\
\text { DIS- } \\
\text { SOLVED } \\
\text { (TONS } \\
\text { PER } \\
\text { AC-FT) }\end{array}$ & $\begin{array}{l}\text { SOLIDS, } \\
\text { DIS- } \\
\text { SOLVED } \\
\text { (TONS } \\
\text { PER } \\
\text { DAY) }\end{array}$ & $\begin{array}{c}\text { NITRO- } \\
\text { GEN, } \\
\text { NITRATE } \\
\text { DIS- } \\
\text { SOLVED } \\
\text { (MG/L } \\
\text { AS N) }\end{array}$ & $\begin{array}{c}\text { NITRO- } \\
\text { GEN, } \\
\text { NITRITE } \\
\text { DIS- } \\
\text { SOLVED } \\
\text { (MG/L } \\
\text { AS N) }\end{array}$ \\
\hline $\begin{array}{c}\text { MAR } 1988 \\
07 \ldots\end{array}$ & & 369 & 1,500 & 15 & 0.2 & 12 & 2,520 & 2,590 & 3.43 & -- & 1.82 & 0.28 \\
\hline $\begin{array}{l}\text { APR } \\
12 \ldots\end{array}$ & & 328 & 1,700 & 12 & .2 & 10 & 2,800 & 2,690 & 3.81 & 2.04 & 6.99 & .01 \\
\hline$\underset{\text { JNNE }}{11} \cdots$ & & 350 & 1,700 & 13 & .3 & 9.8 & 2,870 & 2,720 & 3.90 & 1.12 & 7.07 & .03 \\
\hline$\underset{A U G}{08 \ldots}$ & & 375 & 1,700 & 15 & .4 & 7.5 & 2,830 & 2,710 & 3.85 & -- & 10.9 & .11 \\
\hline $\operatorname{Nov}^{31 \ldots}$ & & 405 & 1,600 & 10 & .2 & 11 & 2,730 & 2,600 & 3.71 & .37 & -- & $<.01$ \\
\hline $\begin{array}{l}15 \ldots \\
\text { APR } 1989\end{array}$ & & 395 & 1,600 & 16 & .2 & 8.8 & 2,600 & 2,550 & 3.54 & .34 & -- & $<.01$ \\
\hline$\underset{\text { JNE }}{05 \ldots}$ & & 373 & 1,900 & 16 & .3 & 10 & 2,890 & 2,950 & 3.93 & 3.82 & 13.0 & .03 \\
\hline$\underset{\text { SEPT }}{06 . .}$ & & 408 & 1,800 & 14 & .2 & 6.3 & 2,940 & 2,830 & 4.00 & .79 & $\cdots$ & -- \\
\hline $18 \ldots$ & & 335 & 1,700 & 19 & .2 & 17 & 2,720 & 2,640 & 3.70 & -- & -- & -- \\
\hline
\end{tabular}


Table 26.--Water-quality analyses for springs and lysimeters--Continued

\begin{tabular}{|c|c|c|c|c|c|c|c|c|c|c|c|}
\hline DATE & $\begin{array}{l}\text { NITRO- } \\
\text { GEN, } \\
\text { NITRITE } \\
\text { DIS- } \\
\text { SOLVED } \\
(\mathrm{MG} / \mathrm{L} \\
\left.\text { AS } \mathrm{NO}_{2}\right)\end{array}$ & $\begin{array}{l}\text { NITRO- } \\
\text { GEN, } \\
\mathrm{NO}_{2}+\mathrm{NO}_{3} \\
\text { DIS- } \\
\text { SOLVED } \\
\text { (MG/L } \\
\text { AS N) }\end{array}$ & $\begin{array}{l}\text { NITRO- } \\
\text { GEN, } \\
\text { AMMONIA } \\
\text { DIS- } \\
\text { SOLVED } \\
\text { (MG/L } \\
\text { AS N) }\end{array}$ & $\begin{array}{l}\text { NITRO- } \\
\text { GEN, } \\
\text { AMMONIA } \\
\text { DIS- } \\
\text { SOLVED } \\
\text { (MG/L } \\
\text { AS NH4) }\end{array}$ & $\begin{array}{l}\text { NITRO- } \\
\text { GEN, } \\
\text { ORGANIC } \\
\text { DIS- } \\
\text { SOLVED } \\
\text { (MG/L } \\
\text { AS N) }\end{array}$ & $\begin{array}{l}\text { NITRO- } \\
\text { GEN,AM- } \\
\text { MONIA + } \\
\text { ORGANIC } \\
\text { DISSOLVED } \\
\text { (MG/L } \\
\text { AS N) }\end{array}$ & $\begin{array}{l}\text { PHOS- } \\
\text { PHORUS } \\
\text { DIS- } \\
\text { SOLVED } \\
\text { (MG/L } \\
\text { AS P) }\end{array}$ & $\begin{array}{l}\text { PHOS- } \\
\text { PHORUS } \\
\text { ORTHO, } \\
\text { DIS- } \\
\text { SOLVED } \\
\text { (MG/L } \\
\text { AS P) }\end{array}$ & $\begin{array}{l}\text { PHOS- } \\
\text { PHORUS } \\
\text { ORGANIC } \\
\text { DIS- } \\
\text { SOLVED } \\
\text { (MG/L } \\
\text { AS P) }\end{array}$ & $\begin{array}{c}\text { ALU- } \\
\text { MINUM, } \\
\text { DIS- } \\
\text { SOLVED } \\
\text { ( } \mu \mathrm{G} / \mathrm{L} \\
\text { AS AL) }\end{array}$ & $\begin{array}{c}\text { ARSENIC } \\
\text { DIS- } \\
\text { SOLVED } \\
\text { ( } \mu G / L \\
\text { AS AS) }\end{array}$ \\
\hline \multicolumn{12}{|c|}{ SITE IDENTIFICATION - SCSS87-SPRING--Continued } \\
\hline $\begin{array}{c}\text { MAR } 1988 \\
07 \ldots \\
\text { APR }\end{array}$ & 0.92 & 2.10 & 0.08 & 0.1 & 0.52 & 0.6 & $<0.01$ & $<0.01$ & -- & $<10$ & $<1$ \\
\hline${ }_{\mathrm{MAY}}^{12 \ldots}$ & .03 & 7.00 & .08 & .1 & .62 & .7 & $<.01$ & $<.01$ & -- & $<10$ & $<1$ \\
\hline INE & .1 & 7.10 & .11 & .14 & 1.7 & 1.8 & .01 & $<.01$ & 0.01 & $<10$ & $<1$ \\
\hline$\underset{A U G}{08} \ldots$ & .36 & 11.0 & .12 & .15 & 1.3 & 1.4 & $<.01$ & $<.01$ & -- & $<10$ & $<1$ \\
\hline${ }_{\text {Nov }}^{31 \ldots}$ & -- & .85 & .10 & .13 & .4 & .5 & $<.01$ & $<.01$ & -- & $<10$ & $<1$ \\
\hline $\begin{array}{l}15 \ldots \\
\text { APR } 1989\end{array}$ & -- & .55 & .11 & .14 & .29 & .4 & $<.01$ & $<.01$ & -- & $<10$ & $<1$ \\
\hline JUNE & .1 & 13.0 & .08 & .1 & .72 & .8 & $<.01$ & $<.01$ & -- & 20 & $<1$ \\
\hline$\underset{\text { SEPT }}{06 \ldots}$ & - & -- & -- & - & -- & -- & - & -- & -- & $<10$ & $<1$ \\
\hline $18 \ldots$ & -- & -- & -- & - & -- & -- & -- & -- & -- & 10 & 2 \\
\hline DATE & & $\begin{array}{l}\text { BARIUM, } \\
\text { DIS- } \\
\text { SOLVED } \\
(\mu G / L \\
\text { AS BA) }\end{array}$ & $\begin{array}{l}\text { BERYL- } \\
\text { IIUM, } \\
\text { DIS- } \\
\text { SOLVED } \\
(\mu G / L \\
\text { AS BE) }\end{array}$ & $\begin{array}{l}\text { BORON, } \\
\text { DIS- } \\
\text { SOLVED } \\
(\mu G / L \\
\text { AS B) }\end{array}$ & $\begin{array}{c}\text { CADMIUM } \\
\text { DIS- } \\
\text { SOLVED } \\
(\mu G / L \\
\text { AS CD) }\end{array}$ & $\begin{array}{l}\text { CHRO- } \\
\text { MIUM, } \\
\text { DIS- } \\
\text { SOLVED } \\
\text { ( } \mu \text { G/L } \\
\text { AS CR) }\end{array}$ & $\begin{array}{l}\text { COBALT, } \\
\text { DIS- } \\
\text { SOLVED } \\
(\mu G / L \\
\text { AS CO) }\end{array}$ & $\begin{array}{l}\text { COPPER, } \\
\text { DIS- } \\
\text { SOLVED } \\
(\mu G / L \\
\text { AS CU) }\end{array}$ & $\begin{array}{l}\text { IRON, } \\
\text { DIS- } \\
\text { SOLVED } \\
\text { ( } \mu G / L \\
\text { AS FE) }\end{array}$ & $\begin{array}{l}\text { LEAD, } \\
\text { DIS- } \\
\text { SOLVED } \\
(\mu G / L \\
\text { AS PB) }\end{array}$ & $\begin{array}{c}\text { MANGA- } \\
\text { NESE, } \\
\text { DIS- } \\
\text { SOLVED } \\
(\mu G / L \\
\text { AS MN) }\end{array}$ \\
\hline MAR 1988 & & & & & & & & & & & \\
\hline$\underset{A P R}{07 \ldots}$ & & 43 & $<2$ & 200 & 4 & $<20$ & $<9$ & $<30$ & 34 & $<30$ & 320 \\
\hline $\mathrm{MAY}^{12 \ldots}$ & & 47 & $<2$ & 190 & $<3$ & $<20$ & $<9$ & $<30$ & 12 & $<30$ & 77 \\
\hline JUNE & & 43 & $<2$ & 210 & $<3$ & $<20$ & $<9$ & $<30$ & 22 & $<30$ & 73 \\
\hline $\begin{array}{l}08 \ldots \\
\text { AUG }\end{array}$ & & 35 & $<.5$ & 210 & $<1$ & $<5$ & $<3$ & $<10$ & 7 & $<10$ & 88 \\
\hline $\begin{array}{l}31 \ldots \\
\text { Nov }\end{array}$ & & 38 & $<.5$ & 200 & 5 & 8 & $<3$ & $<10$ & 31 & $<10$ & 150 \\
\hline $\begin{array}{c}15 \ldots \\
\text { APR } 1989\end{array}$ & & 37 & $<.5$ & 200 & $<1$ & $<5$ & $<3$ & $<10$ & 19 & $<10$ & 170 \\
\hline $\begin{array}{l}05 \ldots \\
\text { JUNE }\end{array}$ & & 39 & $<2$ & 220 & 3 & $<20$ & $<9$ & $<30$ & 120 & 30 & 55 \\
\hline SEPT & & 35 & $<2$ & 220 & $<3$ & $<20$ & $<9$ & $<30$ & 41 & $<30$ & 110 \\
\hline $18 \ldots$ & & 46 & $<1$ & 200 & $<2$ & $<10$ & $<6$ & $<20$ & 150 & $<20$ & 840 \\
\hline
\end{tabular}


Table 26.--Water-quality analyses for springs and lysimeters--Continued

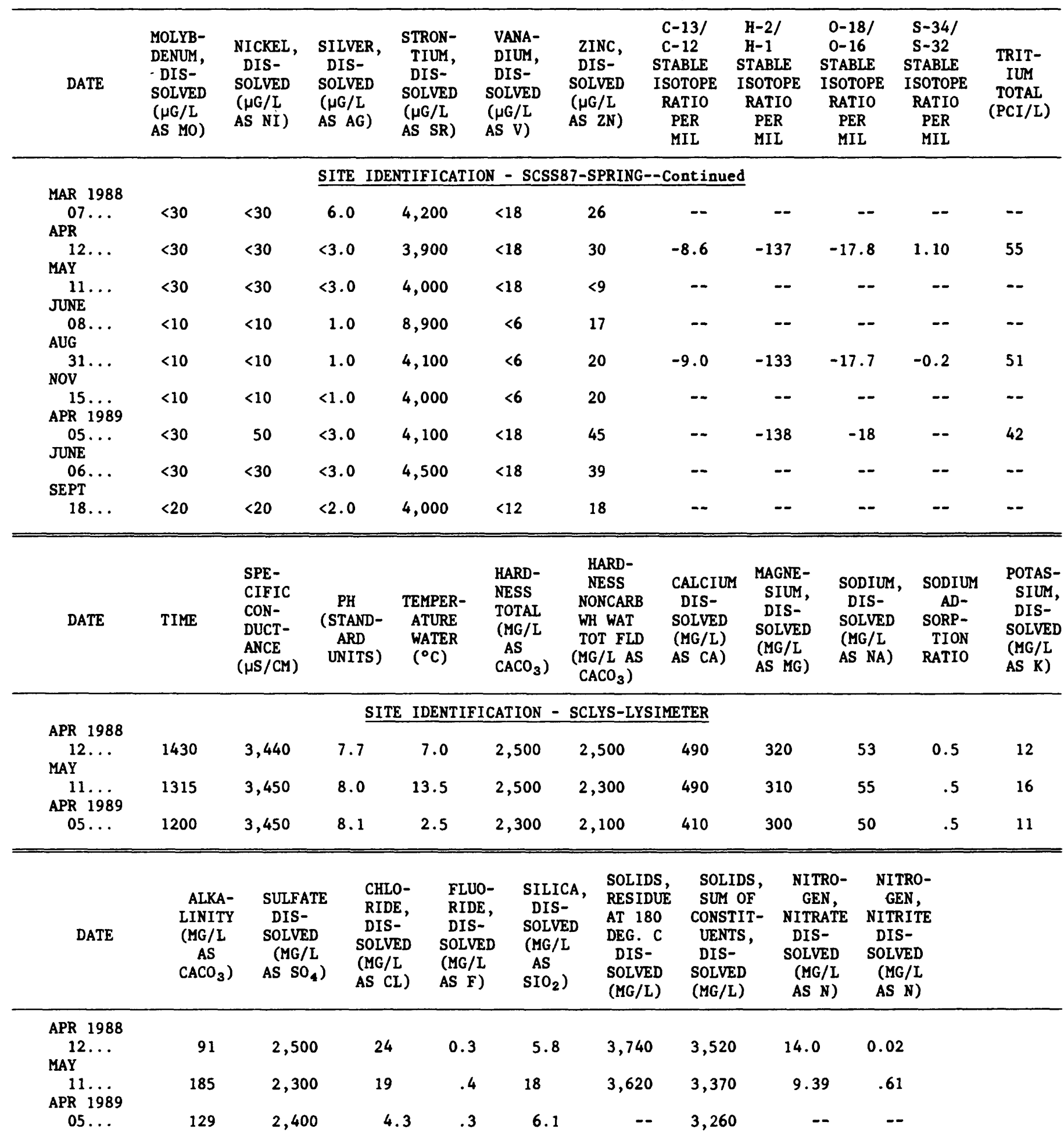


Table 26.--Water-quality analyses for springs and lysimeters--Continued

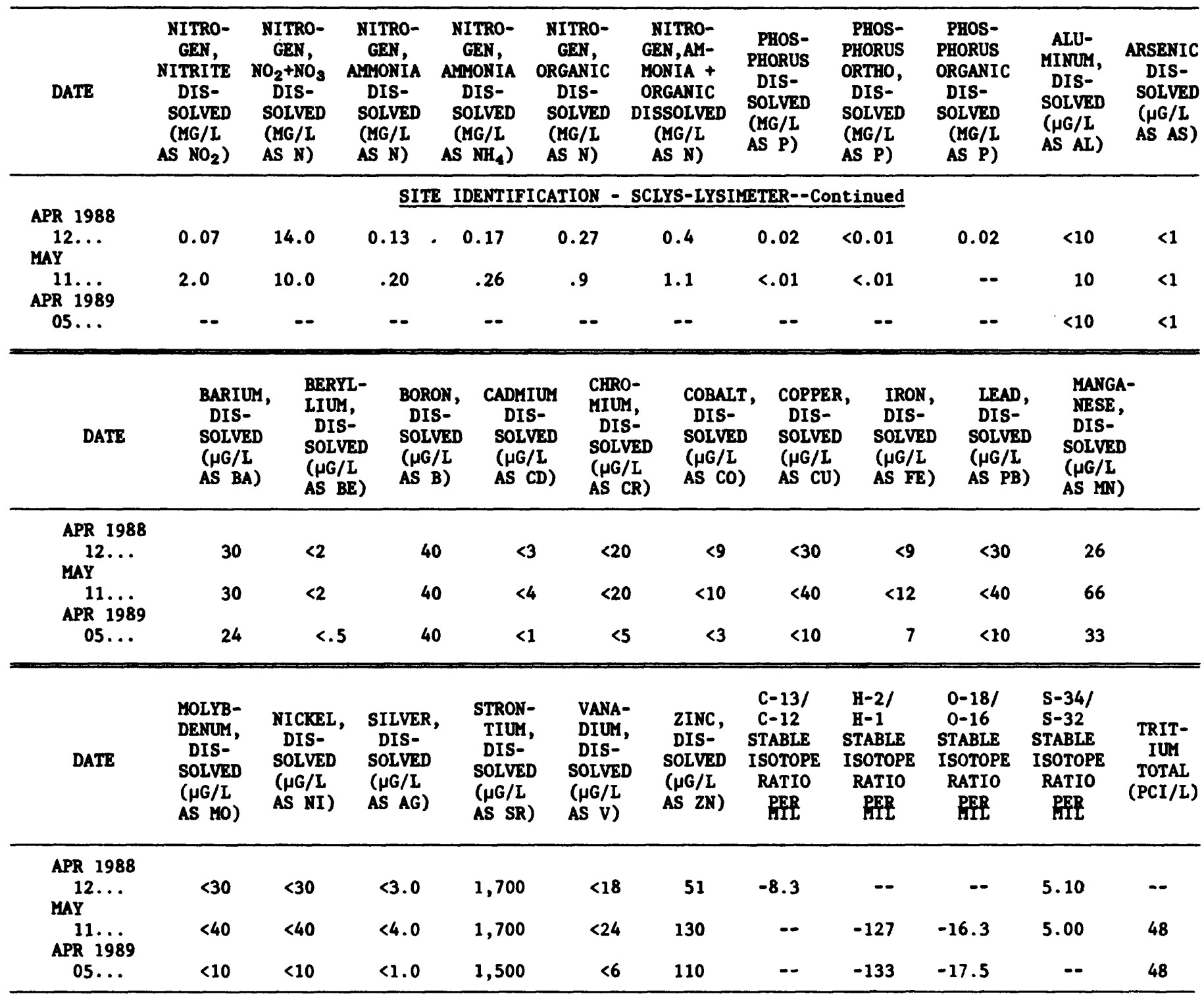


Table 27.--Water-quality information from cow Camp Creek batch-mixing samples collected after 90 days of mater-rock contact

$\left[\mu \mathrm{S} / \mathrm{CM}\right.$, microsiemens per centimeter at 25 degrees Celsius; ${ }^{\circ} \mathrm{C}$, degrees Celsius; MG/L, milligrams per liter; $\mu G / L$, micrograms per liter; <, less than]

\begin{tabular}{|c|c|c|c|c|c|c|c|c|c|c|c|}
\hline $\begin{array}{l}\text { LITHO- } \\
\text { LOGIC } \\
\text { UNIT } \\
\text { (SEE } \\
\text { FIGURE } \\
28)^{1}\end{array}$ & \multicolumn{2}{|c|}{$\begin{array}{c}\text { SAMPLE } \\
\text { DEPTH } \\
\text { (FEET) }\end{array}$} & $\begin{array}{c}\text { SPE- } \\
\text { CIFIC } \\
\text { CON- } \\
\text { DUCT- } \\
\text { ANCE } \\
(\mu S / C M)\end{array}$ & $\begin{array}{c}\text { PH } \\
\text { (STAND- } \\
\text { ARD } \\
\text { UNITS) }\end{array}$ & $\begin{array}{l}\text { TEMPER- } \\
\text { ATURE } \\
\text { WATER } \\
\left({ }^{\circ} \mathrm{C}\right)\end{array}$ & $\begin{array}{l}\text { CALCIUM } \\
\text { DIS- } \\
\text { SOLVED } \\
\text { (MG/L } \\
\text { AS CA) }\end{array}$ & $\begin{array}{l}\text { MAGNE- } \\
\text { SIUM, } \\
\text { DIS- } \\
\text { SOLVED } \\
\text { (MG/L } \\
\text { AS MG) }\end{array}$ & $\begin{array}{l}\text { SODIUM, } \\
\text { DIS- } \\
\text { SOLVED } \\
\text { (MG/L } \\
\text { AS NA) }\end{array}$ & $\begin{array}{c}\text { ALKA- } \\
\text { LINITY } \\
\text { (MG/L } \\
\text { AS } \\
\left.\mathrm{CACO}_{3}\right)\end{array}$ & $\begin{array}{l}\text { SULFATE } \\
\text { DIS- } \\
\text { SOLVED } \\
\left(\mathrm{MG} / \mathrm{L} \mathrm{SO}_{4}\right) \\
\text { AS }\end{array}$ & $\begin{array}{c}\text { CHLO- } \\
\text { RIDE, } \\
\text { DIS- } \\
\text { SOLVED } \\
\text { (MG/L } \\
\text { AS CL) }\end{array}$ \\
\hline $\begin{array}{l}\text { SSO1 } \\
\text { CLO2 } \\
\text { SS03 } \\
\text { SH04 }\end{array}$ & \multicolumn{2}{|c|}{$\begin{array}{l}18.4-18.7 \\
22.6-22.9 \\
27.9-28.2 \\
36.7-37.0\end{array}$} & $\begin{array}{l}6,000 \\
6,400 \\
1,100 \\
1,200\end{array}$ & $\begin{array}{l}2.58 \\
1.91 \\
7.73 \\
7.45\end{array}$ & $\begin{array}{l}24.2 \\
23.7 \\
24.7 \\
24.6\end{array}$ & $\begin{array}{r}490 \\
30 \\
55 \\
100\end{array}$ & $\begin{array}{r}250 \\
14 \\
100 \\
52\end{array}$ & $\begin{array}{l}1.6 \\
39 \\
44 \\
86\end{array}$ & $\begin{array}{l}0.0 \\
0.0 \\
350 \\
160\end{array}$ & $\begin{array}{r}6,100 \\
3,900 \\
340 \\
520\end{array}$ & $\begin{array}{l}2.8 \\
3.1 \\
4.6 \\
3.2\end{array}$ \\
\hline $\begin{array}{l}\text { SS05 } \\
\text { SS06 } \\
\text { SH07 } \\
\text { SS08 }\end{array}$ & \multicolumn{2}{|c|}{$\begin{array}{l}39.2-39.5 \\
49.0-49.3 \\
61.0-61.3 \\
66.1-66.5\end{array}$} & $\begin{array}{r}1,400 \\
760 \\
980 \\
700\end{array}$ & $\begin{array}{l}7.48 \\
7.88 \\
8.26 \\
8.02\end{array}$ & $\begin{array}{l}25.1 \\
24.1 \\
24.6 \\
24.8\end{array}$ & $\begin{array}{r}180 \\
65 \\
18 \\
32\end{array}$ & $\begin{array}{l}78 \\
42 \\
10 \\
34\end{array}$ & $\begin{array}{r}35 \\
33 \\
190 \\
70\end{array}$ & $\begin{array}{l}150 \\
180 \\
260 \\
260\end{array}$ & $\begin{array}{l}790 \\
240 \\
240 \\
120\end{array}$ & $\begin{array}{l}3.4 \\
2.3 \\
2.0 \\
4.3\end{array}$ \\
\hline $\begin{array}{l}\text { SH09 } \\
\text { CL10 } \\
\text { SS12 } \\
\text { SS13 }\end{array}$ & \multicolumn{2}{|c|}{$\begin{array}{l}73.5-73.7 \\
76.0-76.4 \\
87.1-87.4 \\
89.7-90.0\end{array}$} & $\begin{array}{r}3,600 \\
230 \\
670 \\
860\end{array}$ & $\begin{array}{l}7.25 \\
7.63 \\
7.72 \\
8.13\end{array}$ & $\begin{array}{l}24.7 \\
24.1 \\
24.3 \\
24.4\end{array}$ & $\begin{array}{r}370 \\
18 \\
16 \\
19\end{array}$ & $\begin{array}{c}180 \\
9.0 \\
14 \\
12\end{array}$ & $\begin{array}{r}300 \\
19 \\
130 \\
160\end{array}$ & $\begin{array}{r}77 \\
120 \\
190 \\
380\end{array}$ & $\begin{array}{c}2,100 \\
5.0 \\
140 \\
80\end{array}$ & $\begin{array}{l}3.6 \\
1.6 \\
3.5 \\
2.7\end{array}$ \\
\hline $\begin{array}{l}\text { SH14 } \\
\text { SH16 } \\
\text { SS } 17 \\
\text { SH18 }\end{array}$ & \multicolumn{2}{|c|}{$\begin{array}{c}90.9-91.2 \\
98.3-98.6 \\
106.2-106.5 \\
118.8-119.2\end{array}$} & $\begin{array}{r}1,400 \\
2,400 \\
820 \\
1,600\end{array}$ & $\begin{array}{l}7.91 \\
3.19 \\
7.75 \\
7.95\end{array}$ & $\begin{array}{l}23.7 \\
24.1 \\
23.8 \\
23.6\end{array}$ & $\begin{array}{r}18 \\
150 \\
56 \\
22\end{array}$ & $\begin{array}{l}9.0 \\
44 \\
40 \\
10\end{array}$ & $\begin{array}{r}290 \\
260 \\
86 \\
380\end{array}$ & $\begin{array}{l}450 \\
0.0 \\
200 \\
370\end{array}$ & $\begin{array}{r}300 \\
1,500 \\
240 \\
480\end{array}$ & $\begin{array}{l}1.9 \\
3.8 \\
2.6 \\
1.7\end{array}$ \\
\hline $\begin{array}{l}\text { LITHO- } \\
\text { LOGIC } \\
\text { UNIT } \\
\text { (SEE } \\
\text { FIGURE } \\
28 \text { ) }\end{array}$ & $\begin{array}{l}\text { IRON, } \\
\text { DIS- } \\
\text { SOLVED } \\
\text { (MG/L } \\
\text { AS FE }\end{array}$ & $\begin{array}{c}\text { SILICA, } \\
\text { DIS- } \\
\text { SOLVED } \\
\text { (MG/L } \\
\text { AS } \\
\text { SI) }\end{array}$ & $\begin{array}{l}\text { DIS- } \\
\text { SOLVED } \\
\text { SOLIDS } \\
\text { SUM } \\
(\mathrm{MG} / \mathrm{L})\end{array}$ & $\begin{array}{l}\text { ALU- } \\
\text { MINUM } \\
\text { DIS- } \\
\text { SOLVED } \\
(\mu G / L \\
\text { AS AL) }\end{array}$ & $\begin{array}{l}\text { BARIUM, } \\
\text { DIS- } \\
\text { SOLVED } \\
(\mu G / L \\
\text { AS BA) }\end{array}$ & $\begin{array}{c}\text { BERYL- } \\
\text { LIUM, } \\
\text { DIS- } \\
\text { SOLVED } \\
(\mu G / L \\
\text { AS BE) }\end{array}$ & $\begin{array}{c}\text { BORON, } \\
\text { DIS- } \\
\text { SOLVED } \\
(\mu G / L \\
\text { AS B) }\end{array}$ & $\begin{array}{l}\text { CADMIUM } \\
\text { DIS- } \\
\text { SOLVED } \\
\text { ( } \mu G / L \\
\text { AS CD) }\end{array}$ & $\begin{array}{l}\text { CHRO- } \\
\text { MIUM, } \\
\text { DIS- } \\
\text { SOLVED } \\
(\mu G / L \\
\text { AS CR) }\end{array}$ & $\begin{array}{l}\text { COBALT, } \\
\text { DIS- } \\
\text { SOLVED } \\
(\mu G / L \\
\text { AS CO) }\end{array}$ & $\begin{array}{c}\text { COPPER, } \\
\text { DIS- } \\
\text { SOLVED } \\
(\mu G / L \\
\text { AS CU) }\end{array}$ \\
\hline $\begin{array}{l}\text { SS01 } \\
\text { CLO2 } \\
\text { SS03 } \\
\text { SH04 }\end{array}$ & $\begin{array}{r}1,700 \\
1,100 \\
.10 \\
.10\end{array}$ & $\begin{array}{l}49 \\
6.4 \\
1.8 \\
4.9\end{array}$ & $\begin{array}{r}8,700 \\
5,100 \\
980 \\
980\end{array}$ & $\begin{array}{r}95,000 \\
7,900 \\
<10 \\
<10\end{array}$ & $\begin{array}{l}7.0 \\
10 \\
25 \\
35\end{array}$ & $\begin{array}{l}43 \\
<1.0 \\
<1.0 \\
<1.0\end{array}$ & $\begin{array}{r}1,500 \\
2,600 \\
24 \\
210\end{array}$ & $\begin{array}{r}470 \\
380 \\
<1.0 \\
<1.0\end{array}$ & $\begin{array}{l}220 \\
170 \\
<1.0 \\
110\end{array}$ & $\begin{array}{r}6,800 \\
4,100 \\
<1.0 \\
<1.0\end{array}$ & $\begin{array}{r}160 \\
69 \\
61 \\
22\end{array}$ \\
\hline $\begin{array}{l}\text { SS05 } \\
\text { SS06 } \\
\text { SH07 } \\
\text { SS08 }\end{array}$ & $\begin{array}{l}.04 \\
.03 \\
.70 \\
.03\end{array}$ & $\begin{array}{l}2.8 \\
2.9 \\
3.9 \\
2.2\end{array}$ & $\begin{array}{r}1,300 \\
610 \\
780 \\
580\end{array}$ & $\begin{array}{l}<10 \\
<10 \\
<10 \\
<10\end{array}$ & $\begin{array}{l}28 \\
28 \\
63 \\
77\end{array}$ & $\begin{array}{r}<1.0 \\
4.0 \\
<1.0 \\
2.0\end{array}$ & $\begin{array}{r}160 \\
48 \\
110 \\
150\end{array}$ & $\begin{array}{l}22 \\
19 \\
<1.0 \\
17\end{array}$ & $\begin{array}{l}1.0 \\
42 \\
52 \\
17\end{array}$ & $\begin{array}{l}32 \\
<1.0 \\
<1.0 \\
<1.0\end{array}$ & $\begin{array}{l}95 \\
23 \\
22 \\
83\end{array}$ \\
\hline $\begin{array}{l}\text { SH09 } \\
\text { CL10 } \\
\text { SS12 } \\
\text { SS13 }\end{array}$ & $\begin{array}{l}.06 \\
.07 \\
.14 \\
.07\end{array}$ & $\begin{array}{l}4.7 \\
1.2 \\
4.3 \\
2.8\end{array}$ & $\begin{array}{r}3,600 \\
190 \\
530 \\
730\end{array}$ & $\begin{array}{l}<10 \\
<10 \\
<10 \\
<10\end{array}$ & $\begin{array}{r}16 \\
320 \\
110 \\
145\end{array}$ & $\begin{array}{l}<1.0 \\
<1.0 \\
<1.0 \\
<1.0\end{array}$ & $\begin{array}{r}160 \\
1,100 \\
26 \\
30\end{array}$ & $\begin{array}{l}<1.0 \\
<1.0 \\
<1.0 \\
<1.0\end{array}$ & $\begin{array}{l}33 \\
74 \\
74 \\
<1.0\end{array}$ & $\begin{array}{l}7.0 \\
<1.0 \\
11 \\
10\end{array}$ & $\begin{array}{r}110 \\
68 \\
12 \\
15\end{array}$ \\
\hline $\begin{array}{l}\text { SH14 } \\
\text { SH16 } \\
\text { SS17 } \\
\text { SH18 }\end{array}$ & 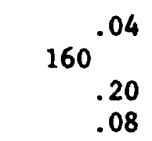 & $\begin{array}{l}4.5 \\
64 \\
58 \\
2.4\end{array}$ & $\begin{array}{r}1,200 \\
2,100 \\
660 \\
1,300\end{array}$ & $\begin{array}{r}<10 \\
5,500 \\
<10 \\
<10\end{array}$ & $\begin{array}{l}58 \\
20 \\
49 \\
45\end{array}$ & $\begin{array}{l}<1.0 \\
59 \\
<1.0 \\
<1.0\end{array}$ & $\begin{array}{r}150 \\
520 \\
21 \\
12\end{array}$ & $\begin{array}{l}<1.0 \\
200 \\
<1.0 \\
8.0\end{array}$ & $\begin{array}{l}24 \\
47 \\
90 \\
91\end{array}$ & $\begin{array}{c}<1.0 \\
4,000 \\
35 \\
<1.0\end{array}$ & $\begin{array}{r}49 \\
280 \\
31 \\
32\end{array}$ \\
\hline
\end{tabular}


Table 27.--Water-quality information from Cow Camp Creek batch-mixing samples collected after 90 days of water-rock contact--Continued

\begin{tabular}{|c|c|c|c|c|c|c|c|c|}
\hline $\begin{array}{c}\text { LITHO- } \\
\text { LOGIC } \\
\text { UNIT } \\
\text { (SEE } \\
\text { FIGURE } \\
28 \text { ) }\end{array}$ & $\begin{array}{l}\text { LEAD, } \\
\text { DIS- } \\
\text { SOLVED } \\
(\mu G / L \\
\text { AS PB) }\end{array}$ & $\begin{array}{l}\text { LITHIUM, } \\
\text { DIS- } \\
\text { SOLVED } \\
\text { ( } \mu G / L \\
\text { AS LI) }\end{array}$ & $\begin{array}{l}\text { MANGA- } \\
\text { NESE, } \\
\text { DIS- } \\
\text { SOLVED } \\
(\mu G / L \\
\text { AS MN) }\end{array}$ & $\begin{array}{l}\text { MOLYB- } \\
\text { DENUM, } \\
\text { DIS- } \\
\text { SOLVED } \\
(\mu G / L \\
\text { AS MO) }\end{array}$ & $\begin{array}{l}\text { NICKEL, } \\
\text { DIS- } \\
\text { SOLVED } \\
(\mu G / L \\
\text { AS NI) }\end{array}$ & $\begin{array}{l}\text { STRONT- } \\
\text { TIUM, } \\
\text { DIS- } \\
\text { SOLVED } \\
(\mu G / L \\
\text { AS SR) }\end{array}$ & $\begin{array}{l}\text { VANA- } \\
\text { DIUM, } \\
\text { DIS- } \\
\text { SOLVED } \\
(\mu G / L \\
\text { AS V) }\end{array}$ & $\begin{array}{l}\text { ZINC, } \\
\text { DIS- } \\
\text { SOLVED } \\
(\mu G / L \\
\text { AS ZN) }\end{array}$ \\
\hline $\begin{array}{l}\text { SS01 } \\
\text { CL02 } \\
\text { SS03 } \\
\text { SH04 }\end{array}$ & $\begin{array}{l}<1.0 \\
71 \\
<1.0 \\
170\end{array}$ & $\begin{array}{r}240 \\
56 \\
59 \\
160\end{array}$ & $\begin{array}{r}63,000 \\
830 \\
28 \\
320\end{array}$ & $\begin{array}{l}<10 \\
<10 \\
<10 \\
<10\end{array}$ & $\begin{array}{r}2,400 \\
500 \\
110 \\
240\end{array}$ & $\begin{array}{r}300 \\
450 \\
1,000 \\
3,200\end{array}$ & $\begin{array}{c}510 \\
360 \\
9.0 \\
27\end{array}$ & $\begin{array}{r}8,300 \\
480 \\
69 \\
59\end{array}$ \\
\hline $\begin{array}{l}\text { SS05 } \\
\text { SS06 } \\
\text { SH07 } \\
\text { SS08 }\end{array}$ & $\begin{array}{l}<1.0 \\
33 \\
<1.0 \\
260\end{array}$ & $\begin{array}{r}76 \\
68 \\
110 \\
67\end{array}$ & $\begin{array}{r}480 \\
100 \\
64 \\
46\end{array}$ & $\begin{array}{l}<10 \\
<10 \\
<10 \\
<10\end{array}$ & $\begin{array}{c}140 \\
4.0 \\
120 \\
63\end{array}$ & $\begin{array}{r}2,500 \\
1,500 \\
780 \\
1,000\end{array}$ & $\begin{array}{l}25 \\
<1.0 \\
29 \\
<1.0\end{array}$ & $\begin{array}{r}120 \\
63 \\
85 \\
90\end{array}$ \\
\hline $\begin{array}{l}\text { SH09 } \\
\text { CL10 } \\
\text { SS12 } \\
\text { SS13 }\end{array}$ & $\begin{array}{l}<1.0 \\
<1.0 \\
<1.0 \\
24\end{array}$ & $\begin{array}{r}230 \\
54 \\
84 \\
91\end{array}$ & $\begin{array}{c}1,700 \\
3.0 \\
140 \\
48\end{array}$ & $\begin{array}{l}<10 \\
<10 \\
<10 \\
<10\end{array}$ & $\begin{array}{r}130 \\
160 \\
180 \\
21\end{array}$ & $\begin{array}{r}8,900 \\
280 \\
640 \\
610\end{array}$ & $\begin{array}{r}19 \\
23 \\
2.0 \\
<1.0\end{array}$ & $\begin{array}{r}150 \\
130 \\
45 \\
51\end{array}$ \\
\hline $\begin{array}{l}\text { SH14 } \\
\text { SH16 } \\
\text { SS17 } \\
\text { SH18 }\end{array}$ & $\begin{array}{l}<1.0 \\
180 \\
<1.0 \\
130\end{array}$ & $\begin{array}{r}160 \\
200 \\
71 \\
200\end{array}$ & $\begin{array}{r}67 \\
290 \\
65 \\
290\end{array}$ & $\begin{array}{r}120 \\
<10 \\
49 \\
34\end{array}$ & $\begin{array}{r}62 \\
4,300 \\
100 \\
190\end{array}$ & $\begin{array}{r}630 \\
4,200 \\
1,100 \\
750\end{array}$ & $\begin{array}{l}<1.0 \\
92 \\
17 \\
25\end{array}$ & $\begin{array}{r}120 \\
13,000 \\
85 \\
63\end{array}$ \\
\hline
\end{tabular}

${ }^{1}$ Lithologic units are identified as follows: the first two letters indicate lithology (SS, sandstone; CL, coal; SH, shale), the last two numbers indicate relative depth ( 1 is shallowest, 18 is deepest). 